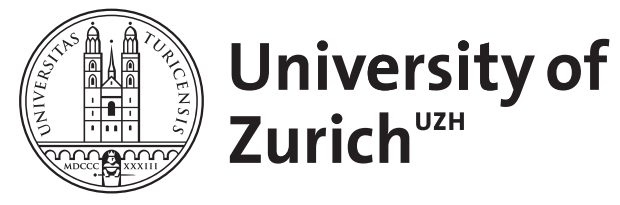

Zurich Open Repository and Archive

University of Zurich

Main Library

Strickhofstrasse 39

CH-8057 Zurich

www.zora.uzh.ch

Year: 2014

Angehörige von drogenabhängigen Menschen : Suchterkrankungen aus einer anderen Perspektive

Ruckstuhl, Lea Anna

Posted at the Zurich Open Repository and Archive, University of Zurich

ZORA URL: https://www.zora.uzh.ch/101639

Dissertation

Published Version

Originally published at:

Ruckstuhl, Lea Anna. Angehörige von drogenabhängigen Menschen : Suchterkrankungen aus einer anderen Perspektive. 2014, Zürich, Faculty of Arts. 


\title{
Angehörige von drogenabhängigen Menschen - Suchterkrankungen aus einer anderen Perspektive
}

\author{
Abhandlung \\ zur Erlangung der Doktorwürde \\ der Philosophischen Fakultät \\ der Universität Zürich
}

vorgelegt von

Lea Anna Ruckstuhl

\begin{abstract}
Angenommen im Frühlingssemester 2014
auf Antrag der Promotionskommission:
\end{abstract}

Prof. Dr. med. Hans-Joachim Haug (Hauptverantwortliche Betreuungsperson)

PD Dr. med. Rudolf Stohler

Zürich, 2014 
gewidmet

meinem Vater

Vinzenz Ruckstuhl 
„Wir wir haben alle das Recht auf das Leben (lacht).“

Dorothea, ${ }^{1}$ jüngere Schwester eines heroinabhängigen Mannes

\footnotetext{
${ }^{1}$ Name der Studienleitung bekannt
} 


\section{Inhaltsverzeichnis}

Vorwort

Dank 8

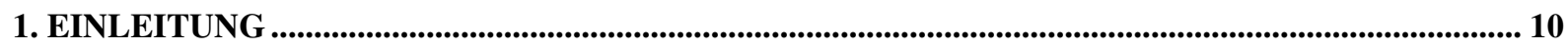

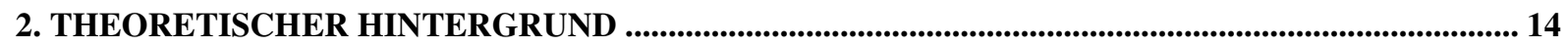

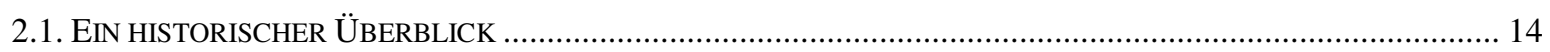

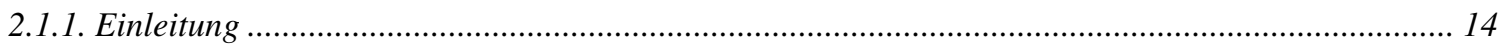

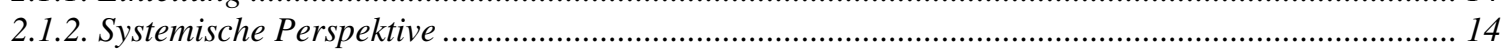

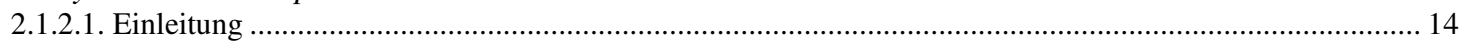

2.1.2.2. Die Rolle der Angehörigen in den Anfängen................................................................................... 14

2.1.2.3. Glaubenssysteme und Rollenverteilungen in suchtbelasteten Familien ...................................................... 15

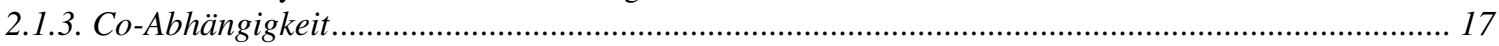

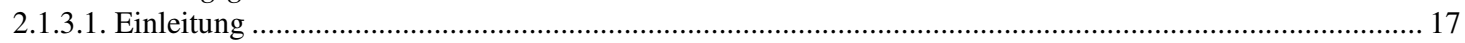

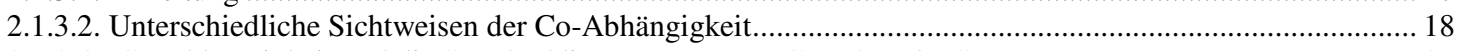

2.1.3.4. Co-Abhängigkeit und die Standarddiagnosesysteme ICD-10 und DSM-IV-TR ........................................... 21

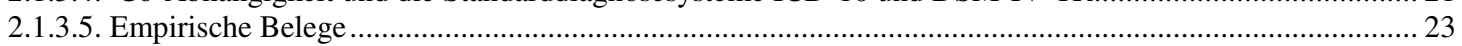

2.2. BELASTUNGSFAKTOREN UND AUSWIRKUNGEN DER SUCHTERKRANKUNG AUF DIE ANGEHÖRIGEN ............ 24

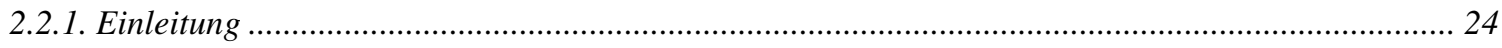

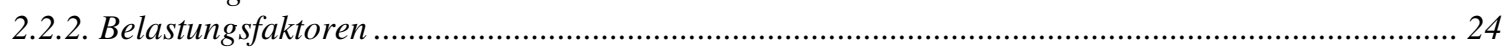

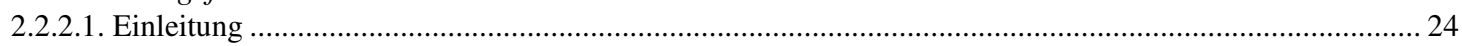

2.2.2.2. Ergebnisse einer qualitativen Studie in England und Mexiko........................................................24

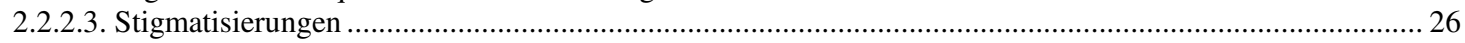

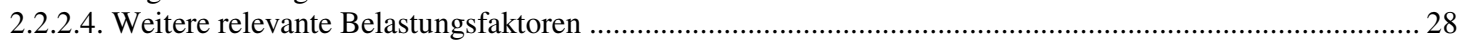

2.2.2.5. Die „Significant Other Checklist“ zur Erfassung der Belastung .............................................................. 29

2.2.3. Auswirkungen der Suchterkrankung auf die Angehörigen ............................................................. 32

2.2.3.1. Einleitung ……………………………………………………………………………………… 32

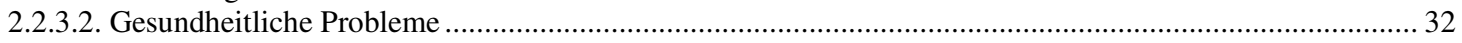

2.2.3.3. Auswirkungen auf das soziale Funktionsniveau der Angehörigen ............................................................. 34

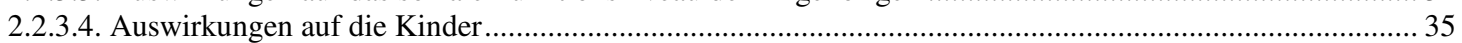

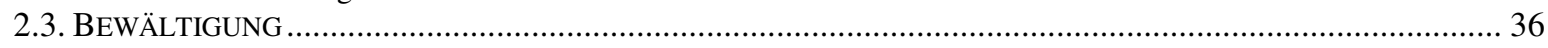

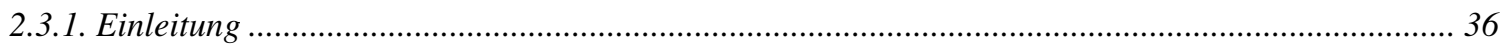

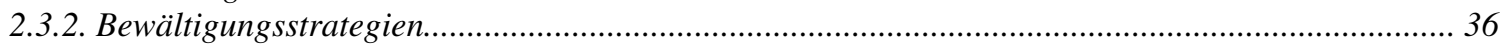

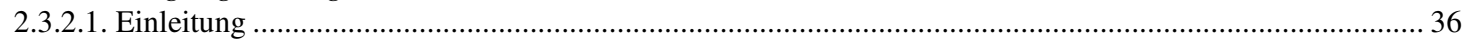

2.3.2.2. Empirisch belegte Copingstruktur ……………………………………………………………….... 37

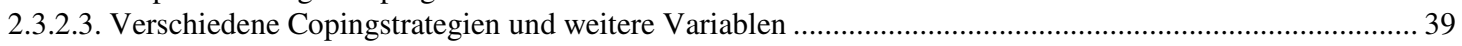

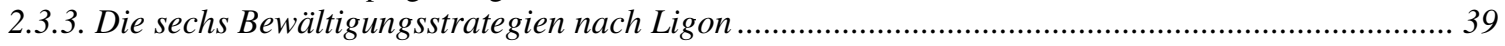

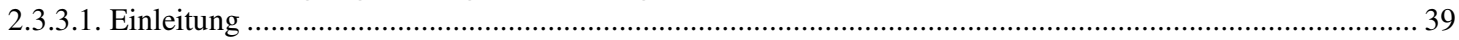

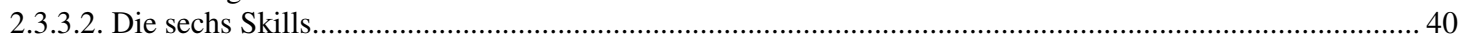

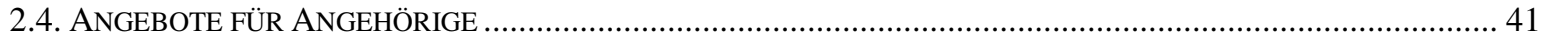

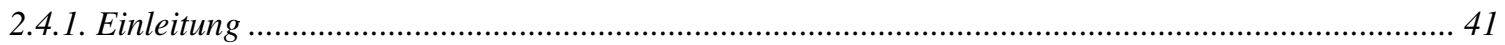

2.4.2. Al-Anon .

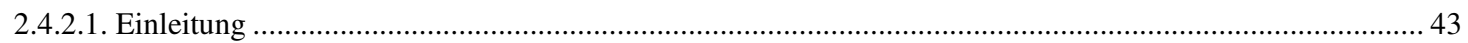

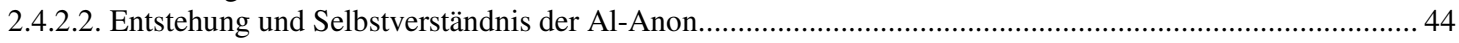

2.4.2.3. Ablauf der Meetings und die 12 Schritte und Traditionen .................................................................. 45

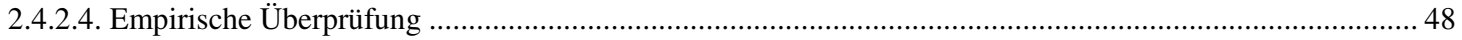

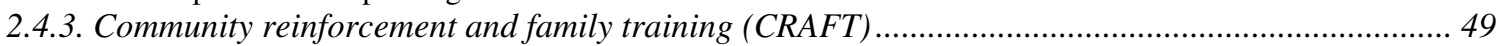

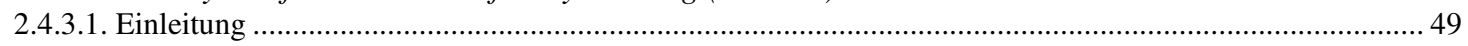

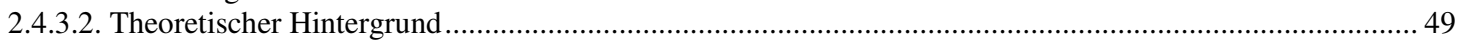

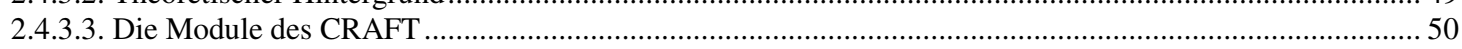

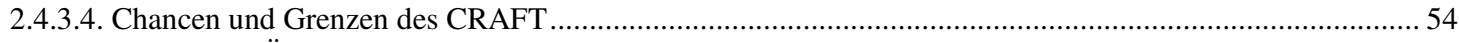

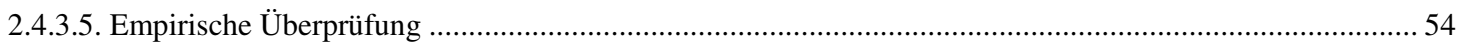

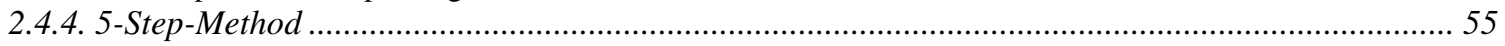

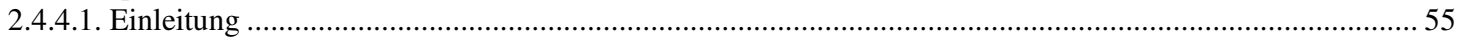

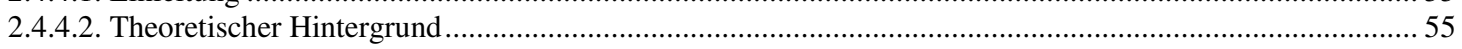

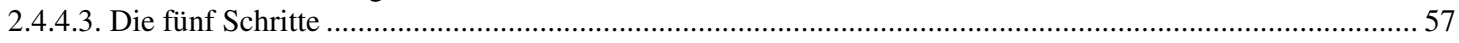

2.4.4.4. Chancen und Risiken der 5-Step-Method ........................................................................................59

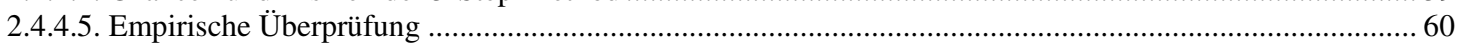

2.4.5. Angehörigenvereinigung Drogenabhängiger Zürich (ada-zh) und Verband der Eltern- und

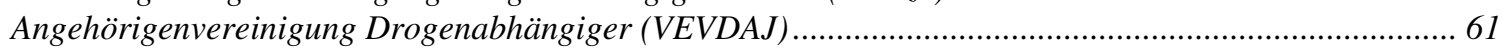

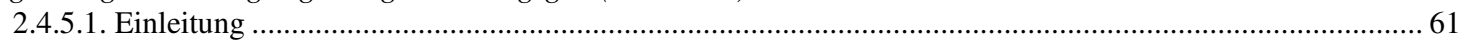

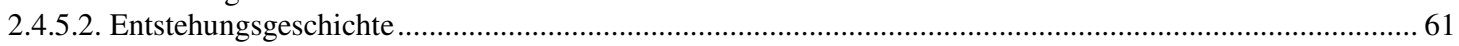


2.4.5.3. VEVDAJ - heute

2.4.5.4. Angehörigenvereinigung Drogenabhängiger Zürich (ada-zh) - heute...

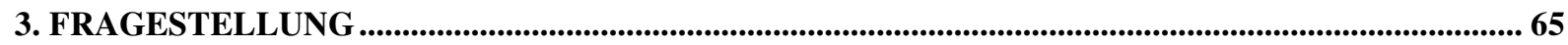

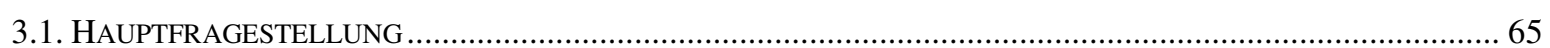

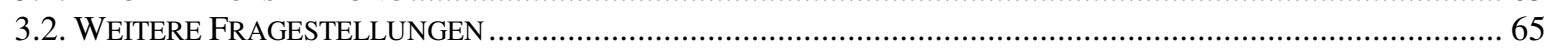

3.2.1. Charakteristika von Angehörigen von drogenabhängigen Menschen ......................................... 65

3.2.2. Belastung durch die Suchterkrankung und deren Auswirkungen ............................................... 65

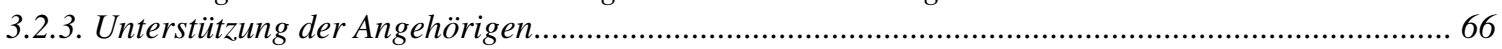

3.2.4. Typische Gruppen von Angehörigen aufgrund der erlebten Belastung ......................................... 66

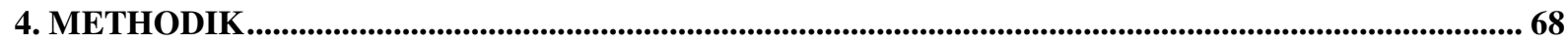

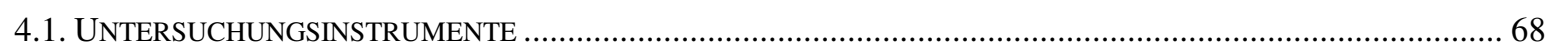

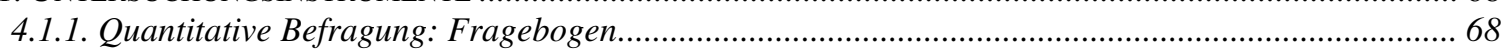

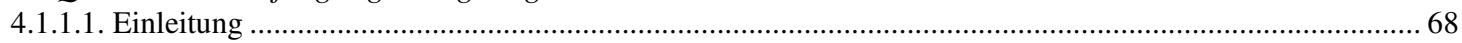

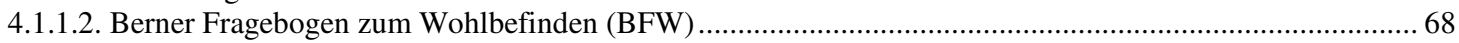

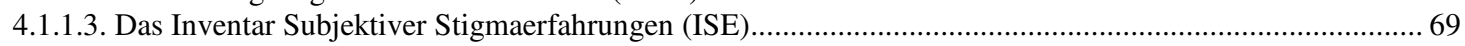

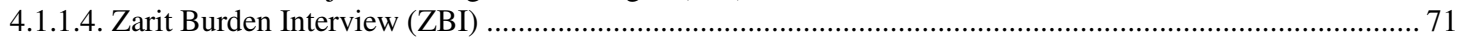

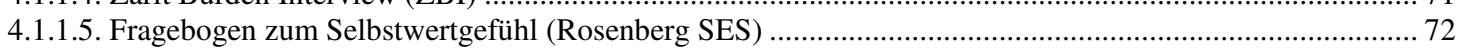

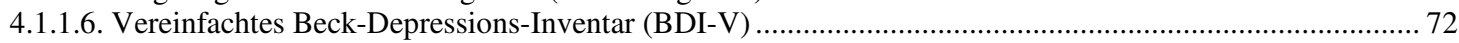

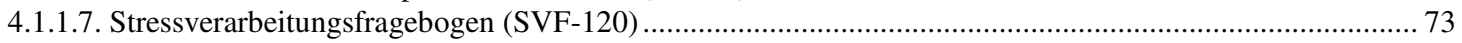

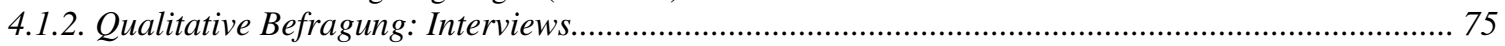

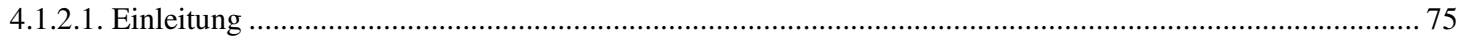

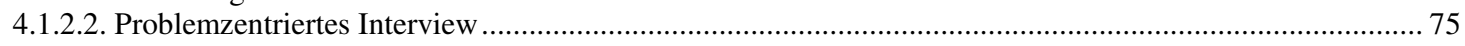

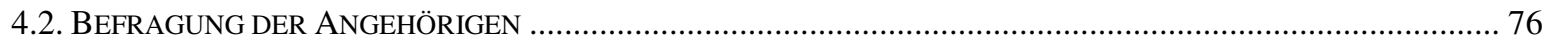

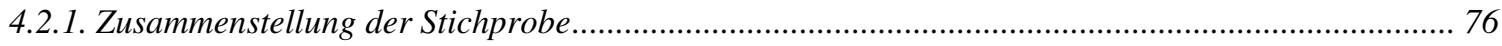

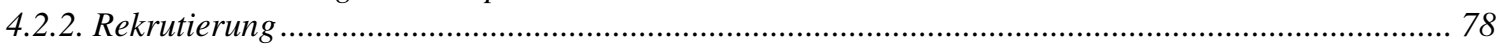

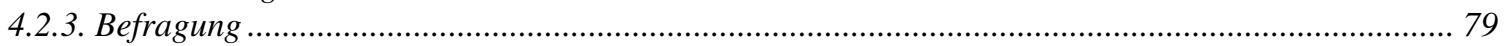

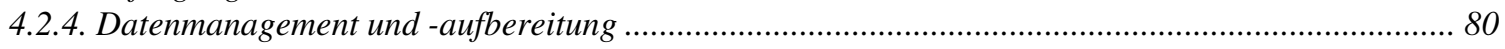

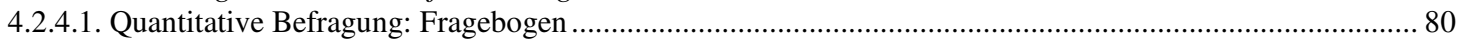

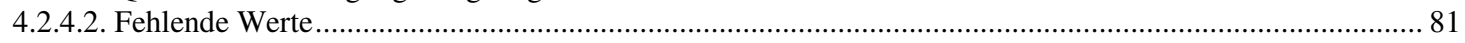

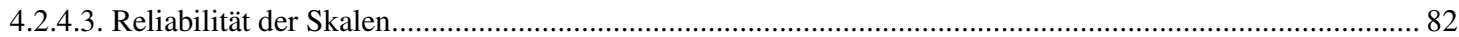

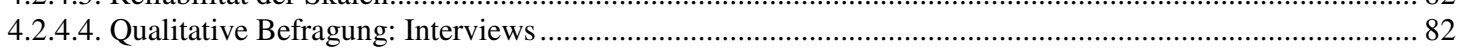

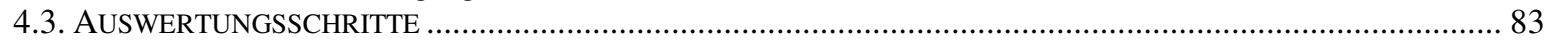

4.3.1. Inventar Subjektiver Stigmaerfahrungen (ISE) ........................................................... 83

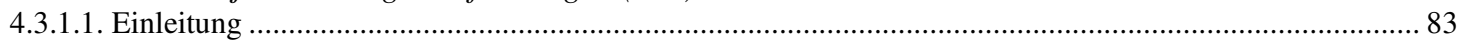

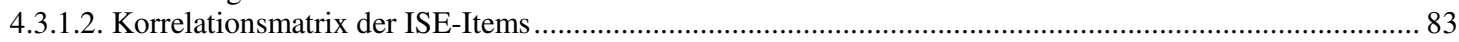

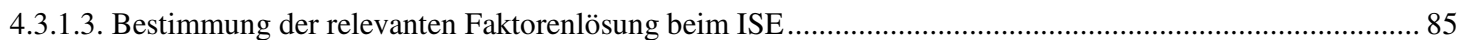

4.3.2. Auswirkungen der Suchterkrankung auf die Angehörigen ........................................................ 87

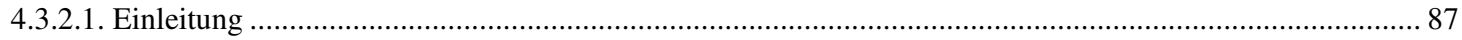

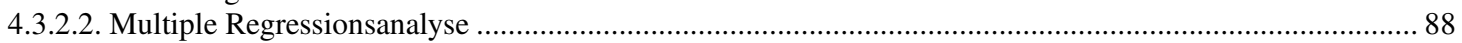

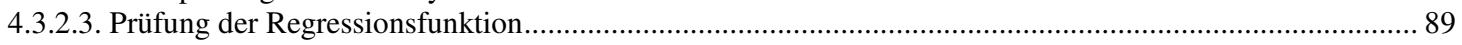

4.3.3. Typische Gruppen von Angehörigen aufgrund der Belastung ................................................ 90

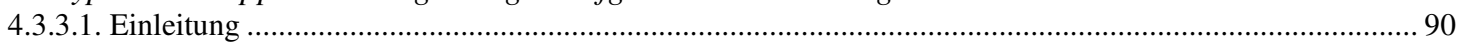

4.3.3.2. Bestimmung der optimalen Clusterlösung ...................................................................................... 91

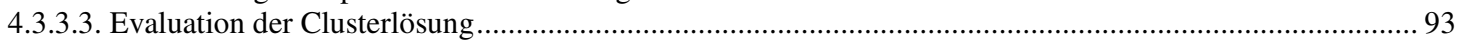

4.3.3.4. Bezug der Clusterlösung zu weiteren Variablen (logistische Regression) ............................................... 94

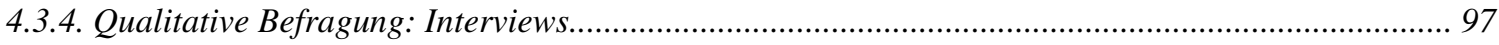

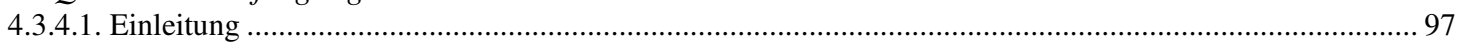

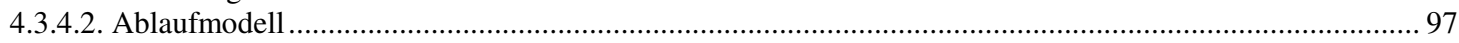

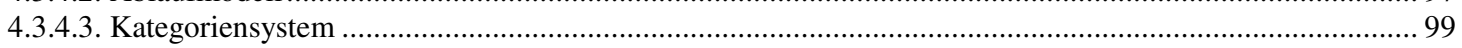

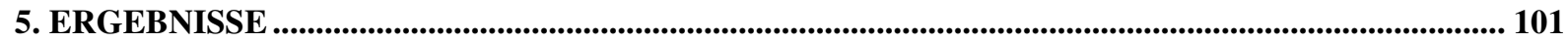

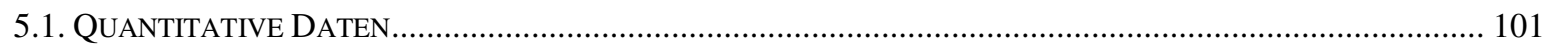

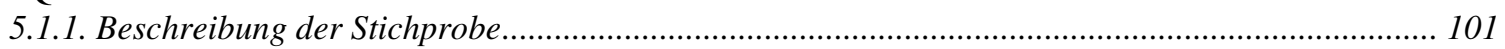

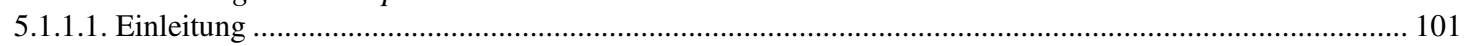

5.1.1.2. Beschreibung der Angehörigen (Studienteilnehmende)............................................................. 101

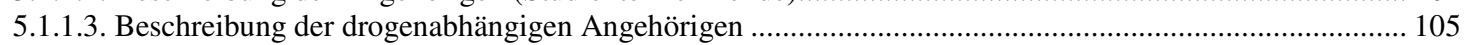

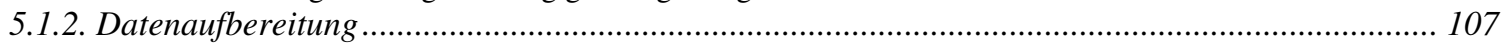

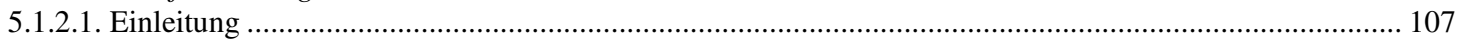

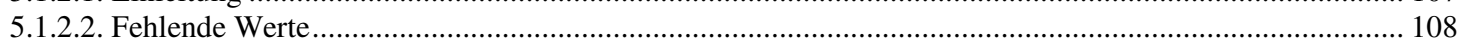

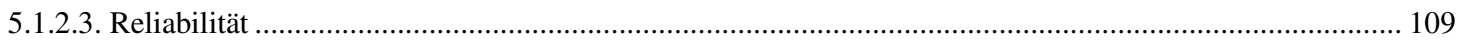

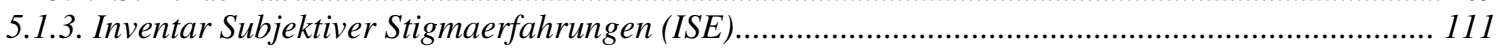

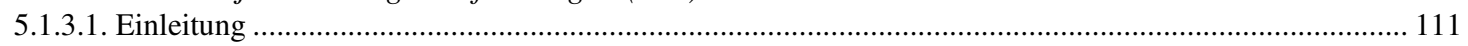

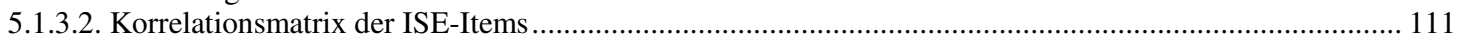




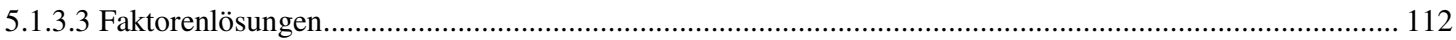

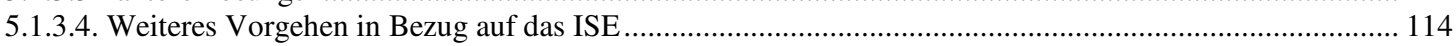

5.1.4. Auswirkungen der Suchterkrankung auf die Angehörigen ..................................................... 114

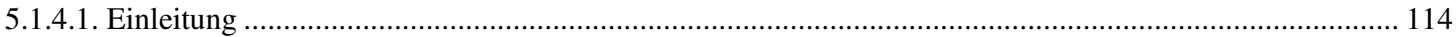

5.1.4.2. Belastung und psychische Gesundheit .............................................................................. 114

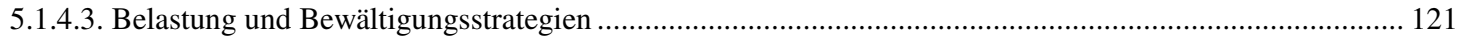

5.1.5. Verschiedene Gruppen von Angehörigen aufgrund der Belastung.............................................. 124

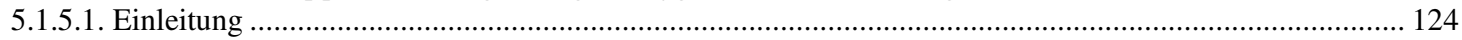

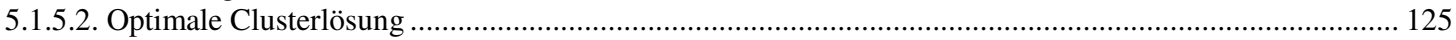

5.1.5.3. Evaluation der Clusterlösung mittels Diskriminanzanalyse .............................................................. 129

5.1.5.4. Bezug der Zwei-Clusterlösung zu anderen Variablen (logistische Regression)..................................... 131

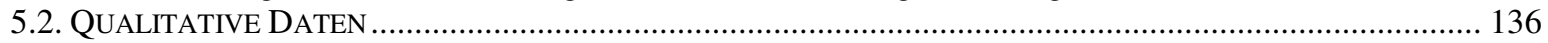

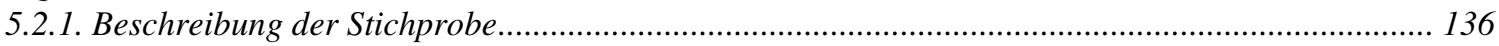

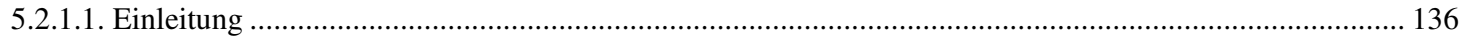

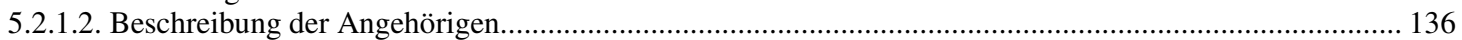

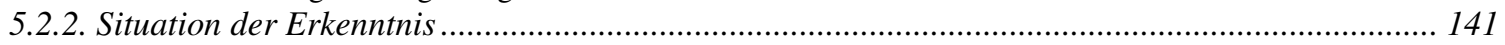

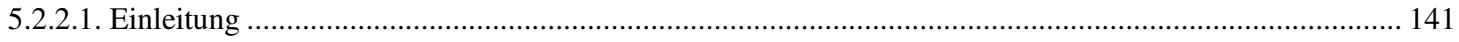

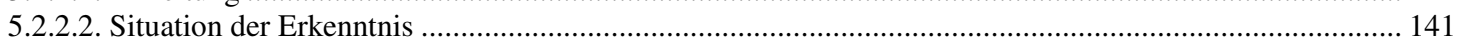

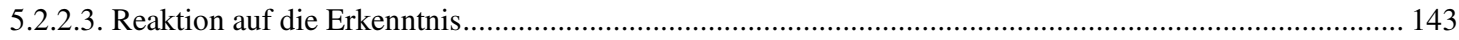

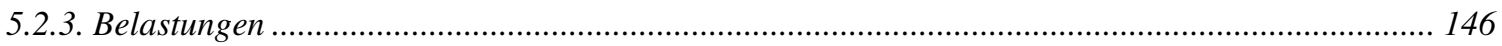

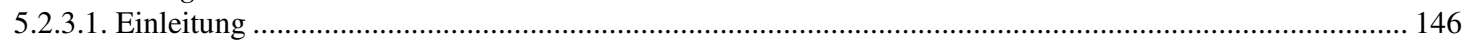

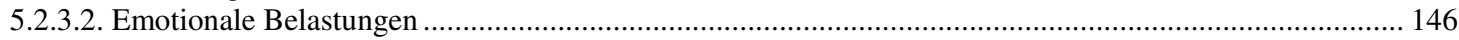

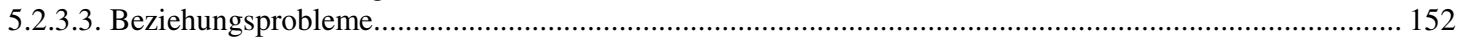

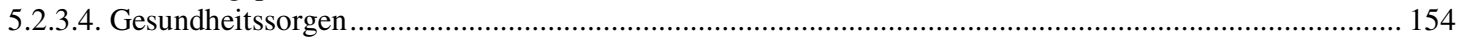

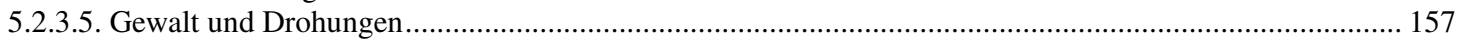

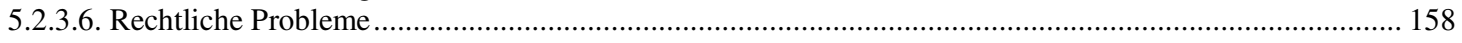

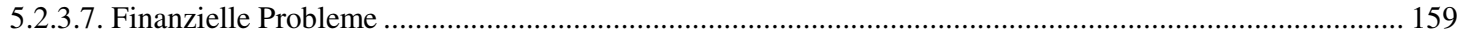

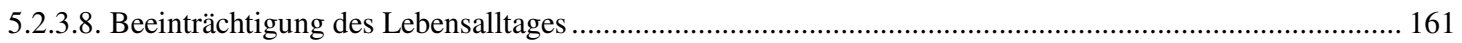

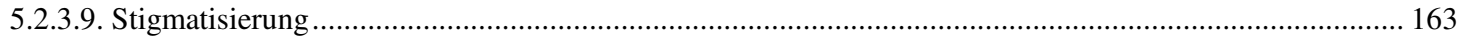

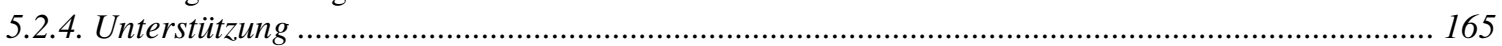

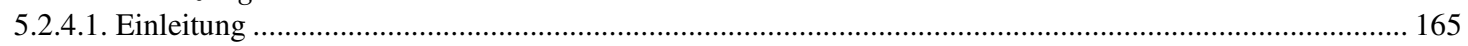

5.2.4.2. Erhaltene Unterstützung, die positiv beurteilt wird ...................................................................... 166

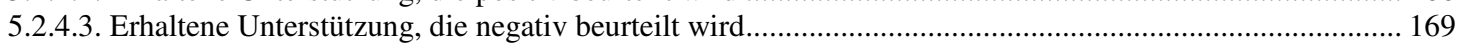

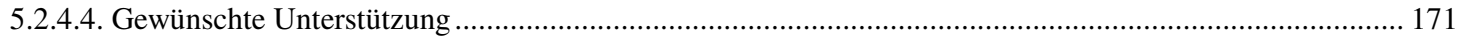

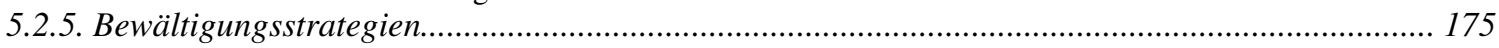

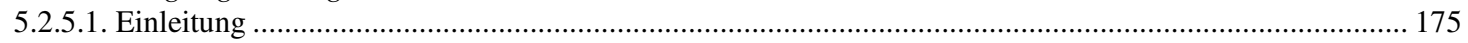

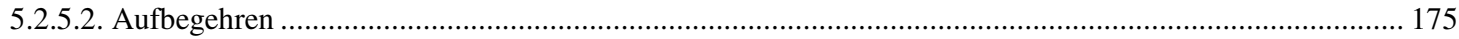

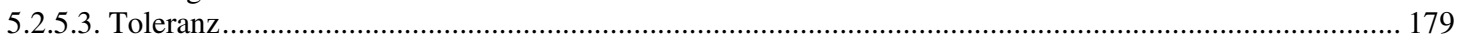

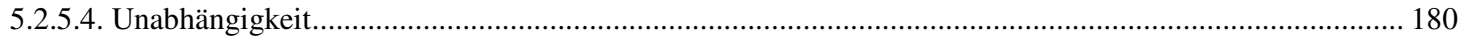

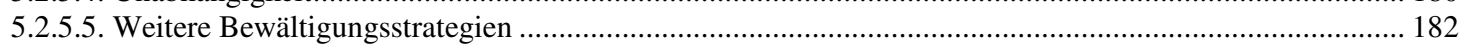

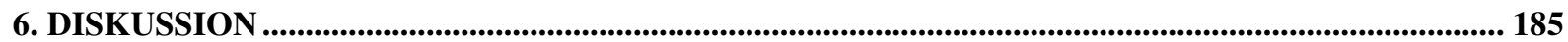

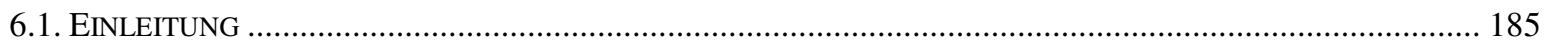

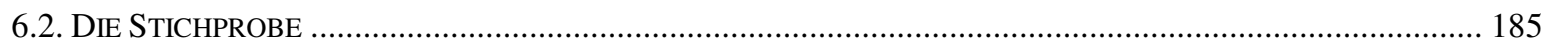

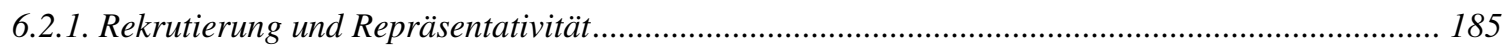

6.2.2. Angehörige und die Schweizer Durchschnittbevölkerung .................................................... 187

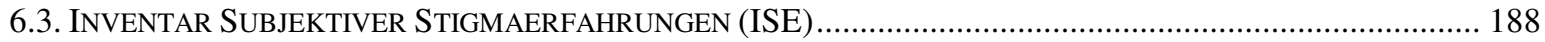

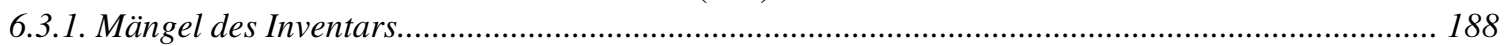

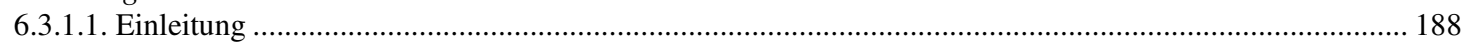

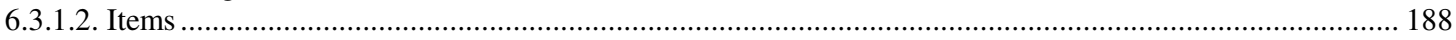

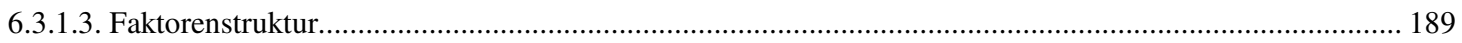

6.3.2. Bedeutung von Stigmatisierung für die Angehörigen .......................................................... 190

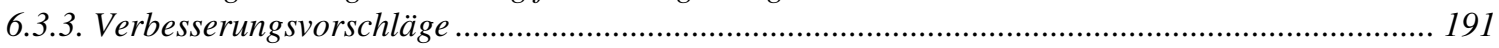

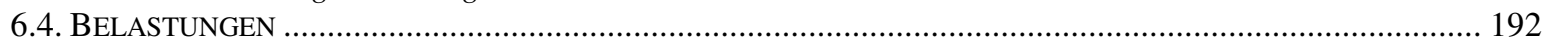

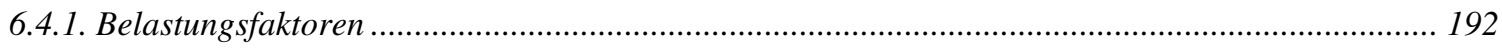

6.4.2. Auswirkungen der Belastungsfaktoren ......................................................................... 197

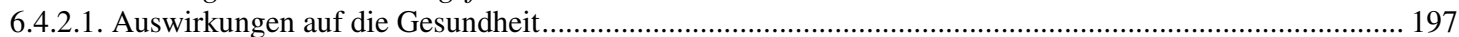

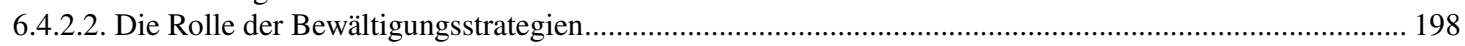

6.4.2.3. Charakterisierung von stark belasteten Angehörigen .................................................................... 200

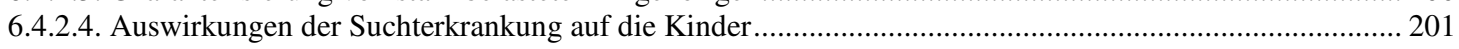

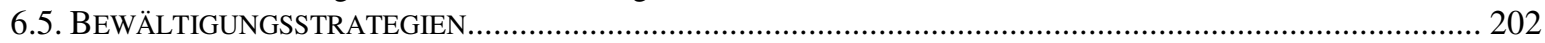

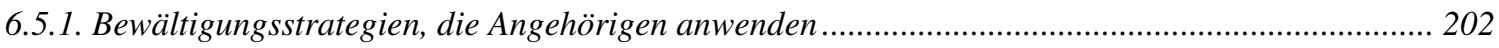

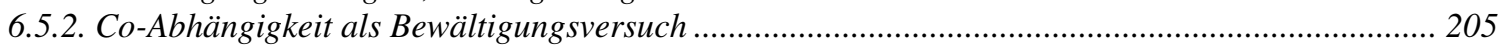

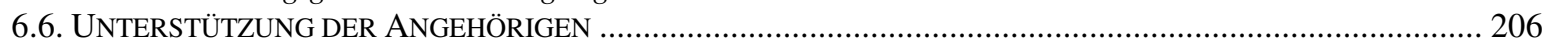

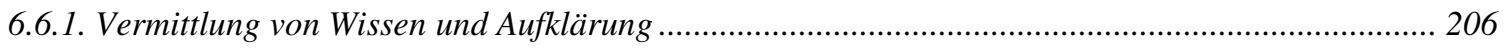


6.6.2. Die Bedeutung von sozialer Unterstützung...................................................................... 207

6.6.3. Therapie des suchtkranken Angehörigen............................................................................. 208

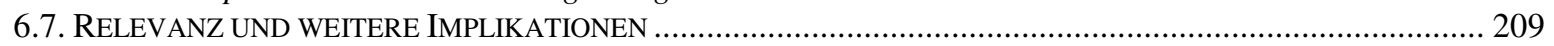

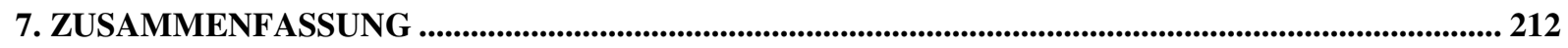

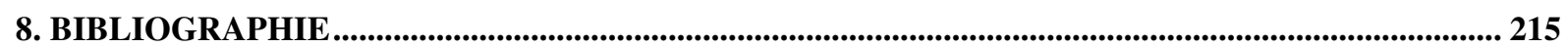

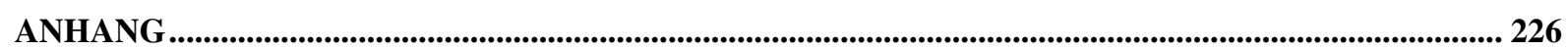

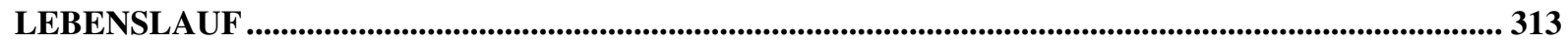




\section{Vorwort}

Im Alter von acht oder neun Jahren kam ich zum ersten Mal in einer Art und Weise mit dem Thema Suchterkrankungen in Berührung, die mich und mein Leben geprägt haben. In jener Zeit existierte die offene Drogenszene auf dem Platzspitz, dem „Needle-Park“, noch. Wir hatten mit der Familie einen Ausflug ins Landesmuseum Zürich gemacht, als ich dort ein Fenster aufmachte, das sich zum Platzspitz hin öffnete. Das Elend, das mir von dort entgegenschlug versetzte mir den Schock meines bis dahin kurzen Lebens. Ich empfand eine unglaubliche Angst und Hilflosigkeit gegenüber dieser Welt der Drogen, die ich bis anhin nicht gekannt hatte. In diesem Moment wurde Sucht für mich zum Schrecklichsten, was einem widerfahren kann und ich hatte in der Folge Angst, dass Menschen, die ich liebe drogenabhängig werden könnten.

Als es dann, viele Jahre später, um die Abschlussarbeit meines Psychologiestudiums ging, bin ich per Zufall - wenn man an solche Zufälle glauben mag - beim Thema Eltern von drogenabhängigen Söhnen gelandet. Mit Überraschung stellte ich damals fest, dass es im deutschsprachigen Raum kaum Forschung zu Angehörigen von suchtkranken Menschen gab. Ein Umstand, der mir unverständlich und inakzeptabel schien, aufgrund der Tatsache, dass diverse Studien im englischsprachigen Raum zeigten, dass Angehörige ein grosses Risiko für gesundheitliche Probleme tragen, die im Zusammenhang mit den Belastungen durch eine Suchterkrankung entstehen.

Aufgrund meines eigenen prägenden Erlebnisses mit der offenen Drogenszene am Platzspitz interessierte mich seither die Frage, wie Angehörige mit einer solchen Belastung umgehen. In dieser Arbeit wollte ich deshalb der Frage nachgehen, welche Belastungen Angehörige in der Schweiz im Zusammenhang mit der Suchterkrankung eines ihrer Familienmitglieder erleben, wie sie mit diesen Belastungen umgehen und welche Auswirkungen dies auf ihre Gesundheit haben kann. Zudem wollte ich in Erfahrungen bringen, wie Angehörige in der Schweiz unterstützt werden und welche weiteren Angebote sie sich für sich selbst wünschen würden. Die vorliegende Arbeit soll einen Beitrag dazu leisten, dass die Situation der Angehörigen mehr beachtet und besser verstanden wird. 


\section{Dank}

Als erstes bedanke ich mich bei meinem Lebenspartner Peter Keller, der mich in vielerlei Hinsicht beim Realisieren der Arbeit unterstützt hat und häufig auch zugunsten dieser zurückgestanden ist. In diesem Sinne geht auch ein Dank an meine Familie und meine Freunde.

Der nächste Dank geht an die beiden Referenten dieser Arbeit: Herrn PD Dr. med Rudolf Stohler, dem ehemaligen Leitenden Arzt des Zentrums für Abhängigkeitserkrankungen der Psychiatrischen Universitätsklinik Zürich und Prof. Dr. med. Hans-Joachim Haug, dem Ordinarius für Psychiatrie, speziell Psychopathologie und psychiatrische Diagnostik an der Universität Zürich und Ärztlichen Direktor der Clienia Gruppe. Sie beide zeigten Interesse für das Projekt und waren bereit, es zu begleiten. Ein besonderer Dank geht an Dr. phil. Lukas Boesch, dem wissenschaftlichen und klinischen Mitarbeiter im Zentrum für Abhängigkeitserkrankungen, der mit seinen kritischen und motivierenden Anmerkungen und seiner Unterstützung bei statistischen Fragen sehr zum Gelingen der Arbeit beigetragen hat. Ein herzlicher Dank geht an lic. phil. Monika Ambauen Pfarrer, Psychologin, die die Arbeit kurzfristig und im Eilzugtempo lektoriert hat. An dieser Stelle bedanke ich mich zudem bei Dr. phil. Armin Krauer, der mich sehr zum Projekt ermutigt hat.

Ein weiterer Dank geht an die Mitarbeiterinnen und Mitarbeiter des Zentrums für Abhängigkeitserkrankung, die bei der Rekrutierung der Angehörigen mitgeholfen haben. In diesem Zusammenhang geht auch ein grosser Dank an die Mitarbeiterinnen und Mitarbeiter der Angehörigenvereinigung Drogenabhängiger Zürich (ada-zh), die beim Rekrutieren von Angehörigen grosses Engagement zeigten. Zudem bedanke ich mich auch beim Präsidenten des VEVDAJ, Herrn Josef Baumgartner, der auf der Homepage des Dachverbandes einen Aufruf für die Studie schaltete. Ein Dank gilt auch den Mitarbeiterinnen und Mitarbeitern der Pendlerzeitung 20minuten und der NZZ, die gratis Werbung für die Studie machten und somit massgeblich dazu beitrugen, dass genügend Angehörige für die Befragung gewonnen werden konnten.

Ein grosser Dank geht nicht zuletzt an meine aktuelle Arbeitgeberin, die Beratungsstelle für Alkoholprobleme Bezirk Hinwil (BAH), die mich beim Fertigstellen der Arbeit sehr unterstützt hat, indem sie mir eine längere Auszeit ermöglichte. 
Schliesslich bedanke ich mich bei allen Angehörigen, die sich unentgeltlich zur Teilnahme an der Studie bereit erklärt hatten und ohne deren grossen Einsatz, das Projekt nicht möglich gewesen wäre. 


\section{Einleitung}

Den Angehörigen von suchtkranken Menschen wird in der Forschung im deutschsprachigen Raum erst seit kurzem etwas mehr Beachtung geschenkt. Dies ist umso erstaunlicher, wenn man bedenkt, dass Angehörige in der Vergangenheit oft nicht nur für die Entstehung der Sucht, sondern auch für deren Aufrechterhaltung verantwortlich gemacht wurden (Uhl \& Puhm, 2007, S. 14). Dabei ist völlig unklar, wie viele Menschen durch die Suchterkrankung eines nahestehenden Menschen überhaupt betroffen sind und welche Kosten für sie und die Gesellschaft durch ihre Mitbetroffenheit entstehen (Copello, Templeton \& Powell, 2010, S. 64). Nichtsdestotrotz wurden verschiedentlich Versuche unternommen, die Zahl der Angehörigen zu schätzen. Konservative Schätzungen gehen davon aus, dass mindestens noch zwei weitere Personen von der Suchterkrankung mitbetroffen sind (Copello, Orford, Velleman, Templeton \& Krishnan, 2000, S. 329). In der Schweiz weisen ca. 328'000 Menschen (4.1\% der Schweizer Bevölkerung) einen chronisch risikoreichen Alkoholkonsum auf, das heisst bei Männern ein Konsum von Reinalkohol > 40 g/Tag und bei Frauen > 20 g/Tag (Gmel, Kuendig, Notari, Gmel \& Flury, 2013, S. 2). Geschätzt wird, dass ca. 250'000 unter ihnen auch abhängig sind (http://www.suchtmonitoring.ch/de.html). Für Abhängigkeit oder Risikokonsum bei den illegalen Drogen ist es dagegen äusserst schwierig, aktuelle verlässliche Zahlen zu finden, weil illegaler Substanzkonsum sozial unerwünscht ist (ebd.). Es ist deshalb davon auszugehen, dass in Umfragen nicht alle ihren Konsum angeben. Im Jahr 2009 machte die damalige Schweizerische Fachstelle für Alkohol- und andere Drogenprobleme noch eine Schätzung, dass nämlich in der Schweiz ca. 60'000 Menschen illegale Drogen konsumieren würden, wovon etwa die Hälfte eine Abhängigkeit aufweisen würden (zit. nach Candrian \& Ruckstuhl, 2009, S. 2). Wird je nach Berechungsgrundlage daraus die Zahl der durch die Sucht Mitbetroffenen berechnet, so ist davon auszugehen, dass in der Schweiz zwischen 500'000 und 800'000 Menschen Angehörige von jemandem mit einem abhängigen oder problematischen Substanzkonsum sind. Wobei mit grosser Wahrscheinlichkeit davon ausgegangen werden muss, dass dieser konservative Schätzwert mit nur zwei Angehörigen pro Betroffenem unter Berücksichtigung der Tatsache, dass eine suchtkranke Person Eltern, Geschwister und eventuell noch einen Partner und Kinder hat, die Realität deutlich unterschätzt. Es ist also anzunehmen, dass eine erheblich grössere Zahl von Angehörigen unter der Suchterkrankung eines nahestehenden Menschen zu leiden hat.

Das Thema „Angehörige von suchtkranken Menschen“ verdient aber in weiterer Hinsicht mehr Beachtung: Copello, Templeton und Powell (2010) betonen, dass Angehörige eines 
suchtkranken Menschen diesen in vielfacher Weise unterstützen und dadurch in unserer Gesellschaft einen erheblichen Beitrag zur Behandlung und zur Deckung von entstehenden Kosten von suchtkranken Menschen leisten (ebd., S. 67). Über die effektiven Kosten der Suchterkrankungen für unsere Gesellschaft zusammen mit dem Beitrag, den Angehörige dazu leisten, ist nur wenig bekannt. So unterstützen Angehörige ihr drogenabhängiges Familienmitglied oft finanziell beim Lebensunterhalt, geben manchmal unbeabsichtigt Geld für Drogen oder erleben Verluste durch den Diebstahl von Geld oder Wertgegenständen (Copello, Templeton \& Powell, 2009; zit. nach Copello, Templeton \& Powell, 2010, S. 69). Dazu kommt, dass Angehörige meist in Krisensituationen für den Suchtkranken da sind, was sie in ihrer wirtschaftlichen Produktivität einschränken kann (ebd.). Durch die Unterstützung in Sorge und Pflege, die Angehörige erbringen, können gleichzeitig Ressourcen in der Gesellschaft und im Gesundheitswesen gespart werden, die sonst zur Verfügung gestellt werden müssten.

Neben der finanziellen Last kommen eine Vielzahl weiterer Belastungen für die Angehörigen dazu (Kirby, Dugosh, Benishek \& Harrington, 2005; Orford, Natera, Davies, Nava, Mora, Rigby et al., 1998c). Diese führen dazu, dass sich das Risiko für die Angehörigen erhöht, selbst psychisch oder psychosomatisch krank zu werden (Lee, Manning, Teoh, Winslow, Lee, Subramaniam et al., 2011; Oreo \& Ozgul, 2007; Orford et al., 1998c; Ray, Mertens \& Weisner, 2007). So weisen Angehörige ein höheres Risiko auf, eine depressive Störung oder selbst eine Abhängigkeitserkrankung zu entwickeln (Lee et al., 2011; Ray et al., 2007). Dazu kommen somatische Beschwerden wie Verspannungen, Bluthochdruck, Kopfschmerzen oder Verdauungsbeschwerden, um nur einige zu nennen (Orford et al., 1998c; Ray et al., 2007). Kolitzus (2006, S. 11) betont in diesem Zusammenhang, dass eine grosse Anzahl Angehöriger wegen psychosomatischen Beschwerden in Behandlung ist, ohne dass diese als Angehörige von suchtkranken Menschen erkannt werden. Durch die gesundheitlichen Probleme der Angehörigen fallen sowohl für die Betroffenen aber auch für die Gesellschaft weitere Kosten an (Ray et al., 2007).

Ein weiterer gewichtiger Belastungsfaktor ist, dass Angehörige aufgrund des chronischen Verlaufs der meisten Abhängigkeitserkrankungen (Sass, op. 2003, S. 246) meist jahrelang betroffen sind. Dabei bleiben sie oft mehrere Jahre ohne Unterstützung. In einer qualitativen Untersuchung gaben die befragten Angehörigen an, dass die Intervention, die im Rahmen der Befragung evaluiert wurde, zu einem früheren Zeitpunkt hilfreicher gewesen wäre (Orford, 
Templeton, Patel, Copello \& Velleman, 2007). Wenn berücksichtigt wird, dass die Studienteilnehmenden durchschnittlich bereits zwischen acht und neun Jahren von der Suchterkrankung ihres Angehörigen betroffen waren, als sie für die Intervention in die Studie aufgenommen wurden (Copello, Templeton, Orford, Velleman, Patel, Moor et al., 2009, S. 54), ist dies nicht erstaunlich. Es ist dringend notwendig, dass Fachpersonen aber auch die Bevölkerung besser über die Situation der Angehörigen aufgeklärt werden, um Angehörige möglichst frühzeitig unterstützen zu können und somit längerfristigen Folgen und Kosten durch die Erkrankung der Angehörigen vorzubeugen.

Ziel der vorliegenden Arbeit ist es zu untersuchen, welche Belastungen Angehörige von drogenabhängigen Menschen in der Schweiz im Zusammenhang mit der Suchterkrankung erleben, wie sie mit diesen Belastungen umgehen und welche Auswirkungen dies auf ihre Gesundheit hat. Zudem interessiert die Frage, wie Angehörige besser unterstützt werden können bei der Bewältigung der mannigfaltigen Belastungen, die sie erleben.

In der vorliegenden Arbeit wird zunächst in Kapitel 2 auf den theoretischen Hintergrund der Angehörigen eingegangen, wobei kurz die historische Entwicklung der Angehörigenarbeit und die systemische Perspektive beschrieben werden, bevor ein längeres Kapitel dem Konzept der Co-Abhängigkeit gewidmet ist. Dieses ist untrennbar mit den Angehörigen von suchtkranken Menschen verbunden. Anschliessend werden Forschungsarbeiten zu den Belastungen, die Angehörige erleben und den Bewältigungsstrategien dargestellt. Darauf folgt schliesslich noch ein Kapitel mit verschiedenen Interventionen, die für Angehörige von suchtkranken Menschen entwickelt wurden. In Kapitel 3 werden dann die Fragen, denen diese Arbeit nachgehen möchte, näher vorgestellt. In Kapitel 4 wird eine Übersicht darüber gegeben, mit welchen Methoden die in Kapitel 3 gestellten Fragen, beantwortet werden sollen. In diesem Zusammenhang werden die Untersuchungsinstrumente vorgestellt, die in der Datenerhebung eingesetzt wurden. Anschliessend wird über die Datenerhebung berichtet und schliesslich werden noch die Auswertungsprozeduren dargestellt, die im Rahmen der Datenauswertung verwendet worden sind.

Das Kapitel 5 ist unterteilt in einen ersten Teil, in dem die quantitativen Ergebnisse vorgestellt werden und in einen zweiten Teil bestehend aus den qualitativen Ergebnissen. Der erste Teil, in dem die quantitativen Ergebnisse zusammengestellt sind, beginnt mit einer detaillierten Beschreibung der Stichprobe. Anschliessend folgt ein Kapitel, in welchem die Ergebnisse der 
Datenaufbereitung berichtet werden, bevor relevante Faktoren, die das Risiko für eine psychische Erkrankung (depressive Störung) erhöhen, diskutiert werden. In einem weiteren Kapitel werden schliesslich basierend auf typischen Gruppen von Angehörigen in Bezug auf die erlebte Belastung Risikofaktoren beschrieben, die dazu führen, dass Angehörige eher der Gruppe von stark belasteten Angehörigen mit einem höheren Risiko für gesundheitliche Probleme angehören. Im zweiten Ergebnisteil wird auf die qualitativen Ergebnisse der Befragung eingegangen. Diese sind unterteilt in folgende Kapitel: Situation, in der die Angehörigen vom Drogenkonsum erfahren haben, Belastungen, die Angehörige im Zusammenhang mit der Abhängigkeitserkrankung angeben, Unterstützung, die Angehörige erhalten hatten oder sich wünschen würden und schliesslich ein letztes Kapitel zu den Bewältigungsstrategien, die Angehörige angeben. Im 6. Kapitel werden die Ergebnisse diskutiert. Die Diskussion ist gegliedert in Belastungen, Bewältigungsstrategien und Unterstützung für die Angehörigen sowie die Relevanz des Themas. Im 7. Kapitel ist der Inhalt der Arbeit kurz zusammengefasst. 


\section{Theoretischer Hintergrund}

\subsection{Ein historischer Überblick}

\subsubsection{Einleitung}

Die Geschichte der Angehörigen von suchtkranken und insbesondere alkoholabhängigen Menschen beginnt Mitte des letzten Jahrhunderts. Es ist eine Geschichte, die vor allem von Schuldzuschreibungen an die Angehörigen geprägt ist (Uhl \& Puhm, 2007, S. 14). In den nachfolgenden beiden Kapiteln soll auf den Ursprung der Arbeit mit Angehörigen, die systemische Perspektive, eingegangen werden gefolgt von einem weiteren Kapitel zu systemischen Theorien zu Angehörigen. Anschliessend wird das Konzept der CoAbhängigkeit beleuchtet, das im Rahmen der Suchtarbeit über viele Jahre eine entscheidende Rolle gespielt hat und es zum Teil immer noch tut.

\subsubsection{Systemische Perspektive}

\subsubsection{Einleitung}

Die Ursprünge der Angehörigenarbeit an sich sind mit grosser Wahrscheinlichkeit im systemischen Denken zu suchen. Um 1950 begannen einige Pioniere, das vertraute Setting von Einzel- und Gruppentherapie aufzulösen zugunsten der Arbeit mit Familien und Paaren (Schlippe \& Schweitzer, 2012, S. 32). „Der Mehrgenerationenansatz in der Familientherapie hat sich aus den psychoanalytischen Wurzeln heraus entwickelt. Er versucht, unsichtbare und belastend wirkende Bindungen und Aufträge bewusst zu machen, die über die Generationen hinweg weitergegeben werden“ (ebd., S. 36). Aus dieser Bewegung heraus folgten schliesslich auch die ersten Interventionen für Angehörige, welche in Kapitel 2.4. vorgestellt werden. In den folgenden beiden Kapiteln werden verschiedene Rollen besprochen, die Angehörige von suchtkranken Menschen aus systemischer Sicht einnehmen können.

\subsubsection{Die Rolle der Angehörigen in den Anfängen}

Die Anfänge der systemischen Perspektive waren vor allem dadurch gekennzeichnet, dass nach möglichen Ursachen von psychischen Störungen in der Familie gesucht wurden (Schlippe \& Schweitzer, 2012, S. 33). Dabei richteten sich die frühen Familienstudien, die in den 40er Jahren entstanden, vor allem auf die Mütter, die schnell als pathogen identifiziert worden sind (ebd.). Begriffe wie die schizophrenogene Mutter stammen aus dieser Zeit. Es überrascht deshalb nicht so, dass eine der ersten Beschreibungen von Partnerinnen von 
Alkoholkranken eher als Schuldzuschreibung und Pathologisierung der Angehörigen ausfiel. Thelma Whalen (1953; zit. nach Uhl \& Puhm, 2007, S. 14) glaubte, basierend auf ihrer beraterischen Tätigkeit von Frauen von alkoholabhängigen Männern, vier Typen von Frauen entdeckt zu haben, die sich einen alkoholkranken Mann als Partner aussuchen, um ihre eigene primäre Pathologie in der Beziehung befriedigen zu können. Sie unterschied folgende vier Typen und versah sie mit eingängigen Namen: Suffering Susan heiratet einen alkoholkranken Mann, weil sie das Bedürfnis hat, sich selbst zu bestrafen. Controlling Catherine dagegen möchte alles kontrollieren und heiratet deshalb einen schwachen, kranken Mann. Wavering Winnifred ist selbst unsicher und schwankend und sucht sich deshalb einen Partner, der von ihrer Fürsorge abhängig ist. Punitive Polly schliesslich findet in ihrem alkoholkranken Mann einen Partner, an dem sie ihre aggressive Impulse ausleben kann.

\subsubsection{Glaubenssysteme und Rollenverteilungen in suchtbelasteten Familien}

Schweizer und Besson (1999, S. 1717) haben aus systemischer Sicht folgende typische Glaubenssysteme und Beziehungsregeln in Familien mit suchtkranken Menschen herausgearbeitet, die vor allem im Zusammenhang mit dem Suchtverhalten Gültigkeit bekommen und verdeutlichen, welchen nachhaltigen Einfluss Sucht auf die Familie haben kann:

- Das Gemeinwohl der Familie ist über das Wohl des Einzelnen zu stellen.

- Ist man sich nah, muss man ganz für den anderen da sein. Nicht-Eingehen auf die Bedürfnisse anderer ist tabu, als Alternative gibt es nur den Bruch.

- Man muss sich kontrollieren können, „ein bisschen“ Regression ist nicht möglich; ist totale Kontrolle nicht möglich, kommt es gleich zum totalen Bruch.

- Übernimmt man Verantwortung, ist diese total und man übernimmt sie für alle, sonst ist man für nichts und niemanden verantwortlich. Man kann sich diesen Pflichten nur durch Krankheit entziehen.

- Die Bedürfnisse nach Nähe und noch viel mehr nach Distanz dürfen keine vom Individuum gesteuerte Realisierung erfahren. Emanzipationswünsche und Autonomiebestrebungen werden schuldhaft erlebt und manchmal mit dem Auseinanderbrechen der Familie oder sogar mit dem Tod in Verbindung gebracht.

- Es gibt nur eine richtige Sichtweise der Dinge, Abweichungen davon könnten die Beziehung gefährden. 
- Hat jemand Probleme, übernimmt man die Verantwortung für ihn.

- Wünsche nach Autonomie oder Zuwendung dürfen als solche nicht direkt ausgesprochen werden. Sie werden aber akzeptiert, wenn man sich krank zeigt (Schweizer \& Besson, 1999, S. 1717).

Besonders gravierend sind die Auswirkungen auf Kinder, deren Eltern alkohol- oder drogenabhängig sind. Dabei wurden verschiedene Rollen identifiziert, die Kinder aus suchtbelasteten Familien einnehmen können (Wegscheider, 1981; zit. nach Rennert, 1990, S. 70ff).

Die Heldenrolle wird meist vom ältesten Kind der Familie eingenommen (Rennert, 1990; S. 70; Rennert, 1993a, S. 31). Dies wurde empirisch auch belegt (Fischer, Pidcock, Munsch \& Forthun, 2005, S. 91). Die Rolle ist dadurch gekennzeichnet, dass das Kind bereits sehr früh viel Verantwortung übernimmt. Es zeichnet sich zudem durch eine hohe Fürsorglichkeit und gutes Organisationstalent aus und ist in seinen Bemühungen sehr engagiert, die familiären Schwierigkeiten auszugleichen, die durch den Substanzkonsum eines Elternteils entstehen.

Kinder, die die Rolle des Sündenbocks übernehmen, zeichnen sich vor allem durch unangenehme Verhaltensauffälligkeiten aus (Rennert, 1990; S. 72; Rennert, 1993a, S. 32). Während der Held überangepasst ist, ist das Kind in der Sündenbockrolle genau das Gegenteil. Es gerät dauernd in Schwierigkeiten und lenkt die negative Aufmerksamkeit von den suchtbelasteten Eltern auf sich.

Ein Kind, welches die Rolle des verlorenen Kindes inne hat, ist meistens nach aussen vollkommen unauffällig (Rennert, 1990; S. 73; Rennert, 1993a, S. 32). Häufig sind die Eltern froh, wenn das Kind überhaupt keine Anforderungen an sie stellt und keine grosse Aufmerksamkeit einfordert. Das Kind ist meist schüchtern und ruhig und eher ein Einzelgänger.

Schliesslich gibt es noch die Rolle des Maskottchens: Dieses Kind zeichnet sich dadurch aus, dass es ständig den Clown macht für die anderen (Rennert, 1990; S. 74; Rennert, 1993a, S. 32). Es trägt dazu bei, dass unangenehme Spannungen in der Familie abgebaut werden können und bekommt dadurch gleichzeitig Aufmerksamkeit. 
Fischer et al. (2005) untersuchten, welche Rolle die familiäre Funktionalität und das Konsumverhalten der Eltern in der Rollenübernahme der Geschwister spielten. Es zeigte sich, dass die Dysfunktionalität in der Familie, wie beispielsweise eine fehlende Fähigkeit zur Entscheidungsfindung oder zur Bewältigung von Konflikten und der Schweregrad des Substanzmissbrauchs eines Elternteils einen Einfluss auf die Rollenübernahme der Kinder hatte. Dabei unterschieden sich die Rollen der Kinder umso stärker, je grösser der Substanzkonsum und je höher die Dysfunktionalität in der Familie war (ebd., S. 91). Das ältere Kind übernahm häufig die Rolle des Helden, während sein jüngeres Geschwister oft die Rolle des verlorenen Kindes einnahm.

\subsubsection{Co-Abhängigkeit}

\subsubsection{Einleitung}

Wer sich mit Angehörigen von suchtkranken Menschen beschäftigt, kommt nicht darum herum, sich auch mit dem Konzept der Co-Abhängigkeit auseinanderzusetzen. Der Begriff ist in den 70er Jahren in der Beratungsszene der Vereinigten Staaten entstanden (Beattie, 2011, S. 43). Wer den Begriff allerdings genau ins Leben gerufen hatte, bleibt unklar, obwohl es einige Autoren gibt, die spekulieren, dass der Begriff aus der Angehörigenselbsthilfe (AlAnon) stammen könnte (Andorfer \& Schmidt, 2007, S. 41; Beattie, 2011, S. 43; Uhl \& Puhm, 2007, S. 13; Rennert, 2005, S. 45). Tatsache ist, dass das Konzept sich vor allem in den 80er Jahren grosser Popularität erfreute und Eingang in viele populärwissenschaftliche Publikationen und in die Selbsthilfeliteratur fand (Beattie, 2011, Kolitzus, 1997, Kolitzus, 2000, Schaef, 1996). Die meisten dieser Selbsthilfebücher sind auch heute noch in Buchhandlungen erhältlich, so dass das Konzept vielen Angehörigen bekannt ist. Bei der Auseinandersetzung mit der grossen Vielfalt an Literatur zeigt sich jedoch, dass das Konzept überhaupt nicht einheitlich ist und es mehrere verschiedene Definitionen gibt.

Das Konzept der Co-Abhängigkeit erfuhr im Laufe der Zeit in mehrfacher Hinsicht einen Wandel: Sollte der Begriff zuerst der eigenen Mitbetroffenheit von der Suchterkrankung, im Sinne einer sekundären Pathologie, Ausdruck verleihen, wurde er zu einer Primärpathologie, die dazu führte, dass Angehörige plötzlich als Ursache für die Abhängigkeitserkrankung angesehen wurden und dafür verantwortlich gemacht wurden, dass der Substanzkonsum aufrechterhalten wurde (Uhl \& Puhm, 2007, S. 14). In diesem Zusammenhang erfuhr das Konzept auch eine inflationäre Ausweitung. So konnte Co-Abhängigkeit nun auch 
unabhängig von einer Suchterkrankung auftreten und wurde als allgemeines Störungsbild oder gar als Persönlichkeitsstörung vorgeschlagen (Schaef, 1996; Cermak, 1986; zit. nach Uhl \& Puhm, 2007). Im Selbsthilfebuch von Anne Wilson Schaef (1996, S. 80) ist gar zu lesen, dass in unserer pathologischen Gesellschaft $96 \%$ der Bevölkerung co-abhängig seien. Der Begriff bezog sich zudem nicht mehr nur auf Menschen, sondern ganze Institutionen und Organisationen konnten co-abhängig sein (Flassbeck, 2011). Co-Abhängigkeit wurde und wird zudem eher als geschlechterspezifisches Störungsbild angesehen, welches aufgrund der Sozialisation vorwiegend Frauen betrifft (Bärtschi-Waldvogel, 1995).

In den nachfolgenden Kapiteln werden verschiedene Definitionen, Symptome und Verhaltensweisen diskutiert, die als co-abhängig angesehen werden. Zudem wird in einem Kapitel der Bezug zu den Diagnosesystemen ICD-10 und DSM-IV-TR hergestellt. Schliesslich werden in einem letzten Kapitel noch empirische Belege zum CoAbhängigkeitskonzept diskutiert.

\subsubsection{Unterschiedliche Sichtweisen der Co-Abhängigkeit}

Obwohl es verschiedene Definitionen zur Co-Abhängigkeit gibt, sind sich dennoch viele Autoren darin einig, dass die Angehörigen im Verlauf der Suchterkrankung eines nahestehenden Menschen ihre eigenen Bedürfnisse und Gefühle allmählich aus den Augen verlieren, was das Kennzeichen einer Co-Abhängigkeit ist (Flassbeck, 2011; Kolitzus, 1997; Schaef, 1996). Die Angehörigen koppeln ihre eigene Befindlichkeit stark an die abhängige Person: Konsumiert diese, sinkt das Selbstvertrauen der Angehörigen, weil sie glauben, den Substanzkonsum beeinflussen zu müssen und zu können. Rennert (1990, S. 194) definiert CoAbhängigkeit wie folgt:

Co-Abhängigkeit ist ein Problem- und Lebensbewältigungsmuster, das in der Interaktion mit einer suchtkranken Person entwickelt und verstärkt wird. Die Entwicklung co-abhängigen Verhaltens ist gekennzeichnet durch zunehmende Einschränkung in der Wahrnehmung von Verhaltensalternativen bis hin zum Gefühl existenzieller Bedrohung durch jegliche Veränderung. Sie geht mit den gleichen Begleiterscheinungen einher wie eine Entwicklung zur

Drogenabhängigkeit: Verlust von Selbstwert, Unterdrückung von Gefühlen, Verstärkung von Abwehrmechanismen, Kampf um Kontrolle, Verlust der Realität, Beeinträchtigung aller Potentiale der Persönlichkeit (Rennert, 1990, S. 194). 
Rennert (1990, S. 194) sieht in der Co-Abhängigkeit ein komplementäres Verhaltensmuster zur Abhängigkeit. Gemäss Beattie (2007, S.54ff) weisen co-abhängige Personen im Verlauf ihrer Co-Abhängigkeitsentwicklung meist irgendwann folgende Charakteristika auf: sich Sorgen machen, schwaches Selbstwertgefühl, Verdrängung eigener Gedanken und Gefühle, Besessenheit von Pflichten und Verantwortung, Kontrollbedürfnis, Verleugnung von Problemen und der Realität, Abhängigkeit, unzulängliche Kommunikation in Form von Vorwürfen, Betteln, Bestechen und anderem, Wut auf den Suchtkranken, schwache Grenzen und Mangel an Vertrauen. Im fortgeschrittenen Stadium fühlen sich co-abhängige Personen meist nur noch antriebslos, hoffnungslos und deprimiert (ebd.). In diesem Stadium werden Angehörige meist selbst krank oder greifen zu Medikamenten, Alkohol oder Drogen, um sich aus ihrer Situation zu befreien (Kolitzus, 2000, S. 11).

Co-Abhängigkeit wurde zudem anhand von verschiedenen Phasen definiert, wobei die Quelle nicht ganz klar ist, weil verschiedene Autoren es ohne Referenz veröffentlichten (Kolitzus, 1997, S. 47-49; Schmieder, 1992; zit. nach Schmidt, 2007, S. 22). Nachfolgend sind die drei Phasen beschrieben:

\section{Phase des Beschützens und Erklärens}

Der Suchtkranke verändert sich aufgrund des Substanzkonsums zunehmend. Die Angehörigen leugnen und rationalisieren aber zu Beginn diese Veränderungen (Kolitzus, 1997, S. 47). Sie suchen Entschuldigungen und Erklärungen für das krankhafte Verhalten. Gleichzeitig übernehmen die Angehörigen immer mehr Verantwortung und versuchen, den Betroffenen vor den negativen Konsequenzen des Substanzkonsums zu bewahren, indem sie jene abschirmen. Angehörige beginnen in dieser Phase, gegen eigene Regeln und Grundsätze zu verstossen (ebd.). So kann es beispielsweise vorkommen, dass Familienangehörige beim Arbeitgeber anrufen und ihn krank melden, weil dieser aufgrund des Konsums nicht zur Arbeit erscheinen kann.

\section{Phase des Helfens und Kontrollierens}

Wenn die erste Phase nicht zum Erfolg führt, beginnen Angehörige in einem zweiten Schritt, das Verhalten des Suchtkranken zu kontrollieren (Kolitzus, 1997, S. 48). Sie suchen nach Anzeichen, die darauf hinweisen, dass ihr Angehöriger konsumiert haben könnte. Sie suchen mögliche Verstecke nach Substanzvorräten ab und werfen diese fort oder übernehmen je nachdem die Verwaltung der Drogen oder trinken mit dem Alkoholiker mit, um den Konsum 
so steuern zu wollen. Irgendwann merken Angehörige, dass ihre Bemühungen nicht fruchten, was bei ihnen Gefühle von Angst, Ärger, Scham, Trauer und Wut auslöst (ebd.). Dies veranlasst Angehörige dazu, den Suchtkranken mehr unter Druck zu setzen. In dieser Phase werden deshalb oft auch Drohungen ausgesprochen, die dann aber nicht in die Realität umgesetzt werden (ebd.).

\section{Phase der Anklage und Phase der Desorganisation}

Die Angehörigen erkennen irgendwann, dass sie gegenüber der Suchterkrankung machtlos sind und all ihre Bemühungen nichts gebracht haben (Kolitzus, 1997, S. 49). Die Angehörigen fühlen sich mittlerweile ausgelaugt und sind häufig selbst krank geworden. An diesem Punkt kommt es schliesslich zur Anklage, d.h. die suchtkranke Person wird für alle Probleme verantwortlich gemacht. Dies ist häufig der Punkt, an dem es in Partnerschaften zur Trennung kommt oder der Betroffene im Rahmen des beruflichen Umfeldes seine Stelle verliert (ebd.).

Neben den Phasen der Co-Abhängigkeit wurden aber auch typische suchtfördernde Verhaltensweisen bei den Angehörigen gefunden und definiert. Gemäss Rennert (1990) gibt es in Familien mit Drogenabhängigen „erfahrungsgemäss ein Mitglied, das besonders unter der Situation leidet und mit ganz spezifischen Verhaltensweisen darauf reagiert Verhaltensweisen, die den Konsum und die Entwicklung der Abhängigkeit erst recht stabilisieren oder sogar fördern“ (S. 53). Im Englischen werden diese Personen, die dem Betroffenen emotional meist sehr nahe sind, „Enabler“ genannt. Nelson (1985; zit. nach Rennert, 1990, S. 53ff) konnte sechs verschiedene, voneinander abgrenzbare „Enabler“-Stile unterscheiden. Er belegte diese sogar empirisch (Nelson, 1985; zit. nach Rennert, 1990, S. 53ff). Nachfolgend sind die „Enabler“-Stile zusammengestellt:

Angehörige versuchen, analog der ersten der drei Phasen der Co-Abhängigkeit, ihr suchtkrankes Familienmitglied vor den negativen Konsequenzen seines Substanzkonsums zu schützen. Ein solches Verhalten wird als Vermeiden und Beschützen bezeichnet (Rennert, 1990, S. 54)

Die Angehörigen unternehmen in irgendeiner Art und Weise Versuche, den Konsum des Abhängigen zu kontrollieren. Sie versuchen alles, um insgesamt Kontrolle über ihr suchtkrankes Familienmitglied zu bekommen. Mögliche Strategien der Angehörigen können sein: Verweigerung des sexuellen Verkehrs oder heftige Liebeszuwendungen (Schmidt, 2007, 
S. 23). Es können aber auch Drohungen ausgesprochen werden, die Angehörige jedoch oft nicht in die Tat umsetzen.

Im Verlauf der Suchterkrankung übernehmen die Angehörigen immer mehr Aufgaben, für die früher der drogenabhängige Angehörige verantwortlich war (Übernahme von Verantwortlichkeit). Dies soll den Betroffenen entlasten, führt aber dazu, dass dieser immer weiter entmündigt wird und ihm somit auch ein Stück Halt entzogen wird (Rennert, 1990, S. 55).

Unter Rationalisieren und Akzeptieren werden dabei Verhaltensweisen verstanden, mit denen Angehörige ihre Akzeptanz gegenüber dem Substanzkonsum zum Ausdruck bringen oder diesen rationalisieren (Rennert, 1990, S. 54). So können Angehörige beispielsweise ihre Akzeptanz ausdrücken, indem sie finden, der Konsum sei ja gar nicht so gefährlich oder ihr Angehöriger müsse aufgrund der grossen beruflichen Belastung konsumieren, um sich entspannen zu können.

Gemäss Rennert (1990) gehört zur Verhaltensweise Kooperation und Kollaboration ,jegliche Unterstützung oder Beteiligung der co-abhängigen Person bei der Beschaffung, dem Verkauf, der Zubereitung und dem Gebrauch der Droge“ (S. 54). Im weiteren Sinne gehört dazu auch die finanzielle Unterstützung, die viele Angehörige leisten, um den vollständigen Absturz des Suchtkranken zu verhindern.

Angehörige stellen ihre Bedürfnisse ganz und gar in den Hintergrund, um sich voll auf die Unterstützung und Rettung des Suchtkranken zu konzentrieren. Sie zeigen sich dabei häufig sehr unterwürfig, halten den Suchtkranken mit ihrem Verhalten aber auch in einer Abhängigkeit (Schmidt, 2007, S. 23). Diese Verhaltensweise wird als Retten und sich dem Abhängigen nützlich machen bezeichnet.

\subsubsection{Co-Abhängigkeit und die Standarddiagnosesysteme ICD-10 und DSM-IV-TR}

In der Vergangenheit wurden wiederholt Versuche unternommen, das Konzept der CoAbhängigkeit so $\mathrm{zu}$ definieren, dass es sich auch als psychische Störung in die Diagnosesysteme ICD oder DSM aufnehmen liesse. Cermak (1986) schlug in den 80er Jahren erstmals diagnostische Leitlinien für eine co-abhängige Persönlichkeitsstörung vor (Cermak, 1986). Ein neuerer Versuch, Co-Abhängigkeit als Diagnose zu definieren, unternahm 
Flassbeck (2011), wobei er davon ausging, dass mehrheitlich Frauen von Co-Abhängigkeit betroffen sind. Er operationalisierte die Diagnose „Co-Abhängigkeitssyndrom“ wie folgt:

1. Die Betroffene ist im Erleben übermässig durch die Sucht eines nahestehenden Menschen und das zwanghafte Verlangen, ihm zu helfen und ihn kontrollieren zu wollen, eingenommen. Das Eingenommensein ist durch weitere abhängigkeitsspezifische Merkmale gekennzeichnet: Zunahme der Hilfebemühungen, trotz schädlicher Folgen zum Partner halten, Vernachlässigung eigener Aktivitäten und Interessen, Bagatellisierung/Verleugnung der Sucht des anderen, Trennung löst Unwohlsein aus.

2. Das Verhalten der Person ist durch einen ausgeprägten Scham- und Schuldkomplex geprägt. Weitere sozioemotionale Störungen kommen hinzu: Stimmungsschwankungen zwischen Euphorie und Larmoyanz, inkonsequente Verhaltensweisen, starke Hemmung von Ärger und Aggressivität, ständige Feindseligkeit/Höflichkeit, hohe Leistungsansprüche/Perfektionismus, Selbstzweifel/Selbstwertproblematik, übermässige Verantwortungsübernahme.

3. Zusätzliche psychische Auffälligkeiten z.B. depressive Erschöpfung und Freudlosigkeit, soziale Ängste/Unsicherheiten, psychosomatische Beschwerden oder traumatische Gleichgültigkeit und Misstrauen sind typisch.

4. Die Beschwerden sind nicht nur vorübergehend, bessern sich nicht oder verschlimmern sich und weisen einen Schweregrad auf, der zu starkem Leiden und/oder Beeinträchtigung des gewohnten Lebensvollzuges führt. (Flassbeck, 2011, S. 61)

Flassbeck (2011, S. 61) schlägt vor, dass alle vier Kriterien erfüllt sein müssen, damit die Diagnose „Co-Abhängigkeitssyndrom“ vergeben werden kann. Er betont zudem, dass der Leidensdruck und die Beeinträchtigung häufig nicht offen festzustellen seien, weil diese von den Betroffenen verleugnet würden. Zudem seien Co-Abhängige häufig hoch funktionsfähig in Bezug auf Arbeit, Haushalt und Familie. Die meisten würden dagegen vor allem soziale Kontakte und eigene Interessen und Aktivitäten vernachlässigen (ebd., S. 62).

Bis heute ist Co-Abhängigkeit keine psychiatrische Diagnose und lässt sich auch in der neuen Version des DSM-V nicht finden. Grund dafür dürften die fehlenden empirischen Belege sein, auf die im nachfolgenden Kapitel eingegangen wird. 


\subsubsection{Empirische Belege}

Empirische Belege für eine co-abhängige Störung gibt es bisher keine, obwohl immer wieder Versuche unternommen wurden, diese zu operationalisieren. Klein (2002) schreibt dazu Folgendes:

Da die grosse Heterogenität der Gruppe der Angehörigen bislang zu wenig erfolgreich in Form empirisch abgesicherten Subtypen erfasst wurde, konnten noch keine verlässlichen Subgruppen von Angehörigenverhaltensweisen festgestellt werden. Entsprechend ist das Konzept „Co-Abhängigkeit“ nicht verlässlich diagnostizierbar. Das Co-Abhängigkeitskonzept ist daher als wissenschaftlich wenig sinnvolle Kategorie anzusehen, die zunächst nur heuristischen und klinischen Wert besitzen. Es erscheint insgesamt eher plausibel, dass Co-Abhängigkeit ein Muster unterschiedlicher Interaktionsverhaltensweisen und Persönlichkeitseigenschaften darstellt, die sich zu jeweils unterschiedlichen Mustern suchtkranken Verhaltens in optimaler Weise ergänzen, so dass kontinuierliche und stabile Interaktionssequenzen entstehen (Klein, 2002, S. 384).

Trotz fehlender empirischer Belege wurde das Konzept bis heute nicht fallen gelassen und es wird vor allem im amerikanischen und australischen Raum noch immer viel zu CoAbhängigkeit geforscht. Im amerikanischen Raum ist das Konzept nach wie vor akzeptiert (Orford, Templeton, Velleman \& Copello, 2005, S. 1612). In Australien wurde 2012 sogar ein neuer Fragebogen, der Co-Abhängigkeit erfassen soll, publiziert (Marks, Blore, Hine \& Dear, 2012). Es zeigt sich allerdings, dass neuere Forschung, die sich oft auf Kinder aus suchtbelasteten Familien fokussiert, die zum Teil bereits erwachsen sind, neben dem Substanzkonsum eines Elternteils auch familiäre Dysfunktionalität berücksichtigt (Fischer et al., 2005, Harkness, Manhire, Blanchard \& Darling, 2007, Knudson \& Terrell, 2012). Die meisten dieser Studien zeigen, dass familiäre Dysfunktionalität wie beispielsweise häufige Konflikte stärker mit Co-Abhängigkeit assoziiert sind als der Substanzkonsum der Eltern (Fischer et al., 2005, S. 91; Knudson \& Terrell, 2012, S. 254). Dieser Befund spricht eher dagegen, dass es sich bei Co-Abhängigkeit um ein Phänomen handelt, das nur im Zusammenhang mit Suchterkrankungen auftritt.

Neuere Ansätze wie die ,,5-Step-Method“ und das zugrunde liegende Stress-Strain-CopingSupport (SSCS) Modell (siehe Kap. 2.4.4.2) lehnen dagegen das Co-Abhängigkeitskonzept deutlich ab. 


\subsection{Belastungsfaktoren und Auswirkungen der Suchterkrankung auf die Angehörigen}

\subsubsection{Einleitung}

Die Suchterkrankung ist für die Angehörigen mit einer Vielzahl von Belastungsfaktoren assoziiert (Kirby et al., 2005; Nieuwenboom, 2012; Oreo \& Ozgul, 2007; Orford et al., 1998c; Rennert, 1993c). Auf diese wird in den nächsten Kapiteln näher eingegangen. Es ist zudem erwiesen, dass die Belastungen, die durch die Abhängigkeit einer nahestehenden Person entstehen, auch Auswirkungen auf die Gesundheit der Angehörigen hat (Lee et al., 2011; Oreo \& Ozgul, 2007; Orford et al., 1998c; Ray et al., 2007) und massgeblich die kindliche Entwicklung (Bilke-Hentsch \& Gremaud, 2013) und die Funktionsfähigkeit der Angehörigen (Hudson, Kirby, Firely, Festinger \& Marlowe, 2002) beeinflusst.

\subsubsection{Belastungsfaktoren}

\subsubsection{Einleitung}

Es wurden bereits verschiedene Versuche unternommen, Belastungen, die Angehörige von suchtkranken Menschen erleben, zu erheben und zu beschreiben. Dazu wurden verschiedene Zugänge gewählt. Während einige Forschungsgruppen empirisch vorgingen und Angehörige ohne direkten theoretischen Hintergrund $\mathrm{zu}$ ihren Belastungen befragten (Orford et al., 1998c), wählten andere einen theoretischen Zugang (Kirby et al., 2005). Nachfolgend werden die Belastungen vorgestellt, die Angehörige im Zusammenhang mit der Suchterkrankung eines ihnen nahestehenden Menschen erleben können.

\subsubsection{Ergebnisse einer qualitativen Studie in England und Mexiko}

Ein wichtiges Charakteristikum von Angehörigen ist es, wie bereits mehrfach erwähnt, dass sie auf vielfältigste Weise Belastungen ausgesetzt sind. Im Rahmen einer qualitativen Studie wurden deshalb in England und Mexiko Angehörige von drogen- und alkoholabhängigen Menschen zu ihren Belastungen befragt (Orford et al., 1998c). Die Autoren gingen dabei davon aus, dass es vier Bereiche gibt, in denen Angehörige Belastungen erleben, die universell gültig sein dürften (ebd.). Alle Angehörigen aus England und Mexiko sagten, dass das Zusammensein und Zusammenleben mit dem suchtkranken Familienmitglied mit Schwierigkeiten verbunden sei. Dazu gehörten Beschimpfungen und Drohungen durch die substanzkonsumierende Person. Gleichzeitig wurde aber auch von Stimmungsschwankungen und leichter Reizbarkeit beim suchtkranken Angehörigen berichtet. Orford et al. (1998c) 
ordneten der Kategorie zudem Kommunikationsschwierigkeiten seitens des suchtkranken Familienmitglieds wie Lügen zu. Auch Beziehungsschwierigkeiten wie beispielsweise sexuelle Probleme fallen unter diese Kategorie.

In einer zweiten Kategorie, die ebenfalls als universell gültig angesehen wurde, wurden Sorgen in Bezug auf den suchtkranken Angehörigen erfasst (Orford et al., 1998c). Beinahe alle Angehörigen (23 von 24) erzählten, dass sie sich Sorgen machen würden wegen der psychischen und physischen Gesundheit ihres konsumierenden Familienangehörigen. Gleichzeitig machten sie sich Sorgen in Bezug auf die Arbeitsfähigkeit oder um die Ausbildung des Betroffenen. Es beschäftigte sie, dass ihr Angehöriger mit anderen suchtkranken Menschen Umgang pflegte oder sozial isoliert war. Ein weiteres Thema war auch die Vernachlässigung der Körperpflege durch den Substanzkonsum (ebd.)

Eine grosse Last für die Mehrheit der Angehörigen (20 von 24) und somit wiederum von universeller Gültigkeit waren finanzielle Schwierigkeiten (Orford et al., 1998c). Angehörige erzählten, dass ihr suchtkrankes Familienmitglied, Dinge von ihnen leihen und sie dann nicht mehr zurückgeben würde. Einige berichteten auch von Diebstahl, der ebenfalls dieser Kategorie angerechnet wurde oder dass das Familienmitglied keinen Beitrag zu den Lebenshaltungskosten leisten würde. Angehörige machten sich aber auch grundsätzlich Sorgen um die finanzielle Situation des Betroffenen (ebd.).

Die Mehrheit (19 von 24) gab an, dass sie durch die Auswirkungen des Substanzkonsums auf die Familie allgemein und auf das Zuhause belastet seien (Orford et al., 1998c). Sie fanden es schwierig, dass die familiäre Atmosphäre allgemein und andere Familienangehörige unter der Suchterkrankung litten. Angehörige mussten häufig Aufgaben und Pflichten des abhängigen Familienangehörigen übernehmen und sie wurden je nachdem Zeugen von Drogengeschäften, was ihren Lebensalltag beeinträchtigte (ebd.).

Weitere Themen, die Angehörige als belastend ansprachen, die aber keine universelle Gültigkeit haben dürften, waren folgende: Aussenstehende Personen oder Behörden wie beispielsweise die Polizei werden involviert (17 von 24), Sorgen in Bezug auf die Menge, Häufigkeit und Art des Konsums (16 von 24), der suchtkranke Angehörige ist vorübergehend nicht mehr auffindbar (12 von 24), Beeinflussung der sozialen Kontakte der Angehörigen 
durch die Abhängigkeitserkrankung (10 von 24) und Krisen und Zwischenfälle, die im Zusammenhang mit dem suchtkranken Familienmitglied auftreten (9 von 24).

Neben diesen Bereichen, welche diese Studie erfasst hat (Orford et al., 1998c), dürften allerdings noch weitere Belastungsfaktoren für Angehörige relevant sein. Auf diese wird im nächsten Kapitel näher eingegangen.

\subsubsection{Stigmatisierungen}

Ein Aspekt, der am Rande von Orford et al. (1998c) angesprochen wird, nämlich die Beeinflussung der sozialen Kontakte durch die Suchterkrankung, bezieht sich auf Stigmatisierungen, die bei Angehörigen eine Rolle spielen könnten (Nieuwenboom, 2012). Link und Phelan (2001, S. 382) definieren Stigmatisierung wie folgt: Stigmatisierung liegt vor, wenn Elemente von Etikettierung, Stereotypisierung, Ausgrenzung, Statusverlust und Diskriminierung gemeinsam in einem Machtgefälle vorliegen, welches einen Stigmatisierungsprozess möglich macht. Diskriminierung dagegen ist „eine strukturelle oder unmittelbare Benachteiligung oder Ungleichbehandlung aufgrund der angenommenen Zugehörigkeit des oder der Diskriminierten zu der stigmatisierten Gruppe“ (Nieuwenboom, 2012, S. 19). Stigmatisierung erfolgt vor allem aufgrund von Verhaltensweisen oder einem bestimmten Merkmal anhand dessen auf eine bestimmte Eigenschaft geschlossen wird. Ein Beispiel dafür wäre, dass suchtkranke Menschen zugeschrieben wird, dass sie gewalttätig und unberechenbar seien. Das Stigma, welches Angehörige von stigmatisierten Personengruppen trifft, wird als „Courtesy Stigma“ oder assoziiertes Stigma bezeichnet (Goffman, 1967; zit. nach Nieuwenboom, 2012, S. 19). Es erklärt, dass das Stigma, welches suchtkranke Menschen trifft, ein Stück weit auch auf ihre Angehörigen zurückfällt.

Stigmatisierungen bei Angehörigen von suchtkranken Menschen wurden bisher nur wenig untersucht (Nieuwenboom, 2012, S. 19). Die bestehenden Studien konzentrierten sich vorwiegend auf Angehörige von schizophrenen Patienten (Angermeyer, Schulze \& Dietrich, 2003, Schulze \& Angermeyer, 2003). Link und Phelan (2001, S. 372ff) unterscheiden vier Ebenen der Diskriminierung, auf denen Angehörige Stigmatisierungen erleben können:

Auf der Ebene der interpersonellen Interaktion gaben Angehörige von schizophrenen Patienten im Kontakt mit professionellen Helfern an, dass sie das Gefühl hatten, mit einer technischen Sprache auf Distanz gehalten zu werden oder dass grundsätzlich kein Interesse 
für sie da war (Angermeyer et al., 2003, S. 595). Diese Angehörigen berichteten aber auch über Schuldzuschreibungen aus ihrem Umfeld. Sie hätten sich sozial immer mehr zurückzogen oder ausgeschlossen gefühlt wegen diskriminierenden Bemerkungen oder dem Geschwätz der Leute. Ähnlich dürfte es sich auf dieser Ebene auch bei Angehörigen von suchtkranken Menschen verhalten, die mittels des Co-Abhängigkeitskonzepts zum Teil direkt verantwortlich gemacht werden für die Suchterkrankung des ihnen Nahestehenden (Nieuwenboom, 2012, S. 20).

Strukturelle Diskriminierung zeichnet sich dadurch aus, dass über gesellschaftliche oder institutionelle Strukturen eine Personengruppe benachteiligt oder ungleich behandelt wird (Link \& Phelan, 2001, S. 372). So wird beispielsweise für die Forschung zu einer stigmatisierten Krankheit weniger Geld gesprochen als für andere Krankheiten und es werden folglich auch weniger Gelder für die Behandlung zur Verfügung gestellt. Bezüglich der Angehörigen stellt die strukturelle Diskriminierung deren Vernachlässigung bei der Behandlung des Patienten dar (Angermeyer et al., 2003; Schulze \& Angermeyer, 2003). Dass Angehörige sich mehr Unterstützung und Aufklärung von Seiten der psychiatrischen Kliniken wünschen, zeigte eine Angehörigenbefragung in Deutschland (Schmid, Spiessl, Vukovich \& Cording, 2003; Spiessl, Schmid, Vukovich \& Cording, 2003). Schmid et al. (2003, S. 122) konnten aufzeigen, dass Angehörige, wenn sie sich Unterstützung suchen, auf ein lückenhaftes Versorgungssystem stossen. Weitere Aspekte der strukturellen Diskriminierung finden sich beispielsweise im Bereich der Finanzen. So gibt es in der Schweiz die Verwandtenunterstützungspflicht: Ab einem bestimmten Einkommen und Vermögen werden Angehörige dazu verpflichtet, für ihre Angehörigen aufzukommen (www.verwandtenunterstuetzung.ch).

Der dritte Bereich der Stigmatisierung ist das öffentliche Bild, welches zu Suchterkrankungen vorherrscht (Link \& Phelan, 2001). So sind Abhängigkeitserkrankungen häufig assoziiert mit Selbstverschuldung, Unberechenbarkeit und Gewalttätigkeit (Nieuwenboom, 2012, S. 20). Diese Ansichten und Vorurteile werden über die Medien transportiert und betreffen letztlich auch die Angehörigen. Schliesslich führen Stigmata aber auch dazu, dass beispielsweise niemand eine Suchthilfeeinrichtung oder Psychiatrie in seiner Nachbarschaft haben möchte.

Der letzte Bereich der Stigmatisierung ist der Zugang zu sozialen Rollen. Der erhöhte Betreuungsaufwand, den Angehörige übernehmen müssen, erschwert es ihnen weitere soziale 
Rollen auszuüben (Nieuwenboom, 2012, S. 20). So kann es beispielsweise zu mehr Fehlzeiten bei der Arbeit kommen, wofür den Angehörigen kein Verständnis entgegengebracht wird. Zudem können Reklamationen und Beschwerden der Nachbarn auch die Angehörigen treffen (ebd.).

Die Studie zu Angehörigen von schizophrenen Patienten zeigte, dass Angehörige vor allem über strukturelle Diskriminierungen und Stigmatisierungen im öffentlichen Bild berichteten (Angermeyer et al., 2003, S. 600). Diese Ergebnisse können sehr wahrscheinlich auch auf Angehörige von suchtkranken Menschen übertragen werden.

Eine wichtige Rolle im Umgang mit Stigmatisierungen spielt die Selbststigmatisierung (Link \& Phelan, 2001, S. 373-374; Nieuwenboom, 2012, S. 20-21). Unter Selbststigmatisierung wird verstanden, dass Angehörige oder auch suchtkranke Menschen die Vorurteile, die sich gegen ihre Gruppe richten, teilen. Dies kann dazu führen, dass sich die betroffene Person immer mehr zurückzieht und sie ihr Selbstbewusstsein verliert (ebd.). Selbststigmatisierungen, die häufig auch mit Gefühlen der Scham verbunden sind, können dazu führen, dass sich die Betroffenen keine Hilfe suchen (Nieuwenboom, 2012), was mit grosser Wahrscheinlichkeit auch für die Angehörigen von suchtkranken Menschen gilt.

\subsubsection{Weitere relevante Belastungsfaktoren}

Ein weiterer wesentlicher Belastungsfaktor für Angehörige sind Rückfälle (Oreo \& Ozgul, 2007, S. 80; Rennert, 1993c, S. 329). Angehörigen müssen im Verlauf der Erkrankung ihrer Familienmitglieder oft eine unendliche Serie von Entzügen, Entwöhnungen und Rückfällen miterleben. Sie haben im Zusammenhang mit der Suchterkrankung die Erfahrung gemacht, dass sich ihr Angehöriger immer mehr veränderte, dass er sie belog und betrog. Folglich ist für sie der Rückfall oft der Inbegriff der Katastrophe und zerstört alles, was mit einer Therapie und der Entwöhnung hart erarbeitet worden ist (Rennert, 1993c, S. 329). Angehörige leben deshalb meist in Phasen der Abstinenz oder bei einer Stabilisierung der Abhängigkeitserkrankung ihres Angehörigen in einer ständigen Angst vor einem Rückfall oder einer Verschlechterung des Zustandes.

Oreo und Ozgul (2005) untersuchten in diesem Zusammenhang, welche Trauer Eltern von einem erwachsenen suchtkranken Kind erleben. Sie konzeptualisierten dabei Trauer als eine kognitive, emotionale und behaviorale Reaktion auf einen Verlust (ebd., S. 73). Sie betonen 
dabei, dass Verlust und Trauer ähnlich dem Rückfall wiederkehrende Themen für die Eltern seien. Der Verlust bezieht sich dabei auf einen Verlust an Beziehung aber gleichzeitig auch einen Verlust an möglichen Lebensentwürfen, die in dem Kind gesehen wurden (ebd., S. 79). Dabei erleben Eltern keinen realen Verlust, weil das Kind nicht verstirbt. Sie bleiben in der Beziehung zu einem erwachsenen Kind, welches in der Persönlichkeit verändert ist. Durch diesen häufig jahrelang andauernden Umstand können die Eltern den Trauerprozess um das erwachsene suchtkranke Kind häufig nicht abschliessen.

\subsubsection{Die „Significant Other Checklist“ zur Erfassung der Belastung}

Einen etwas anderen Zugang wählten die Autoren der „Significant Other Checklist“ (Benishek, Dugosh, Faranda-Diedrich \& Kirby, 2006; Kirby et al., 2005). Sie gingen davon aus, dass Angehörige von suchtkranken Menschen in ähnlichen Bereichen Probleme und Belastungen erleben wie die Betroffenen selbst (Kirby et al., 2005, S. 32). Sie zogen deshalb den Addiction Severity Index (ASI) (McLellan, Luborsky, Woody \& O'Brien, 1980) heran, um Problembereiche $\mathrm{zu}$ definieren. Der ASI erhebt in folgenden Bereichen Beeinträchtigungen: Gesundheit, Bildung/Arbeitssituation, Substanzkonsum (Alkohol und Drogen), rechtliche Schwierigkeiten, familiäre und soziale Beziehungen und psychiatrische Symptome. Analog wurden für die Angehörigen Problembereiche definiert, die den Schweregrad der Beeinträchtigung der Angehörigen über eine vierstufige Likertskala erhebt.

Analog zum ASI wurde davon ausgegangen, dass Angehörige sowohl im sozialen als auch im familiären Bereich Beziehungsprobleme erleben. Diese sind durch verschiedene Aspekte gekennzeichnet (Kirby et al., 2005, S. 45). In Familien mit Suchtkranken kommt es vermehrt zu Streitereien. Dies kann sich in Auseinandersetzungen mit dem Betroffenen selbst zeigen, aber auch in Meinungsverschiedenheiten zwischen Familienmitgliedern in Bezug auf den suchtkranken Angehörigen, weil sie sich beispielsweise uneinig darin sind, wie man sich dem drogenkonsumierenden Familienmitglied gegenüber verhalten soll (ebd.). $\mathrm{Zu}$ den Beziehungsproblemen gehört zudem, dass die suchtmittelmissbrauchende Person häufig weniger Zeit mit den Angehörigen verbringt. In eine ähnliche Richtung weist auch die Tatsache, dass die Angehörigen oft von ihrem suchtkranken Familienmitglied belogen werden (ebd.). Die Abhängigkeitserkrankung hat zudem massive Auswirkungen auf die Kinder, die häufig vernachlässigt werden, weil ihre Eltern mit der Suchtmittelbeschaffung und dem Konsum beschäftigt sind (ebd.). 
Es ist offensichtlich, dass die Suchterkrankung für Angehörige auch eine emotionale Belastung darstellt. Aus diesem Grund wurde analog zu den psychiatrischen Symptomen ein Themenbereich mit emotionalen Belastungen der Angehörigen definiert. Angehörige leiden häufiger unter Schlafstörungen, weil sie sich Gedanken und Sorgen machen über ihr suchtkrankes Familienmitglied (Kirby et al., 2005, S. 45). Sie müssen zudem oft Enttäuschungen wegstecken, weil sie von ihrem Angehörigen versetzt oder Versprechungen nicht eingehalten werden. Angehörige haben häufig Schuldgefühle in Bezug auf die Suchterkrankung der ihnen nahestehenden Person (ebd.).

Nicht nur für den suchtkranken Menschen selbst stellt die Abhängigkeitserkrankung eine grosse finanzielle Belastung dar, sondern auch für die ihnen nahestehenden Menschen (Kirby et al., 2005, S. 45). Sie unterstützen ihre abhängigen Familienmitglieder finanziell, indem sie ihnen Geld geben, Schulden oder Bussen übernehmen. Manchmal kommen sie aber auch in die Situation, dass die Schulden ihrer Angehörigen auf sie zurückfallen und sie diese übernehmen müssen. Dabei machen sie oft die Erfahrung, dass die Schulden, die die Angehörigen bei ihnen machen, nicht mehr zurückbezahlt werden (ebd.). Weiter kann es vorkommen, dass sie von ihren suchtkranken Familienangehörigen bestohlen werden (ebd.).

Suchtkranke Menschen haben häufig soziale Probleme: Sie verlieren ihren Job oder die Wohnung aufgrund des Substanzkonsums. Dies fällt letztlich auch auf ihre Angehörigen zurück, die dadurch eine Beeinträchtigung ihres Lebensalltages erleben (Kirby et al., 2005, S. 45). Wenn suchtkranke Menschen ihre Unterkunft verlieren, bringt das die Angehörigen häufig in die Situation, dass sie den Suchtkranken bei sich aufnehmen müssen. Dies führt dazu, dass sie damit leben müssen, dass ihr Angehöriger Drogen oder Alkohol in ihrem Zuhause konsumiert (ebd.). Zudem übernehmen die Angehörigen im Zusammenleben häufig auch mehr Pflichten und Aufgaben, weil der von der Suchterkrankung Betroffene seinen Verpflichtungen nicht mehr nachkommen kann (ebd.). Eine weitere, nicht zu unterschätzende Beeinträchtigung des Lebensalltages der Angehörigen kommt hinzu, wenn der Suchtkranke das Auto der Angehörigen nimmt.

Durch die Suchterkrankung können Betroffene rechtliche Probleme bekommen, in die schliesslich auch ihre Angehörigen involviert werden (Kirby et al., 2005, S. 45). Zu den rechtlichen Problemen gehören Verhaftungen und Hausdurchsuchungen, die Angehörige miterleben oder über sich ergehen lassen müssen, wenn sie mit einem drogenabhängigen 
Menschen den Haushalt teilen. Sie müssen je nachdem Anwälte organisieren und bezahlen, um ihre Angehörigen wieder aus dem Gefängnis holen zu können. Dazu kommt, dass Angehörige sich Sorgen machen, wenn ihr suchtkranker Angehöriger auf Bewährung ist (ebd.).

Im Rahmen des ASI werden suchtkranke Menschen gefragt, ob es unter Substanzeinfluss zu Gewaltdelikten gekommen sei (McLellan et al., 1980). Analog dazu wurde in der „Significant Other Checklist" eine Kategorie Gewalt und Drohungen definiert (Kirby et al., 2005, S. 45). In Familien von suchtkranken Menschen kommt es häufig zu Gewalt (Moore \& Stuart, 2004; Murphy, O'Farrell, Fals-Stewart \& Feehan, 2001). Dies kann bedeuten, dass die Angehörigen vom Suchtkranken bedroht oder sogar körperlich attackiert werden (Kirby et al., 2005, S. 45). Es ist aber nicht so, dass nur der Suchtkranke seine Angehörigen körperlich angreift, sondern auch umgekehrt die Angehörigen manchmal zuschlagen.

Analog zu den gesundheitlichen Problemen, die häufig mit dem Substanzkonsum der Betroffenen assoziiert sind, machen sich Angehörige häufig Gesundheitssorgen (Kirby et al., 2005, S. 45). Zu den Gesundheitssorgen gehören Unfälle oder Verletzungen, die durch die Suchterkrankung zustande kommen und eine medizinische Versorgung notwendig machen. Häufig machen sich Angehörigen Sorgen wegen sexuell übertragbarer Krankheiten, die der Betroffene bekommen und die dann auch auf sie zurückfallen könnten (ebd.). Angehörige kümmern sich zudem oft um den kranken Angehörigen, wenn es ihm nicht gut geht, er krank oder high ist.

Kirby et al. (2005, S. 38) stellten im Rahmen ihrer Befragung von Angehörigen mit der „Significant Other Checklist“" fest, dass alle Angehörigen seit Beginn der Suchterkrankung mindestens einmal Beziehungsprobleme, emotionale, finanzielle oder gesundheitliche Sorgen im Zusammenhang mit der Abhängigkeitserkrankung erlebt hatten. Beinahe alle Angehörigen gaben zudem an, dass ihr Alltag durch die Erkrankung massgeblich beeinträchtigt worden sei. Neben der Beeinträchtigung in Bezug auf die Suchterkrankung insgesamt wurden - ebenfalls analog zum ASI - die Belastung in den letzten 30 Tagen erhoben (Kirby et al., 2005, S. 33). Dabei zeigte sich, dass diese Zahlen von den Langzeitdaten nur geringfügig abwichen. Alle Angehörigen gaben an, dass es in den letzten 30 Tagen zu Beziehungsproblemen sowie emotionalen Belastungen gekommen war. $95 \%$ berichteten zudem über finanzielle Belastungen in den letzten 30 Tagen. $96 \%$ gaben gesundheitliche Sorgen und $89 \%$ eine 
Beeinträchtigung ihres Lebensalltages durch die Suchterkrankung an (ebd., S. 38). Es zeigte sich zudem, dass Angehörige, die mit dem Suchtkranken zusammenlebten, stärker belastet waren, als diejenigen, die dies nicht taten (ebd., S. 41). Diese markanten Zahlen verdeutlichen eindrücklich, wie Angehörige in diversen Bereichen stark belastet sind.

\subsubsection{Auswirkungen der Suchterkrankung auf die Angehörigen}

\subsubsection{Einleitung}

Die mannigfaltigen Belastungen, die Angehörige erleben, lassen bereits vermuten, dass die Suchterkrankung schwerwiegende Auswirkungen auf die Angehörigen hat. Im nachfolgenden Kapitel wird auf die Auswirkungen auf die Gesundheit der Angehörigen eingegangen. Neben den gesundheitlichen Risiken hat die Suchterkrankung noch weitere Auswirkungen auf die Angehörigen. Sie beeinträchtigt besonders das soziale Funktionsniveau der Angehörigen (Hudson et al., 2002). In besonderer Weise betroffen durch eine Abhängigkeitserkrankung sind die Kinder von suchtkranken Eltern oder die jüngeren Geschwister von Substanzmissbrauchern. Ihnen und den Auswirkungen, die die Suchterkrankung auf sie hat, ist ein weiteres Kapitel gewidmet.

\subsubsection{Gesundheitliche Probleme}

Diverse Studien haben belegt, dass Angehörige von suchtkranken Menschen durch die Belastungen, welche die Suchterkrankung eines ihnen Nahestehenden mit sich bringt, ein höheres Risiko haben, selbst psychisch oder psychosomatisch zu erkranken (Lee et al., 2011; Oreo \& Ozgul, 2007; Orford et al., 1998c; Ray et al., 2007). So berichteten Angehörige in der Studie von Orford et al. (1998c, S. 13) nebst physischen Symptomen wie Rückenschmerzen, Asthma, Verdauungsproblemen, Kopfschmerzen inklusive Migräne, Bluthochdruck und anderen, die in einem direkten Zusammenhang mit der Suchterkrankung ihres Angehörigen standen, über eine generelle Verschlechterung des Gesundheitszustandes durch eine erhöhte Anfälligkeit für vielerlei Beschwerden. $\mathrm{Zu}$ den gesundheitlichen Problemen gehörten auch sexuell übertragbare Krankheiten, mit denen sich Partner bei ihren drogenabhängigen Angehörigen angesteckt hatten (ebd.)

In einer asiatischen Studie wurden Angehörige von suchtkranken Menschen mit einer gematchten Kontrollgruppe verglichen in Bezug auf Depressivität, Stress, psychiatrische Morbidität und Wohlbefinden (Lee et al., 2011). Die Ergebnisse zeigten deutlich, dass die 
Angehörigen signifikant höhere Depressivitätswerte erreichten, dass sie deutlich höhere Werte auf der Stresserlebensskala angaben und eine höhere psychiatrische Morbidität aufwiesen als die Kontrollgruppe (ebd., S. 443). Zudem war eine klare Beeinträchtigung des Wohlbefindens feststellbar. Mittels hierarchischen linearen Regressionsmodell konnten die Autoren nachweisen, dass der einzige Prädiktor, der psychiatrische Morbidität vorhersagen konnte, das Stresserleben der Angehörigen war (ebd., S. 445).

Ray et al. (2007) untersuchten die gesundheitlichen Probleme und in diesem Zusammenhang auch die Gesundheitskosten von Angehörigen von drogen- und alkoholabhängigen Menschen im Vergleich zu einer Kontrollgruppe. Dabei wurde zwischen Kindern und erwachsenen Angehörigen unterschieden. Die grössten Unterschiede zwischen Angehörigen von suchtkranken Menschen und den Kontrollgruppen zeigten sich sowohl bei den Kindern als auch bei den Erwachsenen bei den Störungen durch psychotrope Substanzen (OR: 1.9 für Erwachsene, 2.5 für Kinder) und bei den depressiven Störungen (OR: 2.2 für Erwachsene, 2.8 für Kinder), die bei Angehörigen deutlich häufiger auftraten (ebd., S. 119). Angehörige litten aber auch signifikant häufiger unter anderen gesundheitlichen Problemen wie Rückenschmerzen, Kopfschmerzen oder Traumatisierungen. Sie zeigten häufiger Verhaltensauffälligkeiten und bei Kindern aus suchtbelasteten Familien wurde signifikant häufiger eine Aufmerksamkeitsdefizitstörung diagnostiziert als bei den Kontrollgruppen (OR: 2.1) (ebd., S. 121). Dieses erhöhte Risiko für gesundheitliche Probleme widerspiegelte sich auch in den Gesundheitskosten. So hatten erwachsene Angehörige durchschnittlich $31 \%$ höhere Gesundheitskosten als nicht durch eine Abhängigkeit betroffene Menschen (ebd., S. 119). Bei Kindern, die aus suchtbelasteten Familien stammten, lagen die Kosten $28 \%$ über den Kosten von Kindern aus nicht suchtbelasteten Familien (ebd.).

Oreo und Ozgul (2005) hatten sich in ihrer Untersuchung auf Eltern von erwachsenen drogenabhängigen Kindern konzentriert. Sie hatten in diesem Zusammenhang untersucht, welche Auswirkungen das Erleben von Trauer und Schmerz aufgrund der Erkrankung des Kindes auf die Gesundheit der Eltern hat. Sie stellten in diesem Zusammenhang fest, dass Trauer und Schmerz mit mehr gesundheitlichen Problemen wie Depression, Angst oder psychosomatischen Beschwerden assoziiert waren (ebd., S. 80). Je stärker ein Elternteil Trauer und Schmerz im Zusammenhang mit der Abhängigkeitserkrankung des Kindes erlebt, desto grösser ist das Risiko für eine psychiatrische Störung (ebd.). 


\subsubsection{Auswirkungen auf das soziale Funktionsniveau der Angehörigen}

Die Drogenabhängigkeit eines Angehörigen hat nicht nur Auswirkungen auf die Gesundheit der Angehörigen, sondern beeinflusst auch ihr soziales Funktionsniveau (Hudson et al., 2002). Hudson et al. (2002) untersuchten als erste, ob sich die Angehörigen von Drogenkonsumenten insgesamt und in Bezug auf verschiedene Funktionsbereiche wie der Arbeit, dem wirtschaftlichen Funktionsniveau, sozialen Aktivitäten und Freizeitgestaltung, in Eltern- und Paarbeziehungen von der Durchschnittbevölkerung und ihren drogenabhängigen Angehörigen unterschieden. Die Autoren stellten fest, dass sich die Angehörigen signifikant von ihren suchtkranken Familienmitgliedern unterschieden (ebd., S. 177). Sie zeigten in allen Bereichen ein höheres soziales Funktionsniveau.

In der Studie wurde zudem das soziale Funktionsniveau von Eltern erwachsener drogenabhängiger Kindern und von Partnern von Drogenkonsumenten verglichen (Hudson et al., 2002, S. 172). Dabei zeigte sich, dass die Partner von drogenabhängigen Menschen über ein insgesamt schlechteres soziales Funktionsniveau berichteten als die Eltern. Dieser Unterschied war vor allem auf die Paarbeziehung und die wirtschaftliche Situation zurückzuführen, in denen Partner erheblich mehr Beeinträchtigungen erlebten als Eltern (ebd., S. 177). Dass die Beziehungsqualität in Paarbeziehungen durch den Substanzkonsum eines Partners negativ beeinflusst wird, hatte sich bereits in vorausgehenden Studien gezeigt (FalsStewart, Birchler \& O'Farrell, 1999). Im Vergleich zur Durchschnittsbevölkerung gaben alle Angehörigen, Partner als auch Eltern, eine höhere Einschränkung ihres sozialen Funktionsniveaus an. Es zeigte sich, dass sich diese Einschränkung nicht nur auf das Familienleben beschränkte, sondern dass sich die Drogenabhängigkeit auch auf soziale Aktivitäten, die Freizeitgestaltung und das Berufsleben auswirkte (ebd., S. 178).

Diese Befunde machen eines deutlich, nämlich dass die Suchterkrankung eines nahestehenden Menschen nicht nur direkte Auswirkungen auf das familiäre Zusammenleben hat, sondern auch Einschränkungen in den Freizeitaktivitäten, im Berufsleben und im Sozialleben nach sich zieht. Insgesamt zeigte sich, dass die Suchterkrankung auf Partner, die häufig mit dem drogenabhängigen Menschen zusammenleben, die stärksten Auswirkungen hatte. 


\subsubsection{Auswirkungen auf die Kinder}

Kinder sind diejenigen, die durch Suchterkrankungen am stärksten betroffen sind. Die Studie von Ray et al. (2007) hat bereits gezeigt, dass Kinder durch die Suchterkrankung der Eltern selbst ein höheres Risiko haben, eine Störung durch psychotrope Substanzen zu entwickeln. Dabei ist das Risiko für Kinder besonders dann gross, wenn die Mutter von einer Abhängigkeitserkrankung betroffen ist (Yule, Wilens, Martelon, Simon \& Biederman, 2013).

Abgesehen vom Risiko selbst suchtkrank zu werden, beeinträchtigt die Abhängigkeit eines oder beider Elternteile die Entwicklung des Kindes massiv. So konnte wiederholt belegt werden, dass der Substanzmissbrauch der Eltern mit einer Vernachlässigung und einem Missbrauch der Kinder assoziiert ist (Jaudes Kienberger \& Ekwo, 1995; Shulman, Shapira \& Hirshfield, 2000). Zudem beeinflusst die permanente Beschäftigung mit der Beschaffung und dem Konsum die Fähigkeit der Mütter, auf ihre Kinder in einer konsistenten und warmen Weise einzugehen (Schuler, Nair \& Blach, 2002). Es ist deshalb nicht überraschend, dass Kinder von drogenabhängigen Eltern häufiger unsicher oder desorganisiert gebunden sind (Goodman, Hans \& Cox, 1999, S. 67). In der Studie von Shulman et al. (2000, S. 1931) zeigten alle Kinder von drogenabhängigen Eltern, die in eine Studie eingeschlossen worden waren, in irgendeiner Weise spezielle Bedürfnisse. Bei der Mehrheit der Kinder (75\%) wurde eine leichte Beeinträchtigung der kognitiven Fähigkeiten und der Sprachentwicklung festgestellt. Bei $16 \%$ der Kinder zeigten sich Auffälligkeiten im emotionalen Bereich oder im Verhalten (ebd.). Im Fall einer schweren Vernachlässigung oder bei schwerem Missbrauch müssen Kinder zudem oft noch eine Fremdplatzierung durchmachen.

Nicht ausser Acht gelassen werden dürfen aber auch jüngere Geschwister eines adoleszenten älteren Geschwisters mit einem problematischen Substanzkonsum. Diese jüngeren Geschwister befinden sich manchmal erst in der Latenzphase, also im Alter zwischen sieben und zwölf Jahren. Sie reagieren auf den Substanzkonsum des älteren Geschwisters häufig überangepasst und versuchen dem Problem auszuweichen (Bilke-Hentsch \& Gremaud, 2013, S. 32). Bilke-Hentsch und Gremaud (2013) erklären in diesem Zusammenhang weiter: „Sie [Diese Kinder] negieren auffällig jegliches Interesse an Suchtmitteln und versuchen so durch eine sog. kontraphobische Abwehr, die die eigentliche Angst umkehrt, sich den familiären Problemen nicht stellen zu müssen. Diese Verhaltensweisen können oft bis in die mittlere Jugend „durchgehalten“ werden, machen dann aber ggf. einem intensiven eigenen Gebrauch bis hin zur Abhängigkeit Platz“ (S. 32). Das Risiko eines Substanzmissbrauchs beim jüngeren 
Geschwister darf keinesfalls vergessen werden: In einer Studie konnte nachgewiesen werden, dass der Einfluss eines älteren Geschwisters auf den Substanzkonsum bei einem jüngeren Geschwister grösser ist als eine vorhandene Abhängigkeit bei den Eltern, wenn keine gute Beziehung zu den Eltern besteht (Brook, Brook \& Whiteman, 1999, S. 462-463).

Durch die Unauffälligkeit des jüngeren Kindes in der Latenzphase wird es und erste auftretende Warnzeichen wie Schlafstörungen oder depressive Anteile oft übersehen (BilkeHentsch \& Gremaud, 2013). Die Familie ist in den meisten Fällen stark damit beschäftigt, sich um das auffällige Kind mit dem problematischen Substanzkonsum zu kümmern. Im Sinne einer Prävention sollten aber auch jüngere Geschwister im Auge behalten und dabei unterstützt werden, adaptive Bewältigungsstrategien auf Belastungssituationen zu erlernen (ebd.).

\subsection{Bewältigung}

\subsubsection{Einleitung}

Die Bewältigungsstrategien, die Angehörige im Umgang mit den Belastungen, die sie durch die Suchterkrankung erleben, anwenden, sind stark von den Umständen geprägt, in denen sich Angehörige befinden (Orford, Velleman, Copello, Templeton \& Ibanga, 2010, S. 54). In den nachfolgenden Kapiteln werden Bewältigungsstrategien vorgestellt, die empirisch erhoben worden sind (Orford, 1994; Orford, Natera, Davies, Nava, Mora, Rigby et al., 1998e; Orford,

Rigby, Miller, Tod, Bennett \& Velleman, 1992). Schliesslich widmet sich ein Kapitel noch den Bewältigungsstrategien, die für eine erfolgreiche Bewältigung der Belastungen vorgeschlagen wurden (Ligon, 2004). Eine Form der Bewältigung stellt auch die CoAbhängigkeit dar, die in Kapitel 2.1.3. bereits beschrieben wurde. Das Konzept geht davon aus, dass Angehörige mit ihren co-abhängigen Verhaltensweisen versuchen, die Situation in einer dysfuntionalen Weise zu bewältigen.

\subsubsection{Bewältigungsstrategien}

\subsubsection{Einleitung}

Unabhängig von einem theoretischen Hintergrund wurden in England in den 90er Jahren Angehörige von drogenabhängigen Menschen befragt, wie sie mit den Belastungen, mit denen sie konfrontiert sind, umgehen (Orford et al., 1992). Die gewonnen Interviewdaten wurden 
qualitativ ausgewertet und acht Bewältigungsstrategien definiert (ebd.). In einer Nachfolgestudie wurde schliesslich in einer Kombination von qualitativen und quantitativen Auswertungsmethoden nach einer Copingstruktur gesucht, die besser darüber Auskunft geben sollte, wie Angehörige von drogenabhängigen Menschen die Belastungen bewältigen (Orford et al., 1998e). Im nachfolgenden Kapitel werden die Bewältigungsstrategien, die Angehörige anwenden und die auf empirischen Erkenntnissen beruhen, zusammengestellt.

\subsubsection{Empirisch belegte Copingstruktur}

Es gibt grundsätzlich drei mögliche Positionen, die Angehörige in Bezug auf die Suchterkrankung einnehmen können (Orford \& Velleman et al., 2010, S. 51). Sie können die Situation erdulden (Toleranz). Sie können versuchen ein Stück weit die Kontrolle zurückzugewinnen und standfest zu bleiben (Aufbegehren) oder sie können sich von der Problematik zurückziehen (Unabhängigkeit). Die nachfolgende Abbildung (Abb. 1) veranschaulicht die drei Positionen und die acht Bewältigungsstrategien der Angehörigen.

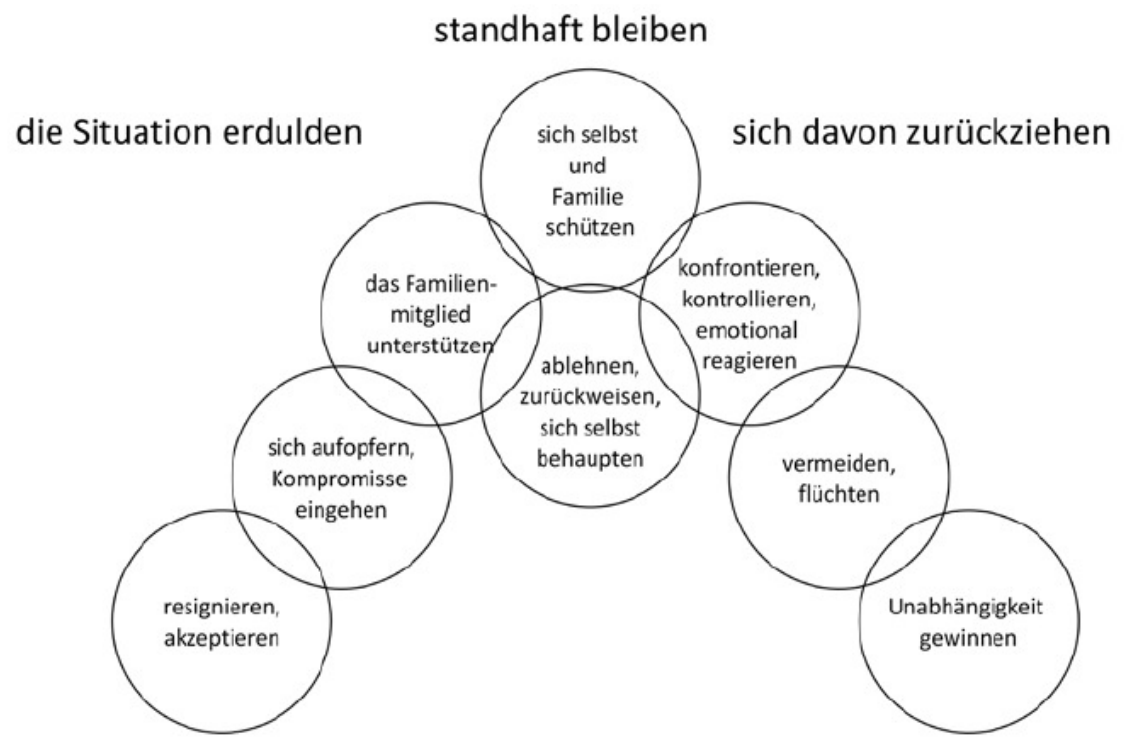

Abb. 1: Drei Hauptpositionen und Bewältigungsstrategien von Angehörigen gemäss Kläusler-Senn und Stohler (2012, S. 7) modifiziert nach Orford \& Velleman et al. (2010)

Die Abbildung (Abb. 1) macht deutlich, dass jede der drei Positionen auf weitere spezifischere Formen der Bewältigung heruntergebrochen werden kann. Orford, Velleman et al. (2010, S. 51) betonen, dass die acht Bewältigungsstrategien ineinander überlaufen und dass es keine klaren Grenzen zwischen den einzelnen Strategien gibt. Dies hat folgende 
Implikationen: Angehörige können mehrere Bewältigungsstrategien gleichzeitig anwenden. Zugleich ist es aber auch Ausdruck für das Dilemma, in dem sich Angehörige häufig befinden, nämlich der Frage, wie sie sich denn nun am besten verhalten sollen. Orford, Velleman et al. (2010, S. 51) machen deutlich, dass es für Angehörige oft schwierig ist, die richtige Balance zu finden. Angehörige, die sich selbst und die Familien schützen wollen und gleichzeitig den suchtkranken Angehörigen unterstützen möchten, geraten unwillkürlich in einen Konflikt, weil sie nicht beides im gleichen Mass tun können.

Die Position „die Situation erdulden“ fächert sich in verschiedene Facetten auf (Orford \& Velleman et al., 2010, S. 54). Angehörigen können angesichts der grossen Probleme in Passivität verfallen und resignieren. Andere wiederum vertreten die Haltung, dass sie die Dinge so akzeptieren, wie sie sind. Wiederum andere Angehörige investieren ihre ganze Energie dahingehend, für den Betroffenen da zu sein. Die Gründe, weshalb Angehörige die Situation erdulden, sind vielschichtig und stark von den Umständen geprägt (ebd.). So kann es beispielsweise sein, dass das suchtkranke Familienmitglied Druck auf die Angehörigen ausübt oder dass die Angehörigen schlichtweg finden, dass es für sie zu schwierig sei, hart zu bleiben. Weitere Gründe können sein, dass der Angehörige glaubt, dass seine Bemühungen sowieso nichts bringen oder sie betonen, dass es ihnen wichtig ist, dass sie den suchtkranken Angehörigen unterstützen können. Es kann aber auch sein, dass sie die Situation der Familie zuliebe akzeptieren und deshalb eine erduldende Haltung einnehmen (ebd.).

Angehörige, welche die Position „sich vom Problem zurückziehen“ einnehmen, versuchen Distanz zwischen sich und dem Betroffenen und seinem Suchtproblem zu schaffen (Orford \& Velleman et al., 2010, S. 54). Dies kann entweder eine räumliche oder emotionale Trennung bedeuten. Eine räumliche Trennung kann zeitlich begrenzt sein, wenn Angehörige beispielsweise vorübergehend die Wohnung verlassen oder sich in ein Zimmer zurückziehen, um alleine $\mathrm{zu}$ sein. Es kann aber auch sein, dass Angehörige ausziehen oder ihren drogenabhängigen Angehörigen bitten, sich eine andere Unterkunft zu suchen. Eine andere Art Distanz zu schaffen, stellt eine gewisse emotionale Ablösung dar (ebd.). Angehörige betonen dabei, dass sie ihre eigene Lebensqualität und ihre eigenen Wünsche und Bedürfnisse mehr ins Zentrum ihres Lebens stellen wollen.

Angehörige, die „standfest bleiben“, versuchen nicht, Distanz zwischen sich und ihren Angehörigen $\mathrm{zu}$ bringen, sondern setzen sich dafür ein, dass der Umgang mit der 
Suchterkrankung und dem Betroffenen nach neuen, anderen Regeln stattfindet (Orford \& Velleman et al., 2010, S. 55). Standfestigkeit ist mit einem grossen Energieaufwand für die Angehörigen verbunden. Angehörige versuchen auf diese Weise oft auch eine Veränderung des Konsumverhaltens herbeizuführen. Dazu gehören auch Verhaltensweisen wie das Kontrollieren oder das Konfrontieren des Angehörigen (ebd.).

\subsubsection{Verschiedene Copingstrategien und weitere Variablen}

Eine Studie untersuchte schliesslich, wie die erlebte Belastung, die Bewältigungsstrategien und die Gesundheit der Angehörigen zusammenhängen (Orford, Natera, Velleman, Copello, Bowie, Bradbury et al., 2001). Es zeigte sich dabei deutlich, dass ein tolerantes und inaktives Coping mit den grössten gesundheitlichen Problemen assoziiert war (ebd., S. 769; Lee et al., 2011, S. 445). Dazu gehören Verhaltensweisen wie das Verleugnen, Verheimlichen und Entschuldigen des Substanzkonsums oder das Nachgeben in Konfliktsituationen, welche Ausdruck von Inaktivität sind (Orford et al., 2001, S. 769). Unter Toleranz werden dagegen Verhaltensweisen verstanden, die entweder eine aktive Unterstützung des Substanzkonsums meinen, indem Angehörige beispielsweise die Droge oder den Alkohol für den Konsumenten besorgen oder eine passive Form der Akzeptanz, indem Angehörige keine Ansprüche stellen und hinnehmen, was ihr suchtkrankes Familienmitglied tut (ebd.).

Orford et al. (2001, S. 770) konnten nachweisen, dass vor allem Partnerinnen von alkoholund drogenabhängigen Männern zu einem inaktiven und toleranten Coping neigen. Dagegen zeigte sich, dass Eltern von drogenabhängigen Kindern eher zu einem aufbegehrenden, standhaft bleibenden Coping tendierten (ebd., S. 771). Sie stellten zudem fest, dass die Wahrscheinlichkeit von einem inaktiven, toleranten Coping zunimmt, je grösser die Belastungen für die Angehörigen sind (ebd., S. 772).

\subsubsection{Die sechs Bewältigungsstrategien nach Ligon}

\subsubsection{Einleitung}

Ligon (2004) konzentrierte sich in seiner Arbeit auf Bewältigungsstrategien, die die Belastung für die Angehörigen reduzieren sollen. Insgesamt hat er sechs Skills zusammengestellt, die die Angehörigen sowohl in Phasen von Abstinenz als auch bei Rückfällen unterstützen sollen (Ligon, 2004, S. 96). Die sechs Strategien richten sich als therapeutische Leitlinien vor allem an Therapeuten, die mit Angehörigen von suchtkranken Menschen arbeiten. Nichtsdestotrotz 
handelt es sich bei den Skills auch um Bewältigungsstrategien, die Angehörige häufig selbst unabhängig von einer Beratung - anwenden (Candrian \& Ruckstuhl, 2009). Die Strategien werden im folgenden Kapitel vorgestellt.

\subsubsection{Die sechs Skills}

Ligon (2004) stellte sechs Skills zusammen, die die Angehörigen dabei unterstützen sollen, mit den Belastungen umzugehen und diese erfolgreich zu bewältigen. Nachfolgend werden die sechs Strategien kurz vorgestellt:

\section{Separation vom Problem, aber nicht von der Person}

Ligon (2004, S. 96) betont, dass es für Angehörige wichtig ist, zwischen der Suchterkrankung und dem suchtkranken Angehörigen differenzieren zu lernen. Dabei ist es von entscheidender Bedeutung, dass sie sich von der Suchterkrankung abgrenzen, ohne jedoch den Kontakt zum Betroffenen abzubrechen. Ligon (2004, S. 96) gibt folgendes Beispiel: Die drogenmissbrauchende Person wird zu einer Gefängnisstrafe verurteilt. Die Familie soll in diesem Fall ihren Angehörigen besuchen, aber keinesfalls Versuche unternehmen, diesen vorzeitig aus dem Vollzug herauszuholen.

\section{Setzen von klaren Regeln und Grenzen}

Angehörige sollen im Umgang mit dem drogenabhängigen Familienmitglied klare Regeln festlegen, die sie diesem auch mitteilen (Ligon, 2004, S. 97). Eine klare Regel könnte sein, dass Eltern beispielsweise dem drogenabhängigen Sohn verbieten, in die Familienwohnung zu gehen, wenn niemand zu Hause ist. Mit klaren Regeln setzen Angehörige Grenzen, die einen Schutz darstellen und gleichzeitig ihre eigenen Bedürfnisse widerspiegeln. Ligon (ebd.) merkt in diesem Zusammenhang an, dass gerade Eltern von erwachsenen suchtkranken Kindern häufig wieder in eine Rolle als Eltern von kleinen Kindern zurückfallen.

\section{Konsequentes Durchsetzen von Regeln}

Einmal aufgestellte Regeln sollten eingehalten werden und allenfalls durch entsprechende Massnahmen durchgesetzt werden (Ligon, 2004, S. 97). Ein konsequentes Durchsetzen von Regeln hilft dabei nicht nur den Angehörigen, sondern auch dem Suchtkranken, weil er dadurch weiss, wo seine Familie steht. Im Sinne des obigen Beispiels könnte es bedeuten, dass Eltern dem Sohn den Schlüssel für die Wohnung abnehmen oder das Schloss 
auswechseln lassen, wenn der Sohn entgegen der Regeln der Eltern trotzdem alleine in die Wohnung geht.

\section{Unterstützung von Abstinenz}

Angehörige sollen die Abstinenz ihrer suchtkranken Familienmitglieder unterstützen (Ligon, 2004, S. 97), d.h. wenn sich die drogenabhängige Person dazu entscheidet, in eine Therapie zu gehen, sollten Angehörige diese Bemühungen fördern. Es geht dabei auch darum, dass Angehörige sich darüber Gedanken machen, welche Verhaltensweisen die Suchterkrankung eher unterstützen und welche nicht (ebd.). Beispielsweise ist das Geben von Geld nicht sinnvoll, auch wenn es in bestimmten Situationen angemessen erscheint.

\section{Setzen von kleinen Zielen, die erreichbar sind}

Wichtig für Angehörige ist, dass sie in kleinen Schritten eine Veränderung einleiten (Ligon, 2004, S. 98). Sie sollen sich selbst deshalb nur Ziele setzen, die sie auch realisieren können. So macht es beispielsweise keinen Sinn, dass Angehörige die Regel aufstellen, dass bei ihnen zu Hause nicht konsumiert werden darf, wenn sie anschliessend nicht bereit sind die angedrohte Konsequenz, den drogenabhängigen Angehörigen rauszuwerfen, falls es zum Konsum kommt, umzusetzen. Angehörige sollen sich idealerweise in dem Bereich (Separation vom Problem, aber nicht der Person, Setzen von klaren Regeln und Grenzen, konsequentes Durchsetzen von Regeln, eigene körperliche und geistige Gesundheit nicht aus den Augen verlieren) Ziele setzen, wo sie den grössten Handlungsbedarf sehen (ebd.)

\section{Eigene körperliche und geistige Gesundheit nicht aus den Augen verlieren}

Für Angehörige ist es zur erfolgreichen Bewältigung der Belastungen wichtig, dass sie sich selbst und ihre eigenen Bedürfnisse nicht aus den Augen verlieren (Ligon, 2004, S. 98). Dazu gehört, dass sie genügend schlafen und essen und sich hin und wieder auch mal etwas Gutes tun oder etwas Schönes gönnen.

\subsection{Angebote für Angehörige}

\subsubsection{Einleitung}

Gemäss Peukert (2011, S. 66) können Interventionen für Angehörige und Familien im Bereich der Störungen durch psychotrope Substanzen in Bezug auf das primäre Ziel, das sie verfolgen, in drei Kategorien unterschieden werden:

- Ziel ist es, den Suchtkranken für eine Behandlung zu motivieren. 
- Angehörige werden im Rahmen der Behandlung des Suchtkranken einbezogen, z.B. zur Verbesserung der interaktionellen Schwierigkeiten, um den Behandlungserfolg des Suchtkranken längerfristig zu sichern.

- Mit der Intervention sollen vor allem die Angehörigen entlastet werden (Peukert, 2011, S. 66).

Die Ursprünge für die Unterstützung der Angehörigen liegen in der Selbsthilfe und sind ganz klar der letzten Kategorie zuzuordnen. Die Al-Anon Familiengruppen waren die ersten, die in den 50er Jahren Angehörigen von suchtkranken Menschen Hilfe anboten und mit einem 12Schritte-Programm ein erstes Angebot hatten, mit dem die Familienmitglieder entlastet werden konnten. Ansonsten gibt es bis jetzt praktisch keine Interventionen, die ihren primären Fokus auf die Belastungsreduktion der Angehörigen legen. Eine Ausnahme stellt dabei lediglich die ursprünglich aus England stammende „5-Step-Method“ dar (Copello, Ibanga, Orford, Templeton \& Velleman, 2010, S. 205).

Ungefähr zur gleichen Zeit als die Al-Anon Familiengruppen entstanden, breitete sich auch das systemische Denken in Therapie und Beratung aus. Die ersten Pioniere der Systemtheorie begannen, mit Paaren und Familien zu arbeiten (Schlippe \& Schweitzer, 2012, S. 32). Daraus entwickelten sich in der Folge diverse paar- und familientherapeutische Ansätze im Rahmen der Suchtbehandlung, die der zweiten Kategorie, dem Einbezug der Angehörigen in die Suchtbehandlung zugeordnet werden können. Dazu gehört beispielsweise die multidimensionale Familientherapie (Gantner, 2011), deren Zielgruppe drogenmissbrauchende und delinquente Jugendliche und ihre Familien sind oder die verhaltenstherapeutische Paartherapie (BCT) (O'Farrell \& Fals-Stewart, 2003; O'Farrell \& Clements, 2012; Peukert, 2011). Für beide dieser systemischen Ansätze liegen Studien (ebd.) vor, die ihnen eine positive Wirksamkeit bestätigen, einerseits in Bezug auf den Substanzkonsum des Betroffenen, andererseits aber auch in Bezug auf eine verbesserte Beziehungsqualität zwischen der substanzkonsumierenden Person und den Angehörigen.

In den 60er Jahren entstand schliesslich mit der Johnson Intervention der erste Ansatz, der mit Hilfe der Angehörigen die Betroffenen für eine Therapie gewinnen wollte (Fernandez, Begley \& Marlatt, 2006, S. 208). Mit der Intervention sollen therapieunwillige Substanzkonsumenten von ihrer Familien, Freunden oder Arbeitskollegen über Konfrontationen zur Behandlung motiviert werden. Der Ansatz ist vor allem in den Vereinigten Staaten sehr populär, konnte 
sich aber bei uns in Europa nie durchsetzen. Gründe dafür könnten kultureller Art sein. Das Community Reinforcement and Family Training (CRAFT), welches einige Jahre später entwickelt wurde, steht ebenfalls in dieser Tradition, wobei allerdings im Gegensatz zur Johnson Intervention zusätzlich auch die Befindlichkeit der Angehörigen Beachtung findet (Bischof, 2012, S. 30; Smith \& Meyers, 2013, S. 58ff).

In der Schweiz entstand unabhängig von diesen Interventionen ein eigenes Angebot. Aufgrund der starken Zunahme des Heroinkonsums in den 80er Jahren (Grob, 2012) entwickelte sich aus der Angehörigen-Selbsthilfe in Zürich eine professionelle Beratungsstelle, die sich ausschliesslich auf die Unterstützung von Angehörigen spezialisiert hat (Suhner \& Beck, 2012). Die Angehörigenvereinigung Drogenabhängiger Zürich (ada-zh) ist ebenfalls ein Angebot, das der letzten Kategorie, nämlich der primären Entlastung der Angehörigen, zugeordnet werden kann.

Im Rahmen der vorliegenden Arbeit sollen einige Ansätze und Angebote für Angehörige detaillierter vorgestellt werden. Die Al-Anon Familiengruppen, das CRAFT und die Angehörigenvereinigung Drogenabhängiger Zürich (ada-zh) sind Angebote, die es in der Schweiz bereits gibt. Sie werden deshalb in den nachfolgenden Kapiteln besprochen. Bei der „5-Step-Method“ handelt es sich um eine gut handhabbare Intervention, die den primären Fokus auf die Angehörigen selbst richtet (Copello, Ibanga \& Orford et al., 2010, S. 205). Aus diesem Grund soll auch sie an dieser Stelle besprochen werden, auch wenn es dieses Angebot in der Schweiz bis jetzt noch nicht gibt.

\subsubsection{Al-Anon}

\subsubsection{Einleitung}

Der Ursprung der Angehörigenselbsthilfe liegt bei den Al-Anon Familiengruppen. Heute sind die Al-Anon Familiengruppen die grösste, weltweit verbreitete Selbsthilfeorganisation für Angehörige von suchtkranken Menschen (Fernandez et al., 2006; O'Farrell \& Fals-Stewart, 2003). In der Schweiz sind sie seit über 40 Jahren tätig. Weltweit existieren mehr als 28'000 Al-Anon Familiengruppen in 115 Ländern (Timko, Young \& Moos, 2012, S. 282). Gemäss Fernandez et al. (2006, S. 208) verdienen die Al-Anon Familiengruppen aber auch noch aus weiteren Gründen Beachtung: Ihre Techniken und Arbeitsweisen bilden häufig die Grundlage für neue Zugänge und Ansätze zur Unterstützung von Angehörigen. Zudem werden die Al- 
Anon Familiengruppen immer wieder in Wirksamkeitsstudien als Kontrollgruppen herangezogen (ebd.). In den nachfolgenden Kapiteln sollen deshalb die Entstehung der Gruppen sowie die Technik, mit der sie arbeiten und die Wirksamkeit dargestellt werden.

\subsubsection{Entstehung und Selbstverständnis der Al-Anon}

Die Al-Anon Familiengruppen waren 1951 aus den Anonymen Alkoholikern hervorgegangen (Al-Anon Familiengruppen, Timko et al., 2012). Sie hatten sich als Reaktion auf die Anonymen Alkoholiker gebildet, weil die Frauen von Alkoholikern festgestellt hatten, dass auch sie aufgrund der Abhängigkeitserkrankung ihrer Männer selbst schwer belastet waren. Die Al-Anon übernahmen dabei die 12 Schritte der Anonymen Alkoholiker und adaptierten diese für eigene Zwecke (Rennert, 1990, S. 92; Timko et al., 2012, S. 281). Bereits kurz nachdem die Al-Anon Familiengruppen gegründet worden waren, entstanden weitere, so beispielsweise die Alateens für Kinder und Jugendliche von alkoholkranken Eltern oder die Nar-Anon für Angehörige von drogenabhängigen Menschen (Timko et al., 2012, S. 281).

Die Al-Anon Familiengruppen sind wie die meisten Selbsthilfeorganisationen als Verein organisiert. Ziel der Gruppen ist es in erster Linie, das Wohlbefinden und das Selbstwertgefühl der Angehörigen zu steigern und gleichzeitig eine grössere Unabhängigkeit vom alkoholkranken Angehörigen zu erreichen (Fernandez et al., 2006, S. 208). Dabei gehen die Mitglieder der Al-Anon Familiengruppen davon aus, dass sie an einem eigenen Krankheitsbild leiden, das es zu überwinden gilt (Al-Anon Familiengruppen). Der Begriff der Co-Abhängigkeit wird bei den Al-Anon jedoch nicht verwendet, obwohl inhaltlich eine grosse Übereinstimmung zwischen dem Krankheitsbegriff der Al-Anon und dem CoAbhängigkeitskonzept besteht wie folgender Beschrieb aus der Pressemitteilung der Al-Anon zeigt (ebd.):

Angehörige von Alkoholikern versuchen, den Konsum des Alkoholikers zu kontrollieren, decken sein übermässiges Trinken nach aussen, entschuldigen ihn, nehmen ihm die Verantwortung für sein Verhalten ab, indem sie sich in falsch verstandener Fürsorge um ihn kümmern. Dabei fixieren sie sich zwanghaft auf die Bedürfnisse des Alkoholikers, verlieren den Kontakt zu ihren eigenen Gefühlen und Bedürfnissen und entwickeln infolgedessen häufig krankhafte Verhaltensweisen, depressive und psychosomatische Störungen (Al-Anon Familiengruppen). 
Das Verständnis der Al-Anon Familiengruppen gegenüber den Abhängigkeitserkrankungen ist systemisch. Dadurch werden Suchterkrankungen in einem grösseren Zusammenhang verstanden, wobei sich das Individuum in einem System über mehrere Generationen zu verstehen versucht (Ruckstuhl, 2012, S. 16). Auf diese Weise kann die häufig sehr belastende Frage der Schuld relativiert werden.

\subsubsection{Ablauf der Meetings und die 12 Schritte und Traditionen}

Die Treffen beginnen häufig damit, dass einer oder mehrere der 12 Schritte und Traditionen vorgelesen werden (Timko et al., 2012, S. 282). Danach fokussiert sich die Gruppe auf einen der Schritte, den der Gruppenleiter zur Besprechung vorgibt. Die Teilnehmenden sind nicht dazu verpflichtet, in den Gruppen etwas zu sagen. Wenn sie sich aber dazu entscheiden, sich mitzuteilen, werden sie ermuntert, dies zu tun, ohne dass sie unterbrochen werden und ohne dass ihnen Ratschläge erteilt werden. Auf diese Weise entsteht eine respektvolle und vorurteilsfreie Atmosphäre.

Die 12 Schritte sollen die Angehörigen auf dem Weg der Besserung unterstützen. Sie sind darauf ausgelegt, dass sie in einem Jahr bearbeitet werden können. Nachfolgend sind die 12 Schritte der Al-Anon zur Veranschaulichung zusammengestellt (Al-Anon Familiengruppen, 1996a; www.al-anon.ch):

1. Wir haben zugegeben, dass wir Alkohol gegenüber machtlos sind und unser Leben nicht mehr meistern können.

2. Wir kamen zu dem Glauben, dass eine Macht, grösser als wir selbst, uns unsere geistige Gesundheit wiedergeben kann.

3. Wir fassten den Entschluss, unseren Willen und unser Leben der Sorge Gottes, wie wir Ihn verstanden, anzuvertrauen.

4. Wir machten eine gründliche und furchtlose Inventur von uns selbst.

5. Wir gestanden Gott, uns selbst und einem anderen Menschen die genaue Art unserer Verfehlung ein.

6. Wir wurden vorbehaltlos bereit, unsere Charakterfehler von Gott beseitigen zu lassen.

7. Demütig baten wir Ihn, uns von unseren Mängeln zu befreien.

8. Wir machten eine Liste aller Personen, denen wir Unrecht zugefügt hatten, und nahmen uns vor, es ihnen allen wieder gut zu machen. 
9. Wo immer möglich, bemühten wir uns aufrichtig um direkte Wiedergutmachung an ihnen, ausgenommen, es würde ihnen oder anderen Schaden daraus entstehen.

10. Wir fuhren fort, persönliche Inventur zu machen, und wenn wir Unrecht hatten, gaben wir es sofort zu.

11. Durch Gebet und Meditation suchten wir unseren bewussten Kontakt zu Gott - wie wir Ihn verstanden - zu verbessern. Wir baten Ihn nur, uns Seinen Willen für uns wissen zu lassen und uns die Kraft zu geben, den auszuführen.

12. Nachdem wir durch diese Schritte ein inneres Erwachen erlebt hatten, versuchten wir, diese Botschaft an andere weiterzugeben und uns in allen unseren Angelegenheiten nach diesen Grundsätzen zu richten.

Die 12 Schritte machen deutlich, dass die Al-Anon Familiengruppen stark die Machtlosigkeit gegenüber dem Substanzkonsum betonen. Angehörige können den Substanzkonsum ihrer abhängigen Familienmitglieder nicht kontrollieren und haben auch keinen Einfluss darauf, ob dieser seine Situation verändert oder nicht (Timko et al., 2012, S. 283). Folglich müssen sie lernen, loszulassen und sich auf sich selbst und ihre Bedürfnisse zu fokussieren (Denning, 2010, S. 165; Rennert, 1990, S. 100). Die Al-Anon Familiengruppen betonen die persönliche Verantwortung von jedem Individuum (Timko et al., 2012, S. 284). Von grosser Bedeutung bei den Al-Anon ist dabei das Vertrauen auf eine höhere Macht (Rennert, 1990, S. 96). Die 12 Schritte können beim Betrachter auf den ersten Blick den Eindruck erwecken, dass es sich bei den Al-Anon Familiengruppen um eine Gemeinschaft von religiösen Spinnern handelt. Dies ist jedoch nicht der Fall. Eine Meinung, die auch von der Verfasserin dieser Arbeit geteilt wird aufgrund der Teilnahme an einem offenen Meeting der Al-Anon Familiengruppen. Die Al-Anon Familiengruppen bezeichnen sich selbst auch nicht als religiöse, sondern als spirituelle Gemeinschaft (Al-Anon Familiengruppen, 2003). Gemäss Rennert (1990) handelt es sich bei der höheren Macht um Spiritualität ,als eine Qualität, die dem Leben eine Bedeutung, einen Sinn gibt“" (S.97). In diesem Sinne ist das Vertrauen auf eine höhere Macht auch mit Fragen wie nach dem Sinn des Lebens und eigenen Moral- und Wertvorstellungen verbunden und soll Angehörige dazu anregen, dass es Kräfte ausserhalb des eigenen Selbst gibt, sprich dass es nicht in ihrer Macht liegt, ob ihr Angehöriger wieder gesund wird oder nicht.

Eine wichtige Rolle spielen auch die 12 Traditionen. Diese wurden aufgestellt, um die Gruppen vor äusseren Einflüssen zu schützen, die sie davon abhalten könnten, ihre Ziele zu 
verfolgen (Timko et al., 2012). Folgende 12 Traditionen, die zur Veranschaulichung zusammengestellt sind, gelten bei den Al-Anon Familiengruppen (Al-Anon Familiengruppen, 1996a, Al-Anon Familiengruppen, 1996b):

1. Unser gemeinsames Wohlergehen sollte an erster Stelle stehen: persönlicher Fortschritt für möglichst viele hängt vom Einigsein ab.

2. Für das Anliegen unserer Gruppe gibt es nur eine Autorität: einen liebenden Gott wie Er sich im Gewissen der Gruppe zu erkennen geben mag. Unsere Sprecher und Sachbearbeiter sind dienende Vertrauensleute, sie herrschen nicht.

3. Wenn sich Verwandte von Alkoholikern zur gegenseitigen Hilfe versammeln, können sie sich Al-Anon Familiengruppe nennen, vorausgesetzt, dass sie - als Gruppe - keine andere Bindung haben. Die einzige Voraussetzung für die Zugehörigkeit ist, dass bei einem Verwandten oder Freund ein Alkoholproblem besteht.

4. Jede Gruppe sollte selbständig sein, ausser in Dingen, die eine andere Gruppe, AlAnon oder AA als Ganzes berührt.

5. Jede Al-Anon Familiengruppe hat nur ein Anliegen: den Familien von Alkoholkranken zu helfen. Wir tun dies dadurch, dass wir selbst die 12 Schritte der AA praktizieren, dadurch dass wir unsere alkoholkranken Angehörigen ermutigen und verstehen, und dadurch, dass uns Familienmitglieder von Alkoholikern willkommen sind und wir sie trösten.

6. Unsere Familiengruppen sollten niemals ein aussenstehendes Unternehmen unterstützen, finanzieren oder mit unserem Namen decken, damit uns nicht Geld-, Eigentums- oder Prestigeprobleme von unserem eigentlichen geistigen Ziel ablenken. Obwohl wir eine eigenständige Gemeinschaft sind, sollten wir doch immer mit den Anonymen Alkoholikern zusammenarbeiten.

7. Jede Gruppe sollte sich selbst erhalten und von aussen kommende Zuwendungen ablehnen.

8. Al-Anon Arbeit im 12. Schritt sollte für immer ausserberuflich bleiben, unsere Dienstzentren dürfen jedoch Fachkräfte anstellen.

9. Unsere Gruppen als solche sollten niemals organisiert werden. Aber wir dürfen Dienstgremien oder Komitees bilden, die denjenigen direkt verantwortlich sind, denen sie dienen. 
10. Die Al-Anon Familiengruppen nehmen zu Fragen ausserhalb ihrer Gemeinschaft nicht Stellung. Folglich sollte unser Namen nie in öffentliche Auseinandersetzungen hineingezogen werden.

11. Unser Auftreten in der Öffentlichkeit stützt sich mehr auf Anziehung als auf Werbung. Wir haben stets persönliche Anonymität gegenüber Presse, Rundfunk, Film und Fernsehen zu wahren. Wir müssen mit ganz besonderer Sorgfalt die Anonymität aller Anonymen Alkoholiker schützen.

12. Anonymität ist die geistige Grundlage aller unserer Traditionen, die uns immer daran erinnern soll, Prinzipien über Personen zu stellen.

Im Vordergrund der Traditionen steht, dass die Anonymität der Mitglieder geschützt wird (Al-Anon Familiengruppen, 1996a). So sprechen sich die Gruppenteilnehmenden immer nur mit Vornamen an und die Organisation tritt nie mit Namen von ihren Mitgliedern in die Öffentlichkeit. Obwohl die Al-Anon Familiengruppen grossen Wert auf Anonymität legen, zeichnen sie sich dennoch durch eine grosse Offenheit aus. So werden in regelmässigen Abständen offene Meetings veranstaltet, bei denen Interessierte teilnehmen können, ohne sich selbst als betroffen outen zu müssen.

Ein weiterer wichtiger Grundsatz, der in den Traditionen der Al-Anon Familiengruppen verankert ist, ist die Unabhängigkeit der Gruppen (Al-Anon Familiengruppen, 1996a). Die Gruppen erhalten sich selbst über freiwillige Spenden, die jeweils am Ende des Meetings über einen Spendenkorb gesammelt werden. Beiträge oder Spenden von ausserhalb der Gemeinschaft werden abgelehnt.

\subsubsection{Empirische Überprüfung}

Die Wirksamkeit der Al-Anon Familiengruppen ist gut belegt, weil sie in Studien, die die Wirksamkeit anderer Verfahren belegen wollten, oft als Kontrollgruppe eingesetzt wurden (Kirby, Marlowe, Festinger, Garvey \& LaMonaca, 1999; Meyers, Miller, Smith \& Tonigan, 2002; Miller, Meyers \& Tonigan, 1999). Alle diese Studien belegen die positive Wirksamkeit der Al-Anon Familiengruppen. Rychtarik und McGillicuddy (2005) stellten in ihrer Untersuchung fest, dass sich die Lebenssituation (Abnahme der Depressionswerte) der Angehörigen unabhängig von der Situation des Suchtkranken verbesserte (Rychtarik \& McGillicuddy, 2005). Miller et al. (1999) konnten zudem nachweisen, dass die Teilnahme an 
einer Al-Anon Familiengruppe zu einer Verbesserung der familiären Beziehungen führte und eine Reduktion von depressiven Symptomen, Konflikten und Wut zur Folge hatte.

Die qualitative Analyse eines Online-Al-Anon-Meetings zeigte zudem, dass gerade die Spiritualität eine entscheidende Rolle dabei spielt, dass sich die Situation der Angehörigen verbessert (Roth \& Tan, 2007; Roth \& Tan, 2008).

\subsubsection{Community reinforcement and family training (CRAFT)}

\subsubsection{Einleitung}

Das CRAFT ist eine im deutschsprachigen Raum neuere Methode. Es war im Rahmen des Community Reinforcement Approach (CRA) entwickelt worden (Meyers, Miller \& Smith, 2001). Der Ansatz erfreut sich mittlerweile im deutschsprachigen Raum grosser Popularität. Seit kurzem ist das Manual deshalb auch in deutscher Sprache erhältlich (Smith \& Meyers, 2013) und es werden auch Schulungen im deutschsprachigen Raum angeboten (www.gkquest.de/seminare). Dies hat dazu geführt, dass der Ansatz Fachleuten in der Zwischenzeit gut bekannt ist und auch aktiv angewendet wird. Das Training ist zudem wissenschaftlich sehr gut untersucht. In den nachfolgenden Kapiteln werden der theoretische Hintergrund, die Module des verhaltenstherapeutischen Manuals und die empirischen Belege des CRAFT vorgestellt.

\subsubsection{Theoretischer Hintergrund}

Der Community Reinforcement Approach (CRA), von dem das CRAFT eine Teilanwendung ist, ist in den 70er Jahren entwickelt worden (Meyers \& Godley, 2001, S.1). Der verhaltenstherapeutische Ansatz geht davon aus, dass die Faktoren für den Verlauf der Suchterkrankung in der Umwelt des Betroffenen zu suchen sind. Dabei soll über positive Verstärkung und negative Konsequenzen ein abstinentes Verhalten gegenüber einem Konsumverhalten gestärkt werden. Bis zu Beginn der 80er Jahre gab es keinen Ansatz für Angehörige, der sie dabei unterstützte, das therapieunwilligen Familienmitglied zur Behandlungsaufnahme zu motivieren (Meyers \& Miller, 2001, S. 147). In diesem Zusammenhang vertrat der CRA einen anderen Standpunkt, nämlich dass Angehörige eine entscheidende Rolle in der Therapie von suchtkranken Menschen spielen (ebd.). Das CRAFT, welches für Angehörige entwickelt wurde, griff dabei auf die Konzepte des CRA (Vermittlung von Fertigkeiten, die die Wahrscheinlichkeit einer Behandlungsaufnahme beim 
Betroffenen erhöhen und die Lebensqualität der Angehörigen verbessern) zurück und richtet sich an Angehörige von Suchtkranken, die aktuell nicht zu einer Behandlung bereit sind.

Mit dem CRAFT werden folgende drei Ziele verfolgt (Bischof, 2012, S. 30; Smith \& Meyers, 2013, S. 58ff):

1. Reduktion des Substanzkonsums beim Betroffenen

2. Behandlungsaufnahme der substanzkonsumierenden Person

3. Verbesserung der Lebensqualität der Angehörigen unabhängig von der Behandlungsaufnahme der betroffenen Person

Das Training der Angehörigen erfolgt normalerweise in einem ambulanten Einzelsetting, wobei die Intervention auf 12 Sitzungen ausgelegt ist (Bischof, 2012, S. 32). Gemäss Bischof (ebd.) gibt es derzeit noch keine strukturierten Manuale für Gruppensettings. In den Vereinigten Staaten wurde allerdings bereits ein Versuch unternommen, das Manual in einer Gruppe einzusetzen (Manuel, Austin, Miller, McCrady, Tonigan, Meyers et al., 2012). Der Versuch brachte positive Erfahrungen mit sich. Das CRAFT ist aber auch als Selbsthilfebuch erhältlich.

\subsubsection{Die Module des CRAFT}

Nachfolgend werden die einzelnen Module, die während der CRAFT-Intervention bearbeitet werden, kurz besprochen.

\section{Motivieren des Angehörigen}

Bevor mit dem eigentlichen Training begonnen wird, wird zuerst Zeit investiert, bei den Angehörigen eine Motivation aufzubauen, damit sie regelmässig am Programm teilnehmen und eine Zuversicht entwickeln, dass sie mittels CRAFT etwas bei ihren suchtkranken Angehörigen verändern können (Bischof \& Freyer, 2006, S. 54; Bischof, 2012, S. 30; Bischof, Iwen, Müller, Freyer-Adam \& Rumpf, 2007, S. 55; Brueck, 2011, S. 69; Meyers et al., 2001, S. 151; Smith \& Meyers, 2013, S. 30ff). Bei dieser Vermittlung von Zuversicht wird bewusst auf die positiven Ergebnisse aus den Studien hingewiesen. Gleichzeitig ist es aber auch eine wichtige Aufgabe während des ganzen Trainings, den Angehörigen immer wieder bewusst zu machen, dass sie nicht für das Verhalten des Substanzmissbrauchers verantwortlich sind. Der Therapeut nimmt in den Sitzungen eine unterstützende, empathische 
und unvoreingenommene Haltung ein und setzt immer wieder verbale Verstärkungen im Gespräch ein.

\section{Gewaltprävention}

In Familien mit Alkohol- und Drogenmissbrauch kommt es häufig zu häuslicher Gewalt (Moore \& Stuart, 2004; Murphy et al., 2001; Mattson, O'Farrell, Lofgreen, Cunningham \& Murphy, 2012) (siehe Kapitel 2.2.2.5.). Durch eine Verhaltensänderung kann das Risiko für Gewalt noch zunehmen. Aus diesem Grund ist es unerlässlich, dass Angehörige auf Gewalterfahrungen, die sie im Zusammenhang mit ihrem suchtkranken Angehörigen bereits erlebt haben, angesprochen werden. Ist es in der Familie bereits zu Gewalt gekommen, macht der CRAFT-Therapeut mit dem Klienten gemeinsam eine funktionale Verhaltensanalyse, um Muster von aggressivem Verhalten erkennbar und besser vorhersehbar zu machen (Bischof \& Freyer, 2006, S. 54; Bischof, 2012, S. 31; Bischof et al., 2007, S. 56; Brueck, 2011, S. 70; Meyers et al., 2001, S. 152; Smith \& Meyers, 2013, S. 114ff). Mit den Angehörigen, die in der Vergangenheit von Gewalt betroffen waren, werden Handlungsstrategien erarbeitet, die sie bei drohender Gewalt anwenden können. Gleichzeitig wird mit den Angehörigen aber auch ein Notfallplan erarbeitet, zu dem auch soziale Unterstützungssysteme gehören, die in einer Krisensituation aktiviert werden könnten.

\section{Funktionale Analyse des Konsums}

Die funktionale Verhaltensanalyse des Substanzkonsums stellt ein Kernstück der Intervention dar (Bischof \& Freyer, 2006, S. 54; Bischof, 2012, S. 31; Bischof et al., 2007, S. 56; Brueck, 2011, S. 70; Meyers et al., 2001, S. 154; Smith \& Meyers, 2013, S. 68ff). Dabei wird gemeinsam mit den Angehörigen nach einer typischen Konsumsituation gesucht, die schliesslich auf äussere und innere Auslöser hin untersucht wird. Zusätzlich werden auch kurzfristig positive und langfristig negative Konsequenzen erhoben. In dieser Analyse zeigen sich meist bereits erste Momente, die den Konsum auslösen und die beeinflusst werden können (ebd.). Beachtet werden dabei auch die Bedingungen, die den Konsum verstärken und mögliche Gründe, die zu einer Veränderung führen könnten, wobei die Änderungsgründe auch der substanzkonsumierenden Person bewusst und für sie von Bedeutung sein müssen. Meist wird die Verhaltensanalyse in einem ersten Schritt mit offenen Fragen erhoben und dann in einem zweiten Schritt auf einem Arbeitsblatt schriftlich festgehalten. Zu diesem Zeitpunkt wird noch keine Verhaltensänderung angestrebt, dies geschieht erst nachdem weitere Module bearbeitet wurden. 


\section{Kommunikationstraining}

Das Kommunikationstraining soll einen Beitrag dazu leisten, dass es zu einer allgemeinen Verbesserung der Lebensqualität kommt. Daneben sind aber die Auseinandersetzungen zwischen Angehörigen und ihren abhängigkeitserkrankten Familienmitgliedern häufig durch negative und undifferenzierte Kommunikationsmuster geprägt, die durch das Kommunikationstraining verändert werden sollen (Bischof \& Freyer, 2006, S. 54; Bischof, 2012, S. 31; Bischof et al., 2007, S. 56; Brueck, 2011, S. 70; Meyers et al., 2001, S. 155; Smith \& Meyers, 2013, S. 152ff). Zudem es ist wichtig, dass Angehörige über positive kommunikative Fertigkeiten verfügen bei der Verfolgung der Ziele des CRAFT. Die neuen kommunikativen Fertigkeiten werden psychoedukativ vermittelt und über Rollenspiele eingeübt.

\section{Positive Verstärkung}

Eine der zentralen Strategien, um eine Verhaltensänderung beim substanzmissbrauchenden Angehörigen $\mathrm{zu}$ erreichen ist die positive Verstärkung im Sinne einer operanten Konditionierung nach Skinner (Bischof \& Freyer, 2006, S. 54; Bischof, 2012, S. 31; Bischof et al., 2007, S. 57; Brueck, 2011, S. 70; Meyers et al., 2001, S. 155ff; Smith \& Meyers, 2013, S. 184ff). Dabei erhöhen die Konsequenzen eines Verhaltens, die als positiv erlebt werden, die Wahrscheinlichkeit, dass ein Verhalten wiederholt wird. Positive Konsequenz des Substanzkonsums kann beispielsweise Entspannung sein. Es gilt nun positive Verstärker zu definieren, die eingesetzt werden können, wenn der suchtkranke Angehörige nicht konsumiert hat, dazu gehören beispielsweise Lob, Zuwendung oder gemeinsame Aktivitäten (ebd.). Nach der Wahl der geeigneten Verstärker geht es schliesslich auch darum, Verhaltensweisen zu identifizieren, die verstärkt werden sollen, wobei diese möglichst unvereinbar mit dem Substanzkonsum sein sollten. Es ist wichtig, dass dem substanzkonsumierenden Angehörigen der Zusammenhang zwischen abstinentem Verhalten und positiver Verstärkung deutlich aufgezeigt wird. Das Kommunikationstraining unterstützt die Angehörigen dabei.

\section{Nutzung negativer Konsequenzen}

„Das Prinzip der Nutzung negativer Konsequenzen bezieht sich sowohl auf die eigene Interaktion mit der substanzmissbrauchenden Person als auch darauf, natürliche Konsequenzen des Substanzmissbrauchs nicht abzumildern (indem z.B. ein alkoholassoziierter Fehltag bei der Arbeit durch den Angehörigen nicht gedeckt wird)“ (Bischof \& Freyer, 2006, S. 54). Auf der Interaktionsebene bedeutet die Nutzung negativer 
Konsequenzen, dass bei Konsumverhalten positive Verstärker entzogen werden, die für den suchtkranken Angehörigen bedeutsam sind und durch die Angehörigen ohne grössere Schwierigkeiten zeitnah umgesetzt werden können (ebd.). So könnte beispielsweise eine positive, geplante Aktivität wieder abgesagt werden oder Angehörige unternehmen alleine etwas Schönes. Auch hier wird dem substanzkonsumierenden Angehörigen der Zusammenhang zwischen negativer Konsequenz und Substanzkonsum deutlich aufgezeigt.

Beim Zulassen der natürlichen negativen Konsequenzen des Substanzkonsums müssen Angehörigen lernen, dass sie nichts unternehmen dürfen, um die negativen Folgen des Konsums in irgendeiner Form abzumildern (Bischof \& Freyer, 2006, S. 55; Bischof, 2012, S. 31; Bischof et al., 2007, S. 57; Brueck, 2011, S. 71; Meyers et al., 2001, S. 157ff; Smith \& Meyers, 2013, S. 233ff). Den Angehörigen wird dabei vermittelt, dass das Verhindern von negativen Konsequenzen die Wahrscheinlichkeit reduziert, dass jemand sein Verhalten verändert, wie es bereits bei diversen Co-Abhängigkeitskonzepten postuliert worden ist. Notwendige Strategien werden dabei mit Rollenspielen eingeübt.

\section{Strategien zur Verbesserung der Lebensqualität}

Eines der Ziele des CRAFT ist die Verbesserung der Lebensqualität der Angehörigen. In einem ersten Schritt geht es dabei darum, bei den Angehörigen Lebensbereiche zu identifizieren, in denen Schwierigkeiten bestehen (Bischof \& Freyer, 2006, S. 55; Bischof, 2012, S. 31; Bischof et al., 2007, S. 58; Brueck, 2011, S. 71; Meyers et al., 2001, S. 158; Smith \& Meyers, 2013, S. 264ff). Dazu gehören beispielsweise finanzielle oder rechtliche Schwierigkeiten, Belastungen in Beziehungen, das eigene emotionale Erleben und weitere. Mit dem Angehörigen werden dabei konkrete Ziele definiert und Kriterien für den Grad der Zielerreichung festgelegt. Ziel ist es, das soziale Unterstützungssystem der Angehörigen zu vergrössern und die Angehörigen zu mehr angenehmen und eigenständigen Aktivitäten zu motivieren.

\section{Die suchtkranke Person zur Inanspruchnahme von Hilfe motivieren}

Ein weiteres zentrales Ziel des CRAFT ist es, den suchtkranken Angehörigen zu einer Behandlungsaufnahme zu motivieren (Bischof \& Freyer, 2006, S. 55; Bischof, 2012, S. 3132; Bischof et al., 2007, S. 58; Brueck, 2011, S. 71; Meyers et al., 2001, S. 158ff; Smith \& Meyers, 2013, S. 298ff). Aus diesem Grund werden parallel zum CRAFT Behandlungsmöglichkeiten angeschaut, um bei einer Behandlungsbereitschaft des 
Suchtkranken sofort Vorschläge machen zu können. Mit Angehörigen wird zudem besprochen, welche Situationen sich besonders eignen, um mittels positiver Kommunikation eine Behandlungsaufnahme anzusprechen (ebd.). Gleichzeitig werden aber die Angehörigen auch darauf vorbereitet, dass sich ihr Familienmitglied zu einer Behandlungsaufnahme weigern könnte oder dass es zu einem Therapieabbruch kommen könnte.

\subsubsection{Chancen und Grenzen des CRAFT}

Während die Art der Beziehung, in der die Angehörigen zum Suchtkranken stehen, nicht relevant ist, ist dagegen eine der Voraussetzungen für das Training, dass Angehörige einen ausreichenden Kontakt zum Betroffenen haben. Gemäss Bischof (2012, S. 32) sind in Studien mind. 20 Stunden pro Woche für die erfolgreiche Durchführung als notwendig erachtet worden. In der Studie von Kirby et al. (1999, S. 87) mussten die Angehörigen mindestens dreimal in der Woche Kontakt zum Suchtkranken haben, um in die Studie aufgenommen zu werden. Nur wenn Angehörige einen genügend engen Kontakt pflegen, können sie Auskunft über das Konsumverhalten für die Verhaltensanalyse geben und gleichzeitig auch das Verhalten des Suchtkranken über positive Verstärkung und negative Konsequenzen beeinflussen. CRAFT stösst deshalb an Grenzen, wenn die Angehörigen nur wenig Kontakt zum Suchtkranken haben oder wenn es ihnen in erster Linie um eine bessere Abgrenzung von diesem geht (Bischof, 2012, S. 32).

Nichtsdestotrotz ist der Ansatz insbesondere für Angehörige, die aktiv etwas unternehmen möchten, um ihren suchtkranken Angehörigen zur Therapie zu motivieren, sehr gut geeignet und schliesst an dieser Stelle auch eine Angebotslücke.

\subsubsection{Empirische Überprüfung}

Das CRAFT wurde in mehreren kontrollierten Studien bereits überprüft (Kirby et al., 1999, Meyers, Miller, Hill \& Tonigan, 1998, Meyers et al., 2002, Miller et al., 1999). Es zeigt insbesondere eine hohe Überlegenheit gegenüber anderen Ansätzen wie der Johnson Intervention und den Al-Anon Familiengruppen, die meist als Kontrollbedingungen herangezogen wurden. Diese hohe Überlegenheit bezieht sich insbesondere auf die Behandlungsaufnahme der suchtmittelmissbrauchenden Person, wobei sich das Training bei verschiedenen Substanzen bewährt hat. So zeigte sich beispielsweise in der Studie von Kirby et al. (1999, S. 91), dass $64 \%$ der Angehörigen ihren drogenabhängigen Angehörigen 
während der zehnwöchigen Intervention zu einer Behandlungsaufnahme hatten motivieren können. Gleichzeitig bestätigten alle Studien (Kirby et al., 1999, Meyers et al., 1998, Meyers et al., 2002, Miller et al., 1999), dass nicht nur die Wahrscheinlichkeit der Behandlungsaufnahme durch das Training beeinflusst werden konnte, sondern dass es auch den Angehörigen selbst nach der Intervention besser ging.

In der Zwischenzeit existiert zudem eine Metaanalyse, in der allerdings nur vier Studien eingeschlossen wurden (Roozen, Waart \& van der Kroft, 2010). In allen Studien, die in die Metaanalyse eingeschlossen worden waren, wurden die Interventionen Al-Anon versus CRAFT und Johnson Intervention versus CRAFT in Bezug auf die Behandlungsaufnahme des suchtkranken Angehörigen und das Funktionsniveau der Angehörigen verglichen. Wie bereits erwähnt zeigte sich, dass CRAFT gegenüber den beiden anderen Ansätzen deutlich überlegen war, was die Behandlungsaufnahme der substanzkonsumierenden Person betrifft (ebd., S. 1734). Innerhalb von sechs Monaten liess sich zudem eine deutliche Verbesserung in den familiären Beziehungen, bei den depressiven Symptomen und der Wut gegenüber dem Suchtkranken feststellen (ebd.). Dies war unabhängig davon, ob der Betroffene eine Behandlung aufnahm oder nicht. Die Ergebnisse in Bezug auf das soziale Funktionsniveau der Angehörigen waren vergleichbar mit denjenigen der Al-Anon Familiengruppen und der Johnson Intervention.

\subsubsection{5-Step-Method}

\subsubsection{Einleitung}

Ein neuerer Ansatz stellt die ,5-Step-Method“ dar. Diese Intervention ist einer der ersten Ansätze, der den primären Fokus auf den Angehörigen von suchtkranken Menschen legt (Copello, Ibanga \& Orford et al., 2010, S. 205). In den folgenden Kapiteln sind der theoretische Hintergrund, die einzelnen Schritte der Methode und die empirischen Befunde der Intervention beschrieben.

\subsubsection{Theoretischer Hintergrund}

Die 5-Step Intervention wurde basierend auf wissenschaftlichen Befunden zu Angehörigen von suchtkranken Menschen entwickelt (Copello et al., 2000, S. 331). Den theoretischen Hintergrund bildete das Stress-Coping-Health Modell (ebd.), welches zum Stress-StrainCoping-Support (SSCS) Modell (Orford, Templeton, Velleman \& Copello, 2010; Orford, 
Copello, Velleman \& Templeton, 2010) weiterentwickelt wurde (siehe Abb. 2). Das Modell ist in der Tradition der Stress-Coping Modelle zu sehen und postuliert, dass Angehörige normale Menschen sind, die eine äusserst belastende Situation bewältigen müssen. Mit dem Stress-Strain-Coping-Support Modell grenzen sich die Autoren (Orford \& Copello et al., 2010, S. 38) deutlich von älteren Modellen ab, die davon ausgehen, dass Angehörige von suchtkranken Menschen über eine eigene Pathologie verfügen, die die Abhängigkeitserkrankung erst ermöglicht und somit auch mit verursacht hat (siehe Kap. 2.1.3 zu Co-Abhängigkeit).

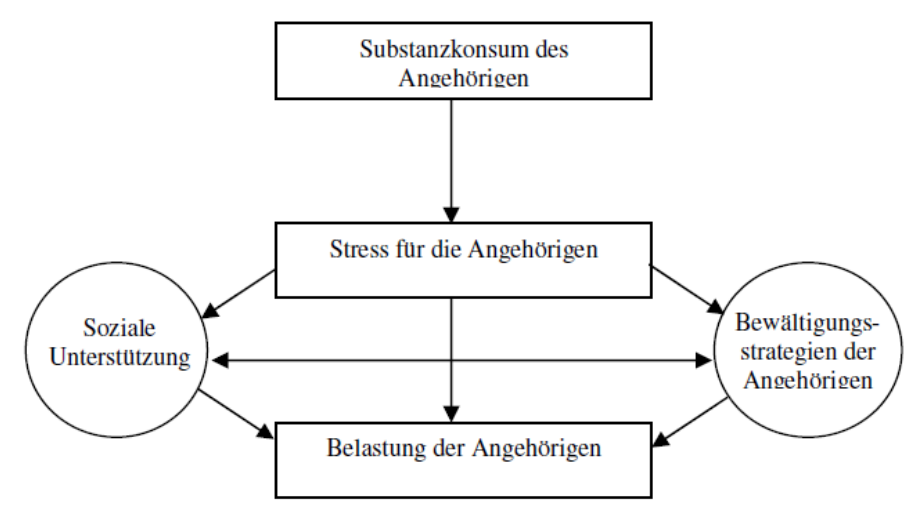

Abb. 2: Stress-Strain-Coping-Support (SSCS) Modell modifiziert nach Orford \& Copello et al. (2010)

Das Stress-Strain-Coping-Support Modell betont insbesondere die gesundheitlichen Auswirkungen, die die Suchterkrankung auf die Angehörigen hat (Orford \& Copello et al., 2010, S. 40). Das Modell postuliert dabei einen klaren Zusammenhang zwischen Ursache und Effekt. Es geht nämlich davon aus, dass die Abhängigkeitserkrankung als chronischer Stressor für Angehörige das Risiko mit sich bringt, dass sie selbst gesundheitliche Probleme bekommen (ebd.). Diese Annahme stützt sich auf Studien, die bestätigt haben, dass Angehörige von suchtkranken Menschen eine höhere Rate an physischen und psychischen gesundheitlichen Problemen haben (Orford \& Velleman et al., 2010, S. 50; Ray et al., 2007) (siehe auch Kap. 2.2.3.2).

Das SSCS Modell geht davon aus, dass Angehörige oft nicht wissen, wie sie am besten auf den Substanzkonsum und ihr betroffenes Familienmitglied reagieren sollen. Die dritte Komponente des Modells befasst sich deshalb mit den Bewältigungsstrategien (Orford \& Copello et al., 2010, S. 41). Die Angehörigen suchen nach Möglichkeiten der Bewältigung, die für sie persönlich den Stress und folglich auch die Belastungen reduzieren. Die Umstände, in denen Angehörige leben und die Ressourcen, die ihnen zur Verfügung stehen, beeinflussen 
dabei massgeblich die Bewältigungsstrategien (ebd.). Das Stress-Strain-Coping-Support Modell geht von der Annahme aus, dass Angehörige nicht vollkommen machtlos sind, sondern etwas tun können, um ihre gesundheitlichen Risiken zu reduzieren und gleichzeitig auch Einfluss auf ihren suchtkranken Angehörigen nehmen können.

Der vierte Aspekt des SSCS Modells ist die Unterstützung, insbesondere die soziale Unterstützung. Forschung hat in der Vergangenheit gezeigt, dass gute soziale Unterstützung einen entscheidenden Einfluss auf die Gesundheit hat (Cohen \& Wills, 1985). Folglich gingen auch Orford, Copello et al. (2010, S. 41) davon aus, dass eine gute soziale Unterstützung eine wichtige Ressource für die Bewältigung des Stresses der Angehörigen darstellt. Coping und soziale Unterstützung sind deshalb im Modell auch miteinander verknüpft. Wichtig bei der sozialen Unterstützung ist die Qualität und nicht nur die Grösse des sozialen Netzwerkes. Häufig bestehen viele Barrieren, die das Nutzen von sozialer Unterstützung verunmöglichen, beispielsweise dadurch, dass sich verschiedene Familienangehörige nicht einig sind, wie man sich gegenüber dem suchtkranken Familienmitglied verhalten soll (ebd.).

Die 5-Step Intervention, die basierend auf dem SSCS Modell entwickelt wurde, ist grundsätzlich von jedem Mitarbeiter im Gesundheitssystem anwendbar, ohne dass es ein langes Training oder eine therapeutische Weiterbildung voraussetzen würde (Copello, Templeton, Orford \& Velleman, 2010b, S. 89). Ziel ist es, dass mit der Intervention möglichst viele Angehörige auf unkomplizierte Art erreicht werden können, um so die gesundheitlichen Risiken der Angehörigen zu reduzieren.

\subsubsection{Die fünf Schritte}

Die ,5-Step-Method“ ist wie es der Name bereits andeutet in fünf Schritte unterteilt. Sie sind nachfolgend kurz zusammengefasst:

\section{Durch vorurteilsfreies Zuhören Problem- und Belastungsbereiche explorieren}

In einem ersten Schritt geht es darum, den Angehörigen kennen zu lernen und in Erfahrung zu bringen, in welchen Bereichen die grössten Belastungen vorliegen und wie sich diese äussern (Copello et al., 2000, S. 332ff; Copello, Templeton \& Orford et al., 2010b, S. 89-90). Dabei ist es hilfreich, wenn die Person, die die Intervention durchführt, weiss, in welchen Bereichen, Angehörige von suchtkranken Menschen Belastungen erleben können. Es ist ihre Aufgabe in einem ersten Gespräch mögliche Problembereiche anzusprechen und gleichzeitig den 
Eindruck zu vermitteln, dass diese Probleme normal sind und häufig in suchtbelasteten Familien vorkommen (ebd.). Der Interviewer sollte im Gespräch aktiv und vorurteilsfrei zuhören und Angehörige ermuntern zu berichten und ihre Gefühle auszudrücken. Wichtig ist zudem die empathische Haltung des Therapeuten. Den Angehörigen sollte im Gespräch ein realistischer Optimismus vermittelt werden, dass sie ihre Situation verändern können (ebd.).

\section{Vermittlung relevanter Informationen}

Copello et al. (2000, S. 333; 2010b; 90-91) betonen, dass fehlendes oder falsches Wissen zu Alkohol, Drogen und Abhängigkeitserkrankungen einen erheblichen Stressfaktor darstellen. Es ist deshalb im zweiten Schritt wichtig, mit den Angehörigen zu klären, über welches Wissen sie zu den Substanzen, die die ihnen nahestehende Person konsumiert, verfügen. Es gilt abzuklären, ob sie die Auswirkungen des ständigen Konsums kennen und ob sie über die Entstehung eines Abhängigkeitssyndroms Bescheid wissen. Beim Vermitteln der Informationen muss darauf geachtet werden, dass den Angehörigen nicht zu wenige Informationen abgegeben werden, was Anlass zu weiteren Sorgen geben könnte. Gleichzeitig sollten aber auch nicht zu viele Informationen abgegeben werden, weil die Angehörigen dadurch überfordert werden könnten (ebd.).

\section{Diskussion unterschiedlicher Möglichkeiten von Coping}

Gemäss Copello, Templeton, Orford et al. (2010, S. 91) werden unter Coping alle Gefühle, Verhaltensweisen und Haltungen verstanden, die Angehörige als Reaktion auf die Suchterkrankung angeben. Dabei werden folgende drei Arten der Bewältigung unterschieden: Toleranz, Entzug (Unabhängigkeit) und Aufbegehren (Orford et al., 1998e). Diese drei Strategien lassen sich weiter differenzieren und wurden in Kap. 2.3.2.2 ausführlich beschrieben. Alle drei Bewältigungsstrategien sind dabei mit Vor- und Nachteilen verbunden. Dies löst bei den Angehörigen eine Ambivalenz und Unsicherheit aus bei der Frage, wie sie sich am besten verhalten sollen. Ziel des dritten Schrittes ist es deshalb, mit den Angehörigen die Vor- und Nachteile ihres aktuellen Copings zu besprechen und die Vor- und Nachteile anderer alternativer Bewältigungsstrategien zu diskutieren (Copello et al., 2000, S. 335; Copello, Templeton \& Orford et al., 2010b, S. 91). Dabei ist es auch wichtig, dass die Angehörigen darüber aufgeklärt werden, dass die Bewältigungsstrategien der Toleranz gegenüber dem Substanzkonsum mit den grössten psychischen und physischen Gesundheitsrisiken assoziiert sind (Orford et al., 2001). Grundsätzlich soll aber den 
Angehörigen freigestellt werden, welche Art des Umgangs mit der Suchterkrankung für sie passend ist.

\section{Exploration von sozialer Unterstuitzung}

Die ,5-Step-Method“ betont die Bedeutung von sozialer Unterstützung für den Umgang mit den Belastungen und die Entlastung der Angehörigen allgemein (Copello et al., 2000, S. 336; Copello, Templeton \& Orford et al., 2010b, S. 94ff). Im vierten Schritt geht es deshalb darum, mit den Angehörigen zu explorieren, wo sie soziale Unterstützung bekommen und wie sie allenfalls ihr Netzwerk an sozialer Unterstützung weiter ausbauen können (ebd.). Copello, Templeton, Orford et al. (2010, S. 94) empfehlen in diesem Zusammenhang auch ein soziales Netzwerkdiagramm zu erstellen.

\section{Exploration weiterer Bedürfnisse und Vermittlung weiterer Unterstützung}

Copello et al. (2000, S. 338; 2010b, S. 96) betonen, dass in den meisten Fällen diese Kurzintervention ausreichend ist und ein grosser Teil der Angehörigen keine weitere Unterstützung braucht. Nichtsdestotrotz kann es vorkommen, dass es nach dem Durchführen der ,5-Step-Method“ noch offene Fragen gibt, die eine Vermittlung der Angehörigen an eine weitere Stelle notwendig machen. Folgende Szenarios sind dabei möglich (Copello et al., 2000, S. 338; Copello, Templeton \& Orford et al., 2010b, S. 96):

- Weitere Unterstützung für den Angehörigen

- Weitere Unterstützung für den Suchtkranken

- Weitere Unterstützung für die ganze Familie

- Weitere Unterstützung für andere Familienangehörige, die nicht an der Intervention teilnahmen

So können Angehörige beispielsweise den Wunsch äussern, dass sie sich weiter damit auseinandersetzten möchten, wie sie sich besser abgrenzen können. Eine weitere Möglichkeit wäre aber auch, dass Angehörige sich gerne in Bezug auf finanzielle Angelegenheiten beraten lassen würden usw.

\subsubsection{Chancen und Risiken der 5-Step-Method}

Die 5-Step Intervention ist so entwickelt worden, dass sie in Institutionen mit unterschiedlichem Hintergrund und verschiedenen Settings eingesetzt werden kann (Copello, 
Templeton \& Orford et al., 2010b, S. 88). Grundsätzlich ist es auch möglich die Intervention, bei der ein Selbsthilfemanual abgegeben wird, in einer einzigen Sitzung durchzuführen (Copello et al., 2009). Dabei hatte sich allerdings gezeigt, dass besonders der erste Schritt des vorurteilsfreien Zuhörens von grosser Bedeutung ist und genügend Zeit darauf verwendet werden sollte. Der Vorteil des Verfahrens liegt folglich ganz klar darin, dass ohne grossen Aufwand, sprich ohne ein jahrelanges Training und ohne spezielle therapeutische Weiterbildung die Intervention durchgeführt werden kann.

Obwohl der Ansatz leicht zu erlernen ist und auch so entwickelt wurde, dass Personen, die in der primären Gesundheitsversorgung arbeiten, den Ansatz anwenden können, besteht vor allem die Schwierigkeit in der Implementierung des Ansatzes in Institutionen und Diensten, die ihre primäre Aufgabe nicht in der Beratung und Unterstützung der Angehörigen sehen (Orford, Templeton, Copello, Velleman \& Ibanga, 2010). Orford, Tepmleton, Copello et al. (2010) konnten aber zeigen, dass sich die Haltung der Institutionen, die sich zum Einsatz der 5-Step Intervention bereit erklärten hatten, veränderte und Angehörige mehr eingeladen und einbezogen wurden (Orford, Templeton, Patel, Velleman \& Copello, 2007; Orford, Tepmleton, Copello, Velleman, Ibanga \& Binnie, 2009), was grundsätzlich als sehr positiv zu beurteilen ist.

\subsubsection{Empirische Überprüfung}

Die ,5-Step-Method“ ist ein Ansatz, der nebst theoretischen Überlegungen auch basierend auf wissenschaftlichen Erkenntnissen entwickelt worden ist. Aus diesem Grund ist er auch empirisch sehr gut untersucht und positiv belegt (Copello, Templeton, Krishnan, Orford \& Velleman, 2000; Copello, Templeton \& Orford et al., 2009; Copello, Templeton, Orford \& Velleman, 2010a; Orford et al., 2007; Velleman, Orford, Templeton, Copello, Patel, Moore et al., 2011).

In den bisherigen Studien konnte gezeigt werden, dass sich durch die „5-Step-Method“ das Coping bei den Angehörigen signifikant verbesserte und dass sie signifikant tiefere Belastungssymptome angaben (Copello et al., 2009; Velleman, Arcidiacono, Procentese, Copello \& Sarnacchiaro, 2008; Velleman et al., 2011). Velleman et al. (2011) konnten zudem nachweisen, dass die Verbesserung der Situation der Angehörigen über den Verlauf von 12 Monaten stabil blieb. 


\subsubsection{Angehörigenvereinigung Drogenabhängiger Zürich (ada-zh) und Verband der Eltern- und Angehörigenvereinigung Drogenabhängiger (VEVDAJ)}

\subsubsection{Einleitung}

Die Angehörigenvereinigung Drogenabhängiger (ada-zh) und der Verband der Eltern- und Angehörigenvereinigung Drogenabhängiger (VEVDAJ) stehen für ein spezifisches Angebot für Angehörige in der Schweiz. Die beiden Vereine haben eine gemeinsame Entstehungsgeschichte. Der VEVDAJ als Dachverband der Eltern- und Angehörigenvereinigungen ist vor allem auf politischer Ebene und in der Vernetzung und Kooperation seiner Mitglieder tätig (Hälg, 2013, S. 44). Die ada-zh als Mitglied des VEVDAJ dagegen stellt als einzige professionelle Beratungsstelle, die Angehörige von suchtkranken Menschen berät, ein einmaliges Angebot in der Schweiz dar (Suhner \& Beck, 2012, S. 26). In den folgenden Kapiteln werden die Entstehungsgeschichte der beiden Vereine sowie die Aktivitäten beschrieben, denen sie sich verschrieben haben.

\subsubsection{Entstehungsgeschichte}

Ende der 60er und Anfang der 70er Jahre begannen sich die ersten Drogenszenen in der Schweiz zu bilden (Grob, 2012, S. 16ff). Als Reaktion darauf schlossen sich 1974 bereits die ersten betroffenen Eltern in Zürich zusammen und riefen die erste Selbsthilfegruppe ins Leben. Aus diesen Selbsthilfegruppen wurde schliesslich die Elternvereinigung DAJ („DrogenAbhängige Jugendliche“) gegründet. 1976 wurde der erste DAJ in Zürich ins Leben gerufen (Kauer, 1996, S.5-6). Daraufhin folgten weitere DAJ-Gründungen in den Städten Basel (1977), Bern (1977), Luzern (1978) und schliesslich im Kanton Aargau, in Glarus und St. Gallen (ebd.). In Zürich wurden die Bemühungen der Eltern sehr vom Drop-In der Psychiatrischen Universitätsklinik unterstützt, indem den Angehörigen Räumlichkeiten für ihre Zusammenkünfte zur Verfügung gestellt wurden (Suhner \& Beck, 2012, S. 26). 1980 gründet der DAJ Zürich schliesslich einen offiziellen Verein mit dem Ziel folgende Aufgaben zu erfüllen: „Elterngespräche betroffener Eltern, Öffentlichkeitsarbeit, Beratungstätigkeit, Schulung von Gruppenleitern, Kontakt- und Koordinationsstelle für alle DAJ der deutschen Schweiz“ (Kauer, 1996, S. 5). Die Beratungsstelle, die daraufhin 1981 an der Stampfenbachstrasse eröffnet wurde, wurde zum damaligen Zeitpunkt noch von selbstbetroffenen Leitern der Selbsthilfegruppen geführt (Suhner \& Beck, 2012, S. 26). Sie stellten sich den ratsuchenden Eltern rund um die Uhr mit ihren privaten Nummern zur Verfügung. Die stetig steigende Nachfrage auf der Beratungsstelle konnte aufgrund der zunehmenden Heroinwelle in der Schweiz irgendwann von den Gruppenleitenden nicht mehr 
bewältigt werden. Dank finanzieller Unterstützung seitens der Stadt Zürich und diverser Spenden und Gönnerbeiträge konnte schliesslich 1987 die Beratungsstelle professionalisiert werden und die ersten beiden Berater wurden beim DAJ Zürich angestellt (ebd.).

Die Selbsthilfegruppen hatten untereinander von Anfang an einen intensiven Austausch gepflegt und auch immer wieder Netzwerktagungen zu verschiedenen Thema organisiert (Kauer, 1996, S. 5). Im Verlauf der Zeit wurde der Wunsch nach einem Dachverband laut, der die Selbsthilfegruppen besser vernetzen würde. Daraufhin wurde 1986 schliesslich der Dachverband VEVDAJ gegründet, der auch heute noch aktiv ist und sich für die Belange der Angehörigen und ihrer drogenabhängigen Familienmitglieder einsetzt (ebd.).

Die Beratungsstelle DAJ Zürich durchlief im Laufe der Zeit einige Veränderungen. Die Stelle wurde ausgebaut auf drei Fachpersonen, die auf der Beratungsstelle im Teilzeitpensum tätig waren. Schliesslich veränderten sich auch zunehmend die Klienten, die auf der Stelle beraten wurden (Suhner \& Beck, 2012, S. 26). Während zu Beginn vor allem Eltern von drogenabhängigen Jugendlichen Unterstützung suchten, meldeten sich mit der Zeit immer mehr Geschwister, Bekannte und Partner von suchtkranken Menschen. Dies hatte zur Folge, dass die Beratungsstelle 2005 umbenannt wurde in „Angehörigenvereinigung DrogenAbhängiger Zürich“ (ada-zh). Durch eine Veränderung des Konsumverhaltens (Abnahme der Inzidenz bei Heroin) hat sich die Beratungsstelle in der Zwischenzeit auch für Angehörige von alkoholkranken Menschen geöffnet.

\subsubsection{VEVDAJ - heute}

Der VEVDAJ ist ein politisch und konfessionell neutraler Verband, der sich aus den Elternund Angehörigenvereinigungen DAJ in der deutschen und italienischen Schweiz

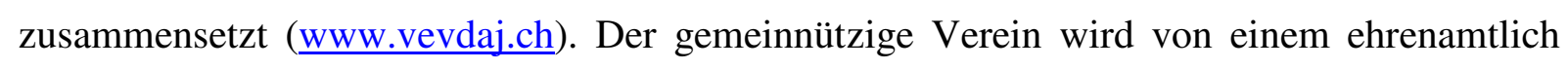
tätigen Vorstand geleitet. Die Finanzierung erfolgt durch das Bundesamt für Gesundheit (BAG), mit welchem eine Leistungsvereinbarung besteht, aber auch über Mitgliederbeiträge und Spenden (Hälg, 2013, S. 44).

Der Dachverband der Eltern- und Angehörigenvereinigungen setzt sich bei Behörden und auf eidgenössischer Ebene für die Belange von drogenabhängigen Menschen ein. Der VEVDAJ engagiert sich für eine umfassende kontrollierte Drogenabgabe im Sinne einer Schadensminderung und dafür, dass drogenkonsumierende Menschen nicht kriminalisiert 
werden (www.vevdaj.ch). Weitere Ziele sind die Schaffung von Arbeits- und Wohnmöglichkeiten für suchtkranke Menschen, die eine soziale Reintegration ermöglichen. Daneben koordiniert und vernetzt der Dachverband aber auch die bestehenden Vereinigungen in den verschiedenen Kantonen, unterstützt neue Selbsthilfegruppe bei der Gründung und organisiert Aus- und Weiterbildungen für Gruppenleiter und Netzwerktagungen zu aktuellen Themen (ebd; Hälg, 2013, S. 44).

Neben diesen Aktivitäten arbeitet der VEVDAJ aber auch mit vielen suchtpolitischen Organisationen zusammen. Auf nationaler Ebene besteht eine Zusammenarbeit mit der NAS (Nationale Arbeitsgemeinschaft Suchtpolitik), der SSAM (Schweizerische Gesellschaft für Suchtmedizin), dem Fachverband Sucht, der Arud (Arbeitsgemeinschaft für den risikoarmen Umgang mit Drogen), der EKDF (Eidgenössische Kommission für Drogenfragen), Infodrog und weiteren (Hälg, 2013, S. 44). Der VEVDAJ pflegt aber auch Kontakte ins Ausland zu anderen Eltern- und Angehörigenvereinigungen.

Der VEVDAJ engagiert sich nach wie vor vorwiegend im Bereich der illegalen Drogen. Es ist allerdings momentan eine Prüfung im Gange, dass sich der Verband und die Vereinigungen allgemein für Angehörige von suchtmittelabhängigen Menschen öffnen (Hälg, 2013, S. 44).

\subsubsection{Angehörigenvereinigung Drogenabhängiger Zürich (ada-zh) - heute}

Die Angehörigenvereinigung Drogenabhängiger Zürich ist wie der VEVDAJ ein politisch und konfessionell neutraler Verein und wird von einem ehrenamtlichen Vorstand, der durch eine Geschäftsführerin auf der Beratungsstelle vertreten wird, geführt (www.ada-zh.ch). Die Angehörigenvereinigung ist Mitglied des VEVDAJ. Die Stelle hat sich auf die Beratung und therapeutische Begleitung von Angehörigen eines suchmittelgefährdeten oder -abhängigen Menschen spezialisiert. Die Beratungen werden zu vergünstigten einkommensabhängigen Tarifen angeboten (ebd.). Neben der Unkostenbeteiligung der ratsuchenden Angehörigen wird die Beratungsstelle von der Stadt und vom Kanton Zürich finanziell unterstützt (www.adazh.ch). Die restlichen Aufwendungen müssen über Spendengelder gedeckt werden.

Während in den Anfängen noch Fachpersonen aus dem Bereichen Sozialpädagogik/Psychotherapie und Erwachsenenbildung angestellt worden waren, werden in der Zwischenzeit aufgrund der zunehmenden psychotherapeutischen Problemstellungen heute bei der Beratungsstelle nur noch Psychologen mit psychotherapeutischer Weiterbildung 
eingestellt (Suhner \& Beck, 2012, S. 26). Das Angebot der Beratungsstelle ist sehr umfassend und beinhaltet Informationen $\mathrm{zu}$ Sachfragen, Kriseninterventionen, lösungsorientierte Kurzzeitberatung, längerfristige therapeutische Begleitung, Familien- und Paarberatung und Telefon- und Email-Beratungen. Daneben werden von der Beratungsstelle auch immer wieder neue Selbsthilfegruppen initiiert, die im ersten Jahr von einem Mitglied der Beratungsstelle begleitet werden bis nach einem Jahr jemand von den Gruppenmitgliedern die Gruppenleitung übernimmt. Die Berater orientieren sich in ihrem Handeln an verschiedenen Ansätzen und integrieren auch neue Angebote in ihrer Arbeit. So bietet die Stelle neu auch eine CRAFTSelbsthilfegruppe an (www.ada-zh.ch) .

Seit 2008 besteht zwischen der Angehörigenvereinigung Drogenabhängiger Zürich und der Arud (Arbeitsgemeinschaft für risikoarmen Umgang mit Drogen) eine Kooperation (Suhner \& Beck, 2012, S. 27). 


\section{Fragestellung}

\subsection{Hauptfragestellung}

Die Situation von Angehörigen von drogenabhängigen Menschen wurde im deutschsprachigen Raum und der Schweiz bisher nur wenig untersucht. Die vorliegende Studie hatte deshalb einen ausgeprägten explorativen Charakter. Im Zentrum der Untersuchung stand dabei folgende Frage:

Welche Auswirkungen hat die Suchterkrankung eines nahestehenden Menschen auf die Angehörigen in Bezug auf die erlebten Belastungen und den Umgang mit diesen?

Die weiteren Fragestellungen sind dieser Hauptfragestellung untergeordnet.

\subsection{Weitere Fragestellungen}

Die weiteren Fragestellungen sollen dazu beitragen die Hauptfragestellung weiter auszudifferenzieren.

\subsubsection{Charakteristika von Angehörigen von drogenabhängigen Menschen}

Durch welche soziodemographischen und -ökonomischen Eigenschaften sind Angehörige von drogenabhängigen Menschen charakterisiert? (F1)

- Verfügen Angehörige von Drogenabhängigen in der vorliegenden Stichprobe über das Bildungsniveau der Schweizer Durchschnittsbevölkerung? (F1.1)

- Sind Angehörige in der vorliegenden Stichprobe bezüglich des Erwerbsstatus' im gleichen Mass erwerbstätig wie die Schweizer Durchschnittbevölkerung? (F1.2)

\subsubsection{Belastung durch die Suchterkrankung und deren Auswirkungen}

Welche Auswirkungen haben die erlebten Belastungen durch die Suchterkrankung auf die psychische Gesundheit der Angehörigen? (F2)

- Über welche Belastungen berichten Angehörige von drogenabhängigen Menschen? $(\mathrm{F} 2.1)$

- Gibt es einen allfälligen Zusammenhang zwischen der erlebten Belastung und der psychische Gesundheit (Depressivität) der Angehörigen? (F2.2) 
- Gibt es einen allfälligen Zusammenhang zwischen der erlebten Belastung und den Bewältigungsstrategien, die Angehörige im Umgang mit der Suchterkrankung anwenden? (F2.3)

- Gibt es einen allfälligen Zusammenhang zwischen der erlebten Belastung und den Charakteristika der Suchterkrankung des Angehörigen (Schweregrad, Status der Erkrankung im Vergleich zum Vorjahr)? (F2.4)

- Welche Rolle spielt die Beziehung, in der die Angehörigen zum Suchtkranken stehen, für ihr Erleben von Belastungen? (F2.5)

\subsubsection{Unterstützung der Angehörigen}

Wie können Angehörige von drogenabhängigen Menschen unterstützt werden bei der Bewältigung der Belastungen, die für sie durch die Suchterkrankung ihres Angehörigen entstehen?

- Welche Art der Unterstützung erhalten Angehörige von drogenabhängigen Menschen bereits? (F3.1)

- Wie beurteilen Angehörige das aktuell bestehende Unterstützungsangebot? (F3.2)

- Welche weitere Unterstützung könnte es aus Sicht der Angehörigen von drogenabhängigen Menschen noch geben, die Ihnen den Umgang mit den Belastungen der Suchterkrankung erleichtern würde? (F3.3)

\subsubsection{Typische Gruppen von Angehörigen aufgrund der erlebten Belastung}

Gibt es aufgrund der erlebten Belastung durch die Suchterkrankung typische Gruppen von Angehörigen? (F4)

- Gibt es zwischen allfällig typischen Gruppen von Angehörigen Unterschiede bezüglich ihrer Lebensqualität? (F4.1)

- Gibt es zwischen allfällig typischen Gruppen von Angehörigen Unterschiede bezüglich ihrer Bewältigungsstrategien? (F4.2)

- Gibt es zwischen allfällig typischen Gruppen von Angehörigen Unterschiede bezüglich ihres Selbstwertes? (F4.3)

- Gibt es zwischen allfällig typischen Gruppen von Angehörigen Unterschiede bezüglich der Suchterkrankung ihrer Angehörigen (Schweregrad)? (F4.4) 
- Gibt es zwischen allfällig typischen Gruppen von Angehörigen Unterschiede bezüglich soziodemographischer Eigenschaften wie Bildung, Alter, Geschlecht? (F4.5) 


\section{Methodik}

\subsection{Untersuchungsinstrumente}

\subsubsection{Quantitative Befragung: Fragebogen}

\subsubsection{Einleitung}

Über eine Fragebogenerhebung wurden die Lebensqualität bzw. das Wohlbefinden, die Belastung der Angehörigen sowie deren Bewältigungsstrategien erhoben.

Nachfolgend werden die einzelnen Verfahren, welche im Rahmen der Erhebung zum Einsatz kamen, kurz vorgestellt. Die Befindlichkeit wurde über den Berner Fragebogen zum Wohlbefinden (BFW) erfasst. Zur Erfassung der mannigfaltigen Belastungen, mit denen sich die Angehörigen von Suchtpatienten konfrontiert sehen, wurden das Inventar Subjektiver Stigmaerfahrungen (ISE) und das Zarit Burden Interview (ZBI) eingesetzt. Um die Auswirkungen der Belastungen auf die psychische Gesundheit der Angehörigen zu untersuchen, wurde den Angehörigen das vereinfachte Beck-Depressions-Inventar (BDI-V) vorgelegt. Aufgrund der bestehenden theoretischen Assoziation der CoAbhängigkeitsentwicklung mit einer Beeinträchtigung des Selbstwertgefühls wurde zusätzlich die Rosenberg Self-Esteem Scale (Rosenberg SES) eingesetzt. Mögliche Bewältigungsstrategien im Umgang mit der Belastung sollten über den Stressverarbeitungsfragebogen (SVF-120) und Teile des Inventars Subjektiver Stigmaerfahrungen (ISE) erfasst werden. Der Fragebogen, der den Angehörigen vorgelegt wurde, befindet sich im Anhang.

\subsubsection{Berner Fragebogen zum Wohlbefinden (BFW)}

Der Berner Fragebogen zum Wohlbefinden (BFW) erfasst über die beiden Faktoren zweiter Ordnung „Zufriedenheit“ und „Negative Befindlichkeit“ das subjektive Wohlbefinden (Grob, Lüthi, Kaiser, Flammer, Mackinnon, Wearing, 1991; Grob, 2003). Anhand von 39 Items werden sechs Primärfaktoren erhoben (ebd.), wobei der Zufriedenheitsfaktor sich aus den Faktoren „Positive Lebenseinstellung“, „nicht vorhandene depressive Stimmung“, „Selbstwert“ und „Lebensfreude“ zusammensetzt. Der Sekundärfaktor „Negative Befindlichkeit“ besteht dagegen nur aus den beiden Faktoren „Probleme und Sorgen“ und „Körperliche Beschwerden“. 
Die Items für den Fragebogen wurden teilweise aus bestehenden Instrumenten übernommen und teilweise neu konstruiert (Grob et al., 1991, S. 66). Die Autoren definierten dabei folgende Bedingungen als massgebend für die Itemauswahl bei der Erfassung des subjektiven Wohlbefindens: „,die Befriedigung alltäglicher Bedürfnisse, ein guter Gesundheitszustand, frei sein von Sorgen, eine sinnvolle Verwendung der Zeit, das Erreichen selbst- und fremdbestimmter Ziele sowie der erfolgreiche Umgang mit divergierenden Zielen, die Überlegenheit des Prozesses über den Zustand der Zielerreichung und das Erfüllen allgemeiner gesellschaftlich definierter Normen“ (Grob et al., 1991, S. 67).

Die Primärfaktoren weisen gemäss Literatur interne Konsistenzen (Cronbach's $\alpha$ ) von .60 bis .82 auf, während die für die Sekundärfaktoren „Zufriedenheit“ und „Negative Befindlichkeit“ Koeffizienten von .82 resp. .77 geschätzt wurden (Grob, 2003, S. 58). Trotz der etwas niedrigen internen Konsistenz der Lebensqualitätsskala von .60 kann die Reliabilität als ausreichend gut beurteilt werden unter anderem auch aufgrund der guten Kennwerte für die Sekundärfaktoren.

Der BFW/E zur Erhebung des Wohlbefindens wurde ausgewählt, weil er neben emotionalen auch kognitive Aspekte bei der Erfassung des subjektiven Wohlbefindens berücksichtigt (Grob, 2003, S. 56). Zudem machten die von den Autoren definierten Bedingungen, welche das subjektive Wohlbefinden beeinflussen, deutlich, dass das Konstrukt Wohlbefinden differenziert erfasst wird mittels BFW/E, was auch in den sechs Primärfaktoren zum Ausdruck kommt. Über den Vergleich mit diversen anderen Skalen wie beispielsweise der Well-being-Skala des CPI, der Self-Value-Skala des MMPI und anderen ist die Kriteriumsvalidität des Fragebogens in der Zwischenzeit gut belegt (ebd., S. 58).

\subsubsection{Das Inventar Subjektiver Stigmaerfahrungen (ISE)}

Beim Inventar Subjektiver Stigmaerfahrungen (ISE) handelt es sich um ein neueres Verfahren, über welches subjektive Stigmaerfahrungen sowohl quantitativ als auch qualitativ erfasst werden können. Das Inventar liegt in zwei verschiedenen Versionen vor: einer Patienten- und einer Angehörigenversion.

Das ISE wurde mit dem Ziel entwickelt, das Ausmass und die Intensität von Stigmatisierung aus der Sicht der Betroffenen erfassen zu können (Stuart, Milev \& Koller, 2005, S. 33). Ursprünglich wurde es in englischer Sprache entwickelt und publiziert (Stuart et al., 2005). 
2009 folgte die deutschsprachige Übersetzung und Validierung der Patientenversion des ISE (Schulze, Stuart \& Riedel-Heller, 2009). Das Inventar wurde bereits bei einer Stichprobe von Borderline- und Schizophrenie-Patienten eingesetzt, um deren spezifischen Stigmaerfahrungen und Coping-Strategien im Umgang mit Stigmatisierung zu erfassen (Schulze, Janeiro \& Kiss, 2010). Dabei konnten die Autoren interessante Unterschiede in den Erfahrungen und im Umgang mit Stigmatisierung zwischen den beiden Patientengruppen herausarbeiten. Zum Zeitpunkt der Planung der vorliegenden Studie war auch die Validierung der deutschen ISE-Angehörigenversion geplant.

Das ISE weist sowohl in der englisch- als auch der deutschsprachigen Version eine zweifaktorielle Struktur auf: Mit 10 Items wird in der englischsprachigen Version das Ausmass der Stigmaerfahrungen (SES) in verschiedenen Lebensbereichen erfragt (Schulze et al., 2009, S. e20; Stuart et al., 2005, S. 35). Die Items sind durch eine offene Frage ergänzt, in der der Betroffene dazu aufgefordert wird, die gemachte Angabe durch ein Beispiel zu beschreiben. Im Vergleich zur englischsprachigen Version verfügt die deutschsprachige jedoch in der Stigma-Erfahrungs-Skala (SES) nur über 9 Items. In den weiteren sieben Items wird auf der zweiten Skala die Intensität der Auswirkungen von Stigma (SAS) auf verschiedene Bereiche wie soziale Kontakte, familiäre Beziehungen, die Lebensqualität und den Selbstwert erhoben (ebd.).

Die Skalen des ISE verfügen sowohl in der englischsprachigen als auch in der deutschsprachigen Version über gute bis sehr gute interne Konsistenzen (SES: KuderRichardson-Koeffizient von .74; SAS: Cronbach's $\alpha$ von .86) und mittlere Trennschärfen (SES: .41; SAS: .63) (Schulze et al., 2009, S. e22). Das Instrument kann sowohl als Selbstbeschreibungsverfahren als auch als halbstandardisiertes Interview eingesetzt werden (ebd., S. e20).

Die im Rahmen der vorliegenden Arbeit erhobenen Daten sollten einen Beitrag zur Validierung der deutschsprachigen Angehörigenversion des ISE leisten. Während in weiteren Projekten zur Validierung das ISE als halbstandardisiertes Interview eingesetzt wurde, wurde den Studienteilnehmenden dieser Studie die Angehörigenversion des ISE als Fragebogen zum Selbstausfüllen vorgelegt. Über das ISE sollte mit den von den Angehörigen berichteten Stigmaerfahrungen ein möglicher belastender Aspekt erfasst werden. Ausserdem erfragte das ISE Copingstrategien im Umgang mit Stigmatisierung. 


\subsubsection{Zarit Burden Interview (ZBI)}

Das Zarit Burden Interview wurde ursprünglich entwickelt, um die Belastung von Angehörigen zu erfassen, welche Alzheimer-Patienten zu Hause betreuen (Bachner \& O'Rourke, 2007, S. 679). In der Zwischenzeit wurde das Interview in diverse Sprachen übersetzt und bei Angehörigen von Patienten mit diversen Störungen und Krankheiten angewendet (ebd.; Ramos-Cerqueira, Torres, Torresan, Negreiros \& Vitorino, 2008). Bei der Erhebung der subjektiven Belastung berücksichtigt das ZBI physische, psychologische, emotionale, soziale und finanzielle Probleme, welche im Zusammenhang mit der Krankheit einer nahestehenden Person entstehen können (Schreiner, Morimoto, Arai \& Zarit, 2006, S. 108).

Die deutsche Version des ZBI ist relativ neu und wurde an einer Stichprobe pflegender Ehepartner von Demenzkranken validiert (Braun, Scholz, Hornung \& Martin, 2010). Braun et al. (2010, S. 116) bestimmten die Reliabilität über den Cronbach's- $\alpha$-Koeffizienten und die Testhalbierungsmethode nach Spearman-Brown. Diese beiden Koeffizienten konnten mit .91 bzw. 93 als sehr gut beurteilt werden. Die Kriteriumsvalidität der deutschen Version wurde über signifikante Korrelationen zwischen dem ZBI und einer Lebenszufriedenheits- und Depressivitätsskala bestätigt (ebd., S. 116). Schreiner et al. (2006, S. 109) konnten im Rahmen ihrer Untersuchung zudem zeigen, dass das ZBI eine sehr gute Vorhersagevalidität hat und somit auch für den klinischen Alltag von Nutzen sein kann. Bei Studienteilnehmenden, welche einen Gesamtwert zwischen 24 und 26 auf der Skala erreichten, konnte ein signifikant höheres Risiko für eine Depression nachgewiesen werden (ebd.).

Obwohl das ZBI ursprünglich für Angehörige von Demenzpatienten entwickelt wurde, wurde der Einsatz des Fragebogens im Rahmen der vorliegenden Arbeit aus folgenden Gründen eingeplant: Das ZBI ist ein gut untersuchtes Instrument, welches bei Angehörigen von Patienten mit verschiedenen Krankheits- und Störungsbildern weit verbreitet eingesetzt wird und deren Belastung erfasst. Bachner und O'Rourke (2007) konnten in einer Metaanalyse zur Reliabilität des ZBI zudem zeigen, dass das Instrument in unterschiedlichen Stichproben von Angehörigen vergleichbare Reliabilitätskoeffizienten aufwies, so dass der Einsatz sowohl bei Angehörigen von Demenzpatienten als auch von anderen physisch und psychisch Kranken gerechtfertigt ist (ebd., S. 683). 


\subsubsection{Fragebogen zum Selbstwertgefühl (Rosenberg SES)}

Die Rosenberg-Skala zum Selbstwertgefühl wurde 1965 von Rosenberg (1965) vorgelegt und gehört in der Zwischenzeit zu den am meisten eingesetzten Verfahren zur Erfassung des Selbstwertgefühles (Gray-Little, Williams \& Hancock, 1997). Anhand von 10 Items wird nach neusten Untersuchungen zur internen Struktur der Skala über die beiden Faktoren positives und negatives Selbstbild ein globaler Selbstwert ermittelt (Roth, Decker, Herzberg \& Brähler, 2008, S. 195).

Die Rosenberg SES wurde 1996 erstmals in einer deutschsprachigen Version überprüft und publiziert (Ferring \& Filipp, 1996). Seit 2003 liegt zudem eine von Collani und Herzberg (2003) revidierte Fassung der deutschsprachigen Skala vor, in der das Item Nr. 4 neu übersetzt und der Fragebogen neu überprüft wurde (Collani \& Herzberg, 2003). Obwohl die Trennschärfe des Items Nr. 4 durch die Neuübersetzung massgeblich verbessert werden konnte, hatte sie keine Verbesserung der Kennwerte der Gesamtskala zur Folge, weil die übrigen Items bereits gute bis sehr gute Kennwerte aufwiesen (ebd., S. 6). Gemäss Roth et al. (2008, S. 195) wurden für die deutschsprachige Rosenberg-Skala ein Cronbach's $\alpha$ Koeffizient von .88 und für die beiden Subskalen Cronbach's $\alpha$-Koeffizienten von .81 (RSESpos.) und .86 (RSES-neg.) ermittelt.

Gemäss Grey-Little et al. (1997) handelt es sich bei der Rosenberg SES im Vergleich zu anderen Skalen zur Erfassung des Selbstwertes um eine grosse Ausnahme: Einerseits wurde die Skala bereits in diversen Studien von ganz unterschiedlichen Populationen eingesetzt und andererseits sind ihre psychometrischen Kennwerte gut untersucht (ebd., S. 443). In ihrer umfangreichen Itemanalyse kamen die Autoren zudem zum Schluss, dass es sich um ein sehr reliables und intern konsistentes Instrument handelt. Ein weiterer Grund, weshalb die Skala in der vorliegenden Studie eingesetzt wurde, ist ihre bestechend kurze Form.

\subsubsection{Vereinfachtes Beck-Depressions-Inventar (BDI-V)}

Das Beck-Depressions-Inventar (BDI) gehört $\mathrm{zu}$ den am meisten verwendeten Depressionsskalen (Schmitt, Altstötter-Gleich, Hinz, Maes \& Brähler, 2006, S. 51; Schmitt \& Maes, 2000, S. 38). Das Inventar erfragt 21 Symptome der klinischen Depression, wobei pro Symptom vier Aussagen mit zunehmenden Schweregrad der Symptomatik gemacht werden. 
Dem Verfahren wurde in der Vergangenheit wiederholt eine hohe Reliabilität bescheinigt und seine Validität gilt als belegt. Schmitt und Maes (2000) kritisierten jedoch aufgrund der hohen Itemzahl die Ökonomie des Verfahrens und schlugen eine Vereinfachung vor, indem sie pro Symptom nur noch ein Item vorgaben, welches über eine sechsstufige Likert-Skala eingeschätzt werden kann. Zudem wurde das Symptom „Gewichtsverlust“ aufgrund der geringen Trennschärfe aus der Skala ausgeschlossen (ebd., S. 39), so dass das BDI-V nur noch aus 20 Items besteht.

Eine Untersuchung zur Messgüte des vereinfachten Beck-Depressions-Inventar (BDI-V) legte den Schluss nahe, dass die vereinfachte Form des BDI „etwas homogener und etwas zuverlässiger als das Original“ ist (Schmitt, Beckmann, Dusi, Maes, Schiller \& Schonauer, 2003, S. 154). Zudem konnten die Autoren im Vergleich mit anderen Depressionsskalen aufzeigen, dass die Originalversion und der BDI-V höher miteinander korrelieren als mit anderen Depressionsskalen. 2006 folgten schliesslich die Normwerte zum BDI-V, wobei die Autoren als Cut-off-Wert für die klinisch orientierte Diagnose der Depression den BDI-VWert von 35 empfehlen (Schmitt et al., 2006, S. 58).

Das BDI ist eine weit verbreitete Depressionsskala, die unter anderem auch in der Praxis häufig eingesetzt wird. Aus zeitökonomischen Gründen wurde im Rahmen dieser Arbeit die vereinfachte BDI-Version in der Datenerhebung eingesetzt.

\subsubsection{Stressverarbeitungsfragebogen (SVF-120)}

Der Stressverarbeitungsfragebogen (SVF-120) gehört in der Forschung im deutschsprachigen Raum zu den am meisten eingesetzten Verfahren (Bodenmann \& Gmelch, 2009; Weyers, Ising, Reuter \& Janke, 2005, S. 208). Der Fragebogen wird auch von den Autoren selbst vorwiegend für die Forschung empfohlen (Janke, Erdmann, Kallus \& Boucsein, 1997, S. 715).

Der SVF-120 misst anhand von 20 Subtests und insgesamt 120 Items die habituelle Stressreaktion (Amelang \& Schmitz-Atzert, 2006, S. 283). Dabei werden folgende Subtests als mögliche Bewältigungsformen im Umgang mit Stress unterschieden: „Bagatellisieren“, „,herunterspielen“, „Schuldabwehr“, „Ablenkung“, „Ersatzbefriedigung“, „Suche nach Selbstbestätigung“, „Entspannung“, „Situationskontrolle“, „Reaktionskontrolle“, „positive Selbstinstruktion“, „soziales Unterstützungsbedürfnis“, „Vermeidung“, „Flucht“, „soziale 
Abkapselung“, „gedankliche Weiterbeschäftigung“, „Resignation“, „Selbstbemitleidung“, „Selbstbeschuldigung“, „Aggression“, „Pharmakaeinnahme“ (ebd., S. 284ff). Aus diesen Primärskalen lassen sich über Additionsbildungen auf höherer Ordnung „Positiv“- und „Negativ“-Strategien abbilden. Wobei die wertende Bezeichnung bewusst gewählt wurde, um deutlich zu machen, dass die Positiv-Strategien eher zu einer Verminderung und NegativStrategien eher zu einer Verstärkung der Stressreaktion führen dürften (ebd.).

Der Test wird als mehrheitlich reliabel und zeitstabil beurteilt (Amelang \& Schmitz-Atzert, 2006, S. 285; Beutel \& Brähler, 2004, S. 166; Janke et al., 1997, S. 715). Die internen Konsistenzen (Cronbach's $\alpha$ ) variieren für die einzelnen Skalen um .80 mit Ausnahme der Extremskalen Pharmakaeinnahme (.65) und gedankliche Beschäftigung (.91) (Amelang \& Schmitz-Atzert, 2006, S. 285). Die Retestreliabilität mit einem Zeitinvervall von 4 Wochen liegt zwischen $\mathrm{r}_{\mathrm{tt}}=.70$ und .86 , wodurch die ursprüngliche Annahme der Autoren, dass es sich bei der im Verfahren erfassten Stressbewältigung um eine zeitstabile und habituelle Persönlichkeitseigenschaft handelt, bestätigt werden konnte.

In verschiedenen Rezensionen wurde wiederholt kritisiert, dass die Normen zwar aktuell seien, aber dass es grundsätzlich wünschenswert wäre, die Normen über eine grössere Zahl von Probanden abzustützen (Amelang \& Schmitz-Atzert, 2006, S. 286; Beutel \& Brähler, 2004, S. 167). Dennoch schien der Fragebogen für die Befragung aus folgenden Gründen als geeignet: Über eine Vielzahl von Subskalen werden verschiedene mögliche Bewältigungsstrategien im Umgang mit Stress abgebildet, welche relativ situationsunabhängig sind. Dies war im Sinne der vorliegenden Arbeit, die der Frage nachgeht, wie Angehörige von Suchtpatienten im Allgemeinen mit der Belastung umgehen. Aufgrund der weiten Verbreitung des SVF-120 wurde die Validität des Instrumentes inzwischen gut belegt (Amelang \& Schmitz-Atzert, 2006, S. 286) und das Instrument ist gut akzeptiert. 


\subsubsection{Qualitative Befragung: Interviews}

\subsubsection{Einleitung}

Die Situation von Angehörigen von drogenabhängigen Menschen wurde im deutschsprachigen Raum bisher nur wenig wissenschaftlich untersucht. Entsprechend standen für die quantitative Datenerhebung nur wenige Instrumente zur Verfügung, die spezifisch die Situation von Angehörigen erhoben. Aus diesem Grund wurden - in Ergänzung zur Fragebogenerhebung - Angehörige mit einem problemzentrierten Interview zu ihrer spezifischen Situation befragt. Die qualitative Datenerhebung bot den Vorteil, dass Angehörige ihre subjektive Perspektive selbst darlegen konnten (Mayring, 2002, S. 68), ohne dass ihre Antworten an Vorgaben gebunden waren.

\subsubsection{Problemzentriertes Interview}

Gemäss Mayring (2002, S. 67) werden unter dem Begriff problemzentriertes Interview alle Formen der qualitativen Datenerhebung zusammengefasst, bei der die Interviewten offen bzw. halbstrukturiert befragt werden. Ziel des problemzentrierten Interviews ist es, dass die Befragten möglichst frei zu Wort kommen (offenes Gespräch), während der Interviewer gleichzeitig auf bestimmte Problemstellungen fokussiert und das Gespräch entsprechend lenkt (Problemzentrierung).

Das problemzentrierte Interview wird basierend auf einem Interviewleitfaden durchgeführt, in dem die wesentlichen Aspekte, auf die in der Befragung eingegangen werden soll, zusammengestellt sind (Mayring, 2002, S. 67). Durch den Interviewleitfaden ist eine teilweise Standardisierung der Befragung möglich. Auf diese Weise wird die Vergleichbarkeit von mehreren Interviews erleichtert. In der vorliegenden Arbeit wurde der Interviewleitfaden auf der Basis der vorhandenen Literatur sowie aufgrund von Erfahrungen aus einer früheren Befragung von Müttern von heroin- und kokainabhängigen Söhnen (Candrian \& Ruckstuhl, 2009) zusammengestellt.

Anhand der problemzentrierten Interviews wurden die verschiedenen Bereiche, in denen Angehörige potenziell Belastungen erleben können, genauer exploriert. Neben den Belastungen sollte auch die Frage geklärt werden, welche Art der Unterstützung die befragten Angehörigen erhalten hatten, wie sie diese beurteilten und welche Unterstützung sie sich des Weiteren wünschen würden. Über das problemzentrierte Interview wurde zudem erhoben, 
welche Bewältigungsstrategien Angehörige im Zusammenhang mit den Belastungen durch die Suchterkrankung angaben. Die Angehörigen wurden auch zur Anfangszeit der Suchterkrankung befragt, wobei insbesondere nach Vorahnungen, Gedanken und Gefühlen im Zusammenhang mit der Erkenntnis, dass der betroffene Angehörige einen problematischen Drogenkonsum hat, gefragt wurde. Dabei wurde davon ausgegangen, dass das Vorwissen, das Angehörige über Suchterkrankungen besassen, relevant sein könnte für das Erleben und den Umgang mit Belastungen (Interviewleitfaden im Anhang).

\subsection{Befragung der Angehörigen}

\subsubsection{Zusammenstellung der Stichprobe}

Zur Grundgesamtheit (Population) der Angehörigen von drogenabhängigen Menschen sind bisher nur wenige Kenntnisse vorhanden. Wie einleitend bereits erwähnt, bestehen bis anhin keine verlässlichen Schätzungen, wie viele Personen effektiv durch die Drogenabhängigkeit eines Angehörigen betroffen sind und durch welche Charakteristika diese Population gekennzeichnet ist (Copello, Templeton \& Powell, 2010, S. 64). Die Grundgesamtheit konnte deshalb nicht herangezogen wurden, um eine möglichst repräsentative Stichprobe zu ziehen. Für die Studienteilnahme wurden deshalb nach Ermessen folgende Einschlusskriterien definiert: Als Angehörige von drogenabhängigen Menschen galten Eltern, Geschwister, erwachsene Kinder, Lebens- bzw. Ehepartner und -partnerinnen oder nahestehende Freunde und Freundinnen. Alle Studienteilnehmenden mussten zum Zeitpunkt der Befragung volljährig und mündig sein und über ausreichende Deutschkenntnisse verfügen. Sie mussten zudem jemanden in ihrem nahen Umfeld mit einem Abhängigkeitssyndrom oder einem problematischen Konsum von Heroin oder Kokain haben, wobei der suchtkranke Angehörige auch weitere zusätzliche Substanzen konsumieren konnte. Der drogenabhängige Angehörige konnte sich zum Zeitpunkt der Befragung in Behandlung befinden, sei dies eine Substitutionsbehandlung mit Methadon, Subutex etc., eine heroingestützte Behandlung oder eine Entzugsbehandlung. Er durfte auch abstinent sein, jedoch nicht länger als ein Jahr oder er durfte von der Gasse konsumieren. Zudem musste der drogenabhängige Angehörige zum Zeitpunkt der Befragung noch am Leben sein. Ausschlusskriterien waren ungenügende Deutschkenntnisse und fehlende Volljährigkeit und Mündigkeit.

Bezüglich der geplanten Stichprobengrösse wurden folgende Überlegungen gemacht. Im Rahmen der Auswertung sollte mit linearen Regressionsmodellen gearbeitet werden. Aus 
diesem Grund wurde basierend auf der Annahme, dass in ein Modell höchstens fünf Prädiktoren eingeschlossen werden sollten, mit $G^{*}$ Power die optimale Stichprobengrösse bestimmt (siehe Abb. 3) (Faul, Erdfelder, Lang \& Buchner, 2007; Faul, Erdfelder, Buchner \& Lang, 2009).

Die Abbildung 3 zeigt deutlich, dass bei einer Stichprobengrösse zwischen 90 und 100 Personen bei fünf Prädiktoren mit einem multiplen linearen Regressionsmodell ein mittlerer Effekt aufgedeckt werden kann. Aus diesem Grund wurde die Stichprobengrösse auf etwa 90 Personen festgelegt.

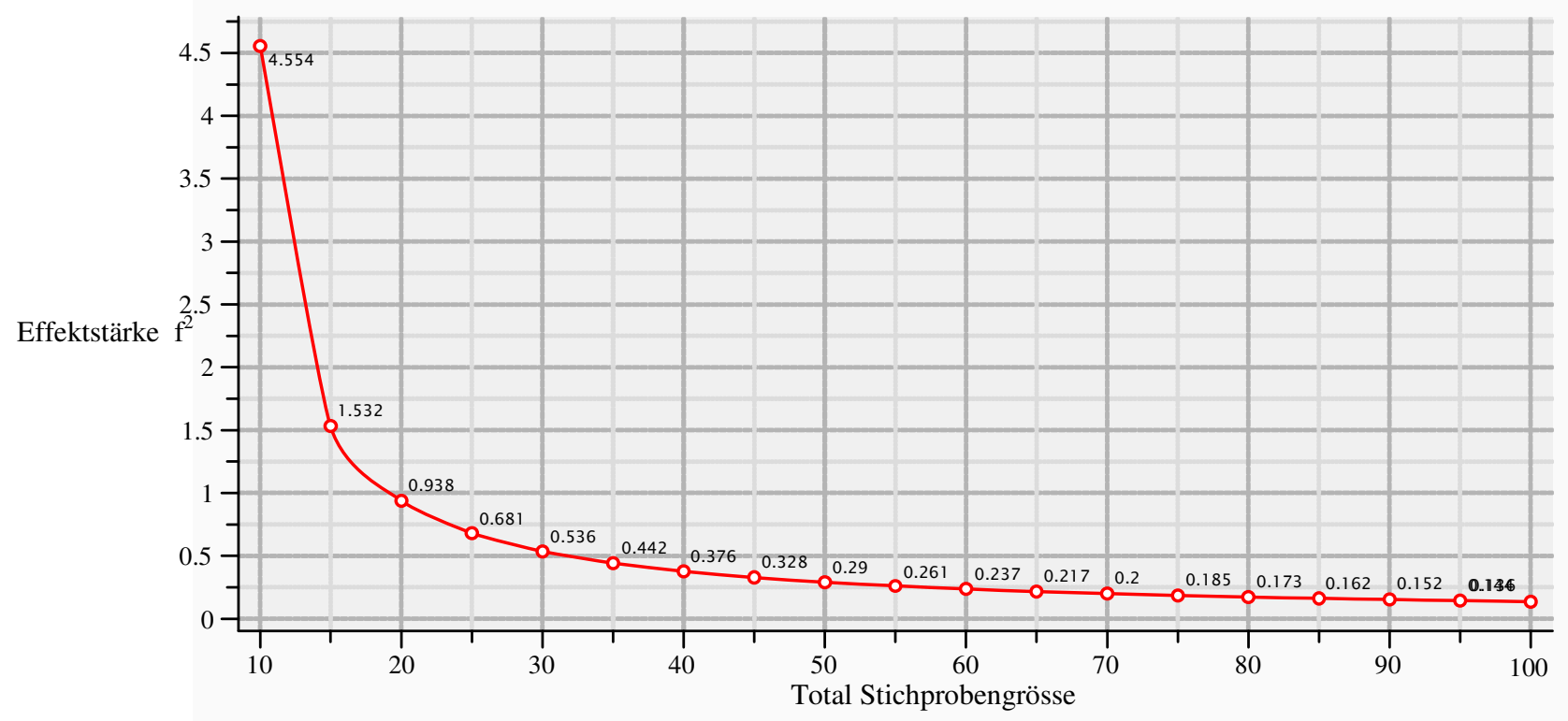

Abb. 3: G*Power: Multiples lineares Regressionsmodell: Anzahl der Prädiktoren $=5, \alpha=.05$, Power $=.80$

Nach der Befragung von 71 Studienteilnehmenden wurde eine erste Zwischenauswertung vorgenommen. In dieser Zwischenanalyse stellte sich heraus, dass die Gruppe der Eltern in der Stichprobe deutlich überrepräsentiert waren. Aus diesem Grund richtete sich die Rekrutierung in der Folge nur noch auf Geschwister und Partnerinnen und Partner aus, weil die Zwischenauswertung ausserdem vermuten liess, dass die Beziehungsart allenfalls relevant sein könnte für die erlebte Belastung. 


\subsubsection{Rekrutierung}

Die Rekrutierung der Angehörigen stellte eine grosse Herausforderung dar, da es in der Schweiz nur wenige Institutionen und Vereine gibt, die sich auf die Anliegen von Angehörigen von Drogenabhängigen konzentriert haben. Entsprechend erfolgte die Rekrutierung der Studienteilnehmenden über mehrere Quellen und mit unterschiedlichen Strategien. Grundsätzlich liessen sich dabei drei Bereiche unterscheiden: Rekrutierungen über Institutionen oder Vereine, die Kontakt zu Angehörigen hatten, Rekrutierungen über die Medien und Rekrutierungen über Mund-zu-Mund-Werbung von Angehörigen.

Zuerst soll kurz auf die Institutionen eingegangen werden, die das Projekt bei der Rekrutierung unterstützten. An erster Stelle ist die Angehörigenvereinigung Drogenabhängiger (ada-zh) zu nennen. Die Rekrutierung der Angehörigen über die Angehörigenvereinigung Drogenabhängiger (ada-zh) erfolgte in einem ersten Schritt als Informationsveranstaltung im Rahmen der Generalversammlung des Vereins. In einem zweiten Schritt wurden die Therapeutinnen und Therapeuten der Beratungsstelle gebeten, ihre aktuellen Klientinnen und Klienten für eine Studienteilnahme anzufragen. In diesem Zusammenhang wurde den potentiellen Studienteilnehmenden eine Information zur Studie mit den Kontaktdaten der Studienleitung abgegeben. Eine weitere Vereinigung, über die Angehörige rekrutiert wurden, war der Verband der Eltern- \& Angehörigenvereinigung Drogenabhängiger (VEVDAJ). Einerseits konnten im Rahmen einer Präsentation zum Thema an der Delegiertenversammlung des VEVDAJ Angehörige für die Studie gewonnen werden, andererseits schaltete der VEVDAJ auf der Homepage einen Aufruf. Unterstützung bei der Rekrutierung kam zudem vom Zentrum für Anhängigkeitserkrankungen der Psychiatrischen Universitätsklinik Zürich, wo ebenfalls die Therapeuten und Therapeutinnen Angehörige für eine mögliche Studienteilnahme anfragten.

Eine weitere wichtige Quelle der Rekrutierung stellten die Medien dar. Dazu gehörten zwei Aufrufe in der Angehörigenzeitschrift FORUM, die vierteljährlich von der Angehörigenvereinigung Drogenabhängiger (ada-zh) und dem VEVDAJ gemeinsam herausgegeben wird. Ein kleiner Aufruf erschien in der Neuen Zürcher Zeitung NZZ und zwei kurze Artikel wurden in der Pendlerzeitung 20minuten publiziert. 
Nicht zuletzt sei die Mund-zu-Mund-Werbung erwähnt, die viele Angehörige für die Studie machten und somit auch dazu beitrugen, dass Personen als Probanden gewonnen werden konnten.

\subsubsection{Befragung}

In einem ersten Schritt wurden alle Studienteilnehmenden, die sich meldeten, telefonisch über den Zweck und den Ablauf der Studie informiert. In diesem Zusammenhang wurde jeweils auch bereits abgeklärt, ob sie die Kriterien für die Studienteilnahme erfüllten. War dies der Fall und die Angehörigen erklärten sich zur Teilnahme an der Studie bereit, wurde ein Termin für die Befragung vereinbart. Bei diesem persönlichen Treffen wurden den Studienteilnehmenden vor Befragungsbeginn eine schriftliche Probandeninformation abgegeben, in der sie nochmals über den Zweck der Studie und den Ablauf der Befragung aufgeklärt wurden. Alle Studienteilnehmenden wurden gebeten, diese Probandeninformation sowie die schriftliche Einverständniserklärung sorgfältig durchzulesen und offene Fragen vor Studienbeginn zu klären. Nach Unterzeichnung der schriftlichen Einverständniserklärung durch den jeweiligen Studienteilnehmenden und die Interviewerin wurden den Probanden die Fragebögen zum Ausfüllen abgegeben. Die Angehörigen wurden dabei gebeten, die Fragebögen den Instruktionen entsprechend möglichst vollständig auszufüllen. Auch wurden sie darauf hingewiesen, dass sie sich bei Unklarheiten jederzeit an die Interviewerin wenden könnten. Nach dem Ausfüllen der Fragebögen fand eine kurze Nachbesprechung statt, in der die Angehörigen bei Interesse über die erhobenen Konstrukte der einzelnen Fragebogen aufgeklärt wurden und in der sie um eine kurze Rückmeldung zu den einzelnen Fragebogeninstrumenten gebeten wurden, da es sich bei den eingesetzten Instrumenten bis auf das ISE nicht um spezifische Fragenbogeninstrumente für Angehörige von drogenabhängigen Menschen handelte. Mit denjenigen Angehörigen, die sich zusätzlich zur Teilnahme für das problemzentrierte Interview bereit erklärten, wurde ein zweiter Termin vereinbart. Die Studienteilnehmenden erhielten keine Aufwandentschädigung.

Der erste Angehörige wurde Anfang September 2011 befragt und die letzte Befragung fand Ende August 2012 statt. Insgesamt konnten 88 Angehörige mit den Fragebögen befragt werden. Von diesen 88 Personen erklärten sich 13 für ein zusätzliches problemzentriertes Interview bereit. Die Befragungen wurden entweder in der Angehörigenvereinigung Drogenabhängiger (ada-zh), im Zentrum für Abhängigkeitserkrankungen der Psychiatrischen Universitätsklinik Zürich oder bei den Angehörigen zu Hause durchgeführt. 


\subsubsection{Datenmanagement und -aufbereitung}

\subsubsection{Quantitative Befragung: Fragebogen}

Die Daten wurden in einem Datenfile von SPSS (Version 20) aufbereitet. Vor Auswertungsbeginn wurden die Daten über Häufigkeitstabellen auf fehlende und falsche Werte überprüft und entsprechend korrigiert. Neben der Bildung der Skalenwerte basierend auf den Manualen der Fragebögen wurden für die Datenauswertung zusätzlich diverse Variablen neu gebildet:

Dauer der Suchterkrankung: Die Variable wurde aus der Differenz der Variablen Alter des suchtkranken Angehörigen und der Variablen Alter, in dem beim suchtkranken Angehörigen erstmals Anzeichen der Suchterkrankung auftraten, gebildet.

Dauer bis zur ersten Behandlung: Die Variable wurde gebildet aus der Differenz der beiden Variablen Alter des Angehörigen bei der ersten Behandlung und Alter, in dem erstmals Anzeichen der Suchterkrankung auftauchten.

Lebenssituation: Ursprünglich umfasste die Variable Lebenssituation, die darüber Auskunft gibt, ob und mit wem die Angehörigen zusammenlebten, sechs Kategorien. Die Zahl der Kategorien wurde für die weitere Auswertung auf drei reduziert und vereinfacht. Die neue Variable umfasste die Kategorien: 1. allein, 2. mit (Ehe-)Partner und 3. andere, wobei in der Kategorie andere auch Kinder erfasst wurden.

Mit dem suchtkranken Angehörigen zusammenlebend: Die Variable hatte ursprünglich drei Kategorien und wurde für die weitere Auswertung vereinfacht. Es wurde eine neue Variable mit den Ausprägungen „mit dem suchtkranken Angehörigen zusammenlebend“ versus „,nicht mit dem suchtkranken Angehörigen zusammenlebend“" gebildet.

Höchster Schulabschluss (Bildung): Die Variable bestand ursprünglich aus zehn Kategorien. Für die weitere Auswertung wurde die Zahl der Kategorien auf fünf reduziert: 1. Grundschule, 2. Berufslehre, 3. Höhere Fachschule, 4. Universität bzw. Fachhochschule und 5. Sonstiges. Die Angehörigen wurden dabei derjenigen Kategorie zugeordnet, deren höchsten Abschluss sie erfolgreich erreicht hatten. 
Beschäftigungssituation: Im Fragebogen wurde die Variable über sieben Kategorien erhoben. Diese wurde für die weitere Auswertung auf fünf reduziert und somit vereinfacht: 1. arbeitslos, 2. Hausfrau bzw. -mann, 3. in Ausbildung, 4. erwerbstätig und 5. pensioniert.

Für die Stichprobenbeschreibung wurden zudem sämtliche Variablen kategorisiert, die eine Zeitdauer abbildeten. Mit den folgenden Variablen wurde so verfahren: Alter der Studienteilnehmenden, Alter des suchtkranken Angehörigen, Alter bei den ersten Anzeichen der Suchterkrankung, Dauer der Suchterkrankung und Dauer bis zur ersten Behandlung.

Alle Auswertungen, die in der vorliegenden Arbeit mit den quantitativen Fragebogendaten vorgenommen wurden, wurden mit SPSS (Version 20) durchgeführt.

\subsubsection{Fehlende Werte}

Im Rahmen der Überprüfung der Werte in den Häufigkeitstabellen wurde auch eine Analyse der fehlenden Werte durchgeführt. Dies geschah aus folgenden Gründen: Der durch die fehlenden Werte reduzierte Datensatz führt zu einem Verlust an Effizienz bei der Schätzung der Parameter (Lüdtke, Robitzsch, Trautwein \& Köller, 2007, S. 103). Aus diesem Grund schien es sinnvoll abzuschätzen, wie gross der Verlust an Power durch den reduzierten Datensatz war. Zudem verlangen die meisten statistischen Auswertungsverfahren vollständige Datensätze, so dass in der Auswertung entschieden werden muss, wie mit den unvollständigen Daten vorgegangen werden soll (ebd.). Schliesslich besteht die Gefahr und darin liegt wohl das grösste Risiko, dass die fehlenden Werte nicht zufällig zustande gekommen sind, sondern dass ihnen eine gewisse Systematik zugrunde liegt. In diesem Fall bestünde die Gefahr, dass es in der statistischen Parameterschätzung zu Verzerrungen kommen könnte und somit die Ergebnisse verfälscht würden. Gemäss Lüdtke et al. (2007, S. 103) ist es momentan in der Forschung nach wie vor gängige Praxis, dass Personen mit fehlenden Werten aus der Auswertung ausgeschlossen werden oder dass die fehlenden Werte durch Mittelwerte ersetzt werden. Dies obwohl diese beiden Vorgehensweisen mit einigen Risiken verbunden sind.

Der fallweise Ausschluss von fehlenden Werten ist gemäss Lüdtke et al. (2007, S. 106) nur dann zulässig, wenn nur wenige Fälle betroffen sind $(<5 \%)$, weil sonst der Verlust an Power zu gross ist. Der fallweise Ausschluss bietet den Vorteil, dass es nicht zu einem Bias bei der Parameterschätzung kommt und wird von den Autoren vor allem für explorative Analysen empfohlen. In der vorliegenden Arbeit wurde deshalb in einem ersten Schritt untersucht, wie 
viele Missings bei den einzelnen Fragebögen auftraten. Bei einer nur geringen Zahl von Ausfällen sollte der fallweise Ausschluss in Betracht gezogen werden.

Während Lüdtke et al. (2007, S. 106) ausser von der multiplen Imputation, die von SPSS nicht angeboten wird, eher von imputationsbasierten Verfahren abraten, da sie mit einem Bias in der Paramaterschätzung verbunden sein könnten, sollte in der vorliegenden Arbeit bei einer grossen Zahl von Missings in einem Fragebogen dennoch über ein imputationsbasiertes Verfahren nachgedacht werden. Um das Risiko eines Bias ein Stück weit zu kontrollieren, wurden basierend auf praktischen Überlegungen verschiedene Imputationen (Einsetzen von Mittelwerten bzw. Einsetzen von konservativen Schätzwerten) vorgenommen und die Ergebnisse miteinander verglichen.

\subsubsection{Reliabilität der Skalen}

Vor Beginn der Auswertungen wurde für sämtliche Skalen die Reliabilität über die interne Konsistenz bestimmt. Die interne Konsistenz ist sehr einfach zu berechnen und wird folglich in der Praxis häufig eingesetzt, um über die Reliabilität einer Skala Auskunft zu geben (Schermelleh-Engel \& Werner, 2012, S. 132).

Bei der internen Konsistenz wird jedes Item einer Skala oder eines Tests als separater Testteil angesehen (Schermelleh-Engel \& Werner, 2012, S. 130). Aus der Zusammenhangsstruktur dieser Items kann die Reliabilität einer Skala geschätzt werden. Im Vorfeld wurde festgelegt, dass die Skalen mindestens eine Reliabilität von Cronbach's $\alpha>.50$ aufweisen sollten, um in die weitere Auswertung eingeschlossen zu werden (George \& Mallery, 2003, S. 231). Gemäss George und Mallery (2003) ist ein Cronbach's $\alpha>.50$ zwar ein schlechtes Reliabilitätsmass, aber noch akzeptabel für eine Auswertung, während interne Konsistenzen unter .50 inakzeptabel und unbrauchbar sind. Skalen, die nicht über eine ausreichende Reliabilität verfügten, sollten in der weiteren Auswertung nicht berücksichtigt werden.

\subsubsection{Qualitative Befragung: Interviews}

Die Interviews wurden mit dem Aufnahmegeräte eines Handys aufgezeichnet und schliesslich als MP3-Daten auf dem Computer abgespeichert. Anschliessend wurden die Interviews in Microsoft Word transkribiert nach den Regeln der Ulmer Textbank (Mergenthaler, 1992) mit einigen kleinen Adaptationen, wie sie von Boothe, Grimmer, Luder, Luif, Neukom und 
Spiegel (2002) vorgeschlagen worden sind (Boothe et al., 2002) (siehe Anhang). Wie von Kuckartz (2010, S. 47) empfohlen wurden die Interviews zunächst wörtlich transkribiert und korrigiert und erst anschliessend anonymisiert. Im Anonymisierungsprozess wurden sämtliche Namen, Orte und Daten mit Platzhaltern ersetzt, die „für das Verständnis wesentliche Bedeutungen transportierten“ (Kuckartz, 2010, S.47). Beispielsweise wurden Ortschaften mit Bezeichnungen wie *Dorf codiert. Für die Entschlüsselung der Codes wurde eine separate Übersichtstabelle erstellt. Jedem Angehörigen wurde zudem ein Deckname zugeteilt.

Die in Microsoft Word aufbereiteten Interviews wurden anschliessend in MAXQDA, einem Programm zur qualitativen Datenauswertung, importiert.

\subsection{Auswertungsschritte}

\subsubsection{Inventar Subjektiver Stigmaerfahrungen (ISE)}

\subsubsection{Einleitung}

Bei der Angehörigenversion des ISE handelt es sich um einen Fragebogen, der ursprünglich in Kanada entwickelt und neu aus der englischen Sprache ins Deutsche übersetzt wurde. $\mathrm{Zu}$ Beginn des vorliegenden Projektes war die Validierung des Fragebogens geplant, wobei die Ergebnisse aus der vorliegenden Stichprobe einen Beitrag zur Validierung leisten sollten. Da es sich um ein neues, nicht etabliertes Instrument handelte, erschien es sinnvoll, die von den Autoren in der englischen Originalversion vorgeschlagene Zwei-Faktorenstruktur des ISE in der vorliegenden Stichprobe zu überprüfen, bevor weitere Analysen mit dem Fragebogen vorgenommen wurden.

\subsubsection{Korrelationsmatrix der ISE-Items}

Die Ergebnisse einer Faktorenanalyse sind sehr stark von der Qualität der Daten beeinflusst (Backhaus, Erichson, Plinke \& Weiber, 2011, S. 236). Dabei ist es von zentraler Bedeutung, dass neben der Tatsache, dass die erhobenen Variablen für das gesuchte Konstrukt relevant sind, die einzelnen Variablen auch miteinander korrelieren. Folglich wurde im Vorfeld der Faktorenanalyse der Zusammenhang zwischen den einzelnen Variablen, sprich die Korrelationsmatrix der ISE-Items, untersucht. Für die Überprüfung der Korrelationen zwischen den einzelnen Merkmalen stehen verschiedene Prüfkriterien zur Verfügung. Im 
Rahmen dieser Arbeit wurde die Korrelationsmatrix anhand der folgenden zwei Verfahren überprüft:

Der „Bartlett Test of Sphericity“ prüft, mit welcher Wahrscheinlichkeit die untersuchte Stichprobe aus einer Population stammt, in der die einzelnen Variablen nicht korreliert sind (Backhaus et al., 2011, S. 341). Dabei postuliert die Nullhypothese $\left(\mathrm{H}_{0}\right)$, dass die erhobenen Merkmale (Items des ISE) in der Grundgesamtheit unkorreliert sind, während die Alternativhypothese $\left(\mathrm{H}_{1}\right)$ vom Gegenteil ausgeht, nämlich dass die Variablen in der Population korreliert sind. Voraussetzung für eine Faktorenanalyse ist, dass die Merkmale miteinander korrelieren. Um eine Faktorenanalyse mit den Items des ISE durchführen zu können, musste deshalb die Nullhypothese zu Gunsten der Alternativhypothese verworfen werden können.

Als weiteres Gütekriterium für die Korrelationsmatrix wurde die Prüfgrösse von Kaiser, Meyer und Olkin „measure of sampling adequacy (MSA)“ herangezogen (Backhaus et al. 2011, S. 342). Neben der Beurteilung der Angemessenheit der Gesamtstichprobe (Korrelationsmatrix) in Bezug auf eine Faktorenanalyse kann der MSA-Wert auch darüber Auskunft geben, welche Items sich für die Faktorenanalyse eignen und welche besser im Vorfeld aus der Analyse ausgeschlossen werden sollten. Der Wert für die MSA-Statistik kann zwischen 1 und 0 liegen, wobei bei einem Wert nahe 0 davon auszugehen ist, dass die Faktorenanalyse wenig reliable Faktoren liefert (Backhaus et al, 2011, S. 342; Field, 2009, S. 647). Gemäss Kaiser und Rice (Kaiser \& Rice, 1974; zit. nach Backhaus et al., 2011, S. 343) kann das Kaiser-Meyer-Olkin (KMO-)Kriterium, wie das MSA-Kriterium ebenfalls bezeichnet wird, folgendermassen interpretiert werden:

$$
\begin{array}{ll}
\text { MSA } \geq .90 & \text { marvelous (,,erstaunlich“) } \\
\text { MSA } \geq .80 & \text { meritorious (,,verdienstvoll“) } \\
\text { MSA } \geq .70 & \text { middling (,,ziemlich gut“) } \\
\text { MSA } \geq .60 & \text { mediocre (,,mittelmässig“) } \\
\text { MSA } \geq .50 & \text { miserable (,,kläglich“) } \\
\text { MSA }<.50 & \text { unacceptable (,untragbar“) }
\end{array}
$$

In der Auswertung sollte das MSA-Kriterium mindestens .60 betragen, damit die Faktorenanalyse verlässlich interpretiert werden konnte. Basierend auf den Empfehlungen 
von Field (2009, S. 659) sollten zudem die Items des ISE ebenfalls bezüglich des KMOKriteriums untersucht werden. In der vorliegenden Arbeit wurde bei Items, deren MSA-Werte untragbar waren, ein Ausschluss aus der Faktorenanalyse in Betracht gezogen und geprüft.

\subsubsection{Bestimmung der relevanten Faktorenlösung beim ISE}

Ziel der Faktorenanalyse war es, die von Stuart et al. (2005) postulierte Zwei-Faktorenstruktur des ISE zu bestätigen, die sich gemäss den Autoren in eine Stigma-Erfahrungs- und eine Stigma-Auswirkungs-Skala trennen liess. $\mathrm{Zu}$ diesem Zweck wurde eine Hauptkomponentenanalyse gerechnet. Die Wahl des Verfahrens orientierte sich an den kanadischen Autoren, die bei der Bestimmung der Faktorenstruktur ebenfalls eine Hauptkomponentenanalyse gerechnet hatten (ebd., S. 202).

Gemäss Field (2009, S. 638) ist die Hauptkomponentenanalyse nicht im eigentlichen Sinne als Faktorenanalyse zu bezeichnen. Im Gegensatz zu anderen faktorenanalytischen Methoden schätzt die Hauptkomponentenanalyse keine Faktoren, sondern das Koordinatensystem mit den zu faktorisierenden Variablen wird so rotiert, dass die neu gefundenen Achsen maximale Varianz erklären, das heisst auf einer der neuen Achsen maximal streuen (Bortz \& Schuster, 2010, S. 397). Dabei werden die Koordinaten (Item-Werte) eines Studienteilnehmenden auf der neuen Achse als Linearkombination der ursprünglichen Koordinaten dargestellt. Gemäss Backhaus et al. (2011) lautet die zentrale Frage bei der Interpretation der Faktoren einer Hauptkomponentenanalyse: „Wie lassen sich die auf einem Faktor hoch ladenden Variablen durch einen Sammelbegriff zusammenfassen?“ (S. 357). Ziel des Verfahrens ist es folglich, die Variablen zu gruppieren.

Die Rotation der Achsen erfolgte orthogonal mit dem Varimax-Kriterium, so dass die Items nur substantiell auf einem Faktor luden (Hauptladung) und auf den anderen Faktoren (Nebenladungen) gering (Field, 2009, S. 644). Bei der Wahl der Rotationsmethode wurden die Empfehlungen der Autoren der deutschen ISE-Version berücksichtigt (Schulze et al., 2009, S. e21).

Bei der Bestimmung der Anzahl relevanter Faktoren standen verschiedene Kriterien zur Verfügung. Eines dieser Kriterien war das Kaiser-Kriterium (Backhaus et al., 2011, S. 359). Dabei wird die Zahl der zu extrahierenden Faktoren durch die Zahl der Faktoren mit einem Eigenwert grösser eins bestimmt. Nach dem Kaiser-Kriterium werden diejenigen Faktoren als 
relevant erachtet, die mehr Varianz erklären als ein einzelnes Item. Gemäss Field (2009, S. 641) überschätzt aber das Kaiser-Kriterium die Zahl der relevanten Faktoren häufig. Es gibt allerdings Hinweise darauf, dass das Kaiser-Kriterium akkurate Ergebnisse liefert, wenn die Zahl der zu faktorisierenden Variablen unter 30 liegt und alle Kommunalitäten (= Ausmass der Varianz in einer Variablen, die sich durch die Faktoren erklären lässt) einen Wert grösser .70 aufweisen (ebd.).

Eine Alternative zum Kaiser-Kriterium stellt der Scree-Test dar (Cattell, 1966; zit. nach Bortz \& Schuster, 2010, S. 416; Backhaus et al., 2011, S. 359). Beim Scree-Test wird der Verlauf der Eigenwerte der Faktoren graphisch dargestellt (y-Achse: Eigenwert; x-Achse: Nummer des einzelnen Faktors). An der Stelle, an der sich die Differenz zwischen zwei Faktoren vergrössert, entsteht ein Knick in der Graphik. Der erste Knick von links in der Graphik kann herangezogen werden zur Bestimmung der Anzahl relevanter Faktoren. In der vorliegenden Arbeit wurde anhand des Scree-Tests versucht, die optimale Faktorenzahl zu finden, wobei allerdings abhängig vom Ergebnis auch dem Kaiser-Kriterium Beachtung geschenkt wurde. Bei der Bestimmung der relevanten Faktorenanzahl wurden vor allem Kriterien wie Interpretierbarkeit und Generalisierbarkeit berücksichtigt, da eines der Ziele in der vorliegenden Arbeit war, einen Beitrag zur Validierung des ISE zu leisten.

Bei der Beurteilung der Faktorenstruktur wurden die von Guadagnoli und Velicer (1988; zit. nach Bortz \& Schuster, 2010, S. 396) aufgestellten Bedingungen herangezogen. Gemäss den beiden Autoren kann eine Faktorenstruktur als generalisierend interpretiert werden, wenn folgende Bedingungen erfüllt sind:

- Wenn in der Planungsphase dafür gesorgt wurde, dass auf jedem zu erwartenden Faktor zehn oder mehr Variablen entfallen, ist ein Stichprobenumfang von $\mathrm{n} \approx 150$ ausreichend.

- Wenn auf jedem bedeutsamen Faktor mindestens vier Variablen Ladungen über .60 aufweisen, kann die Faktorenstruktur ungeachtet der Stichprobengrösse generalisierend interpretiert werden.

- Das Gleiche gilt für Faktorenstrukturen mit Faktoren auf denen jeweils zehn bis zwölf Variablen Ladungen um .40 oder darüber aufweisen.

- Faktorenstrukturen mit Faktoren, auf denen nur wenige Variablen geringfügig laden, sollten nur interpretiert werden, wenn $n \geq 300$ ist. Für $n<300$ ist die Interpretation 
der Faktorenstruktur von den Ergebnissen einer Replikation abhängig zu machen. (Guadagnoli \& Velicer, 1988; zit. nach Bortz \& Schuster, 2010, S. 396)

In der vorliegenden Arbeit waren vor allen die letzten drei Bedingungen relevant, falls sich die Zwei-Faktorenstruktur mit den beiden Skalen Stigma-Erfahrung und Stigma-Auswirkung nicht bestätigen liesse. Sollte sich die Struktur des Inventars nicht bestätigen, sollten die Bedingungen von Guadagnoli und Velicer (1988; zit. nach Bortz \& Schuster, 2010, S. 396) herangezogen werden, um eine alternativ gefundene Faktorenstruktur zu beurteilen in Bezug auf deren Interpretier- und Generalisierbarkeit.

Bei der Beurteilung der gefundenen Faktorenlösung sollte zudem untersucht werden, wie gut diese die Daten repräsentierte (goodness of fit) (Field, 2009, S. 662ff). Im Rahmen von SPSS lässt sich eine Korrelationsmatrix basierend auf dem Modell berechnen. Diese Modell-Matrix kann anschliessend mit der Korrelationsmatrix der Daten verglichen werden. Je geringer die Differenzen zwischen der Korrelationsabweichungen zwischen Modell und Daten ist, desto besser ist das Modell oder in anderen Worten die gefundene Faktorenlösung in Bezug auf die vorhandenen Daten. Als Richtwert gibt Field (2009, S. 664) an, dass der Anteil der nicht redundanten Residuen $>.05$ nicht mehr als $50 \%$ der Residuen ausmachen sollte, damit das Modell die Daten ausreichend erklärt. Die vorliegende Arbeit orientierte sich an diesem Kriterium. Damit die Daten durch die gefundene Faktorenlösung ausreichend erklärt würden, wurde ein Anteil von nicht redundanten Residuen > .05 von unter $50 \%$ festgelegt.

\subsubsection{Auswirkungen der Suchterkrankung auf die Angehörigen}

\subsubsection{Einleitung}

Eine der zentralen Fragen der Arbeit lautete, welche Auswirkungen die Suchterkrankung auf die Angehörigen hat. Mittels multipler linearer Regressionsmodelle wurde dieser Frage nachgegangen, indem untersucht wurde, welche Faktoren die psychische Gesundheit von Angehörigen beeinflussen und welche Bewältigungsstrategien beim Umgang mit den Belastungen besonders hilfreich sind. Zusätzlich wurde über den Cut-off-Wert des BDI-V untersucht, wie das Risiko für psychische Störungen durch die Belastungen beeinflusst wird. 


\subsubsection{Multiple Regressionsanalyse}

Die multiple Regressionsanalyse erlaubt es, die Beziehungen zwischen mehreren Prädiktorvariablen und einer abhängigen Variablen zu untersuchen (Bortz \& Schuster, 2010, S. 342). Im vorliegenden Fall wurde als abhängige Variable der BDI-V gewählt, während als Prädiktorvariablen Variablen und Skalen basierend auf Zero-Order-Korrelationen und sachlogischen Überlegungen gewählt wurden. In der Regressionsanalyse erfolgt die Schätzung der Beziehung zwischen der Outcome- (abhängigen Variablen) und den Prädiktorvariablen (unabhängigen Variablen) über eine Regressionsfunktion, die der Annahme unterliegt, dass die Beziehung zwischen unabhängiger und abhängiger Variable linear ist (Field, 2009, S. 221). Die lineare Beziehung zwischen Prädiktor- und Outcomevariable ist dabei eine zentrale Grundvoraussetzung für die Analyse der Daten mit einem linearen Regressionsmodell.

Die Regressionsfunktion ist charakterisiert durch ein konstantes Glied $b_{0}$, die Regressionskoeffizienten $b_{j}$ und dem Residualwert $\varepsilon$ (Field, 2009, S. 199). Die Regressionsfunktion wird dabei so definiert, dass die Summe der quadrierten Residuen minimal wird (Methode der kleinsten Quadrate) (Backhaus et al., 2011, S. 67; Field, 2009, S. 200). Im Rahmen der Regressionsfunktion besitzen insbesondere die Regressionskoeffizienten eine wichtige inhaltliche Bedeutung, da sie den Effekt der Änderung einer unabhängigen Variablen auf die abhängige Variable angeben.

Die Güte der Regressionsfunktion ist neben der bereits genannten linearen Beziehung zwischen unabhängiger und abhängiger Variablen von weiteren Voraussetzungen abhängig. Verletzungen dieser Voraussetzungen führen $\mathrm{zu}$ unpräzisen Schätzungen bei der Regressionsfunktion und den Regressionskoeffizienten. Folgende Annahmen sollten erfüllt sein (Backhaus et al., 2011, S. 85 ff; Field, 2009, S. 220 ff):

- Zwischen zwei oder mehreren Prädiktorvariablen darf keine lineare Abhängigkeit bestehen (keine perfekte Multikollinearität). Zur Prüfung wurde in der vorliegenden Arbeit die Toleranz der einzelnen Variablen herangezogen. Werte kleiner .20 bilden dabei Grund zur Besorgnis (Menard, 1995; zit. nach Field, 2009, S. 224).

- Die Residuen für sämtliche Beobachtungen sollten unabhängig sein, d.h. sie sollten unkorreliert sein (keine Autokorrelation). Dieses Kriterium ist gemäss Backhaus et al. 
(2011, S. 103) allerdings nur relevant für Zeitreihendaten und wurde deshalb in den vorliegenden Daten nicht berücksichtigt.

- Die Varianz $\sigma^{2}$ der Residuen sollte für alle Prädiktorvariablen konstant sein (Homoskedastizität). Die Homoskedastizität wurde mittels Streudiagramm untersucht, wobei die aufgrund der Regressionsgleichung geschätzten standardisierten Werte auf der $\mathrm{x}$-Achse und die standardisierten Residuen für die einzelnen Beobachtungswerte auf der y-Achse abgetragen wurden. Lassen sich im Rahmen solcher Streudiagramme Muster erkennen, muss davon ausgegangen werden, dass die Voraussetzung verletzt ist und Heteroskedastizität vorliegt. Gemäss Backhaus et al. (2011, S. 92) kann Heteroskedastizität in den Daten auch einen Hinweis auf Nichtlinearität geben. Insofern konnte die Interpretation aus den Streudiagrammen auch herangezogen werden, um die Linearität zwischen Prädiktor- und Outcomevariablen zu prüfen.

- Die Residuen sollten normalverteilt sein. Basierend auf dem zentralen Grenzwertsatz wurde davon ausgegangen, dass die Annahme erfüllt war. Folglich soll im weiteren Verlauf der Arbeit nicht mehr darauf eingegangen werden.

- Die Residuen sollten einen Erwartungswert gleich Null haben. Grundsätzlich geht das Regressionsmodell davon aus, dass sich Schwankungen in den Residuen letztlich ausgleichen und somit gleich Null sind. In der vorliegenden Arbeit wurde aufgrund der Objektivität bei der Durchführung nicht davon ausgegangen, dass es zu einem systematischen Messfehler in der Erhebung gekommen war, wodurch eine Verletzung der Annahme möglich gewesen wäre. Aus diesem Grund soll im weiteren Verlauf nicht weiter darauf eingegangen werden.

\subsubsection{Prüfung der Regressionsfunktion}

Die geschätzte Regressionsfunktion kann anhand von zwei Kriterien auf ihre Güte, d.h. die Passung des Modells auf die erhobenen Daten, überprüft werden (Backhaus et al., 2011, S. 72). Die Prüfung kann über die Regressionsfunktion global erfolgen, wobei es um die Frage geht, ob und wie gut das Regressionsmodell die abhängige Variable erklären kann. Schliesslich ist aber auch eine Prüfung der einzelnen Regressionskoeffizienten möglich, wobei die Klärung des Einflusses einer einzelnen Prädiktorvariablen im Regressionsmodell auf die abhängige Variable im Vordergrund steht. 
In der vorliegenden Arbeit sollten das Bestimmtheitsmass $\left(R^{2}\right)$ und die F-Statistik herangezogen werden, um die Güte der Regressionsfunktion global zu bestimmen. Die Basis für das Bestimmtheitsmass $R^{2}$ bilden die Residuen, die durch die Abweichungen zwischen empirischen Werten und den geschätzten Werten der Regressionsfunktion zustande kommen (Backhaus et al., 2011, S. 72). Das Bestimmtheitsmass $R^{2}$ wird dabei aus den quadrierten Korrelationen zwischen den beobachteten und geschätzten Y-Werten gebildet und gibt Auskunft über den Anteil der Varianz, die das Regressionsmodell in den empirischen Daten zu erklären vermag. Im Gegensatz zum Bestimmtheitsmass bezieht die F-Statistik auch die Stichprobengrösse bei der Beurteilung des gesamten Regressionsmodells heran und prüft das Modell beziehungsweise die Regressionskoeffizienten auf Signifikanz (ebd., S. 76). Es wird folgende Nullhypothese $\left(\mathrm{H}_{0}\right)$ formuliert: Es besteht kein Zusammenhang zwischen den unabhängigen und der abhängigen Variablen und somit ist die Grundgesamtheit der Regressionskoeffizienten gleich Null. Wird der F-Test signifikant, kann von einem durch die Regressionsbeziehung postulierten Zusammenhang mit einer vorbestimmten Irrtumswahrscheinlichkeit $(\alpha<.05)$ ausgegangen werden.

Wenn die Prüfung der Regressionskoeffizienten durch den F-Test ergeben hat, dass nicht alle Regressionskoeffizienten Null sind, kann in einem zweiten Schritt untersucht werden, welche Regressionskoeffizienten signifikant von Null abweichen $\left(\mathrm{H}_{0}: \beta_{j}=0\right)$ (Backhaus et al., 2011, S. 81). Die Prüfung von $\mathrm{H}_{0}$ erfolgt mittels einer t-Statistik. Muss die Nullhypothese $\left(\mathrm{H}_{0}\right)$ verworfen werden, dann kann davon ausgegangen werden, dass unter einer vorbestimmten Irrtumswahrscheinlichkeit $(\alpha<.05)$ die unabhängige Variable einen signifikanten Einfluss auf die abhängige Variable hat.

\subsubsection{Typische Gruppen von Angehörigen aufgrund der Belastung}

\subsubsection{Einleitung}

Eine der zentralen Fragen der Arbeit lautete, wie Angehörige mit den Belastungen, die durch die Suchterkrankung eines nahestehenden Menschen entstehen, umgehen und welche Auswirkungen dies auf ihr Wohlbefinden, ihre psychische Gesundheit und ihre Bewältigungsstrategien hat. Es war deshalb auch Ziel der Arbeit, diejenigen Angehörigen zu identifizieren, die angaben, durch die Suchterkrankung am stärksten belastet zu sein. Im Rahmen einer künftigen Prävention ist es äusserst wichtig, Angehörige charakterisieren zu können, die ein erhöhtes Risiko haben, krank zu werden. Die Gruppierung der Angehörigen 
erfolgte mit clusteranalytischen Verfahren, wobei als Datenbasis alle Itemwerte des BDI-V und des ZBI sämtlicher Studienteilnehmender für die Analyse herangezogen wurden. Jeder Angehörige war dabei durch ein eigenes Belastungsmuster charakterisiert, das sich durch die Itemwerte in den beiden Fragebögen BDI-V und ZBI definierte. Für die Analyse wurden der BDI-V und das ZBI gewählt, da das ZBI einerseits direkt die Belastungen erhebt, die die Angehörigen im Zusammenhang mit ihrem drogenabhängigen Angehörigen erleben und der BDI-V in Form der Depressivität andererseits die direkten Auswirkungen der Belastungen erfasst.

Clusteranalytische Verfahren gruppieren Objekte oder im vorliegenden Fall Angehörige von drogenabhängigen Menschen so, dass die Unterschiede zwischen den Angehörigen eines Clusters möglichst klein und die Unterschiede zwischen Angehörigen unterschiedlicher Cluster möglichst gross sind (Bortz \& Schuster, 2010, S. 458). Dabei kann zwischen hierarchischen und nicht-hierarchischen (partitionierenden) Verfahren unterschieden werden. Während hierarchische Verfahren gut geeignet sind, eine inhaltlich plausible Clusteranzahl zu finden, bringen nicht-hierarchische Verfahren den Vorteil, dass sie aufgrund des schrittweisen Verschiebens von Angehörigen nach festgelegten Kriterien die beste Zuteilung der Angehörigen zu der vorgegebenen Clusteranzahl ermöglichen. In der vorliegenden Arbeit sollte deshalb wie von Bortz und Schuster (2010, S. 461) empfohlen ein hierarchisches Verfahren in Form der Ward-Methode und ein nicht-hierarchisches Verfahren in Form der k-

Means-Methode kombiniert werden, um die optimale Clusterlösung und -zuteilung der Angehörigen zu finden.

\subsubsection{Bestimmung der optimalen Clusterlösung}

In einem ersten Schritt wurde ausgehend von der Rohdatenmatrix, die anhand der 88 Studienteilnehmenden und der Itemwerte des ZBI und des BDI-V jedes Studienteilnehmenden charakterisiert war, die Ähnlichkeit beziehungsweise Distanz zwischen den einzelnen Studienteilnehmenden durch die quadrierte Euklidische Distanz quantifiziert (Backhaus et al, 2011, S. 399). Die quadrierte Euklidische Distanz wurde berechnet, indem für jedes Studienteilnehmendenpaar die Differenzwerte jeder Variablen quadriert und anschliessend addiert wurde. Durch die Bestimmung der Proximität mittels der quadrierten Euklidischen Distanz wurde die Rohdatenmatrix in eine Distanzmatrix überführt. Dabei waren Angehörige umso ähnlicher, je kleiner ihr Distanzmass war. Diese Distanzmatrix 
bildete die Grundlage für die Clusteralgorithmen, die zum Ziel hatten die Angehörigen in verschiedenen Gruppen zusammen zu fassen (ebd., S. 417).

In einem zweiten Schritt wurde mit dem Ward-Verfahren die Zahl der relevanten Cluster bestimmt. Bei der Ward-Methode handelt es sich um ein hierarchisch agglomeratives Verfahren, d.h. jedem Studienteilnehmenden wurde zu Beginn der Analyse ein eigenes Cluster zugeordnet (Backhaus et al., 2011, S. 418). Ausgehend von diesen einzelnen Clustern wurde über die quadrierte Euklidische Distanz die Proximität zwischen den einzelnen Angehörigen bestimmt. Dabei geht es beim Ward-Verfahren nicht darum, diejenigen Objekte zusammenzufassen, die die geringste Distanz zueinander aufweisen, sondern diejenigen durch deren Zusammenfügen sich das Heterogenitätsmass, welches durch die Fehlerquadratsumme charakterisiert ist, am wenigsten vergrössert. Die Ward-Methode hat folglich zum Ziel, möglichst homogene Gruppen innerhalb der Distanzmatrix zu identifizieren. Das Bilden von Gruppen (Clustern) erfolgt sequentiell. Konkret bedeutet dies, dass basierend auf der berechneten Distanzmatrix nur diejenigen Cluster zusammengeführt werden, die die Fehlerquadratsumme am wenigsten erhöhen. Danach werden die Distanzen zwischen den neuen und den bestehenden Clustern nochmals bestimmt und der Vorgang der Clusterbildung über die kleinste Fehlerquadratsumme wird wiederholt bis alle Studienteilnehmenden in einer grossen Gruppe zusammengefasst sind.

Nach der Clusterbildung ging es in einem dritten Schritt darum, die ideale Clusterlösung zu identifizieren. Backhaus et al. (2011, S. 436ff) geben an, dass eine Veränderung des Heterogenitätsmasses, welches sich in Form eines Sprungs in den Fehlerquadratsummen anzeigt, herangezogen werden kann, um die optimale Clusterlösung zu identifizieren. Es empfiehlt sich deshalb, die Entwicklung des Heterogenitätsmasses in einem Struktogramm aufzuzeichnen (y-Achse: Zuwachs der Fehlerquadratsumme; x-Achse: Anzahl Cluster). Zeigt sich in der Grafik ein deutlicher Sprung in der Entwicklung der Fehlerquadratsumme, kann dies ein Entscheidungskriterium für die zu wählende Clusteranzahl sein. Backhaus et al. (2011, S. 437) sprechen in diesem Zusammenhang vom „Elbow-Kriterium“. Sie empfehlen zudem, dass beim Erstellen des Diagramms, die Ein-Cluster-Lösung nicht berücksichtigt werden sollte, da von der Ein- zur Zwei-Clusterlösung meist der grösste Heterogenitätssprung stattfindet. 
In einer Untersuchung konnte Bergs (1981; zit. nach Backhaus et al., 2011, S. 430ff) zeigen, dass das Ward-Verfahren im Vergleich zu anderen Clusteralgorithmen sehr gute Clusterlösungen findet. Nach Bestimmung der optimalen Clusterzahl über das WardVerfahren wurden anschliessend die Cluster mit der nicht-hierarchischen k-Means-Methode gebildet. Dabei wurde die durch die Ward-Methode gefundene optimale Clusteranzahl als Startpartition vorgegeben. Über Euklidische Distanzen, die sich durch das Ziehen der Quadratwurzel aus der quadrierten Euklidischen Distanz bilden liessen, wurde dabei die Distanz aller Angehörigen zu allen Clusterschwerpunkten bestimmt. Hatte ein Angehöriger zum eigenen Clusterschwerpunkt eine grössere Distanz als zu einem anderen, wurde er in dasjenige Cluster verschoben, zu dessen Schwerpunkt er die geringste Distanz aufwies. Daraufhin wurden die Clusterschwerpunkte neu gerechnet und der Vorgang wurde wiederholt bis jeder Angehörige dem Cluster zugeteilt war, zu dessen Schwerpunkt er die geringste Distanz aufwies.

Aufgrund von Vorüberlegungen waren mit grosser Wahrscheinlichkeit zwei Cluster zu erwarten, nämlich eine Gruppe von stark belasteten und eine Gruppe von weniger stark belasteten Angehörigen. Bei mehreren Clusterlösungen wurde allerdings im Vorfeld festgelegt, dass möglichst wenige Cluster mit ähnlicher Probandenzahl pro Gruppe gewählt werden sollten. Auf diese Weise sollte sichergestellt werden, dass im Hinblick auf weitere statistische Auswertungen den einzelnen Gruppen genügend Probanden zugeteilt worden waren.

\subsubsection{Evaluation der Clusterlösung}

Gemäss Bortz und Schuster (2010, S. 465ff) kann trotz der positiven Eigenschaften, die der Ward-Methode und der k-Means-Methode in Grundlagenstudien bestätigt wurden, nicht ausgeschlossen werden, dass ein anderer Clusteralgorithmus allenfalls ein besseres Ergebnis gebracht hätte. Die Diskriminanzanalyse kann an dieser Stelle helfen, die Güte der gefundenen Clusterlösung zu bestätigen (ebd., S. 469). In der vorliegenden Arbeit sollte deshalb die Clusterlösung mittels einer Diskriminanzanalyse überprüft werden. Neben der Prüfung, ob sich zwei oder mehrere Gruppen tatsächlich signifikant unterschieden, war es mittels Diskriminanzanalyse auch möglich zu untersuchen, welche der Items des BDI-V und des ZBI am besten trennten zwischen den Gruppen von Angehörigen bezüglich ihrer Belastungen, die durch die k-Means-Methode definiert worden waren. Dabei entsprachen die Items des BDI-V und des ZBI den unabhängigen Variablen und die Gruppen der Angehörigen 
bezüglich ihrer Belastungen (Clusterlösung) der abhängigen Variablen. Die Bestimmung der Diskriminanzfunktion, derjenigen Achse, die die maximale Unterscheidung zwischen den Gruppen von Angehörigen ermöglichte, erfolgte über Gewichtungskoeffizienten für die einzelnen Variablen (Fragebogen-Items) (Bortz \& Schuster, 2010, S. 490). Diese Gewichtungskoeffizienten entsprachen den Ladungen der einzelnen Items auf der Diskriminanzfunktion, die durch eine Linearkombination der Messwerte gefunden wurde. Folglich gaben die Gewichtungskoeffizienten, welche durch Korrelationen zwischen den einzelnen Items mit dem Diskriminanzfaktor (Diskriminanzfunktion) definiert wurden, darüber Auskunft, welche Items des BDI-V und des ZBI besonders bedeutsam waren für die Unterscheidung zwischen den Gruppen von Angehörigen bezüglich ihrer Belastungen und welche Items eher eine geringere Rolle spielten (ebd.).

Bortz und Schuster (2010, S. 469) merken zudem an, dass es in manchen Fällen sinnvoll sein kann, die gefundene Clusterlösung an externen Variablen zu validieren. Im vorliegenden Fall wurde die Clusterlösung zusätzlich am BDI-V Cut-off-Wert validiert, der mit einer hohen Wahrscheinlichkeit zwischen einer klinisch relevanten Depression und klinischer Unauffälligkeit trennt. Im Falle von mehreren Gruppen (Clustern) sollte zumindest die Gruppe von Angehörigen, die am stärksten belastet war, deutlich über dem Cut-off liegen. Diese Validierung erfolgte über eine Kreuztabelle und unabhängig von einer Diskriminanzanalyse.

\subsubsection{Bezug der Clusterlösung zu weiteren Variablen (logistische Regression)}

Aufgrund des explorativen Charakters der Studie war auch von Interesse, wie sich die verschiedenen Gruppen von Angehörigen (Cluster) bezüglich ihrer Copingstrategien, ihres Selbstwertes und der verschiedenen Facetten der Lebensqualität voneinander unterschieden. Für eine allfällige Unterstützung oder Prävention bei Angehörigen ist es wichtig, die entscheidenden Faktoren, durch die stark belastete Angehörige charakterisiert sind, zu kennen.

Als Zugang wurde eine logistische Regression gewählt. Die logistische Regression bietet den Vorteil, dass sie im Gegensatz zur Diskriminanzanalyse an weniger Voraussetzungen gebunden ist und ihre Ergebnisse somit als robuster angesehen werden können (Backhaus et al., 2001, S. 250). Im Gegensatz zur linearen Regression, die Korrelationen zwischen einer abhängigen metrischen Variablen und einer oder mehreren unabhängigen Variablen 
nachweist, wird in der logistischen Regression die Eintrittswahrscheinlichkeit von zwei oder mehr kategorialen abhängigen Variablen in Abhängigkeit von einer oder mehreren unabhängigen Variablen bestimmt (ebd., S. 254). Im Fall einer binären Ausprägung wird unterschieden zwischen $\mathrm{y}=1$ als „Ereignis tritt ein“ (stark belastete Angehörige) und y =0 als „Ereignis tritt nicht ein“ (weniger stark belastete Angehörige). Um die Wahrscheinlichkeit für das Eintreten des Ereignisses $\mathrm{y}=1$ bestimmen $\mathrm{zu}$ können, wird eine Variable $\mathrm{Z}$ angenommen, deren Eigenschaften nicht empirisch beobachtbar sind. Über diese latente Variable Z, von der angenommen wird, dass sie durch eine Linearkombination der unabhängigen Variablen erzeugt wird, wird die Verbindung zwischen der kategorialen abhängigen Variablen und den unabhängigen Variablen hergestellt (ebd., S. 255). Unter Verwendung der logistischen Funktion wird schliesslich die Stärke des Einflusses (LogitKoeffizient) jeder unabhängigen Variablen auf die Eintrittswahrscheinlichkeit des Ereignisses (Zugehörigkeit der Angehörigen zu einer bestimmten Gruppe) berechnet. Wie bei der linearen Regressionsanalyse ist es bei der logistischen Regressionsanalyse ebenfalls möglich, die Signifikanz einzelner Koeffizienten innerhalb des Modells zu überprüfen. Dabei wird geprüft, ob die unabhängige Variable einen Einfluss auf die Trennung zwischen den Kategorien der abhängigen Variable hat (ebd., S. 280). Die Wald-Statistik gab in diesem Zusammenhang Aufschluss darüber, welche Variablen einen signifikanten Einfluss auf die Zuteilung der Angehörigen zu den verschiedenen Gruppen von Angehörigen hatten.

Neben der Beurteilung der einzelnen Variablen im Modell ist es auch möglich, das Gesamtmodell zu beurteilen. Dabei steht folgende Frage im Vordergrund: „Wie gut können die Parameterschätzungen in ihrer Gesamtheit das definierte Regressionsmodell abbilden?“ (Backhaus et al., 2011, S. 267). Zu diesem Zweck können verschiedene Gütemasse herangezogen werden. In der vorliegenden Arbeit wurde auf zwei unterschiedliche Gütekriterien zurückgegriffen:

Die Pseudo-R-Quadrat-Statistik versucht, den Anteil der erklärten Varianz im Modell zu quantifizieren (Backhaus et al., 2011, S. 269). Zur Bestimmung der Statistik werden in einem ersten Schritt alle Regressionskoeffizienten des Modells auf Null $\left(\mathrm{LL}_{0}\right)$ und schliesslich mit dem vollständigen Modell ( $\left.L_{V}\right)$ ins Verhältnis gesetzt. Ist die absolute Differenz zwischen der Devianz des Nullmodells $\left(\mathrm{LL}_{0}\right)$ und der des vollständigen Modells $\left(\mathrm{LL}_{\mathrm{V}}\right)$ gross, kann davon ausgegangen werden, dass die unabhängigen Variablen massgeblich zur Unterscheidung zwischen den Kategorien der abhängigen Variable, im vorliegenden Fall die 
verschiedenen Gruppen von Angehörigen, beitragen. Zur Beurteilung des Gesamtmodells sollte wie von Backhaus et al. (2011, S. 270) empfohlen Nagelkerke- $R^{2}$ herangezogen werden, weil Nagelkerke- $R^{2}$ eine eindeutige inhaltliche Interpretation erlaubt. Ein Modell kann als akzeptabel beurteilt werden, wenn Werte grösser .2 erreicht werden. Als gut beurteilt werden Werte ab .4 und als sehr gut Werte ab .5 (ebd., S. 276).

Eine weitere Möglichkeit die Passung des Modells zu überprüfen, bietet die Beurteilung der Klassifikationsergebnisse (Backhaus et al., 2011, S. 271). Dabei werden die beobachteten Gruppenzugehörigkeiten mit den Zuteilungen zu den Gruppen verglichen, die basierend auf der Regressionsgleichung berechnet wurden. Ziel der geschätzten logistischen Regressionsfunktion sollte es sein, möglichst viele Fälle korrekt zu klassifizieren. Die logistische Regressionsfunktion ist nur dann brauchbar, wenn sie bei der Zuteilung der Fälle zu den Kategorien der abhängigen Variablen eine höhere Trefferquote erzielt als dies bei einer Zufallszuteilung möglich wäre. Als Prüfgrössen kann einerseits die Klassifikationsmatrix herangezogen werden, die Auskunft gibt über den Prozentsatz korrekter Zuteilungen anhand der logistischen Regressionsfunktion. Andererseits gibt der Hosmer-Lemeshow-Test Auskunft darüber, ob die Zuteilung zu den Gruppen zufällig zustande kam. In der vorliegenden Arbeit wurden an ein Modell folgende Voraussetzungen gestellt: Es sollte eine möglichst gute Modellpassung (hohes Nagelkerke- $R^{2}$ ) bei einer gleichzeitig hohen Trefferquote bei der Klassifikationszuteilung erreicht werden.

Zur Entwicklung von geeigneten logistischen Regressionsmodellen wurde zuerst auf der Basis der einzelnen Skalen der Fragebogen des SVF-120, der SES und des BFW/E ein ZeroOrder-Vergleich zwischen den gefundenen Clusterlösungen durchgeführt. Neben diesen ZeroOrder-Vergleichen, die Hinweise auf mögliche Modelle gaben, spielten aber gleichzeitig auch theoretische und sachlogische Überlegungen für die Entwicklung von Modellen eine wichtige Rolle. Es war erwartet worden, dass weniger belastete Angehörige über ein deutlich höheres Wohlbefinden und einen höheren Selbstwert berichteten. Gleichzeitig wurde angenommen, dass weniger belastete Angehörige häufig Copingstrategien anwendeten, die zu einer grösseren Entlastung führten. Zudem bestand die Vermutung, dass Faktoren wie der Schweregrad der Erkrankung, der beispielsweise an einem Konsum von gleichzeitig mehreren Substanzen festgemacht werden konnte, oder der aktuelle Status der Suchterkrankung (gebessert, gleich geblieben, verschlechtert) für die erlebte Belastung relevant sein könnten. 


\subsubsection{Qualitative Befragung: Interviews}

\subsubsection{Einleitung}

Inhaltsanalysen zeichnen sich vor allem durch die Theoriegeleitetheit und die Regelgeleitetheit der Interpretation aus (Mayring, 2010, S. 57). Die von Mayring (2010) vorgeschlagene Methode der qualitativen Inhaltsanalyse greift diese Aspekte auf. Dies bedeutet, dass die Analyse einer konkreten theoretisch und inhaltlich begründeten Fragestellung folgt, wobei bisherige Erfahrungen zum Gegenstand (Thema) herangezogen werden, um einen Erkenntnisfortschritt zu erreichen (Theoriegeleitetheit). Die Analyse selbst wird in einzelne Interpretationsschritte zerlegt, die im Vorfeld der Auswertung genau festgelegt werden (Regelgeleitetheit). Auf diese Weise kann gewährleistet werden, dass der Prozess der Analyse für andere nachvollziehbar wird (ebd.). In den nachfolgenden Kapiteln werden das Ablaufmodell, in dem die einzelnen Analyseschritte definiert sind, sowie die Entwicklung des Kategoriensystems vorgestellt.

\subsubsection{Ablaufmodell}

Im Ablaufmodell werden die einzelnen Schritte der Analyse und ihre Reihenfolge definiert. Das Ablaufmodell der Analyse ist die Grundlage dafür, dass der Analyseprozess für andere nachvollziehbar und somit auch intersubjektiv überprüfbar wird. Gemäss Mayring (2010, S. 59) wird die qualitative Inhaltsanalyse dadurch zur wissenschaftlichen Methode. Nachfolgende Abbildung 4 zeigt das Ablaufmodell der Analyse dieser Arbeit. 


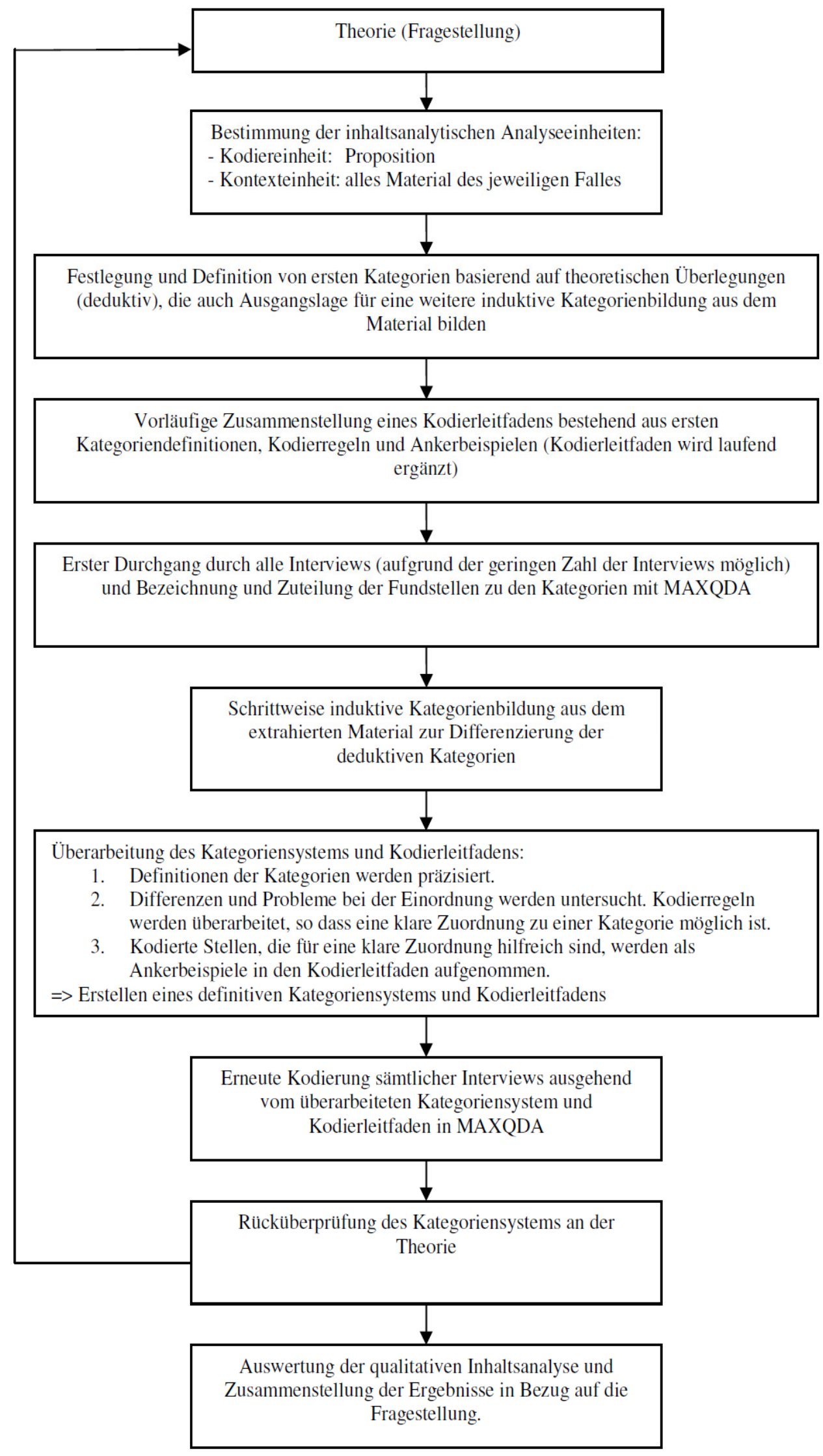

Abb. 4: Ablaufmodell qualitative Inhaltsanalyse Dissertationsprojekt „Angehörige von drogenabhängigen Menschen“ 


\subsubsection{Kategoriensystem}

Das Kategoriensystem bildet das zentrale Instrument der Inhaltsanalyse. Die einzelnen Kategorien werden durch genaue Definitionen, Kodierregeln und Ankerbeispiele beschrieben. Auf diese Weise wird der Prozess der Zuordnung von Textstellen zu einzelnen Kategorien nachvollziehbar und transparent.

Das Ablaufmodell verdeutlichte bereits, dass in der vorliegenden Arbeit das Kategoriensystem in einer Kombination von deduktivem und induktivem Vorgehen entwickelt wurde. In einem ersten Schritt wurden im Vorfeld der Auswertung, ausgehend von der Fragestellung, mehrere Hauptkategorien gebildet. Grundlage für die deduktiven Kategorien bildete die Literatur und theoretische Überlegungen, anhand derer auch der Interviewleitfaden entwickelt worden war.

Für die Bildung der deduktiven Kategorien zu den Belastungen, die Angehörige aufgrund der Drogenabhängigkeit erleben, wurden die Kategorien der „Significant Other Checklist“ (Kirby et al., 2005) herangezogen. So wurden im Vorfeld die Kategorien „Beziehungsprobleme“, „Emotionale Belastungen“, „Gesundheitssorgen“, „Gewalt und Drohungen“, „Rechtliche Probleme“, „Beeinträchtigung des Lebensalltages“ und „Finanzielle Belastung“ deduktiv gebildet. Aufgrund von inhaltlichen Überlegungen wurden die von Kirby et al. (2005) vorgegebenen Kategorien leicht adaptiert. Beispielsweise wurden Schlafschwierigkeiten und -störungen nicht den emotionalen Belastungen zugeordnet sondern den Gesundheitssorgen. Während sich bei Kirby et al. (ebd.) die Gesundheitssorgen vorwiegend auf die drogenkonsumierende Person beziehen, wurden in der vorliegenden Arbeit auch die gesundheitlichen Probleme, die bei Angehörigen im Zusammenhang mit der Suchterkrankung auftraten, in dieser Kategorie kodiert. Die Kategorien wurden grundsätzlich offen gestaltet, dass sie durch weitere relevante Aspekte ergänzt werden konnten. Nach einem ersten Durchgang durchs Datenmaterial wurden die Kategorien überarbeitet und über Paraphrasierungen und Generalisierungen induktiv weitere relevante Kategorien gebildet.

Die Bildung der deduktiven Kategorien zu den Bewältigungsstrategien orientierte sich an den von Copello et al. (2010b, S. 91ff) vorgeschlagenen Copingstrategien. Copello et al. (ebd.) schlagen drei verschiedene Arten des Umgangs mit der Suchterkrankung vor: Involviert und gegen die Situation aufbegehrend, tolerant und den Substanzkonsum akzeptierend und sich der Situationen entziehen und Unabhängigkeit gewinnen. Die Autoren hatten diese drei Arten 
des Copings weiter differenziert (siehe Kap. 2.3.2.2). Für die Auswertungen in der vorliegenden Arbeit wurden in einem ersten Schritt aber nur die drei Hauptkategorien „Unabhängigkeit“”, „Aufbegehren“ und „Toleranz“ deduktiv gebildet. Die Differenzierung der Kategorien und die Ergänzung durch weitere relevante Aspekte erfolgten erst in einem zweiten Schritt über eine induktive Kategorienbildung.

Ausgehend von der Fragestellung, welches Vorwissen Angehörige hatten, als sie vom Drogenkonsum erfuhren, wurde eine Kategorie „Situation der Erkenntnis“ gebildet. Diese Hauptkategorie wurde zusätzlich ergänzt durch „Vorahnungen“, die Angehörige im Vorfeld der Situation der Erkenntnis hatten und durch eine Kategorie „Reaktion auf die Erkenntnis“, in der Textstellen kodiert wurden, die Auskunft darüber gaben, was Angehörige dachten, taten oder fühlten, nachdem sie erfahren hatten, dass jemand in ihrem nahestehenden Umfeld eine Abhängigkeitsproblematik hat.

Eine wichtige Frage der Arbeit lautete, wie Angehörige von drogenabhängigen Menschen besser unterstützt werden können. In diesem Zusammenhang wurden folgende Kategorien gebildet: „Gründe für die Inanspruchnahme von Hilfe“, „gewünschte Unterstützung“ und „erhaltene Unterstützung“. Die einzelnen Kategorien wurden wo nötig im Rahmen eines zweiten Durchgangs über die Bildung von induktiven Kategorien differenziert.

Unter jedem thematischen Schwerpunkt wurde zudem eine Kategorie „Diverses“ gebildet, um relevante Aspekte, die sich nicht ohne weiteres in eine der deduktiven Kategorien zuordnen liessen, ebenfalls miterfassen zu können.

Der Kodierleitfaden mit Ankerbeispielen befindet sich im Anhang der Arbeit. 


\section{Ergebnisse}

\subsection{Quantitative Daten}

\subsubsection{Beschreibung der Stichprobe}

\subsubsection{Einleitung}

Über die Charakteristika der Angehörigen von suchtkranken und insbesondere drogenabhängigen Menschen ist bisher wenig bekannt (Copello, Templeton \& Powell, 2010, S. 64). Da die vorliegende Untersuchung einen stark explorativen Charakter hatte, soll an dieser Stelle ausführlich auf die Beschreibung der Stichprobe eingegangen werden. Dazu gehören auf der einen Seite die Angaben zu den Angehörigen selbst, aber auf der anderen Seite auch die Informationen zu ihren drogenabhängigen Angehörigen, durch die sie ebenfalls charakterisiert sind.

\subsubsection{Beschreibung der Angehörigen (Studienteilnehmende)}

Im Kapitel 4.2.2 wurden die Quellen, über die die Angehörigen für die Studie gewonnen wurden, bereits kurz beschrieben. Nachfolgende Darstellung (Tab. 1) zeigt neben der Anzahl der Angehörigen, die über die einzelnen Quellen für die Studie rekrutiert werden konnten auch die Zahl der Rückmeldungen von potentiell interessierten Studienteilnehmenden.

Tab. 1: Angaben zu den Rückmeldungen und der Rekrutierung aus den Rekrutierungsquellen

\begin{tabular}{|c|c|c|}
\hline & $\begin{array}{c}\text { Rekrutierungen } \\
\text { n }(\%)\end{array}$ & $\begin{array}{c}\text { Rückmeldungen } \\
\text { n }(\%)\end{array}$ \\
\hline $\begin{array}{l}\text { Institutionen } \\
\text { • Angehörigenvereinigung Drogenabhängiger (ada-zh) } \\
\text { • Zentrum für Abhängigkeitserkrankungen } \\
\text { - VEVDAJ }\end{array}$ & $\begin{array}{cl}31 & (\mathbf{3 5 . 2}) \\
21 & (23.8) \\
5 & (5.7) \\
5 & (5.7)\end{array}$ & $\begin{array}{cl}42 & (\mathbf{2 6 . 9 )} \\
28 & (17.9) \\
6 & (3.8) \\
8 & (5.2)\end{array}$ \\
\hline $\begin{array}{cl}\text { Medien } & \\
\text { - } & \text { Pendlerzeitung 20minuten } \\
\text { - Neue Zürcher Zeitung } \\
\text { - } & \text { Angehörigenzeitschrift FORUM }\end{array}$ & $\begin{array}{cl}\mathbf{5 6} & (\mathbf{6 3 . 6}) \\
39 & (44.3) \\
2 & (2.3) \\
15 & (17.0)\end{array}$ & $\begin{array}{cc}111 & (71.2) \\
91 & (58.3) \\
3 & (1.9) \\
17 & (11.0)\end{array}$ \\
\hline Mund-zu-Mund-Werbung & 1 & 3 (1.9) \\
\hline Total & $88(100)$ & $156(100)$ \\
\hline
\end{tabular}

Insgesamt brachten die Aufrufe in den Zeitungen und Zeitschriften die grössten Erfolge bei der Rekrutierung von Studienteilnehmenden. $63.6 \%$ der befragten Angehörigen konnten auf diese Weise gewonnen werden. Am erfolgreichsten waren dabei die beiden Inserate in der 
Pendlerzeitung 20minuten, auf die sich insgesamt 91 Personen meldeten, was $58.3 \%$ aller Rückmeldungen ausmachte. Von diesen insgesamt 91 Anfragen konnten 39 Personen schliesslich für die Studie befragt werden. Bei den Institutionen konnten am meisten Angehörige über die Angehörigenvereinigung Drogenabhängiger (ada-zh) rekrutiert werden. 21 der 88 befragten Angehörigen waren durch die ada-zh vermittelt worden.

Die Gründe dafür, dass von den 156 Anfragen nur 88 in die Studie aufgenommen werden konnten, lagen vorwiegend darin, dass sich viele Angehörige meldeten, die in der Vergangenheit betroffen gewesen waren. Ihr drogenabhängiger Angehöriger war häufig aufgrund seiner Drogenabhängigkeit verstorben oder seit mehreren Jahren abstinent. Bei Partnerinnen gab es zudem die Situation, dass sie sich von ihrem suchtkranken Partner getrennt hatten bzw. hatten scheiden lassen. Weiter kam es bei einigen wenigen Probanden zu Terminabsagen, weil sie sich zu stark belastet fühlten, um über die Problematik zu sprechen oder weil sie Angst davor hatten, dass ihre Anonymität nicht gewahrt bleiben könnte. Vereinzelt gab es auch Personen, die auf die Inserate reagierten, die einen Angehörigen hatten, der einen problematischen oder abhängigen Cannabis- oder Alkoholkonsum hatte, aber niemals Heroin oder Kokain konsumiert hatte. Schliesslich gab es noch einige wenige Angehörige, die sich auf die Aufrufe gemeldet hatten, nachdem die Befragungen bereits abgeschlossen waren und deshalb nicht mehr einbezogen wurden.

Bei der Rekrutierung der Angehörigen stellte insbesondere die Rekrutierung von Partnern und Partnerinnen eine grosse Herausforderung dar. Das gesetzte Ziel von $n=20$ bei den Partnern bzw. Partnerinnen konnte nicht erreicht werden, da sich auch nach einem gezielten Aufruf nach Partner und Partnerinnen über die Pendlerzeitung 20minuten nicht genügend meldeten. Bei vielen Rückmeldungen handelte es sich um ehemalige Partnerinnen von Drogenabhängigen (sechs Frauen) und fünf noch betroffene Partnerinnen, die jedoch nicht mehr zur Teilnahme bereit waren, als sie die genaueren Details zur Studie erfuhren. Insbesondere die Tatsache, dass damit ein persönliches Treffen verbunden war, hielt sie davon ab. Zudem meldeten sich nur zwei Männer, die berichteten eine drogenabhängige Partnerin $\mathrm{zu}$ haben bzw. gehabt $\mathrm{zu}$ haben. Beide konnten letztlich nicht für eine Studienteilnahme gewonnen werden.

In der nachfolgenden Darstellung (Tab. 2) sind die soziodemographischen Angaben der Angehörigen, die an der Studie teilgenommen haben, zusammengestellt: 


\begin{tabular}{|c|c|}
\hline Charakteristika & N $(\%)$ \\
\hline $\begin{array}{l}\text { Geschlecht } \\
\text { - Männer } \\
\text { - Frauen }\end{array}$ & $\begin{array}{l}14(15.9) \\
74(84.1)\end{array}$ \\
\hline 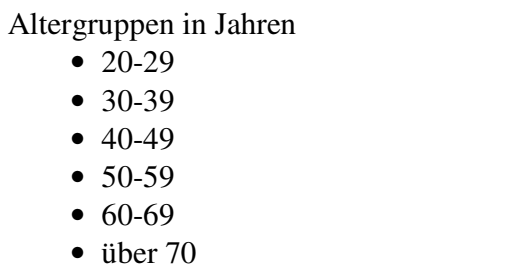 & $\begin{array}{cl}8 & (9.1) \\
17 & (19.3) \\
17 & (19.3) \\
22 & (25.0) \\
16 & (18.2) \\
8 & (9.1)\end{array}$ \\
\hline $\begin{array}{l}\text { Beziehung zur drogenabhängigen Person } \\
\text { - Eltern } \\
\text { - Geschwister } \\
\text { - (Ehe-)Partnerinnen } \\
\text { - Kinder } \\
\text { - andere }\end{array}$ & $\begin{array}{ll}44 & (50) \\
21 & (23.9) \\
15 & (17) \\
5 & (5.7) \\
3 & (3.4)\end{array}$ \\
\hline $\begin{array}{l}\text { Lebenssituation } \\
\text { - allein } \\
\text { - mit (Ehe-)Partnerinnen } \\
\text { - } \text { andere }\end{array}$ & $\begin{array}{l}23(26.1) \\
39(44.3) \\
26(29.5)\end{array}$ \\
\hline $\begin{array}{l}\text { Mit drogenabhängigem Angehörigen zusa } \\
\qquad \quad \text { ja } \\
\bullet \quad \text { nein }\end{array}$ & $\begin{array}{l}21(23.9) \\
67(76.1)\end{array}$ \\
\hline $\begin{array}{l}\text { Höchster Abschluss (Bildung) } \\
\text { - Grundschule } \\
\text { - Berufslehre / Maturität } \\
\text { - Höhere Fachschule } \\
\text { - Universität bzw. Fachhochschule } \\
\text { - Sonstiges }\end{array}$ & $\begin{array}{cc}4 & (4.5) \\
43 & (48.9) \\
18 & (20.5) \\
15 & (17.0) \\
8 & (9.1)\end{array}$ \\
\hline $\begin{array}{l}\text { Beschäftigungssituation/Arbeitssituation } \\
\text { - Arbeitslos } \\
\text { - Hausfrau bzw -mann } \\
\text { - in Ausbildung } \\
\text { - Erwerbstätig } \\
\text { - Pensioniert }\end{array}$ & $\begin{array}{cc}1 & (1.1) \\
3 & (3.4) \\
2 & (2.3) \\
71 & (80.7) \\
11 & (12.5)\end{array}$ \\
\hline
\end{tabular}

Die befragten Angehörigen waren vorwiegend weiblichen Geschlechts (84.1\%). Dies war allerdings nicht sonderlich überraschend, weil in anderen Studien zu Angehörigen der Anteil der Frauen ebenfalls deutlich über dem der Männer lag. In der Studie von Oreo und Ozgul (2007, S. 74) lag der Frauenanteil bei $85.7 \%$ und in der Befragung von Lee et al. (2011, S. 443) bei $81 \%$.

Beim Alter zeigte sich eine grosse Bandbreite. Der jüngste Studienteilnehmende war 22 Jahre alt und der älteste 81 Jahre zum Zeitpunkt der Befragung. Das Durchschnittsalter in der Stichprobe lag bei 49.8 Jahren $(\mathrm{SD}=14.61)$. 
Die Stichprobe bestand aus 44 Eltern (50\%), 21 Geschwistern (23.9\%), 15 Partnerinnen (17 \%), fünf erwachsenen Kindern von drogenabhängigen Eltern (5.7\%) und drei nahestehenden Freunden (3.4 \%). Über die Repräsentanz bezüglich der Population konnte keine Auskunft gegeben werden. Nichtsdestotrotz schien die hohe Zahl von Eltern auffällig in der Stichprobe. Dies war aber mit der Tatsache erklärbar, dass die meisten Vereinigungen für Angehörige, die sich im Zuge der Ausbreitung des Heroins ab den 1970er Jahren in der Schweiz gebildeten hatten und über die zu einem grossen Teil auch rekrutiert wurde, vorwiegend von Eltern gegründet worden waren (Suhner \& Beck, 2012, S. 26). Vereinigungen wie der VEVDAJ und die ada-zh waren ursprünglich Elternvereinigungen. Zudem wird die Angehörigenzeitschrift FORUM von diesen beiden Vereinigungen in Kooperation herausgegeben.

$26.1 \%$ der befragten Angehörigen gaben an, dass sie alleine leben würden. $44.3 \%$ lebten zum Zeitpunkt der Befragung mit einem Partner zusammen und $29.5 \%$ gaben an mit irgendwelchen anderen Menschen zusammenzuleben. In diese Kategorie fielen auch andere Angehörige wie Kinder oder Eltern. Mit dem suchtkranken Angehörigen lebten $23.9 \%$ der Studienteilnehmenden zusammen.

Bezüglich des Bildungsniveaus zeigte sich, dass mit grosser Wahrscheinlichkeit Angehörige der Mittelschicht an der Studie teilgenommen hatten. Flückiger und Falter (2004) unterscheiden in ihrer Analyse der Daten der Eidgenössischen Volkszählung aus dem Jahr 2000 folgende drei Aggregationsstufen bezüglich des Bildungsniveaus:

1. Sekundarstufe I: tiefes Bildungsniveau. Diese Kategorie umfasst die obligatorische Schule sowie die Diplommittelschule (bis 2 Jahre).

2. Sekundarstufe II: mittleres Bildungsniveau. Zu dieser Stufe gehören Lehrabschluss (EFZ), Maturität, Abschluss von Vollzeitberufsschulen sowie Lehrerpatent.

3. Tertiärstufe: hohes Bildungsniveau. Die höhere Ausbildung umfasst universitäre Hochschulen (UH) und Fachhochschulen (FH), höhere Fachschulen sowie die höhere Fach- und Berufsausbildung (Flückiger \& Falter, 2004, S.10).

Das Bildungsniveau der Studienteilnehmenden konnte als hoch beurteilt werden. $37.5 \%$ der Angehörigen, die an der Studie teilnahmen, verfügten über einen Abschluss auf Tertiärstufe. Gemäss Flückiger und Falter (2004, S. 10) gaben im Jahr 2000 lediglich $18.3 \%$ der 
Bevölkerung an, über einen tertiären Abschluss zu verfügen. Es ist allerdings nicht auszuschliessen, dass das Bildungsniveau in der Schweiz seit 2000 allgemein gestiegen ist und in der Zwischenzeit ein höherer Prozentsatz über einen tertiären Abschluss verfügt. $40.9 \%$ der befragten Angehörigen in der Studie gaben an, dass sie über einen Lehrabschluss verfügten. Dieser Prozentsatz ist vergleichbar mit demjenigen der Volkszählung aus dem Jahr 2000, wo $40.3 \%$ der Bevölkerung angaben, dass sie eine Lehre abgeschlossen hatten (ebd.). Während in der Volkszählung 200029.7 \% der Bevölkerung nach der obligatorischen Schule keine weitere Ausbildung gemacht hatten, verfügten in der vorliegenden Studie lediglich vier Personen $(4.5 \%)$ nur über den Grundschulabschluss.

Das durchschnittlich hohe Bildungsniveau der Studienteilnehmenden, welches mit einer niedrigeren Arbeitslosigkeit assoziiert ist (NEETs, 2012, S. 7), spiegelte sich auch in der Beschäftigungssituation wider. $80.7 \%$ der Studienteilnehmenden ging zum Zeitpunkt der Befragung einer Erwerbstätigkeit nach, während nur eine Person angab, derzeit auf Arbeitssuche zu sein, was in der Stichprobe einer Arbeitslosenquote von $1.1 \%$ entsprach.

Diese Quote lag deutlich unter der Arbeitslosenquote der Schweizer Bevölkerung im November 2013 mit $3.2 \%$ (Schweizerische Eidgenossenschaft Staatssekretariat für Wirtschaft SECO, 2013).

\subsubsection{Beschreibung der drogenabhängigen Angehörigen}

Die Schwierigkeiten, mit denen sich Angehörige von drogenabhängigen Menschen auseinandersetzen müssen, sind massgeblich auch durch ihre suchtkranken Angehörigen definiert. Aus diesem Grund soll an dieser Stelle auf die Charakteristika der drogenabhängigen Angehörigen eingegangen werden. Diese sind in Tab. 3 zusammengefasst.

Tab. 3: Charakteristika der drogenabhängigen Angehörigen basierend auf den Angaben der Studienteilnehmenden

\begin{tabular}{lc}
\hline Charakteristika & N $(\%)$ \\
\hline Geschlecht & \\
• Männer & $73(83.0)$ \\
- Frauen & $15(17.0)$ \\
Alter in Jahren zum Zeitpunkt der Befragung & $2(2.3)$ \\
• unter 20 & $23(26.4)$ \\
- $20-29$ & $26(29.9)$ \\
- $30-39$ & $28(32.2)$ \\
- $40-49$ & $8(9.2)$ \\
- $50-59$ & \\
\hline
\end{tabular}


Alter in Jahren bei ersten Anzeichen der Suchterkrankung

- unter 15

- 15-19

- $20-29$

- 30-39

$3(3.4)$

- $40-49$

Dauer der Suchterkrankung in Jahren

- $<1$

- $1-9$

- 10-19

- 20-29

- 30-39

Dauer bis zur ersten Behandlung in Jahren

- $0-2$

- 3-5

- 6-9

- 10-19

- keine Angabe

Substanz, die momentan im Vordergrund steht

- Alkohol

- Cannabis

- Benzodiazepine

- Kokain

- Opiate/Opioide (inkl. Substitutionsbehandlungen)

- Stimulanzen

- Halluzinogene

Weitere psychische Störungen

- Schizophrenie

- Bipolare Störung

- Depressive Störung

- Angststörung

- Borderline Persönlichkeitsstörung

- Dissoziale Persönlichkeitsstörung

Im Vergleich zu vor einem Jahr hat sich die Suchterkrankung ...

$83 \%$ der drogenabhängigen Angehörigen waren männlichen Geschlechts. Beim Alter des suchtkranken Angehörigen zeigte sich eine ähnlich grosse Bandbreite wie bei den Studienteilnehmenden. Der jüngste suchtkranke Angehörige war zum Zeitpunkt der Befragung 17 Jahre alt und der älteste 58 Jahre. Die Mehrheit unter ihnen (61.4 \%) zeigten bereits vor dem 20. Lebensjahr erste Anzeichen für eine Suchterkrankung oder erkrankten im Alter zwischen 20 und 29 Jahren (30.7\%). Der häufig sehr frühe Beginn der Suchterkrankung führte dazu, dass viele Angehörige bereits seit Jahrzehnten, mit der Krankheit konfrontiert waren. In der vorliegenden Stichprobe waren die drogenabhängigen Angehörigen 
durchschnittlich seit 17 Jahren $(\mathrm{SD}=9.51)$ krank. Zwei Angehörige berichteten in diesem Zusammenhang, dass die Suchterkrankung bereits seit 36 Jahren bestand.

Die Angaben zu der bei ihrem Angehörigen im Vordergrund stehenden konsumierten Substanz beruhten auf dem aktuellen Kenntnisstand der Angehörigen zum Zeitpunkt der Befragung. In den telefonischen Vorgesprächen mit den Angehörigen, in denen die Ein- und Ausschlusskriterien abgeklärt worden waren, gaben alle einen Opiat- bzw. Kokainkonsum an. Dieser wurde jedoch nicht immer als am problematischsten erlebt, was sich in den Ergebnissen widerspiegelt. Die Angaben zum Substanzkonsum machen deutlich, dass sich bei den befragten Personen um Angehörige von meist schwer suchtkranken Menschen handelte. Beinahe ein Drittel aller Studienteilnehmenden (32.1\%) gab an, dass bei ihren suchtkranken Angehörigen mehr als eine Substanz im Vordergrund stand. Ein weiteres Drittel (36.4 \%) nannte vorwiegend Opiate und Opioide als Hauptproblemsubstanz und ein letztes Drittel nannte weitere Substanzen wie Kokain (14.8\%), Alkohol (5.7\%), Benzodiazepine (3.4 \%), Cannabis $(2.3 \%)$ und Stimulanzen $(2.3 \%)$ als die zum Zeitpunkt der Befragung im Vordergrund stehende problematische Substanz.

Beinahe die Hälfte (46\%) der Studienteilnehmenden gab in der Befragung an, dass sich die Suchterkrankung im Vergleich zu vor einem Jahr gebessert habe. $39.1 \%$ berichteten, dass die Erkrankung etwa gleich geblieben sei und nur ein kleiner Prozentsatz von Angehörigen $(14.9 \%)$ sagte, dass es zu einer Verschlechterung gekommen sei.

In Bezug auf weitere psychische Störungen bei den suchtkranken Angehörigen wurden von den Studienteilnehmenden mit $29.5 \%$ am häufigsten depressive Störungen genannt. Darauf folgten Borderline Persönlichkeitsstörungen (10.2\%), Angststörungen (9.1\%), Schizophrenie $(6.8 \%)$ und Bipolare Störungen $(6.8 \%)$.

\subsubsection{Datenaufbereitung}

\subsubsection{Einleitung}

Im Vorfeld der Datenauswertung wurden die Daten auf fehlende Werte untersucht, die Reliabilität der Skalen bestimmt und der zu validierende ISE einer Faktorenanalyse unterzogen. Auf diese Weise wurde sicher gestellt, dass die Daten über eine ausreichende 
Qualität verfügten, bevor sie einer weiteren statistischen Analyse unterzogen wurden. Die Ergebnisse dieser Datenaufbereitung sind in den folgenden Kapiteln dargestellt.

\subsubsection{Fehlende Werte}

Insgesamt konnte die Zahl der fehlenden Werte relativ gut kontrolliert werden. Dies war unter anderem darauf zurückzuführen, dass die Angehörigen während der Befragung die Möglichkeit hatten bei Unklarheiten nachzufragen. Nichtsdestotrotz liessen sich fehlende Werte nicht vollständig vermeiden.

Wenige fehlende Werte generierten insbesondere der ZBI mit nur einem Fall $(1.1 \%$ aller Fälle) und der BFW/E, die Rosenberg SES und der SVF-120 mit je drei fehlenden Werten (je $3.4 \%$ der Fälle). Aufgrund der geringen Fallzahlen mit Missings konnte davon ausgegangen werden, dass den fehlenden Werten keine Systematik zugrunde lag. Mit den fehlenden Werten wurde deshalb wie folgt verfahren: Beim ZBI wurde in der Folge auf die Schätzung eines fehlenden Wertes verzichtet. Beim BFW/E, der Rosenberg SES und dem SVF-120 bestand allerdings das Risiko, dass bei den weiteren Auswertungen, in denen teilweise mehrere Fragebögen gleichzeitig eingeschlossen wurden, die Ausfälle $>5 \%$ aller Fälle sein könnten. Aus diesem Grund wurde bei den Skalen, bei denen es zu mehr als einem Ausfall gekommen war, die fehlenden Werte geschätzt. Die Schätzung der fehlenden Werte erfolgte über Mittelswertsschätzungen mit SPSS. Da die Zahl der fehlenden Werte in den einzelnen Skalen nur sehr gering war, konnte davon ausgegangen werden, dass der Bias in den Parameterschätzungen sich ebenfalls in Grenzen hielt.

Beim BDI-V liessen sich dagegen vermehrt fehlende Werte feststellen. Bei sieben Items kam es bei sieben Personen zu Missings ( $8.0 \%$ aller Fälle), wobei einige Personen mehr als einen fehlenden Wert aufwiesen (insgesamt 9 fehlende Werte). Aus diesem Grund wurden diese Ausfälle einer genaueren Analyse unterzogen. Es zeigte sich, dass vier von diesen sieben Personen den Cut-off von 35 überschritten, bei dem gemäss Schmitt et al. (2006, S. 58) mit 90prozentiger Sicherheit eine klinisch relevante Depression vorliegt, wenn die fehlenden Werte konservativ mit 0 (= Symptom gar nicht vorhanden) ersetzt wurden. Aufgrund dieses Ergebnisses konnte ein systematischer Fehler, nämlich dass die fehlenden Werte vom BDI-V selbst abhängig waren, nicht ausgeschlossen werden. Ein Ausschliessen dieser Fälle hätte eine zu tiefe Schätzung der Depressivität in der Stichprobe zur Folge gehabt. Um die Ergebnisse weiter abzusichern, wurden deshalb in einem zweiten Schritt die fehlenden Werte noch durch 
Mittelwertsschätzungen ersetzt. Es zeigte sich, dass sich das Ergebnis nicht veränderte und vier der sieben Personen mit fehlenden Werten den Cut-off von 35 überschritten. Um die Belastung nicht zu überschätzen, wurden in der weiteren Analyse mit den Werten aus der konservativen Schätzung gerechnet, in der die fehlenden Werte mit 0 (=Symptom gar nicht vorhanden) ersetzt worden waren.

Ein besonderes Problem stellte das ISE dar, das sehr viele fehlende Werte generierte. Insgesamt kam es zu 67 fehlenden Werten, die über 21 Fälle verteilt waren (23,9 \% aller Fälle). Dabei kam es bei einzelnen Items (ISE_Nr. 27: „Haben Ihre Erfahrungen mit Vorurteilen Ihre Lebensqualität oder die Lebensqualität Ihrer Familie beeinträchtigt?“) bis zu sechs Missings. In den Befragungen war bereits der Verdacht entstanden, dass das ISE ein erhebliches Akzeptanz- und Validitätsproblem haben könnte, weil sich viele der Angehörigen während und nach den Befragungen negativ zum ISE äusserten und die Ambiguität einiger Items kritisierten. Der Verdacht, dass beim ISE ein Akzeptanz- und Validitätsproblem vorliegen könnte, erhärtete sich noch durch die hohe Zahl der fehlenden Werte. Beim ISE handelt es sich um ein neues Instrument, zu dessen Validierung die vorliegende Untersuchung einen Beitrag leisten sollte. Aus diesem Grund wurden in einem ersten Schritt die fehlenden Werte aufgrund der Ambiguität einiger Items nicht geschätzt, sondern zuerst die Faktorenstruktur überprüft, bevor über ein geeignetes Verfahren zur Schätzung der fehlenden Werte nachgedacht wurde.

\subsubsection{Reliabilität}

In der nachfolgenden Tab. 4 sind die Reliabilitäten sämtlicher Skalen zusammengefasst. Mit Cronbach's $\alpha=.67$ wiesen die beiden Skalen „Probleme“ des BFW und „Bagatellisierung“ des SVF-120 die tiefsten internen Konsistenzen auf. Die Reliabilität dieser beiden Skalen befand sich in einem Bereich, der als fragwürdig einzustufen war. Gemäss Schermelleh-Engel und Werner (2012, S. 135) sind aber bei Persönlichkeitsfragebogen Reliabilitätsmasse um .70 durchaus häufig und somit akzeptabel. In diesem Sinne wurden keine der Skalen aus der weiteren Auswertung ausgeschlossen.

Die internen Konsistenzen der restlichen Skalen befanden sich in einem Bereich, der als akzeptabel bis exzellent bezeichnet werden konnte. Insbesondere der BDI-V und einzelne Skalen des SVF-120 verfügten mit Cronbach's $\alpha>.90$ über exzellente interne Konsistenzen. 
Der Vergleich der internen Konsistenzen mit denjenigen der Literatur zeigte deutlich, dass sich sämtliche Reliabilitäten in dem Bereich bewegten, der in der Literatur berichtet worden war.

Tab. 4: Schätzung der Reliabilität mittels interner Konsistenz

\begin{tabular}{|c|c|c|c|}
\hline Fragebogen & Skala & Cronbach's $\alpha$ & Cronbach's $\alpha$ Literatur \\
\hline BDI-V & & .91 & .93 (Schmidt et al., 2003) \\
\hline Rosenberg SES & & .84 & .88 (Roth et al., 2008) \\
\hline ZBI & & .82 & .86 (Bachner \& O’Rourke, 2007) \\
\hline \multirow[t]{8}{*}{$\mathrm{BFW} / \mathrm{E}$} & Positive Lebenseinstellung & .90 & $.82($ Grob, 2003) \\
\hline & Probleme & .67 & \\
\hline & Körperliche Beschwerden & .72 & \\
\hline & Selbstwert & .78 & \\
\hline & Depressive Stimmung & .80 & \\
\hline & Lebensfreude & .75 & $.60($ Grob, 2003) \\
\hline & Zufriedenheit & .74 & \\
\hline & Negative Befindlichkeit & .76 & \\
\hline \multirow[t]{20}{*}{ SVF-120 } & Bagatellisierung & .67 & .71 (Beutel \& Bähler, 2004) \\
\hline & Herunterspielen & .80 & .85 (ebd.) \\
\hline & Schuldabwehr & .78 & .71 (ebd.) \\
\hline & Ablenkung & .75 & .75 (ebd.) \\
\hline & Ersatzbefriedigung & .73 & .81 (ebd.) \\
\hline & Selbstbestätigung & .78 & .85 (ebd.) \\
\hline & Entspannung & .88 & .84 (ebd.) \\
\hline & Situationskontrolle & .74 & .75 (ebd.) \\
\hline & Reaktionskontrolle & .81 & .77 (ebd.) \\
\hline & Positive Selbstinstruktion & .83 & .79 (ebd.) \\
\hline & Soziales Unterstützungsbedürfnis & .94 & .90 (ebd.) \\
\hline & Vermeidung & .86 & .88 (ebd.) \\
\hline & Flucht & .87 & .84 (ebd.) \\
\hline & Soziale Abkapselung & .93 & .88 (ebd.) \\
\hline & Gedankliche Weiterbeschäftigung & .91 & .91 (ebd.) \\
\hline & Resignation & .85 & .84 (ebd.) \\
\hline & Selbstbemitleidung & .85 & .83 (ebd.) \\
\hline & Selbstbeschuldigung & .91 & .83 (ebd.) \\
\hline & Aggression & .79 & .83 (ebd.) \\
\hline & Pharmakaeinnahme & .71 & .65 (ebd.) \\
\hline
\end{tabular}




\subsubsection{Inventar Subjektiver Stigmaerfahrungen (ISE)}

\subsubsection{Einleitung}

Aufgrund der geringen Erfahrungen mit dem ISE und der noch ausstehenden Validierung im deutschsprachigen Raum wurde die Struktur des Instrumentes vor der weiteren Analyse untersucht. Zu diesem Zweck wurde in einem ersten Schritt die Korrelationsmatrix der ISEItems untersucht, um festzustellen, ob die Voraussetzungen für eine Faktorenanalyse gegeben waren. In einem zweiten Schritt wurde dann die relevante Struktur des ISE bestimmt und mit derjenigen der kanadischen Autoren (Stuart et al., 2005) verglichen.

\subsubsection{Korrelationsmatrix der ISE-Items}

Vor der Durchführung der Faktorenanalyse wurde untersucht, ob die 20 ISE-Items der 88 Angehörigen ausreichend miteinander korrelierten, um die Analyse durchführen zu können.

Der Bartlett Test (test of sphericity), der darüber Auskunft gab, ob die 20 Items des ISE miteinander korrelierten, war hochsignifikant $\left(\chi^{2}=759.24\right.$, df $\left.=190, \mathrm{p}<.001\right)$. Folglich konnte davon ausgegangen werden, dass die 20 ISE-Items in der Population miteinander korrelierten und somit konnte die Nullhypothese $\left(\mathrm{H}_{0}\right)$ zugunsten der Alternativhypothese $\left(\mathrm{H}_{1}\right)$ verworfen werden. Gemäss dem Bartlett Test konnte davon ausgegangen werden, dass die Voraussetzungen für eine Faktorenanalyse gegeben waren.

Das Mass der Stichprobeneignung (MSA) erreichte unter Einschluss aller 20 quantitativen Items des ISE den Wert von MSA $=.718$, was grundsätzlich als ziemlich gut zu beurteilen war (Kaiser \& Rice, 1974; zit. nach Backhaus et al., 2011, S. 343). Neben der Überprüfung der KMO-Statistik für die Stichprobeneignung empfiehlt Field (2009, S. 659), auch die KMOStatistik der einzelnen Items genau zu analysieren und allenfalls einen Ausschluss der Items in Betracht zu ziehen, die untragbare MSA-Werte aufweisen. Im Gegensatz zum Mass der Stichprobeneignung zeigte sich auf der Itemebene, dass vier Items Werte von MSA $<.50$ hatten. Es handelte sich um das Item Nr. 16 „Glauben Sie, dass die Leute weniger von Menschen halten, die eine Suchterkrankung haben?“ (MSA = .474), das Item Nr. 30 „Haben Sie schon einmal versucht, Vorurteile abzubauen, indem Sie Ihre Freunde oder Familie über Suchterkrankungen aufklären?“ (MSA = .249), das Item Nr. 31 „Haben Ihre Erfahrungen mit Vorurteilen Sie oder ein Mitglied Ihrer Familie motiviert, sich für die Rechte von Menschen mit einer Suchterkrankung einzusetzen?“ (MSA = .357) und das Item Nr. 36 „Denken Sie, 
dass Vorurteile gegenüber Suchtkranken irgendwelche Vorteile für die Betroffenen oder die Gesellschaft haben?“ (MSA = .268). Insbesondere die Items Nr. 30, 31 und 36 zeigten sehr schlechte MSA-Werte. In der englischen Version sind diese drei Items nicht relevant für die Bildung einer Skala. Aus diesem Grund wurde die Analyse unter Ausschluss dieser drei Items nochmals wiederholt. Item Nr. 16 wurde allerdings beibehalten, da es in der englischen Originalversion für die Bildung einer Skala relevant ist und sein MSA-Wert in der Analyse nur knapp unter .50 lag.

Beim zweiten Durchgang zeigte sich, dass das Item 32 „Haben Ihre Erfahrungen mit Vorurteilen Sie oder ein Mitglieder Ihrer Familie motiviert, bei Projekten zur Aufklärung der Öffentlichkeit über Suchterkrankungen oder psychische Erkrankungen allgemein mitzuwirken?“, welches in der englischen Originalversion ebenfalls nicht relevant ist für die Bildung einer Skala, mit einem MSA-Wert von .422 ebenfalls einen untragbaren Wert aufwies. Gleichzeitig zeigte sich, dass sich der MSA-Wert des Items Nr. 16 erheblich verbesserte und mit MSA = .70 sogar als ziemlich gut eingestuft werden konnte. Aus diesem Grund wurde eine dritte Analyse gerechnet. Konkret bedeutete dies, dass neben Item 30, 31 und 36 auch Item Nr. 32 aus der dritten Analyse ausgeschlossen wurde, während alle restlichen Items beibehalten wurden.

Der dritte Durchgang brachte schliesslich beim Mass der Stichprobeneignung einen Wert MSA = .825. Gemäss Kaiser und Rice (1974; zit. nach Backhaus et al., 2011, S. 343) konnte dieser Wert als verdienstvoll beurteilt werden. Auf Itemebene zeigte sich zudem, dass sämtliche Items MSA-Werte zwischen .672 und .890 aufwiesen und somit von mittelmässig bis verdienstvoll beurteilt werden konnten. Die Faktorenlösung wurde schliesslich basierend auf dieser dritten Lösung bestimmt.

\subsubsection{Faktorenlösungen}

Die Hauptkomponentenanalyse extrahierte vier Komponenten mit einem Eigenwert grösser eins. Nach dem Kaiser-Kriterium wäre deshalb eine Vier-Faktorenlösung naheliegend gewesen (Backhaus et al., 2011, S. 359). Gemäss Field (2009, S. 641) besteht allerdings die Tendenz, dass mit dem Kaiser-Kriterium die Zahl der relevanten Faktoren überschätzt wird. Als verlässlich kann das Kaiser-Kriterium nur angesehen werden, wenn die Zahl der Variablen unter 30 liegt und die resultierenden Kommunalitäten alle über .70 liegen (ebd.). Die Zahl der Items lag zwar unter 30 in der vorliegenden Untersuchung, aber gleichzeitig 
erreichte nur die Hälfte der ISE-Items eine Kommunalität, die über .70 lag. Ein weiteres Problem, das im Zusammenhang mit einer Vier-Faktorenlösung auftauchte, war die Tatsache, dass das Inventar über zu wenige Items verfügte, um die Faktorenstruktur generalisieren zu können. Pro Skala luden teilweise nur drei Items eindeutig, so dass die Anforderungen von Guadagnoli und Velicer (1988; zit. nach Bortz \& Schuster, 2010, S. 396) nicht erfüllt waren. Die beiden Autoren fordern, dass mindestens vier Items eine Ladung grösser .60 aufweisen müssen, um die Skala generalisieren zu können. Eine inhaltliche Interpretation der vier Komponenten schien zudem aufgrund der starken Überschneidungen der einzelnen Faktoren äusserst schwierig, da die Hälfte der Items auf mehr als einem Faktor grösser .40 luden.

Abb. 5 zeigt den Verlauf der 16 Eigenwerte. Die Interpretation des Screeplots legte eine Einoder Zwei-Faktorenlösung nahe (Knick beim zweiten und dritten Faktor), wobei eine VierFaktorenlösung ebenfalls möglich gewesen wäre (Knick beim fünften Faktor). Die ZweiFaktorenlösung wäre im Sinne der englischen Version des ISE naheliegend gewesen. Bei der Zwei-Faktorenlösung konnten dem zweiten Faktor allerdings nur fünf Items mit einer Ladung grösser .40 zugeordnet werden, wobei nur drei eine Ladung grösser .60 auf dem Faktor hatten. Folglich stellte sich das gleiche Problem wie bei der Vier-Faktorenlösung. Die ZweiFaktorenstruktur liess sich nicht generalisieren. Zur Tatsache, dass die Ladungen der Items auf den beiden Faktoren in keiner Weise mit den Ladungen der englischen Originalversion übereinstimmten, kam noch hinzu, dass zwei der fünf Items in etwa gleich hoch auf dem ersten Faktor luden wie auf dem zweiten.

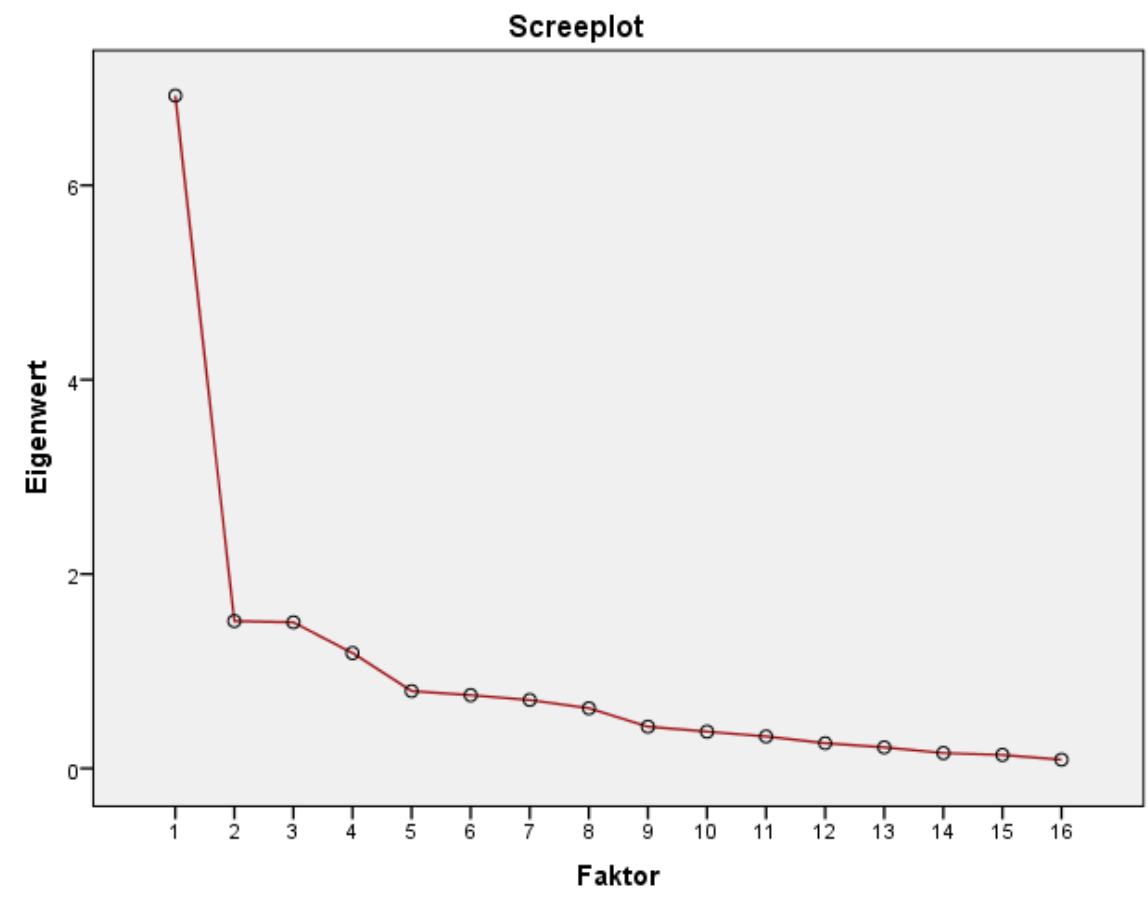

Abb. 5: Screeplot für die 16 Eigenwerte 
Da sich weder die Vier- noch die Zweifaktorenlösung als haltbar erwiesen hatte, schien die Ein-Faktorlösung die beste Option. Bei der Ein-Faktorlösung luden 14 der 16 Items mit einer Ladung grösser .40 auf dem Faktor. Es zeigte sich allerdings bei der Überprüfung des Modells anhand des Vergleichs zwischen den beobachteten Korrelationen in der Korrelationsmatrix und den vorhergesagten Korrelationen basierend auf dem Modell, dass die Ein-Faktorlösung eine hohe Anzahl nicht redundanter Residuen (67\% mit einem Wert höher .05) produzierte. Dies wies darauf hin, dass die Ein-Faktorlösung in Bezug auf die Passung auf die Daten höchst fraglich war und grundsätzlich ein anderes Modell idealer wäre.

Bei der Analyse der nicht redundanten Residuen der Vier- und Zwei-Faktorenlösung zeigte sich, dass lediglich die Vier-Faktorenlösung die Anforderungen bezüglich des Goodness of fit des Modells erfüllte. Mit $46 \%$ nicht redundanten Residuen > 05 war die VierFaktorenlösung das einzige Modell, dass die geforderte Rate von $50 \%$ nicht überschritt.

\subsubsection{Weiteres Vorgehen in Bezug auf das ISE}

Das ISE wurde aus der weiteren Analyse in der vorliegenden Untersuchung ausgeschlossen, da sich sowohl in der Erhebung als auch in der Auswertung erhebliche Mängel gezeigt hatten (siehe weitere Ausführungen in Kap. 6.3.1.).

\subsubsection{Auswirkungen der Suchterkrankung auf die Angehörigen}

\subsubsection{Einleitung}

In den nachfolgenden Kapiteln wird der Frage nachgegangen, welche Auswirkungen die Suchterkrankung eines nahestehenden Menschen auf die Angehörigen hat. Mittels einer BDIV-Erhebung wurde die Depressivität in der Stichprobe geschätzt, die die Frage klären sollte, ob die Suchterkrankung mit einer höheren Depressivität bei den Angehörigen einhergeht. Über lineare Regressionsmodelle wurde zudem untersucht, welche weiteren Variablen die Belastungen und den Umgang mit den Belastungen, die zu einer erhöhten Depressivität führen, beeinflussen.

\subsubsection{Belastung und psychische Gesundheit}

In der Literatur wurde bereits verschiedentlich darauf hingewiesen, dass Angehörige von suchtkranken Menschen ein höheres Risiko haben, selbst psychisch krank zu werden (Lee et 
al., 2011; Oreo \& Ozgul, 2007; Orford et al., 1998c; Ray et al., 2007). Im Rahmen der vorliegenden Studie wurde deshalb mittels BDI-V die Depressivität in der Stichprobe erhoben. Nachfolgende Tabelle (Tab. 5) zeigt deutlich, dass sich der Befund mit dem erhöhten Risiko für psychische Störungen und insbesondere einer klinisch relevanten Depression auch in der vorliegenden Stichprobe bestätigte. $27.3 \%$ der befragten Angehörigen erreichten oder überschritten zum Zeitpunkt der Befragung den Cut-off-Wert von 35 und erfüllten somit die Kriterien für eine klinisch relevante Depression. Gemäss Schmitt et al. (2006, S. 58) kann bei einem BDI-V-Wert von 35 oder mehr mit 90prozentiger Sicherheit von einer klinisch relevanten Depression ausgegangen werden. Der Prozentsatz von $27.3 \%$ lag erheblich über der erwarteten 12-Monatsprävalenz von rund $7 \%$ der Schweizer Bevölkerung (Baer, 2013, S. 10).

Tab. 5: Depressivität in der Stichprobe

\begin{tabular}{|c|c|c|c|c|c|}
\hline & & Häufigkeit & Prozent & Gültige Prozente & $\begin{array}{c}\text { Kumulierte } \\
\text { Prozente }\end{array}$ \\
\hline \multirow{3}{*}{$\begin{array}{l}\text { Cut-off } \\
\text { BDI-V }\end{array}$} & $<35$ & 64 & 72.7 & 72.7 & 72.7 \\
\hline & $\geq 35$ & 24 & 27.3 & 27.3 & 100.0 \\
\hline & Gesamt & 88 & 100.0 & 100.0 & \\
\hline
\end{tabular}

Ausgehend von Zero-Order-Vergleichen und theoretischen Überlegungen wurde schliesslich nach einem linearen Regressionsmodell gesucht, welches erklärende Faktoren zusammenfasste, die zu höheren BDI-V-Werten bei den Angehörigen führten. In der nachfolgenden Tabelle (Tab. 6) sind die Zero-Order-Vergleiche von verschiedenen Variablen und Skalen in Bezug auf die BDI-V Scores zusammengestellt.

Tab: 6: Zero-Order-Vergleiche verschiedener Variablen in Bezug auf den BDI-V-Score

\begin{tabular}{|c|c|c|c|}
\hline \multicolumn{4}{|l|}{ Variablen } \\
\hline \multicolumn{4}{|l|}{ ANOVA (post hoc) } \\
\hline & Teststatistik (df) & Signifikanz & Effekt \\
\hline Wohnsituation & $F(2,85)=1.190$ & $\mathrm{p}=.309$ & $\mathrm{r}=.17$ \\
\hline Bildung & F-Welch $(4,15.243)=1.438$ & $\mathrm{p}=.204$ & $\mathrm{r}=.25$ \\
\hline Berufliche Situation & $F(4,83)=.630$ & $\mathrm{p}=.642$ & $\mathrm{r}=.17$ \\
\hline Hauptproblemsubstanz & $F(6,77)=.955$ & $\mathrm{p}=.462$ & $\mathrm{r}=.26$ \\
\hline Beziehungsart & $\mathrm{F}(4,83)=1.146$ & $\mathrm{p}=.341$ & $\mathrm{r}=.23$ \\
\hline
\end{tabular}


ANOVA (post hoc)

Teststatistik (df)

Status der Erkrankung

(gebessert, gleich, verschlechtert)
$\mathrm{F}(2,84)=2.978$

Signifikanz

Effekt

$\mathrm{p}=.056$

$\mathrm{r}=.26$

t-Test für unabhängige Stichproben

$\begin{array}{lllll} & \text { Mittelwerte } / \mathrm{SD} & \text { Test-Statistik }(\boldsymbol{d} \text { f }) & \text { Signifikanz } & \text { Effekt } \\ \begin{array}{l}\text { Drogenabhängigen zusammenlebend } \\ \text { (ja - nein) }\end{array} & \begin{array}{l}\mathrm{j}(21: \mathrm{M}=30 ; \mathrm{SD}=14.48) \\ \mathrm{n}(67: \mathrm{M}=26.34 ; \mathrm{SD}=15.11)\end{array} & \mathrm{t}(86)=-1.142 & \mathrm{p}=.257 & \mathrm{r}=.12 \\ \text { Geschlecht } & \begin{array}{l}\mathrm{m}(14: \mathrm{M}=30 ; \mathrm{SD}=17.29) \\ \mathrm{w}(74: \mathrm{M}=26.86 ; \mathrm{SD}=14.60)\end{array} & \mathrm{t}(86)=.715 & \mathrm{p}=.476 & \mathrm{r}=.08 \\ & \begin{array}{l}\mathrm{j}(60: \mathrm{M}=27.57 ; \mathrm{SD}=14.57) \\ \mathrm{n}(28: \mathrm{M}=26.93 ; \mathrm{SD}=16.14)\end{array} & \mathrm{t}(86)=-.185 & \mathrm{p}=.854 & \mathrm{r}=.02 \\ \begin{array}{l}\text { Unterstützung } \\ \text { (ja-nein) }\end{array} & & & \end{array}$

\section{Pearson Korrelation}

\begin{tabular}{lll} 
& Korrelationskoeffizient & Signifikanz \\
Alter der Studienteilnehmenden & $\mathrm{r}=.058$ & $\mathrm{p}=.592$ \\
BFW: Positive Lebenseinstellung & $\mathrm{r}=-.698$ & $\mathrm{p}<.001$ \\
BFW: Problembewusstheit & $\mathrm{r}=.607$ & $\mathrm{p}<.001$ \\
$\begin{array}{l}\text { BFW: Körperliche Beschwerden und } \\
\text { Reaktionen }\end{array}$ & $\mathrm{r}=.505$ & $\mathrm{p}<.001$ \\
$\begin{array}{l}\text { BFW: Selbstwert } \\
\text { BFW: Depressive Stimmung }\end{array}$ & $\mathrm{r}=-.632$ & $\mathrm{p}<.001$ \\
BFW: Lebensfreude & $\mathrm{r}=-.529$ & $\mathrm{p}<.001$ \\
$\begin{array}{l}\text { BFW: Zufriedenheit } \\
\text { (Sekundärfaktor) }\end{array}$ & $\mathrm{r}=-.712$ & $\mathrm{p}<.001$ \\
$\begin{array}{l}\text { BFW: Negative Befindlichkeit } \\
\text { (Sekundärfaktor) }\end{array}$ & $\mathrm{r}=.657$ & $\mathrm{p}<.001$ \\
Rosenberg SES & $\mathrm{r}=-.575$ & $\mathrm{p}<.001$ \\
ZBI & $\mathrm{r}=.582$ & $\mathrm{p}<.001$ \\
\hline
\end{tabular}

Die zusammengestellten Ergebnisse zeigen deutlich einen signifikanten Zusammenhang zwischen den BDI-V Werten und allen Skalen des BFW/E, dem ZBI und der Rosenberg SES. Die Skalen „Problembewusstheit“, „Körperliche Beschwerden und Reaktionen“ und „negative Befindlichkeit“ standen alle in einem positiven Zusammenhang zum BDI-V. Konkret bedeutet dies, dass Angehörige, die über mehr Sorgen berichteten und die mehr körperliche Beschwerden wie Schlafstörungen, Herzklopfen, Kopfschmerzen und Weiteres angaben, tendenziell höhere BDI-V Scores erreichten. In die gleiche Richtung ging auch der 
Zusammenhang zwischen dem ZBI und dem BDI-V: Je belasteter sich Angehörige durch die Suchterkrankung fühlten, desto höher waren auch ihre Depressivitätswerte. Dagegen zeigte sich ein deutlicher negativer Zusammenhang zwischen dem BDI-V Score und den Skalen „Positive Lebenseinstellung“, „Lebensfreude“, „Zufriedenheit“ allgemein und dem Selbstwert. So hatten beispielsweise Angehörige, die über einen höheren Selbstwert berichteten, tendenziell tiefere BDI-V Werte.

Bei den soziodemographischen Variablen und den Variablen, die die Suchterkrankung kennzeichneten, liessen sich keine signifikanten Unterschiede in Bezug auf den BDI-V Score nachweisen. Nichtsdestotrotz liess sich nicht ausschliessen, dass gewisse Variablen einen Einfluss auf die BDI-V Scores hatten. Dass sich keine signifikanten Effekte zeigten, konnte mit der Tatsache erklärt werden, dass sich die Gruppen in den t-Tests und den ANOVAs zum Teil deutlich in ihrer Grösse unterschieden, was die Power der Tests beeinflusste. Die berechneten Effektstärken liessen aber vermuten, dass die Variablen zum Status der Erkrankung (gebessert, gleich, verschlechtert) und zur Substanz, die beim drogenabhängigen Angehörigen im Vordergrund stand, bedeutsam sein könnten für das Risiko eine klinisch relevante Depression zu entwickeln. Weiter könnten aber auch die Wohnsituation und die berufliche Situation eine Rolle spielen. Zudem schien es auch wahrscheinlich, dass die Art der Beziehung, in der jemand zu einem drogenabhängigen Menschen steht, relevant sein könnte für die erlebte Belastung und das Risiko einer psychischen Störung. Nachfolgende Abbildung (Abb. 6) veranschaulicht, dass in der vorliegenden Stichprobe Kinder von drogenabhängigen Eltern und (Ehe-)Partnerinnen die höchsten Werte auf dem BDI-V erreichten. Ausser der Gruppe der Eltern waren allerdings die Gruppen von Geschwistern (21), (Ehe-)Partnerinnen (15), Kinder (5) und Freunde (3) zu klein, um mit der ANOVA einen signifikanten Effekt nachweisen zu können. 


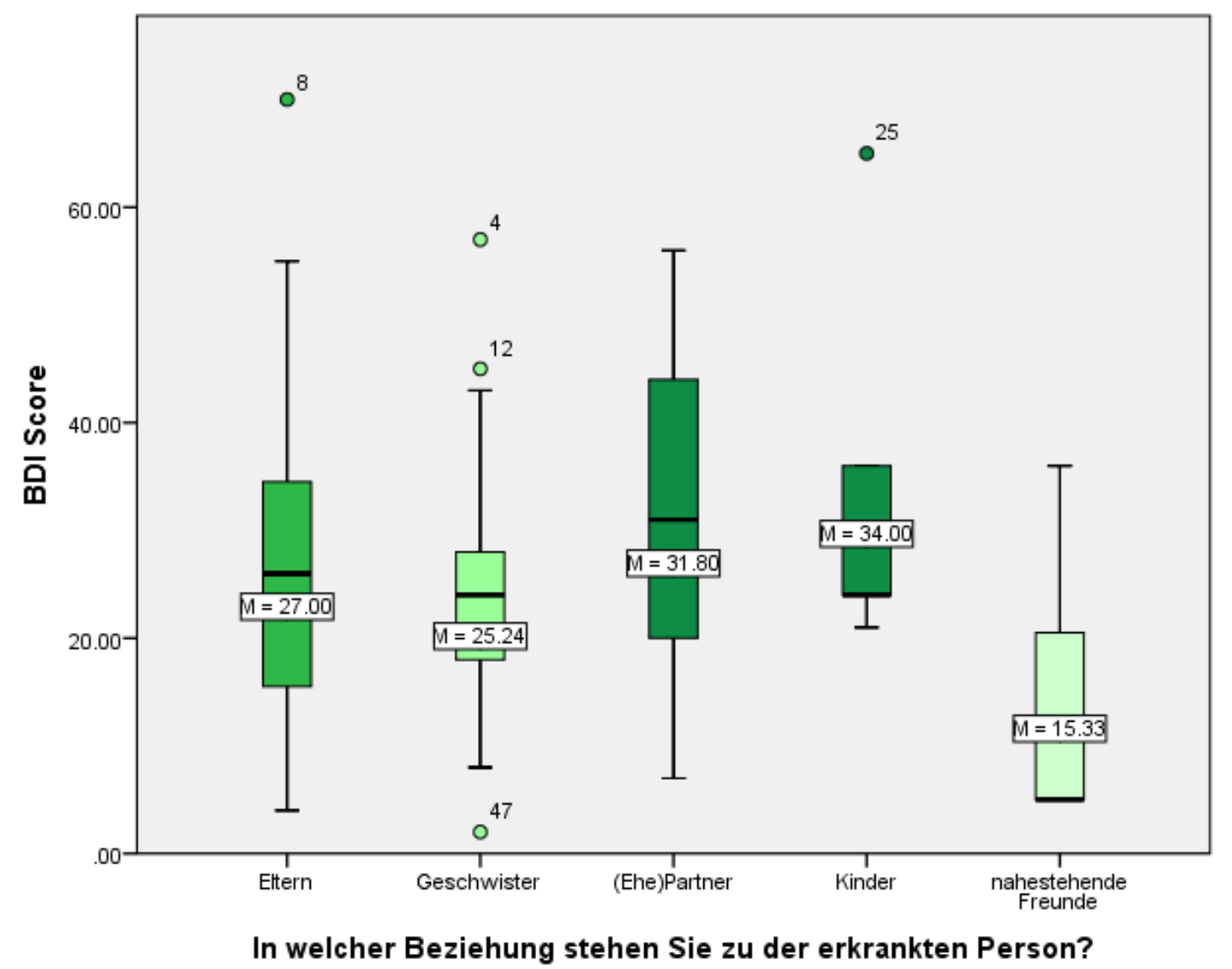

Abb. 6: Streuung der BDI-V Scores in den Gruppen von Angehörigen bezüglich ihrer Beziehung zum suchtkranken Angehörigen

Hingegen zeigte sich beim Alter der Studienteilnehmenden, beim Geschlecht und bei der Unterstützung praktisch kein Zusammenhang mit dem BDI-V.

Aus diesen Zero-Order-Vergleichen wurde schliesslich nach einem multiplen linearen Regressionsmodell gesucht, das erklärende Faktoren zusammenfasste, die zu höheren BDI-V Werten bei den Angehörigen führten. Im Modell wurden insbesondere Variablen berücksichtigt, die äussere Belastungsfaktoren darstellen und zum Teil direkt mit der Suchterkrankung assoziiert sind. Folgende Variablen wurden dabei als relevant erachtet: Der Status der Suchterkrankung (gebessert, gleich, verschlechtert), wobei der Post hoc-Vergleich gezeigt hatte, dass insbesondere diejenigen Angehörigen, die eine Besserung im Vergleich zum Vorjahr angaben, über tiefere BDI-V Werte berichteten. Aus diesem Grund wurde die Variable „Status der Suchterkrankung“ in eine bivariate Variable (gebessert vs. gleich/verschlechtert) umgewandelt. Obwohl sich kein grosser Effekt gezeigt hatte, schien es aufgrund von theoretischen Überlegungen auch wichtig zu erfassen, ob die Angehörigen mit dem Drogenabhängigen zusammenlebten oder nicht. Dies hatte sich zumindest in der Studie von Kirby et al. (2005) als relevant gezeigt. Naheliegend war zudem auch, dass die Belastung 
(ZBI-Werte), die die Angehörigen angaben, im Modell berücksichtigt wurde. In ähnlicher Weise verhielt es sich mit der Skala „BFW: Problembewusstheit“, in der diverse Sorgen wie Beziehungsprobleme, gesundheitliche, arbeitsbedingte oder finanzielle Probleme erfasst werden. Zudem wurde die Variable zur Wohnsituationen in eine bivariate Variable (allein lebend vs. mit jemandem zusammenlebend) umgewandelt und ins Modell aufgenommen. In einem ersten Modell wurde der Schweregrad der Erkrankung über eine bivariate Variable zum Substanzkonsum (Polytoxikomanie/Heroin vs. andere Substanzen) im Modell berücksichtigt. Es zeigte sich allerdings, dass die Variable nur einen geringen Beitrag in der Varianzaufklärung leistete. Aus diesem Grund wurde sie aus dem Modell schliesslich ausgeschlossen. Die Prädiktorvariablen wurden alle gleichzeitig ins Modell eingeschlossen, weil bei einer schrittweisen Aufnahme der Prädiktoren ins Modell das Risiko besteht, dass es zu einem „over-fitting“ (zu viele Variablen im Modell) oder zu einem „under-fitting“ (relevante Prädiktoren ausgeschlossen) kommen kann (Field, 2009, S. 213).

Die Überprüfung der Voraussetzungen der linearen Regressionsanalyse brachte folgende Ergebnisse: Keiner der Toleranzwerte fiel unter den kritischen Wert von 0.2. Folglich konnte Multikollinearität ausgeschlossen werden. Die Prüfung der geschätzten standardisierten Werte und der standardisierten Residuen für die einzelnen Beobachtungswerte auf Homoskedastizität und Linearität im Streudiagramm brachten keine Hinweise, dass die Voraussetzung verletzt war (Abb. 7). In der nachfolgenden Abbildung lässt sich innerhalb der Daten kein Verteilungsmuster erkennen, weshalb davon auszugehen war, dass die Varianz der Residuen für alle Prädiktorvariablen konstant war und zwischen Prädiktor- und OutcomeVariable eine lineare Beziehung bestand. 


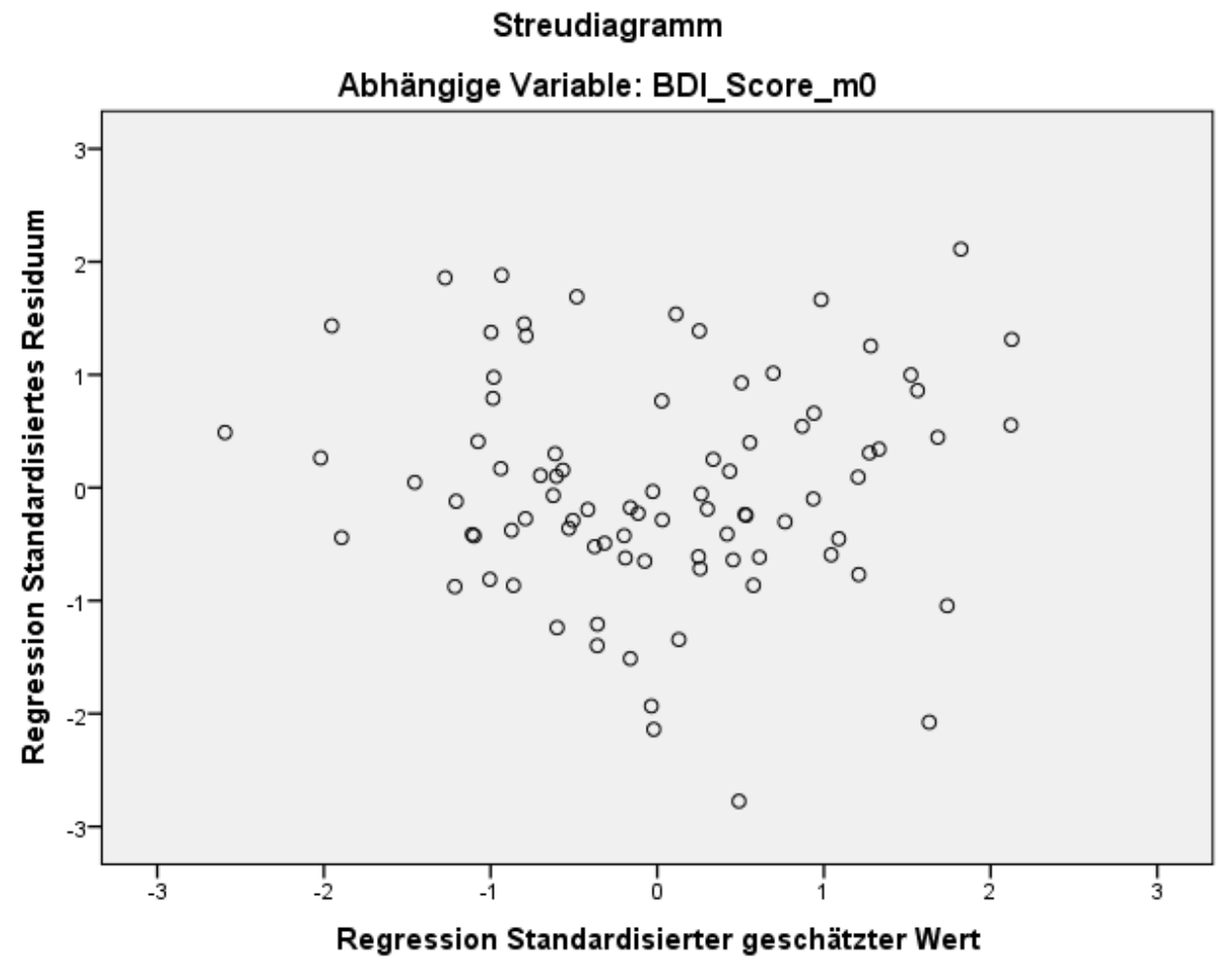

Abb. 7: Prüfung auf Linearität und Homoskadastizität mittels Streudiagramm mit BDI-V als abhängige Variable

In Tab. 7 sind die Ergebnisse des multiplen linearen Regressionsmodells zusammengestellt.

Tab. 7: Multiples lineares Regressionsmodell: BDI-V (AV) und relevanten Belastungsfaktoren (UV)

\begin{tabular}{|c|c|c|c|c|c|c|}
\hline Skala & $\begin{array}{l}\text { Regressions- } \\
\text { koeffizient B }\end{array}$ & $\begin{array}{l}\text { Standard- } \\
\text { fehler }\end{array}$ & $\boldsymbol{\beta}$ & $\mathbf{T}$ & Signifikanz & Toleranz \\
\hline Konstante & -4.685 & 4.285 & & -1.093 & .278 & \\
\hline $\begin{array}{l}\text { Status Suchterkrankung } \\
\text { (gleich/verschlechtert vs. gebessert) }\end{array}$ & -5.792 & 2.372 & -.193 & -2.441 & .017 & .915 \\
\hline ZBI & .657 & .172 & .355 & 3.828 & .000 & .663 \\
\hline $\begin{array}{l}\text { Mit dem suchtkranken Angehörigen } \\
\text { zusammenlebend }\end{array}$ & 6.392 & 2.921 & .180 & 2.188 & .032 & .839 \\
\hline $\begin{array}{l}\text { Wohnsituation } \\
\text { (alleine vs. mit jemandem zusammen) }\end{array}$ & 5.575 & 2.778 & .163 & 2.006 & .048 & .869 \\
\hline BFW: Problembewusstheit & 7.377 & 1.723 & .393 & 4.282 & .000 & 679 \\
\hline
\end{tabular}

Die von den Angehörigen erlebte Belastung im Zusammenhang mit der Suchterkrankung (ZBI) sowie die Skala „BFW: Problembewusstheit“ wurden im Modell hochsignifikant und zeigten die höchste Wirkung auf den BDI-V Score (ZBI: $\beta=.355$; BFW: $\beta=.393$ ) von allen fünf Variablen. Der ZBI Score zeigte folgenden Zusammenhang zum BDI-V Score auf: Je stärker belastet sich Angehörige durch die Suchterkrankung fühlten, desto höhere Werte 
erreichten sie auch auf dem BDI-V. Der Regressionskoeffizient b gab dabei an, dass bei der Erhöhung des ZBI Wertes um eine Einheit sich der BDI-V Wert um .657 Einheiten erhöhte. In ähnlicher Weise verhielt es sich mit den Sorgen, die sich Angehörige in verschiedenen Lebensbereichen machten: Je mehr Angehörige über Sorgen berichteten, desto höhere Werte erreichten sie auf dem BDI-V. Neben der erlebten Belastung (ZBI) und der Skala des „BFW: Problembewusstheit" zeigten auch die weiteren Variablen Status der Suchterkrankung, $(\beta=-.193, \mathrm{p}<.05)$, die Variable, ob jemand mit dem suchtkranken Angehörigen zusammenlebte $(\beta=.180, \mathrm{p}<.05)$ und die Variable, ob jemand alleine lebte $(\beta=.163$, $\mathrm{p}<$.05), im Regressionsmodell einen signifikanten Zusammenhang mit dem BDI-V Score. Dies bedeutete konkret, dass diejenigen Angehörigen, die über eine Besserung der Suchterkrankung im vergangenen Jahr berichteten mit grösserer Wahrscheinlichkeit niedrigere BDI-V Werte erreichten. Dagegen waren das Alleine- oder das Zusammenleben mit dem drogenabhängigen Angehörigen mit höheren BDI-V Werten assoziiert.

Das Bestimmtheitsmass $R^{2}$ besagte, dass $54.4 \%$ der Variation der Scores des BDI-V durch die fünf unabhängigen Variablen erklärt werden konnte. Die F-Statistik, die die Nullhypothese $\left(\mathrm{H}_{0}\right)$ prüfte, dass die Grundgesamtheit der Regressionskoeffizienten gleich Null war, wurde hochsignifikant $(\mathrm{F}(5,80)=19.056, \mathrm{p}<.001)$. Folglich konnte davon ausgegangen werden, dass zwischen den unabhängigen und der abhängigen Variablen ein systematischer Zusammenhang bestand.

\subsubsection{Belastung und Bewältigungsstrategien}

Eine zentrale Frage der Arbeit lautete, wie Belastungen und Bewältigungsstrategien zusammenhingen. In der nachfolgenden Tabelle (Tab. 8) sind die Korrelationen der Skalen des SVF-120 zusammengestellt, die im Zero-Order-Vergleich nach Anpassung des Signifikanzniveaus auf $\mathrm{p}=.0014$ (Bonferoni-Korrektur: $\alpha^{\prime}=.05 / 35$ ) signifikant wurden.

Tab. 8: Signifikante Ergebnisse der Zero-Order-Korrelationen zwischen dem BDI-V-Score und SVF-120

\begin{tabular}{lll}
\hline Skala & $\begin{array}{l}\text { Korrelation } \\
\text { (Pearsons's r) }\end{array}$ & Signifikanz \\
\hline SVF: Flucht & .389 & $\mathrm{p}<.001$ \\
SVF: Soziale Abkapselung & .401 & $\mathrm{p}<.001$ \\
SVF: Gedankliche Weiterbeschäftigung & .340 & $\mathrm{p}=.001$ \\
\hline
\end{tabular}


Fortsetzung Tab. 8: Signifikante Ergebnisse der Zero-Order-Korrelationen zwischen dem BDI-V-Score und SVF-120

\begin{tabular}{lll}
\hline Skala & $\begin{array}{l}\text { Korrelation } \\
\text { (Pearsons's r) }\end{array}$ & Signifikanz \\
\hline SVF: Resignation & .466 & $\mathrm{p}<.001$ \\
SVF: Selbstbemitleidung & .517 & $\mathrm{p}<.001$ \\
SVF: Selbstbeschuldigung & .449 & $\mathrm{p}<.001$ \\
\hline
\end{tabular}

Die Zusammenstellung macht deutlich, dass vor allem ein positiver Zusammenhang zwischen negativen Bewältigungsstrategien, die das Stresserleben allgemein eher erhöhen, und dem BDI-V Score bestand. Konkret bedeutet dies, dass Angehörige, die auf dem BDI-V eine höhere Depressivität angaben, auch eher über negative Bewältigungsstrategien berichteten. Bei den positiven Bewältigungsstrategien, die das Stresserleben eher reduzieren, zeigte sich dagegen kein Zusammenhang zu den BDI-V-Werten.

Ausgehend von theoretischen Überlegungen, die sich sehr stark an Symptomen orientierten, die im Rahmen des Co-Abhängigkeitssyndroms als relevant diskutiert werden, und den ZeroOrder-Vergleichen wurde ein Regressionsmodell entwickelt. Folgende Variablen und Skalen wurden im Modell berücksichtigt: Als relevant erachtet wurden vor allem die Skalen „Selbstbeschuldigung“, „Resignation“ (Gefühl der Hilf- und Hoffnungslosigkeit) und „,soziale Abkapselung“. Zudem schien es auch wichtig zu beachten, ob die Angehörigen mit dem suchtkranken Angehörigen zusammenlebten oder nicht, da dies die Bewältigungsstrategien beispielsweise den soziale Rückzug ebenfalls mit beeinflussen könnte. Zusätzlich wurde die Variable zum Status der Suchterkrankung (gebessert vs. gleich/verschlechtert) ins Modell aufgenommen, da davon auszugehen war, dass Kennzeichen der Suchterkrankung die Bewältigungsstrategien beeinflussen könnten. Sämtliche Variablen wurden gleichzeitig ins Modell eingeschlossen.

Die Überprüfung der Annahmen zur Durchführung einer linearen Regressionsanalyse brachte folgende Ergebnisse: Aufgrund der Toleranzwerte, die alle über dem kritischen Wert von 0.2 lagen, konnte Multikollinearität ausgeschlossen werden. Die Prüfung der geschätzten standardisierten Werte und der standardisierten Residuen für die einzelnen Beobachtungswerte auf Heteroskedastizität und Non-Linearität im Streudiagramm brachten keine Hinweise, dass die Voraussetzung für Homoskedastizität und Linearität verletzt war (Abb. 8). In den Daten liess sich kein spezielles Verteilungsmuster erkennen, wodurch davon 
auszugehen war, dass die Varianz der Residuen für alle Prädiktorvariablen konstant war und zwischen Prädiktor- und Outcomevariable eine lineare Beziehung bestand.

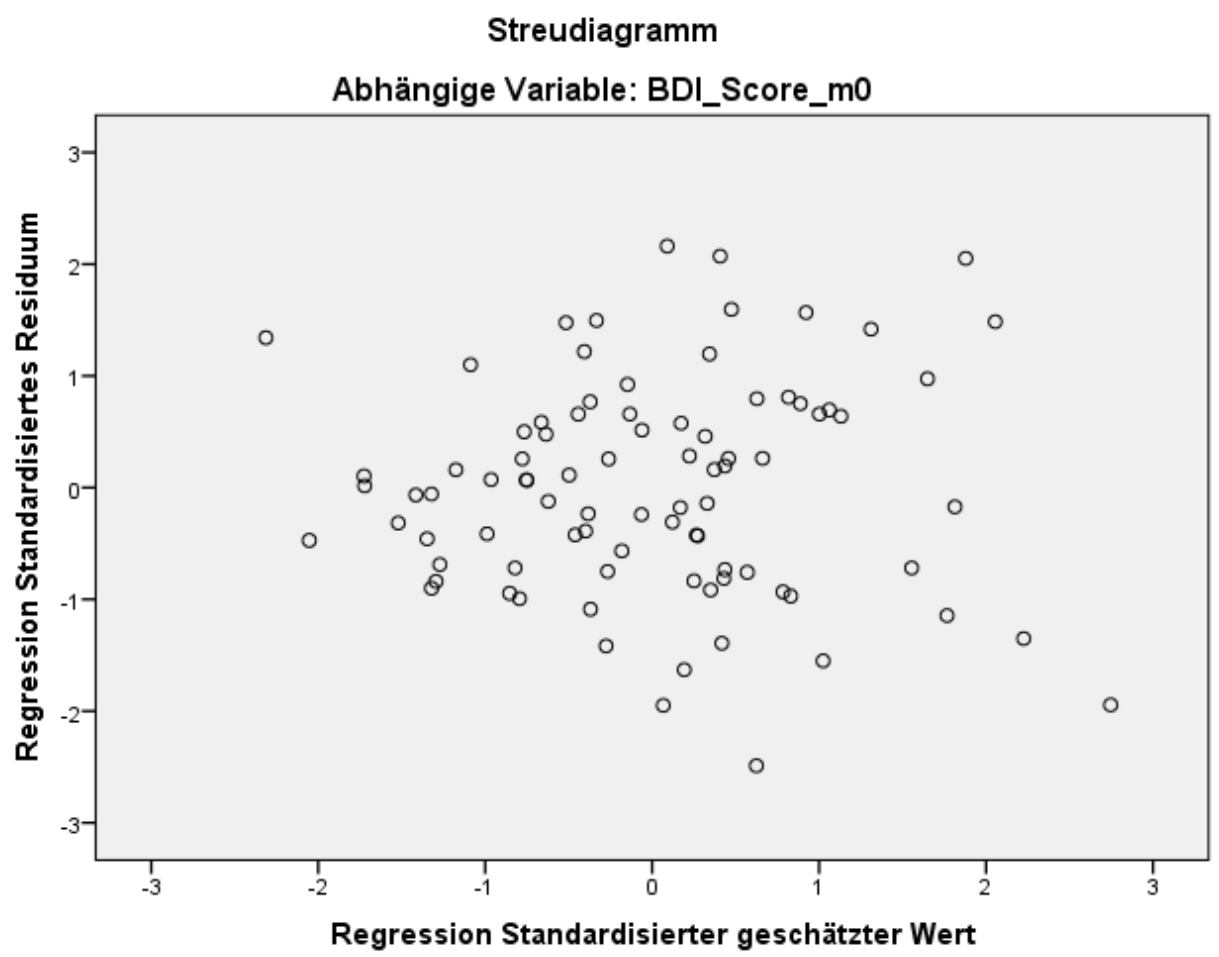

Abb. 8: Prüfung auf Linearität und Homoskadastizität mittels Streudiagramm mit BDI-V als abhängige Variable

In der nachfolgenden Tabelle (Tab. 9) sind die Ergebnisse des multiplen linearen Regressionsmodells zusammengestellt.

Tab. 9: Multiples lineares Regressionsmodell: BDI-V (AV) und relevante Faktoren der Bewältigung (UV)

\begin{tabular}{lcccccc}
\hline Skala & $\begin{array}{l}\text { Regressions- } \\
\text { koeffizient B }\end{array}$ & $\begin{array}{l}\text { Standard- } \\
\text { fehler }\end{array}$ & $\boldsymbol{\beta}$ & T & Signifikanz & Toleranz \\
\hline Konstante & 5.097 & 4.191 & & 1.216 & .227 & .838 \\
$\begin{array}{l}\text { Status der Suchterkrankung } \\
\text { (gebessert vs. gleich/verschlechtert) }\end{array}$ & -3.943 & 2.787 & -.131 & -1.415 & .161 & .949 \\
$\begin{array}{l}\text { Mit dem suchtkranken Angehörigen } \\
\text { zusammenlebend }\end{array}$ & 4.569 & 3.052 & .131 & 1.497 & .138 & .710 \\
SVF: Resignation & .703 & .333 & .213 & 2.114 & $\mathbf{. 0 3 8}$ & .850 \\
SVF: Soziale Abkapselung & .685 & .237 & .267 & 2.893 & $\mathbf{. 0 0 5}$ & .770 \\
SVF: Selbstbeschuldigung & .910 & .279 & .316 & 3.259 & $\mathbf{. 0 0 2}$ & .710 \\
\end{tabular}

Die Prädiktoren „mit dem suchtkranken Angehörigen zusammenlebend“, „Resignation“, „Soziale Abkapselung“ und „Selbstbeschuldigung“ hatten alle eine positive Beziehung zur abhängigen Variablen BDI-V. Dies bedeutete, dass die Erhöhung der unabhängigen Variablen 
auch eine Erhöhung der BDI-V Werte zur Folge hatte. Die Beziehung zwischen dem Status der Suchterkrankung und den BDI-V Scores dagegen war umgekehrt. Angehörige, die berichteten, dass sich die Suchterkrankung ihres Angehörigen im vergangen Jahr gebessert hatte, gaben eher tiefere BDI-V Scores an. Im Regressionsmodell wurde der Einfluss folgender Skalen signifikant: „SVF: Selbstbeschuldigung“ $(p<.01)$, „SVF: Resignation“ ( $\mathrm{p}<.05)$ und „SVF: Soziale Abkapselung“ $(\mathrm{p}<.01)$, wobei die Skala „SVF: Selbstbeschuldigung“" mit $\beta=.316$ am stärksten auf den BDI-V Werte wirkte. Die Erhöhung um eine Einheit (= ein Punkt) auf der Selbstbeschuldigungsskala hatte die Erhöhung um .910 Punkte auf dem BDI-V Score zur Folge. Gefolgt wurde die Skala „SVF: Selbstbeschuldigung“ von der Skala „SVF: Soziale Abkapselung“ $(\beta=.267)$ und „SVF: Resignation“ ( $\beta=.213$ ) in der Wirkung auf den BDI-V Score. Der Einfluss der beiden Variablen „Status der Suchterkrankung“ und „mit dem suchtkranken Angehörigen zusammenlebend" wurden nicht signifikant.

Das Bestimmtheitsmass $R^{2}$ gab an, dass $41.4 \%$ der Variation der Scores des BDI-V durch die fünf unabhängigen Variablen erklärt werden konnte. Die F-Statistik wurde hochsignifikant. Folglich konnte die Nullhypothese $\left(\mathrm{H}_{0}\right)$, die postulierte, dass die Grundgesamtheit der Regressionskoeffizienten gleich Null war, verworfen werden $(\mathrm{F}(5,81)=11.454, \mathrm{p}<.001)$. Ein systematischer Zusammenhang zwischen den Prädiktorvariablen und den Outcomevariablen konnte angenommen werden.

Modelle mit Bewältigungsstrategien, die das Stresserleben eher reduzierten, brachten keine signifikanten Ergebnisse, was den Schluss nahelegte, dass in Bezug auf das Erleben der Belastung insbesondere negative Bewältigungsstrategien eine Rolle spielen könnten. Dieser Befund deckte sich auch mit den Zero-Order-Korrelationen zu den Skalen des SVF-120 und dem BDI-V Score.

\subsubsection{Verschiedene Gruppen von Angehörigen aufgrund der Belastung}

\subsubsection{Einleitung}

Im Rahmen der Arbeit wurde unter anderem der Frage nachgegangen, ob sich bezüglich der Belastung verschiedene Gruppen von Angehörigen identifizieren lassen, um in einem weiteren Schritt den Bezug zu weiteren Variablen herzustellen. Zu diesem Zweck wurde eine clusteranalytische Auswertung vorgenommen, die in diesem Kapitel vorgestellt werden soll. 
Anschliessend wurden mittels Diskriminanzanalyse die Ergebnisse der Clustanalyse überprüft. Schliesslich wurde mit einer logistischen Regressionsanalyse untersucht, welche Variablen insbesondere schwer belastete Angehörige charakterisieren.

\subsubsection{Optimale Clusterlösung}

In der Tabelle (Tab. 10) sind die letzten zehn Fusionierungsschritte des Ward-Verfahrens dargestellt. Jedem Fusionierungsschritt lag als Heterogenitätsmass der geringste Zuwachs der Fehlerquadratsumme $\left(\mathrm{QS}_{\text {Fehler }}\right)$ zugrunde. Basierend auf den Fehlerquadratsummen $\left(\mathrm{QS}_{\text {Fehler }}\right)$ wurden für die letzten zehn Fusionierungsschritte die zu erwartenden FehlerquadratsummenZuwächse $\left(\Delta \mathrm{QS} \mathrm{S}_{\mathrm{Fehler}}\right)$ berechnet, wie ebenfalls in Tabelle 10 ersichtlich ist.

Ausgehend von den Fehlerquadratsummen-Zuwächsen $\left(\Delta \mathrm{QS}_{\text {Fehler }}\right)$ liess sich das Struktogramm zur Bestimmung der optimalen Clusteranzahl zur Gruppierung der Angehörigen bezüglich ihrer Belastungen erstellen. Gemäss Backhaus et al. (2011, S. 437) wird die Ein-Cluster-Lösung normalerweise im Struktogramm nicht berücksichtigt, da sich aufgrund des grössten Heterogenitätssprunges zwischen der Ein- und Zwei-Clusterlösung an dieser Stelle immer ein „Elbow“ abzeichnet.

Bei der Analyse des Struktogramms wird diejenige Clusteranzahl extrahiert, bei der ein Sprung in der Grafik stattfindet (Backhaus et al., 2011, S. 437). Die Abb. 9 zeigt keinen all zu deutlichen „Elbow“, sprich die Fehlerquadratsummen-Zuwächse $\left(\Delta \mathrm{QS} \mathrm{S}_{\text {Fehler }}\right)$ waren nur noch gering nach der Zwei-Clusterlösung. Dies kann gemäss Backhaus et al. (2011, S. 444) als Indikator für eine Zwei-Clusterlösung gesehen werden. Nichtsdestotrotz wurden zur Absicherung der Zwei-Clusterlösung eine Drei- und Fünf-Clusterlösung überprüft, da sich an dieser Stelle im Struktogramm leichte Fehlerquadratsummen-Zuwächse ( $\left.\Delta Q S_{\text {Fehler }}\right)$ andeuteten. Bei der Entscheidung für die optimale Clusterlösung sollte neben der Homogenitätsanforderung die Handhabbarkeit der Lösung ebenfalls berücksichtigt werden. 
Tab 10: Zuordnungsübersicht für die letzten 10 der 86 Zuordnungsschritte

\begin{tabular}{|c|c|c|c|}
\hline Schritt & Clusteranzahl & Heterogenitätsmass $\left(\mathbf{Q S}_{\text {Fehler }}\right)$ & $\Delta \mathbf{Q S}_{\text {Fehler }}$ \\
\hline 77 & 10 & 2'278.584 & \\
\hline 78 & 9 & 2'371.049 & 92.5 \\
\hline 79 & 8 & $2^{\prime} 474.853$ & 103.8 \\
\hline 80 & 7 & 2'594.812 & 120.0 \\
\hline 81 & 6 & $2^{\prime} 723.035$ & 128.2 \\
\hline & & & 137.4 \\
\hline 82 & 5 & $2^{\prime} 860.393$ & 164.3 \\
\hline 83 & 4 & $3^{\prime} 024.648$ & 207.1 \\
\hline 84 & 3 & 3'231.793 & 237.6 \\
\hline 85 & 2 & 3'469.393 & 709.4 \\
\hline 86 & 1 & 4'178.805 & \\
\hline
\end{tabular}

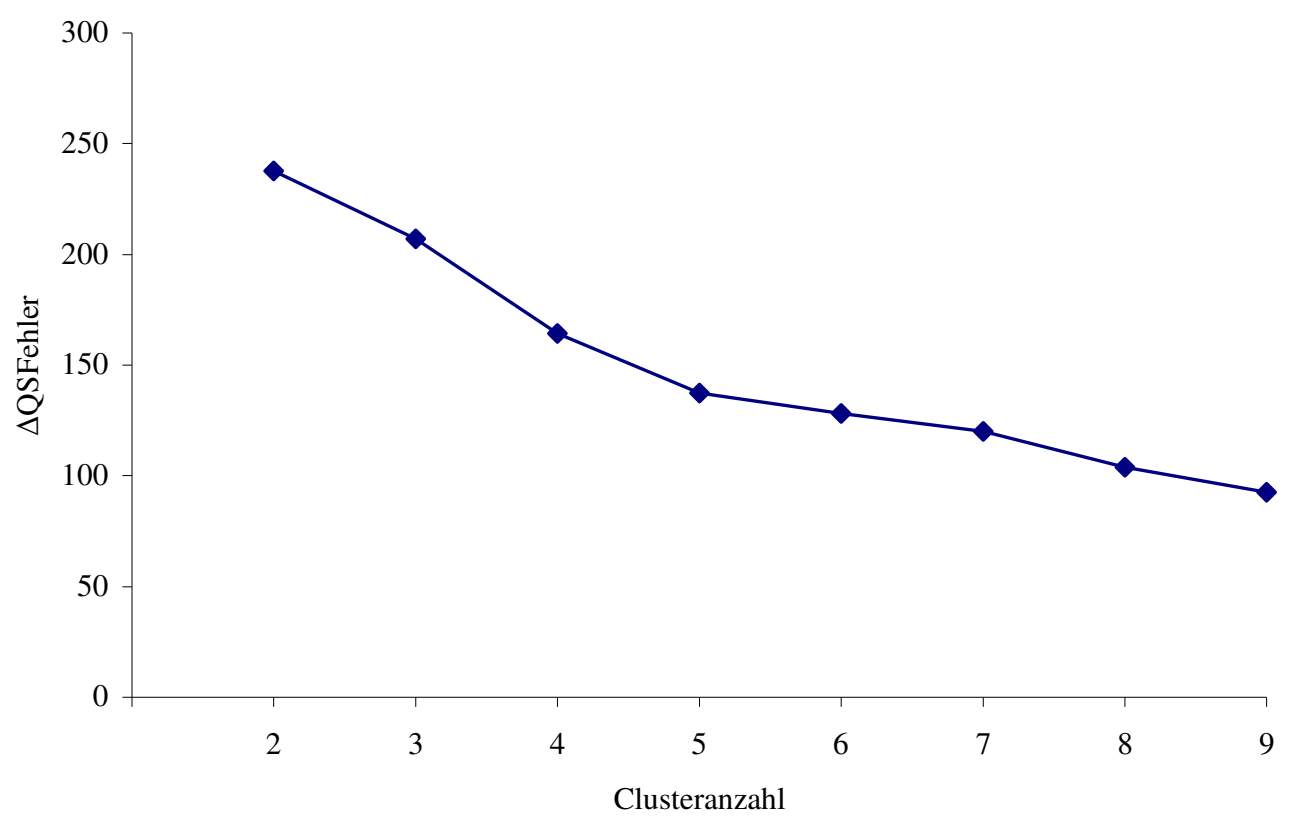

Abb. 9: Struktogramm des Ward-Verfahrens

Anschliessend wurden mit der Clusterzentrenanalyse nach der nicht-hierarchischen k-MeansMethode die einzelnen Angehörigen aufgrund ihres Belastungsmusters aus den ZBI- und BDI-V-Items den einzelnen Clustern zugeordnet. Die Clusteranzahl wurde dabei auf die aus dem Ward-Verfahren bestimmte Clusteranzahl festgelegt. Es wurde folglich eine Zwei-, Dreiund Fünf-Clusterlösung berechnet. 
In Tab. 11 ist die Anzahl der Fälle, die den einzelnen Clustern zugeordnet wurden, für eine Fünf-Clusterlösung zu entnehmen. Die Tabelle zeigt deutlich, dass dem ersten Cluster nur vier Probanden und dem fünften Cluster nur neun Angehörige zugeordnet wurden. Infolgedessen machte es wenig Sinn, eine Fünf-Clusterlösung zu übernehmen.

Tab. 11: Anzahl Probanden pro Cluster (5)

\begin{tabular}{cc}
\hline 5 Cluster: & Anzahl Angehörige: \\
\hline 1 & 4 \\
2 & 21 \\
3 & 34 \\
4 & 19 \\
5 & 9 \\
Total & $\mathbf{8 7}$ \\
\hline
\end{tabular}

Tab. 12: Anzahl Probanden pro Cluster (3)

\begin{tabular}{cc}
\hline 3 Cluster: & Anzahl Angehörige: \\
\hline 1 & 31 \\
2 & 11 \\
3 & 45 \\
& \\
& \\
& $\mathbf{8 7}$ \\
\hline
\end{tabular}

Tab. 13: Anzahl Probanden pro Cluster (2)

\begin{tabular}{cc}
\hline 2 Cluster: & Anzahl Angehörige: \\
\hline 1 & 63 \\
2 & 24 \\
& \\
Total & $\mathbf{8 7}$ \\
\hline
\end{tabular}

Bei der Drei-Clusterlösung (siehe Tab. 12) zeigten sich ähnlich Probleme wie bei der FünfClusterlösung. Während in den Clustern eins und drei die Angehörigen ausgewogen zugeteilt wurden, wurden dem Cluster zwei nur elf Angehörige zugeordnet.

Die Zwei-Clusterlösung brachte bezüglich der Handhabbarkeit die beste Lösung (siehe Tab. 13). So wurde die Stichprobe geteilt in eine kleinere Gruppe von 24 schwer belasteten Angehörigen und einer grösseren Gruppe von 63 Angehörigen, die weniger stark belastet waren. In den nachfolgenden beiden Abb. 10 und 11 sind die beiden Gruppen bezüglich ihrer ZBI- und BDI-V Werte dargestellt. Die beiden Gruppen schwer belasteter Angehöriger $(M=46.46 ; S D=9.69)$ und weniger stark belasteter Angehöriger $(M=20.32 ; S D=9.27)$ wurden zudem im t-Test für unabhängige Stichproben über ein hochsignifikantes Ergebnis $(t(85)=-11.613, p<.001)$ beim BDI-V Score bestätigt. Die beiden Gruppen (stark belastet: 
$\mathrm{M}=26.67, \mathrm{SD}=6.45$; weniger stark belastet: $\mathrm{M}=16.84, \mathrm{SD}=7.18$ ) bestätigten sich aber auch über einen hochsignifikanten t-Test für unabhängige Stichproben beim ZBI Score $(\mathrm{t}(85)=-5.858, \mathrm{p}<.001)$.

Da bereits im Vorfeld die Vermutung bestand, dass eine Zwei-Cluster-Lösung wahrscheinlich ist, wurde für den weiteren Verlauf dieser Arbeit, die Zwei-Clusterlösung beibehalten. Zur weiteren Absicherung der Zwei-Clusterlösung wurde wie von Bortz und Schuster (2010, S. 469) empfohlen noch eine Diskriminanzanalyse gerechnet.

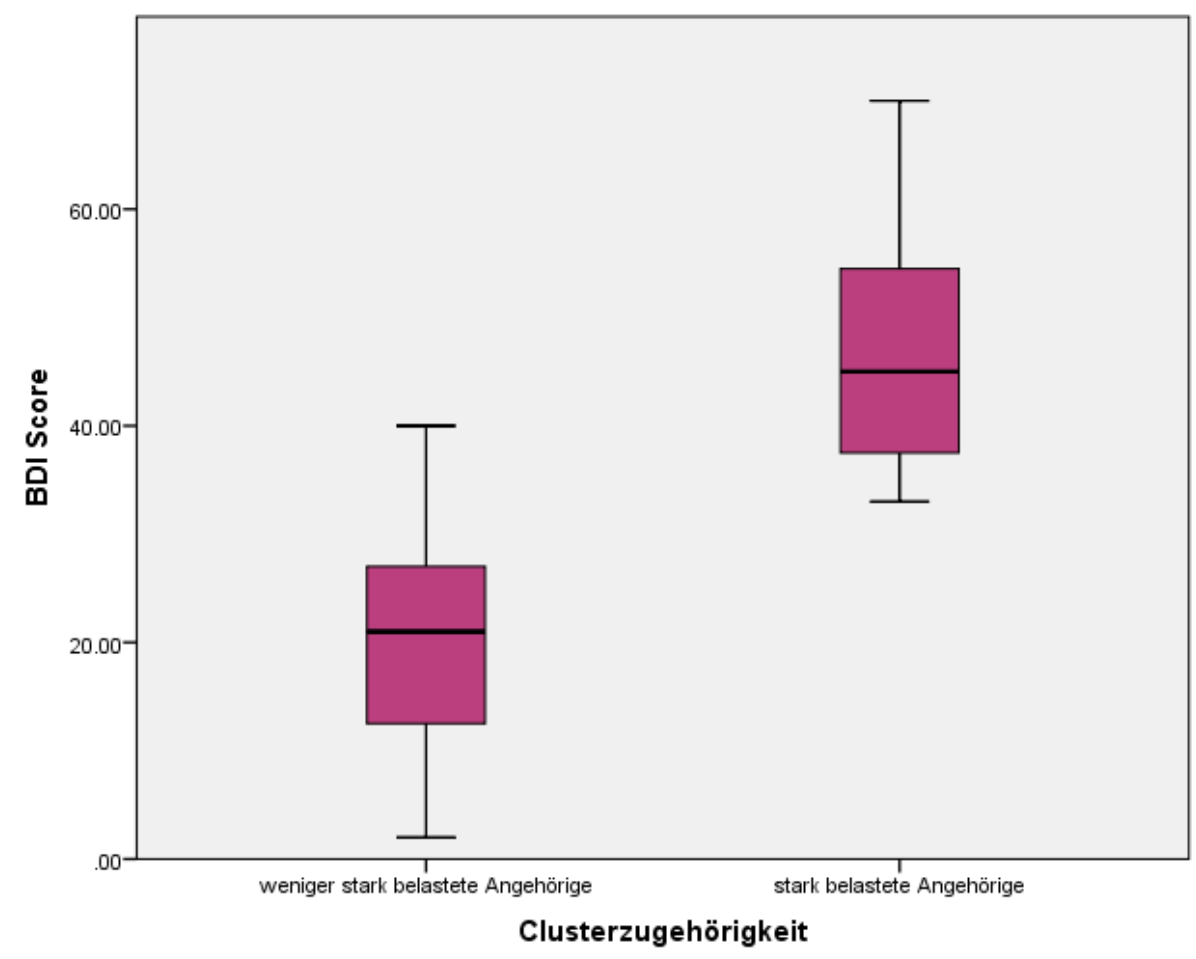

Abb. 10: Boxplot zum BDI-V Score der stark und weniger stark belasteten Angehörigen 


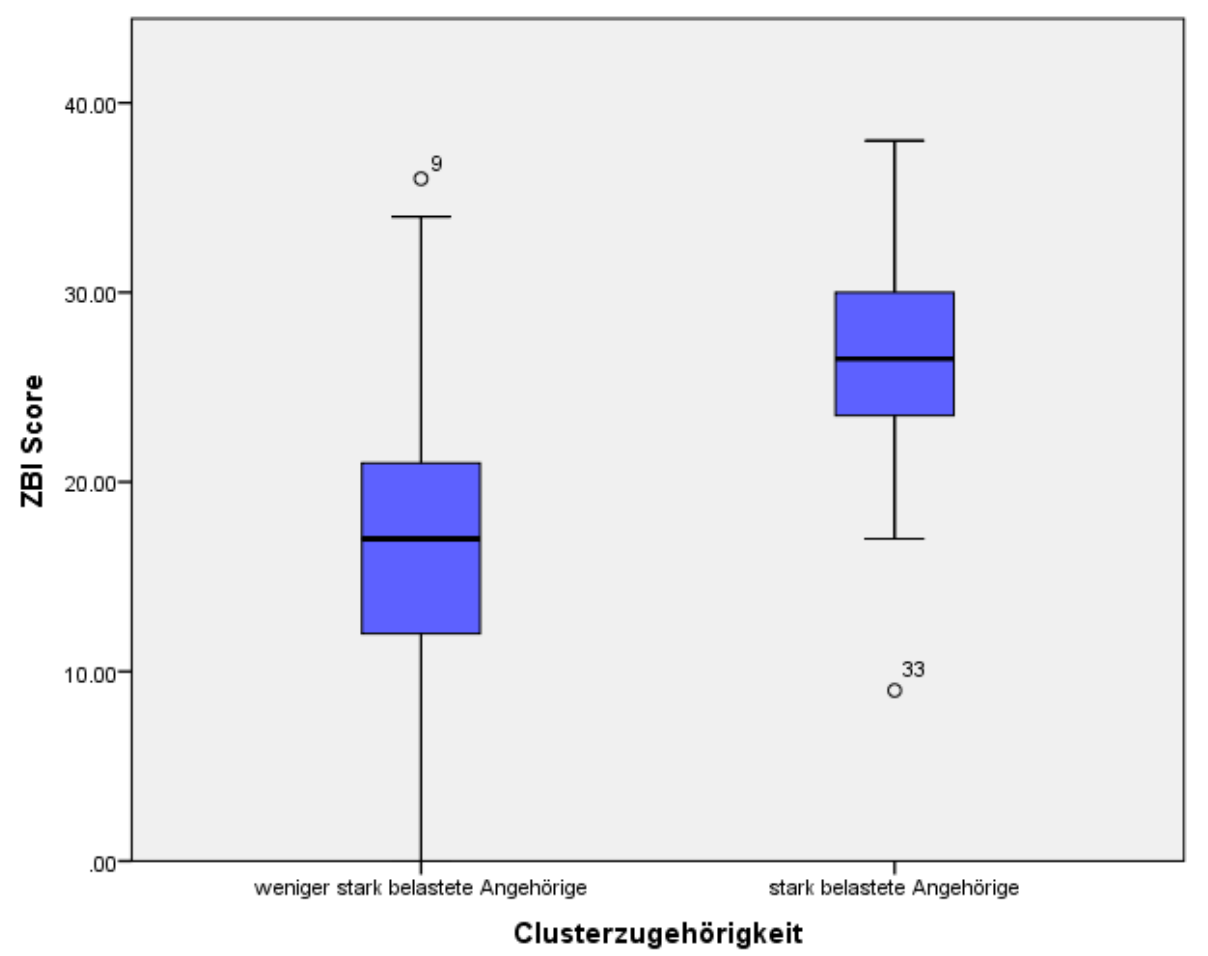

Abb. 11: Boxplot zum ZBI Score der stark und weniger stark belasteten Angehörigen

\subsubsection{Evaluation der Clusterlösung mittels Diskriminanzanalyse}

Basierend auf den Berechnungen, die im vorangegangenen Kapitel vorstellt wurden, wurde für die weiteren Berechnungen nur noch die Zwei-Clusterlösung berücksichtigt. Aufgrund der Zwei-Clusterlösung wurde die Zahl der Diskriminanzfunktionen auf eine beschränkt $(2-1=1)$. Die Tab. 14 zeigt deutlich, dass die berechnete Diskriminanzfunktion hochsignifikant war $(\mathrm{p}<.001)$ und somit davon ausgegangen werden konnte, dass sich die beiden gefundenen Gruppen von Angehörigen bezüglich ihrer Belastungen signifikant voneinander unterscheiden. In diesem Sinne konnte die Zwei-Clusterlösung für die vorliegende Stichprobe bestätigt werden, nachdem bereits die t-Tests für unabhängige Stichproben hochsignifikante Ergebnisse gebracht hatten (siehe Kap. 5.1.5.2).

Tab. 14: Kennwerte der Diskriminanzfunktion

\begin{tabular}{ccccc}
\hline Diskriminanzfunktion & Wilks-Lambda & Chi-Quadrat & df & Signifikanz \\
\hline 1 & .205 & 109.264 & 32 & .000 \\
\hline
\end{tabular}


Die Zuteilung der Angehörigen basierend auf der Diskriminanzfunktion zu den beiden Gruppen von Angehörigen erfolgte zudem überzufällig häufig korrekt wie nachfolgende Tabelle 15 zeigt. $98.9 \%$ aller Fälle wurden der korrekten Gruppe zugeteilt.

Tab. 15: Klassifizierung der Angehörigen basierend auf der Diskriminanzfunktion

\begin{tabular}{|c|c|c|c|c|c|}
\hline & & \multirow[t]{2}{*}{ Cluster-Nr. des Falls } & \multicolumn{2}{|c|}{ Vorhergesagte Gruppenzugehörigkeit } & \multirow[t]{2}{*}{ Gesamt } \\
\hline & & & Weniger Belastete & Stärker Belastete & \\
\hline \multirow{4}{*}{ Original } & Anzahl & Weniger Belastete & 63 & 0 & 63 \\
\hline & & Stärker Belastete & 1 & 23 & 24 \\
\hline & \multirow{2}{*}{$\%$} & Weniger Belastete & 100.0 & .0 & 100.0 \\
\hline & & Stärker Belastete & 4.2 & 95.8 & 100.0 \\
\hline
\end{tabular}

a. $98.9 \%$ der ursprünglich gruppierten Fälle wurden korrekt klassifiziert.

Die gefundene Zwei-Clusterlösung wurde zusätzlich mittels BDI-V Cut-off-Wert an einem externen Merkmal validiert (Bortz \& Schuster, 2010, S. 469). In der nachfolgenden Kreuztabelle (Tab. 16) zeigte sich, dass die gefundene Zwei-Clusterlösung hochsignifikant $\left(\chi^{2}(1)=77.28, \mathrm{p}<.001\right)$ zwischen denjenigen Angehörigen, die den Cut-off-Wert für eine klinisch relevante Depression überschritten, von denjenigen Angehörigen trennte, die klinisch unauffällig eingestuft wurden. Dieses Ergebnis bestätigte die Güte der gefundenen ZweiClusterlösung zusätzlich, da davon ausgegangen wurde, dass stark belastete Angehörige ein erhöhtes Risiko hatten, selbst psychisch krank zu werden.

Tab. 16: Kreuztabelle Zwei-Clusterlösung und BDI-V Cut-off

\begin{tabular}{|c|c|c|c|c|}
\hline \multirow{5}{*}{ Cluster } & \multirow{5}{*}{$\begin{array}{l}\text { wenig belastete Angehörige } \\
\text { stark belastete Angehörige }\end{array}$} & \multicolumn{2}{|c|}{ BDI-V Cuf-off } & \\
\hline & & nicht überschritten & überschritten & \\
\hline & & 62 & 1 & 63 \\
\hline & & 1 & 23 & 24 \\
\hline & & 63 & 24 & 87 \\
\hline
\end{tabular}

In der nachfolgenden Tabelle 17 ist die Struktur-Matrix der zehn Items zusammengefasst, die am stärksten mit dem Diskriminanzfaktor (Diskriminanzfunktion) korrelierten und somit die höchsten Gewichtungskoeffizienten aufwiesen. Es handelt sich folglich um diejenigen Items, die am besten zwischen den beiden Gruppen von Angehörigen diskriminierten. Bei den Gewichtungskoeffizienten handelte es sich um Korrelationen zwischen den Items des BDI-V 
und des ZBI und der standardisierten kanonischen Diskriminanzfunktion. Die Items sind nach ihrer Grösse der Korrelation geordnet.

Tab. 17: Struktur-Matrix der Diskriminanzfunktion

Es zeigte sich deutlich, dass insbesondere die Items des BDI-V am besten zwischen den beiden Gruppen von Angehörigen bezüglich ihrer Belastungen diskriminierten. Die beiden BDI-V-Items (Nr. 17) „Ich bin müde und lustlos.“ und (Nr. 2) „Ich sehe mutlos in die Zukunft.“ korrelierten dabei am stärksten mit der Diskriminanzfunktion. Diejenigen Items, die daraufhin am besten zwischen den zwei Gruppen trennten, konnten eher dem somatischen Bereich zugeordnet werden. So gaben stark belastete Angehörige auf den Items (BDI-V 16) „Ich habe Schlafstörungen“ und (BDI-V 18) „Ich habe keinen Appetit.“ eine höhere Beeinträchtigung an als die weniger stark belasteten.

\subsubsection{Bezug der Zwei-Clusterlösung zu anderen Variablen (logistische Regression)}

Vor der Analyse der Daten mit einer logistischen Regression wurde zuerst ein Zero-OrderVergleich zwischen den beiden Gruppen von Angehörigen bei sämtlichen Skalen (SES, SVF120 und BFW/E) durchgeführt. Aus diesem Grund wurden in einem ersten Schritt sämtliche Skalen auf ihre Normalverteilung hin untersucht, um anschliessend in einem zweiten Schritt mittels t-Test für unabhängige Stichproben bezüglich signifikanter Unterschiede verglichen zu werden. In der nachfolgenden Tabelle 18 sind diejenigen Skalen zusammengestellt, in denen sich die weniger belasteten Angehörigen von den stärker belasteten nach Anpassung des Signifikanzniveaus auf $p=.0014$ (Bonferoni-Korrektur: $\alpha^{\prime}=.05 / 35$ ) signifikant unterschieden. 
Tab. 18: Signifikante Ergebnisse des t-Tests für unabhängige Stichproben für die Clusterlösung

\begin{tabular}{|c|c|c|c|c|}
\hline Skala & Mittelwerte & SD & (df)T-Werte & Signifikanz \\
\hline $\begin{array}{l}\text { SVF: Negativ-Strategien } \\
\text { (Sekundärfaktor) }\end{array}$ & $\begin{array}{l}10.32 \text { (weniger belastete) } \\
13.79\end{array}$ & $\begin{array}{l}3.53 \\
2.08\end{array}$ & $(70.20)-5.659$ & $\mathrm{p}<.001$ \\
\hline SVF: Flucht & $\begin{array}{l}9.33 \text { (weniger belastete) } \\
13.21\end{array}$ & $\begin{array}{l}5.01 \\
4.51\end{array}$ & $(85)-3.316$ & $\mathrm{p}<.001$ \\
\hline $\begin{array}{l}\text { SVF: Resignation } \\
\text { (Hoffnungslosigkeit, Hilflosigkeit) }\end{array}$ & $\begin{array}{l}8.65 \text { (weniger belastete) } \\
12.33\end{array}$ & $\begin{array}{l}4.39 \\
3.89\end{array}$ & $(85)-3.602$ & $\mathrm{p}=.001$ \\
\hline BFW: Positive Lebenseinstellung & $\begin{array}{l}4.72 \text { (weniger belastete) } \\
3.57\end{array}$ & $\begin{array}{l}.58 \\
.82\end{array}$ & $(32.24) 6.310$ & $\mathrm{p}<.001$ \\
\hline BFW: Problembewusstheit & $\begin{array}{l}2.35 \text { (weniger belastete) } \\
3.30\end{array}$ & $\begin{array}{l}.68 \\
.68\end{array}$ & $(85)-5.804$ & $\mathrm{p}<.001$ \\
\hline $\begin{array}{l}\text { BFW: Körperliche Beschwerden und } \\
\text { Reaktionen }\end{array}$ & $\begin{array}{l}2.04 \text { (weniger belastete) } \\
3.22\end{array}$ & $\begin{array}{l}.84 \\
.83\end{array}$ & $(85)-5.884$ & $\mathrm{p}<.001$ \\
\hline BFW: Selbstwert & $\begin{array}{l}4.95 \text { (weniger belastete) } \\
3.92\end{array}$ & $\begin{array}{l}.69 \\
.70\end{array}$ & $(85) 6.198$ & $\mathrm{p}<.001$ \\
\hline BFW: Depressive Stimmung & $\begin{array}{l}1.95 \text { (weniger belastete) } \\
2.99\end{array}$ & $\begin{array}{l}.71 \\
.85\end{array}$ & $(85)-5.812$ & $\mathrm{p}<.001$ \\
\hline BFW: Lebensfreude & $\begin{array}{l}4.59 \text { (weniger belastete) } \\
3.55\end{array}$ & $\begin{array}{r}.85 \\
1.21\end{array}$ & $(32.15) 3.860$ & $\mathrm{p}=.001$ \\
\hline $\begin{array}{l}\text { BFW: Zufriedenheit } \\
\text { (Sekundärfaktor) }\end{array}$ & $\begin{array}{l}4.81 \text { (weniger belastete) } \\
3.74\end{array}$ & $\begin{array}{l}.53 \\
.63\end{array}$ & $(85) 8.003$ & $\mathrm{p}<.001$ \\
\hline $\begin{array}{l}\text { BFW: Negative Befindlichkeit } \\
\text { (Sekundärfaktor) }\end{array}$ & $\begin{array}{l}2.20 \text { (weniger belastete) } \\
3.26\end{array}$ & $\begin{array}{l}.58 \\
.56\end{array}$ & $(85)-7.659$ & $\mathrm{p}<.001$ \\
\hline SES & $\begin{array}{l}25.20 \text { (weniger belastete) } \\
18.67\end{array}$ & $\begin{array}{l}3.80 \\
5.05\end{array}$ & $(85) 6.528$ & $\mathrm{p}<.001$ \\
\hline
\end{tabular}

Im Zero-Order-Vergleich zeigt sich, dass stärker belastete Angehörige durchschnittlich deutlich niedrigere Werte auf der Rosenberg SES erreichten, was den Hinweis darauf gab, dass sie tendenziell eher über einen tieferen Selbstwert berichteten als weniger stark belastete Angehörige.

Es zeigte sich im Zero-Order-Vergleich zudem, dass sich stärker belastete Angehörige deutlich unterschieden von den weniger stark belasteten Angehörigen in sämtlichen Skalen des Wohlbefindens. So berichteten sie signifikant häufiger von körperlichen Beschwerden wie Herzklopfen, Schlafstörungen, Kopfschmerzen und Appetitlosigkeit. Sie erreichten durchschnittlich höhere Werte auf der Skala Problembewusstsein, was Hinweis darauf gab, dass sie sich häufiger Sorgen machten wegen der Arbeit, der Gesundheit, finanziellen Schwierigkeiten aber auch in Bezug auf ihre sozialen Beziehungen. Neben dem erhöhten 
Problembewusstsein zeichneten sie sich auch durch eine weniger positive Lebenseinstellung aus als weniger belastete Angehörige. Es schien den stärker belasteten Angehörigen schwerer $\mathrm{zu}$ fallen, das Gute in Ereignissen $\mathrm{zu}$ sehen. Sie gaben zudem weniger positive Gefühlserfahrungen im Zusammenhang mit ihren persönlichen Eigenschaften und Fähigkeiten an, was sich in der Skala „Lebensfreude“ an durchschnittlich signifikant tieferen Werten zeigte. Die durchschnittlich höheren Werte auf der Skala „Depressivität“ des BFW/E bestätigten die Ergebnisse des BDI-V, der zur Clusterbildung herangezogen worden war. Die tiefen Werte auf der „Selbstwertskala“ des BFW/E waren analog der „Rosenberg SES“ zu sehen.

Die Hypothese, dass weniger stark belastete Angehörige vermehrt Coping-Strategien anwenden würden, die das Stresserleben reduzieren, bestätigte sich im Zero-Order-Vergleich nicht. Dafür zeigte sich, dass stärker belastete Angehörige signifikant häufiger Stressverarbeitungsweisen angaben, die im Allgemeinen das Stresserleben erhöhen. Sie berichteten insbesondere signifikant häufiger über die Tendenz einer Belastungssituation entkommen zu wollen (Flucht) und über ein Aufgeben und Resignieren mit Gefühlen von Hoffnungslosigkeit und Hilflosigkeit in Belastungssituationen. Bezüglich der soziodemographischen Variablen wie Geschlecht, Bildung, Alter usw. und den Charakteristika der Suchterkrankung liessen sich keine Unterschiede zwischen den beiden Gruppen feststellen.

Die Zero-Order-Vergleiche wurden neben theoretischen Überlegungen anschliessend herangezogen, um Modelle für die logistische Regressionsanalyse zu entwickeln. In einem ersten Schritt wurden die folgenden Skalen ins Modell eingeschlossen: „BFW: Problembewusstheit“, „BFW: Körperliche Beschwerden und Reaktionen“ und „Rosenberg SES“. Die Skala „Problembewusstheit“ wurde mit der Überlegung übernommen, dass stärker belastete Angehörige mehr Sorgen in verschiedenen Lebensbereichen (Arbeit, Gesundheit, Finanzen, Beziehungen) erleben als weniger belastete Angehörige. Die Diskriminanzfunktion hatte erste Hinweise geliefert, dass schwerer belastete Angehörige deutlich häufiger somatische Symptome angaben als weniger belastete Angehörige. Aus diesem Grund wurde die Skala „BFW: Körperliche Beschwerden und Reaktionen“ ins Modell aufgenommen. Das Modell wurde schliesslich durch das Zufügen einzelner Variablen aufgrund von theoretischen Überlegungen modifiziert und überprüft. Insbesondere die Skalen des Stressverarbeitungsfragebogens wurden auf ihre Wirkung hin untersucht. Es zeigte sich 
allerdings, dass keine der Skalen einen signifikanten Einfluss auf die Eintretenswahrscheinlichkeit einer hohen Belastung leisten konnte. Aus diesem Grund wurde letztlich der Sekundärfaktor „SVF: Negativ-Stragien“ ins Modell eingeschlossen, obwohl dieser ebenfalls keinen signifikanten Beitrag leisten konnte. In der nachfolgenden Tabelle 19 sind die Ergebnisse der logistischen Regressionsanalyse dargestellt.

Tab. 19: Logistische Regressionsanalyse

\begin{tabular}{lcccccccc}
\hline Skala & $\begin{array}{l}\text { Regressions- } \\
\text { koeffizient B }\end{array}$ & $\begin{array}{l}\text { Standard- } \\
\text { fehler }\end{array}$ & Wald & df & Sig. & Odds ratio & 95\% CL OR \\
\hline Rosenberg SES & -.312 & .105 & 8.795 & 1 & $\mathbf{. 0 0 3}$ & .732 & .596 & .900 \\
BFW: Problembewusstheit & 1.383 & .598 & 5.354 & 1 & $\mathbf{. 0 2 1}$ & 3.985 & 1.236 & 12.855 \\
BFW. Körperliche Beschwerden & 1.455 & .464 & 9.844 & 1 & $\mathbf{. 0 0 2}$ & 4.283 & 1.726 & 10.628 \\
SVF: Negativ-Strategien & .173 & .153 & 1.288 & 1 & .256 & 1.189 & .882 & 1.605 \\
Konstante & -3.952 & 3.510 & 1.268 & 1 & .260 & .019 & & \\
\hline
\end{tabular}

Das Modell legte folgende Zusammenhänge nahe: Je niedriger die Angehörigen ihr eigenes Selbstwertgefühl einschätzten, desto wahrscheinlicher war es, dass sie zur Gruppe der schwer belasteten Angehörigen gehörten. Bei den restlichen Variablen im Modell zeigte sich der umgekehrte Zusammenhang. Je stärker jemand angab, unter Sorgen oder körperlichen Beschwerden wie Kopf- und Magenschmerzen, Schlafstörungen, Herzklopfen und anderen zu leiden, desto grösser war das Risiko zu der Gruppe von schwer belasteten Angehörigen zu gehören. In die gleiche Richtung wirkten auch negative Bewältigungsstrategien, die das Stresserleben grundsätzlich eher erhöhen. Dieser Zusammenhang wurde aber im Modell nicht signifikant.

Die fünf Variablen des logistischen Regressionsmodells erklärten $80 \%$ (Nagelkerke- $R^{2}$ ) der Varianz bezüglich der Gruppenzugehörigkeit. Die nachfolgende Klassifizierungstabelle (siehe Tab. 20) zeigt zudem deutlich, dass das Modell eine korrekte Zuordnung zu den Gruppen macht, die höher ist als es das Zufallsprinzip erwarten liesse. $90.8 \%$ der Angehörigen wurden den Gruppen korrekt zugeordnet. 
Tab. 20: Klassifizierungstabelle

\begin{tabular}{|lc|r|r|r|}
\hline \multirow{2}{*}{ Beobachtet } & \multicolumn{3}{|c|}{ Vorhergesagt } \\
\cline { 3 - 5 } & & \multicolumn{2}{|c|}{ Clusterzugehörigkeit } & \multirow{2}{*}{ Prozentsatz der } \\
\cline { 3 - 5 } & & weniger Belastete & stärker Belastete & Richtigen \\
\hline \multirow{2}{*}{ Clusterzugehörigkeit } & weniger Belastete & 62 & 1 & 98.4 \\
& stark Belastete & 7 & & 17 \\
Gesamtprozentsatz & & & & 70.8 \\
& & & & 90.8 \\
\hline
\end{tabular}

Der Hosmer-Lemeshow-Test wurde nicht signifikant $\left(\chi^{2}(8)=.684, \mathrm{p}=1.000\right)$, wodurch davon auszugehen war, dass die Abweichungen zwischen den beobachteten Zuteilungen zu den beiden Gruppen von Angehörigen und den durch die logistische Regressionsfunktion berechneten Zuteilungen nicht häufiger als durch Zufall auftraten. 


\subsection{Qualitative Daten}

\subsubsection{Beschreibung der Stichprobe}

\subsubsection{Einleitung}

Aufgrund der aufwendigen Datenaufbereitung und -auswertung, die mit einer qualitativen Analyse verbunden sind, wurden nur einige wenige Angehörigen $\mathrm{zu}$ einem problemzentrierten Interview eingeladen. Im nachfolgenden Kapitel sind die Kriterien zusammengefasst, nach denen die Angehörigen für das Interview ausgewählt wurden. Zudem werden die einzelnen Interviewpartner anhand der Beziehung zu ihrem suchtkranken Angehörigen, ihrer aktuellen Wohn- und Lebenssituation sowie der Unterstützung, die sie sich suchten und erhielten, kurz vorgestellt.

\subsubsection{Beschreibung der Angehörigen}

Insgesamt wurde mit 13 Angehörigen, die auch an der Fragebogenerhebung teilgenommen hatten, ein problemzentriertes Interview durchgeführt. Die Angehörigen, die in die qualitative Datenerhebung eingeschlossen wurden, wurden selektiv angefragt, ob sie $\mathrm{zu}$ einem persönlichen Interview bereit wären. Bei der Anfrage der Angehörigen wurde darauf geachtet, dass sie bezüglich der Beziehung, in der sie zum drogenabhängigen Angehörigen standen, etwa in ähnlicher Weise wie in den quantitativen Daten repräsentiert waren wie nachfolgende Tabelle (Tab. 21) zeigt. Gleichzeitig wurde beim Sampling darauf geachtet, ob und welche Art der Unterstützung die Angehörigen erhalten hatten, um möglichst verschiedene Perspektiven berücksichtigen zu können.

Tab. 21: Verhältnis der qualitativen zu den quantitativen Daten

\begin{tabular}{|c|c|c|}
\hline & Qualitative Daten & Quantitative Daten \\
\hline Eltern & $8(61.5)$ & $44 \quad(50.0)$ \\
\hline Geschwister & $2(15.4)$ & 21 \\
\hline Partner(innen) & $2(15.4)$ & $15(17.0)$ \\
\hline Kinder & $1 \quad(7.7)$ & $\begin{array}{ll}5 & (5.7)\end{array}$ \\
\hline Andere & - & $3 \quad(3.4)$ \\
\hline Total & $13 \quad(100)$ & $88 \quad(100)$ \\
\hline
\end{tabular}

Alle Angehörigen erhielten einen Decknamen zugeteilt. Nachfolgend sollen die Angehörigen, die sich für die Teilnahme zu einem Interview bereit erklärten, kurz vorgestellt werden. 


\section{Annabelle:}

Annabelle war die Ehepartnerin eines 49-jährigen Mannes, der nach einem Rückfall vor 10 Jahren Heroin wieder von der Gasse konsumierte. Annabelle kannte ihren Mann seit 25 Jahren. Er hatte ihr kurz nach dem Kennenlernen erzählt, dass er heroinabhängig gewesen sei. Sie hatte mit ihrem Mann zwei gemeinsame Kinder im Jugend- und frühen Erwachsenenalter. Die Kinder wussten nicht von der Heroinabhängigkeit ihres Vaters. Annabelle hatte sich aufgrund von finanziellen Schwierigkeiten, die durch den Drogenkonsum ihres Mannes entstanden waren, Hilfe gesucht und war zur Angehörigenvereinigung Drogenabhängiger (ada-zh) gegangen. Sie hatte dort vor allem Einzelberatungen in Anspruch genommen. Sie hatte zudem auf Anraten der ada-zh Unterstützung bei einer Rechtsberatung gesucht.

\section{Andrea:}

Andrea war die Mutter eines Sohnes, der zum Zeitpunkt der Befragung 30 Jahre alt war. Vor ca. 10 Jahren hatten sich erste Anzeichen für eine Suchterkrankung gezeigt, als der Sohn begonnen hatte, Partydrogen zu konsumieren. Im Laufe der Zeit waren immer weitere Substanzen dazu gekommen, so dass er neben Stimulanzen auch regelmässig Heroin, Benzodiazepine, Kokain und Alkohol konsumierte. Andrea lebte alleine, hatte aber ihren drogenabhängigen Sohn immer wieder vorübergehend bei sich aufgenommen, wenn er keinen festen Wohnsitz hatte. Andrea hatte sich auf Drängen der Tochter und des drogenabhängigen Sohnes Hilfe bei der Angehörigenvereinigung Drogenabhängiger (ada-zh) gesucht. Sie hatte dort Einzelgespräche in Anspruch genommen und war in eine Selbsthilfegruppe gegangen.

\section{Anke:}

Anke hatte einen 34-jährigen Sohn, der neben einem problematischen Alkoholkonsum auch regelmässige Kokain-Konsumereignisse hatte. Ihr Sohn hatte ihr vom Kokainkonsum etwa zwei oder drei Jahren vor dem Interview berichtet. Von der Alkoholproblematik hatte sie dagegen schon länger gewusst. Zum Zeitpunkt der Befragung hatte sich seine Situation etwas gebessert. Anke lebte mit ihrem Ehepartner zusammen, der jedoch nicht der leibliche Vater ihres Sohnes war. Der suchtkranke Sohn bewohnte eine Einlegerwohnung in der Liegenschaft der Eltern. Anke hatte sich sehr schnell Unterstützung gesucht, sobald sie vom Kokainkonsum ihres Sohnes erfahren hatte. Auch sie besuchte bei der Angehörigenvereinigung Drogenabhängiger (ada-zh) sowohl Einzelgespräche als auch eine Selbsthilfegruppe. 


\title{
Angelika:
}

Angelika war die Mutter eines 41-jährigen Sohnes, der im Alter von 15 Jahren begonnen hatte, Heroin zu konsumieren. Angelikas Sohn befand sich in einem Methadonprogramm, konsumierte aber zusätzlich noch diverse weitere Substanzen. Sie lebte mit ihrem Ehepartner zusammen, der nicht der leibliche Vater ihres Sohnes war. Angelika hatte sich bereits vor über 20 Jahren das erste Mal Unterstützung gesucht. Sie war in verschiedenen Selbsthilfegruppen unter anderem auch für Eltern von drogenabhängigen Kindern gewesen. Als der Kokainkonsum bei ihrem Sohn zunahm, meldete sie sich für Einzelgespräche bei der Angehörigenvereinigung Drogenabhängiger (ada-zh) an.

\begin{abstract}
Albert:
Albert hatte eine 28-jährige Tochter, die zum Zeitpunkt der Befragung regelmässig Kokain i.v. konsumierte und sich deswegen auch in Therapie befand. Der Kokainkonsum war etwa vor sechs Jahren auffällig geworden. Neben Kokain konsumierte sie zudem missbräuchlich Benzodiazepine. Albert war verheiratet und lebte mit seiner Ehefrau zusammen. Die Tochter war gemeinsam mit ihrem Kind in einer betreuten Wohngemeinschaft untergebracht. Sie verbrachte aber die Wochenenden und Ferien regelmässig bei ihren Eltern. Albert nahm bei der Angehörigenvereinigung Drogenabhängiger (ada-zh) Einzelgespräche in Anspruch.
\end{abstract}

\section{August:}

August war der Vater von drei heroinabhängigen Söhnen und seit über 20 Jahren betroffener Angehöriger. Einer seiner Söhne war vor einigen Jahren verstorben aufgrund einer Folgeerkrankung der Sucht. Die beiden anderen Söhne lebten gemeinsam und befanden sich beide in einer heroingestützten Behandlung $(\mathrm{HeGeBe})$. August lebte mit seiner Ehepartnerin zusammen. Er und seine Frau hatten sich, unmittelbar nachdem sie vom Heroinkonsum der Söhne erfahren hatten, Unterstützung gesucht. Sie hatten neben Einzelgesprächen auch eine Selbsthilfegruppe besucht.

\section{Dagmar:}

Dagmar war die Partnerin eines 33-jährigen Mannes, der sich aufgrund seiner Heroinabhängigkeit in einem Methadonprogramm befand. Zum Zeitpunkt der Befragung stand vor allem der Benzodiazepinmissbrauch (Dormicum ${ }^{\circledR}$ ) im Vordergrund. In der Beziehung zwischen Dagmar und ihrem Partner war es in der Vergangenheit immer wieder zu Beziehungsunterbrüchen gekommen. Als die Befragung stattfand war das Paar gerade 
frisch wieder zusammengekommen. Dagmar lebte mit ihrer Tochter aus einer anderen Beziehung und zeitweise mit ihrem Partner zusammen, der neben ihr auch noch bei der Mutter wohnte. Aufgrund von Schwierigkeiten (Gewalt, Streitereien), die sich im Zusammenleben ergaben, hatte Dagmar Unterstützung bei einer Familienberatung und einer Suchtberatungsstelle gesucht.

\section{Doris:}

Der Sohn von Doris war zum Zeitpunkt der Befragung 39 Jahre alt. Er konsumierte seit 18 Jahren regelmässig Kokain und Alkohol. Er war verheiratet mit einer Frau, die ebenfalls eine Suchtproblematik hatte. Die beiden hatten gemeinsam ein Kind. Doris war verheiratet und lebte mit ihrem Ehemann. Sie kümmerte sich zudem regelmässig um ihr Enkelkind. Unterstützung erhielt Doris vor allem vom Psychiater ihres Sohnes, zu dem sie einen losen, aber regelmässigen Kontakt unterhielt. Sie war zudem kurzzeitig zu zwei Psychotherapeuten gegangen, um sich Entlastung zu verschaffen, was sie allerdings nicht positiv erlebt hatte.

\section{Dana:}

Dana lebte mit ihren beiden Kindern zusammen. Der 17-jährige Sohn von Dana konsumierte seit zwei Jahren Kokain und Stimulanzen. Nachdem er im Rahmen einer Therapie Ritalin verschrieben bekommen hatte, begann er dieses i.v. zu konsumieren. Danas Sohn befand sich zum Zeitpunkt der Befragung in stationärer Behandlung. Dana hatte sich einer Selbsthilfegruppe (VASK) angeschlossen. Im Vorfeld hatte sie sich zudem Hilfe bei der Familienberatung und beim KJPD gesucht. Sie fühlte sich zudem unterstützt von der aktuellen stationären Therapie des Sohnes.

\section{Dorette:}

Dorette lebte mit ihrem 40-jährigen, heroinabhängigen Sohn zusammen. Ihr Sohn konsumiert Heroin von der Gasse. Er hatte an mehreren Methadonprogrammen teilgenommen, die er aber immer wieder abgebrochen hatte. Er war zum Zeitpunkt der Befragung nicht bereit, ein therapeutisches Angebot in Anspruch zu nehmen. Dorette war seit über 20 Jahren von der Drogenabhängigkeit ihres Sohnes betroffen. Sie erhielt keine Unterstützung. Sie hatte wiederholt erlebt, dass Hilfsangebote mit Vorwürfen und Schuldzuweisungen verbunden waren. 


\section{Dylan:}

Dylan war der Sohn von heroinabhängigen Eltern. Der Vater befand sich in einem Methadonprogramm, trank regelmässig Alkohol und konsumierte gelegentlich weitere Substanzen. Zur Mutter bestand zum Zeitpunkt des Interviews kein Kontakt, weil es zu einem Konflikt wegen deren neuem Partner, der ebenfalls drogenabhängig war, gekommen war. Dylan hatte zudem einen jüngeren Bruder, zu dem er eine enge Bindung hatte, der aber begonnen hatte, gelegentlich Kokain zu konsumieren. Dylan lebte mit seiner Partnerin und dem gemeinsamen Kind zusammen. Er war bei den Eltern aufgewachsen und hatte keine Unterstützung erhalten.

\section{Darina:}

Darina war die sechs Jahre ältere Schwester eines 24-jährigen Mannes, der Heroin konsumierte. Sie erfuhr vor einem Jahr von der Suchterkrankung, nachdem ihr Bruder den Eltern seine Heroinabhängigkeit gestanden hatte und sich für ein Methadonprogramm angemeldet hatte. Darina lebte mit ihrem Lebenspartner zusammen. Sie oder ihre Familie hatten bisher keine Unterstützung gesucht oder erhalten.

\section{Dorothea:}

Dorothea hatte einen acht Jahre älteren Bruder, der seit 16 Jahren Heroin konsumierte. Sie war etwa acht oder neun Jahre alt gewesen, als der Bruder mit dem Heroinkonsum begonnen hatte. Ihr Bruder war zum Zeitpunkt der Befragung 31 Jahre alt. Dorothea lebte mit ihrem Lebenspartner zusammen. Sie befand sich in einer Psychotherapie, um ihre eigene Geschichte und Entwicklung aufzuarbeiten, die von der Suchterkrankung des Bruders stark geprägt und beeinflusst worden war.

In der nachfolgenden Abbildung 12 sind die BDI-V Werte der Studienteilnehmenden, die an der qualitativen Befragung teilnahmen, dargestellt. Dylan und Dorothea, die beide als Kinder bereits von der Suchterkrankung betroffen waren, überschritten den Cut-off von 35 für eine klinisch relevante Depression erheblich. Daneben erreichten oder überschritten Dana und Doris den Cut-off. Die Depressivitätswerte in der qualitativen Stichprobe waren folglich mit $30.77 \%$ vergleichbar mit derjenigen der quantitativen Stichprobe $(27.3 \%)$. 


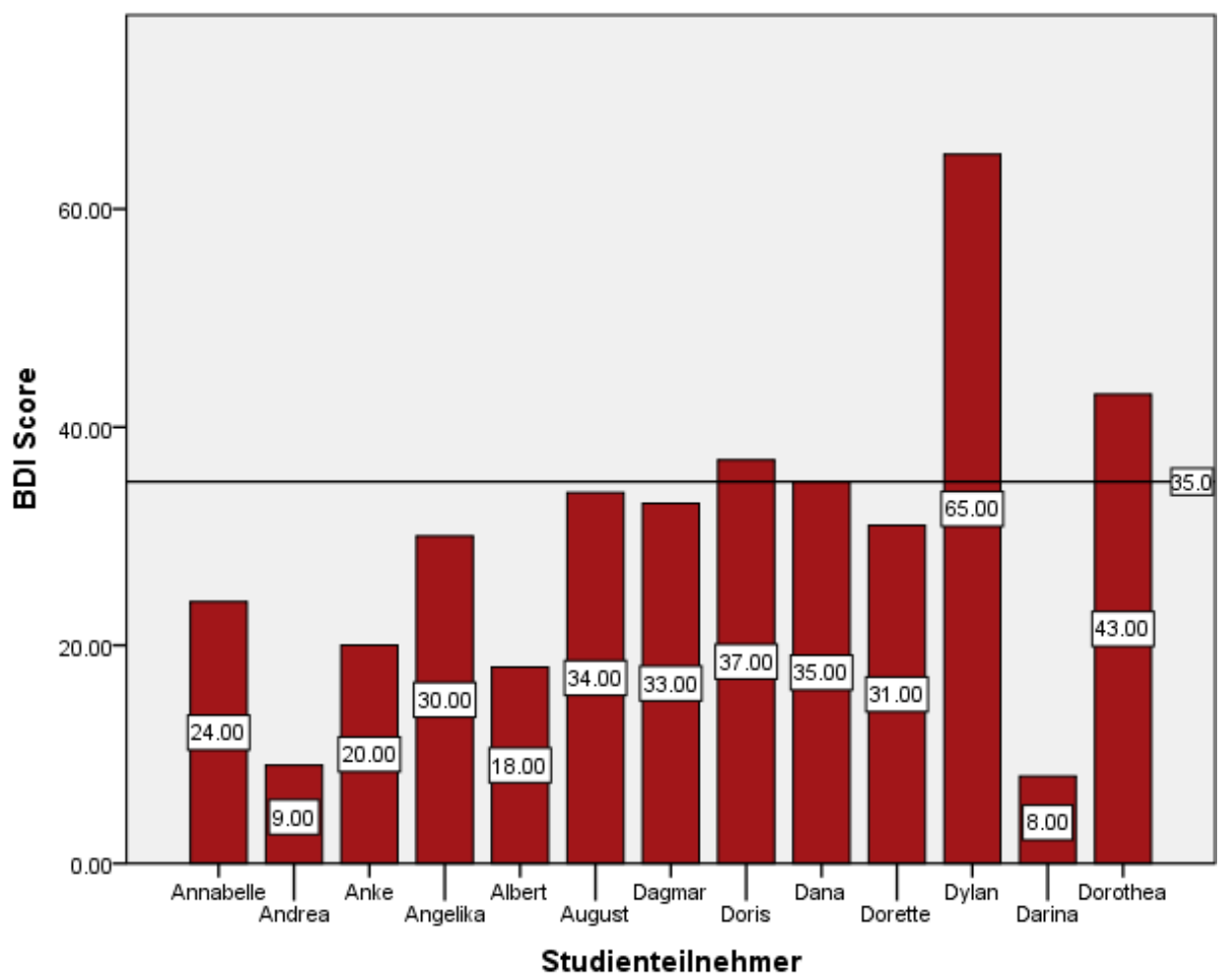

Abb. 12: BDI-V Score der mittels halbstrukturiertem Interview befragten Studienteilnehmenden

\subsubsection{Situation der Erkenntnis}

\subsubsection{Einleitung}

Eine der Fragestellungen lautete, welches Vorwissen Angehörige haben, wenn sie vom Drogenkonsum der nahestehenden Person erfahren. Die meisten befragten Angehörigen hatten Vorahnungen im Vorfeld der Erkenntnis. Diese zeigten sich in einem diffusen Gefühl, dass irgendetwas nicht stimmte (7), sich die substanzkonsumierende Person immer mehr veränderte (7) oder dass sie in der Ausbildung oder finanziell Schwierigkeiten (5) hatte. In den nachfolgenden beiden Kapiteln ist zusammengefasst, wie die Angehörigen von der Abhängigkeitserkrankung erfahren und wie sie darauf reagiert hatten.

\subsubsection{Situation der Erkenntnis}

Die Auswertung der Interviews zeigte deutlich, dass es für viele Angehörige nicht die eine Situation der Erkenntnis gab. Vielmehr zogen sie mehrere Informationsquellen heran, um schliesslich zu einer definitiven Erkenntnis zu kommen und sich ein Bild über das Ausmass des Drogenkonsums zu verschaffen. So erfuhr Andrea beispielsweise von ihrer Tochter, dass ihr Sohn Kokain konsumiert. Andrea holte sich aber anschliessend noch die Bestätigung von ihrem Sohn, den sie direkt darauf ansprach. Ähnlich wie Andrea machten auch andere 
Angehörige in verschiedenen Kategorien Angaben dazu, wie sie von der Suchterkrankung erfahren hatten.

Die meisten Angehörigen waren irgendwann von den Betroffenen informiert worden, dass sie ein Drogenproblem hätten (8). Entweder hatten die Angehörigen schon einen Verdacht gehabt und bei ihren Familienmitgliedern nachgefragt oder diese hatten von sich aus zugegeben, dass sie Drogen konsumieren würden. August, Andrea und Angelika sprachen ihre Söhne direkt an und bekamen so die definitive Bestätigung. Dagegen klärten die Kinder von Anke und Albert von sich aus ihre Eltern über die Suchterkrankung auf. Annabelle und Dagmar erzählten, dass ihre Partner ihnen nach ein paar Treffen deren Abhängigkeitserkrankung offenbart hatten, als sie noch nichts von der Sucht geahnt hatten.

Bei einigen wenigen Angehörigen (3) spielten auch Funde oder Situationen eine entscheidende Rolle, die zur Erkenntnis führten, dass ihr Familienmitglied ein Drogenproblem haben oder nach langer Zeit wieder rückfällig geworden sein musste. Annabelle, die zwar wusste, dass ihr Mann mal ein Problem mit Heroin hatte, fand irgendwann Spritzen im Abfall, die ihr bestätigten, dass ihr Mann wieder rückfällig geworden war. Dagegen berichteten Albert und August von Situationen, in denen für sie klar wurde, wie schwer ihre Kinder in der Abhängigkeit drinsteckten. Der minderjährige Sohn von August hatte beispielsweise dem Vater das Auto gestohlen, als die Eltern in den Ferien waren, um sich so Heroin zu beschaffen und hatte anschliessend auch noch einen Unfall verursacht.

Die Auswertung der Interviews zeigte ausserdem, dass einige Angehörige über eine Drittperson vom Substanzkonsum erfahren hatten (6). Bevor Andrea ihren Sohn auf seinen Drogenkonsum ansprach, hatte ihr ihre Tochter erzählt, dass dieser Kokain konsumiere. Darina war von einem ihrer Brüder telefonisch über die Heroinabhängigkeit ihres Bruders informiert worden, nachdem dieser der Familie gesagt hatte, dass er in ein Methadonprogramm eintreten werde. Hingegen hatte Dorothea als Kind ihre Eltern belauscht und so erfahren, dass ihr Bruder ein Drogenproblem hatte. Doris hatte sich an einen Kollegen bei der Drogenfahndung gewandt, der ihr dann sagte, dass ihr Sohn ein Problem mit Kokain habe. 
Einige wenige Angehörige konnten sich nicht mehr erinnern, wie sie vom Drogenkonsum erfahren hatten (3). Dorette mutmasste, dass ihr Sohn wahrscheinlich irgendwann die Wahrheit gesagt hatte und auch Dana konnte nicht mehr genau sagen, unter welchen Umständen sie vom Substanzkonsum erfahren hatte. Besonders eindrücklich waren Dylans Ausführungen in diesem Zusammenhang:

Das ist noch eine gute Frage. Weil das habe ich mich selbst schon viel gefragt. Ich habe das eigentlich - man hat es einfach gewusst. Ich kann es nicht sagen. Woher. Aber vielleicht, weil sie sich ein bisschen versteckt haben ab und zu. Oder vielleicht auch ihre Art. Wenn sie vielleicht konsumiert gehabt haben oder nicht. Oder; [...] Ja. Aber ich ich, das habe ich mich selbst auch schon viel gefragt. Aber ich kann Dir; Ich weiss nur noch. Ich habe es einfach gewusst. Ohne+ dass ich eigentlich gewusst habe. Was Drogen sind. Also was was das ist eigentlich. Habe ich gewusst. Die haben etwas konsumiert. Also ich habe gewusst. Dass sie Drogen genommen haben. Aber woher eigentlich? Kann ich nicht sagen. (lacht leicht) (Z10-20)

Obwohl er, als er älter war, von seinen Eltern aufgeklärt wurde, hatte er vorher schon gewusst, dass die Eltern Drogen nahmen. Er hatte bereits als kleines Kind eine Vorstellung vom Substanzkonsum seiner Eltern gehabt.

\subsubsection{Reaktion auf die Erkenntnis}

Die Reaktionen auf die Erkenntnis waren unterschiedlich. Andrea, Anke und Darina waren im ersten Moment sehr erleichtert (3). Anke hatte den Eindruck, dass ihr Sohn durch das Schweigen belastet gewesen war und war deshalb erleichtert. Dagegen hatten Andrea und Darina sehr unter der Ungewissheit gelitten. Sie waren deshalb vor allem froh, dass sie endlich Klarheit darüber hatten, was los war. Andrea beschrieb die Situation wie folgt:

Von dem Moment wo ich eigentlich gewusst habe dass er dass er äh Coci nimmt, dann habe ich hat ma- habe ich habe ich gefunden. Ich kann etwas machen. Obwohl ich ja das gar nicht; im Nachhinein weiss ich das liegt gar nicht drin. Aber ich habe dann das Gefühl gehabt. Jetzt +ka-; [...] Was es ist. Jetzt kann ich etwas machen mit ihm. (Z318-324)

Die obige Textstelle zeigt zudem einen weiteren Aspekt auf, mit dem Angehörige oft auf die Erkenntnis des Drogenkonsums reagierten: Sie aktivierten all ihre Kräfte und Möglichkeiten, damit ihr Familienmitglied aufhört zu konsumieren (6). So informierten sich Angelika, Albert und Darina sofort über Therapieangebote und was zu tun sei in einer solchen Situation. Dorothea, die noch ein Kind war, konnte sich daran erinnern, dass sie das Gefühl hatte, dass nun alle zurückstehen mussten, damit dem heroinabhängigen Bruder geholfen werden konnte. Die beiden Partnerinnen Annabelle und Dagmar waren beide im ersten Moment 
optimistisch und glaubten, dass sie die Situation gemeinsam mit dem Lebenspartner meistern könnten. Annabelle berichtete, dass sie Folgendes gedacht hatte, als ihr Mann ihr seine Drogenvergangenheit gestanden hatte:

vielleicht ist das eher so ein bisschen -- Das Helfende wo mich gereizt hat. Ahh (=Ton der Begeisterung) da ist einer ähm ja. Dem kann man doch ein bisschen zeigen wie schön das Leben ist oder äh; (Z40-42)

Dagegen gaben nur zwei Angehörige an, sie hätten sich sofort für sich selbst Unterstützung gesucht. Während Anke sich sehr schnell bei der Angehörigenvereinigung Drogenabhängiger (ada-zh) gemeldet hatte, hatte Darina einer Kollegin vom Heroinkonsum ihres Bruders erzählt, um sich zu entlasten.

Vier Angehörige erzählten im Interview, dass die Gewissheit, dass ein ihnen nahestehender Mensch ein Drogenproblem hatte, unvorstellbar schlimm gewesen sei. Den Schreck, den Albert, August, Dorette und Darina im Moment der Erkenntnis verspürten, beschrieben sie zum Teil wie Albert in Bildern, die erahnen lassen, wie schlimm es für sie gewesen sein muss:

Ja. Und die, die, also den! Moment. Den vergesse ich nie mehr. Wo sie mir das gesagt hat. [...] Da reisst es einem ein Stück Fleisch, aus dem Körper. Das ist ähm - das ist äh, unvorstellbar. Das ist wie wenn man äh - ein eigenes ä- ein eigenes Stück Fleisch zum Körper heraus,reisst oder. Und nicht mehr kann äh; Also mit dem habe ich gar nicht umgehen können. Das ist, das hat mich einfach völlig, heruntergerissen. Ich meine ich; es hat mich schon heruntergerissen dass sie Konka- Kokain konsumiert! Was klar ist. Aber. Dass sie dann noch spritzt oder. (Z50-54)

Drei Mütter gaben im Interview an, dass sie im ersten Moment nicht erschrocken gewesen seien, sondern nur nicht hätten glauben wollen, dass ihre Söhne drogenabhängig seien. Angelika war zwar im ersten Moment erschrocken, hatte dann aber gemeint, dass es nicht so schlimm sei und es sich schon wieder einrenken würde. Auch Doris hatte die Tatsache von sich weggeschoben, weil sie nicht glauben wollte, dass ihr Sohn Kokain konsumierte. Dorothea, die noch ein Kind war, als sie von der Sucht ihres Bruders erfuhr, hatte dagegen gar nicht einordnen können, was das überhaupt bedeutete:

Jäh. Jäh. (sehr leidend) Es ist wirklich einfach das -- vielleicht nicht nicht wissen was jetzt los ist. Nicht nicht wissen was das jetzt bedeutet. Also, macht er das jetzt extra? Oder ist das; ist er jetzt krank? Oder um was geht es? (Z14-16)

Angelika und Anke hatten sich zudem schwere Vorwürfe gemacht, als sie vom Substanzkonsum ihrer Söhne erfuhren. Anke hatte Schuldgefühle, weil sie nicht schon früher 
gemerkt hatte, was mit ihrem Sohn los war und Angelika machte sich grosse Vorwürfe, weil bereits der Vater ihres Sohnes drogenabhängig gewesen war und sie den Kontakt zwar nicht gefördert, aber dennoch nicht unterbunden hatte:

Ich habe mir angefangen schwere Vorwürfe machen. Und habe mich absolut schuldig gefühlt weil ich ihm; sein Vater ist ja schon drogenabhängig gewesen. Und ich bin ja, von diesem Vater weg. Also das heisst, eigentlich, schon bevor *Sohn zur Welt gekommen ist. Und, habe auch, den Kontakt, von diesen beiden nicht gefördert. (Z98-100)

In etwas andere Richtung gingen die Schuldgefühle von Annabelle. Sie hatte den Eindruck etwas falsch gemacht $\mathrm{zu}$ haben, als ihr Mann nach mehreren Jahren Beziehung und Methadonprogramm wieder rückfällig wurde und wieder anfing Heroin von der Gasse zu konsumieren.

Neben Schuldgefühlen kämpften fünf Angehörige auch mit Ängsten, die bei ihnen hochkamen. Anke sagte beispielsweise, dass sie sich Sorgen gemacht hätte, dass der Kokainkonsum irgendwelche Langzeitschäden verursachen könnte oder dass ihr Sohn schwer abhängig werden könnte. Sie hatte aber auch Angst vor der Substanz selbst. Die Ängste der anderen Eltern gingen in eine ähnliche Richtung. Darina dagegen hatte vor allem Angst, dass ihr Bruder wieder einen Rückzieher machen oder sogar abhauen könnte, weil er nicht bereit war, etwas zu verändern. Dagmars Ängste hingegen bezogen sich weniger auf die Auswirkungen eines Drogenkonsums auf ihren Partner sondern mehr auf sie selbst. Sie befürchtete, dass sich ihr Freund beim Fixen eine sexuell übertragbare Krankheit holen könnte, die dann letztlich auch auf sie übertragen werden könnte:

Es ist jetzt nicht gewesen wo ich Ahh (imitiert einen verschreckten Laut) [...] Nein! Die ersten Bedenken sind einfach gewesen äh, was ich natürlich gehabt habe ist ähm schon mit den Spritzen. (Z93-95)

Drei Frauen gaben an, dass sie sofort versucht hätten sich zu informieren. Nachdem Dorothea erfahren hatte, dass ihr Bruder Heroin konsumierte, hatte sie begonnen sich intensiv damit auseinander zu setzen und Informationen zu sammeln. Ähnlich hatten auch Anke und Darina reagiert. 


\subsubsection{Belastungen}

\subsubsection{Einleitung}

Die von Kirby et al. (2005) vorgeschlagenen Problembereiche „Beziehungsprobleme“, „emotionale Belastungen“, „Gesundheitssorgen“, „Gewalt und Drohungen“, „Rechtliche Probleme“, „Beeinträchtigung des Lebensalltages“ und „Finanzielle Belastung“ wurden in der vorliegenden Arbeit als Grundlage herangezogen, um die Belastungen der Angehörigen deduktiv zu kategorisieren. Einige Kategorien wurden anhand des Materials ergänzt und differenziert. Die Auswertung der Interviews machte deutlich, dass auch „Stigmatisierungen“ für die Angehörigen eine Belastung darstellten. Dazu gehörten neben real erlebten Vorurteilen gegenüber Suchtkranken und ihren Angehörigen auch antizipierte negative Vorstellungen über Suchterkrankungen. In den nachfolgenden Kapiteln wird auf die Belastungen der Angehörigen im Detail eingegangen.

\subsubsection{Emotionale Belastungen}

Es ist klar, dass die Drogenabhängigkeit für Angehörige eine grosse emotionale Belastung darstellt. Folglich ist es auch nicht erstaunlich, dass alle befragten Angehörigen angaben, dass die Suchterkrankung für sie eine grosse psychische Belastung war.

Kirby et al. (2005) ordneten den emotionalen Belastungen folgende Problembereiche zu: Schuldgefühle gegenüber dem Suchtkranken, Enttäuschungen, weil der drogenabhängige Angehörige Versprechungen nicht einhält oder Schlafstörungen wegen Sorgen. Aufgrund inhaltlicher Überlegungen wurden die Schlafstörungen den Gesundheitssorgen zugeteilt. In Kapitel 4.3.4.3. wurde bereits darauf eingegangen. Die Auswertung des Interviewmaterials macht deutlich, dass die emotionalen Belastungen sehr vielschichtig sind.

Drei Angehörige (Darina, Dana, August) berichteten, dass es für sie sehr belastend sei, dass andere Angehörige, die selbst keine Drogen konsumierten, unter der Suchterkrankung leiden würden. Sie äusserten den Wunsch, ihre Angehörigen ein Stück weit zu entlasten und ihnen ihr Leid abnehmen zu können. Darina erzählte in diesem Zusammenhang von ihren Eltern, die mit dem heroinabhängigen Bruder zusammenlebten:

Ähm mehr wo es nachher die ganze Familie betroffen hat. Speziell meine Eltern. Wo ich für sie; eben ich bin ein bisschen weit weg gewesen. Ich habe gemerkt. Dass es sie enorm belastet. Und ich habe nicht genau gewusst. Wie ich ihnen helfen kann. Ähm das ist, also das ist das schwierigste gewesen. (Z227-240) 
Eine grosse Sorge für Angehörige war zudem, dass weitere Drittpersonen durch die Suchterkrankung (3) betroffen werden könnten. So hatten Darina und Dylan beispielsweise beide Angst, dass ihre Geschwister auch drogenabhängig werden könnten. Darina erzählte, dass ihr jüngster Bruder sehr viel Zeit mit dem heroinabhängigen Bruder verbrachte, was sie und ihre Eltern beunruhigte:

Ja.+ Genau. Weil sie möchten auch dass er nicht zu nahe ist zu meinem kleineren Bruder. Ähm weil weil die sind so nahe zusammen. Also zum Beispiel mein kleiner Bruder. Der schläft jetzt die ganze Zeit bei bei meinem; über die Sommerferien. Bei meinem drogenabhängigen Bruder im Zimmer. Und dann schauen sie vielleicht einen Film. (lacht) Und ähm schlafen im gleichen Zimmer. Und meine Eltern möchten, ein bisschen aufpassen dass sie nicht zu nahe sind. Der Rhythmus von meinem drogenabhängigen Bruder ist nicht unbedingt gut jetzt für meinen kleinsten Bruder. Wo wo eine Lehrstelle suchen sollte und so Sachen. [...] Ja. ja ja. Und ich glaube sie müssen schon ein bisschen aufpassen. Weil mein kleinster Bruder der hat auch, mal einen Verweis gehabt wegen wegen; es ist jetzt nur das Kiffen. Aber ähm, eben wenn man so einen Fall hat wie einen, einen heroinabhängigen Bruder ist es, sieht es, schrillen schon alle Alarmglocken. (Z450-462)

Dorette dagegen fürchtete eher, dass andere Leute über den Kontakt mit ihrem Sohn in Versuchung kommen könnten, harte Drogen zu probieren. Aus diesem Grund lud sie keine jungen Menschen zu sich nach Hause ein.

Zwei Mütter erzählten, dass es sie sehr belastete, wenn sie sahen, dass ihre Söhne aufgrund der häufig jahrelangen Erkrankung immer einsamer wurden. Angelika und Doris gaben beide an, dass es sie traurig gemacht hätte zu sehen, wie ihre Söhne immer mehr Freunde verloren hätten. Insbesondere Angelika beschäftigten die Vereinsamung aber auch die Enttäuschungen sehr, die ihr Sohn erleben musste. Sie thematisierte diese auch wiederholt im Interview:

Aber eben. Ich habe ihnen am Anfang gesagt dass ich ihm wieder zwanzig Stutz gegeben habe. Also ich nehme es, ich nehme es als elendiglich wahr. Es tut mich sehr beelenden. $\mathrm{Zu}$ sehen, wie einsam! er! ist. Das, was, was ihm, am Leben abgeht. Das tut mir so weh:. Er ist so, eigentlich ein hübscher Mann. Und, er häer hätte sicher Chancen bei den Frauen. (Z450-454)

Die Auswertungen zeigten, dass sich viele Angehörige in verschiedenen Bereichen in einer ständigen Ambivalenz befanden, die eine grosse Belastung darstellte (8). Einige erzählten von einem ständigen Wechselbad der Gefühle von Hoffnung und Hoffnungslosigkeit (2). Andere dagegen gaben an, dass sie in gewissen Situationen entgegen ihren eigenen Grundsätzen gehandelt hätten (2). August sagte im Interview, dass er einem seiner Söhne einen letzten Schuss bezahlt hätte, damit dieser mit in eine stationäre Therapie gekommen 
sei. Auch Angelika machte nach mehr als 20 Jahren Betroffenheit immer noch die Erfahrung, dass es ihr nicht immer gelang, die Regeln, die sie aufgestellt hatte, einzuhalten. So gab sie ihrem Sohn wieder CHF 20.00, obwohl sie sich vorgenommen hatte, ihm kein Geld mehr zu geben, was sie anschliessend sehr bereute:

I: Haben sie es nachher, bereut dass sie ihm +die zwanzig Franken gegeben haben? A: Ja. Ja.+ Ich habe es bereut, ja. (beide lachen). Und zwar nicht wegen den zwanzig Stutz. - I: Ja ja! A: Sondern, dass dass ich nicht konsequent gewesen bin. (Z491-494)

Die Ambivalenz wurde nicht immer so direkt angesprochen, sondern zeigte sich oft in den Themen der Angehörigen. So hatten Angehörige beispielsweise oft Angst, dass ihr suchtkranker Angehöriger plötzlich aufgrund einer Überdosis oder den Verhältnissen, in denen er lebte, versterben könnte. Acht Angehörige sprachen diese Verlustangst in den Interviews an, während August auch über den realen Verlust eines Sohnes berichtete:

Äh aber im ersten Moment ist man mit einem Todesfall alleine. +Das ist klar. Aber es hat schon sehr sehr viel gebracht. Und sie haben sich alle Mühe gegeben. Aber es bleibt! halt. Was soll ich jetzt sagen. Man ist - dort immer wund. Das ist so eine Stelle wo: nie ganz, verheilt. (Z392-396)

Während Angehörige häufig Angst haben, dass ihr suchtkranker Angehöriger versterben könnte, kann es in sehr belastenden Situationen aber auch vorkommen, dass Angehörige sich wünschen, dass ihr suchtkranker Angehöriger sterben möge. Dass Angehörige sich einerseits wünschten, dass ihr Angehöriger stirbt und andererseits aber auch davor Angst hatten, einen geliebten Menschen zu verlieren, zeigt ebenfalls die innere Zerrissenheit, in der sie oft lebten. Es ist deshalb nicht überraschend, dass vier Frauen (Annabelle, Andrea, Doris, Dorothea), die Verlustängste geäussert hatten, auch darüber sprachen, dass sie sich schon gewünscht hätten, dass der Betroffene sterbe, damit das Leiden für sie ein Ende hat. Die nachfolgende Textstelle stammt aus dem Interview mit Dorothea:

Und auf der anderen Seite irgendwie manchmal auch schon fast ein bisschen. Lasst es doch endlich fertig sein! Und - also dieser dieser Zwiespalt macht einen fertig. Und man nachher plötzlich irgendwo durch realisiert dass man dass man manchmal seinem seinem Bruder schon fast den Tod an an den Hals wünscht. Dass -- ja das, das das macht einen fertig. (Z491-494)

Die Ambivalenz zeigte sich noch in weiteren Bereichen. Viele Angehörige stellten früher oder später fest, dass es wichtig war, sich ein Stück weit von ihren suchtkranken Angehörigen abzugrenzen. Die Regulierung von Nähe und Distanz zum Drogenabhängigen war in sechs Interviews Thema (Darina, Dorothea, Dana, August, Albert, Andrea) und war mehr oder weniger belastend für die Angehörigen. Angehörige erlebten es nicht nur als 
belastend, wenn sie sich nicht abgrenzen konnten, sondern auch, wenn sie es taten. Albert berichtete davon, wie er und sein Frau der kokainabhängigen Tochter gesagt hätten, dass sie ausziehen müsse:

Eben. Aber ich denke - eben wenn die Tochter achtundzwanzig ist. Und, ein eigenes Kind hat. Und immer noch, weiter macht. Da kannst du gar nicht anders. Und wenn es dich noch so verreisst. Innerlich +oder. (Z615-617)

Während sich Angehörige häufig etwas mehr Distanz zur Drogen konsumierenden Person wünschten, fühlten sie sich gleichzeitig stark belastet, wenn sie ihre suchtkranken Angehörigen nicht erreichen konnten oder diese für eine unbestimmte Zeit verschwanden. Häufig machten sie sich dann Sorgen, dass etwas Schwerwiegendes passiert sein könnte. Sechs befragte Angehörige gaben an, dass sie eine solche Situation schon einmal erlebt hätten (Andrea, Angelika, Albert, August, Doris, Dorothea). Dorothea erzählt, dass es für sie sehr schlimm gewesen sei, wenn der Bruder beispielsweise nach einem Streit einfach eine Woche weg gewesen sei:

Und nachher hockt man einfach. Und und wartet. Oder? -- Einmal einmal ist er wirklich ja eine Woche lang einfach weg gewesen. (Z212)

In ähnlicher Weise verhielt es sich mit dem Gefühl, für den suchtkranken Angehörigen verantwortlich zu sein, das in einem Widerspruch zum Wunsch, sich abzugrenzen steht. Annabelle erzählte beispielsweise davon, dass die Familie ihres Mannes, froh gewesen sei, als sie die Beziehung zu ihrem Partner eingegangen sei und ihr damit ein Stück weit auch die Verantwortung übergeben wurde:

Und ich denke mir nachdem er ähm aus dieser *Institution1 ausgetreten ist. Bin ich eigentlich so ein bisschen in seiner Verwandtschaft so ein bisschen, als die Retterin! angeschaut worden. Ah super! Da kommt*Annabelle. Und jetzt ist alles paletti“. (Z255-257)

Aber auch andere Angehörige gaben an, dass sie sich aus verschiedenen Gründen verantwortlich fühlen würden: Einerseits hatten sie wie Annabelle den Eindruck, dass sie schauen müssten, dass ihr drogenabhängiges Familienmitglied wieder gesund würde (4). Andererseits hatten sie aber auch das Gefühl, dass sie, sobald ihr Angehöriger in Schwierigkeiten geraten war, bereit sein müssten, um zu helfen. Dies führte dazu, dass sie sich in einem dauernden Zustand der Alarmbereitschaft befanden. Ständig kam es zu irgendwelchen Notfällen. Insbesondere Eltern berichteten, dass jedes Telefonklingeln zur Belastung geworden sei, weil das meistens ein Hinweis dafür gewesen sei, dass wieder 
irgendeine Notsituation eingetreten war (Andrea, Albert, August, Doris, Dana, Dorette). Albert berichtet in diesem Zusammenhang, dass er sein Telefon verschenkt hätte:

Zum Beispiel ein Telefonton. - [...] Einfach wenn das Telefon geläutet hat. Dieser Ton. Der der hat mich äh so fertig gemacht. I- ich habe ich habe mein Telefon: verschenkt. Und habe noch ein anderes Telefon gekauft. Mit einem, anderen Ton. Ähm, weil, es ist wieder irgendein Spital am Telefon. Oder äh, ich bin dort, kommst du mich holen. Oder äh, äh ihre Tochter ist äh bewusstlos auf der Strasse gefunden worden. Und ist jetzt im *Instiution05. Oder, äh wir haben sie in die Zelle einsperren müssen. Weil sie erwischt worden ist beim Spritzen irgendwo in einem, WC oder was. Und+, einfach das, das Ganze drum herum oder. Das sind soviel kleine, Sachen wo: wo einen psychisch belasten. (Z541551)

Dass Angehörige sich dermassen verantwortlich fühlten, hatte zum Teil auch damit zu tun, dass sie ihren suchtkranken Angehörigen gegenüber oft Schuldgefühle hatten. Ursache für Schuldgefühle waren beispielsweise negative Gefühle gegenüber dem Angehörigen (1), aber auch das Gefühl, für Abstürze oder den Konsum verantwortlich zu sein (3) oder nicht alles unternommen zu haben, um die Erkrankung zu verhindern (2). Dagmar äusserte zudem auch Schuldgefühle ihrer Tochter gegenüber, weil sie einen drogenabhängigen Partner hatte. Insbesondere Eltern und Partnerinnen kämpften mit Schuldgefühlen. August sprach im Interview von den Schuldgefühlen, die er und seine Frau empfanden:

In einem Kreis. Da ist immer „,sochli“. Äh diese Schuldgefühle. Erdrücken einen eben auch ,ächli“. Was haben wir alles falsch! gemacht? Wir haben etwas falsch gemacht. Was ist es denn genau? Und so weiter. Ist es denn nur bei uns? Und so (Z1165-1169)

Die Textstelle von August verdeutlicht neben Schuldgefühlen einen weiteren wichtigen Aspekt, nämlich die Frage nach der Ursache. Insbesondere die Eltern belastete die Frage, weshalb ihre Kinder ein Drogenproblem hatten. Bis auf Doris machten sich alle Eltern im Interview Gedanken darüber, was denn die Suchterkrankung verursacht haben könnte. Sie sahen mögliche Ursachen in der Scheidung (1), einer zu strengen Erziehung, gegen die die Kinder opponierten (1), einer $\mathrm{zu}$ beschützenden Erziehung (1) oder verunsichernde Lebenserfahrungen, wie der frühe Tod des Vaters (1). Daneben gab es einige, die die Veranlagung (2) oder den Kollegenkreis und den aktuellen Lebensstil unserer Gesellschaft (2) für den Drogenkonsum verantwortlich machten.

Angehörige und vor allem Eltern machen sich häufig Sorgen, was die Zukunft ihrer drogenabhängigen Familienmitglieder anbelangt. Sie machen sich darüber Gedanken, welche längerfristigen Auswirkungen die Suchterkrankung auf ihre Kinder haben könnte oder was 
einmal sein wird, wenn sie selbst nicht mehr leben. Drei Mütter (Anke, Andrea, Dorette) und eine Schwester (Darina) äusserten Zukunftsängste. Anke berichtete über die Sorge vor möglichen Spätfolgen:

Ich hoffe nicht dass das spätere Folgen hat wenn er alt ist. Oder weil man sagt. Er ist natürlich nicht verheiratet. Er hat zwar eine Freundin. Wo: geschieden ist und Kinder hat. Aber, ähm sie leben nicht zusammen. Man weiss auch dass Männer wo älter sind und alleinstehend, mehr psychische Probleme haben als andere. Und das kann natürlich Spätfolgen haben. Ich hoffe nicht dafür dass wir das dann durch,machen. Aber bis dann bin ich nicht mehr da. (Z231-135)

Im Laufe der Suchterkrankung verändert sich der betroffene Angehörige häufig stark. Er beginnt die Angehörigen zu belügen, hält Vereinbarungen nicht mehr ein und verstösst gegen ursprünglich geteilte Normen und Werte. Diese Veränderung der Persönlichkeit ist für Angehörige meist sehr schwierig. Acht von 13 befragten Angehörigen gaben an, dass es sie belaste, dass ihr Angehöriger sich durch die Erkrankung verändert habe. Dana erzählte, dass sie am meisten belaste, dass der Sohn sie belüge und sie ihm nicht mehr vertrauen könne:

Das Lügen. (spricht sehr leise) [...] Vertrauensmiss- also dass man nicht mehr vertrauen kann. Weil durch die Drogen das ist; eben man hat keine Achtung mehr. Nicht einmal vor der Mutter. Wenn es um das eigene! Ding, - um die Suchtbeschaffung oder äh ja (Z314-321)

Angehörige möchten ihren suchtkranken Angehörigen in den meisten Fällen helfen. Oft machen sie dabei die Erfahrung, dass sie nicht helfen können, wenn ihr Angehöriger nichts verändern möchte und die Hilfe gar nicht wünscht. Diese Erfahrung löst Gefühle von Ohnmacht aus. Sie können ihren Angehörigen nur hilflos zuschauen, wie es ihnen schlechter geht oder wie es immer wieder zu Rückfällen kommt. Es ist deshalb nicht erstaunlich, dass sieben Angehörige Gefühle von Hilflosigkeit und Ohnmacht als sehr belastend angaben wie das Beispiel von Doris zeigt:

Es ist, man ist ohnmächtig! Man ist richtig, man weiss nicht was machen. (Z74)

Neben dem Gefühl der Hilflosigkeit und Ohnmacht stellt sich bei vielen Angehörigen auch immer wieder ein Gefühl der Resignation und Hoffnungslosigkeit ein. Sie erleben im Zusammenhang mit der Drogenabhängigkeit ihrer suchtkranken Angehörigen immer wieder Momente, in denen sie nicht wissen, wie es weitergehen soll, weil die Situation ausweglos und unveränderbar erscheint. Je länger die Suchterkrankung andauert, desto stärker wird das Gefühl der Resignation, weil Angehörige erkennen, dass ihre Angehörigen ein Leben lang krank bleiben werden. Die Angehörigen, die Gefühle von Resignation ansprachen, waren alles Eltern, die bereits länger als fünf Jahre betroffen waren (Andrea, Angelika, Albert, 
August, Dorette). Dorette, die selbst seit über 20 Jahren betroffen war, sprach über die Trostlosigkeit, die sie erlebte:

Oder äh und u- u- eben besonders! belastend ist einfach das Trostlose. Also ich finde dass, dass es einfach, ja dass es einfach trostlos ist. Es wird immer schlimmer! (Z202)

Im Laufe der Suchterkrankung machen Angehörige häufig die Erfahrung, dass nach Phasen der Besserung wieder Rückfälle folgen, in denen der Betroffene wieder zu konsumieren beginnt. Neben den Rückfällen müssen sie aber oft auch mit der Tatsache fertig werden, dass die ihnen nahestehende Person beginnt noch weitere psychoaktive Substanzen zu missbrauchen, die Erkrankung sich allgemein verschlimmert oder ihre Angehörigen Therapien abbrechen. Es ist deshalb nicht erstaunlich, dass sich neun Angehörige belastet fühlten durch Rückfälle und Therapieabbrüche, die $\mathrm{zu}$ einer immer weiteren Verschlechterung der Erkrankung führten. Andrea war eine der Mütter, die hatte mit ansehen müssen, wie ihr Sohn immer wieder Abstürze hatte:

Das hat er dann aber normalerweise ,amel“ (=jeweils) mehr genommen oder weniger genommen aber dann hat er dann im *414 hat er dann auch wieder geschafft. Da ist er ja wieder schaffen gegangen. Dann wäre ja wieder das Thema gekommen in eine WG zu gehen. [...] Ein betreutes Wohnen. Das ist dann eigentlich gewesen aber dann hat er wieder; (seufzt) Da habe ich dann auch wieder gemerkt. Er geht wieder nicht schaffen. Und dann habe ich auch längere Zeit nichts gehört. (Z175-179)

\subsubsection{Beziehungsprobleme}

In Familien, in denen jemand von einer Suchterkrankung betroffen ist, kommt es auch vermehrt zu Konflikten oder allgemein zu Beziehungsproblemen (Kirby et al., 2005, S. 45). Es ist deshalb nicht erstaunlich, dass alle bis auf Anke über Beziehungsprobleme berichteten. Die meisten erzählten von Auseinandersetzungen mit dem Betroffenen selbst (9). Dorothea, die jahrelang Verständnis für ihren Bruder aufbrachte, schilderte, dass ihr Bruder sich vor allem dann noch bei der Familie meldete, wenn er etwas brauchte, was sie wütend machte:

Und, es regt mich halt auch auf. Dass er nicht ge- wieso dass er nicht irgendwie ein bisschen die Augen auftun kann wenn es um um um um seine Mitmenschen geht (lacht leicht). Also vor allem ja. Eben die Familie. Also ich glaube. Der sieht uns - irgendwie nicht mehr. Und wenn dann ist es einfach immer gerade das Zapfbild das er von uns hat. Oder einfach Abbild also quasi familiärer Geldautomat. (Z529-531)

Dorothea hatte aufgrund ihres momentanen Empfindens vorübergehende den Kontakt zu ihrem Bruder abgebrochen. Sie war nicht die einzige, die angab, dass es aufgrund der 
gehäuften Konflikte zu vorübergehenden Kontaktabbrüchen gekommen war (4). Dagmar trennte sich wiederholt von ihrem Partner wie die nachfolgende Textstelle belegt:

Ah!+ Wir haben uns mehrmals getrennt! (lacht dabei) Mal mehr und mal weniger. Also jetzt haben wir uns gehabt ähm, Ich habe mich von ihm getrennt gehabt im (schnaubt)*Jahreszahl. (Z345)

Angehörige berichteten aber auch, dass es zu Kontaktabbrüchen gekommen war, weil der drogenabhängige Angehörige eine Beziehung zu einer Person eingegangen war, die ebenfalls eine Suchtproblematik hatte. Sowohl Dylan als auch Doris machten die Erfahrung, dass die Konflikte durch den neuen Partner ihres suchtkranken Angehörigen noch zunahmen. Nachdem sich die Eltern von Dylan getrennt hatten, ging seine Mutter eine neue Beziehung zu einem ebenfalls drogenabhängigen Mann ein, mit dem Dylan nicht auskam. Als es zu einem Streit kam, bei dem sich Dylan wehrte, musste er innerhalb eines Tages aus der Wohnung, die dem Freund der Mutter gehörte, ausziehen, wie das folgende Textbeispiel zeigt:

Am nächsten Tag. Wo ich dann am Mittag heim komme nach dem Schaffen heisst es. Ja. Heute Abend musst du dann - verreisen. Oder? Du musst dann selber schauen was du machst. Ich so. Ja. Ich bin in der Lehre und so. Mann! Du hilfst mir nicht einmal. Zu meiner Mutter. Oder? Er schlägt mich. Und du hilfst mir nicht. Gibst ihm noch Recht. Ich sei ja selber Schuld. - Dann habe ich auf jeden Fall; Dann habe ich gedacht. Ja. Ich gehe sicher nicht! und so. Du kannst die Polizei holen von mir aus. Die sollen dann schauen und so. Dann habe ich gewartet oben. Und Tatsache! haben sie; hat der andere der Polizei angerufen. Dass ich von Daheim raus müsse. Oder? (Z761-763)

Die Textstelle macht, neben dem Ausmass den Konflikte annehmen können, deutlich, dass suchtkranke Menschen ihre Verantwortung als Eltern nicht in dem Mass wahrnehmen können wie gesunde Eltern. Sie sind häufig mit dem Beschaffen und dem Konsum ihrer Droge beschäftigt (Kirby et al., 2005, S. 45). Dylans Mutter stand in der obigen Konfliktsituation zwischen ihrem minderjährigen Sohn und dem drogenabhängigen Partner, der sie gleichzeitig auch mit Stoff versorgte. Dylan schilderte in diesem Zusammenhang an anderer Stelle treffend, dass er und sein Bruder immer erst nach der Sucht gekommen seien:

Ja dass ich eigentlich äh die Drogen wichtiger gewesen sind als ich. [...] Das ist eigentlich; Ja.+ Aber sie können ja nichts dafür in dem Sinn. Weil sie ja krank sind. Und das ist ja das ist ja, das ist ja der Scheiss von der Sucht. Oder? Das ist einfach, du bist süchtig. Und und, zuerst kommt ja die Sucht. Und wenn du das nicht besiegen kannst dann; das heisst wenn er seine Sucht hat befriedigen konnte. Dann bist auch noch du gekommen. Also das ist; ich denke das ist sicher mal eines gewesen. (Z473-481) 
Neben der Tatsache, dass seine drogenabhängigen Eltern stark mit ihrer Sucht beschäftigt waren, kam erschwerend hinzu, dass sie häufig aufgrund der psychoaktiven Wirkung der Droge nicht richtig ansprechbar waren für Dylan:

Ja nur ich meine. Es ist auch etwas anderes. Trinkst du mal ein Bier. Und bist halt ein bisschen angetrunken. Oder bist du auf Heroin. Wenn einer auf Heroin ist dann bei der ersten halben Stunde nachdem er konsumiert. Dann kannst du ja; dann bist du ja weg vom Fenster. Oder? (Z531-535)

Dylan war aber nicht der einzige, der die Erfahrung machte, dass seine suchtkranken Eltern nicht verlässlich oder nicht einmal ansprechbar waren. Auch die beiden Partnerinnen Annabelle und Dagmar erzählten, dass sie sich häufig nicht auf ihre Männer verlassen könnten, wenn sie sie bräuchten. Annabelle meinte, dass sich ihr Mann vollkommen aus dem Familienleben ausgeklinkt hätte. Dagmar hatte Ähnliches erlebt. Nach einer schweren Erkrankung konnte ihr drogenabhängiger Freund ihr keine Unterstützung geben, weil er mit der Situation überfordert war. Dies obwohl er ihr im Vorfeld versprochen hatte, für sie da zu sein.

Es kommt allerdings auch vor, dass es zwischen Angehörigen zu Auseinandersetzungen in Bezug auf das drogenabhängige Familienmitglied kommt (4). Angehörige sind sich beispielsweise darin nicht einig, wie man sich dem Suchtkranken gegenüber verhalten soll (Kirby et al., 2005, S. 45). Gerade Eltern berichteten besonders oft, dass die Drogenabhängigkeit ihres Kindes die Paarbeziehung stark belastetet hatte. Neben Angelika, von der das nachfolgende Textbeispiel stammt, erzählten auch Doris und August von vermehrten Konflikten in der Beziehung zum Ehepartner:

Manchmal schlafe ich wieder schlecht und dann rufe ich an. Und sage. Wie geht es dir? - Mein Mann sagt dann ,amigs“ (=jeweils). Ou! (imitiert die genervte Stimme des Mannes) Lass ihn doch mal, selber! anrufen. (Z470)

Nichtsdestotrotz kannten auch andere Angehörige das Problem, dass die Abhängigkeitserkrankung die Partnerschaft belastete. So erzählte auch Dylan, dass seine Freundin manchmal kein Verständnis für die Geduld hatte, die er für seine Eltern aufbrachte.

\subsubsection{Gesundheitssorgen}

Im Bereich der Gesundheitssorgen fassten Kirby et al. (2005, S. 45) vorwiegend gesundheitliche Probleme der substanzkonsumierenden Person zusammen. Die Aspekte wurden in Kap. 2.2.2.5 beschrieben. Der Einbezug eines weiteren Blickwinkels schien aber 
im Vorfeld der Auswertungen bereits relevant: Die Suchterkrankung eines nahestehenden Menschen hat häufig auch Auswirkungen auf die Gesundheit der Angehörigen. Die Belastungen führen dazu, dass die Angehörigen selbst psychisch oder psychosomatisch erkranken ((Lee et al., 2011; Oreo \& Ozgul, 2007; Orford et al., 1998c; Ray et al., 2007). Während bereits die Auswertung des BDI-V gezeigt hat, dass Angehörige ein hohes Risiko tragen eine klinische Depression zu entwickeln (siehe Kap. 5.1.4.2.), bestätigte sich auch in den Interviews, dass Angehörige einen gewissen Zusammenhang zwischen ihrer Gesundheit und der Suchterkrankung ihres Angehörigen annahmen. Nur Annabelle gab an, dass die Suchterkrankung ihres Mannes keine gesundheitlichen Auswirkungen gehabt hätte. Sie erwähnte allerdings in einem anderen Zusammenhang, dass sie täglich kiffe, weil sie sonst die Situation mit ihrem Mann nicht aushalten würde. In eine ähnliche Richtung gingen auch die Angaben von Dorette, die sich selbst als esssüchtig bezeichnete, weil sie in Belastungssituationen zu Essen griff. Besonders häufig berichteten Angehörige, dass sie unter Schlafstörungen gelitten hätten oder leiden würden (7). Das Textbeispiel von Dana macht deutlich, dass sie diese Schlafstörungen im Zusammenhang mit der Drogenabhängigkeit ihres Sohnes sah:

Also das hat ja auch angefangen. Ich konnte nicht mehr schlafen. Oder ich bin wach geblieben um ihn, um zu schauen ob er eben wieder abhaut. Äh oder wann er wieder heim kommt. Ich: bin dann auch äh, man schläft nicht mehr gut weil man denkt äh. Passiert etwas. Äh oder wird er von der Polizei äh gefilzt? Oder äh stellt er etwas an? Macht er Einbrüche? (Z171-175)

Je nachdem können sich die Ängste und Sorgen der Angehörigen auch in Alpträumen äussern. Darina berichtete, dass sie zu Beginn, als sie ganz frisch vom Heroinkonsum ihres Bruders gewusst hatte, oft Alpträume gehabt hätte. Diese Träume veränderten sich im Laufe der Zeit und begannen sich auf den jüngsten Bruder zu beziehen:

Also jetzt geht es besser. Ich habe dann plötzlich noch wie wie so Alpträume gehabt dass; also ich habe ja drei Brüder. Dass der jüngste wo sehr nahe ist bei meinem mittleren Bruder wo drogenabhängig ist. Dass ähm, dass ihm etwas Ähnliches widerfährt. +Oder so. Und ja. Ganz komisch in der Nacht wenn man manchmal so Träume hat und dann aufwacht. Dann hat man das Gefühl. Es ist real. Also dann muss man muss man irgendwie ja. Halt ganz aufwachen. Und dann äh +merkt man. Dass die Situation+ eigentlich nicht so ist. (Z406-412)

Neben Schlafstörungen berichteten Angehörige von weiteren Symptomen, die im Zusammenhang mit einer depressiven Episode auftreten können (5). Angelika erzählte beispielsweise, dass sie in einer besonders belastenden Phase der Suchterkrankung ihres Sohnes unter Appetitlosigkeit gelitten und dadurch auch Gewicht verloren hätte. Weiter 
wurden Suizidgedanken und -versuche berichtet (2). Albert, August und Dorothea gaben alle an, dass die Belastungen eine depressive Episode ausgelöst hätte, was eine psychiatrische Behandlung notwendig gemacht hätte.

Die hohe chronische Belastung, in der sich Angehörige meist über lange Zeit befanden, führte nicht nur dazu, dass Angehörige häufig unter psychischen Beschwerden litten, sondern im Laufe der Zeit auch psychosomatische Symptome entwickelten (5). Von Nacken- und Rückenschmerzen bis hin zu Zähneknirschen im Schlaf, drückendem Schmerz in der Brust oder stressbedingtem Bluthochdruck wurden verschiedene körperliche Symptome genannt, wobei die Beschwerden meist so schwerwiegend waren, dass sie eine Behandlung notwendig machten, wie dass Beispiel von Doris zeigt:

Nein. Ich d-, nicht direkt. Ich habe, einen schweren Rückenpr-, Rücken,probleme gehabt. Und wenn es mit nicht gut gegangen ist. Mit dem Rücken. Dass ich eine Spritze habe machen müssen. Dann hat Frau Doktor auch immer gerade gewusst dass etwas nicht richtig läuft. (Z536-538)

Dagmar gab als Partnerin von einem heroinabhängigen Mann an, dass sie auch Angst vor sexuell übertragbaren Krankheiten hätte (Kirby et al., 2005, S. 45). Ihr Partner hatte früher einmal eine gebrauchte Spritze verwendet und sich in diesem Zusammenhang mit Hepatitis C angesteckt. Dies machte ihr immer wieder Sorgen, wie aus der folgenden Aussage hervorgeht:

Also von dem her, also es ist so, die einzige Sorge die ich immer wieder hatte war, hoffentlich ähm nimmt er eine Spritze. Und da er ja noch Benzo dazu genommen hat immer. Und sich die Hälfte nicht erinnern konnte. Und ich so. Ja bist du sicher? Du kannst dich ja an die Hälfte nicht erinnern. Weisst du das sicher dass du? Er hat immer gesagt. Nein das würde er nie mehr machen. Nie eine gebrauchte Spritze benutzen. Und ja irgendwo musst du es dann halt glauben. Weil das bringt ja sonst nichts. Das einzige wenn er ,amigs“ (=jeweils) abstürzen gegangen ist ist meine grösste Sorge gewesen. [...] Ja also dass es dann halt auf mich zurückkommt. (Z123-129)

Albert nannte ähnliche Sorgen. Er fürchtete, dass seine Tochter auf dem Drogenstrich anschaffen gehen könnte, um sich ihre Sucht $\mathrm{zu}$ finanzieren und dass dies weitere Auswirkungen auf ihre Gesundheit haben könnte. Neben Albert gaben zwei weitere Eltern Sorgen wegen weiteren gesundheitlichen Problemen an, die ihre Kinder hatten und die aufgrund der Suchterkrankung entstanden waren (August, Dorette). 


\subsubsection{Gewalt und Drohungen}

In Familien, in denen jemand von einem Suchtproblem betroffen ist, sind Gewalt und Drohungen häufig ein Thema (Mattson et al., 2012; Moore \& Stuart, 2004; Murphy et al., 2001). In der vorliegenden Stichprobe berichteten zehn Angehörige, dass sie mit Gewalt oder Drohungen in irgendeiner Form bereits konfrontiert worden waren. Dabei waren Angehörige oft sehr gut im Stande, einen Zusammenhang zwischen verschiedenen Substanzen und der Gewalt oder den Drohungen herzustellen. In den meisten Fällen, in denen es zu Aggressionen zwischen Angehörigen und dem betroffenen Suchtkranken gekommen war, hatte der suchtkranke Angehörige Alkohol oder Kokain konsumiert. Drei Angehörige zeigten diesen Zusammenhang zwischen Substanz und aggressivem Verhalten klar auf. Das folgende Textbeispiel stammt aus dem Interview mit Dorette:

Ja das ist; und er; ich muss halt natürlich sagen. Wir, ich habe natürlich immer gesagt. Also lieber Drogen als als Alkohol. Weil Alkohol machen ihn aggre- also macht ihn aggressiv. Ist er enorm aggressiv. Und, jaa. Schlägt, dann, auch sonst irgendwo drein. Also. Eben. (Z172-174)

Fünf Angehörige berichteten, dass es in der Beziehung zum Betroffenen bereits zu körperlicher Gewalt gekommen war. Während drei Angehörige nur einmalige Erfahrungen gemacht hatten, erlebten Dagmar und Dylan wiederholt körperliche Auseinandersetzungen in der Beziehung zu ihren Angehörigen. Dagmar hatte sich regelmässig mit ihrem Freund handgreiflich gestritten. Dylan dagegen machte Gewalterfahrungen mit verschiedenen drogenabhängigen Personen. Er wurde sowohl von den Eltern als auch vom neuen Partner der Mutter geschlagen. In der nachfolgenden Textstelle berichtete er davon, wie er als Kind vor seinem Vater Angst hatte:

Ja und einfach vom vom Gefühl her von mir. Von der Angst! ist einfach riesig immens gewesen. Oder? Wenn [...] er ausgetickt ist. Oder? Und dann habe ich schon gedacht gehabt. Oh! Jetzt kommst du weiss auch nicht wie dran. (Z205209)

Angehörige erlebten aber nicht nur körperliche Gewalt, sondern sehr oft auch Drohungen, die von ihren suchtkranken Angehörigen ausgesprochen wurden (4). Dabei war die Bandbereite der Drohungen sehr breit. Während Dagmar Mordandrohungen von ihrem Partner erhielt, erlebten andere wie beispielsweise Andrea viel subtilere Drohungen von ihrem Sohn. Er schob ihr indirekt die Verantwortung für sein Verhalten zu, wenn sie nicht machte, was er wollte:

Ja. Ja.+ Genau. Oder Drohungen auch so. Ja. Dann dann gehe ich halt! und dann mache ich halt! Oder; Ja. So in denen; Drohungen +eigentlich. (Z406) 
Neben Andrea erzählten auch Darina und Albert, dass ihre Angehörigen sehr fordernd oder mit Drohungen aufgetreten seien. August, dessen Söhne Heroin konsumieren, berichtete nicht von Gewalt gegenüber ihm oder seiner Frau, sondern vielmehr von Gewalt gegen einen seiner Söhne auf der Gasse, der beinahe erschossen worden wäre. Ähnliche Erfahrungen hatte auch Dana gemacht, deren Sohn gedealt hatte und anschliessend von einer Gang bedroht worden war.

\subsubsection{Rechtliche Probleme}

Im Zusammenhang mit dem Konsum von illegalen Drogen geraten die Betroffenen zwangsläufig in einen Konflikt mit dem Gesetz. Folglich ist es nicht überraschend, dass elf Interviewpartner davon berichteten, dass ihre Angehörigen rechtliche Probleme hatten oder dem nur durch Glück entgangen waren. Drei Mütter erzählen, dass sie irgendwann festgestellt hätten, dass ihre Söhne gedealt hätten, wobei es beim Sohn von Dorette auch zu einer Anzeige gekommen war.

Drei Eltern hatten zudem Hausdurchsuchungen durch die Polizei erlebt. August berichtete davon, dass die Polizei noch eine Hausdurchsuchung gemacht hatte, als der Sohn bereits nicht mehr bei den Eltern gewohnt hatte:

Ja. Oder eine Hausdurchsuchung habe ich auch einmal; heute im Nach- heute würde ich mich wehren. Würde ich sagen äh. Der lebt schon so lange nicht mehr da. Ich lasse es nicht zu. - Rechtsanwalt daher. So ja. Also man hat schon noch I: Also wo bei ihnen +ist die Wohnung durchsuchen gekommen? A: Ja ja.+ Weil er doch ab und zu noch bei uns; letzte Adresse. - Jä ist auch wieder so ein Staatsanwalt gewesen. Aus *Ort01. Wo da wahrscheinlich äh das Gefühl gehabt hat er müsse jetzt da, einfach Wohnungen inspizieren. (Z1025-1029)

Für Dorette, die mehr als einmal die Polizei in ihrer Wohnung hatte, war dies die schlimmste Erfahrung überhaupt im Zusammenhang mit der Drogenabhängigkeit ihres Sohnes.

Sieben Angehörige berichteten, dass sie schon mitbekommen hätten, dass ihre drogenabhängigen Familienmitglieder verhaftet worden waren und kürzere oder längere Gefängnisaufenthalte hinter sich hätten. Die Gründe, weshalb es zu einer Verhaftung gekommen war, waren unterschiedlich. Während Angelika und Albert davon berichteten, dass ihre Kinder wegen Besitz und Konsum von illegalen Drogen angezeigt oder kurzzeitig festgenommen worden waren, waren die Angehörigen von Dagmar, Dorothea und August 
wegen Raubüberfällen und Diebstahl angezeigt worden. August erzählte davon, dass ihm einer seiner Söhne eine Waffe geklaut und damit bewaffnete Raubüberfälle begangen hatte:

Und beim: Mittleren, ist dann hat es dann so eskaliert - dass er äh mir die Pistole, geklaut hat. Mal bei einem Besuch. Und der Jüngste hat mir dann das mal gesagt. So nebenbei. Der habe dann im Fall meine Waffe. Und dann bin ich ihn anzeigen gegangen. Habe ihn wieder nicht gefunden. Dort ist er eben den ganzen Tag nicht auf dem Campingplatz gewesen. Das Zelt ist zusammengeräumt gewesen. Hat man nicht gewusst. Kommt er wieder oder nicht. Und ich habe eigentlich Schlimmeres verhüten wollen. Und bin das melden gegangen. Er habe äh, er sei irgendwo unterwegs. Habe meine Waffe. Haben sie ihn dann gefunden. Die Waffe haben sie ihm abgenommen. U:nd dann ist aber „gli“ ausgekommen. Dass er äh üblicherweise eben einen äh Raubüberfall begangen hat. Bei einer nahe gelegenen Tankstelle. Und dann äh haben sie ihn halt auch verhaftet. Und dann ist auch noch dort alles, dazugekommen. Also - Gefängnis. Und noch so, ja U-Haft. Obergericht. (Z284-292)

Zwei Angehörige gaben zudem an, dass ihre suchtkranken Angehörigen keine schwerwiegenden Delikte begangen hätten, aber dass es bereits zu Anzeigen werden Fahrens in angetrunkenem Zustand gekommen sei (Anke, Doris).

Nur Annabelle und Darina hatten keine Erfahrung gemacht, dass ihre Angehörigen mit dem Gesetz in Konflikt gekommen waren. Bei Darina könnte eine mögliche Erklärung sein, dass ihr Bruder noch nicht sehr lange Heroin konsumierte.

\subsubsection{Finanzielle Probleme}

Die Suchterkrankung hat häufig grosse finanzielle Auswirkungen auf die Angehörigen (Copello, Templeton \& Powell, 2010; Kirby et al., 2005; Orford et al., 1998c). Es ist deshalb nicht überraschend, dass sämtliche im Interview Befragten angaben, dass sich die Erkrankung auf sie oder auf andere Angehörige finanziell ausgewirkt hätte. Besonders Eltern und Partnerinnen gaben viel Geld für die Drogenabhängigkeit ihrer Angehörigen aus. Nicht alle Angehörigen konnten dabei genau beziffern, wie viel Geld sie in die Angehörigen investiert hatten, weil sie häufig kleinere Zuwendungen von CHF 20.00 machten, die dann nicht notiert wurden. Einige Eltern hatten jedoch irgendwann einmal begonnen aufzuschreiben, wie viel Geld sie meist für Schulden der Betroffenen ausgaben. Albert hatte, nachdem er nach zwei Jahren bei CHF 30'000.00 angelangt war, aufgehört mit dem Aufschreiben und entschieden, der Tochter kein Geld mehr zur Tilgung von Schulden zu geben. Andere wie Doris und ihr Mann hatten bis CHF 200'000.00 Schulden vom gemeinsamen Sohn übernommen. Nochmals andere wie Dorette kamen für den ganzen Lebensunterhalt und zusätzlich noch für die 
Schulden ihres heroinabhängigen Sohn auf. Die nachfolgende Textstelle lässt die enormen Kosten, die sie in den vergangenen Jahren trug, nur erahnen:

(seufzt) Also es ist so dass ich seit äh - also:. Die Krankenkasse. Das Bei-mirwohnen - und, das Essen. - Das habe ich nicht aufgeschrieben. Aber, seit äh seit seit der zweiten Therapie wo er gemacht hat. Habe ich alles aufgeschrieben wo ich ihm gegeben habe. Also wo ich gezahlt habe für ihn. Ausser eben diese Sachen. Also die Krankenkasse und so. Das habe ich nie; und das Bei-mirwohnen und so. Das ist alles, gratis. Äh, das ist einfach ///(?: unverständlich, weil A während des Sprechens lacht). Ist jetzt etwa auf 79'000 Franken. Und das ist glaube ich jetzt etwa, seit 2000. Oder wann ist er ,ächt“ (=wohl), retour gekommen, vom zweiten Mal? - Vielleicht 2002. 2003 so. (Z358-362)

Auch für die Partnerinnen stellte die Suchterkrankung ihrer Männer eine grosse finanzielle Belastung dar. Weder Annabelle noch Dagmar konnten aber beziffern, wie viel sie die Drogensucht ihrer Partner gekostet hatte. Beide gaben jedoch an, dass sie phasenweise zu einem grossen Teil für den Lebensunterhalt aufgekommen waren. Annabelle berichtete, dass sie in einen besser bezahlten Job gewechselt hatte, damit die Familie finanziell besser durchkam. Sie gab zudem an, dass ihr Mann phasenweise die Hälfte seines Lohnes für den Heroinkonsum ausgegeben hatte:

Also weil er immer gesagt hat. Kein Problem und so! Eben wegen dem Finanziellen. Äh wegen seinem Lohn. Er nehme das Geld von seinem, das Erbe das ja ihm gehört! Bis ich dann irgendeinmal gemerkt habe. Hm er zahlt die Rechnungen nicht mehr. Oder ähm, wir hatten früher so eine Abmachung; oder ähm ich habe auch immer geschafft aber einfach als //?(unverständlich). Hat er mir wieder ein bisschen Geld auf mein Konto gegeben. Dass ich auch wieder die Rechnungen bezahlen konnte. Das ist einfach bis ich plötzlich gemerkt habe. Ähm das Geld kommt nicht! Oder er hat irgendwelche Ausreden parat. Ja so ist dann; wo ich mich selbst erkundigt habe bei seinen Unterlagen. Wie viel Geld dass er wirklich braucht. Und das ist dann „öpe“ (=etwa) die Hälfte seines Lohnes gewesen. (Z104-108)

Die beiden Geschwister Dorothea und Darina sowie Dylan, der Sohn von drogenabhängigen Eltern, führten an, dass die Suchterkrankung eher indirekt finanzielle Auswirkungen auf sie gehabt hätte. Dylan erzählte, dass seine Eltern immer Schulden gehabt hätten, was auch auf ihn als Kind zurückfiel. Ähnliches berichtete auch Dorothea, deren Eltern sehr viel Geld für den drogenabhängigen Bruder ausgegeben hatten:

Ja. Ja. Man hat, ja. Wir haben einfach nie irgendwie quasi quasi ein traditionelles Familienleben gehabt. Also Das ähm, Ferien. Was ist das? Ähm ja. - ja (Z346348)

Neben den finanziellen Aufwendungen für Schulden, Bussen und den Lebensunterhalt ihrer Angehörigen werden manche Angehörige auch bestohlen von ihren drogenabhängigen 
Familienmitgliedern, wodurch weitere Kosten entstehen (Kirby et al., 2005, S. 45). Im Rahmen der Befragung gaben vier Angehörige an, dass auch Wertgegenstände weggekommen waren. Dabei verschwanden auch Wertgegenstände, die für Angehörige nicht nur von materiellem sondern auch von grossem emotionalem Wert gewesen waren, wie Dana erzählte:

Weil er hat ja mir auch noch Ware gestohlen. Also Schmuck. Den ganzen Schmuck verhöckered. Und und das ist zum Beispiel als er mir den Schmuck verhökered hat. Das hat mir, das hat mir mega weh getan. [...] Das ist bei mir äh auch noch ein ein tiefer Stich ins Herz gewesen. Weil das sind Sachen von meiner Grossmutter in *Ausland. Und von der Familie Schmuck. Also auch nicht, also auch wertvoll. Und ich denke dass er das unter dem Preis noch verschachered hat damit dass er Geld äh bekommt. Und ja äh das sind so Sachen gewesen. (Z177183)

\subsubsection{Beeinträchtigung des Lebensalltages}

Zehn der 13 Angehörigen berichteten, dass die Drogenabhängigkeit ihres Angehörigen sie in ihrem Lebensalltag beeinträchtigt hätte. Dies war insbesondere der Fall, wenn die Angehörigen mit den Betroffenen zusammen lebten oder gelebt hatten, auch wenn es nur vorübergehend gewesen war. Familienmitglieder kamen oft in die Situation, dass sie ihre drogenabhängigen Angehörigen bei sich zu Hause aufnehmen mussten, wenn diese ihre Unterkunft verloren. Sie standen dann vor der Entscheidung, entweder kam der Betroffene zu ihnen oder er stand auf der Strasse. Angehörige entschieden sich in diesen Situationen sehr oft dafür, den suchtkranken Angehörigen bei sich aufzunehmen. Andrea, Dana und Albert berichteten, dass es für sie jeweils sehr belastend gewesen sei, wenn ihre Angehörigen wieder ihre Unterkunft verloren hätten, weil ihre Kinder dann wieder zu ihnen gekommen seien. Das folgende Beispiel stammt von Andrea, die ihren Sohn mehrmals vorübergehend aufgenommen hatte, wenn er aus einer Institution rausflog:

Und ich habe ja gewusst. Er hat die Wohnung nicht mehr. Er hat sie ja da aufgelöst. Und ich bin auch in einer kleinen Wohnung. Also das ist das ist nicht lustig gewesen. Ich habe immer gedacht. Ja dann steht er bei mir. Und ich habe eine Zweizimmerwohnung. Oder? (Z149-151)

Das Zusammenleben mit einem suchtkranken Menschen ist von vielen Schwierigkeiten gekennzeichnet. Eine Belastung für Angehörige stellt dabei der Konsum selbst dar (4). Neben dem Konsum waren Angehörige mit all den Begleiterscheinungen konfrontiert, die mit dem Konsum einhergehen können. Dorette berichtete in diesem Zusammenhang davon, dass ihr Sohn aufgrund des Folienrauchens jeden Morgen unter einem üblen Husten litt: 
Wenn mich jemand anruft eben. Letzthin hat mir eine Kollegin gesagt. „Läck“! der hat dann Husten. Habe ich gesagt. Ja ich weiss. Dieser Husten. Dieser „Chodder“ (=Auswurf) he:. Das ist natürlich, ,grusig“ (=eklig) zum; und er nimmt sich da nicht sehr gross zusammen. (Z200-202)

$\mathrm{Zu}$ den Begleiterscheinungen zählte auch, dass die Wohnung unordentlicher war oder dass Dinge kaputt gingen, weil die drogenkonsumierende Person aufgrund ihrer Erkrankung den Sachen nicht Sorge trug, wenn sie high war (5). Doris und Dorothea erzählten beide davon, dass auch schon Mobiliar in die Brüche gegangen sei. Bei Dana und Dorette zu Hause war es dafür häufig unordentlich und insbesondere Dana gab an, dass sie meist die Unordnung ihres Sohnes beispielsweise in der Küche wegräumen müsste, weil dieser es selbst nicht machte. Dorette und Dana sagten beide, dass sie deshalb keine Freunde oder Bekannten mehr nach Hause einladen würden, weil sie sich schämen würden, wenn andere die Wohnung sehen würden und weil sie auch nicht wüssten, wie die Wohnung beim Nachhausekommen aussehen würde. Auch Dylan erzählte, dass er nie Freunde mit nach Hause genommen hätte, weil es oft unordentlich gewesen sei. Die folgende Textstelle stammt aus dem Interview mit Dorette:

er nimmt auch, in gewissen Sachen; also nicht be-; eben. Rauchen. Das tut er. Es ist alles dreckig. Also ich habe alles, überall Löcher oder. Also die Tische sind ver- oder verlöchert. Also eben. Nur schon wegen dem kann ich niemanden einladen. (Z408)

Während Angehörige einerseits erwähnten, dass sie sich schämten, jemanden einzuladen, brachten andererseits ihre drogenabhängigen Angehörigen fremde Leute, die häufig selbst aus der Drogenszene stammten, mit in die gemeinsame Wohnung. Diese Erfahrung hatte sowohl Dana als auch Dorette und Doris gemacht. $\mathrm{Zu}$ diesen fremden Leuten konnten auch Drogendealer gehören, die vorbeikamen oder anriefen, weil sie die drogenkonsumierende Person suchten. Doris erzählte in diesem Zusammenhang, dass sie in der Nacht jeweils Telefonanrufe auf den Hausanschluss bekommen hätten, wenn die Dealer ihren Sohn gesucht hätten:

Und darum haben wir ja auch die Nummer gewechselt. - Weil die Dealer haben ja angerufen! Kaum ist Zahltag gewesen haben wir Nächte lang „Telefon“ (=Telefonanrufe) gehabt. Komm! Wir haben eine Party! Und, was weiss ich. Das ist nicht gegangen! (Z469)

Angehörige berichteten zudem von folgenden weiteren Beeinträchtigungen: Dagmar erzählte, dass ihr Partner sich nach der Trennung geweigert hatte, aus ihrer Wohnung auszuziehen. Sie hatte sich schliesslich eine andere Wohnung gesucht und gekündigt. August stellte 
irgendwann fest, dass einer seiner Söhne heimlich im Keller des Mehrfamilienhauses geschlafen hatte, in dem er und seine Frau lebten.

\subsubsection{Stigmatisierung}

Nur wenige Angehörige berichteten davon, dass sie selbst direkt auf Vorurteile gestossen seien (4). Vor allem Eltern, die schon sehr lange betroffen waren, hatten Erfahrungen mit Stigmatisierungen gemacht, die sich auf sie richteten, weil ihre Kinder drogenabhängig waren. Angelika, August und Dorette waren mit solchen Vorurteilen konfrontiert worden. Während Angelika mehr indirekt Stigmatisierung erlebt hatte, in dem unbeteiligte Drittpersonen sie fragten, ob sie den wirklich gar nichts machen könne, erzählte Dorette von direkten Schuldzuweisungen und Abwertungen:

Und dann ist die Polizei auch gekommen. Und dann hat die Polizei, mich auch mitgenommen. In jener Nacht. Und, dort haben sie mir gesagt. Ich sei eine potentielle Mörderin. Und, ja und eben. Die armen, armen Süchtigen haben ja bei uns Ware be-. Also bei uns! Nicht bei mir! natürlich. Aber, a- dort bin ich zuerst einmal wirklich, also habe ich gefunden. Wer bin ich eigentlich. (Z102)

Während solche Schuldzuweisungen oder das Unverständnis von unbeteiligten Drittpersonen für die Situation der Angehörigen für Angehörige sehr belastend sind, dürfte die Stigmatisierung für Kinder von drogenabhängigen Eltern noch weit schwerwiegender sein. Obwohl nur ein Interview mit einem Sohn aufgezeichnet wurde, gaben die anderen erwachsenen Kinder, die nur den Fragebogen ausgefüllt hatten, ebenfalls an, dass sie mit ihren suchtkranken Eltern häufig gleichgesetzt würden. Dylan berichtete wie folgt davon:

Ja. Ja. Schon aber äh (seufzt-lacht) -- ich habe immer erlebt. Dass dass quasi; - Du bist nicht so jemand wie deine Eltern. Aber du bist der Sohn! Du bist genau gleich! Auch wenn du noch nie so etwas gemacht hast. Du hast einfach gerade den Stempel darauf. hm ja. Du wirst gerade in die!, Schublade reingesteckt. Oder? Dann bekommst du auch keine Chance. Ich meine, wenn man jetzt äh; sagen wir mal in der Arbeitswelt. Jetzt, zwei Kandidaten wo schaffen gehen. Ich und ein anderer. Und jetzt der Chef weiss zufällig. Wie es bei dem steht in der Familie. Und beim anderen. Oder? Wem gibt er die Chance? - Mir oder ihm? Wenn er voll sozial ist und oah! und nicht für sein eigenes Ding Wohl schaut und Ultramilliardär ist. Vielleicht gibt er mir eine Chance. Aber wahrscheinlich nimmt er dort wo er sicher ist. (Z569-575)

Weitaus häufiger als Stigmatisierungen, die sich gegen Angehörige richten, erzählten sie von Situationen, in denen sie feststellen mussten, dass Vorurteile vor allem gegenüber suchtkranken Menschen existierten. Dorothea erzählte beispielsweise davon, dass eine Bekannte zu ihr gesagt hätte, dass Drogenabhängigkeit eine Strafe Gottes sei. Ähnliche 
Erfahrungen machte auch Darina. Dorothea und Doris hatten zudem beide schon die Situation erlebt, dass ihnen unbeteiligte Dritte sagten, dass es eigentlich nicht schade wäre, wenn drogenabhängige Menschen sterben würden, wie das Beispiel von Dorothea zeigt:

ja oder oder Aussagen wie, die sollten doch eigen- es ist wäre eigentlich nicht schade wenn wenn die sich den goldigen Schuss setzen. Sie liegen ja sowieso nur allen auf der Tasche. (Z286)

Dagmar berichtete zudem davon, dass ihre Eltern ihren drogenabhängigen Partner nicht akzeptieren würden und dieser nicht zu Familienfesten eingeladen würde.

Eine weitere Last für Angehörige stellen strukturelle Diskriminierungen dar. Unter struktureller Diskriminierung wird verstanden, dass eine bestimmte Personengruppe, im vorliegenden Fall drogenabhängige Menschen und zum Teil auch ihre Angehörigen, über gesellschaftliche Strukturen benachteiligt werden (Bruce \& Phelan, 2001, S. 372). August, Doris und Dylan berichteten im weitesten Sinn von solchen strukturellen Stigmatisierungen, die häufig dazu führen, dass Betroffene in weitere Schwierigkeiten hineingeraten anstatt sich davon zu befreien. Sowohl Doris als auch August erzählten davon, dass ihre Söhne sich massiv bei Kreditinstituten verschuldet hätten und gemahnt und betrieben worden seien. Die verhängten Kreditsperren seien aber von den Banken jeweils wieder übergangen worden, sobald die Angehörigen die Schulden abbezahlt hatten. August berichtete zudem davon, dass Eltern zum Teil ohne rechtliche Grundlage zu Zahlungen für ihre drogenabhängigen Kinder aufgefordert wurden:

Also es gäbe schon noch so: das eine oder andere. „Derig“ (=solche) Probleme sind dann auf einen zugekommen. Steuerrechnungen. Mahnungen. [...] Einerseits bei denen wo alle volljährig gewesen sind äh. W- weil sie den Wohnsitz noch lange bei uns gegolten hat. Und andererseits dann einfach weil sie es probieren. Haben keinen Wohnsitz gefunden. Und dann probieren sie es bei den Eltern. (Z1017-1023)

Neben diesen real erlebten Stigmatisierungen belasteten Angehörige häufig auch Vorurteile, die sie nur antizipierten. Sie nahmen beispielsweise an, dass es negative Auswirkungen auf ihren Job oder in der Nachbarschaft haben könnte, wenn auskam, dass ihr Angehöriger drogenabhängig war. Diese Umstände führten dazu, dass Angehörige es nicht wagten, jemandem von der Suchterkrankung zu erzählen. Gerade bei Partnern von drogenabhängigen Menschen fehlt dem Umfeld häufig das Verständnis, weshalb die Angehörigen die Beziehung aufrecht erhalten. Dagmar erzählte in diesem Zusammenhang, dass sie den 
meisten Freunden und der Familie noch nicht erzählt hätte, dass sie wieder mit ihrem Freund zusammen sei:

Und die! Kolleginnen die ich habe, die einte:, ja nein. Die meisten Leute finden vielleicht schon finden eben schon dass ich sch-, dass ich wieder mit ihm zusammen bin. Darum nicht einmal alle wissen das. Vielen wiss-; also meine Eltern wissen das noch gar nicht. (lacht) Denen muss ich das erst gar nicht sagen. Die meisten, wo ich es jetzt gesagt habe. Die sind schon so, sagen schon so. Ow! Wie kannst du wieder? Spinnst Du? Du weisst doch was er gemacht hat? Du weisst ja wie es gewesen ist? Wieso machst du wieder? Also sie haben schon für das kein Verständnis. (Z411-415)

Weitaus häufiger war jedoch die Angst bei den Angehörigen, dass es negative Konsequenzen für ihre Angehörigen haben könnte, wenn jemand vom Drogenkonsum wüsste. Sie befürchten, dass ihre Angehörigen dadurch allenfalls keinen Job mehr finden könnten. Drei Angehörige gaben an, dass sie die Suchterkrankung unter anderem verheimlichten, um ihren Angehörigen nicht die Zukunft zu verbauen. Annabelle, Dylan und Darina führten zudem an, dass ihre Angehörigen ihnen untersagt hätten, mit jemandem darüber zu sprechen. Dylan war dabei als Kind sehr unter Druck gesetzt worden, niemandem von der Drogenabhängigkeit der Eltern zu erzählen. Aus Angst, von den Eltern weg zu müssen, hielt er sich bis ins Erwachsenenalter an diese Auflage. Es kommt zudem vor, dass in der Familie oder gegenüber dem Betroffenen die Abhängigkeit tabuisiert wird, was viele Angehörige auch als belastend erleben. Darina, Dorothea und Annabelle berichteten davon. Annabelle gab an, dass die gemeinsamen, beinahe erwachsenen Kinder nichts von der Heroinabhängigkeit des Vaters wüssten. Sie hätte den Kindern gerne die Wahrheit gesagt, aber ihr Mann war dagegen gewesen, dass die Kinder informiert wurden:

hm. wo ich auch da hin gekommen bin ist das schon für mich ein grosses Thema gewesen. Dass ich es den Kindern eigentlich gerne hätte sagen wollen. (Z358360)

\subsubsection{Unterstützung}

\subsubsection{Einleitung}

Die Gründe, weshalb Angehörige sich Unterstützung suchten, waren unterschiedlich. Im Rahmen der Interviews wurde auch erhoben, was der Auslöser war, dass sich Angehörige Unterstützung gesucht hatten. Der häufigste Grund war, dass sich die Angehörigen mit der Situation überfordert fühlten oder dass ihnen die Last zu gross wurde (6). Weitere Gründe, die angegeben wurden, waren Gewalt (1), der Wunsch mit jemandem über die

Suchterkrankung sprechen zu können (1) und eine Unterstützung, im Umgang mit der 
Problematik zu haben (3), wobei der Wunsch nach Abgrenzung (3) von betroffenen Angehörigen im Vordergrund stand. Die nachfolgenden Kapitel geben einen Überblick, wie die Angehörigen die erhaltene Unterstützung beurteilten und was sie sich weiter als Entlastung gewünscht hätten.

\subsubsection{Erhaltene Unterstützung, die positiv beurteilt wird}

Drei Angehörige gaben an, dass es ihnen im Austausch mit anderen geholfen habe, die Hoffnung nicht aufzugeben oder zu sehen, dass es noch schlimmer sein könnte. Anke hatte in einer Selbsthilfegruppe erfahren, dass andere Eltern noch eine viel schwierigere Situation mit ihren Kindern hatten als sie. Dies hatte sie stark entlastet. Doris dagegen erzählte, dass sie sich durch den Psychiater ihres Sohnes sehr unterstützt gefühlt habe. Er hatte ihr geholfen, die Hoffnung nicht aufzugeben und auch die positiven Dinge an ihrem Sohn wahrzunehmen, wie sie in nachfolgendem Textbeispiel berichtet:

Ich habe dann das Glück gehabt dass ich dahin gekommen bin. Ich weiss auch nicht. Jetzt, ob es, auf, eine Referenz gewesen ist. Ich glaube es zwar nicht. Und dann bin ich an den Doktor *Name01 geraten. Und er ist eigentlich seit Jahren immer ,ächli“, hat man einen losen Kontakt. Also er hat mir auch gesagt. Und er hat mich auch gelehrt. Die guten Seiten vom *Sohn zu geben zu sehen. Und nicht nur das Schlechte in den Vordergrund zu tun. Es ist jetzt so. Man muss „luege“ (=schauen) dass man das Beste machen kann. (Z76-80)

Etwas, das Angehörige ebenfalls als unterstützend erlebten, war Information. Acht Angehörige gaben in den Interviews an, dass es sie entlastet hatte, mehr Informationen zu Suchterkrankungen, deren Bedeutung in der Gesellschaft, deren gesundheitliche und soziale Folgen und den Zusammenhang zu möglichen komorbiden Störungen zu erfahren. Insbesondere Informationen zu Suchterkrankungen holten sich Angehörige im Internet oder über Fachliteratur (4). Darina erzählte, dass es sie beruhigt habe, Informationen zum Thema zu finden:

Also für mich, ist es immer wenn ich irgendein Problem habe oder so. Ich bin also ich bin ja. Auch bei meinem Bruder. Ich habe versucht so viel herauszufinden wie möglich. Ähm also das ist immer so meine (lacht) meine Taktik ein bisschen. ähm und dann auf dem Internet. Es hat mich schon beruhigt. dass es ähm ein Wissen gehabt habe. (Z134-142)

Albert und Doris dagegen hatten sich Informationen von Drittpersonen geholt, die sie auch dabei unterstützten, wie sie sich gegenüber ihrem Angehörigen oder Drittpersonen bezüglich der Suchterkrankung verhalten sollten. Annabelle berichtete, dass sie von der Beratungsstelle ada-zh sehr ermutigt worden sei, sich auch rechtlich zu informieren, da sie aufgrund der 
Drogensucht ihres Mannes finanzielle Probleme hatte. August erhielt im Zusammenhang mit den steuerrechtlichen Schwierigkeiten seines Sohnes Unterstützung von einem Steuersekretär, der ihm sagte, welche Möglichkeiten er hatte, gegen einen ungerechtfertigten, rechtsgültigen Steuerbeschluss vorzugehen. Dass Informationen hilfreich waren, bestätigte auch Dorothea, die vor allem Erklärungen und Informationen von ihrem älteren drogenabhängigen Bruder bekommen hatte.

Insgesamt neun Angehörige, zu denen alle sechs Angehörigen gehörten, die bei der Angehörigenvereinigung Drogenabhängiger ada-zh Unterstützung gesucht hatten, berichteten, dass es ihnen sehr geholfen hätte, wenn sie und ihre Bedürfnisse wahrgenommen worden seien. Dazu gehörte nicht nur, dass Angehörige in ihren Wünschen wahrgenommen wurden, sondern auch dass ihre schwierige und belastende Situation anerkannt wurde. Albert hatte die Einzelberatung bei der ada-zh wie folgt erlebt und sich dadurch sehr unterstützt gefühlt:

Genau.+ Und, von dem her gesehen, das das das erkennt man ja selber nicht. Wenn man reinkommt. Aber vielleicht, dann im Nachhinein oder. Dass das wichtig ist oder. Dass man äh, eine Unterstützung hat. Dass einen jemand spiegelt oder. Dass einen jemand wahrnimmt. Als äh vollwertiger Mensch. Wo:, in einem Problem, in ein starkes Problem verwickelt ist. (Z525-533)

Neben dem Wahrnehmen der Angehörigen in ihren Bedürfnissen war es für viele wichtig, dass sie in ihren Bedürfnissen auch gestützt und ermuntert wurden, diese ohne schlechtes Gewissen auszuleben. Anke, die an einer geleiteten Selbsthilfegruppe in der ada-zh teilgenommen hatte, erzählte, dass die Psychologin sie sehr darin gestärkte hätte, ihre Leben geniessen zu dürfen:

Und ähm i- im Lauf von diesen Gesprächen ist auch hilfreich gewesen dass die Psychologin, *555 uns unterstützt hat und auch gesagt hat. Es ist wichtig! Dass wir auch wirklich äh d- d- den Teil vom Leben ausleben wo uns gehört. Das ähm auch vor der Gesellschaft das wirklich vertretbar ist. (Z156-160)

Annabelle betonte zudem, dass sie sich auch dadurch unterstützt gefühlt hätte, dass ihr dafür Wertschätzung entgegengebracht worden sei, was sie alles geleistet hatte in ihrer 25-jährigen Partnerschaft mit einem drogenabhängigen Mann:

Hmhm -- Also ich denke mir schon dass ich hier im ada sehr viel äh Unterstützung in Form von, also ja ob ich dem /(?:) sei. Oder dass sie das äh --- Ja dass es nicht selbstverständlich ist, fünfundzwanzig Jahre mit einem Partner zusammen zu sein und auch Kinder zu haben. Und ich denke das ist schon wirklich //(?:viel verlangt) also; (Z182-184) 
Ein wichtiges Ergebnis war, dass alle Angehörigen angaben, dass die soziale Unterstützung von anderen Menschen sie entlastet hätte. Im Rahmen dieser Befragung konnten verschiedene Arten der sozialen Unterstützung unterschieden werden. Diese liess sich in drei verschiedene Bereiche gliedern: Unterstützung, die Angehörige von Familienmitgliedern erhielten, Unterstützung, die sie von Freunden oder anderen Drittpersonen bekamen und Unterstützung, die sie in Selbsthilfegruppen fanden. Andrea, Dorette und Dylan erzählten, dass sie von Familienmitgliedern Unterstützung bekommen hätten. Andrea berichtete, dass ihre Kinder, mit denen sie auch über die Erkrankung sprach, sie sehr ermutigt hätten, sich Unterstützung zu suchen:

Aber das haben mir eigentlich mein Sohn und meine Tochter beide gesagt. Mach doch das einmal. Du Dich doch da irgendwo schauen ob jemand eine Selbsthilfegruppe gibt. [...] Ja. Von beiden aus gekommen. (Z348-350)

Es zeigte sich zudem, dass Angehörige sich selektiv immer wieder Menschen anvertrauten, bei denen sie das Gefühl hatten, dass ihnen Verständnis für ihre Situation entgegengebracht würde. Einige Angehörige machten dabei die Erfahrung, dass auch andere in irgendeiner Weise selbst betroffen waren. Dana erzählte davon, dass sie neben ihren Freundinnen, die sie unterstützten auch eine Freundin hatte, die ebenfalls einen drogenabhängigen Sohn hatte:

Und die einte Kollegin hat eben auch ein Sohn wo Drogen nimmt. Und mit ihr habe ich natürlich auch viel noch, haben wir uns gegenseitig manchmal noch "e chli" unterstützt. Oder dann hat einmal eine geheult. Ja! Ja. Und die anderen Freundinnen haben mich dann auch noch ja, jeweils unterstützt. (Z201-207)

Der Austausch mit anderen Angehörigen, die ebenfalls ein drogenabhängiges Familienmitglied hatten, war für viele sehr wichtig und brachte den Angehörigen in verschiedenen Bereichen grosse Entlastung. Von den 13 befragten Angehörigen hatten fünf Erfahrungen mit Selbsthilfegruppen, was von allen als positiv beurteilt wurde. Neben dem Austausch mit anderen hatten Angehörige das Gefühl, nicht alleine zu sein mit dem Problem. Sie machten die Erfahrung, dass sie in äusserst schwierigen Belastungssituationen von der Gruppe getragen wurden oder konnten teilweise Schuldgefühle anders einordnen. Angelika machte zur Selbsthilfegruppe folgende Angaben:

Ja also für mich ist es, enorm schwierig gewesen. Und ich bin noch so: jung gewesen. Wissen sie. Ich habe, ich habe meinen Sohn ja so früh bekommen. Und ich bin noch sehr! jung gewesen. Und ich habe mich nicht gewusst wie wehren. Also ich bin froh gewesen. Dass ich habe können, ähm, in die Elternvereinigung. Und dort habe ich auch gesehen dass dass nicht alles an mi:r liegt sondern dass normale Ehepaare, auch äh - das Problem haben. Und zwar sogar, mit mehreren Kindern! In einer Familie! Also das das hat mich dann sehr bestätigt darin dass 
das nicht einfach ist weil, mein Sohn unehelich ist. Und äh, keinen Vater gehabt hat. Und i:ch! das, quasi verursacht habe. Also in Anführungszeichen. (Z120-126)

Die Auswertung zeigte, dass nur Eltern Erfahrungen mit Selbsthilfegruppen hatten.

Eine grosse Entlastung für die Angehörigen war, wenn ihr drogenabhängiger Angehöriger in Behandlung war oder sonst Unterstützung erfuhr. Acht der 13 Angehörigen äusserten sich positiv über Behandlungsaufnahmen und Therapien. Andrea erzählte davon, dass sie sehr erleichtert gewesen sei, als sich der Sohn zu einer stationären Therapie entschied:

Und er hat sich einfach extrem zugespitzt. Aber dann hat er gefunden, das ist zwischen Weihnacht und Neujahr gewesen, jetzt müsse er etwas machen. Und dann hat er auch in die Klinik gekonnt nach *412. Bin ich natürlich wieder erleichtert gewesen. Ist gut gewesen. (Z95-99)

Das Wohlbefinden vieler Angehöriger war davon abhängig, wie es ihren drogenabhängigen Familienmitgliedern ging (3). War der Suchtkranke gerade in einer Krise, ging es auch den Angehörigen weniger gut, weil sie stärker belastet waren. Stabilisierte sich die Situation des Betroffenen dagegen, fühlten sich die Angehörigen ebenfalls weniger belastet. Albert erzählte davon, dass Voraussetzung für sein eignes Wohlbefinden sei, dass es der Tochter gut gehe:

Was mir - auch sehr geholfen hat in dieser Zeit ist dass, eben die Tochter, behütet gewesen ist an einem Ort. Wo man sich um sie gekümmert that. Einfach der Gedanke dass dass es ihr gut geht. Ist auch die Voraussetzung, dass es mir gut geht. (Z509-511)

\subsubsection{Erhaltene Unterstützung, die negativ beurteilt wird}

Die Realität war leider so, dass Angehörige manchmal auch negative Erfahrungen machten, wenn sie Unterstützung bekamen. Dies hatte verschiedene Ursachen: Einerseits hatten Angehörige manchmal Erwartungen, die sich nicht realisieren liessen. Andererseits reagierte das Hilfssystem, an das sich Angehörige wandten, manchmal in einer Art und Weise, dass die Belastungen für die Angehörigen erhöht und nicht reduziert wurde. Besonders häufig wurden inadäquate Reaktionen von professionellen Mitarbeitern des Sozialbereiches genannt, an die sich Angehörige gewandt hatten (8). Ein häufiges Problem, das Angehörige in diesem Zusammenhang angaben, war, dass sie sich als Person und in ihren Anliegen nicht wahrgenommen fühlten. So erzählte Annabelle von einem Termin auf einer Suchtberatungsstelle, bei der sie sich in einer Belastungssituation Unterstützung suchen wollte: 
Ja. Ich ich würde jetzt nicht ähm; ich will diese Leute nicht wegen; ähm dass es ist voll Scheisse gewesen. Aber ich denke mir -- also ja. Es tut einfach extrem weh wenn man beim ersten Mal merkt. Sie hat einen eigentlich vergessen. Und sie ist schmudlig (=ungepflegt) und sie raucht und überhaupt. Sie; Scheisse! Es geht ums Thema Sucht. Also so ein bisschen das. Habe ich schon nicht so gut; Habe ich wirklich keine gute Erfahrung gefunden. (Z188-194)

Besonders eindrücklich waren in diesem Zusammenhang auch die Schilderungen von Dagmar und Doris. Doris hatte sich an einen Psychiater gewandt, um über ihre Belastungen unter anderem auch wegen des Enkelkindes zu berichten. Darauf hatte ihr der Psychiater geraten, dass über die Behörden eine Adoption der Enkeltochter eingeleitet werden sollte. Ähnlich ging es auch Dagmar, die bei einer Familienberatung Unterstützung wegen der Konflikte innerhalb der Familie gesucht hatte:

Und einmal äh bin ich zur Familientherapie gegangen. Weil einfach er und meine Tochter, die sind wie zwei Geschwister gewesen wo sich immer ähm, gestritten haben. Und ich wollte schauen was geht. Aber das ist dann eben in die Hosen gegangen. Da sie da wirklich das Gefühl hatten sie müsse da schauen wegen meinem Kind. $\mathrm{Ob}$ das wirklich da in den richtigen Händen sei und so. Und das ist äh ja; Nie wieder! (Z149)

Weitere inadäquate Reaktionen waren beispielsweise Schuldzuweisungen von Therapeuten oder auch Vorschriften für die Angehörigen, wie sie sich zu verhalten hätten. Dorette berichtete von Schuldzuweisen vom Therapeuten ihres Sohnes:

Aber äh also also dass ich Hilfe! bekommen habe. I- im Gegenteil. Eben. Ich habe eher das Gefühl gehabt; und dann habe ich ja dann eben zu Psycholo:gen müssen. Und, ja. Weil man hat ja dann das arme Kind dann oder; (lacht) er ist ja kein Kind mehr gewesen ab-. Hat man ja dann abgeklärt. Und was wirklich los ist. Und, und eben. Ich habe dann eher immer das Gefühl gehabt es ist; wobei ich bin natürlich sehr ein: eher negativer Mensch. Und jede Bemerkung ist, fas- fasse ich negativ auf. Aber äh ich habe dann schon eher das Gefühl gehabt man hat gesagt. Ja sie sind halt schon. Und sie haben halt schon nicht recht. Und das arme Kind hat sich ja nicht entwickeln können mit ihnen. Und, und so wei-; also es sind dann „sochli“ (=so ein Bisschen) diese Sachen +sind dann gekommen. (Z119)

Besonders belastend für Angehörige war auch, wenn sie sich Hilfe suchen wollten und dabei die Erfahrung machten, dass sich niemand zuständig fühlte, sie bei ihren Problemen zu unterstützen. Davon erzählte insbesondere Dana, die sich zuerst an den Hausarzt gewandt hatte und schliesslich zu diversen Behörden gegangen war, um sich Unterstützung zu holen.

Einige wenige Angehörige (4) berichteten zudem, dass sie mit der Therapie ihrer Angehörigen nicht ganz zufrieden seien und die Belastung sich für sie dadurch noch erhöht hätte. Drei Mütter (Andrea, Dana, Doris) erzählten in diesem Zusammenhang, dass ihre 
Söhne Medikamente verschrieben bekommen hätten, von denen sie entweder vollkommen zugedröhnt gewesen seien oder von denen sie eine weitere Abhängigkeit entwickelt hätten. Der Sohn von Andrea bekam im Rahmen einer stationären Therapie Valium verschrieben und begann dies massiv zu missbrauchen:

Aber ich habe gedacht. Ja. Ich ich bin ein bisschen blauäugig gewesen. Muss ich ehrlich sagen. Ich habe einfach immerhin die Hoffnung gehabt. Nenein. Das kommt schon wieder in den Griff. Er ist ja unter Kontrolle. Er ist ja unter Ärzten. Also die checken das schon. (Z133)

Trotz aller Vorteile, die die Teilnahme an einer Selbsthilfegruppe mit sich brachte, wurden auch einige wenige Nachteile erwähnt. So erzählte Anke, die grundsätzlich sehr positiv über die Gruppe sprach, an der sie teilgenommen hatte, dass es zu Beginn einige Schwierigkeiten gegeben hätte, weil nicht alle mit dem gleichen Ziel in die Gruppe gekommen seien und einzelne Teilnehmende sehr viel Raum eingenommen hätten. Von etwas anderen Problemen sprach August, der schliesslich die Leitung einer Gruppe übernahm. Im Rahmen seiner Leitungsfunktion musste er feststellen, dass er häufig zu kurz kam in den Gruppentreffen, weil immer zuerst die anderen berichteten. Zudem merkte er auch an, dass es nicht immer einfach sei, eine gute Gruppe mit seriösem Hintergrund zu finden.

Weitere Erfahrungen mit Unterstützung, die als wenig positiv beurteilt wurden, waren Folgende: Institutionen hatten häufig nicht die Kapazität, Familien oder Angehörige in dem Mass zu betreuen, wie sich diese das manchmal gewünscht hätten. Dana berichtete, dass die zweiwöchentlichen Termine in der Familienberatung in zu grossen Abständen erfolgt seien. Darina sprach zudem ein weiteres Problem an. Sie hatte sich im Internet über Drogenabhängigkeit informiert. Sie meinte, dass gewisse Informationen sie eher belastet als entlastet hätten. Zudem sei es für sie auch schwierig gewesen einzuschätzen, welche Informationen verlässlich seien und welche nicht.

\subsubsection{Gewünschte Unterstützung}

Ein Thema, das Angehörige im Zusammenhang mit gewünschter Unterstützung immer wieder vorbrachten, war der Wunsch, dass vermehrt Aufklärung gemacht würde zu Suchterkrankungen aber auch zur Situation der Angehörigen. Vier Angehörige sagten, dass sie es wichtig fänden, wenn die Bevölkerung besser über die Problematik der Angehörigen Bescheid wüsste und wenn auch die Möglichkeiten bekannter wären, wo sich Angehörige Hilfe holen können. Anke brachte es mit folgenden Worten auf den Punkt: 
Aber das über äh der Fall von Drogensüchtigen oder von sonst Süchtigen - äh - es wird davon geredet. Dass der Alkoholkonsum zunimmt. Es wird davon geredet. Dass ähm vielleicht an einem Fest irgend ein irgendwelche Wohnwagen da sind mit Leuten die Drogen ähm prüfen. Ob sie nicht durchmischt mit anderem. Und so weiter. Aber über eine Hilfe über Angehörige hört man ein bisschen wenig. (Z431)

Ein weiteres wichtiges Thema in diesem Zusammenhang war die Aufklärung über Suchterkrankungen. Andrea war es beispielsweise wichtig, dass die Bevölkerung ein grösseres Bewusstsein dafür entwickelte, dass es sich bei einer Substanzabhängigkeit um eine Krankheit handelt und nicht um eine Willens- oder Charakterschwäche. Durch diesen Abbau von Stigmatisierung und die Akzeptanz von Suchterkrankungen in der Gesellschaft erhoffte sich Angelika, dass betroffene Menschen - und somit auch ihr Sohn - einen Platz in der Gesellschaft finden könnten. In eine ähnliche Richtung ging auch das Anliegen von Annabelle. Sie wünschte sich, dass vermehrt ein realistisches Bild zu Abhängigkeitserkrankungen vermittelt würde, nämlich dass Rückfälle die Regel und nicht Ausnahme seien und dass die meisten Erkrankungen einen chronischen Verlauf nehmen würden. Sie plädierte deshalb dafür, dass die Gesellschaft vermehrt über einen Weg mit Konsum sprechen sollte:

-- hm. also vielleicht auch so ein bisschen die Drogenabstinenz. oder im Sinn von ///(?:Aufnahme schlecht) Umgang damit. Ähm so ein bisschen in den Medien oder so Ist ja schon vielmals Thema eben wegen, Nulltoleranz oder null und nix. Oder es gibt ja auch äh Sendungen wo sich also ehemalige Drogenkonsumenten äussern. Die bin ich halt immer sehr interessiert. Wo wenn ich dann höre. Ah! Es ist drei Jahre her. Total euphorisch! Oder irgendwie. Eben ein kleines Kind und und seit fünf Jahren clean und jetzt Happy Day und so. Dann denke ich mir. Ja. Vielleicht müsste man es auch thematisieren, dass die Welt nicht untergeht. Wenn man es ähm; weil nach drei Jahren ist ja das noch längst nicht gegessen. Und nach fünf nicht! Und nach zehn Jahren nicht! (Z380-386)

Neben der vermehrten Aufklärung, die sich Angehörige wünschten, wurden auch vereinzelt Anliegen an die Institutionen geäussert. Sowohl Dorothea als auch Dana fanden, dass es wichtig wäre, wenn die Angehörigen von den Psychiatrien mehr Unterstützung bekämen. Insbesondere Dana, deren Sohn zum Zeitpunkt der Befragung noch minderjährig war, hätte sich erhofft, dass die Klinikaustritte besser vorbereitet und mit ihr mehr abgesprochen worden wären:

Aber+ äh; und die kämpfen ja auch; Denen geht es ja gleich. Eben in den Kliniken werden die Angehörigen viel zu wenig miteinbezogen. [...] Ja. und auch wenn sie ja Therapien haben. Die werden ja irgendwann wieder einmal in dieses Umfeld zurück entlassen. Und dass man dort auch einen fliessenden Übergang hat und nicht einfach. Wusch! da ist er wieder äh: Schaut jetzt. (Z291-297) 
Dorothea wäre es wichtig gewesen, dass im Rahmen der vielen Hospitalisierungen ihres Bruders, irgendwann mal jemand zu ihren Eltern gesagt hätte, dass sie auch auf sich selbst und die anderen Kinder schauen sollten. Sie ging davon aus, dass es für ihre Familie hilfreich gewesen wäre, wenn sie von aussen zu hören bekommen hätten, dass es okay ist, wenn die restlichen Familienmitglieder auch auf sich selbst schauten:

-- Ja wenn wir einfach; wie gesagt, in dem Moment bräuchte man ja nicht jemanden der einen die ganze Woche deine Händchen hält. Aber einfach - jemand der uns mal sagt. Ja. Lasst ihn ihn sein sein Zeugs durch- durchstehen und schaut für euch. Ich glaube dass ist, das wo wir wo wir manchmal hätten hören sollen. Dass wir auch für uns schauen müssen. Und dass -- und dass das ist irgendwie einfach nicht vorhanden gewesen (lacht leicht) (Z226-230)

Weitere Wünsche an Institutionen kamen von August und Doris. Doris erklärte im Interview wiederholt, sie könnte nicht verstehen, dass nicht massiver gegen Drogendealer vorgegangen würde. Sie zeigte dabei auch Humor:

///(?: Wir sehen das); aber sie! Das hat ja mein: Bekannter auch gesagt. Von der Drogenfahndung. /////(?: ) U:nd, er hat ja auch gesagt. Sie wollen sie eben nicht. Sie wollen die Hintermänner. - Das sind kleine +Fische. [...] Die sollen doch zufrieden sein mit denen wo es hat. (kichert) (Z408-414)

August kritisierte, dass Institutionen, die suchtkranke Menschen betreuen, manchmal disziplinarische Massnahmen anwenden würden wie beispielsweise die Verweigerung der Heroinabgabe, weil jemand einen Termin verpasst hätte. Dies könnte dann dazu führen, dass Betroffene die Behandlung ganz abbrechen würden, was sich letztlich auch wieder auf die Situation der Angehörigen auswirken würde.

Drei Angehörige machten in den Interviews zudem Angaben dazu, wo sie noch Bedarf für eine Angebotserweiterung oder einen Ausbau an Hilfsangeboten für drogensüchtige Menschen sehen würden. Es war jedoch nicht erstaunlich, dass die Wünsche vor allem von Dana, deren Sohn noch minderjährig war und von Dylan kamen, dessen beide Eltern heroinabhängig waren. Denn gerade in diesen beiden Bereichen herrscht die grösste Nachfrage nach weiteren Angeboten. Dana gab an, es wäre ihr wichtig, dass vermehrt ein therapeutisches Angebot für Kinder und Jugendliche mit Abhängigkeitserkrankungen geschaffen würde. Sie fügte hinzu, dass es für sie eine enorme Entlastung wäre, wenn es Institutionen gäbe, die vorübergehende Auszeiten zwischen dem betroffenen suchtkranken Kind und ihren Angehörigen ermöglichen würden. Dylan erwähnte im Gespräch, dass es für Kinder von drogenabhängigen Eltern gut wäre, wenn es vermehrt ein betreutes Wohnen für ganze Familien geben würde, wo nicht nur die Mütter, sondern auch Väter mit der Familie 
leben könnten. Andrea wünschte sich zudem, dass es mehr Beschäftigungsprogramme für drogenabhängige Menschen geben würde; Programme, in denen diese Menschen einer sinnvollen Arbeit nachgehen könnten und somit auch einen Beitrag für die Gesellschaft leisten könnten.

Bei der Unterstützung, die sich Angehörige für sich selbst wünschten, gingen die Meinungen auseinander. Albert und Annabelle teilten die Ansicht, dass das Selbsthilfeangebot für Angehörige weiter ausgebaut werden müsste. Annabelle hätte gerne an eine Partnergruppe teilgenommen. In diesem Zusammenhang meinte auch August, es sei wichtig, dass sich immer wieder neue Gruppen formieren würden, weil sich die Situation laufend verändere und Angehörige, die heute frisch betroffen seien, ganz andere Themen hätten als Familien, die sich seit über 20 Jahren mit der Problematik auseinandersetzen müssten.

August merkte zudem an, dass es für ihn hilfreich gewesen wäre, wenn er neben den therapeutischen Gesprächen auch eine Unterstützung gehabt hätte für all die weiteren Probleme, die auf ihn und seine Frau wegen der Drogenabhängigkeit ihrer Söhne zugekommen waren. Dazu gehörten vor allem rechtliche und finanzielle Fragen.

Dylan, der das einzige interviewte Kind von drogenabhängigen Eltern war, meinte zudem, dass er sich gewünscht hätte, dass er als Junge mehr gefördert worden wäre. Er war erst im Erwachsenenalter zum Schluss gekommen, dass er eigentlich gerne studiert hätte, dass ihm diese Möglichkeit jedoch aufgrund der Krankheit seiner Eltern ein Stück weit verwehrt geblieben war.

Während August sich vermehrt Einzel- und weniger Familiengespräche gewünscht hätte, hätte sich Dagmar mehr Termine als Paar und weniger einzeln gewünscht. Albert dagegen hätte gerne eine Familientherapie gemacht, was aber aus finanziellen Gründen nicht möglich gewesen war. Zusammenfassend lässt sich sagen, dass sich Angehörige ein breites Angebot an Unterstützung wünschen würden, das sich massgeschneidert für ihre Situation nutzen liesse.

Doch auch mit der besten Unterstützung bleibt die Situation für die Angehörigen trotz allem sehr belastend. Vier Angehörige sagten deshalb im Interview auch, dass eine Unterstützung nur in begrenztem Rahmen möglich sei, weil ihnen die Last, dass ein nahestehender Mensch 
schwer erkrankt sei, niemand abnehmen könne. Für einige wie Dorette war dies auch einer der Gründe, weshalb sie sich nie Unterstützung gesucht hatte:

finanziell habe ich einfach geschaut dass ich durchkomme. Und und andererseits habe ich auch nicht. Ja habe ich nicht gewusst was sie mir i- i- auf irgendeine Art helfen könnte oder würden. Und eben. Noch mehr!, Probleme von, anderen Leuten hören. Habe ich dann schon gar nicht mehr mögen oder. Weil ich habe immer in diesem ganzen Zeitraum voll geschafft. Oder also, ich habe - einfach müssen. Ja. Habe das Gefühl! gehabt ich müsse für mich schauen. Und ich habe ich habe auch das Gefühl gehabt. Es kann dir gar niemand helfen. (Z122-128)

Neben dem Gefühl, dass ihnen niemand helfen konnte, empfanden Angehörige häufig auch eine grosse Scham wegen der Suchterkrankung ihres Angehörigen und suchten sich deshalb keine Hilfe. So erzählte Albert, dass er zwar gewusst habe, dass es die Angehörigenvereinigung Drogenabhängiger (ada-zh) gäbe, er sich dort aber zwei Jahre nicht gemeldet hätte, weil er sich geschämte hätte. Dylan ergänzte, dass Unterstützung für ihn auch mit weiteren Problemen verbunden gewesen wäre. Wenn er jemandem erzählt hätte, dass seine Eltern beide drogenabhängig gewesen seien, dann hätte das auch bedeutet, dass er allenfalls den Eltern weggenommen worden wäre. Diese Angst hatte es verhinderte, dass er sich als Kind jemandem anvertraut hatte.

\subsubsection{Bewältigungsstrategien}

\subsubsection{Einleitung}

Im Vorfeld der Auswertung wurden basierend auf den Untersuchungen von Copello et al. (2010b) die drei Hauptkategorien „Aufbegehren“, „Toleranz“, und „Unabhängigkeit“ definiert. Zusätzlich wurde noch eine Kategorie „Diverses“ gebildet, um weitere Bewältigungsstrategien erfassen zu können. Induktiv wurden aus diesen Hauptkategorien Unterkategorien gewonnen. Die Auswertungen zeigten deutlich, dass Angehörige häufig nicht nur eine einzelne Bewältigungsstrategie anwandten, sondern häufig situationsgebunden zwischen verschiedenen Strategien wechselten und in schwierigen Situationen immer wieder überdachten, wie sie sich in Zukunft verhalten möchten. In den nach folgenden Kapiteln sind die Bewältigungsstrategien zusammengestellt, die Angehörige angaben.

\subsubsection{Aufbegehren}

Angehörige wünschen sich normalerweise, dass ihr drogenabhängiges Familienmitglied wieder gesund wird. Sie sind deshalb auch bereit, sich persönlich stark zu engagieren, damit 
sich die Situation der suchtkranken Person verbessert. Die Strategien, die Angehörige dabei anwenden, sind sehr vielfältig und reichen von Unterstützung bis zum Ausüben von massivem Druck. Sämtliche Bewältigungsstrategien, die Angehörige angaben und die deutlich machten, dass sie nicht aufgaben und gegen die Suchterkrankung ankämpften, wurden der Kategorie „Aufbegehren“ codiert.

Die nachfolgenden Bewältigungsstrategien haben eher einen konfrontativen, aggressiven und direktiven Charakter. Eine Möglichkeit zu versuchen, den Substanzkonsum einzudämmen, bietet die Kontrolle. Annabelle und Doris berichteten beide, dass sie die Finanzen ihrer Angehörigen kontrollierten. Nachdem Annabelle feststellen musste, dass ihr Mann die Hälfte seines Lohnes für Heroin ausgegeben hatte, begann sie regelmässig seine Postenauszüge zu kontrollieren, um den Konsum im Überblick zu behalten. Doris dagegen hob das Geld jeweils direkt nach dem Zahltag vom Konto ihres Sohnes ab, damit dieser nicht den ganzen Lohn für Kokain ausgeben konnte:

Ich habe es dann so gemacht. Dass ich, und nach Mitternacht. Am Zahltag. Am Fünfundzwanzigsten, aufgestanden bin. Also am Vierundzwanzigsten nach Mitternacht. Und auf die Bank bin. Und sein Geld abgehoben habe. Und auf mein Konto verschoben. Und einfach nur noch einen kleinen Teil gelassen habe. Dass man alles hat zahlen können und. Dass einfach; es ist, unglaublich! ins Haltlose gelaufen. (Z66-68)

Sechs Angehörige gaben an, dass die Umstände der Suchterkrankung sie manchmal auch wütend machen würden. Die Wut konnte sich dabei direkt auf den Drogenabhängigen richten, der nichts unternahm, um seine Situation $\mathrm{zu}$ verändern oder der sie mit in Schwierigkeiten reinzog, die er verursachte. Annabelle erreichte mit ihrer Wut, dass ihr Mann den Konsum massiv reduzierte:

Also bis ich dann irgendwie. Zwei drei Mal wo ich gefunden habe. Hey! (entrüstet) Jetzt; fluchen; Jetzt gopferdeckel“" jetzt kannst du einfach; So viel Kohle! Das +geht einfach nicht! Scheisse die Kinder kosten. Ich reisse mir den Arsch auf und gehe schaffen! Und also einfach, ein bisschen so. (=Diskussion ging in diese Richtung) Und dann: weiss auch nicht; (Z146-148)

Hingegen richtete sich die Wut von Doris und ihrem Mann gegen Drogendealer und Behörden. Doris erzählte, dass ihr Mann die Drogendealer des Sohnes am liebsten erschossen hätte:

Ah! mein+ Mann hat ihm mal gesagt er knalle ihn ab. Er sei bei den Pistolenschützen. Er ist+ auch gewesen. Hat aber alle Pistolen hat er verkauft. Weil er gesagt hat. Ich kann mir nicht trauen. Wenn ich so einen erwische. Dann 
kenne ich mich nicht mehr. Und ich will nicht noch, hinter Gitter wegen denen (lacht). (Z423-429)

Nebst der Tatsache, dass Angehörige den Drogenabhängigen auch anschrien, versuchten sie oft auch durch das Ausüben von Druck, die Betroffenen zu einer Behandlung oder Veränderung zu bewegen (4). Der Mann von Annabelle hatte sich auf ihren Druck hin kurz nach ihrem Kennenlernen in ein Methadonprogramm begeben, weil sie sich eine Familie gewünscht hatte. Auch Andrea und Dana konnten durch das Ausüben von Druck erreichen, dass ihre Söhne eine Behandlung aufnahmen. Die folgende Textstelle stammt aus der Befragung mit Dana:

Und eben wenn dann der Druck von unserer Seite enorm gewesen ist äh ist er wieder bereit gewesen zum etwas machen. (Z69)

Während die bisher genannten Bewältigungsstrategien des Aufbegehrens eher aggressiv und konfrontativ sind, folgen nun solche, die darauf abzielen, den drogenabhängigen Angehörigen zu unterstützen und $\mathrm{zu}$ entlasten und auf diese Weise eine Veränderung im Substanzkonsum zu erreichen. In den Interviews zeigte sich, dass Angehörige, vor allem zu Beginn der Suchterkrankung, das Gefühl hatten, sie müssten helfen und zeigten viel Verständnis für den Betroffenen (4). Dorothea beschrieb es wie folgt:

Wenn das doch so offensichtlich ist. Da muss man doch helfen. Oder? (Z102)

Mit dem Antrieb ihrem drogenabhängigen Kind zu helfen, nahmen Eltern dieses häufig zu sich nach Hause (3). Sie waren dabei davon überzeugt, dass sie mit Fürsorge, Liebe und Unterstützung zu einer Änderungsmotivation beim Betroffenen beitragen könnten. Albert und seine Frau nahmen die kokainabhängige Tochter - trotz Abraten der Therapeuten wieder bei sich auf:

ich habe dann den Vorschlag gemacht dass, dass ich die Tochter, zu uns nach Hause nehme. Von dort wo sie jetzt wohnt. Und dass wir sie dort betreuen. Also quasi - mit äh - Kommoditäten. Vitaminreich kochen. Fein essen. Ruhiges Zimmer ruhig schlafen. Äh erholen und. Dass wir sie irgendwie bewegen können - äh mit der Ko- mit dem Konsumieren aufzuhören. Und eine solche TheraTherapie anzufangen. (Z271-275)

Auch Doris und Dorette glaubten, dass sie bei ihren drogenabhängigen Söhnen eine Stabilisierung der Situation erreichen könnten, indem sie ihre Söhne bei sich aufnahmen. Aus einer ähnlichen Motivation gaben Angehörige häufig auch Geld (4). Obwohl die meisten Angehörigen in irgendeiner Weise für ihre suchtkranken Angehörigen finanziell aufkamen (siehe Kap. 5.2.3.7), sagten einige explizit, dass sie Geld gegeben hätten, um ihre 
Angehörigen vor weiteren Schwierigkeiten zu bewahren. Das nachfolgende Beispiel stammt von Andrea:

Und für mich ist das irgendwie; ich habe immer wieder probiert das Ganze ein bisschen zu besänftigen. Also ich habe es irgendwie immer probiert - nicht dass es nicht nicht so schlimm wird. Also ich habe dann immer eben ich habe ihm dann wieder Geld zugeschoben. Dass seine Schulden nicht extrem zunehmen. Ähm habe geschaut dass er zu mir zum Essen kommt. Habe geschaut, ich habe geschaut dass wir etwas unternehmen. Habe ihn auch ein bisschen ausgefragt. Aber ich habe schon gemerkt. Eigentlich, habe ich gar keine Chance. (Z81-85)

Drei Angehörige hatten im Interview angegeben, sich aktiv dafür zu engagieren, dass sich die Situation von drogenabhängigen Menschen verbessern würde oder dass sie zur Aufklärung beitragen würden. August hatte sich auch auf politischer Ebene sehr für die Anliegen der Drogenabhängigen und ihrer Angehörigen eingesetzt. Dylan betrieb aktive Aufklärung in seinem Umfeld und äusserte sich wie folgt im Gespräch:

Ja aber das ist so! Das das ist einfach so. Auf diesem Planet werden Drogen verkauft. Drogen werden konsumiert und werden produziert. Und und du kommst ja nicht daran vorbei in dem Sinn. Oder? Man kann es verleugnen. Man kann das Problem wegstossen. Oder man man tut es aktiv probieren etwas zu machen. (Z98-100)

Doris dagegen wählte einen etwas anderen Weg. Ihr war es ein grosses Anliegen, dass gegen die Drogenmafia härter vorgegangen wird. Sie schlich deshalb einem Drogendealer ihres Sohnes nach und erstattete Anzeige gegen ihn, als sie herausfand, wo er wohnte.

Entschied sich einer der drogensüchtigen Angehörigen für eine Therapie, bekam er meist Unterstützung von seinem Umfeld. Andrea und Albert begleiteten beide ihre Kinder zu Terminen im Zusammenhang mit der Therapie oder unterstützten diese aktiv bei der Aufrechterhaltung der Abstinenz, wie das folgende Textbeispiel aus dem Interview mit Andrea zeigt:

Wir haben auch abgemacht. Ich bin ihn immer holen gegangen am Bahnhof. Er hat das auch gewollt am Anfang dass ich ihn abholen komme dass er nicht diesen Leuten begegnet. (Z222)

Neben der Unterstützung von therapeutischen Prozessen versuchten Angehörige ihre suchtkranken Angehörigen, die nicht in Behandlung waren, in Therapien zu vermitteln (2) oder auch Auslandaufenthalte zu organisieren, um Distanz zu dem von Drogen geprägten Umfeld zu gewinnen (2). 
Vier Angehörige erwähnten zudem im Gespräch, sie würden die Hoffnung, dass sich etwas ändern könnte, nicht aufgeben. Diese Hoffnung gab ihnen Kraft und half ihnen bei der Bewältigung der Belastungen. Dana beispielsweise meinte im Interview, dass die Situation noch viel schlimmer sein könnte, was ihr die Zuversicht gab, dass die Situation sich auch wieder bessern könnte. Angelika dagegen hatte die Hoffnung, dass ihr Sohn irgendwann von den Drogen wegkommen könnte, ganz aufgegeben, aber sie hielt daran fest, dass ihr Sohn einen Weg finden könnte, weniger einsam zu sein. Auch Dylan hatte als Kind die Hoffnung geholfen, dass es anders werden würde, wie er erzählte:

Ja.+ Weil das ist eigentlich das, das hat dir Kraft gegeben als Kleiner. Bis bis du ein gewisses Alter gehabt hast. Es wird sich verändern. (Z455)

\subsubsection{Toleranz}

Der Bewältigungsstrategie der Toleranz wurden Textstellen zugeordnet, aus denen deutlich hervorging, dass die Angehörigen den Substanzkonsum oder die Abhängigkeitserkrankung akzeptierten und diese hinnahmen. Dazu gehörten aber auch Verhaltensweisen, mit denen Angehörige versuchten, den Drogenabhängigen nach aussen zu decken und den Konsum zu verheimlichen. Copello et al. (2010b, S. 91) betonen, dass das Coping des Akzeptierens der Suchterkrankung und des Substanzkonsums mit den höchsten gesundheitlichen Risiken einhergeht. Bei Dorothea, die um einige Jahre jüngere Schwester eines heroinabhängigen Bruders, zeigte sich deutlich, dass letztlich das jahrelange stillschweigende Akzeptieren und Tolerieren sie krank gemacht hatte. Sie hatte zwei Suizidversuche begangen und war anschliessend mehrere Monate hospitalisiert gewesen. Sie berichtete davon:

Ja. Es ist ähm wie gesagt. Bis bis vor vor ja anderthalb Jahren ist das für mich nie ein Thema gewesen. Ich habe, keine Ahnung. Oder ich habe ich habe im Gymi Psychologie gehabt als Schwerpunktfach. Ich habe ich habe der Kollegin auch immer wieder gesagt. Eben wenn es Probleme gibt. Es ist ja nichts Schlimmes wenn man zu Psychologen geht. Aber selbst. Hmhm- Ich habe es immer nachher geschluckt. Und so so viel geschluckt bis es einfach nicht mehr gegangen ist. Oder? Und ja. Vor anderthalb Jahren ist es nachher einfach, ja eskaliert. (Z106110)

In ähnlicher Weise wie Dorothea berichtete Dylan, er hatte den Konsum der Eltern einfach akzeptiert und damit gelebt, weil er keine andere Möglichkeit gehabt hatte. Gleichzeitig hatte er aber auch versucht, die Eltern nach aussen zu decken und mit ihnen ein positives Bild bei anderen zu vermitteln:

Solche Sachen dass du eben nicht darüber geredet hast. Oder oder, dass du versucht hast also mit den Eltern quasi so so ein Bild von von der schönen Familie zu machen. Wo alles in Ordnung hat. Oder? (Z237-239) 
In der Auswertung zeigten sich bei der Bewältigungsstrategie der Toleranz aber noch weitere Aspekte. Gerade Angehörige, die seit mehreren Jahrzehnten betroffen waren, hatten eine gewisse Toleranz gegenüber der Suchterkrankung entwickelt. Während sie zu Beginn häufig noch daran glaubten, dass ihr Angehöriger wieder gesund werden würde, waren sie schliesslich nach mehreren Rückfällen zum Schluss gekommen, dass ihr Angehöriger mit der Krankheit leben musste. Sechs der 13 befragten Angehörigen drückten ihre Toleranz gegenüber der Abhängigkeitserkrankung aus. Angelika, deren Sohn seit über 20 Jahren heroinabhängig war, erzählte wie folgt:

Es ist immer das, gelaufen dass ich wieder optimistisch gewesen bin. Und gedacht ja mein Sohn, der schafft es! Und, der kommt da draus heraus. Denke ich heute überhaupt nicht mehr. Das denke ich heute überhaupt nicht mehr. Dass er; ich denke heute. Er kann sich. Mit den Drogen das einrichten. Früher, habe ich, gedacht, es gibt nur, eine Abstinenz. Heute sehe ich dass das auch, einfach viel tiefgreifender ist. Dass man auch muss denken äh - er muss jetzt „,halt“ (=eben) mit dem Methadon leben. Oder irgendeinen Ersatzstoff haben. Wo diese äh, Endorphine wo geholt sind ersetzt! Oder; (Z364-370)

Der häufig jahrelange erfolglose Kampf gegen die Drogen liess Angehörige resignieren und sie begannen sich darüber Gedanken zu machen, wie ein Leben mit dem Konsum möglich sein konnte. In diesem Sinne wäre Toleranz gegenüber der Erkrankung - gerade bei einer jahrelangen Abhängigkeit - durchaus als adaptives Verhalten einzustufen. Annabelle, deren Mann seit 10 Jahren von der Gasse Heroin konsumierte, beschrieb dies wie folgt:

Und ähm; -- vielleicht auch dass es einen Weg gibt mit dem Heroin wo ich jetzt manchmal denke oder ähm ja mich nach zehn Jahren; mit ihm mit Heroin zusammen leben; vielleicht hat ja dort auch noch etwas;. (Z96-98)

\subsubsection{Unabhängigkeit}

Die Bewältigungsstrategie „Unabhängigkeit“ ist dadurch gekennzeichnet, dass Angehörige in irgendeiner Form versuchen, sich von ihrem drogenabhängigen Familienmitglied und dessen Substanzkonsum zu lösen oder zu distanzieren. Dazu gehört aber auch, dass Angehörige sich vermehrt auf ihre eigenen Bedürfnisse konzentrieren oder ihre eigenen Interessen durchsetzen. Dies kann auf unterschiedliche Weise geschehen. Eine Möglichkeit, der unangenehmen Situation zu entkommen, ist die Flucht. Angehörige stürzen sich in die Arbeit oder suchen nach Wegen, um nicht nach Hause gehen zu müssen. August und Dagmar erzählten beide von einer Flucht aus der belastenden Situation, indem sie Aussenbeziehungen eingegangen waren. Die nachfolgende Textstelle stammt aus dem Interview mit August:

Ganz! Ja! Ja.+ Und auch selbst in dieser, Fre- in dieser Beziehung. Wo ich gehabt habe. Ist es mir „glich“ nicht ganz gelungen. Ist es mir sehr wohl gewesen. Ich 
habe, ma- man hat „öppe“ wieder, so äh irgend einen schönen Ort gefunden. Wo man sich wieder mal einen Tag, ,ächli“ hat vers- wie verstecken können. (Z499)

Die Auswertungen zeigten, dass die meisten Angehörigen erst im Verlauf der Erkrankung versuchten mehr an Unabhängigkeit zu gewinnen. Dies lag daran, dass sie irgendwann realisierten, dass ihre Bemühungen nicht fruchteten. Eine gewisse Unabhängigkeit und der Fokus auf die eigenen Bedürfnisse schafft den Angehörigen dann Entlastung, auch wenn ihnen dies zu Beginn oft sehr schwer fällt. Albert erzählte davon, wie schwierig es gewesen sei, der Tochter zu sagen, dass sie ausziehen müsse:

Und - eben das das andere habe ich ja schon erzählt gehabt. Was dann da, gewesen ist. Und ich habe es dann auch gemacht. Und das ist auch wirklich! das gewesen wo, genützt hat. Und alles andere; -- (stöhnt) //(?: sind schon) hilfreich oder. Aber diese zwei Sachen -- weil mit Verständnis und mit Liebe und mit Zuneigung und mit, mit Streit. Mit Reibereien mit einander anschreien. Äh miteinander hassen. Wieder auf gut ja: jetzt tun wir doch. Und komm jetzt trinken wir noch ä- ein Glas Wein und dann und so. Das nützt alles! nichts. - Nützen tut, äh die Eigenverantwortung übertragen. Mit allen Konsequenzen. - Ja:. Das ist das ist ein- eines von den wichtigsten, Punkten gewesen. In dieser ganzen Drogengeschichte. - Mit dem Risiko dass es nicht gut herauskommt. (Z601-611)

Neben Albert betonten noch neun weitere Angehörige die Eigenverantwortung der Drogenabhängigen. Durch das Zurückgeben der Verantwortung gelang es vielen Angehörigen, sich auch ein Stück weit abzugrenzen. Häufig begannen sie in diesem Zusammenhang auch klare Regeln aufzustellen, die sie grösstenteils auch einhielten. So sagten beispielsweise fünf Eltern ganz klar, dass sie ihren Kindern kein Geld mehr geben würden. Doris erzählte, dass sie ihren Sohn finanziell bei seinen Schulden unterstützt hätte, bis sie selbst kein Geld mehr gehabt hätte:

Aber es gibt jetzt nichts mehr von mir. Er hat nämlich jetzt noch eine kleine Schuld bei mir. Wo er dauernd sagt er zahle sie ab. Und es kommt nie etwas. [...] Nein!+ Jetzt ist endgültig auch bei mir fertig. Sonst muss ich dann betteln gehen. [...] Hä! [...] Und ich verkaufe auch nichts. Wegen ihm. Wir haben uns jetzt gesagt. Wir verkaufen nichts! Wenn du halt jetzt, wirklich in den roten Zahlen bist. Dann musst du schauen wie du herauskommst. Und sonst tust du Privatkonkurs anmelden. (Z574-582)

Andere Angehörige liessen die Drogenabhängigen nur noch unter bestimmten Bedingungen bei sich wohnen (Andrea, Dagmar, Dorette). Häufige Regeln, die Angehörige aufstellten, waren, dass sie keinen Konsum bei sich zu Hause akzeptierten oder dass keine fremden Leute mitgebracht werden durften. Andrea, die ihren Sohn zweimal aufnahm, als er seine Unterkunft verloren hatte, forderte ihren Sohn, sobald sie feststellte, dass er in ihrer Wohnung wieder konsumiert hatte, dazu auf auszuziehen und setzte dies auch durch. 
Sechs Angehörige erzählten, dass sie eine gewisse Distanz zur drogenkonsumierenden Person entlasten würde. Einige von ihnen hatten deshalb neben einer räumlichen Distanz auch den Kontakt zum Suchtkranken reduziert. Dagmar erzählte beispielsweise, ihr Partner und sie würden nicht mehr die ganze Woche zusammenleben, sondern er wohne die Hälfte der Woche bei seiner Mutter. Albert dagegen erlaubte seiner Tochter nicht mehr das ganze Wochenende bei ihm und seiner Frau zu verbringen, wie er im Interview berichtete:

Und darum - bleibe ich einfach hart. Also - ich lasse sie z- zu mir kommen. Wenn sie will am Wochenende. Aber ich will nicht dass sie vom Freitagabend bis am Sonntagabend bei uns ist. Sondern, mir „langt“ (=reicht) das wenn sie einmal bei uns schläft. Es darf auch zweimal sein ausnahmsweise. Aber nicht jedes Wochenende. Weil ich meine Zeit brauche. Also ich muss mich irgendwie schützen vor ihr. (Z777-779)

Neben der Distanz zur abhängigen Personen betonten viele Angehörige auch, dass es sehr wichtig sei, dass man sich und die eigenen Bedürfnisse nicht aus den Augen verliere und sich selbst hin und wieder etwas Gutes tue (8). Sie meinten, dass Angehörige sich selbst auch einen Lebensbereich erhalten müssten, in dem sie das Leben geniessen könnten. Anke brachte diese Haltung im Interview deutlich auf den Punkt:

Aber ich möchte eigentlich und das ist das ist immer mehr geworden (lacht dabei). Ich möchte eigentlich mein Leben nicht opfern. Für Sachen die andere falsch machen. Und äh es tut mich betreffen. Es tut mich beschäftigen. Aber ich möchte eigentlich einen Teil von meinem Leben für mich behalten wo ich eigentlich normal bin. Und nicht ///(?: lacht und sehr leise, deshalb nicht verständlich). (Z128-130)

Treffend waren in diesem Zusammenhang auch die Worte von Dorothea, die im Interview sagte, dass auch Angehörige das Recht auf ein eigenes Leben hätten und nicht zurückstehen müssten, weil sie einen kranken Angehörigen haben:

Einfach einfach mal - ja. Sagen können. Es ist sein Leben. Ich muss mich jetzt um mich kümmern. Ich glaub. Das, das hat mir lange lange gefehlt. [...] Ja. - und einfach dass ich auch das Recht dazu habe. Also das ist vor allem, im ja. Wir wir haben alle das Recht auf das Leben (lacht). Also (Z402-408)

\subsubsection{Weitere Bewältigungsstrategien}

Sechs Angehörige sagten im Gespräch, dass sie es wichtig fänden, dass sich Angehörige Hilfe suchten, wenn sie vom Drogenkonsum eines nahe stehenden Menschen erfahren würden. Dieser Rat kam auch von Angehörigen wie Darina, deren Familie sich keine Unterstützung geholt hatte. Andrea beschrieb im Interview, weshalb sie es wichtig fände, 
dass sich Angehörige in Bezug auf den Umgang mit dem Substanzkonsum professionellen Rat einholten:

Also auf jeden Fall äh Unterstützung holen wirklich jemand wo wo wo die Problematik erkennt oder weiss wie man mit dieser Problematik umgeht. Also und zwar so schnell wie möglich. Also eben auch das ada. Also mit jemandem wirklich reden. Nicht einfach nur reden mit äh mit Freunden und so. Sondern wirklich dort wo wo man weiss wie man wie man umgeht mit der ganzen Geschichte. Weil ich denke reden kann man mit jedem über diese Geschichte. Aber Unterstützung - gibt es äh, kann dir niemand geben. Es hilft dir vielleicht im Moment mit jemandem reden. Okay. Dass du dich vielleicht hast können mitteilen. Aber wirklich; ich denke dann musst du dann musst du mit jemandem wo wo das Ganze versteht. (Z684-692)

Neben der professionellen Hilfe fanden neun Angehörige, es sei wichtig, dass man offen über die Suchterkrankung spreche, auch wenn, wie es bei einigen der Fall war, nur ein kleiner Kreis eingeweiht worden war. Darina hatte beispielsweise mit Kolleginnen darüber gesprochen, die weit weg lebten und ihre Familie nicht kannten. Sie erzählte im Interview, dass sie sich dadurch entlasten konnte. Einige Angehörige wie Dorette, Dorothea und August gingen sogar so weit, dass sie nicht mehr versuchten, vor jemandem die Drogenabhängigkeit ihrer Familienmitglieder zu verheimlichen. Dorothea hatte sich aus Selbstschutz dazu entschieden, andere über die Heroinabhängigkeit ihres Bruders zu informieren. Während sie mit nahestehenden Freunden, bei denen sie Verständnis bekam, offen sprach, informierte sie die anderen einfach nur über die Tatsache, dass sie einen drogenabhängigen Bruder hatte:

Es gibt auch es gibt auch Leute da da rede ich eigentlich nicht gerne darüber. Also, informiere sie zwar vielleicht dass sie dass sie nicht die ganze Zeit äh mir auf die Eier „tschalpen“ (=treten) mit mit gewissen Bemerkungen. Aber möchte ich eigentlich nicht darüber reden weil äh das Unverständnis einfach, zu gross ist. Und und nachher macht es einen sauer und nachher (Z264-268)

August hatte sogar bei der Arbeit offensiv seine Angestellten über die schwierige private Situation informiert und dabei positive Erfahrungen gemacht. Die Suchterkrankung seiner Söhne nicht mehr verheimlichen zu müssen, hatte ihn stark entlastet.

Eine etwas andere Art der Bewältigung ist der Humor. Im psychoanalytischen Sinn stellt Humor ,nicht nur eine Form der Kommunikation und der Interaktion dar, sondern eine Eigenschaft der Person, die sich in einer inneren Haltung, einem spezifischen Umgang mit Affekten ausdrückt.“ (Frings, 2002, S. 293). Frings (ebd.) führt in diesem Zusammenhang weiter aus: 
Hauptmerkmal dieser Haltung zur Welt und zu sich selbst ist die Fähigkeit zur Distanzierung von leidvollen Erfahrungen und inneren Konflikten, die sich in einer heiter-gelassenen und akzeptierenden, aber auch in einer zynischen, sarkastischen und aggressiv abwehrenden Einstellung zeigen kann. (S. 293)

Die Möglichkeit, über Humor leidvolle Erfahrungen abzuwehren und mit Konflikten umzugehen, hatte bereits Freud dazu veranlasst, Humor als eine hochstehende Abwehrleistung zu bezeichnen. In den Interviews zeigte sich, dass einige Angehörige Humor zeigten oder lachten über zum Teil schwierige Situationen (4). In der folgenden Textpassage lachten sowohl Doris als auch die Interviewerin:

Ja!+ Was ich jetzt einfach noch sagen will ist äh. - Diese Drogendealer. Die könnte ich alle umbringen. Einzeln! Das ,miech“ mir nichts aus. Aber ich erwische keinen. (A und I lachen beide laut) (Z402-404)

Von Angehörigen wurden weitere vereinzelte Bewältigungsstrategien genannt. So sagte Annabelle beispielsweise, dass sie jeden Tag kiffe, weil sie ihren Mann und die Situation sonst nicht mehr ertrage. Dylan, der erfahren hatte, dass neben seinen Eltern auch der Bruder begonnen hatte, mit Drogen $\mathrm{zu}$ experimentieren, versuchte über Verleugnung und Beschwörungen, mit der Realität umzugehen:

hm. Also da; (seufzt) ich müsste mich schwer täuschen. Aber ich würde jetzt noch die Hand ins Feuer legen. Dass er nie so würde so süchtig werden. Also; Aber, [...] man+ könnte sich irren. (Z405-407)

Darina, die eine Weile im Ausland gelebt hatte, fand es dagegen entlastend, dass sie in die Schweiz zurückgekehrt war. Für sie war es eine Erleichterung, dass sie ihre Familie nun jederzeit besuchen und sich ein Bild von der Situation machen konnte. 


\section{Diskussion}

\subsection{Einleitung}

In den folgenden Kapiteln werden die Ergebnisse dieser Arbeit diskutiert. Zuerst sollen einige Bemerkungen zur Stichprobe gemacht werden, die weitreichende Auswirkungen auf die Interpretation der Ergebnisse haben. Anschliessend werden die Ergebnisse zu den Belastungen, den Bewältigungsstrategien und der Unterstützung der Angehörigen diskutiert. Abschliessend werden die Relevanz der Ergebnisse und weiter Implikationen, welche sich aus der Arbeit ergeben, beschrieben.

\subsection{Die Stichprobe}

\subsubsection{Rekrutierung und Repräsentativität}

Die vielen Rückmeldungen, die auf die verschiedenen Zeitungsinserate erfolgten, sowie die hohe Bereitschaft der Angehörigen ohne Aufwandentschädigung an der Studie teilzunehmen, zeigen deutlich, dass es den Angehörigen ein grosses Anliegen war, dass ihre Situation mehr Beachtung erhält.

Es zeigte sich allerdings, dass bei der Rekrutierung der Stichprobe verschiedene Selektionseffekte gewirkt haben könnten, die die Repräsentativität und folglich die Generalisierbarkeit der Ergebnisse stark einschränken dürften. Die Auswertung machte deutlich, dass beinahe die Hälfte der Angehörigen (46\%) angab, dass sich die Situation der drogenabhängigen Person im vergangenen Jahr gebessert hatte und weitere $39.1 \%$ sagten, dass die Situation gleich geblieben sei. Dagegen gaben nur $14.9 \%$ an, dass sich die Erkrankung verschlechtert hatte. Dies lässt vermuten, dass sich Angehörige vor allem dann bereit erklärten, an der Studie teilzunehmen, wenn die Situation ihres suchtkranken Angehörigen stabil oder gebessert war. Dies hatte sich vereinzelt auch in den Kontaktaufnahmen gezeigt, wo Angehörige sagten, dass sie gerne bei der Befragung mitmachen möchten, aber dass es ihnen momentan zu viel sei und sie sonst zu einem späteren Zeitpunkt an der Befragung teilnehmen würden, wenn das noch möglich wäre. Die Tatsache, dass der Gesundheitszustand des drogenkonsumierenden Angehörigen ein Selektionskriterium dargestellt haben könnte, könnte dazu geführt haben, dass die Befindlichkeit und die Belastung der Angehörigen als zu gut eingeschätzt wurden in der Stichprobe. 
Ein weiterer Selektionseffekt könnte beim Geschlecht gewirkt haben. Obwohl auch andere Forschungsarbeiten (Lee et al., 2011, S. 443; Oreo \& Ozgul, 2007, S. 74) über einen ähnlich hohen Frauenanteil berichten, wie es auch bei der vorliegenden Befragung der Fall ist, nämlich um die $80 \%$, drängt sich dennoch die Frage nach den männlichen Angehörigen auf. So konnte beispielsweise kein einziger Partner einer drogenabhängigen Frau für die Studie gewonnen werden, obwohl sich einige wenige Männer gemeldet hatten, die mit einer suchtkranken Frau in einer Beziehung waren. Die Gründe für die geringe Teilnahme von Männern in der Studie können unterschiedlich sein: Störungen durch psychotrope Substanzen werden deutlich häufiger bei Männern als bei Frauen diagnostiziert (Sass, op. 2003, S. 245), was zwangsläufig die Wahrscheinlichkeit bei der Stichprobenselektion für eine Frau, die einen suchtkranken Partner hat, erhöht, während die Wahrscheinlichkeit für einen Mann mit einer substanzkonsumierenden Partnerin geringer ist. Dies zeigt sich auch in der vorliegenden Stichprobe, wo der Anteil der drogenabhängigen Männer deutlich höher liegt als der der Frauen. Vertreter des Co-Abhängigkeitskonzeptes argumentieren zudem, dass vor allem Frauen sich mit der co-abhängigen und fürsorglichen Rolle identifizieren, während Männer eher eine klarere, abgegrenzte Haltung einnehmen (Flassbeck, 2011, S 99). Dies könnte auch ein höheres Hilfesuchbedürfnis von Frauen erklären. Unter diesem Aspekt könnten sich Frauen stärker durch die Studienaufrufe angesprochen gefühlt haben. Nichtsdestotrotz scheint diese Erklärung etwas unbefriedigend. Die drei Männer, die im halbstrukturierten Interview befragt worden waren, gaben die gleichen Belastungen an wie die Frauen, die befragt worden waren, so dass sich die Vermutung aufdrängt, dass andere Gründe dafür verantwortlich sein dürften, dass Männer weniger an der Befragung teilgenommen haben. Eine mögliche Erklärung könnte im Hilfesuchverhalten und dessen Unvereinbarkeit mit dem männlichen Rollenbild liegen (Shepherd \& Rickard, 2012). So konnte gezeigt werden, dass Männer, die sich mit einem starken männlichen Rollenbild identifizieren, das mit einer starken Erfolgsund Konkurrenzorientierung und einem reduziertem Ausdruck an Emotionen assoziiert ist, eine deutlich negativere Einstellung gegenüber Hilfesuchverhalten angeben (ebd.) als Männer, die dies nicht taten. Männer, die eine negative Einstellung gegenüber dem Ausdruck von Emotionen haben, werden mit grosser Wahrscheinlichkeit auch nicht bereit sein über ihre Gefühle durch die Mitbetroffenheit von einer Suchterkrankung zu sprechen.

Eine weitere Schwierigkeit zeigte sich bei der Rekrutierung von Partnern. Während sich kein Mann mit einer drogenabhängigen Partnerin finden liess, der sich zur Studienteilnahme bereit erklärt hätte, konnten immerhin 15 Partnerinnen für die Befragung gewonnen werden. Das 
ursprünglich gesetzte Ziel von $n=20$ Partnern konnte allerdings auch bei den Frauen nicht realisiert werden - trotz eines spezifischen Aufrufs im der Pendlerzeitung 20minuten. Gründe dafür waren, dass sich viele ehemalige Betroffene gemeldet haben, die sich von ihrem drogenabhängigen Partner getrennt hatten, oder dass viele Frauen nicht zu einem persönlichen Treffen bereit waren. Frauen stossen häufig auf Unverständnis in ihrem Umfeld, weil sie die Beziehung zu ihrem drogenabhängigen Partner aufrechterhalten (siehe Bemerkungen Dagmar in Kap. 5.2.3.9). Dies könnte dazu führen, dass Partner sich mehr noch als andere Angehörige schämen, einen suchtkranken Angehörigen zu haben im Sinne einer Selbststigmatisierung (Nieuwenboom, 2012, S. 21). Diese Gefühle der Scham könnten mit dem persönlichen Treffen, welches für die Befragung notwendig gewesen wäre, nicht vereinbar gewesen sein.

\subsubsection{Angehörige und die Schweizer Durchschnittbevölkerung}

Die Auswertungen machen deutlich, dass die Angehörigen in der vorliegenden Stichprobe im Vergleich zur Schweizer Durchschnittbevölkerung über ein sehr hohes Bildungsniveau verfügen (Flückiger \& Falter, 2004, S. 10), was zur Folge hat, dass auch der Beschäftigungsgrad bei den befragten Personen hoch war (NEETs, 2012). Nur eine Person war zum Zeitpunkt der Befragung auf Arbeitssuche (1.1\%). Dies entspricht einer niedrigeren Arbeitslosenquote als die aktuell gültige Arbeitslosenquote von $3.2 \%$ der Schweizer Bevölkerung (Schweizerische Eidgenossenschaft Staatssekretariat für Wirtschaft SECO, 2013). Obwohl keine Einkommenszahlen vorliegen, ist davon auszugehen, dass die Angehörigen aufgrund des Bildungs- und Beschäftigungsgrades mit grosser Wahrscheinlichkeit der Mittelschicht angehören. Allerdings könnte auch hier ein Selektionseffekt gewirkt haben, nämlich dass nur Angehörige, die über ein gewisses Bildungsniveau verfügten, sich von den Studienaufrufen angesprochen fühlten und sich auch darauf meldeten. Das Bildungs- und Beschäftigungsniveau lässt zudem vermuten, dass die suchtkranken Menschen, deren Angehörige an dieser Studie teilnahmen, ebenfalls mit grosser Wahrscheinlichkeit ursprünglich der Mittelschicht entstammen dürften und nicht aus armen Familien, die einen sozialen Abstieg hinter sich haben (Dodgen \& Shea, 2000; zit. nach Thomasius, Sack, Küstner \& Schindler, 1993, S. 90).

Diese Ausführungen zur Stichprobe sollen in Bezug auf die Diskussion der weiteren Ergebnisse im Hinterkopf behalten werden. Sie haben mit grosser Wahrscheinlichkeit nur Gültigkeit für einen begrenzten Teil der Menschen, die durch eine Suchterkrankung betroffen 
sind, nämlich Frauen, die mindestens eine Berufslehre abgeschlossen haben und in irgendeiner Form einer Erwerbstätigkeit nachgehen.

\subsection{Inventar Subjektiver Stigmaerfahrungen (ISE)}

\subsubsection{Mängel des Inventars}

\subsubsection{Einleitung}

Das ISE ist ein neues Instrument, welches Stigmaerfahrungen von Angehörigen von suchtkranken Menschen erfassen möchte. Die Validierung des Inventars steht im Moment im deutschsprachigen Raum noch aus. Der Fragebogen zeigte in der vorliegenden Auswertung erhebliche Mängel sowohl bei der Durchführung als auch bei der Auswertung. Erste Hinweise, dass das Inventar einer Überarbeitung bedarf, zeigten sich bereits in den Nachbefragungen, in denen sich viele Angehörige negativ zum Fragebogen geäussert hatten. In den folgenden Kapiteln werden die Schwierigkeiten und möglichen Ursachen dafür diskutiert. Gleichzeitig werden Vorschläge gemacht, wie das Inventar verbessert werden könnte.

\subsubsection{Items}

Das Instrument zeigte auf Itemebene in mehrfacher Hinsicht Mängel. Während des Ausfüllens des Inventars kamen wiederholt Rückfragen von Angehörigen, weil ihnen die Fragen inhaltlich unklar waren. Die Problematik soll am Item Nr. 25 „Haben Erfahrungen mit Vorurteilen die Fähigkeit von Ihnen oder Ihrer Familie beeinträchtigt, Freundschaften zu schliessen oder aufrecht zu erhalten?" verdeutlicht werden. Einige Angehörige gaben an, dass Beziehungen in die Brüche gegangen seien, aber sie könnten nicht sagen, welche Bedeutung Vorurteilen dabei zugekommen sei. Die Gründe für die Veränderung von Freundschaften seien häufig multikausal gewesen. So berichteten sie beispielsweise davon, dass sie sich aufgrund der Belastungen zurückgezogen hätten, weil sie schlichtweg keine Lust gehabt hätten, jemanden zu treffen. Sie taten dies nicht zwangsläufig, weil sie sich schämten oder weil sie Angst vor Vorurteilen hatten. Eine Mutter berichtete beispielsweise davon, dass die Drogenabhängigkeit ihres Sohnes sie dazu veranlasst hatte, ihre Beziehungen zu überdenken und in wenige Beziehungen mehr Zeit zu investieren. Das Beispiel macht deutlich, dass zwar relevante Themen für Angehörige im Inventar angesprochen werden, aber immer in Bezug auf Stigmatisierungen, was für viele Angehörige oft nicht eindeutig zu beantworten war. 
Ein weiteres Problem war die Ambiguität einiger Items. Für viele war nicht klar, ob sich die Fragen auf real erlebte oder nur antizipierte Vorurteile bezogen. Während Angehörige zwar im öffentlichen Bild wahrnehmen, dass Vorurteile gegenüber Suchtkranken vorhanden sind, erleben sie diese eher selten direkt. So erzählte eine andere Mutter, dass sie im Rahmen ihrer beruflichen Tätigkeit manchmal mit drogenabhängigen Menschen in Kontakt komme. Dort hätte sie schon erlebt, dass sich Arbeitskollegen, die nichts von ihrer Betroffenheit wüssten, negativ über die Familien dieser Menschen geäussert hätten. Sie hätte aber noch nie direkt Vorurteile erlebt, wenn sie sich geöffnet habe oder wenn jemand von der Drogenabhängigkeit ihres Sohnes erfahren habe.

In der Nachbefragung sagten zudem viele Angehörigen direkt, dass sie die Fragen des ISE im Vergleich zu den anderen Fragebögen irrelevant finden würden für ihre Situation und dass sie nichts damit anzufangen wüssten. Die schlechte Akzeptanz des ISE könnte schliesslich auch dafür verantwortlich gewesen sein, dass das Inventar im Vergleich zu den anderen Fragebögen eine deutlich höhere Zahl an fehlenden Werten produzierte, weil viele nicht wussten, wie sie die Fragen beantworten sollten.

\subsubsection{Faktorenstruktur}

Bei der Überprüfung der Faktorenstruktur zeigten sich schliesslich weitere Probleme. Die in der englischen Originalversion vorgeschlagene Zwei-Faktorenstruktur (Stuart et al., 2005) liess sich nicht bestätigen. Die Faktorenanalyse brachte in der vorliegenden Stichprobe überhaupt keine verlässlichen interpretier- oder generalisierbaren Ergebnisse. Dies könnte ebenfalls mit der von den Angehörigen kritisierten Ambiguität der Items zusammenhängen. Aufgrund der Validitätsprobleme konnte sich die Faktorenstruktur allenfalls nicht herausbilden, weil unklar war, welche Konstrukte durch das Instrument überhaupt erhoben worden sind. Diejenigen Angehörigen, die die Fragen beantworteten, taten dies eventuell nach eigenem Ermessen und Gutdünken und gingen wahrscheinlich nicht immer von der gleichen Annahme aus. Dies lässt sich allerdings nur vermuten - ausgehend von den Rückmeldungen, die zum Instrument gemacht wurden.

Das Instrument ist deshalb wie bereits in Kapitel 5.1.3.4 in den weiteren Auswertungen nicht berücksichtigt. 


\subsubsection{Bedeutung von Stigmatisierung für die Angehörigen}

Obwohl bei der Auswertung der quantitativen Daten des ISE und aufgrund der Rückmeldungen der Angehörigen auf das Inventar der Eindruck entstanden ist, dass Vorurteile und Stigmatisierung für Angehörige nicht relevant sind, zeigte sich bei den qualitativen Daten ein etwas anderes Bild. Im Prozess der induktiven Kategorienbildung zeichnete sich ab, dass Stigmatisierungen für Angehörige durchaus ein wichtiges Thema sind. Über direkte Stigmaerfahrungen, die sich gegen die Angehörigen gerichtet hatten, berichteten allerdings nur vier der 13 Angehörigen. Dazu gehörten einerseits drei Eltern, die bereits lange betroffen waren und mit Schuldzuweisungen in der Vergangenheit konfrontiert worden waren, und andererseits der Sohn drogenabhängiger Eltern, der mit den suchtkranken Eltern gleichgesetzt worden war. Gerade bei Kindern von drogenabhängigen Eltern entstand die Vermutung, dass besonders starke Vorurteile wirken könnten. Neben Dylan, zu dem qualitative Daten vorliegen, berichteten auch die anderen erwachsenen Kinder mit einem drogenabhängigen Elternteil, dass sie auf viele Vorurteile stossen würden, wenn andere erfahren würden, dass sie suchtkranke Eltern hätten.

Eine weitere Gruppe, die von Stigmatisierungen betroffen sein dürfte, sind Partner von drogenabhängigen Menschen. In Kapitel 6.2.1 wurde bereits thematisiert, dass diese Angehörigen besonders schwierig zu rekrutieren waren. Die Bemerkungen, die die Partnerinnen zudem in den Befragungen machten, liessen vermuten, dass sie von ihrem Umfeld eher wenig Verständnis für ihre Situation bekommen.

Anstelle von Stigmatisierungen in interpersonellen Interaktionen erzählten Angehörige dagegen häufiger von Stigmatisierungen, die sich gegen suchtkranke Menschen richteten und die sie als belastend erlebten. Dies konnten einerseits negative Bemerkungen sein, die ihnen gegenüber gemacht wurden oder die sie mitbekommen hatten. Unabhängig davon berichteten Angehörige andererseits von Vorurteilen, die sie gegenüber von Suchtkranken antizipierten. Sie gaben beispielsweise an, dass sie sich Sorgen machen würden, dass ihr Angehöriger keinen Job mehr finden könnte, wenn auskommen würde, dass er drogenabhängig sei. Dabei spielten oft auch Befürchtungen der Suchtkranken selbst eine entscheidende Rolle, die ihre Angehörigen dazu anhielten, niemandem von der Abhängigkeit zu erzählen. Insgesamt ist diese Art der Stigmatisierungen dem Bereich des öffentlichen Bildes (Link \& Phelan, 2001) zuzuordnen. 
Eine weitere Form der Stigmatisierung, die von den Angehörigen in den Interviews thematisiert wurde, ist dem Bereich der strukturellen Diskriminierung (Link \& Phelan, 2001, S. 372) zuzuordnen. So erzählte ein Vater, dass sie als Eltern dazu aufgefordert worden seien, für die Schulden ihrer drogenabhängigen Kinder aufzukommen. Zudem beklagten Angehörige aktuelle Strukturen, die begünstigen würden, dass ihre suchtkranken Familienmitglieder immer tiefer in Schwierigkeiten abrutschen würden, beispielsweise dadurch, dass Kreditinstitute ihren Angehörigen trotz Kreditsperren aktiv wieder Kredite anbieten würden.

Dieser Befund, dass für die Angehörigen vor allem Stigmatisierungen im öffentlichen Bild und strukturelle Diskriminierungen ein Thema sind, deckt sich auch mit den Ergebnissen aus der Befragung von Angehörigen von schizophrenen Patienten (Angermeyer et al., 2003, S. $600)$.

\subsubsection{Verbesserungsvorschläge}

Aufgrund der Ergebnisse der vorliegenden Arbeit scheint eine Überarbeitung des ISE unerlässlich. Das ISE hat einen starken Fokus auf den Stigmatisierungen, die Angehörige erleben. Diese sind aber verglichen mit den Stigmatisierungen, die sie gegenüber ihren suchtkranken Angehörigen wahrnehmen, meist nur wenig relevant. Die Stigmatisierung ihrer Angehörigen ist für sie sehr belastend und macht sie betroffen, aber nicht in dem Sinn, dass sie einen direkten Zusammenhang mit sich selbst herstellten. Aus diesem Grund müsste sich das Instrument allenfalls stärker auf die Ebene des öffentlichen Bildes und der strukturellen Diskriminierung konzentrieren.

Eine allfällige Verbesserung könnte zudem dadurch erreicht werden, dass als Antwortoption „,ich weiss nicht“ oder „kann ich nicht beantworten“ hinzugefügt würde. Einige der Angehörigen merkten während den Befragung an, dass sie einige der Fragen gar nicht beantworten könnten, da sie beispielsweise nicht wüssten, ob andere Familienmitglieder irgendwann in der Vergangenheit auf Vorurteile gestossen seien.

Für die Befragung der Angehörigen mittels ISE könnte zudem relevant sein, in welcher Beziehung die Angehörigen zum Drogenabhängigen stehen. Dies könnte über verschiedene Versionen berücksichtigt werden. Die qualitativen Daten und die Gespräche mit den erwachsenen Kindern von drogenabhängigen Eltern legen den Schluss nahe, dass diese die 
meisten Diskriminierungen erfahren, weil bei ihnen das Courtesy Stigma besonders stark wirkt, sprich die Vorurteile, die ihre Eltern treffen, färben meist eins zu eins auf ihre Kinder ab. In besonderer Weise betroffen von Stigmatisierungen sind mit grosser Wahrscheinlichkeit aber auch Partner von drogenabhängigen Menschen. Dies lassen ebenfalls die qualitativen Daten vermuten. So berichtete Dagmar, die Partnerin eines heroinabhängigen Mannes, dass sie von ihrem Umfeld kein Verständnis bekomme, dass sie mit ihrem Freund zusammen sei. Dies wiederum hat einen entscheidenden Einfluss auf das Belastungserleben und die Bewältigungsmöglichkeiten.

\subsection{Belastungen}

Die vorliegende Arbeit macht deutlich, dass Angehörige von drogenabhängigen Menschen eine grosse Zahl von Belastungen erleben und dass diese auch massgeblich die psychische und somatische Gesundheit der Angehörigen beeinflusst.

\subsubsection{Belastungsfaktoren}

Die im Rahmen der qualitativen Befragung erhobenen Daten bestätigten, dass Angehörige unter einer Vielzahl an Belastungen leiden. Diese dürften trotz der kleinen Stichprobe von nur 13 Personen universelle Gültigkeit haben. Kirby et al. (2005, S. 45) haben verschiedene Belastungsbereiche für Angehörige vorgeschlagen und quantitativ untersucht. Diese Problembereiche wurden in der vorliegenden Arbeit mit einigen wenigen Adaptionen in die qualitative Datenauswertung übernommen und bestätigten sich mehrheitlich.

Alle Angehörigen berichteten über emotionale Belastungen im Zusammenhang mit der Suchterkrankung. Diese sind allerdings viel umfassender als Kirby et al. (2005, S. 45) es vorgeschlagen haben. Während Schlafstörungen, Schuldgefühle und Enttäuschungen in der „Significant Other Checklist“ (Kirby et al., 2005) als relevante emotionale Belastungen angesehen wurden, wurden in der vorliegenden Arbeit viele weitere wichtige Aspekte identifiziert. Dazu gehören Gefühl von Hilflosigkeit und Hoffnungslosigkeit. Für viele Angehörige ist zudem sehr belastend, dass sie sich in einer ständigen Ambivalenz befinden in Bezug auf ihren drogenabhängigen Angehörigen. Dies kann sich auf unterschiedliche Weise zeigen. Einerseits haben sie Angst, den drogenabhängigen Angehörigen zu verlieren und wünschen sich andererseits, dieser möge sterben. Sie möchten sich abgrenzen und fühlen sich gleichzeitig verantwortlich. Sie wünschen sich mehr Distanz und machen sich gleichzeitig 
Sorgen, wenn sie den drogenabhängigen Angehörigen nicht erreichen können, um nur einige Beispiele zu nennen. Eine Belastung, die auch von Orford et al. (1998c) angegeben wird, sind die ständigen Notfälle und die Alarmbereitschaft, in der sich die Angehörigen dauernd befinden. In der vorliegenden Untersuchung schien das ein Aspekt zu sein, der vor allem die Eltern von drogenabhängigen Kindern betraf. Weitere emotionale Belastungen sind die Angst, dass andere Familienmitglieder ebenfalls drogenabhängig werden könnten oder Sorgen in Bezug auf die Zukunft des drogenabhängigen Angehörigen, ebenfalls Belastungen, die Orford et al. (1998c) bereits vorgeschlagen hatten. Hinzu kommt, dass sich der drogenabhängige Angehörige durch die Suchterkrankung in der Persönlichkeit verändert, ein Aspekt den Oreo und Ozgul (2007) in ihrer Arbeit untersucht haben. Dies ist für die Angehörigen ebenfalls mit schmerzlichen Gefühlen verbunden.

Die finanzielle Belastung, die die Drogenabhängigkeit ihres Angehörigen für die Angehörigen selbst bedeutet, wurde von verschiedenen Autoren (Kirby et al., 2005; Orford et al., 1998c) betont. Es war folglich nicht überraschend, dass alle Angehörigen in der vorliegenden Stichprobe ebenfalls von finanziellen Belastungen berichteten. Dazu gehören Kosten für den Lebensunterhalt, die die Angehörigen übernehmen, Rechnungen und Schulden, die sie begleichen, aber auch Diebstahl von Wertgegenständen. Während bisher nur wenig untersucht wurde, was die Suchterkrankung die Angehörigen kostet, wurden sie in der vorliegenden Befragung danach gefragt. Es zeigte sich, dass Angehörige gar nicht so genau wissen, wieviel sie für ihre drogenabhängigen Familienmitglieder ausgeben. Ein Grund hierfür ist, dass nicht alle Zuwendungen aufgeschrieben werden oder dass die Angehörigen nach einer bestimmten Zeit damit aufhören, weil die Kosten so immens sind. Für einige kann das Aufschreiben der Kosten aber auch zur Folge haben, dass sie beschliessen, kein Geld mehr zu geben. Die von den Angehörigen genannten und dokumentierten Beträge, bewegten sich zwischen CHF 30'000.00 und CHF 200'000.00.

Beinahe alle Angehörigen berichteten von Beziehungsproblemen. Angehörige erzählten in diesem Zusammenhang von Konflikten mit dem Betroffenen, aber auch von Streitereien innerhalb der Familie in Bezug auf den Suchtkranken. Dies war besonders häufig Thema bei Eltern, die angaben, dass die Drogenabhängigkeit des Kindes die Partnerschaft stark belastete. Es wurde aber auch genannt, dass der drogenkonsumierende Angehörige nicht verlässlich sei. Dies zeigte sich insbesondere in der Elternschaft von Drogenabhängigen. All diese Aspekte sind in Übereinstimmung mit den von Kirby et al. (2005, S. 45) 
vorgeschlagenen. Ergänzend berichteten Angehörige in der vorliegenden Stichprobe auch von einer Zunahme der Konflikte mit dem drogenabhängigen Partner ihres suchtkranken Angehörigen und von vorübergehenden Beziehungsabbrüchen und -unterbrüchen. Auch Orford et al. (1998c) stellten im Rahmen ihrer Untersuchung fest, dass das Zusammenleben und -sein mit einer suchtkranken Person für die Angehörigen nicht einfach ist.

Zehn der 13 Angehörigen machten Erfahrungen mit Gewalt und Drohungen im Zusammenhang mit ihrem drogenabhängigen Familienmitglied. Fünf von ihnen erlebten dabei schon körperliche Gewalt. Die Erfahrungen mit Drohungen variierten, während die einen direkt bedroht wurden, erlebten andere eher indirekte Drohungen, die sich in Äusserungen wie „Du bist Schuld, wenn ...“ zeigten. Dies ist in Übereinstimmung mit Kirby et al (2005, S. 45). Was sich aber zusätzlich gezeigt hat, ist, dass Angehörige gute Zusammenhänge herstellen können zwischen Gewalt und unterschiedlichen Substanzen, so dass sie ein Konsum von bestimmten Substanzen wie Alkohol oder Kokain als belastender empfanden als einer von Heroin.

Zur Beeinträchtigung des Lebensalltages kommt es vor allem, wenn die Angehörigen mit dem Suchtkranken zusammenleben. Die Angehörigen müssen dann damit umgehen, dass in der gemeinsamen Wohnung konsumiert wird. Lebt die drogenkonsumierende Person normalerweise nicht mit den Angehörigen zusammen und verliert die Unterkunft, dann sind es meist die Angehörigen, die die Betroffenen vorübergehend bei sich aufnehmen. Dies sind Aspekte, die Kirby et al. (2005, S. 45) bereits berichteten und die sich auch in der qualitativen Auswertung der Daten dieser Arbeit zeigten. Ergänzend gaben Angehörige im Rahmen dieser Befragung an, dass sie teilweise keinen Besuch mit nach Hause nehmen würden, weil sie nie wüssten, in welchem Zustand sie die Wohnung beim Nachhausekommen antreffen würden. Während Angehörige eigenen Besuch weniger mitbringen, erleben sie in Kontrast dazu, dass der Betroffene fremde Leute und zum Teil auch Dealer mit zu ihnen nach Hause bringt. Dieses Ergebnis deckte sich somit mit der Befragung von Orford et al. (1998c).

Nicht alle Angehörigen erleben rechtliche Probleme im Zusammenhang mit der Suchterkrankung. Durch den illegalen Substanzkonsum erhöht sich allerdings die Wahrscheinlichkeit erheblich, mit dem Gesetz in Konflikt zu geraten, so dass die grosse Mehrheit der Angehörigen schon in irgendeiner Weise illegale Machenschaften mitbekommen hatte oder direkt davon betroffen gewesen war. In diesem Zusammenhang 
wurden Hausdurchsuchungen genannt, was für diejenigen, die das erlebt hatten, sehr belastend und schlimm gewesen war. Andere hatten mitbekommen, dass ihr Angehöriger verhaftet worden war oder aufgrund von illegalen Geschäften wie Dealen Gefahr lief, verhaftet zu werden. Auch dies steht in Konkordanz mit den Angaben von Kirby et al. (2005, S. 45)

Bei Kirby et al. (2005, S. 45) bezogen sich die Gesundheitssorgen vorwiegend auf den drogenabhängigen Angehörigen. Orford et al. (1998c) hatten ebenfalls angegeben, dass sich Angehörige im Rahmen von diversen Sorgen, die sie sich um die drogenkonsumierende Person machten, auch Sorgen wegen deren Gesundheit machen würden. In der vorliegenden Untersuchung war dies allerdings eher am Rande Thema bei den Angehörigen und wurde nur vereinzelt direkt angesprochen. Vor allem Eltern machten sich Sorgen um die Gesundheit ihrer drogenabhängigen Kinder. Der Fokus lag vielmehr auf den Auswirkungen der Suchterkrankung auf die Gesundheit der Angehörigen. Im nachfolgenden Kapitel 6.4.2.1. werden die Auswirkungen der Suchterkrankung auf die Angehörigen diskutiert.

Als weiterer relevanter Belastungsfaktor wurden Stigmatisierungen identifiziert. Dieser Aspekt wurde bereits in Kapitel 6.3.2 diskutiert. Während bei Kirby et al. (2005) dieser Aspekt gänzlich unbeachtet bleibt, haben Orford et al. (1998c) in ihrer Arbeit darauf aufmerksam gemacht, dass die Abhängigkeitserkrankung eines nahestehenden Menschen die sozialen Beziehungen der Angehörigen beeinträchtigen kann.

In der nachfolgenden Darstellung (Tab. 22) sind relevante Belastungsfaktoren für Angehörige zusammengestellt. In der Tabelle werden die Ergebnisse der vorliegenden Arbeit sowie die Ergebnisse aus anderen Studien (Kirby et al., 2005; Orford et al., 1998c) berücksichtigt.

Tab. 22: Relevante Belastungsfaktoren der Angehörigen von drogenabhängigen Menschen

Emotionale Belastungen

- Schuldgefühle

- Auswirkungen der Suchterkrankung auf Dritte (Belastung, Abhängigkeitserkrankung bei Dritten)

- Enttäuschungen und Vereinsamung des suchtkranken Angehörigen

- Ambivalente Gefühle (Hoffnung vs. Hoffnungslosigkeit, Wunsch, dass der Angehöriger stirbt vs. Angst vor Verlust, Distanz vs. Nähe, etc.)

- Zukunftsängste, die den suchtkranken Angehörigen betreffen (Job, Gesundheit)

- Persönlichkeitsveränderung durch die Suchterkrankung (Lügen, betrügen)

- Hilflosigkeit und Ohnmacht

- Hoffnungslosigkeit / Resignation

- Rückfälle 
Fortsetzung Tab. 22: Relevante Belastungsfaktoren der Angehörigen von drogenabhängigen Menschen

\section{Finanzielle Belastungen}

- Kosten für den Lebensunterhalt, den Angehörige übernehmen

- Schulden, die Angehörige übernehmen, auf sie zurückfallen oder vom Suchtkranken nicht zurückbezahlt werden

- Bussen, die Angehörige übernehmen

- Diebstahl

- Angehörige können sich bestimmte Dinge nicht mehr leisten bzw. müssen verzichten

- Verwandtenunterstützungspflicht

\section{Beziehungsprobleme}

- Streit mit dem drogenabhängigen Angehörigen

- Streit mit weiteren Angehörigen wegen des suchtkranken Familienmitgliedes

- Konflikte mit dem suchtkranken Partner des drogenabhängigen Angehörigen

- Beziehungsab- und -unterbrüche

- Angehöriger ist nicht verlässlich

- Vernachlässigung der Kinder

\section{Rechtliche Probleme}

- Hausdurchsuchungen

- Verhaftungen und Gefängnisaufenthalte

- Illegale Aktivitäten des Angehörigen (dealen), die (noch) nicht zur Anzeige geführt haben

- Anwalt besorgen

- Bewährungen

\section{Gesundheitssorgen}

- Gesundheitssorgen und gesundheitliche Probleme des suchtkranken Angehörigen

- Sorge und Pflege, wenn suchtkranker Angehöriger krank, high oder verletzt ist

- Sexuell übertragbare Krankheiten

- Gesundheitliche Probleme der Angehörigen

Gewalt und Drohungen

- Körperliche Gewalt in der Familie

- Drohungen (direkt oder subtil über Schuldgefühle)

- Gewalt und Drohungen gegenüber dem suchtkranken Angehörigen durch Dritte

- Aggressives Verhalten v.a. im Zusammenhang mit bestimmten Substanzen

\section{Beeinträchtigung des Lebensalltages}

- Bei Wohnungsverlust Unterkunft gewähren

- Substanzkonsum

- Beschädigung von Gegenständen und Mobiliar

- Unordnung / Schmutz

- Fremde Leute in der Wohnung

- Freunde können nicht eingeladen werden

- Übernahme von Haushaltspflichten

\section{Stigmatisierungen}

- Diskriminierung und Stigmatisierung der Angehörigen im interpersonellen Kontakt

- Diskriminierung und Stigmatisierung des drogenabhängigen Angehörigen im interpersonellen Kontakt

- Strukturelle Diskriminierung

- Öffentliches Bild zu Drogenabhängigen und ihren Angehörigen

- Beeinträchtigung im Zugang zu sozialen Rollen 


\subsubsection{Auswirkungen der Belastungsfaktoren}

\subsubsection{Auswirkungen auf die Gesundheit}

Die Ergebnisse der vorliegenden Arbeit bestätigen, dass die Suchterkrankung eines nahestehenden Menschen deutliche Auswirkungen auf die Gesundheit der Angehörigen hat. Dies steht in Konkordanz mit verschiedenen anderen Studien (Lee et al., 2011; Orford et al., 1998c; Ray et al., 2007). So zeigte sich in der vorliegenden Untersuchung, dass $27.3 \%$ der Angehörigen den Cut-off für eine klinisch relevante Depression überschritten. Dieser Wert liegt erheblich über der 12-Monatsprävalenz der Schweizer Bevölkerung, welche auf $7 \%$ geschätzt wird (Baer, 2013, S. 10). Berücksichtigt werden muss bei der Interpretation dieses Ergebnisses, dass sich die Stichprobe mehrheitlich aus Frauen zusammensetzte, so dass grundsätzlich eine höhere 12-Monatsprävalenz zu erwarten war. Denn gemäss Baer (2013, S. 11) sind Frauen deutlich häufiger von Depressionen betroffen als Männer. Die Lebenszeitprävalenz für eine Major Depression für Frauen liegt bei $21 \%$ und diejenige für Männer bei $13 \%$ (ebd.). Nichtsdestotrotz zeigt das Ergebnis deutlich, dass der Anteil der Angehörigen, welche die Kriterien für eine klinisch relevante Depression erfüllten, im Vergleich zur Schweizer Durchschnittsbevölkerung erstaunlich hoch war. Dieser Befund bestätigte sich auch in den qualitativen Daten, wo Angehörige sehr häufig Symptome angaben, die sie auf die Suchterkrankung zurückführten und die mit einer depressiven Episode assoziiert sind. Besonders häufig genannt wurden Schlafstörungen, gefolgt von weiteren Symptomen wie Suizidgedanken und -versuche und Appetitlosigkeit. Weitere somatische Symptome, die Angehörige angaben, waren Verspannungen im Rücken, Zähneknirschen im Schlaf, drückende Schmerzen auf der Brust und stressbedingter Bluthochdruck.

Eine Erklärung für das erhöhte Depressionsrisiko der Angehörigen von suchtkranken Menschen kann das Diathese-Stress-Modell liefern (Rief \& Nanke, 2003, S. 108). Dieses betont das Zusammenspiel von psychosozialen Belastungsfaktoren und biologischer Prädisposition in der Entstehung von Krankheiten (ebd.). Seyle (1956; zit. nach Rief \& Nanke, 2003, S. 109) postulierte, dass bei längerem Fortbestehen der Stresssituation die körperliche Stressreaktion in folgenden drei Phasen verläuft:

1. Alarmreaktion: Initiale Reaktion auf einen Stressor.

2. Widerstandsphase: Mobilisierung komplexer Anpassungsprozesse des Organismus, um das innere Gleichgewicht wieder herzustellen. 
3. Erschöpfungsphase: Bei langer Dauer der Stressreaktion Zusammenbruch des Anpassungsvorgangs. (Seyle, 1956; zit. nach Rief \& Nanke, 2003)

Angehörige sind aufgrund der Suchterkrankung häufig jahrelang chronischem Stress ausgesetzt. Dies wird deutlich, wenn die lange Mitbetroffenheit der Angehörigen durch die Suchterkrankung, die mit massiven Belastungsfaktoren verbunden ist, berücksichtigt wird. In der vorliegenden Untersuchung gaben Angehörige nämlich an, dass ihre Angehörigen durchschnittlich seit 17 Jahren drogenabhängig waren, was eine unglaublich lange Zeitdauer ist. Dass das Diathese-Stress-Modell durchaus seine Gültigkeit haben könnte für Angehörige von suchtkranken Menschen zeigt sich unter anderem darin, dass sich im multiplen linearen Regressionsmodell (siehe Kap. 5.1.4.2.) alle Variablen, die einen signifikanten Einfluss auf den BDI-V hatten, mit dem obigen Modell zu erklären sind. Variablen, die grundsätzlich auf eine Erhöhung des Stresserlebens hindeuten, wie die durch die Angehörigen erlebte Belastung und die Anzahl der Sorgen, die sich Angehörige machten, führten zu einer deutlichen Zunahme der BDI-V-Werte. In die gleiche Richtung wirkte auch die Variable ,mit dem drogenabhängigen Angehörigen zusammenlebend“. Durch das Zusammenleben dürfte die Belastung für die Angehörigen erheblich grösser sein als für Angehörige, die räumlich eine gewisse Distanz halten zu ihren drogenabhängigen Angehörigen, nur schon dadurch dass ihr Lebensalltag weniger beeinträchtigt wird. Dagegen war eine Verbesserung der Suchterkrankung im vergangenen Jahr und somit mit grosser Wahrscheinlichkeit auch eine Abnahme der Belastungsfaktoren mit signifikant tieferen BDI-V-Werten assoziiert. Dies legt den Schluss nahe, dass die Stärke der Belastung für die Angehörigen einen deutlichen Einfluss darauf hat, ob Angehörige psychisch krank werden.

\subsubsection{Die Rolle der Bewältigungsstrategien}

Lazarus und Folkman (1984; zit. nach Rief \& Nanke, 2003) betonen zusätzlich die Bedeutung von kognitiven Bewertungsprozessen im Zusammenhang mit dem Stresserleben. Sie definieren folgende Stufen in ihrem Modell:

1. Phase: Einschätzung der affektiven Bedeutung der Situation: Ist ein Ereignis eine Bedrohung oder unbedeutend? Nur Reize, die ein Mensch als bedrohlich einstuft, sind Stressoren.

2. Phase: Verfügbare Bewältigungsstrategien werden gesucht und ihr erwarteter Effekt eingeschätzt. 
3. Phase: Die Situation wird unter Berücksichtigung der erwarteten Bewältigungsmöglichkeiten und -effekte neu bewertet. (Lazarus \& Folkman, 1984; zit. nach Rief \& Nanke, 2003).

Das Modell macht deutlich, dass für das Stresserleben neben der Einschätzung des Ereignisses auf seine Bedeutung hin auch die Bewältigungsstrategien, die eine Person zur Verfügung hat, und deren Effektivität zur Bewältigung des Stressors relevant sind. In der vorliegenden Studie hatte sich sowohl in den Zero-Order-Vergleichen als auch im Regressionsmodell gezeigt, dass ein signifikanter Zusammenhang zwischen Bewältigungsstrategien, die das Stresserleben erhöhen und den BDI-V Werten bestand. Im multiplen linearen Regressionsmodell (siehe Kap. 5.1.4.3.) zeigte sich, dass „Resignation“, „Soziale Abkapselung“ und „Selbstbeschuldigung“ einen signifikanten Einfluss auf die BDI-V Werte der Angehörigen hatte. Dies kann wie folgt verstanden werden: Durch die jahrelange Betroffenheit, in der Angehörige Phasen der Besserung gefolgt von einer erneuten Verschlechterung der Suchterkrankung durchgemacht haben, kommen viele zur Einsicht, dass ihre Angehörigen ein Leben lang drogenabhängig bleiben werden. Sie resignieren gegenüber der Suchterkrankung. Dies zeigte sich deutlich in den qualitativen Daten, wo Angehörige von „Trostlosigkeit“ und „Hoffnungslosigkeit“ im Zusammenhang mit der Suchterkrankung sprachen. Angehörige sind der Problematik gegenüber zudem sehr machtlos. Die Situation scheint nicht bewältigbar, sondern kann nur hingenommen und ertragen werden.

Viele Angehörigen und insbesondere Eltern fragen sich oft, was sie falsch gemacht haben, dass ihr Kind drogenabhängig geworden ist. Angehörige machen zudem die Erfahrung, dass sie Auslöser für erneuten Konsum sein können, wenn es beispielsweise zu Konflikten mit dem drogenabhängigen Angehörigen gekommen ist. Dies kann Schuldgefühle auslösen. Die qualitativen Daten lassen zudem noch einen weiteren Zusammenhang für die Entstehung von Schuldgefühlen vermuten. Angehörige leben in einer ständigen Ambivalenz. Sie empfinden neben Fürsorge und Mitgefühl immer wieder auch Wut und wünschen ihrem suchtkranken Angehörigen manchmal den Tod an den Hals. Diese negativen Gefühle können auch Schuldgefühle auslösen, da der drogenabhängige Angehörige krank ist.

Angehörige, die sich immer mehr zurückziehen, scheinen ebenfalls ein höheres Risiko zu haben, depressiv zu werden. Durch einen sozialen Rückzug in Belastungssituationen stehen 
ihnen weniger Möglichkeiten zur sozialen Unterstützung zur Verfügung. Dabei scheint gerade die soziale Unterstützung bedeutsam zu sein bei der Bewältigung der Belastungen. Dies postuliert die 5-Step-Intervention (Copello, Templeton \& Orford et al., 2010b) und wird auch in den qualitativen Ergebnissen dieser Arbeit bestätigt, wo alle Angehörigen angaben, dass soziale Unterstützung für sich hilfreich gewesen sei.

Interessanterweise hatten Bewältigungsstrategien, die das Stresserleben reduzieren, keinen Einfluss auf die BDI-V Werte in der vorliegenden Untersuchung. Dies bedeutet konkret, dass vor allem die negativen Bewältigungsstrategien das Risiko für eine klinisch relevante Depression erhöhen, positive Bewältigungsstrategien hingegen keine protektive Wirkung bei den Angehörigen haben, was für Interventionen bei den Angehörigen bedeutsam ist. Allenfalls könnten Methoden der Kognitiven Umstrukturierung bei stark belasteten Angehörigen gute Resultate bringen.

Insgesamt lässt sich aus den Ergebnissen folgende Hypothese formulieren: Menschen, die grundsätzlich zu negativen Bewältigungsstrategien neigen, die das Stresserleben eher erhöhen, tragen in schwierigen, chronischen Belastungssituationen, wie es beispielsweise die Drogenabhängigkeit eines Familienmitgliedes darstellt, eher das Risiko eine klinisch relevante Depression zu entwickeln. Dieses Risiko scheint auch zu bestehen, wenn sie in ähnlicher Weise wie andere positive Bewältigungsstrategien anwenden.

\subsubsection{Charakterisierung von stark belasteten Angehörigen}

In der vorliegenden Arbeit wurden zwei Gruppen von Angehörigen anhand ihrer erlebten Belastungen identifiziert. Die beiden Gruppen unterschieden sich nicht nur in Bezug auf ihre Belastungen signifikant voneinander, sondern auch bezüglich der Depressivität. Alle Angehörigen bis auf eine Person, die der Gruppe der stark belasteten Angehörigen zugeteilt wurde, hatten den Cut-off von 35 des BDI-V für eine klinisch relevante Depression überschritten.

In der weiteren Analyse zeigte sich, dass insbesondere somatische Beschwerden des BDI-V wie Müdigkeit, Schlafstörungen und Appetitlosigkeit besonders gut zwischen den beiden Gruppen von Angehörigen diskriminierten. Auch in der logistischen Regression (siehe Kap. 5.1.5.4.) bestätigte sich dies, wo körperliche Beschwerden wie Kopf- oder Magenschmerzen, Herzklopfen und -stechen, Abgeschlagenheit und Schlafstörungen eine gute Voraussage 
machen konnten, ob jemand zu den stark belasteten Angehörigen gehört oder nicht. Die Vermutung liegt nahe, dass die stark belasteten Angehörigen, die in der vorliegenden Untersuchung auch eine klinisch relevante Depression entwickelt hatten, aufgrund von körperlichen Beschwerden mit grosser Wahrscheinlichkeit in den Hausarztpraxen auftauchen dürften.

Relevant für die Identifizierung von stark belasteten und somit mit grosser Wahrscheinlichkeit auch depressiven Angehörigen, die Behandlung bräuchten, sind neben somatischen Beschwerden die Anzahl der Sorgen, über die sie berichten. Je mehr Schwierigkeiten Angehörige bei ihren Finanzen, in der Arbeit, in ihren Beziehungen und in Bezug auf ihre Gesundheit wahrnehmen, desto grösser ist ihr Risiko, der stark belasteten Gruppe von Angehörigen anzugehören, die psychisch krank werden.

Berichten Angehörige von suchtkranken Menschen über viele somatische Beschwerden und eine grosse Anzahl an Sorgen, scheint es sinnvoll, diese auf eine klinisch relevante Depression hin zu untersuchen.

\subsubsection{Auswirkungen der Suchterkrankung auf die Kinder}

In der vorliegenden Studie gibt es nur wenige Hinweise zu den Auswirkungen der Suchterkrankung auf Kinder. In der Untersuchung hatten nur fünf erwachsene Kinder von drogenabhängigen Eltern teilgenommen. Zudem liegen noch die Daten einer um einige Jahre jüngeren Schwester eines heroinabhängigen Mannes vor, die noch ein Kind war, als der Bruder mit dem Heroinkonsum begann. Die wenigen Daten lassen aber vermuten, dass die Auswirkungen der Suchterkrankung auf Kinder gravierend sind. Obwohl die Ergebnisse aufgrund der kleinen Gruppenzahl in der ANOVA nicht signifikant wurden, zeigte sich in Abb. 6 dennoch deutlich, dass die erwachsenen Kinder von drogenabhängigen Eltern die deutlich höchsten BDI-V Werte erreichten. Dies spricht dafür, dass die Suchterkrankung der Eltern langfristige Auswirkungen auf ihre Kinder hat.

Die Beispiele von Dorothea und Dylan, welche an der qualitativen Befragung teilnahmen, zeigen zudem klar, dass Kinder bereits sehr früh verstehen, dass etwas nicht stimmt, auch wenn sie es noch nicht klar einordnen können. Dorothea konnte sehr genau beschreiben, dass sie die Unmöglichkeit, die Ereignisse richtig einzuordnen, verunsichert hatte. Dadurch wird die Belastung, die für Kinder ohnehin schon sehr gross ist, noch weiter erhöht. Es ist deshalb 
wichtig, dass Kinder altersadäquat darüber aufgeklärt werden, was es bedeutet, wenn jemand suchtkrank ist.

Das Beispiel von Dylan macht zudem deutlich, in welch schwieriger Situation die Kinder von drogenabhängigen Eltern sind. Sie erleben nicht nur, dass Eltern nicht verlässlich sind, sondern sind häufig auch Gewalt ausgesetzt. Dabei befinden sie sich in einem ständigen Loyalitätskonflikt gegenüber ihren Eltern. Die Gespräche mit den erwachsenen Kindern machten weiter deutlich, dass Kinder ihre Eltern schützen und diese nicht verraten, auch wenn die Situation für sie noch so belastend sein mag. Professionelle Helfer müssen sich dessen bewusst sein. Es ist zudem Aufgabe all jener, die mit den drogenabhängigen Eltern zu tun haben, das Kindswohl immer im Hinterkopf zu behalten und im Notfall auch über das Wohl der Eltern zu stellen.

\subsection{Bewältigungsstrategien}

\subsubsection{Bewältigungsstrategien, die Angehörigen anwenden}

In der vorliegenden Arbeit hatten die drei Hauptpositionen „standhaft bleiben“, „die Situation erdulden“ und „sich davon zurückziehen“, die Orford, Velleman et al. (2010) vorgeschlagen hatten, als Grundlage gedient. Diese hatten sich in der Auswertung als sinnvoll bestätigt. Orford, Velleman et al. (2010, S. 51) betonen im Rahmen ihrer Arbeit zudem, dass Angehörige häufig mehrere Bewältigungsstrategien gleichzeitig anwenden - ein Umstand, der sich auch in dieser Arbeit bestätigte. Angehörige nehmen häufig nicht nur eine Position ein, sondern verfolgen gleichzeitig mehrere Bewältigungsstrategien. So können sie ihren Angehörigen sowohl klare Regeln vorgeben, die das Zusammensein betreffen, ihre Angehörigen aber auch unterstützen, wenn diese etwas gegen die Abhängigkeitserkrankung unternehmen möchten und gleichzeitig versuchen, sich hin und wieder selbst etwas Gutes zu tun. Selbstverständlich können nicht alle drei Positionen im gleichen Ausmass vertreten sein und die Balance zu finden ist äusserst schwierig (Orford \& Velleman et al., 2010, S. 51). Dennoch zeigte sich, dass sich die Angehörigen ihren Möglichkeiten entsprechend höchst adaptiv verhalten und ihr eigenes Coping immer wieder hinterfragen.

Die drei Positionen wurden wie bei Orford, Velleman et al. (2010) ebenfalls in der vorliegenden Arbeit weiter differenziert. Bei Orford, Velleman et al. (2010, S. 55) zeichnet sich „standhaft bleiben“ dadurch aus, dass die Angehörigen versuchen, den Umgang mit der 
Suchterkrankung und mit den Betroffenen nach neuen, anderen Regeln stattfinden zu lassen. In der vorliegenden Arbeit wurde die Definition wie folgt verändert: Sämtliche Verhaltensweisen, die Angehörige an den Tag legen, um gegen die Abhängigkeitserkrankung anzukämpfen und eine Abnahme des Substanzkonsums zu erreichen, wurden unter dem Begriff „Aufbegehren“ gegen die Suchterkrankung zusammengefasst. Dabei kann unterschieden werden zwischen eher konfrontierenden-kontrollierenden Verhaltensweisen und unterstützend-verständnisvollen Strategien. $\mathrm{Zu}$ den konfrontierenden-kontrollierenden Strategien zählen das Kontrollieren des Suchtkranken in mehrfacher Hinsicht, das Anschreien des Betroffenen und das Ausüben von Druck. Dies sind Bewältigungsstrategien, die auch Orford, Velleman et al. (2010, S. 55) beschreiben. Zu den unterstützend-verständnisvollen Strategien zählt der Wunsch, helfen zu wollen oder unterstützende Verhaltensweisen wie das Geben von Geld, die Aufnahme des Angehörigen bei sich zu Hause, um so eine Verbesserung beim Drogenkonsum zu erreichen. Diese Verhaltensweisen wurden vor allem im Zusammenhang mit dem Beginn der Suchterkrankung genannt. Eine weitere Strategie, die unterstützend-verständnisvoll ist, ist die Unterstützung, die Angehörige der drogenkonsumierenden Person bieten, wenn es darum geht, therapeutische Hilfe in Anspruch zu nehmen. Angehörige können in solchen Situationen bei der Vermittlung von Therapieangeboten helfen oder auch ihre Angehörigen begleiten. Eine etwas andere Form des unterstützend-verständnisvollen Aufbegehrens, welches bei Orford, Velleman et al (2010) nicht genannt wird, ist das politische Engagement. Angehörige können sich nicht nur für die Belange der Betroffenen und deren Angehörigen einsetzen, sondern engagieren sich manchmal auch für eine bessere Aufklärung.

Die Gründe, weshalb Angehörige sich gegenüber der Suchterkrankung tolerant oder akzeptierend verhalten, sind gemäss Orford, Velleman et al. (2010, S. 54) vielschichtig (siehe Kap. 2.3.2.2.). In der vorliegenden Untersuchung stellte sich die Situation wie folgt dar: Nur etwa die Hälfte der Angehörigen gab an, sich inaktiv oder akzeptierend gegenüber dem Substanzkonsum zu verhalten. Die meisten Angehörigen, die ihre Toleranz gegenüber dem Drogenkonsum zum Ausdruck brachten, waren bereits lange betroffen und hatten wiederholt die Erfahrung von Rückfällen gemacht, so dass sie zum Schluss gekommen waren, dass ein Weg mit dem Konsum gefunden werden musste. Neben Angehörigen, die lange betroffen waren, sprachen vor allem diejenigen Angehörigen von einem toleranten und akzeptierenden Coping gegenüber dem Substanzkonsum, die als Kinder betroffen gewesen waren. Obwohl die Zahl der Befragten zu gering ist, um dieses Ergebnis generalisieren zu können, scheint 
dieser Befund dennoch auf der Hand zu liegen. Kinder sind der Drogenabhängigkeit eines ihnen nahestehenden Menschen stärker ausgeliefert als Erwachsene. Ihre Möglichkeiten zur Unabhängigkeit oder zum Aufbegehren gegenüber dem Substanzkonsum sind begrenzt, so dass ihnen letztlich nichts anderes übrig bleibt, als die Situation so hinzunehmen, wie sie ist. Das Beispiel von Dorothea in der vorliegenden Arbeit zeigt, welche gravierende Folgen, dies für die Betroffenen haben kann. Sie hatte aufgrund des Heroinkonsums ihres Bruders nie gelernt, anders mit Problemen umzugehen, als diese hinzunehmen und runterzuschlucken, was in ihren Augen schliesslich zu einem Suizidversuch geführt hatte. Dylan dagegen hatte den höchsten BDI-V Wert von allen im Interview befragten Angehörigen. Dies bestätigt auf qualitativer Ebene, dass ein tolerantes und inaktives Coping mit höheren gesundheitlichen Risiken verbunden ist (Lee et al., 2011, S. 445; Orford et al., 2001, S. 769).

Die Auswertungen zeigten, dass die grosse Mehrheit der Angehörigen erst im Verlauf der Suchterkrankung beginnen, vermehrt an Unabhängigkeit gegenüber der Abhängigkeitserkrankung und dem betroffenen Angehörigen zu gewinnen. Dazu gehören folgende Strategien: Sie grenzen sich gegenüber ihren drogenabhängigen Angehörigen ab und betonen deren Eigenverantwortung. Sie stellen klare Regeln auf, an die sie sich halten. Dieser Aspekt war bei Orford, Velleman et al. (2010, S. 55) eher der Bewältigungsstrategie des Standhaft-Bleibens und des Aufbegehrens zugeordnet worden. Aufgrund der qualitativen Datenauswertung wurde aber klar, dass die meisten Angehörigen das Setzen und Durchsetzen von klaren Regeln als Ausdruck ihrer eigenen Bedürfnisse sehen, was Ausdruck einer bestimmten Unabhängigkeit ist. Weitere Strategien, die Ausdruck von Unabhängigkeit sind und in Übereinstimmung mit Orford, Velleman et al. (2010) stehen, sind räumliche Distanz schaffen und sich vermehrt auf andere Ziele und Wünsche konzentrieren.

In der vorliegenden Arbeit zeichneten sich noch weitere Bewältigungsstrategien ab, die von Orford, Velleman et al (2010) nicht thematisiert werden. Eine grosse Mehrheit der Angehörigen gab an, sie hätten die Suchtproblematik gegenüber anderen angesprochen. Einerseits um sich soziale Unterstützung zu suchen und andererseits um sich vor negativen Kommentaren, die über drogenabhängige Menschen zum Teil gemacht werden, zu schützen. Die meisten machten positive Erfahrungen, wenn sie anderen von der Suchterkrankung ihres Angehörigen erzählten und waren dadurch entlastet, die Abhängigkeit nicht mehr verheimlichen $\mathrm{zu}$ müssen. Eine weitere Form der Bewältigung war der Humor, den 
Angehörige, in den Interviews zeigten. Sie lachten in Interviews oder berichteten davon, dass gewisse Situationen in ihrer Tragik durchaus auch etwas Lustiges hätten.

\subsubsection{Co-Abhängigkeit als Bewältigungsversuch}

Angesichts der mannigfaltigen Bewältigungsstrategien, die Angehörige anwenden, scheint das Co-Abhängigkeitskonzept nicht haltbar zu sein. Während einige Autoren postulieren, dass Angehörige im Verlauf der Suchterkrankung ihre eigenen Bedürfnisse immer mehr aus den Augen verlieren (Flassbeck, 2011; Kolitzus, 1997; Schaef, 1996), zeigt sich in der vorliegenden Arbeit eher ein entgegengesetztes Bild. Häufig fokussieren die Angehörigen gerade zu Beginn sehr stark auf ihr drogenabhängiges Familienmitglied und sind bereit, alles Mögliche zu tun, um ihren Angehörigen zu helfen. Dies sollte allerdings nicht als irgendeine pathologische Reaktion verstanden werden, sondern als eine normale Verhaltensweise, die ein gesunder Mensch, der einen anderen liebt, an den Tag legen würde (Ruckstuhl, 2012, S. 18). Es liegt in der Natur des Menschen, als soziales Wesen co-abhängig zu sein. Angehörige gaben beispielsweise an, dass sie zu Beginn Verständnis und Fürsorge gezeigt und Schulden übernommen hätten. Einige Eltern hatten sogar ihre drogenkonsumierenden Kinder wieder bei sich aufgenommen, um sie gesund zu pflegen. Interessant ist jedoch, dass die meisten Angehörigen irgendwann realisierten, dass ihre Bemühungen nicht zum Erfolg führten und ihr Angehöriger trotzdem weiter konsumierte. Die meisten begannen dann, sich über alternative Bewältigungsmöglichkeiten Gedanken $\mathrm{zu}$ machen. Dies äusserte sich beispielsweise darin, dass sie aufhörten ihren Angehörigen für alles Mögliche, Geld zu geben oder dass sie begannen, klare Regeln aufzustellen, die das Zusammenleben regeln oder sie baten ihre Angehörigen auszuziehen. Nichtsdestotrotz soll dem Co-Abhängigkeitskonzept zugute gehalten werden, dass es die gravierenden Auswirkungen auf die Angehörigen betont, die tatsächlich ein hohes Risiko tragen als Folge der Belastungen krank zu werden. Es fokussiert allerdings zu stark nur auf einen kleinen Aspekt der Bewältigung der Angehörigen. Im professionellen Kontext kann es zudem dazu führen, dass Angehörigen nicht als das wahrgenommen werden, was sie sind, nämlich Menschen in einer äusserst belastenden Situation, die sie bestmöglich zu bewältigen versuchen. 


\subsection{Unterstützung der Angehörigen}

\subsubsection{Vermittlung von Wissen und Aufklärung}

Ein zentraler Aspekt, der Angehörige im Umgang mit den Belastungen unterstützt, ist eine gute Aufklärung. Die Bedeutung einer adäquaten Wissensvermittlung wird auch von der ,5Step-Method“ im ersten Schritt betont (Copello et al., 2000; Copello, Templeton \& Orford et al., 2010b). Angehörige haben oft, wenn sie vom Drogenkonsum ihres Angehörigen erfahren, nur eine diffuse Ahnung, was dies nun eigentlich bedeutet. Dies zeigte sich deutlich in den qualitativen Ergebnissen. Die emotionalen Reaktionen reichten von Angst, Schreck bis hin zur Erleichterung, endlich zu wissen, was los ist und dem Gefühl, nun etwas tun zu können. Andere erzählten, dass sie vor allem erst mal Schuldgefühle gehabt hätten. Die wenigsten suchen sich gleich zu Beginn Unterstützung. Ihnen ist meist nicht bewusst, dass Abhängigkeitserkrankungen häufig einen chronischen Verlauf nehmen. Diese Erkenntnis kommt meist erst nach mehreren Jahren der Betroffenheit. In diesem Sinne kann es für Angehörige hilfreich sein, wenn sie eine adäquate Aufklärung bekommen, beispielsweise dass Rückfälle die Norm und nicht die Ausnahme sind. Es kann für Angehörige eine Entlastung sein, wenn sie auf mögliche Rückfälle vorbereitet werden. Gleichzeitig sollte den Angehörigen aber auch die Hoffnung vermittelt werden, dass es im Rahmen der Erkrankung auch immer wieder bessere Phasen geben kann. Mehr als die Hälfte der Angehörigen erlebten es deshalb auch als hilfreich, mehr Informationen zur Suchterkrankung, deren Bedeutung in der Gesellschaft, deren gesundheitlichen und sozialen Folgen und zu möglichen komorbiden Störungen zu erfahren. Die Belastungen machen auf einen weiteren Bereich aufmerksam, wo Angehörigen allenfalls Aufklärung bräuchten. Im Verlauf der Abhängigkeitserkrankung werden viele mit rechtlichen und finanziellen Fragen konfrontiert, so dass es für diejenigen, die mit Angehörigen zu tun haben, wichtig ist, über ein entsprechendes Netzwerk zu verfügen, in das Angehörige weiter vermittelt werden können.

Wissen und Aufklärung waren aber auch ein wichtiges Thema bei der gewünschten Unterstützung. Eine grosse Mehrheit der Angehörigen wünschte sich in irgendeiner Form eine bessere Aufklärung. Angehörige wünschen beispielsweise, dass sie mehr Informationen von den Kliniken bekommen, die ihre Angehörigen behandeln. Besonders wichtig war es Angehörigen, dass in der Öffentlichkeit ein realistischeres Bild zu suchtkranken Menschen und ihren Angehörigen vermittelt wird. Es war ihnen ein grosses Anliegen, dass ihre Angehörigen weniger stigmatisiert werden und die Störung als Krankheit mit einem meist 
chronischen Verlauf anerkannt wird. Viele fanden es deshalb wichtig, dass eine Diskussion darüber stattfindet, wie ein Leben mit dem Konsum und der Krankheit aussehen kann.

\subsubsection{Die Bedeutung von sozialer Unterstützung}

Das Stress-Strain-Coping-Support Modell und die darauf aufbauende ,5-Step-Method“ betonen die Bedeutung der sozialen Unterstützung für die erfolgreiche Entlastung der Angehörigen (Copello et al., 2000; Copello, Templeton \& Orford et al., 2010b). Dies deckt sich absolut mit den Ergebnissen dieser Arbeit. Alle Angehörigen sagten, dass soziale Unterstützung für sie in einer Form hilfreich gewesen sei im Umgang mit den Belastungen. Im Gegensatz zu einer englisch-mexikanischen Studie, die die soziale Unterstützung in die Bereiche Familie, Freunde, Professionelle, Nachbarn und Freunde des Suchtkranken (Orford et al., 1998a, S. 410) einteilte, wurde in der vorliegenden Arbeit die Einteilung in Unterstützung durch Familie, Unterstützung von Freunden oder anderen Drittpersonen und Unterstützung von Selbsthilfegruppen vorgenommen. Als besonders hilfreich zeigte sich der Austausch mit anderen Betroffenen. Angehörige fühlten sich dadurch nicht alleine gelassen und in schwierigen Situationen von der Gruppe getragen. Der Austausch mit anderen Angehörigen erlaubte es ihnen zudem teilweise, Schuldgefühle anders einzuordnen. In der vorliegenden Arbeit hatten allerdings nur die Eltern an einer Selbsthilfegruppe teilgenommen. In diesem Sinne müsste der Ausbau von Selbsthilfegruppen für verschiedene Gruppen von Angehörigen noch mehr gefördert werden.

Als besonders hilfreich empfanden Angehörige soziale Unterstützung von professioneller Seite vor allem dann, wenn sie sich in ihren Bedürfnissen wahrgenommen fühlten und der Fokus im Gespräch bei ihnen war. Dazu gehörte, dass Angehörige in ihren Wünschen gesehen wurden und ihre äusserst belastende und schwierige Situation als solche anerkannt wurde. Für Angehörige ist es wichtig, dass sie in ihren Bemühungen Wertschätzung erfahren. Sowohl das CRAFT (Bischof \& Freyer, 2006, S. 54; Bischof, 2012; S. 30) als auch die „5-Step-Method“ (Copello et al., 2000, S. 332ff; Copello, Templeton \& Orford et al., 2010b, S. 89-90) betonen, dass diejenigen, die mit Angehörigen arbeiten, eine vorurteilsfreie, empathische Haltung einnehmen sollten, was sich in den Aussagen der Angehörigen bestätigte. Angehörige finden es zudem hilfreich, wenn ihnen vermittelt wird, dass sie sich ohne schlechtes Gewissen etwas Gutes tun und ihr Leben auch geniessen dürfen. 
Es wird jedoch nicht jede Form der sozialen Unterstützung auch als positiv erlebt (Orford et al., 1998a, S.412). Besonders auffällig waren in diesem Zusammenhang die negativen Angaben, die die Angehörigen zu professionellen Hilfssystemen machten. Mehr als die Hälfte hatte Erfahrungen mit professionellen Helfern gemacht, die durch ihre inadäquate Reaktion eher dazu beigetragen hatten, dass sich die Belastung für Angehörigen noch erhöhte. So erlebten Angehörige häufig, dass sie in ihren Bedürfnissen nach Unterstützung nicht wahrgenommen wurden, wenn sie sich an Fachpersonen wandten. Dies konnte bedeuten, dass Fachspersonen sich nicht zuständig fühlten, dass sie nicht auf die Themen der Angehörigen eingingen oder dass es zu Schuldzuschreibungen kam. Dies macht deutlich, dass besonders Fachpersonen, die mit Angehörigen von suchtkranken Menschen in Kontakt treten, sensibilisiert werden müssten für die Anliegen und Schwierigkeiten der Angehörigen, so dass sie adäquat reagieren können.

\subsubsection{Therapie des suchtkranken Angehörigen}

Das CRAFT wurde für Angehörige entwickelt, die ihr therapieunwilliges Familienmitglied zur Behandlung motivieren möchten (Meyers et al., 2001, S. 147). Die Ergebnisse der vorliegenden Arbeit machen deutlich, dass es für Angehörige in der Tat eine Entlastung darstellt, wenn sich ihr drogenabhängiger Angehöriger in Therapie begibt oder in irgendeiner Form Unterstützung erhält. Einige Angehörige meinten sogar, es sei eine unbedingte Voraussetzung, dass es ihrem suchtkranken Angehörigen gut gehe, damit es auch ihnen gut gehen könne. Erst wenn sich die gesundheitliche Situation ihres Angehörigen verbesserte, ging es auch ihnen besser, weil die Belastungen dadurch abnahmen. Dies macht deutlich, dass die Qualität der Behandlung der Betroffenen auch eine entscheidende Rolle in der Entlastung der Angehörigen spielt. Sie wünschen sich, dass der Mensch, den sie lieben, in guten Händen ist. Passieren in der Behandlung Fehler, die einen Therapieabbruch begünstigen, fällt dies häufig direkt auf die Angehörigen zurück. Ein Umstand, der von einem Vater, dessen Söhne in einer heroingestützten Behandlung waren, ganz klar aufgezeigt wurde.

Dass die Qualität der Behandlung für die Be- bzw. Entlastung der Angehörigen sehr wichtig ist, zeigte sich auch in den Aussagen von weiteren Angehörigen, die feststellen mussten, dass ihre Familienmitglieder in der Behandlung noch kränker wurden. In diesem Zusammenhang erzählten einige, dass ihre Angehörigen Medikamente mit Missbrauchspotential wie Ritalin 
oder Benzodiazepine verschrieben bekommen hätten, was zu einer weiteren Abhängigkeit geführt habe.

Wünsche der Angehörigen waren zudem der Ausbau bestimmter therapeutischer Angebote. Insbesondere im Bereich der Kinder- und Jugendpsychiatrie gibt es nur wenig spezifische Angebote für Kinder und Jugendliche, die einen problematischen Substanzkonsum haben und beispielsweise eine stationäre Behandlung brauchen würden. Eine schwierige und belastende Situation für die Eltern, die Hilfe für ihre Kinder suchen.

\subsection{Relevanz und weitere Implikationen}

Die Arbeit zeigt verschiedene Aspekte zu Angehörigen von suchtkranken und insbesondere drogenabhängigen Menschen auf: Es handelt sich um eine Population, die aufgrund der Belastungen ein höheres Risiko für eine Vielzahl von stressbedingten, gesundheitlichen Problemen hat (Ray et al., 2007) und die gleichzeitig völlig unbeachtet leise vor sich hin leidet (Orford, Velleman, Natera, Templeton \& Copello, 2013, S. 71). Schon allein die Tatsache, dass Angehörige von suchtkranken Menschen durch ihre gesundheitlichen Risiken massiv Kosten in unserem Gesundheitssystem verursachen, lässt es als unverständlich erscheinen, dass Angehörige nicht mehr Beachtung und Unterstützung bekommen. In der Schweiz existieren nur wenige Angebote, die sich den Anliegen und Belastungen der Angehörigen annehmen. Zudem kann das bestehende Angebot höchstens einen Bruchteil der Angehörigen erreichen.

Die Al-Anon Familiengruppen, die zwar in der Schweiz weit verbreitet sind, sprechen mit grosser Wahrscheinlichkeit mit ihrem spirituellen und zum Teil auch ritualisierten Zugang viele Menschen in unserer heutigen säkularisierten Gesellschaft nicht mehr an (Ruckstuhl, 2012, S. 16). Zudem dürfte das Krankheitskonzept der Al-Anon Familiengruppen Angehörige abschrecken, die sich nicht aufgrund der Suchterkrankung ihres Angehörigen pathologisieren lassen wollen.

Das CRAFT, welches durchaus ein sinnvolles Angebot für Angehörige darstellt, ist dagegen nur für einen ganz kleinen Teil der Angehörigen geeignet. Die Voraussetzungen, die mit dem Training verbunden sind, schliessen nämlich bereits einen erheblichen Teil der Angehörigen aus. Längst nicht alle Angehörigen haben dreimal in der Woche Kontakt zu ihrem drogenabhängigen Angehörigen. Hinzu kommt, dass sich das CRAFT an Angehörige von 
suchtkranken Menschen richtet, die aktuell nicht zur Behandlung bereit sind (Meyers et al., 2001). Es gibt allerdings auch einen erheblichen Teil Angehöriger, deren drogenabhängiger Angehöriger in Behandlung ist und die sich dennoch belastet fühlen und sich Unterstützung wünschen würden.

Die Angehörigenvereinigung Drogenabhängiger Zürich (ada-zh), welche ein breites Angebot für suchtkranke Menschen offeriert, ist dagegen nur lokal tätig. Den meisten Angehörigen, die ausserhalb des direkten Einzugsgebietes der Stadt Zürich leben, dürfte der Weg nach Zürich zu weit sein, um sich bei der Angehörigenvereinigung Drogenabhängiger (ada-zh) beraten zu lassen.

Die Ergebnisse der Arbeit machen zudem deutlich, dass Angehörige immer wieder an professionelle Helfer gelangen, die nicht adäquat auf ihre Bedürfnisse eingehen. Aus diesem Grund sollten insbesondere Fachpersonen dem Thema mehr Beachtung schenken und sich der enormen gesundheitlichen Risiken, die Angehörige aufgrund der Belastungen tragen, bewusst werden. Aufgrund der vorliegenden Ergebnisse sollten meiner Meinung nach die Versorgungssysteme, die sich um suchtkranke Menschen kümmern, auch den Auftrag übernehmen, sich den Angehörigen anzunehmen. Mit einem Angebot wie der „5-StepMethod" könnte dies mit geringem Aufwand in vielen Psychiatrien und primären Versorgungssystemen implementiert werden (siehe Kap. 2.4.4.4.). Der Vorteil des Verfahrens ist, dass seine Wirksamkeit mehrfach positiv belegt ist (Copello et al., 2000; Copello et al., 2009; Velleman et al., 2008) und dass es den Fokus auf die Bedürfnisse der Angehörigen legt, was wichtig ist, wie die Ergebnisse dieser Arbeit zeigen. Die stärkere Berücksichtigung der Angehörigen im Rahmen der Psychiatrien könnte zudem gleich mehrere Vorteile mit sich bringen. Auf der einen Seite könnten die gesundheitlichen Risiken der Angehörigen und somit die Folgekosten für die Angehörigen aber auch die Gesellschaft reduziert werden. Auf der anderen Seite können die Angehörigen auch als Ressource in der Behandlung oder der Behandlungsaufnahme genutzt werden (Meyers et al., 2001), so dass sich in den Versorgungssystemen allenfalls der Behandlungserfolg bei den Betroffenen selbst verbessern liesse, wenn die Angehörigen vermehrt einbezogen würden. Dies wäre allerdings in weiteren Forschungsprojekten zu untersuchen und zu belegen.

Die quantitativen Ergebnisse legen den Schluss nahe, dass stark belastete Angehörige vielerlei somatische Symptome zeigen. Es ist deshalb anzunehmen, dass diese Angehörige 
wegen ihrer Beschwerden häufig ihre Hausärzte konsultieren. Bei der klinischen Depression scheinen zudem ebenfalls somatische Symptome im Vordergrund zu stehen. Durch eine Sensibilisierung der Hausärzte für die Thematik könnten Angehörige früher erreicht und an Hilfesysteme weiter verwiesen werden.

Weitere Forschungsprojekte im Bereich der Angehörigenarbeit scheinen zudem zwingend notwendig. Die Begrenzungen der Stichprobe machen deutlich, dass die Ergebnisse mit grosser Wahrscheinlichkeit nicht allgemeine Gültigkeit für die Angehörigen von drogenabhängigen Menschen haben. Die gesundheitlichen Probleme könnten allenfalls unterschätzt worden sein, da beinahe die Hälfte der Studienteilnehmenden über eine Verbesserung der Suchterkrankung im vergangenen Jahr berichtete und der verbesserte Status mit geringeren BDI-V Werten assoziiert war. Zudem sollte genauer untersucht werden, welche Auswirkungen die Drogenabhängigkeit der Eltern auf ihre Kinder hat und wie jüngere Geschwister durch die Suchterkrankung beeinflusst werden.

Abschliessend möchte ich anmerken, dass mir die Arbeit bewusst gemacht hat, dass Angehörige immer nur in einem begrenzten Rahmen Unterstützung bekommen können. Die grösste Belastung, nämlich dass ein nahestehender Mensch, den sie lieben, schwer erkrankt ist, kann ihnen niemand abnehmen. Gerade aus diesem Grund verdienen sie aber die bestmögliche Unterstützung im Umgang mit all den anderen Belastungen, die sie im Zusammenhang mit der Suchterkrankung erleben und die für sie zusätzlich gesundheitliche Risiken mit sich bringen. Angehörige erbringen zudem einen grossen Betrag für unsere Gesellschaft, in dem sie sich ihren suchtkranken Angehörigen annehmen und sich um sie kümmern. Dafür verdienen sie Respekt, Wertschätzung und Annerkennung. 


\section{Zusammenfassung}

Die Situation der Angehörigen von suchtkranken Menschen wurde im deutschsprachigen Raum und insbesondere der Schweiz bisher nur wenig erforscht. Forschung aus dem Ausland zeigt jedoch, dass Angehörige diverse Belastungen erleben (Kirby et al., 2005; Moore \& Stuart, 2004; Orford et al., 1998c; Orford \& Velleman et al., 2010; Rennert, 1993c), die dazu führen, dass Angehörige ein höheres Risiko tragen, selbst psychisch oder psychosomatisch zu erkranken (Orford et al., 1998c; Ray et al., 2007). In der vorliegenden Arbeit wurde deshalb die Situation der Angehörigen von heroin- und kokainabhängigen Menschen in der Schweiz untersucht. Die Arbeit hat einen explorativen Charakter und ging den Fragen nach, welche Belastungen Angehörige im Zusammenhang mit der Drogenabhängigkeit ihrer Angehörigen erleben, wie sich die Belastung auf ihre psychische Gesundheit auswirkt und wie Angehörige mit den Belastungen umgehen. Zusätzlich wurde nach Antworten gesucht, wie Angehörige von drogenabhängigen Menschen besser unterstützt werden können.

Befragt wurden insgesamt 88 Angehörige, die in unterschiedlicher Beziehung zur drogenabhängigen Person standen. Es nahmen 44 Eltern, 21 Geschwister, 15 Partnerinnen, 5 Kinder und 3 nahestehende Freunde an der Befragung teil. Sie wurden mittels folgender Fragebögen befragt: Berner Fragebogen zur Wohlbefinden (BFW/E), Zarit Burden Interview (ZBI), Inventar Subjektiver Stigmaerfahrungen (ISE), Stressverarbeitungsfragebogen (SVF12), Vereinfachtes Beck-Depressions-Inventar (BDI-V) und Rosenberg Self-Esteem Scale (Rosenberg SES). Die mit Fragebögen erhobenen quantitativen Daten wurden mit verschiedenen statistischen Methoden ausgewertet. Als Ergänzung zu den quantitativen Daten wurde mit 13 der 88 Angehörigen ein problemzentriertes Interview geführt, in dem sie direkt zu ihren Belastungen, den Bewältigungsstrategien und der Unterstützung, die sie erhalten hatten und sich wünschen würden, befragt wurden. Die qualitativen Interviewdaten wurden mit einer qualitativen Inhaltsanalyse (Mayring, 2010) ausgewertet.

Die Ergebnisse zeigen, dass Angehörige eine Vielzahl von verschiedenen Belastungen erleben. In Konkordanz mit der bestehenden Forschungsliteratur (Kirby et al., 2005; Orford et al., 1998c) berichteten Angehörige über emotionale Belastungen, Beziehungsprobleme, finanzielle Probleme, rechtliche Probleme, Beeinträchtigungen des Lebensalltages, Gewalt und Drohungen und über Stigmatisierungen. Die Belastungen führen schliesslich dazu, dass Angehörige - wie bereits aus dem Ausland bekannt - ein hohes Risiko für gesundheitliche Probleme tragen (Orford et al., 1998c; Ray et al., 2007). In der vorliegenden Arbeit 
überschritten 27.3\% den Cut-off des BDI-V für eine klinisch relevante Depression. Diese 12Monatsprävalenz liegt erheblich über derjenigen der Schweizer Durchschnittsbevölkerung von 7 \% (Baer, 2013, S. 10). Relevante Prädiktoren, die die Depressivität vorhersagen konnten, waren die Belastung, die die Angehörigen erleben oder wenn sie über eine grosse Zahl von Problemen (Finanzen, Beziehungen, Arbeit, Gesundheit) berichteten. Bedeutsam war zudem, wenn die Angehörigen mit dem Drogenabhängigen zusammenlebten. Dagegen reduzierte sich das Risiko für eine klinisch relevante Depression, wenn die Angehörigen angaben, dass sich die Suchterkrankung im vergangen Jahr gebessert hatte. Bei den Bewältigungsstrategien zeigte sich insbesondere ein Zusammenhang zwischen Strategien, die das Belastungserleben noch erhöhen, wie Resignation, Soziale Abkapselung und Selbstbeschuldigung und den BDI-V Werten.

Die Ergebnisse zu den Bewältigungsstrategien machten deutlich, dass Angehörige im Umgang mit den Belastungen unterschiedliche Bewältigungsstrategien anwenden. Dies stellt insgesamt die Relevanz des Co-Abhängigkeitskonzeptes in Frage. Insgesamt liessen sich die drei Hauptpositionen, die von Orford, Velleman et al. (2010) vorgeschlagen werden, mit kleineren Anpassungen bei den Bewältigungsstrategien, die diesen zugeordnet werden, bestätigen. Es handelt sich um die drei Positionen Aufbegehren, Unabhängigkeit und Toleranz. Diese sind entweder durch eine tolerante, akzeptierende Haltung gegenüber dem Substanzkonsum (Toleranz), eine aufbegehrende und verändernde Haltung gegenüber dem Substanzkonsum (Aufbegehren) oder durch eine räumliche oder emotionale Distanzierung vom Substanzkonsum und der drogenkonsumierenden Person (Unabhängigkeit) gekennzeichnet.

In Bezug auf die Unterstützung zeigte sich, dass insbesondere die soziale Unterstützung durch die Familie, durch Freunde und Drittpersonen und durch Selbsthilfegruppen besonders hilfreich ist im Umgang mit den Belastungen. Die Bedeutung der sozialen Unterstützung für den Umgang mit den Belastungen hatte sich bereits in anderen Forschungsarbeiten gezeigt (Orford et al., 1998a). Damit die Unterstützung von professionellen Helfern als hilfreich erlebt wird, müssen diese eine empathische und vorurteilsfreie Haltung einnehmen, die auf die Bedürfnisse der Angehörigen fokussiert. Angehörige berichteten in der vorliegenden Arbeit mehrfach von professionellen Helfern, die inadäquat auf sie und ihre Situation reagiert hatten. Diesem Aspekt sollte vor allem im klinischen Alltag mehr Beachtung geschenkt werden. Unterstützend empfanden Angehörige zudem eine gute Aufklärung über 
Suchterkrankung. Sie betonten aber auch die Bedeutung der Therapie ihrer suchtkranken Angehörigen zu ihrer Entlastung. Es ist ihnen wichtig zu wissen, dass ihre Angehörigen in guten Händen sind und dass es ihnen gut geht.

Insgesamt legen die Ergebnisse nahe, dass Angehörige in der Schweiz besser unterstützt und in der Forschung als auch im klinischen Alltag mehr Beachtung bekommen müssen. Das bestehende Angebot, welches sich derzeit aus dem CRAFT, den Al-Anon Familiengruppen und weiteren Selbsthilfegruppen und der Angehörigenvereinigung Drogenabhängiger Zürich (ada-zh) zusammensetzt sollte noch weiter ergänzt werden. Eine allfällige Implementierung weiterer Angebote wie beispielsweise der ,5-Step Method“ wäre wünschenswert. 


\section{Bibliographie}

NEETs. Young people not in employment, education or training: characteristics, costs and policy responses in Europe. (2012). Dublin: European Foundation for the Improvement of Living and Working Conditions.

Al-Anon Familiengruppen. Angehörige von Alkoholikern brauchen Hilfe - Al-Anon hilft.

Al-Anon Familiengruppen (Hrsg.). (1996a). Fakten für Fachleute. Informationen über AlAnon und Alateen, Essen.

Al-Anon Familiengruppen. (1996b). Informationen über Al-Anon, Essen.

Al-Anon Familiengruppen. (2003). Information für Neue, Essen.

Amelang, M. \& Schmitz-Atzert, L. (2006). Psychologische Diagnostik und Intervention (4. Aufl.). Heidelberg: Springer.

Andorfer, U. \& Schmidt, E. (2007). Das Angehörigenzentrierte Betreuungsangebot für Angehörige von Suchtkranken am Anton-Proksch-Institut Wien. Wiener Zeitschrift für Suchtforschung, 30 (2/3), 41-46.

Angermeyer, M. C., Schulze, B. \& Dietrich, S. (2003). Courtesy stigma. Social Psychiatry and Psychiatric Epidemiology, 38 (10), 593-602.

Bachner, Y. G. \& O'Rourke, N. (2007). Reliability generalization of responses by care providers to the Zarit Burden Interview. Aging \& Mental Health, 11 (6), 678-685.

Backhaus, K., Erichson, B., Plinke, W. \& Weiber, R. (2011). Multivariate Analysemethoden. Eine anwendungsorientierte Einführung (Springer-Lehrbuch, 13. Aufl.). Berlin [u.a.]: Springer.

Baer, N. (2013). Depressionen in der Schweizer Bevölkerung. Daten zur Epidemiologie, Behandlung und sozial-beruflichen Integration (Obsan Bericht, Bd. 56). Neuchâtel: Schweizerisches Gesundheitsobservatorium.

Bärtschi-Waldvogel, M. (1995). Co-Abhängigkeit als komplementäres Verhaltensmuster bei Frauen. Abhängigkeiten, 1, 37-42.

Beattie, M. (2011). Die Sucht, gebraucht zu werden (Heyne-Bücher, 14. Aufl.). München: Heyne.

Benishek, L., Dugosh, K., Faranda-Diedrich, T. \& Kirby, K. (2006). Development of the Significant Other Survey: an interview for family members of substance users. American Journal of Family Therapy, 34 (1), 33-46.

Bergs, S. (1981). Optimalität bei Cluster-Analysen. Diss. Münster.

Beutel, M. E. \& Brähler, E. (2004). Testinformation. Stressverarbeitungsfragebogen (SVF120) von Wilhelm Janke und Gisela Erdmann (unter Mitwirkung von Markus Ising). Diagnostica, 50 (3), 165-167.

Bilke-Hentsch, O. \& Gremaud, F. (2013). Latenzkinder in suchtgefährdeten Familien. Suchtmagazin, 39 (5), 30-33.

Bischof, G. (2012). Das "Community Reinforcement and Familiy Training" CRAFT. Suchtmagazin, 38 (1), 30-32.

Bischof, G. \& Freyer, J. (2006). Angehörigenarbeit bei Personen mit substanzbezogenen Störungen. Suchttherapie, 7 (2), 52-57. 
Bischof, G., Iwen, J., Müller, C. W., Freyer-Adam, J. \& Rumpf, H.-J. (2007). Das Community Reinforcement Ansatz basierte Familien Training (CRAFT):

Angehörigenarbeit bei Suchtkranken ohne Behandlungsbereitschaft. Wiener Zeitschrift für Suchtforschung, 30 (2/3), 53-62.

Bodenmann, G. \& Gmelch, S. (2009). Stressbewältigung. In S. Schneider \& J. Margraf (Hrsg.), Lehrbuch der Verhaltenstherapie. Band 3: Störungen im Kindes- und Jugendalter (S. 617-629). Berlin: Springer Berlin Heidelberg.

Boothe, B., Grimmer, B., Luder, M., Luif, V., Neukom, M. \& Spiegel, U. (2002). Manual der Erzählanalyse JAKOB. Version 10/02. Berichte aus der Abteilung Klinische Psychologie, Nr. 51. Universität Zürich: Psychologisches Institut, Klinische Psychologie I.

Bortz, J. \& Schuster, C. (2010). Statistik für Human- und Sozialwissenschaftler (7. Aufl.). Berlin [u.a.]: Springer.

Braun, M., Scholz, U., Hornung, R. \& Martin, M. (2010). Die subjektive Belastung pflegender Ehepartner von Demenzkranken. Z Gerontol Geriat, 43 (2), 111-119.

Brook, J. S., Brook, D. W. \& Whiteman, M. (1999). Older sibling correlates of younger sibling drug use in the context of parent-child relations. Genetic, Social, and General Psychology Monographs, 125 (4), 451-468.

Brueck, R. (2011). Community Reinforcement and Family Training (CRAFT). In O. Bilke \& A. Batra (Hrsg.), Praxisbuch Suchttherapie (1. Aufl., S. 69-72). Stuttgart: Thieme.

Candrian, R. \& Ruckstuhl, L. A. (2009). Wie gehen Eltern mit der Sucht ihrer Söhne um? Bewältigungsstrategien von Angehörigen aus Erzählungen.

Cattell, R. (1966). The scree test for the number of factors. Multivariate Behavioral Research, 1, 245-276.

Cermak, T. L. (1986). Diagnostic criteria for codependency. Journal of Psychoactive Drugs, $18(1), 15-20$.

Cohen, S. \& Wills, T. (1985). Stress, social support and the buffering hypothesis. Psychological Bulletin, 98, 310-357.

Collani, G. von \& Herzberg, P. Y. (2003). Eine revidierte Fassung der deutschsprachigen Skala zum Selbstwertgefühl von Rosenberg. Zeitschrift für Differentielle und Diagnostische Psychologie, 24 (1), 3-7.

Copello, A., Ibanga, A., Orford, J., Templeton, L. \& Velleman, R. (2010). The 5-Step Method: future directions. Drugs Edu Prev Pol, 17 (s1), 203-210.

Copello, A., Orford, J., Velleman, R., Templeton, L. \& Krishnan, M. (2000). Methods for reducing alcohol and drug related family harm in non-specialist settings. Journal of Mental Health, 9 (3), 329-343.

Copello, A., Templeton, L., Krishnan, M., Orford, J. \& Velleman, R. (2000). A treatment package to improve primary care services for relatives of people with alcohol and drug problems. Addiction Research, 8 (5), 471-484.

Copello, A., Templeton, L., Orford, J. \& Velleman, R. (2010a). The 5-Step Method: evidence of gains for affected family members. Drugs Edu Prev Pol, 17 (s1), 100-112.

Copello, A., Templeton, L., Orford, J. \& Velleman, R. (2010b). The 5-Step Method: principles and practice. Drugs Edu Prev Pol, 17 (s1), 86-99. 
Copello, A., Templeton, L., Orford, J., Velleman, R., Patel, A., Moore, L. et al. (2009). The relative efficacy of two levels of a primary care intervention for family members affected by the addiction problem of a close relative: a randomized trial. Addiction, 104 (1), 49-58.

Copello, A., Templeton, L. \& Powell, J. (2009). Adult family members and carers of dependence drug users: prevalence, social cost, resource savings and treatment response. Final report to the UK Drugs Policy Commission. London: UK DPC.

Copello, A., Templeton, L. \& Powell, J. (2010). The impact of addiction on the family: estimates of prevalence and costs. Drugs Edu Prev Pol, 17 (s1), 63-74.

Denning, P. (2010). Harm reduction therapy with families and friends of people with drug problems. J. Clin. Psychol., 66 (2), 164-174.

Dodgen, C. \& Shea, W. (2000). Substance use disorders. San Diego: Academic Press.

Fals-Stewart, W., Birchler, G. R. \& O'Farrell, T. J. (1999). Drug-abusing patients and their intimate partners: dyadic adjustment, relationship stability, and substance use. Journal of Abnormal Psychology, 108 (1), 11-23.

Faul, F., Erdfelder, E., Buchner, A. \& Lang, A.-G. (2009). Statistical power analyses using $\mathrm{G}^{*}$ Power 3.1: tests for correlation and regression analyses. Behavior Research Methods, 41 (4), 1149-1160.

Faul, F., Erdfelder, E., Lang, A.-G. \& Buchner, A. (2007). G*Power 3: a flexible statistical power analysis program for the social, behavioral, and biomedical sciences. Behavior Research Methods, 39, 175-191.

Fernandez, A. C., Begley, E. A. \& Marlatt, G. A. (2006). Family and peer interventions for adults: past approaches and future directions. Psychology of Addictive Behaviors, 20 (2), 207-213.

Ferring, D. \& Filipp, S.-H. (1996). Messung des Selbstwertgefühls: Befunde zu Reliabilität, Validität und Stabilität der Rosenberg-Skala. Diagnostica, 42 (4), 284-292.

Field, A. P. (2009). Discovering statistics using SPSS (3. Aufl.). Los Angeles, [Calif.], London: SAGE.

Fischer, J. L., Pidcock, B. W., Munsch, J. \& Forthun, L. (2005). Parental abusive drinking and sibling role differences. Alcoholism Treatment Quarterly, 23 (1), 79-97.

Flassbeck, J. (2011). Co-Abhängigkeit. Diagnose, Ursachen und Therapie für Angehörige von Suchtkranken. Stuttgart: Klett-Cotta.

Flückiger, Y. \& Falter, J.-M. (2004). Bildung und Arbeit. Entwicklung des Arbeitsmarktes in der Schweiz (Statistik der Schweiz. Fachbereich 3, Arbeit und Erwerb, Bd. 4). Neuchâtel: Office fédéral de la statistique.

Gantner, A. (2011). Multidimensionale Familientherapie. In O. Bilke \& A. Batra (Hrsg.), Praxisbuch Suchttherapie (1. Aufl., S. 61-64). Stuttgart: Thieme.

George, D. \& Mallery, P. (2003). SPSS for Windows step by step. A simple guide and reference, 11.0 update (4. Aufl.). Boston: Allyn and Bacon.

Gmel, G., Kuendig, H., Notari, L., Gmel, C. \& Flury, R. (2013). Suchtmonitoring Schweiz Konsum von Alkohol, Tabak und illegalen Drogen in der Schweiz. Lausanne.

Goffman, E. (1967). Stigma. Über Techniken zur Bewältigung beschädigter Identität. Frankfurt a.M.: Suhrkamp. 
Goodman, G., Hans, S. L. \& Cox, S. M. (1999). Attachment behavior and its antecedents in offspring born to methadone-maintained women. Journal of Clinical Child Psychology, 28 (1), 58-69.

Gray-Little, B., Williams, V. S. L. \& Hancock, T. D. (1997). An item response theory analysis of the Rosenberg Self-Esteem Scale. Personality and Social Psychology Bulletin, 23, 443-451.

Grob, A. (2003). BFW Berner Fragebogen zum Wohlbefinden. In J. Schumacher, A. Klaiberg \& E. Brähler (Hrsg.), Diagnostische Verfahren zu Lebensqualität und Wohlbefinden (S. 55-59). Göttingen: Hogrefe.

Grob, A., Lüthi, R., Kaiser, F. G., Flammer, A., Mackinnon, A. \& Wearing, A. J. (1991). Berner Fragebogen zum Wohlbefinden Jugendlicher (BFW). Diagnostica, 37 (1), 66-75.

Grob, P. J. (2012). Zürcher "Needle-Park". Ein Stück Drogengeschichte und -politik 19682008 (2. Aufl.). Zürich: Chronos.

Guadagnoli, E. \& Velicer, W. (1988). Relation of sample size to the stability of component patterns. Psychological Bulletin, 103, 265-275.

Hälg, R. (2013). Selbsthilfeorganisationen im Bereich Sucht stellen sich vor. Suchtmagazin, 39 (4), 38-44.

Harkness, D., Manhire, S., Blanchard, J. \& Darling, J. (2007). Codependent attitude and behavior: moderators of psychological distress in adult offspring of families with alcohol and other (AOD) problems. Alcoholism Treatment Quarterly, 25 (3), 39-52.

Hudson, C. R., Kirby, K. C., Firely, M. L., Festinger, D. S. \& Marlowe, D. B. (2002). Social adjustment of family members and significant others (FSOs) of drug users. Journal of Substance Abuse Treatment, 23, 171-181.

Janke, W., Erdmann, G., Kallus, K. \& Boucsein, W. (1997). Stressverarbeitungsfragebogen (SVF und SVF 120). In Brickenkamp Rolf (Hrsg.), Handbuch psychologischer und pädagogischer Tests (2. Aufl., S. 714-716). Göttingen: Hogrefe.

Jaudes Kienberger, P. \& Ekwo, E. (1995). Association of drug abuse and child abuse. Child Abuse \& Neglect, 19 (9), 1065-1075.

Kaiser, H. \& Rice, J. (1974). Little Jiffy, Mark IV. Educational and Psychological Measurement, 34, 111-117.

Kauer. J. (Juni 1996). Was War, Wann Wo? Ein unvollständiger Fahrplan durch 20 Jahre DAJ Zürich ( Nr. 2). Elternvereinigung Drogenabhängiger Jugendlicher DAJ Schweiz.

Kirby, K. C., Dugosh, K. L., Benishek, L. A. \& Harrington, V. M. (2005). The Significant Other Checklist: measuring the problems experienced by family members of drug users. Addictive Behaviors, 30 (1), 29-47.

Kirby, K. C., Marlowe, D. B., Festinger, D. S., Garvey, K. A. \& LaMonaca, V. (1999). Community reinforcement training for family and significant others of drug abusers: a unilateral intervention to increase treatment entry of drug users. Drug and Alcohol Dependence, 56, 85-96.

Kläuser-Senn, C. \& Stohler, R. (2012). Angehörige und Sucht: Zeit für einen Perspektivenwechsel. Suchtmagazin, 38(1), 5-10.

Klein, M. (2002). Partner von Abhängigen. In J. Fengler (Hrsg.), Handbuch der Suchtbehandlung: Beratung - Therapie - Prävention (S. 377-385). Landsberg: ecomed. 
Knudson, T. M. \& Terrell, H. K. (2012). Codependency, perceived interparental conflict, and substance abuse in the family of origin. The American Journal of Family Therapy, 40 (3), $245-257$.

Kolitzus, H. (1997). Die Liebe und der Suff ... Schicksalsgemeinschaft Suchtfamilie. München: Kösel.

Kolitzus, H. (2000). Ich befreie mich von deiner Sucht. Hilfen für Angehörige von Suchtkranken. München: Kösel.

Kuckartz, U. (2010). Einführung in die computergestützte Analyse qualitativer Daten (Lehrbuch, 3. Aufl.). Wiesbaden: VS, Verl. für Sozialwiss.

Lazarus, R. S. \& Folkman, S. (1984). Stress, appraisal and coping. New York: Springer.

Lee, K. M. T., Manning, V., Teoh, H. C., Winslow, M., Lee, A., Subramaniam, M. et al. (2011). Stress-coping morbidity among family members of addiction patients in Singapore. Drug and Alcohol Review, 30 (4), 441-447.

Ligon, J. (2004). Six Ss for families affected by substance abuse. Journal of Family Psychotherapy, 15 (4), 95-99.

Link, B. G. \& Phelan, J. C. (2001). Conceptualizing Stigma. Annual Review Sociology, 27, 363-385.

Lüdtke, O., Robitzsch, A., Trautwein, U. \& Köller, O. (2007). Umgang mit fehlenden Werten in der psychologischen Forschung. Psychologische Rundschau, 58 (2), 103-117.

Manuel, J. K., Austin, J. L., Miller, W. R., McCrady, B. S., Tonigan, J. S., Meyers, R. J. et al. (2012). Community Reinforcement and Family Training: a pilot comparison of group and self-directed delivery. Journal of Substance Abuse Treatment, 43 (1), 129-136.

Marks, A. D., Blore, R. L., Hine, D. W. \& Dear, G. E. (2012). Development and validation of a revised measure of codependency. Australian Journal of Psychology, 64 (3), 119-127.

Mattson, R. E., O'Farrell, T. J., Lofgreen, A. M., Cunningham, K. \& Murphy, C. M. (2012). The role of illicit substance use in a conceptual model of intimate partner violence in men undergoing treatment for alcoholism. Psychology of Addictive Behaviors, 26 (2), 255-264.

Mayring, P. (2002). Einführung in die qualitative Sozialforschung. Eine Anleitung zu qualitativem Denken (Beltz Studium, 5. Aufl.). Weinheim: Beltz.

Mayring, P. (2010). Qualitative Inhaltsanalyse. Grundlagen und Techniken. Qualitative Inhaltsanalyse.

McLellan, A. T., Luborsky, L., Woody, G. E. \& O'Brien, C. P. (1980). An improved diagnostic evaluation instrument for substance abuse patients: The Addiction Severity Index. Journal of Nervous and Mental Disease, 168, 26-33.

Menard, S. (1995). Applied logistic regression analysis. In Sage university paper series on quantitative applications in the social sciences (S. 7-106). Thousand Oaks, CA: SAGE.

Mergenthaler, E. (1992). Die Transkription von Gesprächen (3. Aufl.). Ulm: Ulmer Textbank.

Meyers, R. J. \& Godley, M. D. (2001). Developing the community reinforcement approach. In R. J. Meyers \& W. R. Miller (Hrsg.), A community reinforcement approach to addiction treatment (International research monographs in the addictions, S. 1-7). Cambridge, New York: Cambridge University Press. 
Meyers, R. J., Miller, W. R., Hill, D. E. \& Tonigan, J. S. (1998). Community reinforcement and family training (CRAFT): engaging unmotivated drug users in treatment. Journal of Substance Abuse Treatment, 10 (3), 291-308.

Meyers, R. J., Miller, W. R. \& Smith, J. E. (2001). Community Reinforcement and Family Training (CRAFT). In R. J. Meyers \& W. R. Miller (Hrsg.), A community reinforcement approach to addiction treatment (International research monographs in the addictions, $\mathrm{S}$. 147-160). Cambridge, New York: Cambridge University Press.

Meyers, R. J., Miller, W. R., Smith, J. E. \& Tonigan, J. S. (2002). A randomized trial of two methods for engaging treatment-refusing drug users through concerned significant others. Journal of Consulting and Clinical Psychology, 70 (5), 1182-1185.

Miller, W. R., Meyers, R. J. \& Tonigan, J. S. (1999). Engaging the unmotivated in treatment for alcohol problems: a comparison of three strategies for intervention through family members. Journal of Consulting and Clinical Psychology, 67 (5), 688-697.

Moore, T. M. \& Stuart, G. L. (2004). Illicit substance use and intimate partner violence among men in batterers' intervention. Psychology of Addictive Behaviors, 18 (4), 385-389.

Murphy, C. M., O'Farrell, T. J., Fals-Stewart, W. \& Feehan, M. (2001). Correlates of intimate partner violence among male alcoholic patients. Journal of Consulting and Clinical Psychology, 69 (3), 528-540.

Nelson, C. E. (1985). The styles of enabling behavior. In D. E. Smith \& D. R. Wesson (Hrsg.), Treating the cocain abuser (S. 49-71). Center City: Hazelden Foundation.

Nieuwenboom, W. (2012). Die Stigmatisierung Angehöriger von SuchtpatientInnen. Suchtmagazin, 38 (1), 19-22.

O'Farrell, T. J. \& Clements, K. (2012). Review of outcome research on marital and family therapy in treatment for alcoholism. Journal of Marital and Family Therapy, 38 (1), 122144.

O'Farrell, T. J. \& Fals-Stewart, W. (2003). Alcohol abuse. Journal of Marital and Family Therapy, 29 (1), 121-146.

Oreo, A. \& Ozgul, S. (2007). Grief experiences of parents coping with an adult child with problem substance use. Addict Res Theory, 15 (1), 71-83.

Orford, J. (1994). Empowering family and friends: a new approach to the secondary prevention of addiction. Drug and Alcohol Review, 13 (13(4)), 417-429.

Orford, J., Copello, A., Velleman, R. \& Templeton, L. (2010). Family members affected by a close relative's addiction: the stress-strain-coping-support model. Drugs Edu Prev Pol, 17 (s1), 36-43.

Orford, J., Natera, G., Davies, J., Nava, A., Mora, J., Rigby, K. et al. (1998a). Social support in coping with alcohol and drug problems at home: findings from mexican and english families. Addiction Research, 6 (5), 395-420.

Orford, J., Natera, G., Davies, J., Nava, A., Mora, J., Rigby, K. et al. (1998c). Stresses and strains for family members living with drinking or drug problems in England and Mexico. Salud Mental, 21 (1), 1-13.

Orford, J., Natera, G., Davies, J., Nava, A., Mora, J., Rigby, K. et al. (1998e). Tolerate, engage or withdraw: a study of the structure of families coping with alcohol and drug problems in South West England and Mexico City. Addiction, 93 (12), 1799-1813. 
Orford, J., Natera, G., Velleman, R., Copello, A., Bowie, N., Bradbury, C. et al. (2001). Ways of coping and the health of relatives facing drug and alcohol problems in Mexico and England. Addiction, 96, 761-774.

Orford, J., Rigby, K., Miller, T., Tod, A., Bennett, G. \& Velleman, R. (1992). Ways of coping with excessive drug use in the family: a provisional typology based on the accounts of 50 close relatives. J. Community. Appl. Soc. Psychol., 2 (3), 163-183.

Orford, J., Templeton, L., Copello, A., Velleman, R. \& Ibanga, A. (2010). Working with teams and organizations to help them involve family members. Drugs Edu Prev Pol, 17 (s1), 154-164.

Orford, J., Templeton, L., Copello, A., Velleman, R., Ibanga, A. \& Binnie, C. (2009). Increasing the involvement of family members in alcohol and drug treatment services: the results of an action research project in two specialist agencies. Drugs Edu Prev Pol, 16 (5), 379-408.

Orford, J., Templeton, L., Patel, A., Copello, A. \& Velleman, R. (2007). The 5-Step family intervention in primary care: I. Strengths and limitations according to family members. Drugs Edu Prev Pol, 14 (1), 29-47.

Orford, J., Templeton, L., Patel, A., Velleman, R. \& Copello, A. (2007). The 5-Step family intervention in primary care: II. The views of primary healthcare professionals. Drugs Edu Prev Pol, 14 (2), 117-135.

Orford, J., Templeton, L., Velleman, R. \& Copello, A. (2005). Family members of relatives with alcohol, drug and gambling problems: a set of standardized questionnaires for assessing stress, coping and strain. Addiction, 100 (11), 1611-1624.

Orford, J., Templeton, L., Velleman, R. \& Copello, A. (2010). Methods of assessment for affected family members. Drugs Edu Prev Pol, 17 (s1), 75-85.

Orford, J., Velleman, R., Copello, A., Templeton, L. \& Ibanga, A. (2010). The experiences of affected family members: a summary of two decades of qualitative research. Drugs Edu Prev Pol, 17 (s1), 44-62.

Orford, J., Velleman, R., Natera, G., Templeton, L. \& Copello, A. (2013). Addiction in the family is a major but neglected contributor to the global burden of adult ill-health. Social Science \& Medicine, 78, 70-77.

Peukert, P. (2011). Angehörigenarbeit, Familien- und Paartherapie. In O. Bilke \& A. Batra (Hrsg.), Praxisbuch Suchttherapie (1. Aufl., S. 65-68). Stuttgart: Thieme.

Ramos-Cerqueira, A. T. d. A., Torres, A. R., Torresan, R. C., Negreiros, A. P. M. \& Vitorino, C. N. (2008). Emotional burden in caregivers of patients with obsessive-compulsive disorder. Depress. Anxiety, 25 (12), 1020-1027.

Ray, T. G., Mertens, J. R. \& Weisner, C. (2007). The excess medical cost and health problems of family members of persons diagnosed with alcohol and drug problems. Medical Care, 45 (2), 116-122.

Rennert, M. (1990). Co-Abhängigkeit. Was Sucht für die Familie bedeutet (2. Aufl.). Freiburg im Breisgau: Lambertus.

Rennert, M. (1993a). Rollenverteilung in belasteten Familien und die Entdeckung von CoAbhängigkeit in Familien von Suchtkranken. In Deutsche Hauptstelle gegen die Suchtgefahren (Hrsg.), Sucht und Familie (S. 27-35). Freiburg im Breisgau: Lambertus. 
Rennert, M. (1993c). Rückfall: Alptraum und Falle für die Angehörigen. In Deutsche Hauptstelle gegen die Suchtgefahren (Hrsg.), Sucht und Familie (S. 329-333). Freiburg im Breisgau: Lambertus.

Rennert, M. (2005). Co-Abhängigkeit. In O. Bilke, U. J. Küstner \& R. Thomasius (Hrsg.), Familie und Sucht. Grundlagen, Therapiepraxis, Prävention (S. 45-51). Stuttgart [u.a.]: Schattauer.

Rief, W. \& Nanke, A. (2003). Psychologische Grundkonzepte der Verhaltensmedizin. In U. Ehlert (Hrsg.), Verhaltensmedizin (Springer-Lehrbuch, S. 95-132). Berlin [u.a.]: Springer.

Roozen, H. G., Waart, R. de \& van der Kroft, P. (2010). Community reinforcement and family training: an effective option to engage treatment-resistant substance-abusing individuals in treatment. Addiction, 105 (10), 1729-1738.

Rosenberg, M. (1965). Society and the adolescent self-image. Princeton, NJ: Princeton University Press.

Roth, J. D. \& Tan, E. M. (2007). Analysis of an online Al-Anon meeting. Journal of Groups in Addiction \& Recovery, 2 (1), 5-39.

Roth, J. D. \& Tan, E. M. (2008). Spirituality and recovery from familial aspects of alcohol and other drug problems: analysis of an online Al-Anon meeting. Alcoholism Treatment Quarterly, 26 (4), 399-426.

Roth, M., Decker, O., Herzberg, P. Y. \& Brähler, E. (2008). Dimensionality and norms of the Rosenberg Self-esteem Scale in a German general population sample. European Journal of Psychological Assessment, 24 (3), 190-197.

Ruckstuhl, L. A. (2012). Angehörigen-Selbsthilfe und Co-Abhängigkeit. Suchtmagazin, 38 (1), 16-18.

Rychtarik, R. G. \& McGillicuddy, N. B. (2005). Coping skills training and 12-step facilitation for women whose partner has alcoholism: effects on depression, the partner's drinking, and partner physical violence. Journal of Consulting and Clinical Psychology, 73 (2), 249-261.

Sass, H. (op. 2003). Diagnostisches und Statistisches Manual Psychischer Störungen, DSM$I V-T R$ (4. Aufl.). Göttingen [etc.]: Hogrefe Verl. für Psychologie.

Schaef, A. W. (1996). Co-Abhängigkeit. Die Sucht hinter der Sucht (Heyne-Bücher 19, Heyne-Sachbuch, Bd. 427, Taschenbuchausg., 6. Aufl., 2. Aufl. dieser Ausg). München: Heyne.

Schermelleh-Engel, K. \& Werner, C. S. (2012). Methoden der Reliabilitätsbestimmung. In H. Moosbrugger \& A. Kelava (Hrsg.), Testtheorie und Fragebogenkonstruktion (SpringerLehrbuch, S. 119-141). Berlin, Heidelberg: Springer.

Schlippe, A. v. \& Schweitzer, J. (2012). Lehrbuch der systemischen Therapie und Beratung I. Das Grundlagenwissen. Göttingen: Vandenhoeck \& Ruprecht.

Schmid, R., Spiessl, H., Vukovich, A. \& Cording, C. (2003). Belastungen von Angehörigen und ihre Erwartungen an psychiatrische Institutionen. Literaturübersicht und eigene Ergebnisse. Fortschritte der Neurologie und Psychiatrie, 71, 118-128.

Schmidt, E. (2007). Gemeinsamkeiten und Unterschiede von Angehörigen von Alkohol-, Drogen-, Spiel- und Internetsüchtigen. Wiener Zeitschrift für Suchtforschung, 30 (2/3), $21-27$.

Schmieder, A. (1992). Alkohol \& Co. Stuttgart: Georg Thieme Verlag. 
Schmitt, M., Altstötter-Gleich, C., Hinz, A., Maes, J. \& Brähler, E. (2006). Normwerte für das Vereinfachte Beck-Depressions-Inventar (BDI-V) in der Allgemeinbevölkerung. Diagnostica, 52 (2), 51-59.

Schmitt, M., Beckmann, M., Dusi, D., Maes, J., Schiller, A. \& Schonauer, K. (2003). Messgüte des vereinfachten Beck-Depressions-Inventars (BDI-V). Diagnostica, 49 (4), 147-156.

Schmitt, M. \& Maes, J. (2000). Vorschlag zur Vereinfachung des Beck-DepressionsInventars (BDI). Diagnostica, 46 (1), 38-46.

Schreiner, A. S., Morimoto, T., Arai, Y. \& Zarit, S. (2006). Assessing family caregiver's mental health using a statistically derived cut-off score for the Zarit Burden Interview. Aging \& Mental Health, 10 (2), 107-111.

Schuler, M. E., Nair, P. \& Blach, M. M. (2002). Ongoing maternal drug use, parenting attitudes, and a home intervention: effects on mother-child interaction at 18 months. Journal of Developmental \& Behavioral Pediatrics, 23 (2), 87-94.

Schulze, B. \& Angermeyer, M. C. (2003). Subjective experiences of stigma. A foucs group study of schizophrenic patients, their relatives and mental health professionals. Social Science \& Medicine, 56, 299-312.

Schulze, B., Janeiro, M. \& Kiss, H. (2010). Das kommt ganz drauf an ... Zeitschrift für Psychiatrie, Psychologie und Psychotherapie, 58 (4), 275-285.

Schulze, B., Stuart, H. \& Riedel-Heller, S. (2009). Das Inventar Subjektiver Stigmaerfahrungen (ISE): Ein neues Instrument zur quantitativen Erfassung subjektiven Stigmas. Psychiat Prax, 36 (08), e19.

Schweizerische Eidgenossenschaft Staatssekretariat für Wirtschaft SECO. (2013, 09. Dezember). Die Lage auf dem Arbeitsmarkt. November 2013 (Schweizerische Eidgenossenschaft Staatssekretariat für Wirtschaft SECO, Hrsg.).

Schweizer, V. \& Besson, J. (1999). Alkoholismus aus systemischer Sicht. Praxis, Schweizer Rundschau für Medizin, 88, 1715-1719.

Seyle, H. (1956). The stress of life. MacGraw-Hill, New York.

Shepherd, C. B. \& Rickard, K. M. (2012). Drive for muscularity and help-seeking: the meditational role of gender role conflict, self-stigma, and attitudes. Psychology of Men \& Masculinity, 13 (4), 379-392.

Shulman, L. H., Shapira, S. R. \& Hirshfield, S. (2000). Outreach developmental services to children of patients in treatment for substance abuse. American Journal of Public Health, 90 (12), 1930-1933.

Smith, J. E. \& Meyers, R. J. (2013). Mit Suchtfamilien arbeiten. CRAFT: Ein neuer Ansatz für die Angehörigenarbeit. Köln: Psychiatrie Verlag.

Spiessl, H., Schmid, R., Vukovich, A. \& Cording, C. (2003). Erwartungen von Angehörigen an die psychiatrische Klinik. Psychiat Prax, 30, 51-55.

Stuart, H., Milev, R. \& Koller, M. (2005). The Inventory of Stigmatizing Experiences: its development and reliability. World Psychiatry, 51 (4), 33-37.

Suhner, D. \& Beck, T. (2012). Professionalisierung der Angehörigen-Selbsthilfe. Suchtmagazin, 38 (1), 26-27. 
Thomasius, R., Sack, P.-M., Küstner, U. J. \& Schindler, A. (1993). Drogenabhängigkeit. In Deutsche Hauptstelle gegen die Suchtgefahren (Hrsg.), Sucht und Familie (S. 81-94). Freiburg im Breisgau: Lambertus.

Timko, C., Young, L. B. \& Moos, R. H. (2012). Al-Anon family groups: origins, conceptual basis, outcomes, and research opportunities. Journal of Groups in Addiction \& Recovery, 7 (2-4), 279-296.

Uhl, A. \& Puhm, A. (2007). Co-Abhängigkeit - ein hilfreiches Konzept? Wiener Zeitschrift für Suchtforschung, 30 (2/3), 13-20.

Velleman, R., Arcidiacono, C., Procentese, F., Copello, A. \& Sarnacchiaro, P. (2008). A 5step intervention to help family members in Italy who live with substance misusers. $J$ Ment Health, 17 (6), 643-655.

Velleman, R., Orford, J., Templeton, L., Copello, A., Patel, A., Moore, L. et al. (2011). 12Month follow-up after brief interventions in primary care for family members affected by the substance misuse problem of a close relative. Addict Res Theory, 19 (4), 362-374. Zugriff am 01.12.2012.

Whalen, T. (1953). Wives of alcoholics: four types observed in a family service agency. Quaterly Journal of Studies on Alcohol, 14, 632-641.

Wegscheider, S. (1981). Another chance - hope and health for the alcoholic family. Palo Alto: Science an Behavior Books.

Weyers, P., Ising, M., Reuter, M. \& Janke, W. (2005). Comparing two approaches for the assessment of coping. Journal of Individual Differences, 26 (4), 207-212.

Yule, A. M., Wilens, T. E., Martelon, M. K., Simon, A. \& Biederman, J. (2013). Does exposure to parental substance use disorders increase substance use disorder risk in offspring? A 5-year follow-up study. The American Journal on Addictions, 22, 460-465.

Internetquellen:

Angebot für CRAFT-Seminare bei QK Quest:

www.gk-quest.de/seminare $(15.12 .2013)$

Angehörigenvereinigung Drogenabhängiger Zürich (ada-zh):

$\underline{\text { www.ada-zh.ch }}(14.12 .2013)$

Information zur Verwandtenunterstützungspflicht:

http://www.verwandtenunterstuetzung.ch/ (23.12.2013)

Selbsthilfegruppe Al-Anon, Schweiz:

$\underline{\text { www.al-anon.ch }}(14.12 .2013)$

Suchtmonitoring Schweiz:

http://www.suchtmonitoring.ch/de.html (24.12.2013) 
Verband der Eltern- und Angehörigenvereinigung Drogenabhängiger (VEVDAJ):

http://www.vevdaj.ch/ (14.12.2013) 


\section{Anhang}

Anhangsverzeichnis:

A1. Self-report Fragebögen der quantitativen Angehörigenbefragung

A2. Interviewleitfaden problemzentriertes Interview für die Angehörigen

A3. Interviewleitfaden problemzentriertes Interview für Kinder drogenabhängiger Eltern

B1. Transkriptionsregeln

C1. Varimax-rotierte Komponentenmatrix der 2-Faktorenlösung der HKA der ISE-Items (nur Ladungen grösser .40 angegeben)

C2. Varimax-rotierte Komponentenmatrix der 1-Faktorenlösung der HKA der ISE-Items (nur Ladungen grösser .40 angegeben)

D1. Zuordnungsübersicht des Ward-Verfahrens

E1. Kodierleitfaden mit Ankerbeispielen des Kategoriensystems

F1. Tabellenverzeichnis

F2. Abbildungsverzeichnis 
A1. Self-report Fragebögen der quantitativen Angehörigenbefragung 
A2: Leidfaden problemzentriertes Interview Angehörige

\section{Interviewleitfaden Angehörige von Drogenabhängigen}

1. Wann haben Sie von der Sucht erfahren? Können Sie mir erzählen, wie Sie damals von der Sucht erfahren haben? In welcher Situation haben Sie davon erfahren? Hatten Sie bereits im Vorfeld die Vermutung, dass ihr Angehöriger Drogen konsumieren könnte?

2. Was haben Sie als erstes gemacht, als Sie von der Sucht ihres Angehörigen erfahren haben? Was haben Sie gedacht/gefühlt?

3. Haben Sie Hilfe in Anspruch genommen? Wenn ja, welche Art von Unterstützung haben Sie gesucht? Und wie beurteilen Sie rückblickend dieses Unterstützungsangebot? Welche Hilfe und Unterstützung würden Sie sich wünschen? Können Sie mir von einer Situation erzählen, in der Sie sich besonders unterstützt gefühlt haben?

4. Was erlebten oder erleben Sie als besonders belastend im Zusammenhang mit der Sucht Ihres Angehörigen? Können Sie mir von einer Situation berichten, in der Sie sich besonders stark belastet gefühlt haben?

5. Welchen Rat würden Sie aufgrund Ihrer eigenen Erfahrungen jemanden geben, der von der Sucht oder dem problematischen Drogenkonsum eines nahe stehenden Menschen erfährt? Was sollte man Ihrer Meinung nach in einer solchen Situation tun?

6. Was hat sich durch die Sucht in Ihrem Leben verändert? (Veränderung von Beziehungen, gesundheitliche Probleme, rechtlich Probleme, finanzielle Probleme, allg. Probleme, die durch die Sucht des Angehörigen entstanden sind)

7. Wie nehmen Sie die aktuelle Beziehung zu Ihren Angehörigen wahr? Wann haben Sie sich das letzte Mal gesehen? Würden Sie mir von diesem Treffen erzählen? 
A3: Leitfaden problemzentiertes Interview Kinder drogenabhängiger Eltern

\section{Interviewleitfaden erwachsene Kinder von drogenabhängigen Eltern}

1. Wann haben Sie erfahren, dass Ihre Eltern suchtkrank sind? In welchem Alter? Wie haben Sie davon erfahren? Sind Sie bei Ihren Eltern aufgewachsen? Haben Ihre Eltern Ihnen das gesagt? Jemand anders?

2. Was haben Sie gedacht / gefühlt als Sie davon wussten?

3. Haben Sie jemals Hilfe oder Unterstützung bekommen? Oder danach gesucht? Welche Art von Hilfe/Unterstützung? Wie beurteilen Sie dies rückblickend? Welche Hilfe und Unterstützung hätten sie sich gewünscht? Können Sie mir von einer Situation erzählen, in der Sie sich besonders unterstützt gefühlt haben?

4. Was erleben oder erlebten Sie als besonders belastend im Zusammenhang mit der Suchterkrankung Ihrer Eltern? Können Sie mir von einer konkreten Situation erzählen, in der sie sich besonders stark belastet gefühlt haben? Sprechen Sie mit anderen Menschen über die Suchterkrankung Ihrer Eltern? (auch ausserhalb der Familie)

5. Welchen Rat würden Sie aufgrund Ihrer eigenen Erfahrungen jemandem geben, der von der Sucht oder dem problematischen Konsum einer nahe stehenden Person erfährt? Was sollte man ihrer Meinung nach in einer solchen Situation tun?

6. Inwiefern hatte die Suchterkrankung ihrer Eltern Auswirkungen auf ihr Leben? Wie hat das Ihr Leben beeinflusst? (Bsp: weniger Freunde (soz. Rückzug), weniger Geld (finanziell), rechtliche Probleme (Eltern inhaftiert), etc., eventl. auch nicht bei den Eltern aufgewachsen).

7. Wie nehmen Sie die Beziehung zu Ihren Eltern jetzt wahr? Wann haben Sie sie zum letzten Mal gesehen? Würden Sie mir davon erzählen? 
B1. Transkriptionsregeln

A: $\quad$ Angehöriger

I: $\quad$ Interviewer

* $\quad$ Pseudonym, verschlüsselte Bezeichnung

$+\quad$ gleichzeitiges Sprechen

///(?: ) unverständlich, Anzahl Schrägstriche = Anzahl Wörter, in Klammern der vermutete Wortlaut

! $\quad$ Betonung, Hervorhebung

: Dehnung

? $\quad$ Frage, steigend oder hoch endende Stimme

Abgeschlossener Gedanke, auf dem Grundton endende Stimme

kurzes Zögern, Gedanke wird jedoch fortgesetzt

abgebrochener Gedanke, gefolgt von einem anderen Gedanken

Wortabbruch (bspw. merk-)

\section{Interjektionen:}

hm oder hmhm Bestätigung

hm? $\quad$ Frage

hm, oder hmhm, $\quad$ Verwunderung

hm. oder hmhm. Ratlosigkeit

hmhm- Verneinung

\section{Pausen:}

2 Sekunden

-- 5 Sekunden

--- $\quad 10$ Sekunden

--- $\quad 15$ Sekunden

---- $\quad 30$ Sekunden

------ 1 Minute

------ 2 Minuten 


\section{C1. Varimax-rotierte Komponentenmatrix der 2-Faktorenlösung der HKA der ISE-Items (nur Ladungen grösser .40 angegeben)}

\begin{tabular}{|c|c|c|}
\hline \multirow[t]{2}{*}{ Item } & \multicolumn{2}{|c|}{ Komponente } \\
\hline & 1 & 2 \\
\hline ISE_34b: Beeinträchtigung der sozialen Kontakte der ganzen Familie durch Vorurteile? & .820 & \\
\hline ISE_33c: Beeinträchtigung der familiären Beziehungen durch Vorurteile? & .819 & \\
\hline ISE_33b: Beeinträchtigung der sozialen Kontakte durch Vorurteile? & .811 & \\
\hline ISE_34a: Beeinträchtigung der Lebensqualität der ganzen Familie durch Vorurteile? & .799 & \\
\hline ISE_34c: Beeinträchtigung der familiären Beziehungen der ganzen Familie durch Vorurteile? & .782 & \\
\hline ISE_33a: Beeinträchtigung der Lebensqualität durch Vorurteile? & .772 & \\
\hline ISE_33d: Beeinträchtigung des Selbstwertgefühles durch Vorurteile? & .596 & \\
\hline $\begin{array}{l}\text { ISE_26: Haben Ihre Erfahrungen mit Vorurteilen Ihre Fähigkeit beeinträchtigt, den Kontakt zu } \\
\text { anderen Familienmitgliedern zu pflegen? }\end{array}$ & .564 & \\
\hline $\begin{array}{l}\text { ISE_25: Haben die Erfahrungen mit Vorurteilen die Fähigkeit von Ihnen oder Ihrer Familie } \\
\text { beeinträchtigt, Freundschaften zu schliessen oder aufrecht zu erhalten? }\end{array}$ & .531 & .525 \\
\hline $\begin{array}{l}\text { ISE_29: Versuchen Sie, Situationen zu vermeiden, in denen Sie oder Ihre Familie auf Vorurteile } \\
\text { stossen könnten? }\end{array}$ & .427 & \\
\hline $\begin{array}{l}\text { ISE_17: Glauben Sie, dass die Leute im Durchschnitt Angst vor jemandem haben, der } \\
\text { unter einer Suchterkrankung leidet? }\end{array}$ & & \\
\hline $\begin{array}{l}\text { ISE_18: Ist Ihr Angehöriger schon einmal aufgrund seiner/ihrer Suchterkrankung auf } \\
\text { Vorurteile gestossen? }\end{array}$ & & .820 \\
\hline $\begin{array}{l}\text { ISE_19: Sind Sie persönlich schon einmal aufgrund der Suchterkrankung Ihres Angehörigen } \\
\text { auf Vorurteile gestossen? }\end{array}$ & & .796 \\
\hline $\begin{array}{l}\text { ISE_20: Sind andere Mitglieder Ihrer Familie aufgrund der Suchterkrankung Ihres Angehörigen } \\
\text { auf Vorurteile gestossen? }\end{array}$ & & .616 \\
\hline $\begin{array}{l}\text { ISE_27: Haben Ihre Erfahrungen mit Vorurteilen Ihre Lebensqualität oder die Lebensqualität } \\
\text { Ihrer Familie beeinträchtigt? }\end{array}$ & .517 & .551 \\
\hline
\end{tabular}




\section{C2. Varimax-rotierte Komponentenmatrix der 1-Faktorenlösung der HKA der ISE-Items(nur Ladungen grösser .40 angegeben)}

Item

$$
\text { Iten }
$$

Komponente

1

ISE_34a: Beeinträchtigung der Lebensqualität der ganzen Familie durch Vorurteile?

ISE_33a: Beeinträchtigung der Lebensqualität durch Vorurteile?

ISE_34b: Beeinträchtigung der sozialen Kontakte der ganzen Familie durch Vorurteile?

ISE_33b: Beeinträchtigung der sozialen Kontakte durch Vorurteile?

ISE_33c: Beeinträchtigung der familiären Beziehungen durch Vorurteile?

ISE_27: Haben Ihre Erfahrungen mit Vorurteilen Ihre Lebensqualität oder die Lebensqualität Ihrer Familie beeinträchtigt?

ISE_25: Haben die Erfahrungen mit Vorurteilen die Fähigkeit von Ihnen oder Ihrer Familie beeinträchtigt, Freundschaften zu schliessen oder aufrecht zu erhalten?

ISE_34c: Beeinträchtigung der familiären Beziehungen der ganzen Familie durch Vorurteile?

ISE_33d: Beeinträchtigung des Selbstwertgefühles durch Vorurteile?

ISE_26: Haben Ihre Erfahrungen mit Vorurteilen Ihre Fähigkeit beeinträchtigt, den Kontakt zu anderen Familienmitgliedern zu pflegen?

ISE_19: Sind Sie persönlich schon einmal aufgrund der Suchterkrankung Ihres Angehörigen auf Vorurteile gestossen?

ISE_20: Sind andere Mitglieder Ihrer Familie aufgrund der Suchterkrankung Ihres Angehörigen auf Vorurteile gestossen?

ISE_18: Ist Ihr Angehöriger schon einmal aufgrund seiner/ihrer Suchterkrankung auf Vorurteile gestossen?

ISE_29: Versuchen Sie, Situationen zu vermeiden, in denen Sie oder Ihre Familie auf Vorurteile stossen könnten?

ISE_17: Glauben Sie, dass die Leute im Durchschnitt Angst vor jemandem haben, der unter einer Suchterkrankung leidet?

ISE_16: Glauben Sie, dass die Leute weniger von Menschen halten, die Suchterkrankungen haben? 
D1: Zuordnungsübersicht des Ward-Verfahrens

Zuordnungsübersicht

\begin{tabular}{|c|c|c|c|c|c|c|}
\hline \multirow[t]{2}{*}{ Schritt } & \multicolumn{2}{|c|}{ Zusammengeführte Cluster } & \multirow[t]{2}{*}{ Koeffizienten } & \multicolumn{2}{|c|}{ Erstes Vorkommen des Clusters } & \multirow[t]{2}{*}{ Nächster Schritt } \\
\hline & Cluster 1 & Cluster 2 & & Cluster 1 & Cluster 2 & \\
\hline 1 & 28 & 73 & 7.500 & 0 & 0 & 4 \\
\hline 2 & 1 & 84 & 15.500 & 0 & 0 & 17 \\
\hline 3 & 59 & 75 & 23.500 & 0 & 0 & 9 \\
\hline 4 & 28 & 47 & 32.000 & 1 & 0 & 11 \\
\hline 5 & 49 & 68 & 41.000 & 0 & 0 & 22 \\
\hline 6 & 14 & 52 & 50.000 & 0 & 0 & 21 \\
\hline 7 & 41 & 71 & 60.000 & 0 & 0 & 16 \\
\hline 8 & 34 & 54 & 71.500 & 0 & 0 & 41 \\
\hline 9 & 59 & 67 & 83.500 & 3 & 0 & 47 \\
\hline 10 & 26 & 60 & 95.500 & 0 & 0 & 16 \\
\hline 11 & 28 & 45 & 107.750 & 4 & 0 & 43 \\
\hline 12 & 17 & 58 & 120.750 & 0 & 0 & 52 \\
\hline 13 & 29 & 74 & 134.250 & 0 & 0 & 32 \\
\hline 14 & 16 & 69 & 148.750 & 0 & 0 & 34 \\
\hline 15 & 21 & 57 & 163.750 & 0 & 0 & 37 \\
\hline 16 & 26 & 41 & 178.750 & 10 & 7 & 32 \\
\hline 17 & 1 & 64 & 194.083 & 2 & 0 & 27 \\
\hline 18 & 5 & 70 & 209.583 & 0 & 0 & 33 \\
\hline 19 & 39 & 51 & 225.083 & 0 & 0 & 63 \\
\hline 20 & 43 & 55 & 241.083 & 0 & 0 & 39 \\
\hline 21 & 14 & 72 & 257.417 & 6 & 0 & 44 \\
\hline 22 & 42 & 49 & 274.417 & 0 & 5 & 34 \\
\hline 23 & 32 & 50 & 291.917 & 0 & 0 & 54 \\
\hline 24 & 31 & 77 & 309.917 & 0 & 0 & 62 \\
\hline 25 & 62 & 63 & 327.917 & 0 & 0 & 56 \\
\hline 26 & 15 & 18 & 346.417 & 0 & 0 & 74 \\
\hline 27 & 1 & 37 & 365.333 & 17 & 0 & 43 \\
\hline 28 & 30 & 87 & 384.333 & 0 & 0 & 44 \\
\hline 29 & 4 & 76 & 403.833 & 0 & 0 & 65 \\
\hline 30 & 3 & 88 & 423.833 & 0 & 0 & 45 \\
\hline 31 & 10 & 13 & 443.833 & 0 & 0 & 53 \\
\hline 32 & 26 & 29 & 464.833 & 16 & 13 & 38 \\
\hline 33 & 5 & 65 & 486.000 & 18 & 0 & 52 \\
\hline 34 & 16 & 42 & 508.700 & 14 & 22 & 60 \\
\hline 35 & 2 & 86 & 531.700 & 0 & 0 & 45 \\
\hline 36 & 12 & 83 & 554.700 & 0 & 0 & 58 \\
\hline
\end{tabular}




\begin{tabular}{|c|c|c|c|}
\hline 37 & 21 & 23 & 578.367 \\
\hline 38 & 26 & 61 & 602.867 \\
\hline 39 & 20 & 43 & 627.533 \\
\hline 40 & 7 & 66 & 652.533 \\
\hline 41 & 27 & 34 & 677.700 \\
\hline 42 & 46 & 48 & 703.700 \\
\hline 43 & 1 & 28 & 730.200 \\
\hline 44 & 14 & 30 & 757.067 \\
\hline 45 & 2 & 3 & 787.567 \\
\hline 46 & 1 & 85 & 818.122 \\
\hline 47 & 26 & 59 & 848.722 \\
\hline 48 & 7 & 53 & 879.722 \\
\hline 49 & 19 & 82 & 912.222 \\
\hline 50 & 11 & 40 & 946.222 \\
\hline 51 & 8 & 25 & 980.222 \\
\hline 52 & 5 & 17 & 1016.156 \\
\hline 53 & 7 & 10 & 1052.156 \\
\hline 54 & 21 & 32 & 1088.389 \\
\hline 55 & 33 & 80 & 1125.389 \\
\hline 56 & 62 & 78 & 1162.722 \\
\hline 57 & 56 & 81 & 1200.222 \\
\hline 58 & 11 & 12 & 1237.722 \\
\hline 59 & 24 & 38 & 1275.722 \\
\hline 60 & 16 & 26 & 1318.989 \\
\hline 61 & 35 & 46 & 1362.989 \\
\hline 62 & 19 & 31 & 1407.239 \\
\hline 63 & 27 & 39 & 1452.272 \\
\hline 64 & 7 & 44 & 1497.939 \\
\hline 65 & 4 & 8 & 1543.689 \\
\hline 66 & 14 & 20 & 1589.572 \\
\hline 67 & 16 & 62 & 1640.561 \\
\hline 68 & 21 & 33 & 1694.590 \\
\hline 69 & 1 & 36 & 1749.834 \\
\hline 70 & 9 & 35 & 1807.084 \\
\hline 71 & 2 & 14 & 1865.167 \\
\hline 72 & 5 & 7 & 1926.446 \\
\hline 73 & 56 & 79 & 1988.946 \\
\hline 74 & 11 & 15 & 2055.113 \\
\hline 75 & 6 & 19 & 2123.163 \\
\hline 76 & 6 & 21 & 2200.851 \\
\hline 77 & 1 & 27 & 2278.584 \\
\hline
\end{tabular}

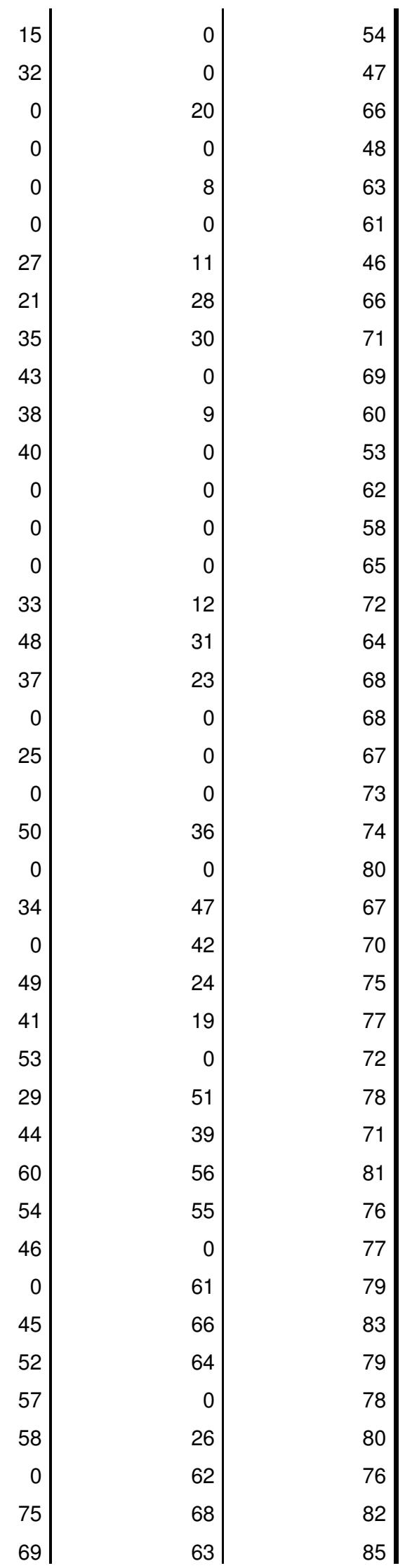




\begin{tabular}{|l|r|r|r|r|r|r|}
78 & 4 & 56 & 2371.049 & 65 & 73 & 84 \\
79 & 5 & 9 & 2474.853 & 72 & 70 & 81 \\
80 & 11 & 24 & 2594.812 & 74 & 59 & 82 \\
81 & 5 & 16 & 2723.035 & 79 & 67 & 83 \\
82 & 6 & 11 & 2860.393 & 76 & 80 & 84 \\
83 & 2 & 5 & 3024.648 & 71 & 81 & 85 \\
84 & 4 & 6 & 3231.793 & 78 & 82 & 86 \\
85 & 1 & 2 & 3469.393 & 77 & 83 & 86 \\
86 & 1 & 4 & 4178.805 & 85 & 84 & 0 \\
\hline
\end{tabular}




\section{E1. Kodierleitfaden mit Ankerbeispielen des Kategoriensystems}

\section{Bewältigungsstrategien}

\subsubsection{3:01:32}

Kategoriendefinition:

Kodierung von Textstellen, in denen deutlich wird, wie Angehörige mit den Belastungen, die durch Suchterkankung entstehen, umgehen.

Die möglichen unterschiedlichen Bewältigungsstrategien werden in den Unterkategorien kodiert.

\section{Diverse}

08.11.2013 16:54:10

Kategoriendefinition:

Kodierung von Textstellen, in denen Angehörige Einzelnennungen angaben, wie sie mit den Belastungen der Suchterkrankung umgehen. Dazu gehören beispielsweise:

a) eigener Substankonsum

b) Selbstbeschwörung

c) Nähe zum Betroffenen

d) Betonung, dass Bewältigungsstrategien sehr unterschiedlich sind

e) und weitere.

Kodierregel:

Nur Textstellen, die keiner anderen Kategorie der Bewältigungsstrategien zugeordnet werden können, werden in dieser Kategorie kodiert.

\section{Humor}

01.11.2013 15:21:31

Kategoriendefinition:

Kodierung von folgenden Textstellen:

1. Angehörige berichten von belastenden und schwierigen Situationen, die sie mit Humor nehmen konnten.

2. Angehörige zeigen im Gespräch Humor, wenn es um den Umgang mit Belastungen geht.

Kodierregel:

Mindestens ein Kriterium der Definition erfüllt.

Zudem sind die Kriterien der Überkategorie "Bewältigungsstrategien" ebenfalls erfüllt.

Ankerbeispiele:

Doris, Z402-404:

"A: Ja!+ Wa ich jetzt einfach noch sagen will ist äh. - Diese Drogendealer. Die könnte ich alle umbringen.

I: hmhm

A: Einzeln! Das „miech“ mir nichts aus. Aber ich erwische keinen. (A und I lachen beide laut)"

\section{Darüber sprechen}

01.11.2013 15:25:55

Kategoriendefinition:

Kodierung von folgenden Textstellen:

1. Angehörigen berichten, dass sie offen über die Suchterkrankung ihres Angehörigen berichten, weil sie davon überzeugt sind, dass es besser ist gleich die Wahrheit zu sagen, weil andere es sowieso rausbekommen würden.

2. Angehörigen sprechen über die Suchterkrankung ihres Angehörigen, weil sie andere an ihren Erfahrungen teilhaben lassen wollen und diese damit auch unterstützen möchten.

3. Angaben dazu, dass Angehörige sich bewusst anderen Menschen anvertrauen, um sich damit zu entlasten.

Kodierregel:

Mindestens ein Kriterium der Definition ist erfüllt.

Zudem sind die Kriterien der Überkategorie "Bewältigungsstrategien" ebenfalls erfüllt.

Ankerbeispiele:

Dylan, Z72-76:

"A: Ich meine; Ich habe die Erfahrung gemacht wenn wenn du den Leuten - noch eben schauspielerst. Ja ja. Ist alles gut. Oder?

I: hmhm

A: Dann ist offensichtlich dass etwas nicht stimmt.

I: hmhm 
A: Dann meinen sie nachher weiss nicht was. Dann sagst du lieber von Anfang weg was Sache ist."

Dorothea, Z254-258:

"Aber ähm inzwischen ja. Er er ist Teil von er ist Teil von meiner Geschichte.

I: hmhm

A: Er ist Teil von von meiner Entwicklung und, ja. - //(?:) meine meine Freunde oder mein mein mein Freund, ja. Sie wollen ja mich kennen. Und

I: hmhm

A: Wie gesagt diese diese Suchterkrankung in unserer Familie ist das Teil von uns."

\section{Hilfe suchen}

01.11.2013 15:34:19

Kategoriendefinition:

1. Die Angehörigen wurden gefragt, was sie jemandem raten würde, der ihnen von der eigenen Betroffenheit als Angehöriger berichten würde. Einige Angehörige gaben in diesem Zusammenhang an, dass sie raten würden, dass sich die Person professionelle Unterstützung holen soll. Die Textstellen werden unter dieser Kategorie kodiert.

Kodierregel:

Kriterium der Kategoriendefinition erfüllt.

Zudem sind die Kriterien der Überkategorie "Bewältigungsstrategien" ebenfalls erfüllt.

Ankerbeispiel:

August, Z724-729:

"I: hmhm Wenn, jetzt jemand würde zu ihnen kommen. Und würde sagen. Ich habe zum Beispiel gestern erfahren. Dass, der Sohn oder die Tochter von mir. Oder m- mein Partner. Oder meine Partnerin, harte Drogen konsumiert. Und offensichtlich ein Problem hat mit dem Konsum. Was würden sie dieser Person raten?

A: Das mache ich ja häufig jetzt. Und ich sage ja immer das Gleiche. Ich schicke sie in die Beratungsstelle.

I: hmhm

A: Ganz klar.

I: Also sich für sich selber Hilfe suchen.

A: Ja! Und und gebe ihnen, also Tipp, ist das falsch-; wir sind nicht Berater. Ab- wir haben Erfahrung +natürlich. Mit dieser Geschichte."

\section{Unabhängigkeit}

04.01.2013 18:52:35

Kategoriendefinition:

Kodierung von Textstellen, in denen deutlich wird, dass Angehörige sich von der Suchterkrankung ihres Angehörigen abgrenzen und auf diese Weise versuchen mit den Belastungen umzugehen. Die Eigenverantwortung der Betroffenen wird betont.

In die Kategorie gehören auch Textstellen, in denen Angehörige betonen, dass sie auf eigene Bedürfnisse und Gefühle achten und diese auch respektieren und ihnen entsprechend handeln.

Kodierregeln:

Die Textstellen werden in den Unterkategorien "Flucht", "Distanz wahren", "Abgrenzung / klare Regeln" und "Autonomie" kodiert.

Flucht

01.11.2013 15:40:10

Kategoriendefinition:

Kodierung von folgenden Textstellen:

1. Angehörige geben an, dass sie sich in etwas geflüchtet (beispielsweise Aussenbeziehung), um den Belastungen durch die Suchterkrankung zu entkommen.

Kodierregel:

Kriterium der Kategoriendefinition erfüllt.

Zudem sind die Kriterien der Überkategorie "Bewältigungsstrategien" und "Unabhängigkeit" ebenfalls erfüllt.

Ankerbeispiel:

August, Z414:

"A: Habe dann eine Beziehung eingegangen."

Distanz, wahren

01.11.2013 16:05:38

Kategoriendefinition: 
Kodierung von folgenden Textstellen:

1. Angehörige geben, dass sie es als hilfreich erlebt haben, wenn sie eine gewisse Distanz (räumlich, Kontakt) zu ihren suchtkranken Angehörigen haben.

Kodierregel:

Kriterium der Definition ist erfüllt.

Zudem sind die Kriterien der Überkategorie "Bewältigungsstrategien" und "Unabhängigkeit" ebenfalls erfüllt.

Ankerbeispiel:

Andrea, Z179-186:

"Mir ist es in dieser Zeit ja gut gegangen wo ich nichts gehört habe.

I: hmhm

A: Weil ich wusste dass die Distanz; habe aber ja schon gewusst es geht ihm ja eigentlich; es, etwas stimmt ja nicht.

Das ist ja nicht mein Sohn.

I: hmhm

A: Aber mir ist es i; ich habe einfach Distanz gehabt.

I: hmhm

A: Eigentlich habe ich sie ja, eh nie."

Dana, Z493:

"A: Genau. Und man hat mehr Energie wenn wenn man ein bisschen einen Abstand hat."

Abgrenzung / klare Regeln

01.11.2013 16:11:19

Kategoriendefinition:

Kodierung von folgenden Textstellen:

1. Angehörige berichten, dass sie gegenüber ihren suchtkranken Angehörigen klare Regeln aufgestellt hätten und diese auch konsequent durchgesetzt hätten.

2. Angehörige berichten, dass sie sich klar gegenüber ihren suchtkranken Angehörigen abgegrenzt haben, beispielsweise gegenüber Forderungen von diesen.

3. Angehörige berichten davon, dass sie Verantwortungen an ihre suchtkranken Angehörigen zurück delegierten. Dazu gehören auch Textstellen, in denen Angehörige angeben, dass sie zur Einsicht gekommen seien, dass ihr Angehörigen nur alleine lösen können oder nicht mehr bereit seien, Probleme der Angehörigen zu lösen.

4. Textstellen, in denen sich Angehörige von der Verantwortung an der Suchterkrankung freisprechen (keine Schuld zu tragen).

Die Textstellen werden auch kodiert, wenn deutlich wird, wie schwierig für die Angehörigen die Abgrenzung gefallen ist und letztlich ein Kopfentscheid war.

Kodierregel:

Mindestens ein Kriterium der Kategoriendefinition ist erfüllt.

Zudem sind die Kriterien der Überkategorie "Bewältigungsstrategien" und "Unabhängigkeit" ebenfalls erfüllt.

Ankerbeispiele:

Doris, Z366

"A: Zuerst haben wir sie nämlich Heim nehmen wollen. Zu uns. Und dann haben wir gefunden. Nein. Diesen! Quatsch! wollen wir nicht mehr. Das halte ich nicht aus (spricht in verschmitztem Tonfall). Sie sollen sich die „Grinder“ (=Köpfe) daheim verschlagen."

Angelika, Z460-464:

"Vielleicht. Ich muss aufhören! Ich muss;

I: hmhm

A: Mein: Wunsch - ä- ist ungültig. Er muss selber das;

I: hmhm

A: //(?: ) wieder distanzieren."

Albert, Z697-699:

"A: Habe dann einfach gesagt. Jetzt ist fertig.

I: hm

A: Jetzt gibt es nichts mehr. Von mir!" 
Autonomie

01.11.2013 16:23:41

Kategoriendefinition:

Kodierung von folgenden Textstellen:

1. Angehörige berichten, dass sie sich von ihrem suchtkranken Angehörigen lösen wollten.

2. Angehörige berichten, dass sie bewusst Dinge machten oder unternahmen, die ihnen Freude bereiteten. Sie berichten, dass sie gerne ihr Leben geniessen möchten.

3. Angehörige versuchen bewusst eigene Bedürfnisse und Wünsche wahrzunehmen (Fokus auf sich selbst), unabhängig davon wie es dem suchtkranken Angehörigen geht.

Dieses Bedürfnis nach Autonomie kann verschieden motivert sein. Während einige Angehörige aus Resignation, nichts beim suchtkranken mit ihren Bemühungen zu erreichen, sich vermehrt auf sich selbst konzentrieren, ist es bei anderen die grundsätzliche Haltung, Anspruch auf ein eigenes Leben zu haben.

Kodierregel:

Mindestens ein Kriterium der Definition ist erfüllt.

Zudem sind die Kriterien der Überkategorie "Bewältigungsstrategien" und "Unabhängigkeit" ebenfalls erfüllt.

Ankerbeispiele:

Dana, Z349-351:

"Es hat eher mich äh verändert --- dass man vielleicht am Anfang nicht so Lust hatte äh zum zum äh Rambazamba, also zum Festen oder äh raus gehen. Dass man vielleicht auch mal ein bisschen (räuspert sich) und dass; Und heute ist es so dass ich äh die Freiheit geniessen möchte und das Leben geniessen.

I: hmhm hmhm

A: Also, ja. Sich an Schönem erfreuen."

Anke, Z128-132:

"Aber ich möchte eigentlich und das ist das ist immer mehr geworden (lacht dabei). Ich möchte eigentlich mein Leben nicht opfern. Für Sachen die andere falsch machen.

I: hmhm hmhm

A: Und äh es tut mich betreffen. Es tut mich beschäftigen. Aber ich möchte eigentlich einen Teil von meinem Leben für mich behalten wo ich eigentlich normal bin. Und nicht ///(?: lacht und sehr leise, deshalb nicht verständlich).

I: hmhm Und wo Sie für sich auch geniessen können.

A: Ja."

Aufbegehren

05.01.2013 17:20:09

Kategoriendefinition:

Kodierung von folgenden Textstellen:

Angaben dazu, dass die Suchterkrankung für die Angehörigen nicht akzeptabel ist und dass sie sich dafür einsetzen, dass der kranke Angehörige suchtfrei wird.

Dies kann sich folgendermassen äussern:

1a) durch emotionales, konfrontierendes Verhalten (bspw. in dem man seinen Emotionen und beispielsweise der Wut freien Lauf lässt)

1b) durch kontrollierendes Verhalten

1c) durch Verhaltensweisen, mit denen der suchtkranke Angehörige entlastet und unterstützt werden soll (Übernahme von Verantwortung) und auf diese Weise wieder gesund werden soll.

1d) Engagement für suchtkranke Menschen

Kodierregel:

Die einzelnen Textstellen werden unter den Unterkategorien kodiert.

Zudem sind die Kriterien der Überkategorie "Bewältigungsstrategien" ebenfalls erfüllt.

Hoffnung

01.11.2013 16:40:33

Kategoriendefinition:

Kodierung von folgenden Textstellen:

1. Angehörige berichten, dass sie Hoffnung nicht aufgeben würden, dass ihre Angehörigen wieder gesund werden.

2. Angaben dazu, welche Hoffnungen Angehörige für ihre suchtkranken Angehörigen allgemein noch haben.

Kodierregeln:

Mindestens ein Kriterium der Kategorie ist erfüllt. 
Zudem sind die Kriterien der Überkategorie "Bewältigungsstrategien" und "Aufbegehren" ebenfalls erfüllt.

Ankerbeispiel:

Doris, Z490-492:

"A: Aber von+ irgendwo hat man immer wieder die Kraft, zum sagen. Nein! Man muss, das Positive vom Negativen sehen. Hat der *Name01 gesagt (lacht). Und das, ist mein Leitfaden geworden.

I: hmhm hmhm hmhm Dass sie, auch immer das Schöne auch noch wieder, +sehen.

A: Ja.+ Dass man einfach, die Hoffnung nie +aufgibt."

\section{Kontrolle}

01.11.2013 17:03:33

Kategoriendefinition:

Kodierung von folgenden Textstellen:

1. Angaben dazu, dass Angehörige ihren suchtkranken Angehörigen in seinem Konsum kontrollieren.

2. Angehörige berichten, was sie tun, um ihren suchtkranken Angehörigen in seinem Konsum zu beschränken und dadurch vor weiteren Schwierigkeiten zu bewahren, beispielsweise in dem Geld oder Drogen verwaltet werden.

Kodierregel:

Mindestens ein Kriterium der Kategorie ist erfüllt.

Zudem sind die Kriterien der Überkategorie "Bewältigungsstrategien" und "Aufbegehren" ebenfalls erfüllt.

Ankerbeispiele:

Annabelle, Z168-170:

"A: Also ich nehme mir die Freiheit heraus, seine äh von der Bank seine Auszüge äh anzuschauen.

I: hmhm

A: Und äh ich weiss wo er seine Sachen holt. Und ich weiss dass er dort Geld herauslässt bei diesem Automat. Und es ist einfach pro Monat nur noch halb so viel."

Doris, Z66-68:

"A: Ich habe es dann so gemacht. Dass ich, und nach Mitternacht. Am Zahltag. Am Fünfundzwanzigsten, aufgestanden bin. Also am Vierundzwanzigsten nach Mitternacht. Und auf die Bank bin. Und sein Geld abgehoben habe. Und auf mein Konto verschoben.

I: hmhm

A: Und einfach nur noch einen kleinen Teil gelassen habe. Dass man alles hat zahlen können und. Dass einfach; es ist, unglaublich! ins Haltlose gelaufen."

Wut

01.11.2013 17:08:43

Kategoriendefinition:

Kodierung von folgenden Textstellen:

Angehörige berichten über Wut im Zusammenhang mit der Suchterkrankung ihres Angehörigen, die verschiedene Ursachen haben kann:

1. Konsum

2. fehlende Bereitschaft beim suchtkranken Angehörigen, etwas zu verändern.

3. Schwierigkeiten, die durch den Konsum entstehen und auf die Angehörige zurückfällt.

4. Behörden, die nicht handeln.

Kodierregel:

Mindestens ein Kriterium der Kategorie ist erfüllt.

Zudem sind die Kriterien der Überkategorie "Bewältigungsstrategien" und "Aufbegehren" ebenfalls erfüllt.

Ankerbeispiele:

Annabelle Z245:

"Also ich denke --- ich hätte ihm „öpe die“ (=öfters mal) mal ein Wallholz über die Rübe ziehen können."

Annabelle, Z146-148:

"A: Also bis ich dann irgendwie. Zwei drei Mal wo ich gefunden habe. Hey! (entrüstet) Jetzt; fluchen; Jetzt gopferdeckel“ jetzt kannst du einfach; So viel Kohle! Das +geht einfach nicht!

I: hmhm

A: Scheisse die Kinder kosten. Ich reisse mir den Arsch auf und gehe schaffen! Und also einfach, ein bisschen so. (=Diskussion ging in diese Richtung) Und dann: weiss auch nicht;"

Druck ausüben 
01.11.2013 17:15:52

Kategoriendefinition:

Kodierung von folgenden Textstellen:

1. Angehörige geben an, dass sie versuchten durch Druck oder Konfrontation den Angehörigen zu einer Änderungsmotivation zu bringen.

Kodierregel:

Kriterium der Kategorie erfüllt.

Zudem sind die Kriterien der Überkategorie "Bewältigungsstrategien" und "Aufbegehren" ebenfalls erfüllt.

Ankerbeispiele:

Andrea, Z694-696:

"Und natürlich mit dieser Person (=drogenabhängige Person) auch reden.

I: hmhm

A: Also immer wieder reden. Auch wenn sie es nicht gerne haben und wenn sie ja; Also das würde ich vorschlagen."

Dana, Z43:

"Bis es dann einfach immer wieder eskaliert hat und ich dann das erste Mal Druck ausgeübt habe. Jetzt müssen wir etwas machen. Oder?"

Unterstützung / Verständnis

01.11.2013 17:20:25

Kategoriendefinition:

Kodierung von folgenden Textstellen:

1. Angehörige berichten ganz allgemein, dass sie ihrem Angehörigen helfen oder diesen unterstützen wollten.

2. Angehörge berichten, dass anderen Suchtkranken helfen und diese unterstützen aufgrund ihrer eigenen Betroffenheit.

Kodierregel:

Mindestens ein Kriterium der Kategorie ist erfüllt.

Zudem sind die Kriterien der Überkategorie "Bewältigungsstrategien" und "Aufbegehren" ebenfalls erfüllt.

Spezifische Verhaltensweisen, mit denen die Angehörigen den Betroffenen unterstützten, werden in den Unterkategorie kodiert.

Ankerbeispiele:

Dorothea, Z102:

"A: Wenn das doch so offensichtlich ist. Da muss man doch helfen. Oder?"

Angelika, Z256:

"A: Ja:.+ Ja ja. hmhm ja:. Gut. Ich ich gebe ihm ,amigs“ (=jeweils), ich gebe ,amigs“ auch wenn wenn ich sehe so ein armer „Cheib“ (=Kerl). Ich gebe ,amigs“ auch einen Fünfliber. Oder - denke es könnte mein Sohn sein. Und der ist jetzt so; also bis einer fragen geht, betteln geht, braucht es ja auch etwas."

Doris, Z370-374:

"A: Also! Mir hat der Doktor *Name01 gelehrt (lacht kurz seufzend)

I: hmhm

A: Ziemlich ,gli“. Ich muss nicht, selber meinen wollen. Ich könne es regeln. Wie ich das gemeint habe am Anfang. I: hmhm

A: Mit Fürsorge. Geborgenheit. Essen und Liebe. Man kann es nämlich nicht mehr steuern."

\section{Politisches Engagement}

01.11.2013 16:57:44

Kategoriendefinition:

Kodierung von folgenden Textstellen:

1. Angehörige berichten, dass sich sich politisch für die Rechte von suchtkranken Menschen engagiert haben.

2. Angehörige berichten, dass sie sich für vermehrte Aufklärung engagieren würden.

3. Angehörige berichten, dass sie sich aktiv dafür einsetzen würden gegen die Verbreitung und Entstehung von Suchterkrankungen.

Kodierregel:

Mindestens ein Kriterium der Kategorie ist erfüllt. 
Zudem sind die Kriterien der Überkategorie "Bewältigungsstrategien" und "Aufbegehren" ebenfalls erfüllt.

Ankerbeispiele:

Dylan, Z98-100:

"A: Ja aber das ist so! Das das ist einfach so. Auf diesem Planet werden Drogen verkauft. Drogen werden konsumiert und werden produziert. Und und du kommst ja nicht daran vorbei in dem Sinn. Oder?

I: hmhm hmhm hmhm

A: Man kann es verleugnen. Man kann das Problem wegstossen. Oder man man tut es aktiv probieren etwas zu machen."

August, Z929:

"A: Bevor da diese SKOS-Richtlinien (Anm.: Schweizerische Konferenz für Sozialhilfe), geändert worden sind. Ich habe noch, noch ziemlich mitgewirkt. Dass da etwas geht."

\section{Abstinenz erreichen \\ 02.11.2013 11:23:11}

Kategoriendefinition:

Kodierung von folgenden Textstellen:

1. Angehörige berichten, dass für ihre suchtkranken Angehörigen Therapieplätze organisierten oder diese in die Therapie schickten. Dazu gehören auch Textstellen, in denen es darum geht, dass Angehörige den Betroffenen darin unterstützten, einen Therapieplatz zu finden.

2. Angehörige berichten, dass sie ihren suchtkranken zur Therapie begleiteten und sich aktiv darüber informierten, was Ziel und Thema der Therapie war.

3. Angehörige berichten, dass sie ihren suchtkranken Angehörigen darin unterstützten, die Abstinenz aufrecht zu erhalten.

Kodierregel:

Mindestens ein Kriterium der Kategorie ist erfüllt.

Zudem sind die Kriterien der Überkategorie "Bewältigungsstrategien", "Aufbegehren" und "Unterstützung/Verständnis" ebenfalls erfüllt.

Ankerbeispiel:

Andrea, Z222:

"Wir haben auch abgemacht. Ich bin ihn immer holen gegangen am Bahnhof. Er hat das auch gewollt am Anfang dass ich ihn abholen komme dass er nicht diesen Leuten begegnet."

Andrea, Z135-139:

"A: Und dann hat das aller- am allersch-; Bevor er dann wieder rein konnte sind wir dann nochmals beim Hausarzt gewesen.

I: hmhm

A: Und dort hat er, mein Sohn gesagt. Mami. Kommst Du mit? Und dann habe ich gesagt. Ja. Ich komme mit.

I: hmhm

A: Es hat mich auch Wunder genommen."

\section{Angehörige aufnehmen}

01.11.2013 17:26:48

Kategoriendefinition:

Kodierung von folgenden Textstellen:

1. Angehörige berichten, dass sie ihren suchtkranken Angehörigen bei sich zu Hause aufgenommen hätten, um sich um diesen zu kümmern und auf diese Weise Änderungsmotivation beim Angehörigen bezüglich seines Substanzkonsums zu erreichen.

2. Angehörige berichten, dass sie den suchtkranken Angehörigen auf der Gasse gesucht hätte, um diesen mit nach Hause zu nehmen.

Kodierregel:

Mindestens ein Kriterium der Kategorie ist erfüllt.

Zudem sind die Kriterien der Überkategorie "Bewältigungsstrategien", "Aufbegehren" und "Unterstützung/Verständnis" ebenfalls erfüllt.

Ankerbeispiel:

Albert, Z271-275:

"ich habe dann den Vorschlag gemacht dass, dass ich die Tochter, zu uns nach Hause nehme. Von dort wo sie jetzt wohnt. 
I: hmhm

A: Und dass wir sie dort betreuen. Also quasi - mit äh - Kommoditäten. Vitaminreich kochen. Fein essen. Ruhiges Zimmer ruhig schlafen. Äh erholen und.

I: hmhm

A: Dass wir sie irgendwie bewegen können - äh mit der Ko- mit dem Konsumieren aufzuhören. Und eine solche Thera- Therapie anzufangen."

\section{Geld geben}

01.11.2013 17:21:11

Kategoriendefinition:

Kodierung von folgenden Textstellen:

1. Angaben dazu, dass Angehörige ihren suchtkranken Angehörigen Geld gaben, um sie vor weiteren Schwierigkeiten zu bewahren und somit den totalen Absturz zu verhindern.

Kodierregel:

Kriterium der Kategorie erfüllt.

Zudem sind die Kriterien der Überkategorie "Bewältigungsstrategien", "Aufbegehren" und "Unterstützung/Verständnis" ebenfalls erfüllt.

Ankerbeispiel:

Dana, Z367-369:

"Das hat auch die Kollegin den Fehler vielleicht gemacht. Äh dass man halt ihm dann trotzdem Geld gegeben hat.

I: hmhm

A: Äh dass er nicht äh kriminell quasi werden muss."

\section{Toleranz}

04.01.2013 18:36:31

Kategoriendefinition:

Kodierung von folgenden Textstellen:

1. Angaben dazu, dass der Substanzkonsum toleriert wird, weil

1a) die Streitereien nichts bringen und man resigniert hat (Resignation).

1b) sich die eigene Einstellung dahingehend verändert hat, dass ein Leben auch mit Substanzkonsum möglich ist (Akzeptanz)

2. Angaben dazu, dass die Angehörigen dem drogenabhängigen Angehörigen helfen, den Konsum zu decken, indem sie nach aussen Ausreden erfinden oder helfen die Fassade aufrecht zu erhalten.

Kodierregel:

Mindestens ein Kriterium der Kategorie ist erfüllt.

Zudem sind die Kriterien der Überkategorie "Bewältigungsstrategien" ebenfalls erfüllt.

Ankerbeispiele:

Dylan, Z237-239:

"A: Solche Sachen dass du eben nicht darüber geredet hast. Oder oder, dass du versucht hast also mit den Eltern quasi so so ein Bild von von der schönen Familie zu machen.

I: hmhm hmhm

A: Wo alles in Ordnung hat. Oder?"

Annabelle, Z290-294:

"Ja. ich denke. Es gibt schon auch einen Weg mit.

I: hmhm

A: mit dem Konsum.

I: hmhm

A: Aber ähm - es müsste es einfach viel offener in der Partnerschaft +thematisiert werden." 


\section{Situation der Erkenntnis}

\subsubsection{6:30:46}

Kategoriendefinition:

Kodierung von folgenden Textstellen:

Angehörige berichten davon, wie sie von der Suchterkrankung erfahren haben, ob sie Vorahnungen im Vorfeld hatten und wie sie auf die Erkenntnis, dass ihr Angehöriger Drogen konsumiert, reagiert haben.

Kodierregeln:

Wie Angehörige von der Suchterkrankung erfahren habe, Vorahnungen im Vorfeld der Erkenntnis und wie Angehörige auf die Erkenntnis reagiert haben, wird in den entsprechenden Unterkategorien kodiert.

\section{Fund/Situation}

05.11.2013 10:52:07

Kategoriendefinition:

Kodierung von folgenden Textstellen:

1. Angehörige berichten von einer spezifischen Situation (Ereignis), in der ihnen bewusst wurde, dass ihr Angehöriger ein schwerwiegendes Suchtproblem hat.

2. Angehörige berichten von einem Fund (Spritzen etc.), die ihnen deutlich machte, dass ihr Angehöriger ein schwerwiegendes Suchtproblem hat.

3. Angehörige (Kinder von drogenabhängigen Eltern) berichten, dass sie bereits immer von der Suchterkrankung gewusst hätten.

Kodierregel:

Mindestens ein Kriterium der Kategoriendefinition ist erfüllt.

Zudem sind die Kriterien der Überkategorie "Situation der Erkenntnis" ebenfalls erfüllt.

Ankerbeispiele:

"Zuerst hat er geraucht dann hat er gesnifft und dann hat er gejunkt. Sich auch einfach immer;

I: hmhm

A: Also gemerkt dass er wieder spritzt habe ich weil er bei Google drin gesehen habe dass er wegen Spritzenautomaten geschaut hat. Wo gibt es Spritzenautomaten?

$\mathrm{I}: \mathrm{hmhm}$

A: Und -- oder auch im Abfall, gefunden habe."

\section{Situation nicht mehr erinnerbar}

\subsubsection{1:15:15}

Kategoriendefinition:

Kodierung von folgenden Textstellen:

1. Angehörige geben an, dass sie sich nicht mehr erinnern können, wie sie erfahren hätten, dass ihr Angehöriger harte Drogen konsumiert.

2. Angehörige stellen Vermutungen an, wie sie von der Suchterkrankung erfahren haben könnten, betonen aber gleichzeitig, dass sie sich nicht mehr daran erinnern könnten, wie sie davon erfahren haben.

Kodierregel:

Mindestens ein Kriterium der Kategoriendefinition ist erfüllt.

Zudem sind die Kriterien der Überkategorie "Situation der Erkenntnis" ebenfalls erfüllt.

Ankerbeispiele:

Dorrette, Z12:

"nein ich denke immer. Er hat es mir gesagt. Irgendwann. Dass es, so ist."

\section{Drittpersonen klären Angehörige auf}

05.11.2013 11:22:45

Kategoriendefinition:

Kodierung von folgenden Textstellen:

1. Angehörige berichten, dass andere Angehörige (beispielsweise Geschwister der betroffenen Person) sie über die Suchterkrankung aufgeklärt hätten.

2. Angehörige berichten, dass sie über eine aussenstehende Drittperson vom Substanzkonsum des Angehörigen erfahren hätten.

Kodierregel:

Mindestens ein Kriterium der Kategorie ist erfüllt. 
Zudem sind die Kriterien der Überkategorie "Situation der Erkenntnis" ebenfalls erfüllt.

Ankerbeispiel:

August, Z20:

"A: Nein. Äh e- i- im Grundsatz haben wir es dann eigentlich eher erfahren durch äh, den Ältesten und den: Jüngsten von diesen Söhnen. Die haben dann natürlich mehr! gewusst."

Doris, Z6-12:

"A: Ja! Das ist „äso“ gewesen. Vor zirka zwanzig Jahren. Weiss es nicht mehr genau. Nach der Lehre. Wo er eigentlich schon sehr einen guten Job gehabt hat. Bei der Bank. Er ist ein Aufsteiger gewesen. Überall. Sehr beliebt. Dann ist er, einfach nicht mehr Heim gekommen. Hat noch bei uns gewohnt!

I: hmhm

A: Ja und dann ist er nicht Heim! gekommen. Zwei drei Tage! - Und dann haben wir einen Bekannten wo bei der Drogenfahndung ist.

I: hmhm

A: Und ich habe dann den angefragt. Ob man eine Vermisstenmeldung herausgebe müsste. Oder was immer. Ich habe keine Ahnung gehabt was los ist. Und dann hat er mir gesagt. Ja es, ist auch wirklich nichts. Aber das ist, Kokain.

I: hmhm

A: - Soweit haben wir es eigentlich dann auf diese Art müssen leider erfahren. - Wir haben es vorher nicht gemerkt. Weil sie können es ja sehr gut kaschieren."

\section{Betroffener informiert Angehörige}

05.11.2013 11:29:03

Kategoriendefinition:

Kodierung von folgenden Textstellen:

1. Die Angehörigen berichten, dass ihr suchtkranker Angehöriger sie von sich aus über ihren Substanzkonsum informiert hatte.

2. Angehörige berichten, dass sie ihren suchtkranken Angehörigen auf den möglichen Drogenkonsum angesprochen hätten und dieser dann vom Angehörigen bestätigt worden sei.

Kodierregeln:

Mindestens ein Kriterium der Kategoriendefinition ist erfüllt.

Zudem sind die Kriterien der Überkategorie "Situation der Erkenntnis" ebenfalls erfüllt.

Ankerbeispiele:

Anke, Z30:

"A: Und etwa vor eineinhalb oder vielleicht auch zwei Jahren. Ich weiss es auch nicht mehr genau. Hat er es mir selbst gesagt. Dann habe ich es natürlich nicht mehr verdrängen können."

Andrea, Z59:

"A: Ja dann habe ich ihn dann darauf angesprochen. Und er hat gesagt. Ja aber selten und, ist nicht so schlimm. Ich habe das schon im Griff und; Aber bald darauf hat er dann selber gesagt. Er kanns; Ich glaub ich muss etwas machen. Und dann bald darauf ist er ja auch ins Ambulatorium."

\section{Vorahnungen}

05.01.2013 16:34:33

Kategoriendefinition:

Kodierung von folgenden Textstellen:

1. Angehörige beschreiben Anzeichen im Vorfeld der Erkenntnis, die ihnen Hinweis darauf gaben, dass ein problematischer Drogenkonsum vorliegen könnte.

2. Angehörige beschreiben, wie sie mit diesen Anzeichen im Vorfeld umgangen sind (Verhalten) und wie sie diese interpretiert (Gedanken) haben.

3. Auch Textstellen, in denen Angehörige angeben, dass sie niemals die Vermutung gehabt hätten, dass ihre Beobachtungen auf einen problematischen Drogenkonsum zurückzuführen wären.

Die Textstellen werden in den Unterkategorien kodiert.

„etwas stimmt nicht“

8.11.2013 09:00:21

Kategoriendefinition:

Kodierung von folgenden Textstellen:

1. Angehörige geben an, dass es Anzeichen oder Andeutungen für den Drogenkonsum ihres Angehörigen gegeben habe, dass sie diese aber verdrängt oder nicht so ernst genommen hätten.

2. Angehörige berichten, dass sie das Gefühl gehabt hätten, dass irgend etwas nicht stimme, aber keine Ahnung 
gehabt hätten, was nicht stimme.

Kodierregel:

Mindestens ein Kriterium der Kategoriendefinition ist erfüllt.

Zudem sind die Kriterien der Überkategorie "Vorahnungen" ebenfalls erfüllt.

Ankerbeispiele:

Darina, Z52:

"A: man hat man hat irgendwie gemerkt. Das ist doch komisch „e so“; Ja. Ja."

Anke, Z24-28:

"A: Und ähm wir haben einen Nachbar gehabt. Wo jung gestorben ist an Drogenmissbrauch. Und der ist dann; hat mit ihm plötzlich wieder Kontakt aufgenommen. Und ich habe gewusst dass der Kokain konsumiert.

I: Ja.

A: Und ich habe ihn ein paar Mal gesehen zu uns kommen. Dann bin ich immer ein bisschen nervös geworden und habe gewusst. Da läuft etwas nicht gut.

I: hmhm

A: Und dann, habe es dann auch wieder, irgendwie verdrängt. Und dann ist derjenige gestorben. Das hat dann äh unser Sohn sehr erschreckt. Und hat sicher eine zeitlang auch aufgehört damit."

Persönlichkeitsveränderung

08.11.2013 09:18:37

Kategoriendefinition:

Kodierung von folgenden Textstellen:

Angehörige erzählen, dass sich ihr suchtkranker Angehöriger im Vorfeld der Erkenntnis verändert, indem

a) er plötzlich weniger verlässlicher war im Vergleich zu früher.

b) er sich an vereinbarte Regeln nicht mehr hielt.

c) er sich grundsätzlich plötzlich ganz anders verhält als früher, indem sich beispielsweise sein Tages- und Nachtrhythmus verändert.

d) er delinquent wird.

Kodierregeln:

Mindestens ein Kriterium der Kategoriendefinition ist gegeben.

Zudem sind die Kriterien der Überkategorie "Vorahnungen" ebenfalls erfüllt.

Ankerbeispiel:

Darina, Z40-46:

"A: Einfach+ sehr abgewendet von uns.

I: hmhm

A: Ähm es ist alles immer nur, wie um die Nacht gegangen. Also ums Raus- in den Ausgang gehen.

I: hmhm

A: Ähm den Tag durch haben wir ihn manchmal kaum gesehen.

I: hmhm

A: Ähm ist er im Zimmer gewesen. Er hat geschlafen. Weil er die ganze Nacht weggewesen ist."

Doris, Z14-18:

"A: Er ist Schaffen gegangen. Täglich. Ist aufgestellt Heim gekommen. Ist einfach am Abend in den Ausgang. Und das hat sich dann vermehrt. Dass er einfach nicht mehr, Heim gekommen ist.

I: hmhm

A: Dass auch das Geschäft angerufen hat. Und gefragt hat. Wo ist der *Sohn?

I: hmhm

A: Und wir sind „schier“ (=fast) Kopfgestanden. Mein Mann und ich. Weil wir es eben auch nicht gewusst haben."

Ausbildungs- und finanzielle Schwierigkeiten

08.11.2013 09:27:38

Kategoriendefinition:

Kodierung von folgenden Textstellen:

1. Angehörige berichten, dass ihr suchtkranker Angehöriger im Vorfeld der Erkenntnis plötzlich vermehrt finanzielle Schwierigkeiten gehabt habe.

2. Angehörige berichten, dass ihr Angehörige im Vorfeld der Erkenntnis, in der Ausbildung oder im beruflichen Umfeld Schwierigkeiten bekommen habe.

Kodierregeln:

Mindestens ein Kriterium der Kategoriendefinition ist erfüllt. 
Zudem sind die Kriterien der Überkategorie "Vorahnungen" ebenfalls erfüllt.

Ankerbeispiele:

August, Z16-18:

"A: Schulaufgaben+, et cetera. Und dann haben wir eben beim Mittleren gemerkt dass etwas nicht mehr stimmt.

I: hmhm

A: Das ist also recht äh, ist schon nicht gut gewesen."

Darina, Z46-48:

"Und dann auch ähm, hat er viel Geld gebraucht.

I: hmhm

A: Und das habe ich jetzt weniger gemerkt. Das habe ich mitbekommen von meinen Eltern. Oder äh sonst, äh von meinen Brüdern. Aber er hat immer wegen Geld gebrauch- gefragt. Und „dernah“ (=je nachdem) in einem in einem Ton wo (lacht leicht) ähm ja. Wo nicht so angenehm gewesen ist."

Keine Vorahnungen

08.11.2013 09:33:04

Kategoriendefinition:

Kodierung von folgenden Textstellen:

1. Angehörige geben an, dass sie im Vorfeld keinerlei Vorahnungen gehabt hätten, dass ihr Angehöriger harte Drogen konsumieren könnte, auch wenn sie im Vorfeld allenfalls einige Veränderung festgestellt hatten.

2. Angabe von Gründen, weshalb die Angehörige gedacht haben, dass der Betroffene keine harten Drogen konsumieren würde.

Kodierregel:

Mindestens ein Kriterium der Kategoriendefinition ist erfüllt.

Zudem sind die Kriterien der Überkategorie "Vorahnungen" ebenfalls erfüllt.

Ankerbeispiel:

Dagmar, Z42-44:

"Ich bin also am Anfang wirklich sehr naiv gewesen, sehr ähm ja

I: Sie haben es auch nicht gewusst.

A: Nein. Ich hätte es jetzt auch nicht vermutet."

Dorette, Z9-12:

"I: Haben sie es einfach vermutet? (räuspert sich)

A: -- Also ich habe es irgendwie nicht gemerkt. Weil er hat ja vorher immer, gekifft.

I: hmhm

A: Und dann getrunken und. Ja. Und ich habe es, eigentlich;"

Reaktion auf Erkenntnis

05.01.2013 16:24:12

Kategoriendefinition:

Kodierung von folgenden Textstellen:

1. Welche Gedanken hatten Angehörige, als sie vom Drogenkonsum erfahren haben?

2. Welche Gefühle hatten Angehörige, als sie vom Drogenkonsum erfahren haben?

3. Was haben Angehörige als erstes gemacht, als sie vom Drogenkonsum erfahren haben?

4. Auch Reaktion auf Abstürze bzw. Rückfälle werden an dieser Stelle kodiert.

Ausschluss:

Achtung: Kodiert werden NUR Gedanken, Gefühle und Verhalten, welches im Zusammenhang mit der Erkenntnis vom Drogenkonsum stehen.

Sonstige Gefühle, Gedanken oder Verhalten werden entweder unter den Bewältigungsstrategien kodiert oder unter den Belastungen.

Kodierregeln:

Die Textstellen werden unter den Unterkategorien kodiert.

Erleichterung, endlich Bescheid zu wissen

05.11.2013 11:39:35

Kategoriendefinition:

Kodierung von folgenden Textstellen:

1. Angehörige gaben an, dass sie im ersten Moment erleichtert gewesen sei, als sie Bescheid gewusst hätten, weil sie 
a) das Gefühl hatten, dass nun etwas machen könnten.

b) das Gefühl hatten, für ihren suchtkranken Angehörigen sei es eine Entlastung gewesen.

c) das Gefühl hatten, die Ungewissheit sei belastender gewesen.

Kodierregel:

Mindestens ein Kriterium der Kategoriendefinition ist erfüllt.

Zudem sind die Kriterien der Überkategorie "Reaktion auf Erkenntnis" ebenfalls erfüllt.

Ankerbeispiele:

Andrea, Z318-324:

"Von dem Moment wo ich eigentlich gewusst habe dass er dass er äh Coci nimmt, dann habe ich hat ma- habe ich habe ich gefunden. Ich kann etwas machen. Obwohl ich ja das gar nicht; im Nachhinein weiss ich dass liegt gar nicht drin.

I: Ja.

A: Aber ich habe dann das Gefühl gehabt. Jetzt +ka-;

I: Ich+ weiss was es ist.

A: Was es ist.

I: Ja.

A: Jetzt kann ich etwas machen mit ihm."

\section{Rettung}

05.11.2013 11:45:01

Kategoriendefinition:

Kodierung von folgenden Textstellen:

1. Angehörige berichten, dass sie, als sie von der Suchterkrankung erfahren hätten, das Gefühl gehabt hätten, dass sie nun selbst zurückstehen und alles unternehmen müssten, um den Angehörigen dabei zu unterstützen, wieder gesund zu werden.

2. Angehörige berichten, dass sie optimistisch gewesen seien, den Angehörigen zu unterstützen, wieder gesund zu werden.

3. Angehörige berichten, sofort Hilfe und Unterstützung für den suchtkranken Angehörige gesucht zu haben.

Kodierregel:

Mindestens ein Kriterium der Kategoriendefinition ist erfüllt.

Zudem sind die Kriterien der Überkategorie "Reaktion auf Erkenntnis" ebenfalls erfüllt.

Ankerbeispiele:

Dorothea, Z16:

"Halt einfach auch, also halt einfach auch sehr früh klar gewesen ist. Ist einfach wirklich dass dass ///(?: noch oft geheissen). Jetzt müssen wir uns um den *Bruder kümmern. Jetzt, jetzt habe ich mich einfach still."

Albert, Z251-255:

"A: - Also wir haben -- eigentlich - in den ersten zwei drei Jahren von dieser Abhängigkeit. Haben wir nicht für uns Hilfe gesucht. Sondern für sie!

I: hmhm

A: Für die Tochter!

I: hmhm

A: Und dann wir haben versucht eine Möglichkeit zu finden. Dass man einen Ort findet. Wo sie hingehen kann. Wo sie kann äh sich erholen. - Und wo sie kann, therapieren."

Annabelle, Z37-42:

"I: hmhm hmhm Wie ist das gewesen für Sie? In dem Moment wo wo; Sie sagen Sie haben sich etwas das dritte oder vierte Mal getroffen gehabt und wo er Ihnen dann gesagt hat. Ich konsumiere Heroin. Wie ist das gewesen für Sie? -- oder ähm was haben Sie gedacht?

A: -- also ich denke es war für mich ähm; ich habe mich schon ein bisschen in diesen Kreisen bewegt gehabt.

I: hmhm

A: Ich habe Kollegen gehabt, Freunde gehabt, ähm: vielleicht ist das eher so ein bisschen -- Das Helfende wo mich gereizt hat.

I: hmhm

A: Ahh (=Ton der Begeisterung) da ist einer ähm ja. Dem kann man doch ein bisschen zeigen wie schön das Leben ist oder äh;" 
Hilfe suchen

05.11.2013 11:57:56

Kategoriendefinition:

Kodierung von folgenden Textstellen:

1. Angehörige berichten, dass sie sich jemandem mitgeteilt und somit Unterstützung gesucht hätten, wobei diese

Unterstützung nicht zwingend professioneller Art sein muss.

Kodierregel:

Kriterium der Kategoriendefinition ist erfüllt.

Zudem sind die Kriterien der Überkategorie "Reaktion auf Erkenntnis" ebenfalls erfüllt.

Ankerbeispiel:

Darina, Z68-70:

"A: Ja: und in in *Ausland habe ich habe ich es sogar ähm, in einer Bürokollegin erzählt.

I: hmhm

A: Und das wird; weil es so weit weggewesen ist habe ich nicht Angst haben müssen. Dass sie es weiter erzählt."

Erkenntnis ist schlimm gewesen

05.11.2013 12:09:21

Kategoriendefinition:

Kodierung von folgenden Textstellen:

1. Angehörige berichten, dass es für sie sehr schlimm gewesen sei, als sie vom problematischen Drogenkonsum ihres Angehörigen erfahren hätten.

2. Angehörige drücken in Form von Metaphern aus, wie schlimm die Erkenntnis, dass ihr Angehöriger Drogen konsumieren, gewesen sei.

Kodierregel:

Mindestens ein Kriterium der Kategoriendefinition ist gegeben.

Zudem sind die Kriterien der Überkategorie "Reaktion auf Erkenntnis" ebenfalls erfüllt.

Ankerbeispiele:

Albert, Z50-54:

"Ja. Und die, die, also den! Moment. Den vergesse ich nie mehr. Wo sie mir das gesagt hat.

I: hmhm Kann ich mir vorstellen.

A: Da reisst es einem ein Stück Fleisch, aus dem Körper.

I: hmhm hmhm

A: Das ist ähm - das ist äh, unvorstellbar. Das ist wie wenn man äh - ein eigenes ä- ein eigenes Stück Fleisch zum Körper heraus,reisst oder. Und nicht mehr kann äh; Also mit dem habe ich gar nicht umgehen können. Das ist, das hat mich einfach völlig, heruntergerissen. Ich meine ich; es hat mich schon heruntergerissen dass sie Konka- Kokain konsumiert! Was klar ist. Aber. Dass sie dann noch spritzt oder."

August, Z100-102:

"Und dann ist eine Welt zusammengebrochen.

I: Ja. Das kann ich mir +vorstellen.

A: Ja.+ Für die Frau und für mich. Ja."

Unverständnis / nicht wahrhaben wollen

05.11.2013 12:14:25

Kategoriendefinition:

Kodierung von folgenden Textstellen:

1. Angehörige geben an, dass sie das Problem nicht hätten einordnen können und nicht verstanden hätten, was das konkret bedeutet.

2. Angehörige berichten, dass sie nicht hätten verstehen können, weshalb ihr Angehöriger Drogen konsumiert und so etwas macht.

3. Angehörige erzählen, dass sie das Problem zu Beginn nicht hätten wahrhaben wollen ("Das kann doch nicht sein!")

Kodierregel:

Mindestens ein Kriterium der Kategoriendefinition ist erfüllt.

Zudem sind die Kriterien der Überkategorie "Reaktion auf Erkenntnis" ebenfalls erfüllt.

Ankerbeispiele:

Andrea, Z57: 
"A: Und ich habe immer gedacht. Das glaube ich nicht. Aber von dort weg ist dann eh klar gewesen."

Dorothea, Z13-16:

"I: hmhm Ähm mögen Sie sich noch erinnern? Wo Sie das anfangen haben zu realisieren. Was haben Sie dort gedacht? Was ist Ihnen durch den Kopf gegangen? Oder ähm;

A: Jäh. Jäh. (sehr leidend) Es ist wirklich einfach das -- vielleicht nicht nicht wissen was jetzt los ist. Nicht nicht wissen was das jetzt bedeutet. Also, macht er das jetzt extra? Oder ist das; ist er jetzt krank?

I: hmhm

A: Oder um was geht es?"

Schuldgefühle

05.11.2013 12:19:59

Kategoriendefinition:

Kodierung von folgenden Textstellen:

1. Angehörige geben an, dass sich selbst Vorwürfe gemacht hätten in der Situation der Erkenntnis, weil

a) sie Probleme nicht früher wahrgenommen hatten.

b) sie das Gefühl hatten, etwas falsch gemacht zu haben, dass es zum Substanzkonsum gekommen ist.

Kodierregeln:

Mindestens ein Kriterium der Kategoriendefinition ist erfüllt.

Zudem sind die Kriterien der Überkategorie "Reaktion auf Erkenntnis" ebenfalls erfüllt.

Ankerbeispiele:

Annabelle, Z95-100:

"I: Wie ist es ihnen in diesem Moment gegangen?

A: Ja. Furchtbar. Fu:rchtbar! Also wirklich schlimm.

I: hmhm

A: Ich habe mir angefangen schwere Vorwürfe machen. Und habe mich absolut schuldig gefühlt weil ich ihm; sein Vater ist ja schon drogenabhängig gewesen. Und ich bin ja, von diesem Vater weg. Also das heisst, eigentlich, schon bevor *Sohn zur Welt gekommen ist.

I: hmhm

A: Und, habe auch, den Kontakt, von diesen beiden nicht gefördert."

Angst / Hilflosigkeit

08.11.2013 07:42:47

Kategoriendefinition:

Kodierung von folgenden Textstellen:

1. Angehörige berichten, dass sie Angst oder Hilflosigkeit empfunden hätten, als sie vom Drogenkonsum erfahren hätten. Die Angst kann sich

a) auf die Substanz beziehen.

b) darauf beziehen, was die Substanz mit den Angehörigen machen könnte.

c) auf sich selbst beziehen, beispielsweise dass Angehörige Angst haben, dass sie sich bei ihrem suchtkranken Angehörigen mit einer sexuell übertragbaren Krankheit anstecken könnten.

Kodierregel:

Kriterium der Kategoriendefinition ist erfüllt.

Zudem sind die Kriterien der Überkategorie "Reaktion auf Erkenntnis" ebenfalls erfüllt.

Ankerbeispiele:

Anke, Z42:

"A: Jah (gelacht). -- Ähm im ersten Moment habe ich Angst gespürt. Weil ich weiss wie das abhängig macht und wie das auch äh die Psyche beeinträchtigen kann."

Informationssuche

08.11.2013 08:06:11

Kategoriendefinition:

Kodierung von folgenden Textstellen:

1. Angehörige geben an, dass sie sich in einem ersten Schritt Informationen zur Substanz, deren Auswirkungen oder Suchterkrankungen gesucht hätten, um sich ein Bild zu verschaffen, was die Erkenntnis konkret bedeutet.

Kodierregel:

Kriterium der Kategoriendefinition ist erfüllt.

Zudem sind die Kriterien der Überkategorie "Reaktion auf Erkenntnis" ebenfalls erfüllt. 
Ankerbeispiele:

Anke, Z46:

"A: Und der zweite Schritt ist dann gewesen dass ich mich informiert äh habe. Also ziemlich genau; Internet und so über den Stoff und die Folgen und wie es Auswirkungen und so weiter."

\section{Unterstützung}

\subsubsection{6:58:46}

Kategoriendefinition:

Kodierung von folgenden Textstellen:

1. Angaben, welche Art der Unterstützung Angehörige erhalten haben und wie sie dieses Unterstützungsangebot beurteilen.

2. Textstellen, in denen deutlich wird, welche Art der Unterstützung, sich Angehörige gewünscht hätten oder wünschen würden.

3. Angaben dazu, aus welchem Grund sich Angehörige Unterstützung gesucht haben.

4. Textstellen, in denen deutlich wird, in welchen Bereichen Angehörige glauben, dass eine Unterstützung nicht möglich ist.

\section{Kodierregeln:}

Die Textstellen werden in den entsprechenden Unterkategorien kodiert, wobei jeweils nur ein Kriterium gegeben sein muss, damit die Textstelle entsprechend kodiert werden kann.

\section{Unterstützung nicht möglich}

25.10.2013 14:04:37

Kategoriendefinition:

Folgende Textstellen werden unter der Kategorie kodiert:

1. Angaben dazu, dass man sich niemals Unterstützung suchte, da eine Unterstützung grundsätzlich nicht als möglich erachtet wird.

2. Angaben dazu, dass trozt erhaltener Unterstützung in gewissen Bereichen keine Unterstützung geboten werden konnte.

3. Angabe von Gründen, weshalb lange Zeit keine Unterstützung gesucht wurde. Angaben insbesondere von Angehörigen, die später Hilfe in Anspruch nahmen.

4. Angaben dazu, dass eine mögliche Unterstützung ambivalent wahrgenommen wurde, in dem die Unterstützung mit positiven und negativen Konsequenzen verbunden war.

\section{Kodierregeln:}

Mindestens eines der Kriterien der Kategoriendefinition ist erfüllt.

Zudem sind die Kriterien der Überkategorie "Unterstützung" ebenfalls erfüllt.

Ankerbeispiele:

August, Z374-378:

"A: Was es, nicht hat können. Und das kann es wahrscheinlich auch nicht. Dass man äh wenn man so so eine Geschichte hinter sich hat. Und vor allem den Verlust hat. Von einem Kind. Dass das halt immer „ume“ (=da) ist.

I: hmhm

A: Und sehr schnell wieder, kommt.

I: hmhm

A: Das äh kann eine Gruppe dann „glich“ (=trotzdem) nicht, ganz auffangen."

Dorrette, Z122-128:

"finanziell habe ich einfach geschaut dass ich durchkomme. Und

I: hmhm

A: und andererseits habe ich auch nicht. Ja habe ich nicht gewusst was sie mir i- i- auf irgendeine Art helfen könnte oder würden. Und eben. Noch mehr!, Probleme von, anderen Leuten hören. Habe ich dann schon gar nicht mehr mögen oder. I: Ja.

A: Weil ich habe immer in diesem ganzen Zeitraum voll geschafft. Oder also, ich habe - einfach müssen. Ja. Habe das Gefühl! gehabt ich müsse für mich schauen.

I: hmhm

A: Und ich habe ich habe auch das Gefühl gehabt. Es kann dir gar niemand helfen."

Gründe für Inanspruchnahme von Hilfe

25.10.2013 13:53:08

Kategoriendefinition:

Kodierung von folgenden Textstellen:

1. Angehörige geben an, was für sie die auslösenden Empfindungen oder Situationen gewesen waren, in denen sie sich 
Unterstützung gesucht haben.

Kodierregeln:

Mindestens ein Kriterium der Kategoriendefinition zutreffend.

Zudem sind die Kriterien der Überkategorie "Unterstützung" ebenfalls erfüllt.

Ausschluss:

Gründe, weshalb sich Angehörige keine Unterstützung/Hilfe gesucht haben, werden unter der Kategorie "Unterstützung nicht möglich" kodiert.

Ankerbeispiele:

Dana, Z166-169:

"Ähm was ist vielleicht auch der Auslöser gewesen dass Sie Hilfe gesucht haben?

A: Ja weil der Zustand Daheim nicht mehr zum aushalten gewesen ist.

I: hmhm + hmhm

A: der Leidens+druck ist gross gewesen."

Albert, Z373-375:

"A: Und ich habe dann gefunden also. Ich schaue jetzt für mich. Fertig! Jetzt gehe ich d- dort hin und lasse mir einfach helfen.

I: hmhm

A: Und das ist dann -- „sochli“ (=ein Bisschen), eben es ist „,sochli“ wie eine Phase! oder. - Wo man da mitmacht. Und man kommt dann irgendwann kommt man zu Phase wo man sagt. So jetzt! Jetzt müssen wir einen Schlussstrich ziehen."

\section{Gewünschte Unterstützung}

05.01.2013 16:05:03

Kategoriendefinition:

Kodiert werden folgende Textstellen, in denen Angehörige Angaben dazu machen, welche Art der Unterstützung sie sich gewünscht hätten oder noch wünschen würden. Die Textstellen werden in den Unterkategorien kodiert.

Gesellschaft

27.10.2013 12:23:29

Kategoriendefinition:

Kodierung von Textstellen, in denen Angehörige angeben, welche gesellschaftlichen Voraussetzungen sie als für sich hilfreich empfinden würden.

Die einzelnen Textstellen werden unter den beiden Unterkategorien "Institutionen" und "Aufklärung" kodiert.

\section{Institutionen}

27.10.2013 12:33:32

Kategoriendefinition:

Kodierung von Textstellen, in denen Angehörige angeben, was sie von Institutionen (Psychiatrien, Polizei, etc.) als hilfreich und unterstützend erleben würde im Umgang mit der Suchterkrankung.

Kodierregel:

Kriterium der Kategoriendefinition ist erfüllt.

Zudem sind die Kriterien der Überkategorien "gewünschte Unterstützung" und "Gesellschaft" ebenfalls erfüllt.

Ankerbeispiel:

Dorothea, Z168-178:

"A: Also - ja. Einfach dass dass wenn wenn wenn der *Bruder mal wieder irgendwo in in eine Einrichtung ist ist eingewiesen worden. Oder eben einen FFE verpasst bekommen hat. Oder sie ihn wieder haben müirgendwo gefunden haben und die Ambulanz kommen musste. Einfach irgendwo halt mal jemand gesagt hätte; Vielleicht mal meine Eltern zusammengenommen hätte und gesagt hätte. Hört zu. Jetzt schauen wir mal für euch.

I: hmhm

A: Dass dass ist nicht einmal! passiert.

I: Also dass die Familie irgendwie mal einbezogen worden wäre?

A: Ja.

I: In eine Behandlung? Oder irgendwo wenn er stationär gewesen ist? Oder dass;

A: hm

I: So meinen Sie?

A: Ja. Weil ja. Ab dem Moment wo er halt 18 gewesen ist äh hat man ihn besuchen gehen können aber -- ja. Weil man kann man kann eh sagen wegen Eigenverantwortung und jeder muss für sich schauen. 
I: hmhm

A: Aber wie gesagt! Also ich habe es bei mir gesehen. Es ist nicht! nicht so einfach. Und -- vielleicht wenn ///(?: unverständlich) Ja vielleicht auch die Eltern mal darauf hingewiesen worden wären dass es - ja vielleicht Gruppen Gruppen gibt mit Angehörigen. Wo man sich einmal wieder austauschen gehen kann."

Doris, Z408-414:

"///(?: Wir sehen das); aber sie! Das hat ja mein: Bekannter auch gesagt. Von der Drogenfahndung. //I//(?:)

I: hmhm

A: U:nd, er hat ja auch gesagt. Sie wollen sie eben nicht. Sie wollen die Hintermänner.

I: hmhm

A: - Das sind kleine + Fische.

I: Ja.+ - Das ist ,äso“.

A: Die sollen doch zufrieden sein mit denen wo es hat. (kichert)"

\section{Aufklärung}

27.10.2013 12:46:28

Kategoriendefinition:

Kodierung von Textstellen, in denen Angehörige angaben, dass eine bessere Aufklärung des Gesellschaft für sie hilfreich und unterstützend wäre. Die Aufklärung kann folgende Bereich betreffen:

1. Aufklärung, dass Sucht eine Krankheit ist.

2. Aufklärung darüber, was es bedeutet, wenn jemand suchtkrank ist, z.B. in dem darüber aufgeklärt wird, dass es auch nach Jahren ohne Substanzkonsum wieder zu Rückfällen kommen kann.

3. Aufklärung der Öffentlichkeit über die Situation der Angehörigen und dass diese ebenfalls der Unterstützung bedürfen.

4. Aufklärung über das Unterstützungsangebot für Angehörige.

Kodierregeln:

Mindestens eines der Kriterien der Kategoriendefinition ist erfüllt.

Zudem sind die Kriterien der Überkategorien "gewünschte Unterstützung" und "Gesellschaft" ebenfalls erfüllt.

Ankerbeispiele:

Anke, Z431:

"A: Aber das über äh der Fall von Drogensüchtigen oder von sonst Süchtigen - äh - es wird davon geredet. Dass der Alkoholkonsum zunimmt. Es wird davon geredet. Dass ähm vielleicht an einem Fest irgend ein irgendwelche Wohnwagen da sind mit Leuten die Drogen ähm prüfen. Ob sie nicht durchmischt mit anderem. Und so weiter. Aber über eine Hilfe über Angehörige hört man ein bisschen wenig."

Andrea, Z712-716:

"A: Und auch vielleicht dass man auch die Leute wo wo so sind. Oder wo die Problematik haben dass das wirklich krank ist. Dass das nicht irgendwie einfach. Das sind faule „Sieche“. Oder es sind äh abgestürzte Personen und die so äh so Randständige sind. Und

I: hmhm

A: Also dass man das einfach ein bisschen mehr kann - an die Öffentlichkeit bringen. Ja. Dass man mehr Verständnis aufbringt für so Sachen.

I: hmhm hmhm dass man es als krank anschaut.

A: Krank an-; Ja. Wirklich als Krankheit anschaut."

\section{Angebote}

27.10.2013 12:58:08

Kategoriendefinition:

Kodierung von Textstellen, in denen Angehörige angeben, welche weiteren Angebote sie sich wünschen würden oder gewünscht hätten und die sie bei der Bewältigung der Belastungen unterstützen könnten oder hätten unterstützen können. Diese Angebote können sich entweder auf

1. Angehörige oder auf

2. Betroffene

beziehen.

Die Textstellen werden in der Unterkategorien "Betroffene" und "Angehörige" kodiert.

\section{Betroffene}

27.10.2013 13:14:26

Kategoriendefinition:

Kodierung von folgenden Textstellen:

1. Angehörige geben an, welches Angebot ihrer Meinung für betroffene suchtkranke Menschen derzeit noch 
fehlt und welches für sie eine Entlastung dastellen würde oder dargestellt hätte in der Vergangenheit.

Ausschluss:

Angebote für Angehörige und Suchtkranke gleichzeitig, werden unter den Angehörigen kodiert. Eine Ausnahme bilden dabei jedoch Angebote für suchtkranke Eltern mit Kindern. Diese werden ebenfalls unter der Kategorie "Betroffene" kodiert.

Kodierregel:

Kriterium der Kategoriendefinition erfüllt.

Zudem sind die Kriterien der Überkategorien "gewünschte Unterstützung" und "Angebote" ebenfalls erfüllt.

Ankerbeispiele:

Dylan, Z305-325:

"A: Eben. Meine einzige Idee wo kommt ist so, betreutes Wohnen vielleicht. Oder so.

I: hmhm

A: Wo so,

I: Also

A: Eltern wo wo sich entscheiden für das dass es; wo auch aufhören wollen.

I: hmhm

A: Dass du die unterstützen kannst.

I: hmhm

A: Quasi äh dass sie einen Entzug machen.

I: hmhm

A: Und langsam oder auch vielleicht mit Methadon. Und immer mehr abbauen.

I: hmhm

A: Und die Kinder ja ,glich“ (=trotzdem) mit denen leben können. Und so.

I: hmhm

A: Und ich denke für Kinder ist es wichtig dass sie aktiv! können an so so einer etwas mitmachen.

I: hmhm

A: Also dass dass sie auch sehen. Ihr Ziel also das was sie da, wo sie sind einfach. Dass das äh wirkt. Also dass dass,

I: hmhm +Also

A: Dass sie+ auch einen Nutzen haben beitragen können. Dass das dass das äh so bessert. Oder?

I: hmhm hmhm Also so wo wie noch jemand äh sagen wir mal Externes wäre wo zusätzlich, ja;

A: Ja. Das wäre eher eine Vormundschaft. Also so beistandsmässig. Oder?"

Dana, Z53-57:

"A: Weil dort ist das Problem, da in *Stadt; eben das *Klinik1 nimmt nicht unter 18-jährigen.

I: hmhm hmhm -

A: Und das überhaupt im ganzen Kanton Zürich ist da einfach noch ein Manko.

I: Okay. +//(?:)

A: Und+ darum ist er dann nach *Entzugsklinik gekommen weil die bekannt sind dass sie auch unter 18jährige nehmen."

Angehörige

27.10.2013 13:24:29

Kategoriendefinition:

Kodierung von folgenden Textstellen:

1. Angaben dazu, welches noch fehlende Unterstützungsangebot sie sich wünschen würden bei der Bewältigung ihrer Belastungen.

2. Angaben dazu, wie ein bestehendes Unterstützungsangebot noch besser auf ihre Bedürfnisse angepasst werden könnte.

3. Angaben dazu, welches Angebot, sie sich gemeinsam für sich und ihren suchtkranken Angehörigen wünschen würden.

4. Angaben dazu, dass sich Angehörige kein weiteres Unterstützungsangebot wünschen.

Kodierregeln:

Mindestens eines der Kriterien der Kategoriendefinition ist erfüllt.

Zudem sind die Kriterien der Überkategorien "gewünschte Unterstützung" und "Angebote" ebenfalls erfüllt.

Ankerbeispiele:

Albert, Z645-647:

"A: Ja. Und ähm -- heute würde ich sagen dass eigentlich so Selbsthilfegruppen etwas, etwas äh sicher sehr Positives und + Gutes sind oder. 
I: hmhm hmhm+

A: Dass man die Möglichkeit hat dass man, irgendwie auch das Angebot versucht äh -- mehr in die Breite zu bringen +oder."

Dagmar, Z241-243:

"A: Ich meine heute sind wir auch ganz anders. Oder?

I: Hmhm hmhm

A: Aber ähm ich würde sagen so therapiemässig wäre es wirklich besser gewesen beide zusammen."

August, Z414-418:

"A: Habe dann eine Beziehung eingegangen. Und äh das wäre vielleicht auch noch gut gewesen. Wenn ich in dieser Zeit. „Ächli“ hätte können diese, Geschichte - auf den Tisch legen. Also.

I: Also dass sie auch die Möglichkeit gehabt hätten. Oder dass das so gewesen wäre. Dass sie vielleicht auch mal alleine, in die Beratung gegangen wären. Nicht nur sie und die Frau zusammen. Oder dann +die ganze Familie.

A: Ja!+ Ja.

I: Dass sie: als, sagen wir als Individuum alleine! Fast ,ächli“ zu kurz gekommen sind. Dann!

A: Ja. Ja."

Erhaltene Unterstïtzung

05.01.2013 16:08:26

Kategoriendefinition:

Kodierung von folgenden Textstellen:

1. Angaben dazu, welche Art der Unterstützung Angehörige bereits erhalten haben, beispielsweise durch Institutionen aber auch von Personen in ihrem privaten Umfeld.

2. Angaben dazu, welche Art der Unterstützung Angehörige aktiv selbst aufgesucht und genutzt haben.

=> Textstellen, in denen Angehörige des Unterstützungsangebots aufzählen, ohne jedoch das Hilfsangebot zu bewerten.

Kodierregeln:

Mindestens ein Kriterium der Kategoriendefinition ist erfüllt.

Ausschluss:

Textstellen, in denen das Hilfsangebot beurteilt wird, werden in den Unterkategorien "positiv" und "negativ" kodiert.

Ankerbeispiele:

Dana, Z45-51:

"A: Da habe ich mir dann auch Hilfe gesucht. Äh zuerst über Drogenfach-äh beratung und die haben mich dann ans KJPD verwiesen.

I: hmhm

A: Dann bin ich dann mit ihm einmal an eine Familie-Jugend-Familieberatung.

I: hmhm

A: Und dann äh, ist er dann; dort ist er eben dann noch mitgekommen ins KJPD. Und dann ist er dann eigentlich immer sehr bereit gewesen.

I: hmhm

A: Und dann haben wir dann zusammen KJPD und Familienberatung; ist er dann eben darauf gekommen dass er nach *Entzugsklinik könnte."

Angelika, Z163-166:

"I: Oder haben sie noch irgend-; also sie haben, auch mit der Jugendanwaltschaft; dort haben sie gesagt haben sie in dem Fall auch immer wieder Hilfe gesucht? Oder Unterstützung gesucht?

A: Ja. Schulärztlicher Dienst.

I: Ja.

A: Bin ich, lange, in einer, in einer Gruppe gewesen."

Negativ

05.01.2013 16:11:33

Kategoriendefinition:

Kodierung von folgenden Textstellen:

1. Die erhaltene oder die gesuchte Unterstützung wird als negativ oder wenig hilfreich beurteilt wird.

2. Trotz Bemühungen, Hilfe oder Unterstützung zu finden, war dies nicht möglich, beispielsweise weil sich der Ansprechspartner nicht verantwortlich fühlte.

3. Aufgrund von negativen Erfahrungen wurde/wird nicht nach Hilfe gesucht.

4. Gründe, weshalb die erhaltene Unterstützung als wenig hilfreich erlebt wurde. 
Kodierregeln:

Mindestens ein Kriterium der Kategoriendefinition ist erfüllt.

Zudem sind die Kriterien der Überkategorie "erhaltene Unterstützung" ebenfalls erfüllt.

Sämtliche Textstellen werden in den Unterkategorien kodiert.

\section{Diverse}

08.11.2013 09:53:55

Kategoriendefinition:

Kodierung von folgenden Textstellen:

1. Angehörige berichten von einer Unterstützung, welche sie in Anspruch genommen haben, die aber nicht ihren Erwartungen entsprach und die sie als wenig unterstützend und hilfreich wahrnahmen.

Kodierregeln:

Kriterium der Kategoriendefinition erfüllt.

Zudem sind die Kriterien der Überkategorien "erhaltene Unterstützung" und "negativ" ebenfalls erfüllt.

Kodiert werden zudem nur diejenigen Textstellen unter der Kategorie diverses, die sich keiner der anderen

Unterkategorien von "negativ" zuordnen lassen.

Ankerbeispiel:

Darina:

"A: Aber ich muss auch sagen. Gerade auf dem Internet hat es hat es ziemlich viel Sachen „ume“. Und oft gerade Erfahrungsberichte ähm die meisten würde ich sagen sind eher die die schlimmeren Erfahrungsberichte. Wo dort ähm aufgeschrieben sind. Vielleicht auch ein bisschen; ich weiss nicht. Sensationslust von den Leuten wo wo so Sachen lesen wollen. Aber ich habe das Gefühl. Es gibt ganz viele Heroinabhängige, wo eigentlich noch ein nor-

I: hmhm

A: also in Anführungszeichen normales Leben führen.

I: hmhm

A: Und die Geschichten sind dann weniger auf dem Internet.

I: hmhm

A: Also, es kann es kann einem auch Angst machen."

\section{Selbsthilfegruppe}

03.11.2013 15:36:34

Kategoriendefinition:

Kodierung von folgenden Textstellen:

1. Angehörige berichten, welche Schwierigkeiten im Zusammenhang mit der Teilnahme an der Selbsthilfegruppe auftreten können:

a) eigene Bedürfnisse kommen zu kurz, weil man sich beispielsweise dazu bereit erklärt hat, den Lead der Gruppe zu übernehmen.

b) unterschiedliche Vorstellungen (kulturelle, persönliche Überzeugungen und Motivationen, an der Gruppe teilzunehmen) können in der Gruppe zu Schwierigkeiten führen.

c) gewisse Themen / Belastungen werden in der Gruppe nicht angesprochen.

2.) Angehörige berichten darüber, dass es schwierig ist ein gutes, seriöses Angebot zu finden.

Kodierregel:

Mindestens ein Kriterium der Kategoriendefinition ist erfüllt.

Zudem sind die Kriterien der Überkategorien "erhaltene Unterstützung" und "negativ" ebenfalls erfüllt.

Ankerbeispiele:

Anke, Z188-200:

"A: Es sind Leute gekommen wo, quasi unsere, aus anderen Kulturen die unsere Art Probleme anzugehen nicht ganz verstanden haben.

I: hmhm

A: Die einte ist schnell weg gegangen. Und es hat nochmals eine Frau gehabt die selbst sehr krank war. Also ich glaube die ist mehr gekommen, weil, weil sie wie, für sich sehr grosse Hilfe gebraucht hat.

I: hmhm

A: Und äh wenn sie etwas erzählt hat ist es immer in einen Monolog äh ausgeartet. Sie hat gar nicht zugehört was die anderen sagen. Sie hat nur sich; ihr Problem ist so gross dass sie nur noch äh das erzählen konnte. Und dann hat sie sich glaub mit der Zeit nicht mehr so verstanden gefühlt.

I: hmhm

A: Weil bei uns ist immer ein Thema im Mittelpunkt gestanden. Wir haben auch Themen gesucht. Und wir haben an denen geschafft. Und sie hat immer wieder, ist ihr Problem; Dann erzählt sie immer das Gleiche.

I: hmhm hmhm 
A: Und sie hat auch so leise geredet dass wir sie fast nicht verstanden haben. Also nicht nur ich wo Hörgeräte trägt sondern auch die anderen.

I: hmhm

A: Und sie ist auch sehr anstrengend gewesen dann.

I: Ja. Ja.

A: Und sie ist dann auch; sie zwar ein bisschen länger gekommen. Aber irgendwann ist sie auch nicht mehr gekommen."

Therapie des Betroffenen

08.11.2013 10:20:53

Kategoriendefinition:

Kodierung von folgenden Textstellen:

1. Angehörige berichten von negativen Erfahrungen, die sie im Zusammenhang mit der Behandlung ihrer suchtkranken Angehörige gemacht haben. Dies kann sein, dass

a) sie beispielsweise nicht in die Behandlung einbezogen wurden oder nicht ausreichend informiert wurden über das Vorgehen.

b) mit der Art der Behandlung nicht einverstanden waren (Medikamente, Vorgehen, Haltung etc.).

Kodierregeln:

Mindestens ein Kriterium der Kategoriendefinition ist erfüllt.

Zudem sind die Kriterien der Überkategorien "erhaltene Unterstützung" und "negativ" ebenfalls erfüllt.

Ankerbeispiele:

Dana, Z219-223:

"A: Aber+ äh; und die kämpfen ja auch; Denen geht es ja gleich. Eben in den Kliniken werden die Angehörigen viel zu wenig mit einbezogen.

I: hmhm hmhm Aber das wäre eigentlich Ihr Hauptwunsch! dass Sie mehr wissen was, was geht?

A: Ja. und auch wenn sie ja Therapien haben. Die werden ja irgendwann wieder einmal in dieses Umfeld zurück entlassen.

I: hmhm

A: Und dass man dort auch einen fliessenden Übergang hat und nicht einfach. Wusch! da ist er wieder äh:

I: hmhm

A: Schaut jetzt."

Dagmar, Z219-223:

"A: Und ähm nachher; jetzt muss ich wieder sagen ich habe wieder ein zwiespältiges Gefühl zum Suchtberater.

I: Hmhm

A: Also es ist; er macht nicht wirklich et-; er sagt einfach. Ich bin da wenn er etwas braucht. Aber wenn er nichts braucht oder nicht will. Ich kann nichts machen. Also er ist einfach da. Aber tut sich nicht verpflichtet; ja also ist wahrscheinlich auch nicht sein Job. Von dem her.

I: hmhm

A: Aber ich habe einfach das Gefühl. Von mir her gesehen. Er hatte mehr mit ihm machen müssen."

\section{Inadäquate Reaktion von Professionellen}

08.11.2013 10:40:11

Kategoriendefinition:

Kodierung von folgenden Textstellen:

Angehörige berichten von negativen Erfahrungen, die sie persönlich im Kontakt mit professionellen Personen gemacht haben:

1. Angehörge werden für die Suchterkrankung verantwortlich gemacht (Schuldzuschreibung).

2. Professionelle gehen nicht auf die Bedürfnisse und Anliegen der Angehörigen ein.

\section{Kodierregeln:}

Mindestens ein Kriterium der Kategoriendefintion ist erfüllt.

Zudem sind die Kriterien der Überkategorien "erhaltene Unterstützung" und "negativ" ebenfalls erfüllt.

Ankerbeispiele:

Doris, Z170-178:

"A: Damit ich mich ,,ächli“ entlasten kann bin ich dann eben auch! zu einem Psychiater. Und der! hat dann gesagt. Gut! Hat den Computer eingestellt. Da! Ähm, jetzt muss ich studieren. Kantona:l. Gesundheitswesen. Der Direktor. Schreiben sie dem dass sie ihr Enkelkind zur Adoption frei geben. - Dann habe ich gesagt. Das meinen sie doch hoffentlich nicht ernst.

I: hmhm 
A: Dann hat er gesagt. Nein. Das das hat keinen Wert.

I: hmhm

A: Dann habe ich gesagt. Gut. In dem Fall muss ich nicht mehr kommen. Solche Sachen brauche ich nie mehr.

I: hmhm

A: Man sollte einfach seine, Erlebnisse, erzählen können. Dass man sie losbringt. Man will sie vielleicht nicht einmal - einen Ratschlag. Was man machen muss. Weil man macht ja sowieso was man will.

I: hmhm

A: Es ist noch schwierig."

Annabelle, Z187-194:

"I: hmhm Und dann hat man Ihnen auf der Suchtberatungsstelle in diesem Fall äh diese Hilfe nicht bieten können? - Oder ab;

A: Ja. Ich ich würde jetzt nicht ähm; ich will diese Leute nicht wegen; ähm dass es ist voll Scheisse gewesen.

I: hmhm

A: Aber ich denke mir -- also ja. Es tut einfach extrem weh wenn man beim ersten Mal merkt. Sie hat einen eigentlich vergessen.

I: hmhm

A: Und sie ist schmudlig (=ungepflegt) und sie raucht und überhaupt. Sie; Scheisse! Es geht ums Thema Sucht.

I: (beide lachen)

A: Also so ein bisschen das. Habe ich schon nicht so gut; Habe ich wirklich keine gute Erfahrung gefunden."

Positiv

05.01.2013 16:21:34

Kategoriendefinition:

Kodierung von folgenden Textstellen:

1. Die erhaltene oder gesuchte Unterstützung wurde oder wird als hilfreich erlebt.

2. Gründe, weshalb die erhaltene Unterstützung als hilfreich erlebt wird oder wurde.

Kodierregeln:

Mindestens ein Kriterium der Kategoriendefinition ist erfüllt.

Zudem sind die Kriterien der Überkategorie "erhaltene Unterstützung" ebenfalls erfüllt.

Sämtliche Textstellen werden in den Unterkategorien kodiert.

\section{Hoffnung / Positives sehen}

08.11.2013 11:10:57

Kategoriendefinition:

Kodierung von folgenden Textstellen:

1. Angehörige geben an, dass es für sie hilfreich gewesen sei, dass ihnen geraten worden sei, niemals die Hoffnung aufzugeben und die positiven Dinge bei ihrem Angehörigen trotzdem noch wahrzunehmen.

2. Angehörige berichten, dass es ihnen geholfen hätte zu sehen, dass die Situation mit einem suchtkranken Angehörigen noch schlimmer sein könnte und es ihrem Angehörigen eigentlich verhältnismässig gut gehe.

Kodierregeln:

Mindestens ein Kriterium der Kategoriendefinition ist erfüllt.

Zudem sind die Kriterien der Überkategorien "erhaltene Unterstützung" und "positiv" ebenfalls erfüllt.

Ankerbeispiel:

Doris, Z76-80:

"A: Ich habe dann das Glück gehabt dass ich dahin gekommen bin. Ich weiss auch nicht. Jetzt, ob es, auf, eine Referenz gewesen ist. Ich glaube es zwar nicht. Und dann bin ich an den Doktor *Name01 geraten. Und er ist eigentlich seit Jahren immer ,,ächli“" hat man einen losen Kontakt. Also er hat mir auch gesagt. Und er hat mich auch gelehrt. Die guten Seiten vom *Sohn zu geben zu sehen.

I: hmhm

A: Und nicht nur das Schlechte in den Vordergrund zu tun.

I: hmhm

A: Es ist jetzt so. Man muss ,luege“ (=schauen) dass man das Beste machen kann."

Dylan, Z110-112:

"A: Und ich weiss noch. Als Kleiner habe ich auch viel versucht; Zum Beispiel die Eltern meine Eltern 
haben mir immer gesagt. Es wird dann schon besser und so. Wir hören ja auf. Dann dann haben wir mehr Geld. Und so und so.

I: hmhm

A: Oder dann haben kannst du auch deine Sachen haben. Und du hast als Kleiner halt immer gehofft. Es wird ja bald; Dann ist quasi der - der Ziel ist immer dann verschoben worden. Ja. Jetzt geht es nochmals zwei Jahre. Jetzt geht es nochmals da bis /(?:)"

\section{Informationen}

08.11.2013 11:22:03

Kategoriendefinition:

Kodierung von folgenden Textstellen:

1. Angehörige berichten, dass fachliche Informationen zu Suchterkrankung und den Substanzen, die ihre Angehörigen konsumieren hilfreich gewesen seien. Diese Informationen können unterschiedlichen Ursprungs sein:

a) Informationen von den Betroffenen

b) Informationen aus Medien (Bücher, Internet, Zeitschriften etc.)

c) Informationen von Fachpersonen.

Kodierregeln:

Mindestens ein Kriterium der Kategoriendefinition ist erfüllt.

Zudem sind die Kriterien der Überkategorien "erhaltene Unterstützung" und "positiv" ebenfalls erfüllt.

Ankerbeispiel:

Darina, Z134-142:

"A: Also für mich, ist es immer wenn ich irgendein Problem habe oder so. Ich bin

I: hmhm

A: also ich bin ja. Auch bei meinem Bruder. Ich habe versucht so viel herauszufinden wie möglich.

I: hmhm

A: Ähm also das ist immer so meine (lacht) meine Taktik ein bisschen.

I: hmhm

A: ähm und dann auf dem Internet. Es hat mich schon beruhigt.

I: hmhm

A: dass es ähm ein Wissen gehabt habe."

Annabelle, Z129-132:

"I: (lacht) und Sie haben dann ähm nachdem dass Sie hier gewesen sind oder auf Grund diesen Gesprächen; oder Sie haben mir per Telefon gesagt; ähm dass Sie Ihren Mann nachher einfach äh deutlich gemacht haben dass er den Konsum reduzieren muss. Dass es auf den finanziellen Grund geht?

A: hmhm

I: Ähm hat das; ist das unabhängig von den Beratung hier gelaufen? Oder oder sind Sie da auch, von hier aus unterstützt worden? Oder hat; verstehen Sie was ich meine?

A: Hmhm Ja. - hm, also ich denke mir. Ich bin hier sehr unterstützt worden auch äh also zum Beispiel auch mich rechtlich zu erkundigen."

Unterstützung für den Suchtkranken

08.11.2013 11:30:13

Kategoriendefinition:

Kodierung von folgenden Textstellen:

1. Angehörige berichten, dass die Therapie ihres suchtkranken Angehörigen für sie eine Entlastung darstelle.

2. Angehörige geben an, dass es sie entlaste, wenn es dem Betroffenen besser gehe.

3. Angehörige fühlen sich entlastet, wenn sie sehen, dass mit suchtkranken Menschen respektvoll umgegangen wird.

\section{Kodierregeln:}

Mindestens ein Kriterium der Kategoriendefinition ist erfüllt.

Zudem sind die Kriterien der Überkategorien "erhaltene Unterstützung" und "positiv" ebenfalls erfüllt.

Ankerbeispiel:

Doris, Z477-482:

"Also ich muss dann noch sagen was ich bewundert habe. Auch da. Diese wenigen Male oder die paar Male wo ich da gewesen bin. Und habe habe warten müssen.

- Diese Leute sind sehr fürsorglich. Und sie machen niemanden herunter. - Diese Betreuer.

I: hmhm

A: Da kannst jemand kommen. „Schier“ (=beinahe) auf den Knien. Oder heulend. Selber erlebt und gesehen 
da auf den Gängen. Verzweifelt! Weil sie ja wissen dass sie es nicht wollen aber „glich“ (=trotzdem) machen. Und, dass sie dann wirklich mit einer Fürsorge betreut werden. Und wieder aufgebaut und nicht hinuntergelassen.

I: hmhm

A: Und nicht; und das hat mir auch geholfen."

Albert, Z511:

"A: Einfach der Gedanke dass dass es ihr gut geht. Ist auch die Voraussetzung, dass es mir gut geht."

Soziale Unterstützung

08.11.2013 11:36:53

Kategoriendefinition:

Kodierung von folgenden Textstellen:

Angehörige geben an, dass sie soziale Unterstützung durch andere Personen als hilfreich und entlastend erlebt hätten. Unterstützung kann von verschiedenen Stellen kommen:

a) aus Selbsthilfgruppe durch den Austausch mit anderen Betroffenen. Aus dieser Betroffenheit können auch neue Freundschaften entstehen.

b) aus bestehenden Freundschaften.

c) von anderen Familienmitgliedern.

Kodierregeln:

Mindestens ein Kriterium der Kategoriendefinition ist erfüllt.

Zudem sind die Kriterien der Überkategorien "erhaltene Unterstützung" und "positiv" ebenfalls erfüllt.

Ankerbeispiele:

Angelika, Z120-126:

"A: Ja also für mich ist es, enorm schwierig gewesen. Und ich bin noch so: jung gewesen. Wissen sie. Ich habe, ich habe meinen Sohn ja so früh bekommen. Und ich bin noch sehr! jung gewesen. Und ich habe mich nicht gewusst wie wehren. Also ich bin froh gewesen. Dass ich habe können, ähm, in die Elternvereinigung. Und dort habe ich auch gesehen dass dass nicht alles an mi:r liegt

I: hmhm

A: sondern dass normale Ehepaare, auch äh - das Problem haben. Und zwar sogar, mit mehreren Kindern!

I: hmhm

A: In einer Familie!

I: hmhm

A: Also das das hat mich dann sehr bestätigt darin dass das nicht einfach ist weil, mein Sohn unehelich ist. Und äh, keinen Vater gehabt hat. Und i:ch! das, quasi verursacht habe. Also in Anführungszeichen."

Albert, Z472-479:

"I: (räuspert sich) Aber sonst sind sie eigentlich direkt hierher gekommen. Oder haben sie sonst noch einmal länger ,naimeds“ Hilfe gesucht gehabt?

A: Privat ja.

I: Einfach bei Freunden.

A: Ja.

I: Ja.

A: Genau. - Also ich habe dann vor allem bei Freunden, Rat gesucht. Wo selber auch - verwickelt gewesen sind in so Kokain-

I: hmhm

A: äh Abhängigkeiten. Und ähm, ich bin dann oft dorthin und habe erzählt äh wie es mir geht."

Fokus auf Bedürfnisse der Angehörigen

27.10.2013 17:27:24

Kategoriendefinition:

1. Mitgefühl, Anteilnahme und Verständnis, welches Angehörige von professionellen Personen erhalten.

2. Wahrnehmen, Wertschätzen und Respektieren der Angehörigen in ihren Bedürfnissen durch professionelle Personen.

3. Anerkennung, dessen was Angehörige alles leisten durch professionelle Personen.

Kodierregeln:

Mindestens ein Kriterium der Kategoriendefinition ist erfüllt.

Zudem sind die Kriterien der Überkategorien "erhaltene Unterstützung" und "positiv" ebenfalls erfüllt.

Ankerbeispiele:

Annabelle, Z267: 
"Und das ist natürlich schon auch in der, in der Gesprächstherapie wo ich dort alleine gegangen gelernt habe ist ähm. Dass ich schon auch ein Recht habe mir Leute auszusuchen wo ich das sagen kann."

Annabelle, Z182-184:

"A: Hmhm -- Also ich denke mir schon dass ich hier im ada sehr viel äh - Unterstützung in Form von, also ja ob ich dem /(?:) sei.

I: hmhm

A: Oder dass sie das äh --- Ja dass es nicht selbstverständlich ist, fünfundzwanzig Jahre mit einem Partner zusammen zu sein und auch Kinder zu haben. Und ich denke das ist schon wirklich //(?:viel verlangt) also;"

Albert, Z505-507:

"A: Und äh in d- in dem Sinn ist mir es einfach da eine Unterstützung gewesen. Dass ich dann, kein schlechtes Gewissen gehabt habe. Irgend- irgendwodurch oder.

I: Das sie bestärkt worden sind.

A: Ja. Dass ich da nichts Falsches mache."

\section{Belastungen}

\subsubsection{7:08:52}

Kategoriendefinition:

Kodierung von sämtlichen Textstellen, in denen Angehörige über Belastungen sprechen, die sie im Zusammenhang mit der Suchterkrankung ihres Angehörigen erleben.

Kodierregeln:

Die Textstellen werden in den Unterkategorien kodiert.

\section{Diverses}

09.11.2013 13:14:17

Kategoriendefinition:

Kodierung von folgenden Textstellen:

1. Angehörige betonen, dass die Belastungen allgemein sehr gross gewesen und vielfältig gewesen seien.

2. Angehörige geben an, dass die Belastungen auch nach Jahren immer noch gross und sehr präsent sind.

3. Angehörige machen deutlich, dass fehlende Aufklärung und Wissen für sie eine Belastung darstellt.

\section{Kodierregeln:}

Mindestens ein Kriterium der Kategoriendefinition ist erfüllt.

Zudem sind die Kriterien der Überkategorien "Belastungen" ebenfalls erfüllt.

Ankerbeispiel:

Doris, Z36-38:

"A: Da hört man immer die unglaublichsten Geschichten.

I: hmhm

A: Aber die unglaublichsten Geschichten, haben sich leider dann mehr bewahrheitet."

August, Z837-847:

"A: So das eine oder andere. Aber. Echt! Was heute lauft und geht. Weiss auch nicht mal ich.

I: hmhm

A: In d-, wie wie professionelle Leute. Diese wo wirklich an der Front, sind. Und äh, in einer Gruppe drin. Ist, das ist vorbei.

I: hmhm

A: Wenn Eltern mal das vor. Ja wie bei uns. *Jahr ist es losgegangen.

I: + hmhm

A: Wenn man sich+ diese Zeit überlegt. Das können wir; das ist ja fast unvorstellbar.

I: +(lacht)

A: Ja weil //(?: unverständlich weil I lacht)+

I: Ist eine lange Zeit. +Ja.

A: Ja!:+ /II//(?:Dafür ist es schnell gegangen). Ist, in der Erinnerung ist das immer noch recht, +nah. Und präsent."

Stigmatisierungen

05.01.2013 16:39:01

Kategoriendefinition:

Kodierung von folgenden Textstellen: 
1. Angaben dazu, dass Angehörige aufgrund der Drogenabhängigkeit ihres Angehörigen stigmatisiert werden, indem sie beispielsweise

1a) kein Verständnis für ihre Situation bekommen.

1b) Vorwürfe gegenüber den Angehörigen, dass sie Schuld an der Suchterkrankung seien.

2. Angaben dazu, dass falsche Annahmen von anderen zu Suchterkrankungen als belastend erlebt werden (beispielsweise Suchterkrankung als Strafe Gottes etc.)

3. Angaben dazu, dass der suchtkranke Angehörige stigmatisiert wurde.

Kodierregeln:

Mindestens ein Kriterium der Kategoriendefinition ist erfüllt.

Zudem sind die Kriterien der Überkategorien "Belastungen" ebenfalls erfüllt.

Vorsicht in der Abgrenzung zur Unterkategorie "Nicht darüber sprechen können"

Sobald es sich um antizipierte Stigmaerfahrungen handelt, werden diese unter der Kategorie "Nicht darüber sprechen können" kodiert.

Nur real erlebte Stigmaerfahrungen werden unter Kategorie "Stigmatisierung" erfasst.

Schuldzuschreibungen, die Angehörige von therapeutischem Personal erfahren, werden unter der Kategorie

"Unterstützung/erhaltene Unterstützung/negativ/inadäquate Reaktion von Professionellen" kodiert.

06.10.2013 14:30:40

Ankerbeispiele:

Dorothea, Z208-212:

"A: Ja einfach. -- Wenn er wenn er irgendwie wieder, eine Woche lang einfach verschwunden ist.

I: hmhm

A: Hat es irgendwie äh einen Streit gegeben. Und nachher ist er eine Woche verschwunden gewesen. -- Und nachher, ja geht man irgendwie bei der Polizei (ironisches Lachen) eine Vermisstenanzeige aufgeben. Und nachher heisst es. Ja. Bei bei dieser Vorgeschichte --

I: Schauen wir nicht.

A: Ja."

Dorothea, Z276-280:

"A: eben - ja. Schul- Schulkollegin wo ich eigentlich, persönlich sehr gut mit ihr ausgekommen bin. +Aber

I: hmhm+

A: sie ist äh in einer ja in einen /(?:)verein gewesen. Sehr sehr christlich. Und äh hat, extrem: extreme Vorurteile gehabt. Wenn es um Drogenkonsum geht. Und hat mir nachher, ja einfach auch; ich bin ich bin Atheistin und zufrieden damit. Und ich will nicht hören dass mein Bruder irgendwie vom Teufel besessen ist.

I: Ja. Das +verstehe ich.

A: Also+ solche Sachen (lacht leicht) und da: ja da habe ich mich halt nachher; das ist einfach ein Tabuthema gewesen zwischen uns. Wir konnten über Filme und über anderes reden aber einfach nicht, über solche Themen (lacht)."

Dorette, Z102-104:

"Und dann ist die Polizei auch gekommen. Und dann hat die Polizei, mich auch mitgenommen. In jener Nacht. Und, dort haben sie mir gesagt. Ich sei eine potentielle Mörderin. Und, ja und eben. Die armen, armen Süchtigen haben ja bei uns Ware be-. Also bei uns! Nicht bei mir! natürlich. Aber, a- dort bin ich zuerst einmal wirklich, also habe ich gefunden. Wer bin ich eigentlich.

I: hmhm

A: Weil, ja ich habe das alles angefangen mitzumachen."

Angelika, Z238:

"Ja und vor allem hat mich immer auch, gestört dass, Leute wo ich dann, geredet habe haben dann gesagt, ja kannst denn du da nichts machen?"

Gesellschaftliche Strukturen (strukturelle Diskriminierung)

09.11.2013 12:08:44

Kategoriendefinition:

Kodierung von folgenden Textstellen:

1. Es wird deutlich, dass gesellschaftliche Strukturen dazu führen, dass suchtkranke Menschen und ihre Angehörigen stigmatisiert wird werden:

a) Angehörige werden dazu aufgefordert, Schulden für die Betroffenen zu übernehmen, obwohl keine rechtliche Grundlage dafür besteht.

2. Gesellschaftliche Strukturen führen dazu, dass suchtkranke Menschen anstatt vor Schwierigkeiten geschützt zu werden, tiefer in Schwierigkeiten geraten:

a) beispielsweise dass Finanzinstitute suchtkranken Menschen wieder Kredite anbieten

3. Gesellschaftliche Strukturen verhindern bis zu einem gewissen Grad, dass Unterstützung oder Hilfe von Betroffenen in Anspruch genommen wird: 
Kodierregeln:

Mindestens ein Kriterium der Kategoriendefinition ist erfüllt.

Zudem sind die Kriterien der Überkategorien "Belastungen" und "Stigmatisierung" ebenfalls erfüllt.

Ankerbeispiele:

Dylan, Z130-132:

"Aber mehr ist einfach die Angst dass es durch das Bewusstsein dass du gehabt hast. Wenn es ja jemand weiss. Dann kannst du sicher sein quasi da da, dann wirst du ja weggenommen von den Eltern.

I: hmhm

A: Wenn sie es wissen. Oder?"

August, Z140-144:

"A: Sie haben natürlich ich würde heute behaupten auch fahrlässig gehandelt. Die Banken. Und zwar weiss, wo ich das heute; ich könnte sogar Belege geben. Weil der eine hat noch nicht einmal; ist ist ist nachher mal auf Gericht. Gelandet. Der Mittlere. Und der ist hat, noch nicht einmal: ist richtig zur, der, „Chischte“ (=Gefängins) hinaus gewesen. Hat er schon von der gleichen! Bank wieder einen Brief bekommen. Ob er wieder Geld wolle.

$\mathrm{I}$ : - Das ist ja schon verrückt.

A: Ja. Also es ist un!vorstellbar. Also die haben ihn ja angeklagt! gehabt. Wegen Kreditbetrug.

I: Und haben ihm je- auf der anderen Seite schon wieder +Kredit angeboten. (lacht)

A: Ja. Ja.+ Ja ist, das ist ungl-. Das ist ein anderes Problem. Aber äh für, also mir ist eine Welt „zämegheit“ (=zusammengebrochen)."

Nicht darüber sprechen können

23.01.2013 10:56:16

Kategoriendefinition:

Kodierung von folgenden Textstellen:

1. Angaben dazu, dass die Angehörigen normalerweise nicht über die Suchterkrankung des Angehörigen spricht, weil

1a) die Angehörigen Angst haben vor Unverständnis für ihre Situation und Stigmatisierung, die sie selbst aber auch den Betroffenen betrifft.

1b) der drogenabhängige Angehörige wünscht, dass nicht über die Suchterkrankung gesprochen wird (Stigmatisierung).

Kodierregel:

Mindestens ein Kriterium der Kategoriendefinition ist erfüllt.

Zudem sind die Kriterien der Überkategorien "Belastungen" und "Stigmatisierung" ebenfalls erfüllt.

06.10.2013 14:34:06

Ankerbeispiele:

Dagmar, Z411-419:

"Und die! Kolleginnen die ich habe, die einte:, ja nein. Die meisten Leute finden vielleicht schon finden eben schon dass ich sch-, dass ich wieder mit ihm zusammen bin. Darum nicht einmal alle wissen das. Vielen wiss-; also meine Eltern wissen das noch gar nicht. (lacht) Denen muss ich das erst gar nicht sagen. Die meisten, wo ich es jetzt gesagt habe. Die sind schon so, sagen schon so. Ow! Wie kannst du wieder? Spinnst Du?

I: $\mathrm{Hm}$

A: Du weisst doch was er gemacht hat? Du weisst ja wie es gewesen ist? Wieso machst du wieder?

I: $\mathrm{Hm}$

A: Also sie haben schon für das kein Verständnis.

I: $\mathrm{Hmhm}$

A: Warum soll ich überhaupt noch mit denen reden. Weil ich weiss! das: ver- verstehen sie nicht. Oder.

I: $\mathrm{Hmhm}$

A: Ich würde es vielleicht wenn ich eine Aussenstehende wäre auch nicht begreifen. Warum macht sie es jetzt schon wieder? Ah ja. Nein. Von dem her. Nein."

Darina, Z173-176:

"I: hmhm Ähm --- Äh gibt es oder was erleben Sie als besonders belastend im Zusammenhang mit der Sucht von Ihrem Bruder?

A: Die Verschwiegenheit. Also dass man dass man nicht darüber reden darf.

I: hmhm

A: Also das ist jetzt auch einerseits äh von meinem Bruder aus. Das ist ein Wunsch von ihm." 


\section{Beziehungsprobleme}

04.01.2013 10:33:26

Kategoriendefinition von Kriby et al.:

Folgende Textstellen werden kodiert:

1. Vermehrt Streitereien mit der drogenabhängigen Person.

2. Vermehrt Streitereien innerhalb der Familie wegen der drogenabhängigen Person. => Beispielsweise darüber, wie man sich verhalten soll?

3. Drogenabhängige Person vernachlässigt die Kinder.

Zudem folgende weitere Textstellen:

4. Aufgrund der Drogenabhängigkeit kommt es oder ist es zu wiederholten Beziehungsunterbrüchen gekommen, beispielsweise zu wiederholten Trennungen in Partnerschaften. Aber auch vorübergehende Kontaktabbrüche in der Familie. 5. Problematische Beziehungen, die der suchtkranke Angehörige eingeht, was zu weiteren Konflikten oder Problemen führt.

\section{Kodierregeln:}

Mindestens ein Kriterium der Kategoriendefinition ist erfüllt.

Zudem sind die Kriterien der Überkategorien "Belastungen" ebenfalls erfüllt.

Ausschluss:

Textstellen, in denen angegeben wird, dass es vermehrt zu Gewalt und Drohungen in der Familie/Partnerschaft kommt, werden unter der entsprechenden Kategorie kodiert.

Kategorien, in denen es nicht um Streitereien, sondern um allgemeine Angaben zur Beziehung zum drogenabhängigen Angehörigen geht, werden unter der Kategorie "Beziehung" codiert.

Ankerbeispiele:

Dagmar, Z345:

"A: Ah!+ Wir haben uns mehrmals getrennt! (lacht dabei) Mal mehr und mal weniger. Also jetzt haben wir uns gehabt ähm, Ich habe mich von ihm getrennt gehabt im (schnaubt) *Jahreszahl."

Dana, Z187:

"Und einfach auch die Streiterei. Wir haben einander eigentlich nur noch +angeschrien."

Angelika, Z208:

"A: Also meine Beziehung zu meinem Mann. Ist immer, durch das! sehr belastet gewesen. Also wenn wir einmal äh Reibereien gehabt haben. Und, Auseinandersetzungen, ist meistens das, hat das mit meinem Sohn zu tun gehabt."

Doris, Z92-94:

"A: Der *Sohn ist dann, wieder einmal in einer Klinik gewesen. In *Ort01. Und hat dann dort seine Frau kennengelernt. Wo wegen dem Alkohol dort gewesen ist.

I: Ah die haben sich in der Klinik + kennengelernt.

A: Ja. hmhm+ Und, es ist dann auch nicht gut gegangen mit diesen Zweien."

\section{Emotionale Belastung}

04.01.2013 10:50:45

Kategoriendefinition:

Kirby et al. (2005) haben in ihrer Checkliste folgende Aspekte von emotionalen Belastungen als relevant erachtet:

1. Schuldgefühle in Bezug auf die Drogenprobleme des Angehörigen. (siehe Kategorie Schuldgefühle/Scham)

2. Enttäuschungen, weil der suchtkranke Angehörige einen "versetzt". (siehe Kategorie Persönlichkeitsveränderung)

Weitere Aspekte, die für wichtig angesehen werden sind weiteren Unterkategorien repräsentiert:

3. Vereinsamung des suchtkranken Angehörigen

4. Abgrenzung vom suchtkranken Angehörigen

5. die Ungewissheit, was mit dem suchtkranken Angehörige ist, wenn er nicht erreichbar ist.

6. Das Gefühl dauernd in Alarmbereitschaft zu sein, weil ständig Notfälle auftreten.

7. Angst, dass der suchtkranken Angehörige sterben könnte oder der Wunsch, dass der Angehörige stirbt

8. der ständige Gefühlswechsel, den viele Angehörige im Zusammenhang mit der Suchterkrankung erleben.

9. Gefühle wie Hilflosigkeit, Ohnmacht, Resignation und Hoffnungslosigkeit

10. das belastende Gefühl, für den suchtkranken Angehörigen verantwortlich zu sein.

11. Ängste, die die Zukunft des suchtkranken Angehörigen betreffen.

12. Sowie Sorgen und Ängste, die andere Drittpersonen betreffen, die durch die Suchterkrankung ebenfalls betroffen sind.

Kodierregeln:

Sämtliche Textstellen werden in den Unterkategorien kodiert.

Zudem sind die Kriterien der Überkategorien "Belastungen" ebenfalls erfüllt. 
Ursache

23.01.2013 12:09:10

Kategoriendefinition:

Kodierung von folgenden Textstellen:

1. Texstellen, in denen deutlich wird, dass sich die Angehörige über die Ursache für Suchterkrankungen im allgemeinen Gedanken machen.

2. Textstellen, in denen Angehörige Vermutungen äussern, weshalb es bei ihrem Angehörigen zu einer Abhängigkeitserkrankung gekommen ist.

2. Textstellen, in denen Angehörige ausdrücken, dass sie nicht verstehen können, weshalb ihr Angehöriger suchtkrank geworden ist.

Kodierregel:

Mindestens ein Kriterium der Kategoriendefintion ist erfüllt.

Zudem sind die Kriterien der Überkategorien "Belastungen" ebenfalls erfüllt.

06.10.2013 14:00:23

Ankerbeispiele:

Dagmar, Z649-651:

"Und ich denke gerade! Drogenabhängige sind eher Menschen wo, manchmal zu sensibel sind.

I: Hmhm

A: Und darum werden sie auch drogenabhängig."

Dorothea, Z64-70:

"A: Jäh. Es ist nachher diese Zeit gewesen wo ich wo ich nachher einfach direkt zum Bruder gegangen bin. Einfach zum Herausfinden. Ich weiss zwar was es ist. Ich weiss was du nimmst. Aber wieso? Keine Ahnung.

I: Also haben Sie Ihren äh Bruder gefragt wo Heroin konsumiert?

A: hmhm Ich habe dann einfach nachher äh viel viel mit ihm auch (seufzt) ja. Wenn also wenn er wenn er mal zu Hause gewesen ist habe ich habe ich immer irgendwie mit ihm darüber reden wollen.

I: hmhm

A: Oder - einfach zum Herausfinden wie; ja.

I: Warum?

A: Warum. Ja. Und: weil ja. Ich ich habe gewusst was es ist. Aber ich ich habe nicht eingesehen. Wieso dass man so, so etwas macht?"

\section{Schuldgefühle / Scham}

04.01.2013 13:57:26

Kategoriendefinition:

Kodierung von folgenden Textstellen:

1. Angaben zu Schuldgefühlen gegenüber dem Suchtkranken.

2. Textstellen, in denen die Frage thematisiert wird, welche Fehler man gemacht haben könnte, die zur Suchterkrankung geführt haben.

3. Angaben dazu, dass Angehörige sich für Abstürze des drogenabhängigen Angehörigen verantwortlich fühlen.

4. Auch Schuldgefühlen anderen gegenüber (bspw. Kindern), die durch die Suchterkrankung mitbetroffen sind und nicht geschützt werden können.

5. Auch Kodierung von Textstellen, in denen Angehörige berichten, wie sie sich von gewissen Schuldgefühlen auch distanzieren konnten.

\section{Kodierregeln:}

Eines oder mehrere Kriterien der Definition sind erfüllt.

Zudem sind die Kriterien der Überkategorien "Belastungen" und "emotionale Belastung" ebenfalls erfüllt.

Ankerbeispiele:

Dagmar, Z437-439:

"A: Und - ja halt das was halt alle haben. Die Schuldgefühle. Ja wenn ich, ich bin halt ein Auslöser immer wieder. Zwangsläufig. Ich bin ein, wie sagt man dem so schön. Irgendwo habe ich das gelesen. Ich bin nicht die Ursache von dem was er macht aber ich bin manchmal der Auslöser. Und das macht Schuldgefühle.

I: $\mathrm{Hmhm}$

A: Ow! Hätten wir jetzt nicht gestritten hätte er das vielleicht jetzt nicht gemacht."

August, Z681:

"Einerseits äh. Im Rückblick. Was haben wir alles, falsch:, und daneben gemacht. Ungeheure 
Selbstvorwürfe."

Dagmar, Z401:

"A: Oder ja. da ist da hat man auch Schuldgefühle. Vor allem halt auch wegen meiner Tochter."

Rückfälle / Abstürze

09.01.2013 13:26:24

Kategoriendefinition:

Kodierung von folgenden Textstellen:

1. Der suchtkranke Angehörige hatte nach einem Entzug einen Rückfall.

2. Der drogenabhängige Angehörige muss vorzeitig aus der Klinik austreten, weil er gegen Regeln verstossen hat.

3. Angehörige berichten, was für sie ein Rückfall bedeutet hat oder bedeuten würde.

Weiter:

4. Angehörige berichten davon, dass der Angehörige im Verlauf der Suchterkrankung begann weitere Substanzen zu konsumieren, von denen er ebenfalls abhängig wurde.

Kodierregeln:

Einer oder mehrere Aspekte der Kategoriendefinition muss erfüllt sein.

Zudem sind die Kriterien der Überkategorien "Belastungen" ebenfalls erfüllt.

Auschluss: Textstellen, in denen Angehörige davon berichten, dass der Angehörige begann Substanzen (Ritalin, Benzos) zu missbrauchen, die im Rahmen einer Behandlung verschrieben wurden, gehören in der Kategorie "Belastung/erhaltene Unterstützung/negativ/Therapie des Betroffenen" kodiert.

Ankerbeispiele:

Dorette, Z248:

"Und, jaa. Und für mich ist halt auch jedesmal wenn er irgendwie einen Entzug macht. Wenn er dann gerade wieder retour kommt. Und es nicht geklappt hat."

Andrea, Z99-117:

"Aber nach drei Tagen kommt ein Telefon. Ich muss gehen. - Er müsse raus.

I: Okay.

A: Ja und es ist ja schon der zweite; sie bekommen ja immer eine Vorwarnung und das ist dann der zweite Vorfall I: Ja.

A: Und ich habe dann dort auch noch nicht gewusst ob er dann; seit dann weiss man es dann. Oder?

I: hmhm

A: Und dann ist er natürlich weil er natürlich so viel Alkohol hatte;

I: Also ist er wegen dem Alkohol haben sie ihn +raus;

A: Nenein+ wegen; nenein auch wegen konsumieren

I: Okay.

A: von; aber dort weiss ich nicht was er konsumiert hat.

I: Ja.

A: Aber das Ritalin ist ja dort noch nicht das Aktuelle gewesen. Das Ritalin ist ja erst nachher gekommen.

I: hmhm

A: Ich nehme an Coci also oder oder oder vielleicht eben auch genügend Alkohol und //(?:) Das weiss ich jetzt nicht genau.

I: hmhm

A: Stimmt nicht einmal. Es ist nicht wegen dem gewesen. Sondern es ist weil er Sport verweigert hat. Fertig gewesen, weil er den Sport verweigert hat.

I: hmhm

A: Das erste Mal ist es Sport verweigern gewesen."

Albert, Z799-805:

"A: Und ich habe ein bisschen Angst dass wenn sie, wieder ins Leben herauskommt. Eine eigene Wohnung hat. Meine Frau vielleicht wieder am Enkel vermehrt „luegt““. Dass sie wieder vermehrt anfängt

I: +konsumieren.

A: konsumieren.+ Und dann wieder

I: abstürzt.

A: „drinine“ (=in die Drogen hinein) kommt. U:nd - da habe ich ihr persönlich gesagt. Wenn du das machst! Dann will ich dich nicht mehr sehen. Ich weiss nicht ob ich das als Vater ihr sagen darf. Und ob ich das dann ertrage oder nicht. Aber, sie ist sich bewusst dass ich das nicht mehr ertragen würde.

I: hmhm hmhm

A: Wenn sie nochmals abstürzen würde." 
Leiden anderer Angehöriger

07.10.2013 09:14:31

Kategoriendefinition:

Kodierung von Textstellen, in denen Angehörige angeben, dass es sie belastet habe, dass andere Familienmitglieder oder Angehörige unter der Suchterkrankung gelitten hätten.

Kodierregel:

Kriterium der Kategoriendefintion ist erfüllt.

Zudem sind die Kriterien der Überkategorien "Belastungen" und "emotionale Belastung" ebenfalls erfüllt.

Ankerbeispiele:

Dana, Z402-403:

"I: Ähm - Gibt es sonst noch irgendwelche Probleme wo entstanden sind durch die Drogenabhängigkeit von Ihrem Sohn?

A: Mit der Tochter wo natürlich, sehr gelitten hat."

Angst, andere könnten betroffen werden

07.10.2013 09:21:03

Kategoriendefinition:

Kodierung von Textstellen, in denen Angehörige angeben, dass sie Angst hätten, dass

1a) andere Angehörige ebenfalls drogenabhängigen werden könnten.

1b) andere Drittpersonen durch den Kontakt mit dem suchtkranken Angehörigen, drogenabhängigen werden könnten.

Kodierregeln:

Mindestens ein Kriterium der Kategoriendefinition ist erfüllt.

Zudem sind die Kriterien der Überkategorien "Belastungen" und "emotionale Belastung" ebenfalls erfüllt.

Ankerbeispiele:

Dylan, Z960-966:

"A: Jaja. Ja. Eben und mein Bruder halt äh; Ich habe es ihm auch schon gesagt. Oder zum Beispiel meiner Mutter mal gesagt. Ich glaube der *Bruder fängt an weiss auch nicht was zu konsumieren! Er geht mit mit mit dem Papi auf *Stadt1 und so. Was soll der Scheiss?

I: hmhm

A: Oder einmal im Monat. Einmal in einem halben Jahr. Das heisst nichts. Oder? - Aber wenn er „all Wuche“ (= jede Woche) und so geht. Äh! (=abwertend) Das meinst Du nur. Hat es geheissen. Und beim ersten Mal sagt sie noch. Wenn es so ist! Ich zeige ihn an! Und so. ///(?:) Sonst hat sie d- quasi äh verstanden und dann; Wahrscheinlich hat sie selber mit meinem Bruder geredet. Und ab und zu hat er mal konsumiert. Sagt er.

I: hmhm

A: Sie glaubt ihm. Und dann vielleicht noch; Es ist nichts. Oder?

I: Ja.

A: Wobei ich bin mir nicht so sicher. (lacht leicht)"

Vereinsamung / Enttäuschungen des suchtkranken Angehörigen

07.10.2013 09:25:37

Kategoriendefinition:

Kodierung von Textstellen, in denen Angehörige angeben, dass es sie belastete,

1.) dass der suchtkranke Angehörige vereinsamt sei beziehungsweise frühere Freundschaften in die Brüche gegangen seien.

2.) wenn der suchtkranke Angehörige Enttäuschungen erlebe.

\section{Kodierregeln:}

Mindestens ein Kriterium der Kategoriendefintion ist erfüllt.

Zudem sind die Kriterien der Überkategorien "Belastungen" und "emotionale Belastung" ebenfalls erfüllt.

Ankerbeispiele:

Angelika, Z450-454:

"A: Aber eben. Ich habe ihnen am Anfang gesagt dass ich ihm wieder zwanzig Stutz gegeben habe.

I: hmhm

A: Also ich nehme es, ich nehme es als elendiglich wahr. Es tut mich sehr beelenden.

I: hmhm

A: Zu sehen, wie einsam! er! ist. Das, was, was ihm, am Leben abgeht. Das tut mir so weh:. Er ist so, eigentlich ein hübscher Mann. Und, er hä- er hätte sicher Chancen bei den Frauen." 
Doris, Z150-152:

"Super Kollegen. -Auch im Ärztebereich. Die haben sich über ihn haben sich alle auch eingesetzt. Für den *Sohn. Haben sich erkundigt. Was können wir machen? Seid ihr gut Heim gekommen? Also - und irgendwann haben sie einfach dann die Nase voll gehabt (lacht).

I: hmhm

A: Der Kontakt ist abgebrochen."

\section{Notfälle}

07.10.2013 09:57:27

Kategoriendefinition:

Kodierung von folgenden Textstellen:

1. Angehörige berichten, dass sie mehr oder weniger immer in Alarmzustand seien oder gewesen seien, weil ständig irgendwelche Notfälle eingetreten seien.

1a) beispielsweise Telefonanrufe werden als extrem unangenehm erlebt

1b) auf dem Weg nach Hause stellen sich bereits unangenehme Gefühle ein.

2. Angehörige berichten, dass sie wiederholt ihre geplanten Aktivitäten unterbrechen mussten, weil es zu einem Notfall wegen des drogenabhängigen Angehörige gekommen war.

\section{Kodierregeln:}

Eines oder beide Kriterien müssen für die Kodierung der Textstelle unter der Kategorie erfüllt sein.

Zudem sind die Kriterien der Überkategorien "Belastungen" und "emotionale Belastung" ebenfalls erfüllt.

Ankerbeispiele:

Doris, Z459-467:

"A: Man ist immer mal auf Stand-by. Für alle Evento- En- - Eventualitäten! Und, man kann auch nicht einmal mehr, locker in die Ferien.

I: hmhm

A: Man, hat, von jedem Telefon Angst. Weil jedes Telefon ist ein Mist über Jahre gewesen. Jedes! Telefon! ist negativ gewesen.

I: hmhm

A: Diesen Kasten! hätte man in die Ecke schiessen können. Und zeitenweise hätte man gewünscht das gar nicht abheben zu müssen.

I: + hmhm

A: Aber+ mein Mann hat immer gesagt. Du kannst es, musst es, abheben. Musst es, annehmen. Weil dann sonst ist es nur verschoben.

I: hmhm + hmhm

A: Dann+ ist es vielleicht noch schlimmer."

Dana, Z189-191:

"A: Dass ich einfach an einen Punkt geko-; oder mi- ich bin am Abend vom Schaffen nach Hause gekommen. Aus dem Bus gestiegen und Richtung Wohnung gelaufen und schon ein Würgen im Hals gehabt. Weil man weil man nicht weiss. Was einen, wieder erwartet.

I: hmhm hmhm

A: wenn man Heim kommt!"

Ambivalenz

06.10.2013 17:31:56

Kategoriendefinition:

Kodierung von Textstellen, in denen Angehörige über widersprüchliche Gefühle im Zusammenhang mit der Suchterkrankung berichten.

1a. Hoffnung vs. Hoffnungslosigkeit

1b. Unterstützung vs. Abgrenzung

2. Kodierung von Textstellen, in denen Angehörige berichten, dass sie entgegen ihrer eigenen moralischen Grunsätze gehandelt hatten.

Dieser Wechsel der Gefühle wird als sehr belastend erlebt in Form eines Hin- und Hergerissenwerdens.

Kodierregeln:

Eines oder mehrere Kriterien der Kategorie sind erfüllt, damit die Textstelle kodiert wird.

Zudem sind die Kriterien der Überkategorien "Belastungen" und "emotionale Belastung" ebenfalls erfüllt.

Ankerbeispiele:

Dana, Z121: 
"Und äh, ja und dann hat es ja auch Phasen gegeben wo er sich wieder ein wenig zusammengerissen hat. Oder auch ein bisschen normal gewesen ist Daheim. Und - dann hat man wieder Hoffnung gehabt! Oder (lacht leicht) dass es besser ist! Bis einfach wieder äh etwas vorgefallen ist wo einem wieder äh das Gegenteil bestätigt hat."

Angelika, Z254:

"A: Ja:. Wi- wir haben ihm jetzt gesagt du kannst zu uns essen kommen. Jederzeit! Das Essen geben wir dir aber Geld geben wir dir nie mehr! Nie mehr! Aber eben das nie mehr, das ist vierzehn Tage gegangen. (beide lachen) Dann habe ich ihm die zwanzig Stutz wieder gegeben."

Angelika, Z491-494:

"I: Haben sie es nachher, bereut dass sie ihm +die zwanzig Franken gegeben haben?

A: Ja. Ja.+ Ich habe es bereut, ja. (beide lachen). Und zwar nicht wegen den zwanzig Stutz. -

I: Ja ja!

A: Sondern, dass dass ich nicht konsequent gewesen bin."

Ungewissheit

07.10.2013 09:44:12

Kategoriendefinition:

Kodierung folgender Textstellen:

1. Angehörige berichten, dass der drogenabhängige Angehörige für eine bestimmte Zeit verschwunden oder nicht mehr erreichbar war. Diese Ungewissheit über den Verbleib und die aktuelle Situation des suchtkranken Angehörigen ist dabei häufig kombiniert mit einem Gefühl der Angst.

Kodierregeln:

Kriterium der Kategoriendefinition ist erfüllt.

Zudem sind die Kriterien der Überkategorien "Belastungen" und "emotionale Belastung" ebenfalls erfüllt.

Ankerbeispiele:

Andrea, Z93-95:

"Er ist auch nicht mehr schaffen gegangen. Oder dann habe ich ihn wieder eine Woche lang nicht erreicht.

I: hmhm

A: Und das Telefon nicht mehr abgenommen."

Doris, Z196-202:

"A: Also viel. Ja. Schon in die Beratung. Und äh, sie hat mir auch gesagt. Das Schlimmste! für alle ist eben diese Warterei immer.

I: hmhm

A: Kommen sie wieder? Kommen sie nicht? Sind sie ganz abgestürzt? Sind sie gestürzt, finden den Weg wieder Heim?

I: hmhm

A: Leben sie überhaupt noch?

I: hmhm

A: Das ist schlimm."

\section{Abgrenzung}

07.10.2013 09:31:53

Kategoriendefinition:

Kodierung von folgenden Textstellen:

1.) Angehörige geben an, dass die Situation so unerträglich war, dass sie den suchtkranken Angehörigen auf die Strasse stellten oder auf die Strasse stellen wollten. Kodiert werden in diesem Zusammenhang auch Textstellen, in denen Fluchtgedanken der Angehörigen zum Ausdruck kommen.

2.) Angehörige thematisieren die Schwierigkeit, sich von drogenabhängigen Angehörigen abzugrenzen.

3.) Angehörige sprechen die Schwierigkeiten an, die durch räumliche Distanz entstehen.

Kodierregeln:

Mindestens ein Kriterium der Kategoriendefinition ist erfüllt.

Zudem sind die Kriterien der Überkategorien "Belastungen" und "emotionale Belastung" ebenfalls erfüllt.

Ankerbeispiele:

Dana, Z195-197:

"A: Äh ja, und dann ist einfach wenn der Leidensdruck; eben dann hat es; - ist man irgendwann einmal an einem Punkt. Ich kann nicht mehr. Ich will so nicht mehr!;

I: hmhm 
A: Ich halt es nicht mehr aus!"

Albert, Z615-617:

"A: Eben. Aber ich denke - eben wenn die Tochter achtundzwanzig ist. Und, ein eigenes Kind hat. Und immer noch, weiter macht. Da kannst du gar nicht anders.

I: hmhm hmhm

A: Und wenn es dich noch so verreisst. Innerlich +oder."

Dorothea, Z220-224:

"A: Dass; und ja wir haben nie nie gelernt wie dass wir abgrenzen konnten

I: hmhm

A: Oder wie wir einfach mal sagen konnten. Bis hier und nachher Schluss. Oder?

I: hmhm

A: Das haben wir; wir haben keine Ahnung gehabt wie. Also"

\section{Verantwortung}

07.10.2013 10:08:20

Kategoriendefinition:

Kodierung von folgenden Textstellen:

1. Angehörige geben an, dass sie das Gefühl gehabt hätten/haben für das Lösen der Probleme der drogenabhängigen Person verantwortlich zu sein. Dieses Gefühl kann unterschiedlich motiviert sein:

1a) Angehörige geben an, dass andere ihnen diese Verantwortung delegiert hatten/haben.

1b) Angehörige fühlen sich verantwortlich aus sich selbst heraus oder weil sie das Gefühl haben, sonst schaut niemand.

Kodierregel:

Eines oder mehrere Kriterien der Kategoriendefinition sind erfüllt.

Zudem sind die Kriterien der Überkategorien "Belastungen" und "emotionale Belastung" ebenfalls erfüllt.

Ankerbeispiele:

Annabelle, Z253-257:

"A: hmhm Also ähm natürlich haben das seine Eltern gewusst. Weil das ist ja auch dieser Zeit wo er auch im

*Institution und überall dabei gewesen ist.

I: hmhm

A: Und ich denke mir nachdem er ähm aus dieser *Institution1 ausgetreten ist. Bin ich eigentlich so ein bisschen in seiner Verwandtschaft so ein bisschen, als die Retterin!

$\mathrm{I}: \mathrm{hmhm}$

A: angeschaut worden. Ah super! Da kommt *Annabelle. Und jetzt ist alles paletti“."

Dylan, Z430-431:

"I: Also ist irgendwie auch jetzt noch so; Hast Du das Gefühl Du bist auch verantwortlich bis zu einem gewissen Grad?

A: (seufzt)"

\section{Verlust}

04.01.2013 14:13:37

Kategoriendefinition:

Kodierung von folgenden Textstellen:

1. Angst, den suchtkranken Angehörigen durch eine Überdosis zu verlieren.

2. Todesfälle, die Angehörige aufgrund der Suchterkrankung erleben mussten.

3. Verlust des Angehörigen an die Suchterkrankung. Es handelt sich nicht um einen realen Verlust, aber Verlust der Möglichkeiten, die im Angehörigen gesehen würden (insbesondere für Eltern relevant).

\section{Kodierregeln:}

Mindestens ein Kriterium muss für die Kodierung der Textstellen erfüllt sein. Es können aber auch mehrere

Kriterien gleichzeitig erfüllt sein.

Zudem sind die Kriterien der Überkategorien "Belastungen" und "emotionale Belastung" ebenfalls erfüllt.

Ankerbeispiele:

Albert, Z553-557:

"Aber, äh, eben das, wo sie erzählt hat. Dass, dass sie Kokain spritzt.

I: hmhm

A: Einfach der Gedanke dass sie in einer Minute tot sein kann. Wenn das Telefon schellt kannst du ,abneh“ (=abheben) und es heisst 
I: hmhm

A: äh wir haben ihre Tochter tot gefunden oder."

August, Z392-396:

"A: Äh aber im ersten Moment ist man mit einem Todesfall alleine. +Das ist klar.

I: Ja!+ Ja!

A: Aber es hat schon sehr sehr viel gebracht. Und sie haben sich alle Mühe gegeben. Aber es bleibt! halt.

Was soll ich jetzt sagen. Man ist - dort immer wund.

I: hmhm

A: Das ist so eine Stelle wo: nie ganz, verheilt."

\section{Wunsch, dass Angehöriger stirbt}

07.10.2013 10:25:27

Kategoriendefinition:

Kodierung von folgenden Textstellen:

1. Angehörige berichten, dass sie sich zwischendurch gewünscht hatten, dass der drogenabhängige Angehörige stirbt, damit das Leiden endlich ein Ende habe.

Kodierregel:

Kriterium der Kategoriendefinition ist erfüllt.

Zudem sind die Kriterien der Überkategorien "Belastungen" und "emotionale Belastung" ebenfalls erfüllt.

Ankerbeispiele:

Annabelle, Z247-249:

"A: Oder für /(?:). Also es hat wirklich auch Zeiten gegeben wo ich gefunden habe. Also weisst du was? Irgendwie. Fahr du doch am besten irgendwo in eine Brücke rein. Und komm gar nicht mehr heim. So ein bisschen. Ja. Dass ich mir zeitenweise eigentlich wirklich auch gewünscht habe. Dass er sich ähm; auf nimmer Wiedersehen.

I: hmhm Wo Sie sich so belastet gefühlt haben?

A: Denk ich mir. Ja. Dass das die Belastung ist;"

Doris, Z484-488:

"A: Habe ich gedacht. Was soll das. Der soll doch.

I: hmhm

A: Soll; ja! Es hat schon Momente gegeben. Wo ich gedacht habe. Jetzt soll er doch in den +Abgrund.

I: hmhm+

A: Von mir aus. Und ich „mage“ (=kann), jetzt dann nicht mehr."

Persönlichkeitsveränderung

04.01.2013 13:39:08

Kategoriendefinition:

Kodierung von folgenden Textstellen:

1. Drogenabhängiger Angehöriger hat sich aufgrund der Suchterkrankung in seiner Persönlichkeit verändert, indem

1a) Angehörige belogen werden.

1b) Angehörige betrogen werden.

1c) der drogenabhängige Angehörige beginnt gegen moralische und ethische Grundsätze, die er oder sie vorher mit den Angehörigen geteilt hat, zu verstossen

2. Drogenabhängiger Angehöriger ist weniger verlässlich als früher.

2a) Angehörige werden bei vereinbarten Terminen versetzt.

2b) Versprechungen werden nicht eingehalten.

Kodierregel:

Mindestens eines der Kriterien der Kategoriendefinition muss für die Kodierung der Textstelle erfüllt sein.

Zudem sind die Kriterien der Überkategorien "Belastungen" und "emotionale Belastung" ebenfalls erfüllt.

Ankerbeispiele:

Doris, Z220-222:

"A: Ja! Verschiedentlich. Natürlich! Wenn, eben diese Situation eingetroffen ist wie ich vorher vorher erwähnt habe. Dass der *Sohn wieder einmal nicht Heim gekommen ist. Dass er, nicht erschienen ist im Geschäft. Dass das Geschäft angerufen hat. Wo ist: der Sohn?

I: hmhm

A: Er hat Kunden. Wir müssen auch Kundentermine absagen. Und so weiter. Das ist ja, das Letzte vom Letzten. ///(?: A spricht extrem undeutlich) mal so:, zuverlässig gewesen ist. Ein Leben lang! Das ist, einfach unakzeptabel!" 
Dana, Z314-321:

"I: hmhm Was erleben Sie oder haben Sie als besonders belastend erlebt im Zusammenhang mit der Sucht von Ihrem Sohn oder der Drogenabhängigkeit?

A: Das Lügen. (spricht sehr leise)

I: Sein Lügen Ihnen gegenüber?

A: Vertrauensmiss- also dass man nicht mehr Vertrauen kann.

I: hmhm hmhm

A: Weil durch die Drogen das ist; eben man hat keine Achtung mehr. Nicht einmal vor der Mutter.

I: hmhm hmhm

A: Wenn es um das eigene! Ding, - um die Suchtbeschaffung oder äh ja"

Hilflosigkeit / Ohnmacht

04.01.2013 14:11:00

Kategoriendefinition:

Kodierung von folgenden Textstellen:

1. Angaben dazu, dass gegenüber der Suchterkrankung des Angehörigen eine grosse Ohnmacht und Hilflosigkeit empfunden wird.

2. Angaben dazu, dass man nicht weiss, was man machen soll.

\section{Kodierregeln:}

Eines oder mehrere Kriterien der Definition sind erfüllt.

Zudem sind die Kriterien der Überkategorien "Belastungen" und "emotionale Belastung" ebenfalls erfüllt.

Ankerbeispiele:

Dorette, Z202-206:

"aber es oder. Es es, man kann eigentlich nicht helfen.

I: hmhm hmhm Also sie müssen so, das Elend oder, die Krankheit von ihrem Sohn einfach mit ansehen +und

A: Mit an-+ man kann nichts machen. Man kann vielleicht mal etwas sagen. In einem guten Moment. Aber, man weiss genau. Es, es nützt nichts.

I: hmhm hmhm

A: Ich denke das ist scho:n, also es ist nicht immer gleich. Aber manchmal dünkt es einen enorm oder."

August, Z244-248:

"A: Wir haben aber gleichzeitig gewusst dass wir - von Daheim vom Stubentisch aus. Einfach. (stöhnt) Ich sage es jetzt ganz wüst. Verdammt wenig machen kann.

I: hmhm + hmhm

A: Effektiv.+ Er geht am Morgen fort. Weil er ja in die, in der Lehre ist. Auf den Bau. Und ob er dann geht oder wieder nicht geht. - Ja +das ist:; nein. Nichts.

I: Da haben sie keine Macht darüber.+

A: Das ist ganz ganz äh schwierig."

Hoffnungslosigkeit / Resignation

04.01.2013 14:15:03

Kategoriendefinition:

Kodierung von folgenden Textstellen:

1. Resignation / Hoffnungslosigkeit gebenüber der Suchterkrankung des Angehörigen.

2. Angaben dazu, dass sich die Situation nicht mehr verändern (verbessern) wird und man mit der Suchterkrankung leben muss im Sinne einer Resignation.

3. Angaben dazu, dass die Angehörigen nicht mehr wissen, wie es weitergehen soll.

Ausschluss:

Wird im Sinne von einer Akzeptanz und einer veränderten Sichtweise über die Suchterkrankung berichtet, beispielsweise dass man damit auch gut leben kann, dann gehört dies eher zu einer Form der Bewältigung und stellt nicht mehr zwingend eine Belastung dar. Textstellen, in denen eine gewisse Akzeptanz gegenüber der Suchterkrankung zum Ausdruck kommt, werden deshalb nicht unter dieser Kategorie kodiert.

Kodierregeln:

Mindestens eines der Kriterien muss für die Kodierung der Textstelle erfüllt sein.

Zudem sind die Kriterien der Überkategorien "Belastungen" und "emotionale Belastung" ebenfalls erfüllt.

Ankerbeispiele:

Dylan, Z114-122:

"A: Es hört ja nie auf. Oder?

I: Jetzt weisst du dass es halt. 
A: Ja.

I: Dass sie immer krank bleiben werden so?

A: Ja. Ja.

I: Okay.

A: Und jetzt ei- jetzt ist schon so weit dass ich eigentlich schon denke. Dass er, er wird immer krank bleiben. Er wird eines Tages daran sterben.

I: hmhm

A: Oder?"

Dorette, Z202:

"Oder äh und $\mathrm{u}-\mathrm{u}$ - eben besonders! belastend ist einfach das Trostlose. Also ich finde dass, dass es einfach, ja dass es einfach trostlos ist. Es wird immer schlimmer!"

Angelika, Z237-238:

"Haben sie manchmal auch das Gefühl gehabt dass sie, nicht mehr gewusst haben wie es weitergehen soll? A: Ja (im Flüsterton). Habe ich viel gehabt."

Zukunftsängste

04.01.2013 14:21:07

Kategoriendefinition:

Kodierung von folgenden Textstellen:

1. Angaben dazu, dass Angehörige sich Sorgen macht in Bezug auf die Zukunft des suchtkranken Angehörigen.

Diese Sorgen beziehen sich auf:

1a) die berufliche Zukunft

1b) die finanzielle Zukunft

1c) die Zukunft allgemein, wenn die Angehörigen nicht mehr da sein sollten.

Kodierregel:

Mind. eines der Kriterien muss erfüllt sein, damit die Textstelle und der Kategorie kodiert werden kann.

Zudem sind die Kriterien der Überkategorien "Belastungen" und "emotionale Belastung" ebenfalls erfüllt.

Ankerbeispiele:

Anke, Z231-235:

"A: Ich hoffe nicht dass das spätere Folgen hat wenn er alt ist.

I: hmhm

A: Oder weil man sagt. Er ist natürlich nicht verheiratet. Er hat zwar eine Freundin. Wo: geschieden ist und Kinder hat. Aber, ähm sie leben nicht zusammen. Man weiss auch dass Männer wo älter sind und alleinstehend, mehr psychische Probleme haben als andere.

I: hmhm

A: Und das kann natürlich Spätfolgen haben. Ich hoffe nicht dafür dass wir das dann durch,machen. Aber bis dann bin ich nicht mehr da."

Andrea; Z266-272:

"A: Und er müsste eigentlich vom Sozialamt müsste er ja 25 Bewerbungen schreiben. Nein! Das stimmt nicht. 13 Bewerbungen schreiben bis am 25igsten. Aber er sagt. Ich gehe nicht jetzt schaffen $100 \%$ weil ich weiss ich schaffe das nicht.

I: hmhm

A: Und auf seinem Beruf sowieso nicht. Weil er ist *Beruf.

I: hmhm

A: Und wenn er natürlich jetzt schon wieder äh wie sagt man äh, Bewerbungen rauslässt im ganzen *Ort und und und es funktioniert einfach grad gar nicht.

I: hmhm

A: Also er hat ja dann eigentlich; und wenn er dann wenn es ihm dann wirklich wieder gut gehen würde. Wenn er wirklich schaffen könnte! hat er einen schlechten Ruf."

\section{Gesundheitssorgen}

04.01.2013 14:26:24

Kategoriendefinition:

Gemäss Kirby et al. (2005) werden folgende Textstellen in der Kategorie kodiert:

1. Drogenabhängige Person erfährt Gewalt beispielsweise auf der Gasse und wird verletzt.

2. Sorgen wegen sexuell und i.v. übertragbaren Krankheiten, die die suchtkranke Person bekommen könnte oder die der Angehörige vom Suchtkranken bekommen könnte. 
Kodierung von weiteren Textstellen, die nicht von Kirby et al. definiert wurden:

3. Gesundheitliche Probleme der Angehörigen, die gemäss den Angehörigen mit der Suchterkrankung assoziert ist (bspw. depressive Störungen, Schlafstörungen, Angststörungen, Verschlechterung einer chronischen Krankheit etc.)

4. Kodierung von Textstellen, in denen Angehörige angeben, dass sie keine gesundheitlichen Probleme bekommen hätten.

Kodierregeln:

Mindestes eines der Kriterien der Kategoriendefinition ist erfüllt.

Zudem sind die Kriterien der Überkategorien "Belastungen" ebenfalls erfüllt.

Ankerbeispiele:

Anke, Z40:

"Weil ich habe dann kaum mehr geschlafen."

Dana, Z171-175:

"A: Also das hat ja auch angefangen. Ich konnte nicht mehr schlafen. Oder ich bin wach geblieben um ihn, um zu schauen ob er eben wieder abhaut.

I: hmhm

A: Äh oder wann er wieder heim kommt. Ich: bin dann auch äh, man schläft nicht mehr gut weil man denkt äh. Passiert etwas.

I: hmhm

A: Äh oder wird er von der Polizei äh gefilzt? Oder äh stellt er etwas an? Macht er Einbrüche?"

August, Z565-571:

"A: Hat er so ein Herpesvirus gewesen. Wo bis ins Hirn.

I: Oje. Oje.

A: Hat ,äfäng“ (=mittlerweile) in sehr äh desolaten Verhältnissen auch gelebt. Und, et cetera.

I: hmhm

A: Hirnhautentzündung. Hirnentzündung.

I: Ja.

A: Also schon nicht gerade eine: schöne Geschichte."

\section{Gewalt und Drohungen}

04.01.2013 14:34:44

Kategoriendefinition:

Gemäss Kirby et al. (2005) Kodierung von folgenden Textstellen:

1. Drogenabhängige Person spricht Drohungen gegenüber von Angehörigen aus. Dazu gehören auch subtilere Formen wie beispielsweise das Induzieren von Schuldgefühlen.

2. Körperliche Gewalt zwischen den Angehörigen und der suchtkranken Person.

Weitere, die allenfalls relevant sein könnten:

3. Drohungen/Gewalt, die der suchtkranke Angehörige erfährt, weil er in kriminelle Geschäfte verwickelt ist.

4. Kodierung von Textstellen, in denen darüber diskutiert wird, dass gewisse Substanzen aggressiver machen als andere.

Kodierregeln:

Mind. eines der Kriterien der Kategoriendefinition muss erfüllt sein.

Zudem sind die Kriterien der Überkategorien "Belastungen" ebenfalls erfüllt.

\section{Ankerbeispiele:}

Dagmar, Z185:

"A: Aber das ist das einzige Mal gewesen wo er mich geschlagen hat. Aber sonst haben wir halt wirklich einfach; Wir sind; ja, so in einer Spirale gefangen gewesen."

Dagmar, Z361-363:

"Und ich habe immer wieder gesagt. Geh geh. Ich meine wir sind immer wieder aneinander geraten.

I: hmhm

A: Ich meine eben äh er ist Borderliner. Also sobald er gemerkt hat es ist wirklich ernst hat er natürlich Telefonterror gemacht. Hat Telefonterror gemacht. Ins ins Geschäft auf meine Natel. Überall hin."

Darina, Z48-50:

"Und „dernah“ (=je nachdem) in einem in einem Ton wo (lacht leicht) ähm ja. Wo nicht so angenehm gewesen ist.

I: Also ford+ernd?

A: Also+ wie. Ähm ich muss jetzt CHF 20.00 haben ähm. Ja. wirklich fordernd! Und"

Dorrette, Z172-174:

"Ja das ist; und er; ich muss halt natürlich sagen. Wir, ich habe natürlich immer gesagt. Also lieber Drogen als als Alkohol. 
Weil Alkohol machen ihn aggre- also macht ihn aggressiv.

I: hmhm

A: Ist er enorm aggressiv. Und, jaa. Schlägt, dann, auch sonst irgendwo drein. Also. Eben."

\section{Rechtliche Probleme}

04.01.2013 14:41:45

Kategoriendefinition:

Gemäss Kirby et al. (2005) werden folgende Textstellen unter der Kategorie kodiert:

1. Angaben dazu, dass Hausdurchsuchungen der Polizei in der Wohnung, die mit der suchtkranken Person geteilt wird/wurde, stattgefunden haben.

2. Festnahme der drogenabhängigen Person durch die Polizei und Besuche im Gefängnis.

3. Sorgen, weil der drogenabhängige Angehörige auf Bewährung ist.

Zusätzlich in dieser Kategorie:

4. Der Suchtkranke ist in kriminelle Geschäfte verwickelt, von denen die Angehörigen wissen oder die sie vermuten, die aber bisher noch zu keiner Anzeige geführt haben.

Kodierregeln:

Mindestens eines der Kriterien der Kategoriendefinition ist erfüllt.

Zudem sind die Kriterien der Überkategorien "Belastungen" ebenfalls erfüllt.

Ausschluss:

Vorsicht bei der Abgrenzung von Gewalt und Drohungen, die bei kriminellen Geschäften ebenfalls eine Rolle spielen können.

Ankerbeispiele:

August, Z1025-1031:

"A: Ja. Oder eine Hausdurchsuchung habe ich auch einmal; heute im Nach- heute würde ich mich wehren. Würde ich sagen äh. Der lebt schon so lange nicht mehr da. Ich lasse es nicht zu. - Rechtsanwalt daher.

I: hmhm

A: So ja. Also man hat schon noch

I: Also wo bei ihnen +ist die Wohnung durchsuchen gekommen?

A: Ja ja.+ Weil er doch ab und zu noch bei uns; letzte Adresse. - Jä ist auch wieder so ein Staatsanwalt gewesen. Aus *Ort01. Wo da wahrscheinlich äh das Gefühl gehabt hat er müsse jetzt da, einfach Wohnungen inspizieren.

I: hmhm hmhm

A: Jaa. Also es gibt schon auch rechtliche, Geschichten."

Dorrette, Z98-100:

"er hat ja dann gedealt. Also Klein- Kleindealer. Aber halt doch viel. Ich habe auch dann; genau! Ich habe ja dann „säb“ (=das) schon auch gesehen. Und irgendwie Nachbarn haben mich dann da, angezeigt.

I: Ah! Nachbarn haben sie +angezeigt?

A: Ich nehme+ es an. Ja. Weil sie haben ihn dann auch beobachtet. Von ihren ihren Wohnungen aus. Also ich vermute, es muss es müssen Nachbarn gewesen sein. Ich weiss nicht wer. Das ist auch gleich."

\section{Beeinträchtigung des Lebensalltages}

04.01.2013 14:51:38

Kategoriendefinition:

Gemäss Kirby et al. (2005) gehören folgende Textstellen in diese Kategorie:

1. Aufnahme der suchtkranken Person in den eigenen Haushalt bei Wohnungsverlust oder fehlende Möglichkeiten, den Angehörigen auf die Strasse zu stellen.

2. Belastung durch den Drogenkonsum der suchtkranken Person im Haushalt.

3. Übernehmen von Verpflichtungen und Aufgaben im Haushalt von der drogenabhängigen Person.

4. Beeinträchtigung des täglichen Lebens durch die Suchterkrankung des Angehörigen. Dies kann verschiedene Ursachen haben:

a) Angehörige können keinen Besuch mehr mit nach Haus bringen, weil die Wohnung häufig in einem unordentlichen Zustand ist aufgrund der Suchterkrankung.

b) Der suchtkranke Angehörige bringt fremde Menschen mit in die gemeinsame Wohnung.

\section{Kodierregeln:}

Mindestens ein Kriterium der Kategoriendefinition muss erfüllt sein.

Zudem sind die Kriterien der Überkategorien "Belastungen" ebenfalls erfüllt.

Ankerbeispiele:

Dana, Z185-187:

"A: Und äh am Morgen wenn ich aufgestanden bin konnte ich zuerst einmal Bergen von Geschirr abwaschen. Weil wenn er dann heim gekommen ist hat er ein Hungerast gehabt und äh - Zeugs zusammengekocht äh wo ja nicht zusammengepasst 
hat.

I: hmhm

A: Auch das hat mich dann, ja;"

Dana, Z193:

"A: Äh dann eben, ich hä- ich konnte auch keine Leute mehr nach Hause nehmen weil ich nicht wusste wie die Wohnung wieder aussieht. Er hat Kollegen nach Hause genommen während ich am schaffen gewesen bin. Wo man nicht gewusst hat äh was für Leute dass das sind. Also man ist sich manchmal noch fremd in der eigenen, Wohnung vorgekommen."

Doris, Z469:

"A: Und darum haben wir ja auch die Nummer gewechselt. - Weil die Dealer haben ja angerufen! Kaum ist Zahltag gewesen haben wir Nächte lang „Telefon“ (=Telefonanrufe) gehabt. Komm! Wir haben eine Party! Und, was weiss ich. Das ist nicht gegangen!"

Dorrette, Z408:

"A: er nimmt auch, in gewissen Sachen; also nicht be-; eben. Rauchen. Das tut er. Es ist alles dreckig. Also ich habe alles, überall Löcher oder. Also die Tische sind ver- oder verlöchert. Also eben. Nur schon wegen dem kann ich niemanden einladen."

\section{Finanzielle Belastung}

04.01.2013 14:56:06

Kategoriendefinition:

In der Kategorie werden diejenigen Textstellen kodiert, in denen angegeben wird, welche Kosten den Angehörigen durch die Drogensucht entstanden sind. Folgende beiden Kategorien werden unterschieden:

- Diebstahl

- Lebensunterhalt

Kodierregeln:

Kodierung der Textstellen in den Unterkategorien.

Lebensunterhalt

04.01.2013 14:58:01

Kategoriendefinition:

Kodierung von folgenden Textstellen:

1. Angaben dazu, dass Schulden/Bussen etc. für die drogenabhängige Person übernommen wurden oder werden mussten.

2. Angaben dazu, dass Angehörige für den Lebensunterhalt (Ausgaben) der drogenabhängigen Person freiwillig aufkommen oder gezwungen werden aufzukommen. Dazu gehört auch das Finanzieren des Drogenkonsums.

3. Aufkommen für Therapien und Behandlungen.

4. Angaben dazu, wieviel Geld die Angehörigen bereits in die Suchterkrankung investiert haben.

Kodierregeln:

Mindestens ein Kriterium der Kategoriendefinition muss erfüllt sein.

Zudem sind die Kriterien der Überkategorien "Belastungen" und "Finanzielle Belastung" ebenfalls erfüllt.

Ankerbeispiele:

Andrea, Z83:

"A: Also ich habe dann immer eben ich habe ihm dann wieder Geld zugeschoben. Dass seine Schulden nicht extrem zunehmen."

Doris, Z560-566:

A: Aber sie haben ihm dann sehr ein kulantes Angebot gemacht. Dass er also das, über eine längere Distanz hat zurückzahlen können.

I: hmhm

A: Aber das nützt im Fall auch nichts.

I: Also da ha- haben sie dann auch wieder bezahlt am Schluss. Oder, hat er das dann selber +retour gezahlt.

A: Er hat es dann+ selber. Also wir haben an die 200'000 Franken gezahlt.

I: hmhm

A: Hätten wir auch sehr gerne für uns gehabt."

Diebstahl

04.01.2013 15:14:59

Kategoriendefinition:

Kodierung von folgenden Textstellen: 
1. Angaben dazu, dass die Angehörigen durch die drogenabhängige Person bestohlen wurden.

2. Angaben, dass Angehörige Angst hatten, vom drogenabhängigen Angehörigen bestohlen zu werden.

3. Angaben dazu, dass Angehörige nie bestohlen wurden.

\section{Kodierregel:}

Mindestens ein Kriterium erfüllt.

Zudem sind die Kriterien der Überkategorien "Belastungen" und "Finanzielle Belastung" ebenfalls erfüllt.

Ankerbeispiele:

Dana, Z379:

"A: Wenn man noch den Schmuck nimmt den er noch verhöckered hat, sind es schon ein paar Tausend Franken."

Doris, Z583-588:

"I: Hat er sie mal bestohlen?

A: Wie?

I: Dass er, dass er sie bestohlen +hätte.

A: Nein.+ Das hat er +nicht.

I: Das hat+ er nie gemacht.

A: hmhm-"

Andrea, Z394-396:

"A: Ja. Ja. Das ist so gewesen. Und dann hat er natürlich /(?:damals) den Schlüssel noch gehabt von der Wohnung. Dann ist natürlich das auch wieder oder?

I: hmhm

A: Ow was ge-; kommt „ächt“ jetzt noch etwas? Es ist ja nie passiert. Aber mit der Zeit fängst du dir dann eben; wo er dann nachher mit Heroin und so extrem geworden ist. Habe ich dann auch Angst gehabt. Ja. Plötzlich ist der Schmuck noch weg. Oder?" 
F1. Tabellenverzeichnis

Tab. 1: Angaben zu den Rückmeldungen und der Rekrutierung aus den Rekrutierungsquellen

Tab. 2: Soziodemographische Daten zu den Angehörigen $(n=88)$

Tab. 3: Charakteristika der drogenabhängigen Angehörigen basierend auf den Angaben der Studienteilnehmenden

Tab. 4: Schätzung der Reliabilität mittels interner Konsistenz

Tab. 5: Depressivität in der Stichprobe

Tab. 6: Zero-Order-Vergleiche verschiedner Variablen in Bezug auf den BDI-V Score

Tab. 7: Multiples lineares Regressionsmodell: BDI-V (AV) und relevante Belastungsfaktoren (UV)

Tab. 8: Signifikante Ergebnisse der zero-order-Korrelationen zwischen BDI-V Score und SVF120

Tab. 9: Multiples lineares Regressionsmodell: BDI-V (AV) und relevante Faktoren der Bewältigung (UV)

Tab. 10: Zuordnungsübersicht für die letzten 10 der 86 Zuordnungsschritte

Tab. 11: Anzahl Probanden pro Cluster (5)

Tab. 12: Anzahl Probanden pro Cluster (3)

Tab. 13: Anzahl Probanden pro Cluster (2)

Tab. 14: Kennwerte der Diskriminanzfunktion

Tab. 15: Klassifizierung der Angehörigen basierend auf der Diskriminanzfunktion

Tab. 16: Kreuztabelle Zwei-Clusterlösung und BDI-V Cut-off

Tab. 17: Struktur-Matrix der Diskriminanzfunktion

Tab. 18: Signifikante Ergebnisse der t-Tests für unabhängige Stichproben für die Clusterlösung Tab. 19: Logistische Regressionsanalyse

Tab. 20: Klassifizierungstabelle

Tab. 21: Verhältnis der qualitativen und quantitativen Daten

Tab. 22: Relevante Belastungsfaktoren der Angehörigen von drogenabhängigen Menschen 
F2: Abbildungsverzeichnis

Abb. 1: Drei Hauptpositionen und Bewältigungsstrategien von Angehörigen gemäss KläuslerSenn \& Stohler (2012, S. 7) modifiziert nach Orford et al. (2010)

Abb. 2: Stress-Strain-Coping-Support (SSCS) Modell modifiziert nach Orford et al. (2010)

Abb. 3: G*Power: Multiples lineares Regressionsmodell, Anzahl der Prädiktoren $=5, \alpha=.05$, Power $=.80$

Abb. 4: Ablaufmodell qualitative Inhaltsanalyse Dissertationsprojekt „Angehörige von drogenabhängigen Menschen“

Abb. 5: Screeplot für die 16 Eigenwerte

Abb. 6: Streuung der BDI-V Scores in den Gruppen von Angehörigen bezüglich ihrer Beziehung zum suchtkranken Angehörigen

Abb. 7: Prüfung auf Linearität und Homoskadastizität mittels Streudiagramm mit BDI-V als abhängige Variable

Abb. 8: Prüfung auf Linearität und Homoskadastizität mittels Streudiagramm mit BDI-V als abhängige Variable

Abb. 9: Struktogramm des Ward-Verfahrens

Abb. 10: Boxplot zum BDI-V Score der stark und weniger stark belasteten Angehörigen Abb. 11: Boxplot zum ZBI Score der stark und weniger stark belasteten Angehörigen Abb. 12: : BDI-Score der mittels halbstrukturiertem Interview befragten Studienteilnehmenden 


\section{Lebenslauf Lea Anna Ruckstuhl}

Geburtsdatum

Zivilstand

Heimatort
14.01.1979

ledig

Pfaffnau/LU

\section{Ausbildung}

2011 - 2014 Strukturiertes Doktoratsprogramm am Psychologischen Institut der Universität Zürich

2003 - 2010 Lizentiatsstudium an der Universität Zürich

Hauptfach: Psychologie

I. Nebenfach: Psychopathologie des Erwachsenenalters

II. Nebenfach:Englische Literaturwissenschaft

2001 - 2003 Vorbereitung für die Eidg. Matura Typus E (Wirtschaft) an der AKAD in ZürichOerlikon

1996 - 1999 Lehre als Kaufmännische Angestellte bei der Neuen Aargauer Bank

\section{Beruflicher Werdegang}

2013 - heute Beraterin/Therapeutin (80\%), Beratungsstelle für Alkoholprobleme Bezirk Hinwil (BAH)

2011 - 2013 Wissenschaftliche Mitarbeiterin (50 \%), Psychiatrische Universitätsklinik Zürich, Forschungsgruppe Substanzstörungen

2004 - 2011 Kaufmännische Angestellte (40 - 60 \%), Neue Aargauer Bank

2003 - 2004 Kaufmännische Angestellte (40 \%), VZ VermögensZentrum Zürich

1999 - 2003 Kaufmännische Angestellte (20 - $100 \%)$, Neue Aargauer Bank

\section{Praktika}

Sept. - Dez. 2008 Forschungspraktikum, Universität Zürich, Lehrstuhl für Klinische Psychologie, Psychotherapie und Psychoanalyse

März - Juni 2008 Psychologiepraktikum (100 \%), Psychiatrische Universitätsklinik Zürich, Psychologischer Dienst 